Forschungszentrum Jülich

Institut für Festkörperforschung

The Jig-saw Puzzle of Crystal Structures:

Alloys, Superconducting Oxides, Semiconductors, Ionic Conductors, Surface Adsorbates and Magnetic Structures

Jürgen Hauck Klaus Mika 


\section{The Jig-saw Puzzle of Crystal Structures: Alloys, Superconducting Oxides, Semiconductors, Ionic Conductors, Surface Adsorbates and Magnetic Structures}

Jürgen Hauck Klaus Mika 
Berichte des Forschungszentrums Jülich ; 3732 ISSN 0944-2952

Institut fürFestkörperforschung Jül-3732

Zu beziehen durch: Forschungszentrum Jülich $\mathrm{GmbH} \cdot$ Zentralbibliothek D-52425 Jülich - Bundesrepublik Deutschland 02461/61-6102-Telefax: 02461/61-6103-e-mail: zb-publikation@fz-juelich.de 
The number of essentially different kinds of constituents in a crystal tends to be small (Pauling's rule of parsimony, 1929) 



\section{Abstract:}

The crystal structures of ordered bcc, fcc or primitive cubic alloys $A_{x} B_{y}$ and related $\mathrm{NaCl}, \mathrm{ZnS}$ or $\mathrm{CaF}_{2}$ derivative structures are characterized by the self-coordination numbers $T_{1}, T_{2}$ of the $\mathrm{A}$ atoms with $\mathrm{A}$ atoms. Structures with identical $T_{1}, T_{2}$ values for all $\mathrm{A}$ atoms are at the corners of $T_{1}, T_{2}$ structure maps and can be analyzed for attractive or repulsive interactions of $\mathrm{A}$ atoms. Most observed structures are at the borders of the structure map and can be obtained by different combinations of structural units. The combination mechanisms explain e.g. the shear structures of $\mathrm{CuAu}$ II or $\mathrm{Nb}_{2} \mathrm{O}_{5}$ and the occurrence of vacancies in $\mathrm{NaCl}$ related structures like $\mathrm{NbO}$. Many other ordered structures like magnetic ordering, adatoms on metal surfaces, colloids and clusters are analyzed by the same method. The very large numbers of possible structures are reduced to a smaller number of structures at the borders of the structure maps, which are stabilized by enthalpy. The other structures are stabilized by entropy. The $T_{1}, T_{2}$ values of DNA sequences in different areas of the structure map are related with the evolution. 



\section{Table of Contents}

1 Introduction 1

2 Analysis of stacking sequences by the one-dimensional Ising 4 model

3 Alloy formation

4 Ordering of atoms in hexagonal and square layers $\quad 15$

5 Hexagonal close-packed structures $\quad 19$

6 Cubic close-packed structures 25

7 Ordered structures of complex close-packed alloys 31

8 Structures with identical powder patterns 32

9 Homologous series of structures $\quad 34$

10 Symmetry of ordered phases 36

11 Ising model 38

12 Characterization of structures by sequences of structural units 41

13 Disordered alloys $\quad 42$

14 Ordered ternary and quaternary compounds 46

15 Enhanced covalent bonding in layered compounds and ordered 48 body-centered alloys

16 Enhanced repulsive interactions 53

17 Valence compounds $\quad 54$

18 Occupation of interstitial sites $\quad 57$

19 Bonding in interstitial alloys $\quad 73$

20 Magnetic interactions $\quad 79$

21 Ordering of I atoms on metal surfaces $\quad 82$

22 Related groups of structures $\quad 85$

23 Distorted structures $\quad 90$

24 Physical and chemical properties of structural units 94

25 Homogeneous sphere packings $\quad 98$

26 Colloids, clusters and DNA 100

27 Structure and interactions of atomic nuclei 107

28 Classification of symmetrical patterns 108

29 Conclusions $\quad 115$

30 Notation 130

31 References 132 
32 Appendix A: Relative ordering numbers, valence electrons, 170 pseudopotential radii and coordination numbers of the elements

33 Appendix B: Reduced unit cells of the hcp, ccp, bcc and pc 174 lattice, structures with a single set of $T_{i}$ values, group-subgroup relations, magic numbers of isotopes and clusters, structures $\mathrm{R}_{n} \mathrm{M}_{\mathrm{x}}$ with invariant $\mathrm{M}$ positions 


\section{Introduction}

Many physical properties like the conductivity of electrons or ions, superconductivity, mechanical strength or thermal properties can be varied in solid solutions. At decreased temperatures the different atoms of the solid solutions are ordered. The variety of materials showing ordering extends from substitutional alloys like $\mathrm{AuCu}_{3}$ to interstitial alloys like $\mathrm{Nb}_{4} \mathrm{C}_{3}, \mathrm{AlFe}_{3} \mathrm{C}$ and $\mathrm{NbO}$ or semiconducting materials like CdIn $\mathrm{Se}_{4}$ or non-metallic compounds like $\mathrm{CaTiO}_{3}$. The $\mathrm{Au}$ atoms of $\mathrm{AuCu}_{3}$ which are distributed randomly on all positions of the face-centered cubic (fcc) $\mathrm{Cu}$ structure above $\approx 390^{\circ} \mathrm{C}$ order at decreased temperatures in such a way that the corners of the cube are occupied by Au atoms and the three face centers by $\mathrm{Cu}$ atoms. The same ordering is obtained for the $\mathrm{NaCl}$ related structure of $\mathrm{Nb}_{4} \mathrm{C}_{3} \widehat{=} \mathrm{Nb}_{4} \square \mathrm{C}_{3}$ with $\mathrm{Nb}$ on $\mathrm{Na}$ positions and an ordering of vacancies $\square$ and $\mathrm{C}$ atoms like $\mathrm{Au}$ and $\mathrm{Cu}$ in $\mathrm{AuCu}_{3}$. A second fcc sublattice containing non-metal atoms is shifted by $a / 2 a / 2$ $a / 2$ in $\mathrm{AlFe}_{3} \mathrm{C} \widehat{=} \mathrm{AlFe}_{3} \mathrm{C}_{3}, \mathrm{NbO} \widehat{=} \square \mathrm{Nb}_{3} \square \mathrm{O}_{3}, \mathrm{CaTiO}_{3} \widehat{=} \mathrm{CaO}_{3} \mathrm{Ti}_{3}$ or $a / 4 a / 4 a / 4$ in $\mathrm{CdIn}_{2} \mathrm{Se}_{4} \widehat{=} \square\left(\mathrm{CdIn}_{2}\right) \mathrm{Se}_{4}$.

The ordered phases show different physical properties like superconducting $\mathrm{NbO}$ or $\mathrm{Ba}(\mathrm{Bi}, \mathrm{Pb}) \mathrm{O}_{3}\left(\mathrm{CaTiO}_{3}\right.$ structure $)$, semiconducting $\mathrm{CdIn}_{2} \mathrm{Se}_{4}$ with interesting properties for solar cells or carbides like $\mathrm{Nb}_{4} \mathrm{C}_{3}$ or $\mathrm{AlFe}_{3} \mathrm{C}$ which are used as abrasives or high temperature materials. Many materials with practical relevance are tailored to achieve the desired ordering by the proper substitution.

The ordering of atoms like $\mathrm{Au}$ in $\mathrm{AuCu}_{3}$ or $\mathrm{Ca}$ in $\mathrm{CaTiO}_{3}$ is related to the interactions between the atoms in the present investigation, to figure out the selection of few structures. The number of observed structures is small compared to the number of all possible structures, which is $2^{n-1}$ for a unit cell with $n$ possible positions of $\mathrm{A}$ and $\mathrm{B}$ atoms.

All reduced unit cells of the fcc, bcc (body-centered cubic), pc (primitive cubic) and other lattices with $n \leq 9$ atom positions were determined and the different structures selected numerically in the present investigation. The theoretical structures can be sorted for different aspects like high 
symmetry, single coordination numbers or the extent to which Pauling's rules are obeyed (Pauling, 1929). Pauling's rule of parsimony is particularly useful for the selection: "The number of essentially different kinds of constituents in a crystal tends to be small. The polyhedra circumscribed about all chemically identical atoms should, if possible, be chemically similar, and similar in the nature of the sharing of corners, edges and faces with other polyhedra". This explanation is similar to the Wiener-Sohncke principle: "Points are disposed around each point in the same way as around every other" (Wiener, 1863; Sohncke, 1879; Brunner, 1971). We have selected the self-coordination numbers $T_{i}$ of $\mathrm{A}$ atoms with $\mathrm{A}$ atoms as parameters. In the fcc lattice each $\mathrm{Cu}$ atom with diameter $d$ for example has $T_{1}=12, T_{2}=6$ and $T_{3}=24$ nearest, next-nearest and third neighbors at distances $R=d, \sqrt{2} d$ and $\sqrt{3} d$. The $T_{i}$ values are reduced in ordered alloys like $\mathrm{AuCu}_{3}$ to $T_{1}=0, T_{2}=6, T_{3}$ $=0$ for $\mathrm{Au}$ atoms. This structure is characterized by the $T_{i}$ values of the minority component and the ratio $\mathrm{r}=\mathrm{y} / \mathrm{x} \geq 1$ of $\mathrm{A}_{\mathrm{x}} \mathrm{B}_{\mathrm{y}}$, e.g. $060 ; 3$ for $\mathrm{AuCu}_{3}$. The structures of $\mathrm{Nb}_{4} \mathrm{C}_{3}, \mathrm{AlFe}_{3} \mathrm{C}, \mathrm{NbO}, \mathrm{CaTiO}_{3}$ and $\mathrm{CdIn}_{2} \mathrm{Se}_{4}$ are characterized by the same self-coordination numbers of the minority component and the translation of both fcc lattices $a / 2 a / 2 a / 2$ or $a / 4$ $a / 4 a / 4$.

The $T_{i}$ values can be plotted in two-dimensional $T_{1}, T_{2}$ structure maps. All possible structures are in a range which is limited by straight lines. Most observed structures like $060 ; 3$ for $\mathrm{AuCu}_{3}$ are at a corner at the end of a straight line. Some other structures like $\mathrm{ZrAl}_{3}$ with the selfcoordination numbers $054 ; 3$ of $\mathrm{Zr}$ atoms (Section 6) are on the line $T_{1}=0$. This structure, which was determined by Brauer (1939), can be assembled from structural elements of the $\mathrm{AuCu}_{3}$ structure $(060 ; 3)$ and the $\mathrm{TiAl}_{3}$ structure $(048 ; 3)$ at the corners of the structure map in a similar way as the architecture of Ruddlesden-Popper phases from $\mathrm{CaTiO}_{3}$ and $\mathrm{NaCl}$ structural elements (Section 18). The structures at the borders of the structure map were projected in different directions to determine the structural units, which are assembled similar to a jig-saw puzzle. Some structural elements are shown in the compilations of Schubert (1964), Sato and Toth (1965), Ogawa (1974), Pearson (1967/1972), Wells (1984), Hyde and Andersson (1989) and Parthé et al. (1993). All structures on a border of the structure map with different numbers of 
structural units form a family of structures, which is named by the authors of the first observed structure like the Ruddlesden-Popper phases. About 5-30 structural units were determined for the ordered structures of the different lattices like fcc, bcc, pc, etc., which is a small number compared to the several million possible structures. The interactions between $A$ can be related with the $T_{i}$ values and vary gradually along a line of the structure map as the number of structural units is varied. The $\mathrm{Au}$ atoms of $\mathrm{AuCu}_{3}$ for example are as far apart as possible for an $\mathrm{AB}_{3}$ alloy with $A$ and $B$ atoms on an fcc lattice indicating repulsive interactions. Structures containing chains or planes of A atoms with attractive interactions are obtained at $T_{1} \neq 0$ and minimum $T_{2}$ values. Some of these structures were also obtained using different Ising methods like Monte Carlo simulations by assuming different interactions between A and $B$ atoms (Section 11) or by the investigation of homogeneous sphere packing (Section 25).

The combination of structural units similar to a jig-saw puzzle can be useful for the search of new compounds with different physical properties and supports the analysis of these compounds by electron diffraction for example. Only few structural units fit the frame of the jig-saw puzzle, which can be determined by electron diffraction.

The interactions between atoms are related to different atomic radii, electronegativity and number of electrons in a very complex manner (Schubert, 1964/1967; Sato and Toth, 1965; Ogawa, 1974; Villars and Hulliger, 1987; Villars et al., 1989; Carlsson and Meschter, 1994; Ellner and Predel, 1994; Villars, 1994; Pettifor, 1994). These parameters or relative ordering numbers (Section 32 ) give rise to another type of structure map with sections, where the $\mathrm{AuCu}_{3}$ or $\mathrm{CaTiO}_{3}$ structures for example can be obtained. 


\section{Analysis of stacking sequences by the one-dimen- sional Ising model}

The use of self-coordination numbers $T_{i}$ and of $T_{1}, T_{2}$ structure maps can be demonstrated for a linear chain of $M$ and $N$ atoms or sequences of bases adenine, cytosine, guanine and thymine in DNA (Section 26). Close-packed layers with a sequence of $M$ and $N$ layers can be analyzed by the one-dimensional Ising model (Section 15). The different stacking of close-packed layers, $\mathrm{c}$ or $\mathrm{h}$ gives rise for a linear chain of stacking symbols for a single element $M$ as will be outlined in this section.

In pure metals, the bonding tendencies of the individual atoms should be identical. The same environment can be expected for each metal atom as e.g. strong directional covalent bonds as in gray $\alpha$-Sn with the diamond structure or a close-packing of spherical metal atoms as in $\mathrm{Mg}$ or $\mathrm{Cu}$. Metal atoms with weak bonding behave like tennis balls packed in a basket. The packing consists of coplanar hexagonal layers (Fig.1). Succesive layers are stacked with centers of spheres at A, B or $\mathrm{C}$ positions. The different stacking sequences of hexagonal layers can be characterized by the sequence of positions. The periodic sequences $A B$ of two layers and ABC of three layers are called hexagonal close-packed (hcp or h) and cubic close-packed (ccp or c), because of the hexagonal and cubic symmetry, respectively. The hcp and ccp metal structures are usually distinguished by the prototype elements $\mathrm{Mg}$ and $\mathrm{Cu}$, the space group and Pearson symbols (Table 1). Most metals (except Eu, U and $\mathrm{Np}$ ) can be obtained in a close-packed modification (Villars and Calvert, 1986). A few metals crystallize in more complex close-packed structures with a maximum of nine layers (Table 1). These structures can be described unambiguously by the stacking symbols $\mathrm{h}$ and $\mathrm{c}$ introduced by Pauling and Jagodzinski (Pearson, 1972; Mardix, 1990). This notation describes a layer as $\mathrm{h}$ or $\mathrm{c}$ type according to whether the layers on each side of it are of the same type, such as for $B$ in $A B A$, or of different types as for $B$ in $A B C$ (Fig.1). The two-layer hcp sequence $A B$ gets the symbol $h \mathrm{~h}$ or $\mathrm{h}$, the three-layer ccp sequence $\mathrm{ABC}$ the symbol ccc or $\mathrm{c}$. The lanthanides $\mathrm{La}$ to $\mathrm{Sm}, \mathrm{Gd}$ and the actinides Am to Cf crystallize in the $\mathrm{ABAC}$ or chch $=(\mathrm{ch})_{2}$ stacking. Sm, Gd and the heavier lan- 


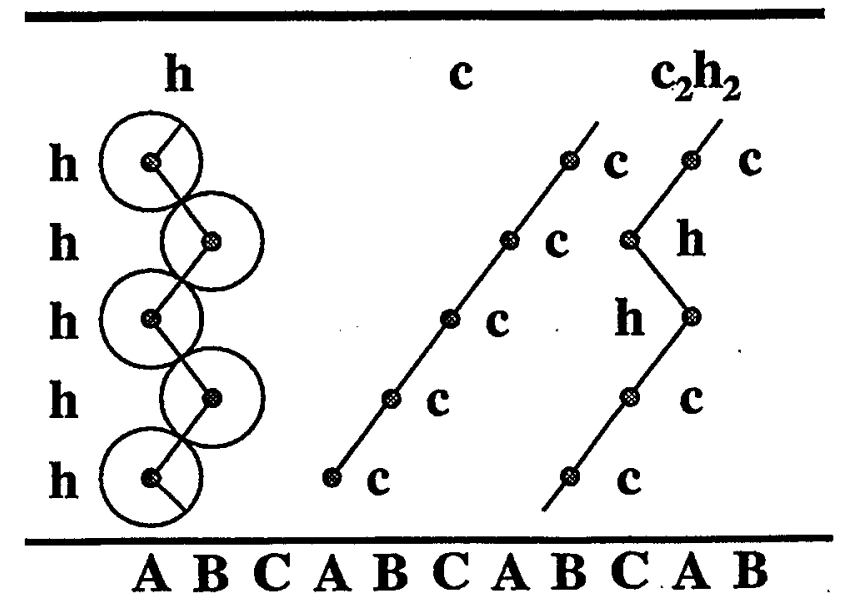

layer \#

\section{ccp}

2

$3 \cdot_{3}^{3}$

$1 \quad \begin{array}{lllll} & 3 & 3 & 2 & \\ & 2 & 1 & & 3 \\ & 3 & 1 & 1 & 3 \\ & 3 & 3 & & \end{array}$

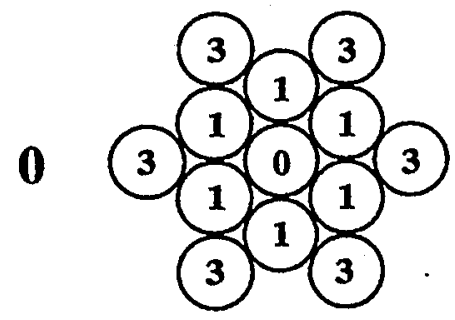

$\begin{array}{rlllll} & & 2 & 3 & 3 \\ -1 & 3 & 1 & 1 & 2 \\ & 3 & & 1 & 2 \\ & & 2 & & 3\end{array}$

$-2 \quad 3_{3}^{3} \cdot 3$ hep

3
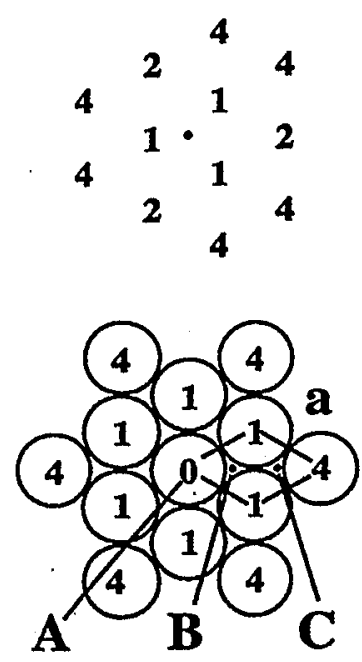

$\begin{array}{llll}4 & 2 & 4 & 4 \\ 4 & 1 & 1 & 2 \\ 4 & 1 & 4\end{array}$

Figure 1: [110] projection (above) of the layer sequence in $h, c_{2} h_{2}$ or $c$ stacking and [001] projection of the different layers with the 12 nearest (1), 6 next-nearest (2) and 24 or 2 third neighbors (3) in ccp or hcp alloys and unit cell with lattice constant $a$. The metal atoms are at different positions $A, B, C$ in layer \# $-2,-1,0,1,2$, respectively. 
thanides $\mathrm{Tb}$ to $\mathrm{Ho}, \mathrm{Tm}, \mathrm{Lu}$ can crystallize also in the $\mathrm{ABABCBCAC}$ or chhchhchh $=(\mathrm{chh})_{3}$ stacking. A comparison of the stacking symbols and population data in Table 1 shows the preference of simple sequences hh, ccc, $(\mathrm{ch})_{2},(\mathrm{hcc})_{2}$ or $(\mathrm{chh})_{3}$. Some more complex sequences are found in alloys $M_{x} N_{y}$ (Table 1), which are discussed in Section 7 .

The stacking sequence can be analyzed by the one-dimensional Ising model. The sequences of layers $\mathrm{h}$ and $\mathrm{c}$ can be compared with a onedimensional chain of atoms with $\oplus$ or $\ominus$ spin (Ising, 1925) or M and N atoms (Ducastelle, 1991). The large variety of chains is characterized by the self-coordination numbers ( $\mathrm{s}-\mathrm{CN}$ ) of $\mathrm{M}$ atoms (or h layers) to construct a structure map (Fig.2).

Each $\mathrm{h}$ layer of the sequence chchch e.g. has no nearest $\mathrm{h}$ layers $\left(T_{1}=\right.$ 0 ), two next-nearest $\mathrm{h}$ layers $\left(T_{2}=2\right)$ and no third-nearest neighbors of $\mathrm{h}$ layers $\left(T_{3}=0\right)$. The sequence of layers is characterized by the three s-CN values $T_{1}=0, T_{2}=2, T_{3}=0$ and the total composition $\mathrm{y} / \mathrm{x}=1$ of $c$ and $h$ layers $c_{x} h_{y}$. The notation $020 ; 1$ for the sequence chchch is identical for $\mathrm{c}$ and $\mathrm{h}$ layers at $\mathrm{y} / \mathrm{x}=1$ and deviates for $\mathrm{y} / \mathrm{x} \neq 1$ as e.g. $002 ; 2$ for $\mathrm{h}$ or $112 ; 0.5$ for $\mathrm{c}$ in the $\mathrm{hc}_{2}$ stacking sequence. The $T_{i}(\mathrm{M})$ values of the minority component and $T_{i}(\mathrm{~N})$ of the majority component give identical Cowley short-range order parameters $\alpha_{i}$ (Ducastelle, 1991; Hauck et al., 1988a).

Structure maps with $T_{i}(\mathrm{M})$ or $\alpha_{i}$ values as parameters can be obtained by the following procedure:

(1) The coordination numbers of each shell are averaged for structures which have different $T_{i}$ values. For example the three $\mathrm{c}$ in the $\mathrm{c}_{3} \mathrm{~h}_{6}$ chain (ccchhhhhh,c) 110,200 and 110 are averaged to $1.30 .70 ; 2$.

(2) The crystal structures characterized by the coordination numbers $T_{1}, T_{2}, T_{3}$ and a fixed composition $\mathrm{y} / \mathrm{x}$ can be plotted as single points in a $T_{1}, T_{2}, T_{3}$ coordinate system or as projection in the $T_{1}, T_{2}$ plane as outlined for $T_{1} T_{2} T_{3} ; 1$ structures in Fig.2. All structures with $\mathrm{y} / \mathrm{x}=1$ are found to fall within a triangle, with the three structures $020 ; 1$ (for ch), $102 ; 1\left(\right.$ for $\left.\mathrm{h}_{2} \mathrm{c}_{2}\right)$ and $222 ;(1)\left(\mathrm{h}_{\infty} \mathrm{c}_{\infty}\right.$ 

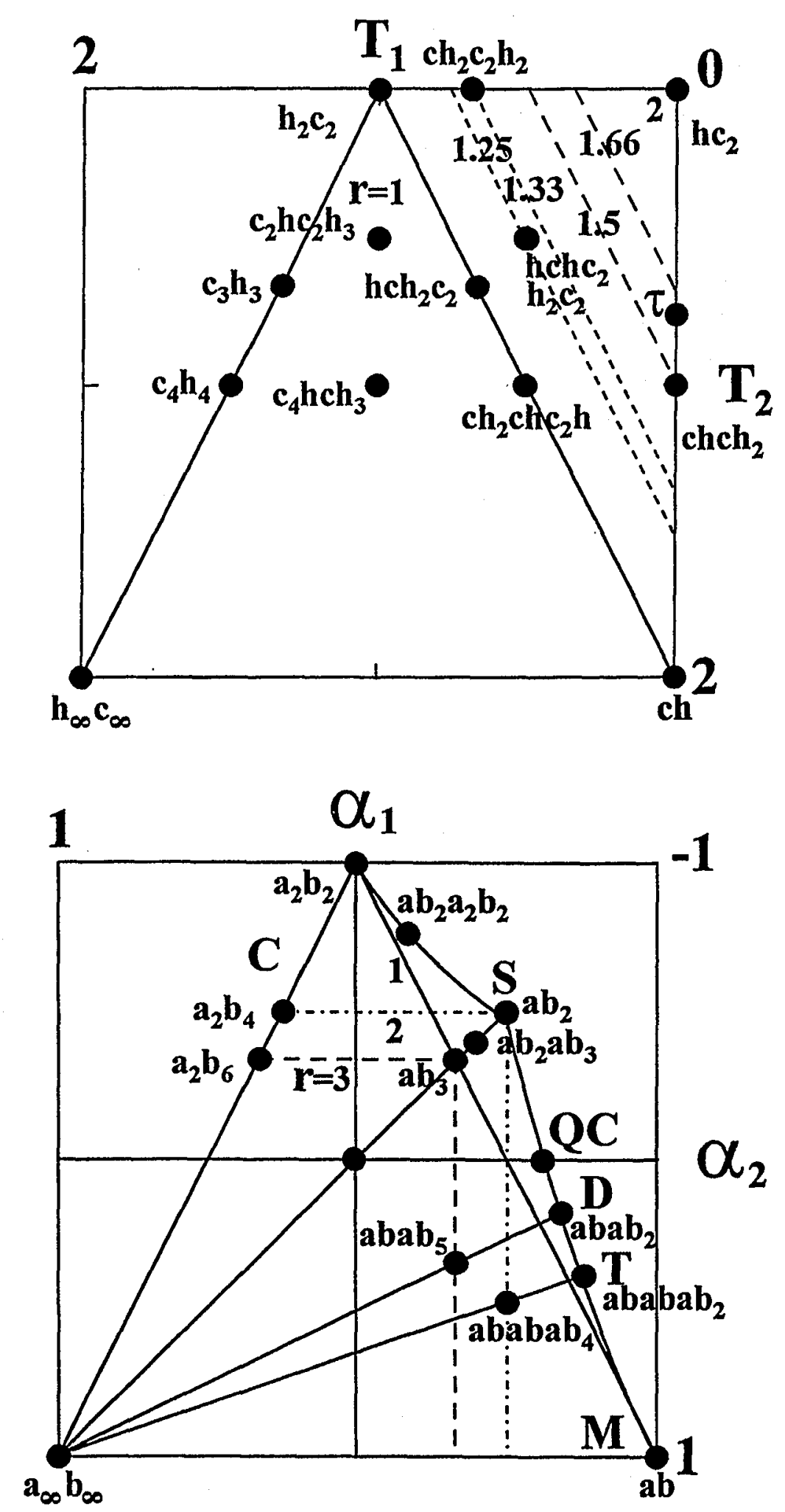

Figure 2: Structure map of a linear chain of $\mathrm{c}$ and $\mathrm{h}$ or $\mathrm{a}$ and $\mathrm{b}$ with the self-coordination numbers $T_{1}, T_{2}$ of nearest and next-nearest neighbors (a), or the short-range order parameters $\alpha_{1}, \alpha_{2}$ as axes. Single (S), double (D), triple (T), multi-alternating units (M) or clusters (C) are at straight lines, a quasicrystal at point QC (b). 
for segregation of $\mathrm{h}$ and $\mathrm{c}$ layers) at the corners. The structure 222 ; (1) with the composition given by (1) in brackets is used to indicate that the structure can only be obtained in the limit of very large stacks and a negligible border between $\mathrm{h}$ and $\mathrm{c}$ layers.

(3) The sequences at the left-hand border of the structure map as e.g. $c_{3} h_{3}$ or $c_{4} h_{4}$ contain increasing segments $h_{n}$ and $c_{n}$, which are segregated at the lower left-hand corner for $n \rightarrow \infty$. The sequences at the right-hand border of the structure map at $y / x=1$ as e.g. $h \mathrm{~h}_{2} \mathrm{c}_{2}$ are a combination of $\mathrm{h}_{2} \mathrm{c}_{2}$ and $\mathrm{hc}(=\mathrm{ch})$ at the upper and lower corners of the border, respectively. The structural units $h_{2}$ and $c_{2}$ can be combined with $h_{3}$ and $c_{3}$ at the left hand border and with $h c_{\mathrm{x}}$ or $\mathrm{ch}_{\mathrm{x}}$ at the right hand border of the structure map (Table 1). The number of theoretical combinations $2^{n-2}$, which is 64 for $n=8$ for example, is reduced to 16 combinations ( 13 at the four borders and 3 interior points). The structures at the four borders with the relation between $T_{1}, T_{2}$ and $\mathrm{r}=\mathrm{y} / \mathrm{x}$ values $T_{2}=2 T_{1}-2$, $T_{2}=0, T_{2}=-2 T_{1}+2(2-\mathrm{r})$ and $T_{1}=0$ (Section 22) correspond to four different structure families with a combination of different structural units.

(4) Structures $T_{1} T_{2} T_{3} ; \mathrm{y} / \mathrm{x}$ with different $\mathrm{y} / \mathrm{x}$ values can be included in the same structure map (Fig.2b) by using Cowley's short-range order parameter $\alpha_{\mathrm{i}}$ (Eqs. (1) and (2) below), which can vary within $-1 \leq \alpha_{i} \leq 1$. The $\alpha_{i}$ values can be obtained from the coordination numbers $T_{i}(\mathrm{M})$ and $T_{i}(\mathrm{~N})$ of $\mathrm{M}$ and $\mathrm{N}$ atoms or from the $T_{i}(\mathrm{M})$ and $\mathrm{r}=\mathrm{y} / \mathrm{x}$ values (Hauck et al., 1988a):

$$
\begin{aligned}
\alpha_{i} & =1-p_{i}(\mathrm{~N}) \mathrm{y}^{\prime} \\
\mathrm{y}^{\prime} & =\mathrm{y} /(\mathrm{x}+\mathrm{y}) \\
T_{i}(\mathrm{~N}) & =T_{i}^{\max }-\left(T_{i}^{\max }-T_{i}(\mathrm{M})\right) \mathrm{x} / \mathrm{y} \\
\alpha_{i} T_{i}^{\max } & =T_{i}(\mathrm{M})+T_{i}(\mathrm{~N})-T_{i}^{\max } \\
\alpha_{i} T_{i}^{\max } & =T_{i}(\mathrm{M})-\left(T_{i}^{\max }-T_{i}(\mathrm{M})\right) \mathrm{x} / \mathrm{y} \\
T_{i}^{\max } & =T_{i}(\mathrm{M})+T_{i}(\mathrm{M}, \mathrm{N}) .
\end{aligned}
$$

$p_{i}(\mathrm{~N})$ is the probability of finding an $\mathrm{N}$ atom in the $i$-th coordination shell of an $\mathrm{M}$ atom. $\mathrm{y}^{\prime}$ is the fraction of $\mathrm{N}$ atoms in $\mathrm{M}_{\mathrm{x}} \mathrm{N}_{\mathrm{y}} \cdot T_{1}^{\max }$ $=T_{2}^{\max }=T_{3}^{\max }=2$ are the maximum ${ }_{\mathrm{s}-\mathrm{CN} \text { values of the linear }}$ 
chain. $T_{i}(\mathrm{M}, \mathrm{N})$ is the coordination number $\mathrm{CN}$ of $\mathrm{M}$ atoms with $\mathrm{N}$ atoms. The self-coordination numbers $T_{i}(\mathrm{M})$ and $T_{i}(\mathrm{~N})$ of $\mathrm{h}$ and c layers are identical at composition $\mathrm{r}=1$ but different at other compositions. The area mapped out by the maximum range of $\alpha_{1}$, $\alpha_{2}$ values is different at different compositions, as outlined for $\mathrm{r}=$ 2 and 3 in Fig.2b.

(5) The $\alpha_{i}$ values are zero for a random distribution of $\mathrm{h}$ and $\mathrm{c}$ layers, because the mean value of $T_{i}(\mathrm{M})+T_{i}(\mathrm{~N})$ equals $T_{i}^{\max }$, which corresponds e.g. to $T_{1}=T_{2}=T_{3}=2 /(\mathrm{r}+1)$ (Fig.2b). Very small $\alpha_{i}$ values are expected for alloys with very weak interactions and in particular at high temperatures. Positive $\alpha_{1}$ values are obtained for attractive interactions of $\mathrm{M}$ atoms, i.e. for cluster formation or segregation. The $102 ; 1\left(\mathrm{~h}_{2} \mathrm{c}_{2}\right)$ structure of Fig.2a consists of double $h$ and $c$ layers. The size of $h$ and $c$ layers is increased e.g. in $c_{3} h_{3}$ or $c_{4} h_{4}$ to complete segregation $h_{\infty} c_{\infty}$ of $h$ and $c$ layers in the 222 ; (1) structure. Negative $\alpha_{1}$ values indicate repulsive interactions between layers, e.g. Coulomb repulsion for alternating layers ch $(020 ; 1)$ at $50 \% \mathrm{~h}$ (Section 11).

(6) Homometric structures (Patterson, 1944) or homologous structures (Hauck and Mika, 1994) have the same $\alpha_{i}$ values (Sections 8,9).

(7) The different structural units of one border of the structure map can also be combined randomly or aperiodically. The $T_{i}(\mathrm{~h})$ values $00.761 .24 ; \tau$ are obtained, for example, if the structural units hc and $\mathrm{hc}_{2}$ in the ratio $\mathrm{hc}_{2} / \mathrm{hc}=\tau \approx 1.62$ vary aperiodically in a Fibonacci sequence (Table 1) (Section 13). The $\alpha_{i}$ values of the onedimensional quasicrystal (QC) are on the boundary of the structure map with $\alpha_{2}=0$ (Fig.2b).

Atoms of close-packed structures with diameter $d=1$ have $T_{1}=6$ nearest neighbors in the same layer at distance $d=a$ (Fig.1) and $T_{1}=6$ neighbors in the two adjacent layers (three in each). The number $T_{2}=6$ of second-nearest neighbors at distance $\sqrt{2} d$ is also identical for all closepacked structures. The atoms of the hh (hcp) structure, however, have $T_{3}=2$ third-nearest neighbors at distance $\sqrt{8 / 3} d$ (in the next-nearest layers) and 18 fourth-nearest neighbors at distance $\sqrt{3} d$, while atoms of 
ccc (ccp) packing have 24 third-nearest neighbors at distance $\sqrt{3} d$ (Fig.1, Table 2). The coordination polyhedra of $T_{1}, T_{2}$ and $T_{3}$ neighbors of the hcp structure correspond to $\mathrm{CN} \mathrm{12',} \mathrm{CN} 6$ (octahedron) and $\mathrm{CN} 2$, and the coordination polyhedra of the ccp lattice to CN 12 (cuboctahedron), CN 6 and CN 24", as shown by Villars (1994). The metal atoms of the sequences $\operatorname{ch}_{n}(n=1,2,3,6), \mathrm{hc}_{2}, \mathrm{~h}_{2} \mathrm{c}_{n}(n=2,3)$ and $\mathrm{ch}_{2} \mathrm{ch}_{3}$ (Table 1) have $M^{i}=2$ different values of $T_{3}, T_{4}$ and higher coordination shells. The more complex structures contain three or more metal atoms with different environments. The averaged $T_{3}$ and $T_{4}$ coordination values of these structures depend on the fraction $f$ of $h$ layers as given by $T_{3}=2 f$ and $T_{4}=24-6 \mathrm{f}$.

The hh hexagonal close-packed and the ccc cubic close-packed structures are the only structure types with a single environment for close and distant neighbors of all atoms $\left(M^{i}=1\right)$. These structures have been observed in 673 binary and ternary systems (Villars and Hulliger, 1987). The sequences of layers with two different environments of metal atoms $\left(M^{i}=2\right)$ (Pauling, 1945/1960) (Table 1) have been found in 66 systems. The metal atoms of the other sequences of layers contain three or more different environments. The tendency to small numbers $M^{i}$ of metal atoms with different environment can be described by Pauling's rule of parsimony: The number of essentially different kinds of constituents in a crystal tends to be small (Pauling, 1929).

The number $M^{i}$ of metal atoms with different sets of $T_{i}$ values is often identical to the number of Wyckoff positions, the number of symmetrically different atom positions. For example, the numbers of Wyckoff positions of the $\mathrm{Mg}, \mathrm{Cu}, \mathrm{Sm}$ and $\mathrm{Nd}$ structures are identical to $M^{i}$, but are larger than $M^{i}$ as e.g. 5 for the $n=8 \mathrm{~b}, 9 \mathrm{~b}\left(M^{i}=3\right)$ structures (Table 1).

The different stacking sequences with $n$ layers in $\mathrm{A}, \mathrm{B}$ or $\mathrm{C}$ positions can be described by hexagonal unit cells with the lattice parameters $a$ $=b=d$ (diameter of atoms) and $c=n \sqrt{2 / 3} d$ (Fig.1). The smallest unit cell of ccc packing containing only one metal atom is rhombohedral with lattice constants $a=d$ and $\alpha=60^{\circ}$. Usually, the ccc structure is 

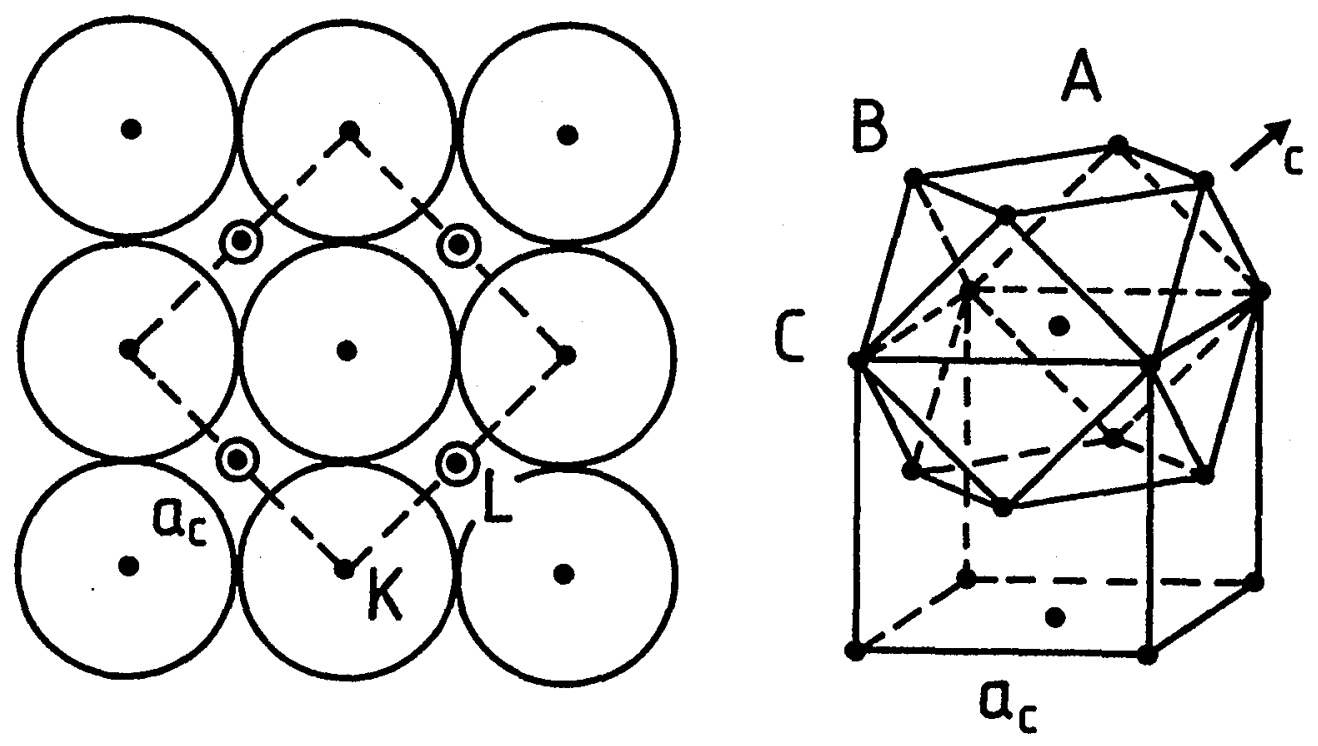

Figure 3: Packing of square layers at $\mathrm{K}$ and $\mathrm{L}$ positions in face-centered cubic metal structure and relation to ccp stacking of hexagonal layers in $A B C$ position.

described as face-centered cubic lattice containing four metal atoms with lattice constant $a_{\mathrm{c}}=\sqrt{2} d$ (Fig.3). The fcc lattice can also be considered as a stacking of square layers in the $K$ and $L$ positions. The $c$ axis of the rhombohedral cell corresponds to one of the four space diagonals of the cubic cell. The only periodic three-dimensional packing of spheres having close-packed hexagonal layers in four inclined directions is ccc. All other sequences are maintained in one direction only.

A comparison of the close-packed structures shows that the interactions between metal atoms, e.g. in the ccc $\mathrm{Cu}$ structure, must be strong enough to stabilize the atoms of the neighboring layers at the proper position. The $\mathrm{M}$ atoms of the hchc 4 structure must be stabilized in the eighth layer.

A participation of $f$ orbitals is suggested for bonding in lanthanides or actinides with $\mathrm{ch}, \mathrm{ch}_{2}$ or $\mathrm{hc}_{2}$ stacking sequences and $M_{i}=2$ different sets of s-CN values for atoms $M$ and $\mathrm{M}^{\prime}$ at $\mathrm{h}$ or c layers.

An interaction between metal atoms with directional bonding can also be deduced from the distortion of some crystal structures. The ratio $c / a$ 
of the lattice constants of the hh hexagonal close-packed structures of $\mathrm{Ca}$ and $\mathrm{Sr}$ is almost ideal with $c / a=n \sqrt{2 / 3} \approx 1.63$ for $n=2$ layers (Sections 15,23). The $c / a$ ratio is slightly decreased to 1.56 for most hcp metals and increased to $c / a=1.86$ and 1.89 for $\mathrm{Zn}$ and $\mathrm{Cd}$, respectively (Pauling, 1945/1960; Laves, 1967; Wyckoff, 1982). The distance between close-packed layers $\sqrt{2 / 3} \approx 0.8$ is also decreased in cubic close-packed $\mathrm{Hg}$, Po and Te with the rhombohedral angle $\alpha=71^{0}-103^{\circ}$ instead of $\alpha=60^{\circ}$ (Villars and Calvert, 1986).

\section{Alloy formation}

All metal atoms for $n=2$ and 3 (hcp and ccp structures) have the same environment $\left(M^{i}=1\right.$ ) (Table 1). The $n=4,5,6 \mathrm{~b}, 8 \mathrm{a}$ and 9a layer stackings of Table 1 contain $M^{i}=2$ metal atoms with different $T_{i}$ values, the other structures 3,4 or 5 different sets of $T_{i}$ values (Table 1). Therefore the structures of the rare earth elements $\alpha-\mathrm{Nd}$ ( $n=4$ layers), Tb HP $(n=6 \mathrm{~b})$ and $\alpha-\operatorname{Sm}(n=9 \mathrm{a})$ with two different $T_{i}$ can be formulated as pseudobinary alloys $\mathrm{NdNd}^{\prime}(n=4,8 \mathrm{a}), \mathrm{TbTb}_{2}^{\prime}$ or $\operatorname{SmSm}_{2}^{\prime}(n=6 \mathrm{~b}$, $9 \mathrm{a})$ and $\mathrm{MN}_{4}(n=5)$. Other stacking sequences are pseudoternary e.g. $\mathrm{M}^{1} \mathrm{M}_{2}^{2} \mathrm{~N}_{3}(n=6 \mathrm{a}), \mathrm{M}^{1} \mathrm{M}_{2}^{2} \mathrm{~N}_{4}(7), \mathrm{M}^{1} \mathrm{M}_{2}^{2} \mathrm{~N}_{5}(8 \mathrm{~b}), \mathrm{M}^{1} \mathrm{M}^{2} \mathrm{~N}_{2}$ (8c, 8e, 8f), $\mathrm{M}^{1} \mathrm{M}_{4}^{2} \mathrm{~N}_{4}(9 \mathrm{~b})$, and $\mathrm{M}_{2}^{1} \mathrm{M}_{3}^{2} \mathrm{~N}_{4}(9 \mathrm{c})$. The population of the different structure types decreases with increasing $M^{i}$. Ordered binary and ternary alloys have not been observed with these structures. The $\mathrm{MN}, \mathrm{MN}_{2}$, $\mathrm{MN}_{4}$ binary alloys e.g. favor other close-packed structures (Section 7).

The $\mathrm{M}$ and $\mathrm{N}$ atoms of most close-packed $\mathrm{M}_{\mathrm{x}} \mathrm{N}_{\mathrm{y}}$ alloys are ordered in different hexagonal or square layers with the composition $M_{x} N_{y}$. These structures can be characterized by the type of the hexagonal or square layer and the stacking of these layers as will be outlined in Sections 4-7. The separation of $\mathrm{M}$ and $\mathrm{N}$ atoms in different layers indicates repulsive interactions. Few structures of $\mathrm{M}_{\mathrm{x}} \mathrm{N}_{\mathrm{y}}$ alloys are composed of two layers with different composition like layers with composition $\mathrm{M}_{\mathrm{x}} \mathrm{N}_{\mathrm{y}^{\prime}}$ and layers with $\mathrm{y}^{\prime \prime} \mathrm{N}$ atoms $\left(\mathrm{y}=\mathrm{y}^{\prime}+\mathrm{y}^{\prime \prime}\right)$. This indicates repulsive interactions only for $\mathrm{M}$ atoms. The attractive interactions between $\mathrm{M}$ and $\mathrm{N}$ atoms are 
increased in $M_{x} N_{y}$ compounds with $x$ layers of $M$ atoms and y layers of $\mathrm{N}$ atoms (Section 15). The distance between the layers of close-packed metals $\sqrt{2 / 3} \approx 0.8$ is decreased to $\approx 0.4$ in the primitive cubic $(\mathrm{pc})$ lattice of Po and to $\approx 0.2$ in the body-centered cubic (bcc) lattice of $W$ as will be outlined in Section 15 .

Some ccp alloys can be characterized by sequences of square layers instead of hexagonal layers (Fig.3). The distance between two identical square layers of ccc packing, which is $a_{\mathrm{c}}=\sqrt{2} d$ for cubic $\mathrm{Cu}$, can be increased up to $1.66 d$ in In, Ga, La or Ce or decreased to $1.0 d$ in Po or $1.15 d$ in W. Tungsten has a body-centered cubic structure. The $74 \%$ density of the close-packed structures however is reduced by hexagonal or tetragonal distortions, e.g. to $68 \%$ for the body-centered cubic structure (Laves, 1967; Pearson, 1972). These strongly distorted structures are considered as different structure types in some compilations (Ho and Douglas, 1968). This is one reason that the population numbers of different structure types are only rough values for comparison. We have taken the maximum values from the compilations of Wyckoff (1982), Pearson (1972) or Villars and Calvert (1986) of experimentally determined crystal structures. The undistorted structures are compared in the present article for the analysis of the self-coordination numbers $T_{i}$.

The atoms $M$ and $N$ e.g. of a binary alloy $M_{x} N_{y}$ have different sizes and a different tendency to exchange electrons, which gives rise to different bonding. The electron density and the radii of the $M$ and $N$ atoms can be varied by charge transfer between the $\mathrm{M}$ and $\mathrm{N}$ atoms. Both parameters do not vary independently for most metals (Fig.4). The electronegativity values of the elements can be related to the electron work function and indicate the tendency of a metal to exchange electrons (Pauling, 1945/1960; de Boer et al., 1988; Sutton, 1993). The electronegativity increases with increasing number of electrons to a maximum for the $8 \mathrm{~b}-10 \mathrm{~b}$ transition elements. Those elements with an almost filled $d$ shell try to accept electrons to complete the $d$ shell with 10 electrons, while elements with few outer electrons like the 1a and 2a alkali and alkaline earth or the $1 \mathrm{~b}$ and $2 \mathrm{~b}$ transition metals with lower electronegativity are ready to donate these electrons. The $1 \mathrm{a}$ and $2 \mathrm{a}$ metals are then stabilized in the spherical $s^{2} p^{6}$ shell of the noble gases, the $1 \mathrm{~b}$ and $2 \mathrm{~b}$ 

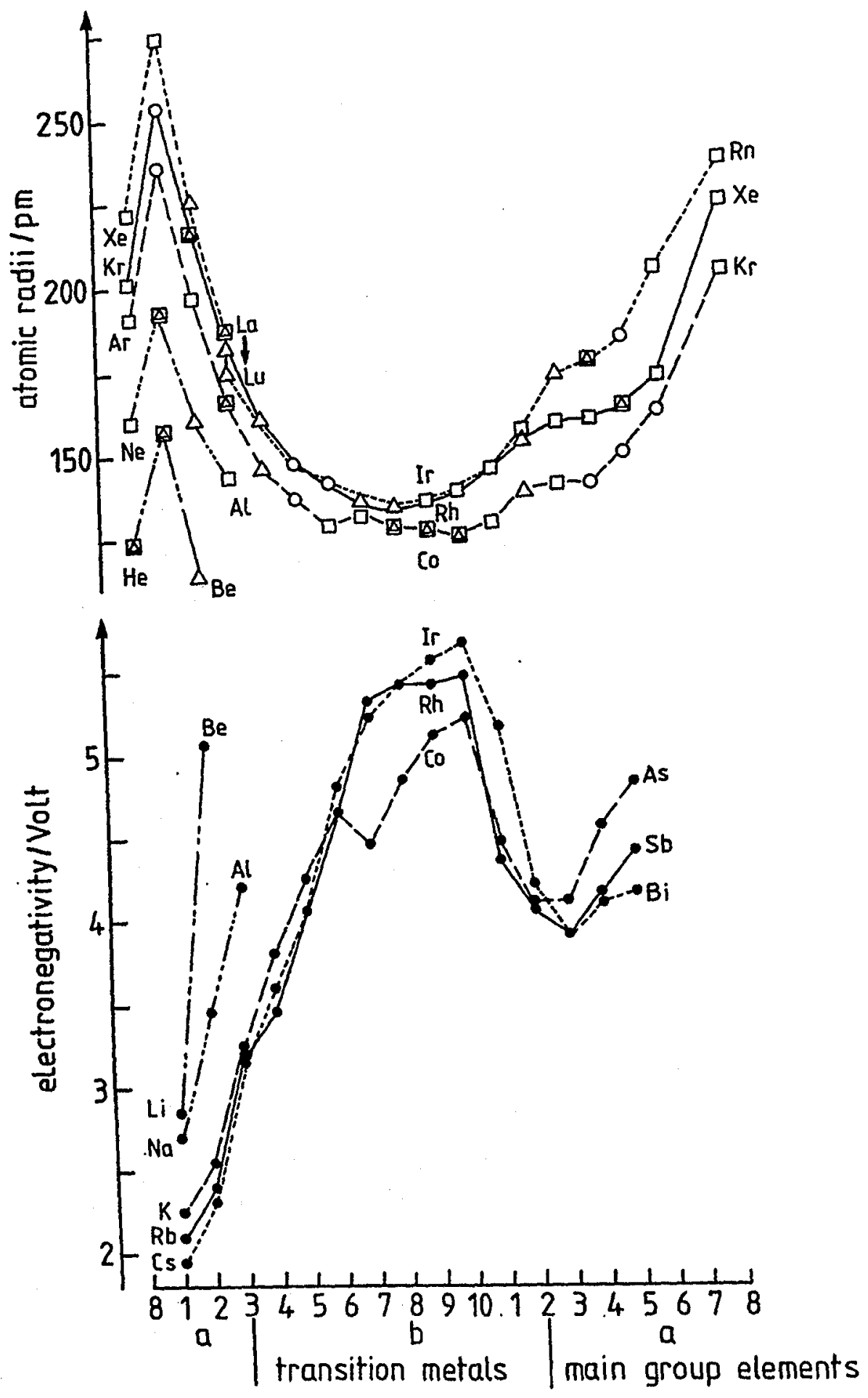

Figure 4: Electronegativity $\phi^{*}$ (de Boer et al., 1988) and radii (Schubert, 1967) of $1 \mathrm{a}-8 \mathrm{a}$ main group and $1 \mathrm{~b}-10 \mathrm{~b}$ transition metals with cubic $(\square)$ or hexagonal $(\triangle)$ close-packed crystal structures. Some metals ( $(0)$ are not observed in a close-packed modification. 
transition metals in the $d^{10}$ shell with decreased radii. The electronegativity increases again for the $4 \mathrm{a}-7 \mathrm{a}$ elements because of the tendency to attain the stable $s^{2} p^{6}$ octet of the noble gases. The radii of metal atoms decrease with increasing number of electrons to a minimum for the stable $d^{10}$ electron configuration (Schubert, 1967). Also the radii of the lanthanides La to Lu are decreased slightly as indicated by the arrow in Fig. 4 for the same effect of the filling of the inner $4 f$ electron shell. The relation between radii and electronegativity indicates the tendency to form spherical metal atoms in alloys.

The interactions between the $M$ metal atoms in $M_{x} N_{y}$ alloys can be attractive, as e.g. with segregation, or repulsive. Both interactions can be distinguished by an analysis of the self-coordination numbers $T_{i}$ of $\mathrm{M}$ atoms with $\mathrm{M}$ atoms. Analysis of the different ways of sphere packing showed the same number $T_{1}=12$ and $T_{2}=6$ of nearest and next-nearest $M$ atoms. These numbers are reduced on the formation of ordered $\mathrm{M}_{\mathrm{x}} \mathrm{N}_{\mathrm{y}}$ alloys and can be used to obtain structure maps (Hauck et al., 1988a/1989; Mika and Hauck, 1990).

The derivation of structure maps will be outlined for the simple examples of the square and hexagonal nets of single metal atom layers. The single square or hexagonal two-dimensional layers of $M$ atoms (Figs. 1,3) are the basic units for describing the three-dimensional close-packed metal structures (Beattie, 1967; Lima-de-Faria and Figueiredo, 1969; Beck, 1969).

\section{Ordering of atoms in hexagonal and square layers}

The hexagonal layer (in h or c position) is the structural unit of the closepacked metals or alloys (see previous section). The $M$ and $N$ atoms of alloys with composition $\mathrm{M}_{\mathrm{x}} \mathrm{N}_{\mathrm{y}}$ are usually not at different $\mathrm{h}$ and $\mathrm{c}$ layers but at different positions within the hexagonal layers (Beattie, 1967; Lima-de-Faria and Figueiredo, 1969; Beck, 1969; Parthé et al., 1993). 
The self-coordination numbers (s-CN) of nearest, next-nearest and third $\mathrm{M}$ atoms within a hexagonal layer were analyzed in a similar procedure as was outlined for the sequence of $h$ and $c$ layers in Section 2. Each metal atom of a hexagonal layer has $T_{1}^{\max }=6$ nearest, $T_{2}^{\max }=$ 6 next-nearest and $T_{3}^{\max }=6$ third neighbors at distances $d, \sqrt{3} d$ and $2 d$, respectively (Table 2 ). The maximum s-CN values are reduced, if the positions are occupied by different atoms $M$ and $N$ in alloys $M_{x} N_{y}$. The different structures are characterized by the s-CN values $T_{1}, T_{2}, T_{3}$ of the minority component $\mathrm{M}$ and the composition $\mathrm{y} / \mathrm{x} \geq 1$. Different structures are obtained by variation of the unit cell, the occupation of different positions by $\mathrm{M}$ atoms to a maximum of $50 \%$ and the determination of the s-CN values of the different $M$ atoms to obtain the averaged values $T_{1} T_{2} T_{3} ; \mathrm{y} / \mathrm{x}$ for the characterization of the structure. The corresponding $\alpha_{1}, \alpha_{2}$ values can be obtained from the $T_{1}, T_{2}$ values by $6 \alpha_{i}=T_{i}-\left(6-T_{i}\right) \mathrm{x} / \mathrm{y}$, as was outlined in Section 2.4 . The $T_{1}, T_{2}$ or $\alpha_{1}, \alpha_{2}$ values of the different structures are plotted in the structure maps. The $\alpha_{1}, \alpha_{2}$ structure map (Fig.5b) contains six structures with a single environment at the corners of an irregular pentagon with curved borderlines: $666 ;(1), 422 ; 1,202 ; 2,226 ; 1,006 ; 3$, and $060 ; 2$. The $226 ; 1$ and $006 ; 3$ structures are homologous with identical $\alpha_{i}$ values (Section 9). The $T_{1}, T_{2}$ structure map (Fig.5a) shows the different borders at varied $y / x$ values. The crystal structures existing along the different borderlines can be obtained by a combination of triangles with three and two $\mathrm{M}$ or $\mathrm{N}$ atoms at the left-hand border (structural units $\mathrm{a}-\mathrm{c}$ ) and triangles with one and two $\mathrm{M}$ or $\mathrm{N}$ atoms at the right-hand border (structural units e - k) (Fig.6). The $060 ; 2$ structure (structural unit e) for example contains triangles with one $\mathrm{M}$ atom and two $\mathrm{N}$ atoms at composition $\mathrm{MN}_{2}$. The dashed areas of the pentagon with the limiting structures $202 ; 2$ and $060 ; 2$ exceed the area at composition $\mathrm{y} / \mathrm{x}=1$, because of the impossibility of $30 T_{3} ; 1$ or $06 T_{3} ; 1$ structures at the corners of a structure map as in Fig.2. The hexagonal layers of close-packed structures are those of Fig.6 like $226 ; 1\left(\mathrm{c}_{2}\right), 060 ; 2$ (e), $006 ; 3(\mathrm{k}), 022 ; 3\left(\mathrm{f}^{\prime}\right)$ and $014 ; 3\left(\mathrm{f}^{\prime} \mathrm{k}\right)$ (Sections $\left.5-7\right)$. The structures at the right-hand border of the map are also common for the ordering of surface atoms or gas atoms at [111] surfaces of ccp metals and are usually characterized by the dimension of the unit cell like $(1 \times 1)$ for the $666 ;(1)$ structure, $(2 \times 2)(006 ; 3)$ or $(\sqrt{3} \times \sqrt{3})(060 ; 2)($ Fig.5) 

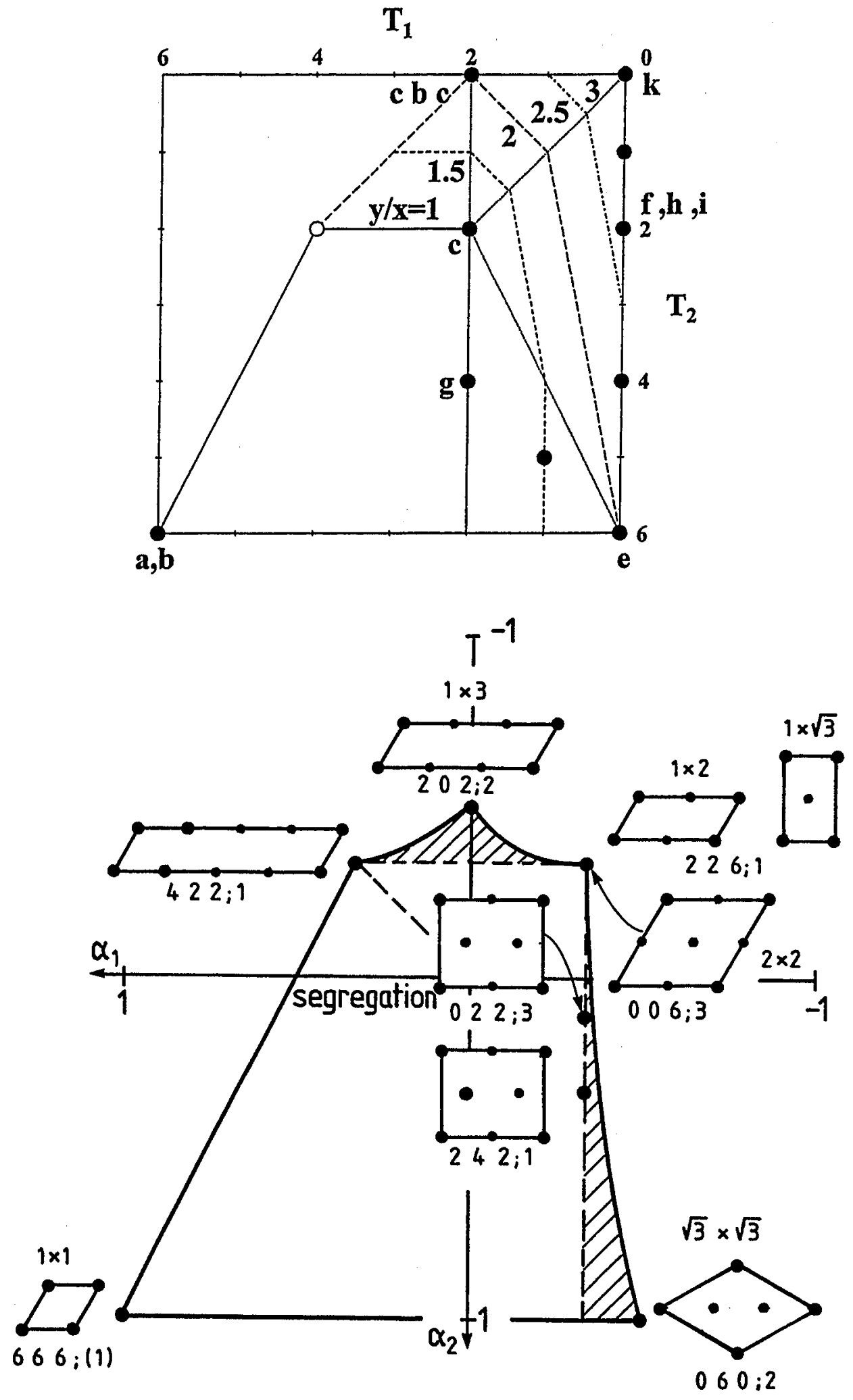

Figure 5: $T_{1}, T_{2}$ (a) and $\alpha_{1}, \alpha_{2}$ (b) structure map of the hexagonal net with different borders for $\mathrm{y} / \mathrm{x}=1,1.5, \ldots, 3$. The structures of Fig. 6 with structural units $\mathrm{a}-\mathrm{i}$ and the surface structures $l_{1} \times l_{2}$ are at the borderline $(\bullet)$. 


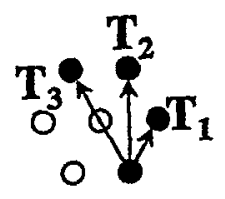

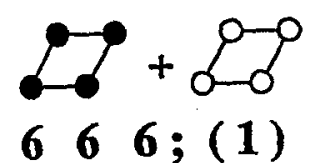

a

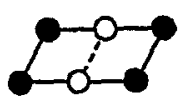

226 ; 1 $c_{2}$

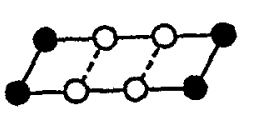

cbc

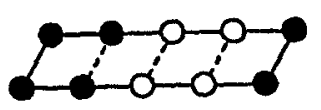

$422 ; 1$

a c b c
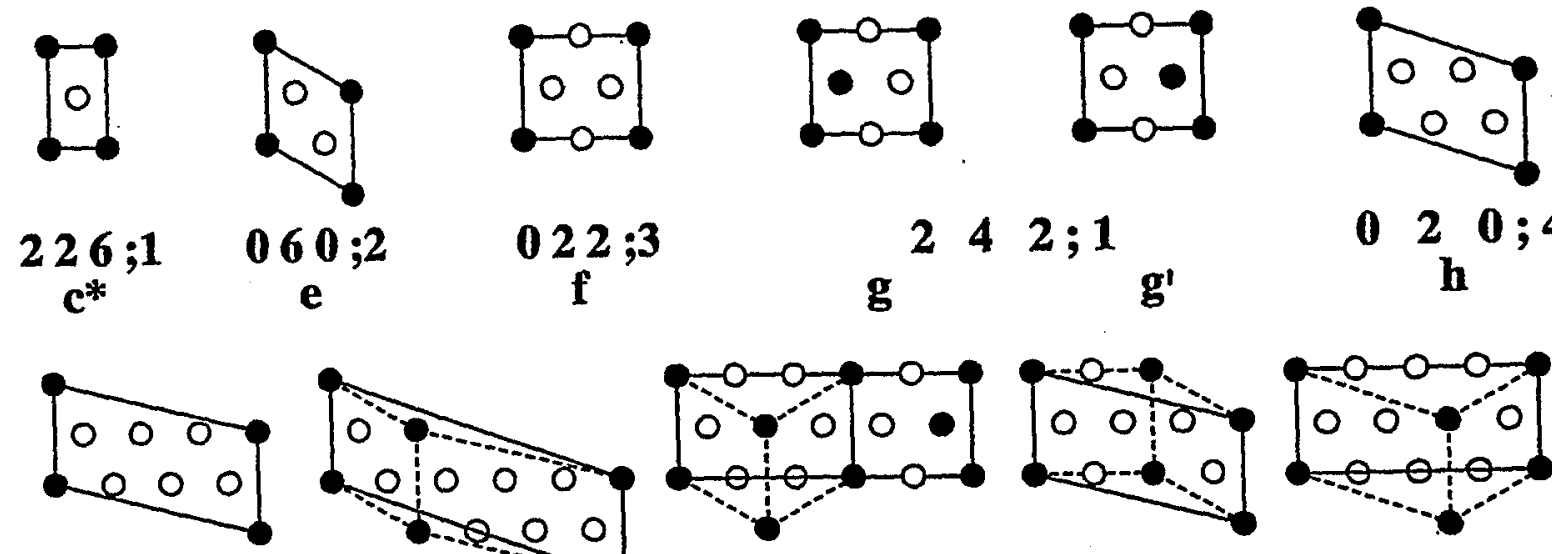

$022 ; 3$

e

$f$

$242 ; 1$

g $\mathbf{g}^{\prime}$

( ${ }_{\mathrm{h}}^{2} 0 ; 4$

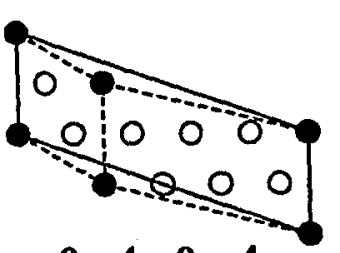

$020 ; 6$

$040 ; 4$

e i
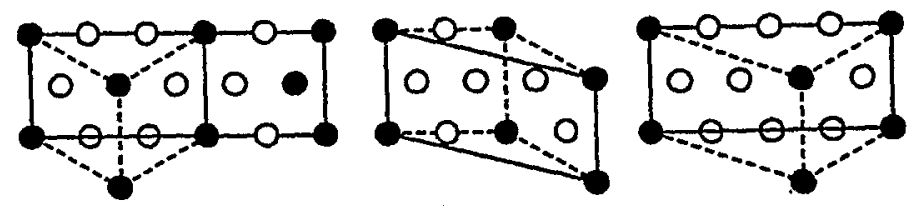

$151 ; 1.5$ e e $\mathbf{g}^{\prime}$

$041 ; 2.5$

fe

$040 ; 3$

he
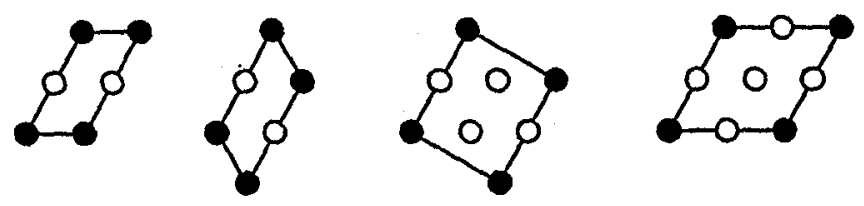

$\begin{array}{lll}2 & 26 ; 1 \\ c^{\prime} & c^{\prime \prime}\end{array}$

$022 ; 3$

$f^{\prime}$

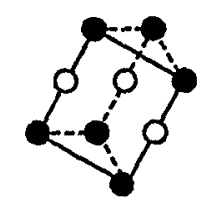

$242 ; 1$

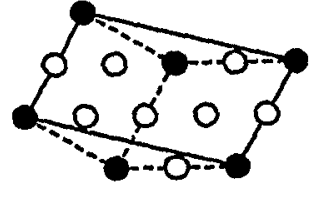

$014 ; 3$ $f^{\prime} k$

Figure 6: Self-coordination numbers $T_{1} T_{2} T_{3} ; \mathrm{y} / \mathrm{x}$ for the hexagonal net of $\mathrm{A}$ atoms in $A_{x} B_{y}$ compounds $(A=\bullet, B=0)$. A atoms with concentration $y / x \geq 1$ are the minority component. The different structural units $a, b, c$, etc. are shown by dashed lines. 
(Watson et al., 1994).

The metal atoms of the square net have $T_{1}^{\max }=4$ nearest, $T_{2}^{\max }=4$ nextnearest and $T_{3}^{\max }=4$ third neighbors at distances $d, \sqrt{2} d$ and $2 d$, respectively (Table 2). The structure map (Fig.7) is similar to the structure map of the chain (Fig.2) with the three structures $T_{1}^{\max } T_{2}^{\max } T_{3}^{\max } ;(1)$ (segregation), $T_{1}^{\max } / 20 T_{3}^{\max } ; 1$ or $T_{1}^{\max } / 20 T_{3}^{\max } / 2 ; 1$ (attraction) and $0 T_{2}^{\max } T_{3}^{\max } ; 1$ or $0 T_{2}^{\max } 0 ; 1$ (repulsion) at the corners of the triangle. The $T_{i}$ values of the three structures at the corners $(222 ;(1), 102 ; 1$ and $020 ; 1$ for the chain and $444 ;(1), 204 ; 1$ and $044 ; 1$ for the square net) show different relations to the $T_{i}^{\max }$ values. The $00 T_{3}^{\max }$ structure is obtained for $y / x=3$ (instead of $y / x=2$ for the chain). The $\mathrm{M}$ atoms of the square net form chains with $T_{1}=2$ nearest $\mathrm{M}$ atoms in the $204 ; 1$ structure containing two squares $\mathrm{c}_{2}$ or $\mathrm{c}^{*}$ with $\mathrm{M}$ atoms in neighboring cis positions. The diagonal trans positions $\left(t_{2}\right.$ or $\left.t_{2}^{*}\right)$ are occupied in the $044 ; 1$ structure and a single position $\left(\mathrm{s}_{4}\right.$ or $\left.\mathrm{s}_{2}^{*}\right)$ in the $004 ; 3$ structure. The structures at the left- and right-hand borders are combinations of structural units $a-c$ and $b, c, s, t\left(o r b^{*}, c^{*}, s^{*}, t^{*}\right)$, respectively (Fig.8).

\section{$5 \quad$ Hexagonal close-packed structures}

The $\mathrm{Mg}$ atom positions of the hexagonal close-packed $\mathrm{Mg}$ structure (Table 1) can be substituted by different $M$ and $N$ atoms in $M_{x} N_{y}$ alloys. The structures of all possible alloys can be obtained, if the unit cell of the $\mathrm{Mg}$ structure is increased in a systematic way (Section 33) (Hauck and Mika, 1999), the metal positions occupied by $\mathrm{M}$ atoms to a maximum of $50 \%$ and the s-CN values $T_{1}, T_{2}, T_{3}$ of all $\mathrm{M}$ atoms averaged, to characterize the structure by $T_{1} T_{2} T_{3} ; \mathrm{y} / \mathrm{x}$. The s-CN values of the $\mathrm{N}$ atoms are different at $\mathrm{y} / \mathrm{x}>1$. The $\alpha_{i}$ values, which can be obtained from the $T_{i}$ values (Section 2.4) are identical for $\mathrm{M}$ and $\mathrm{N}$ atoms in binary $\mathrm{M}_{\mathrm{x}} \mathrm{N}_{\mathrm{y}}$ alloys. In ternary compounds $\mathrm{M}_{\mathrm{x}} \mathrm{N}_{\mathrm{y}} \mathrm{R}_{\mathrm{z}}$ the $\alpha_{i}$ values of $\mathrm{M}, \mathrm{N}$ and $\mathrm{R}$ atoms can be different (Section 14). 


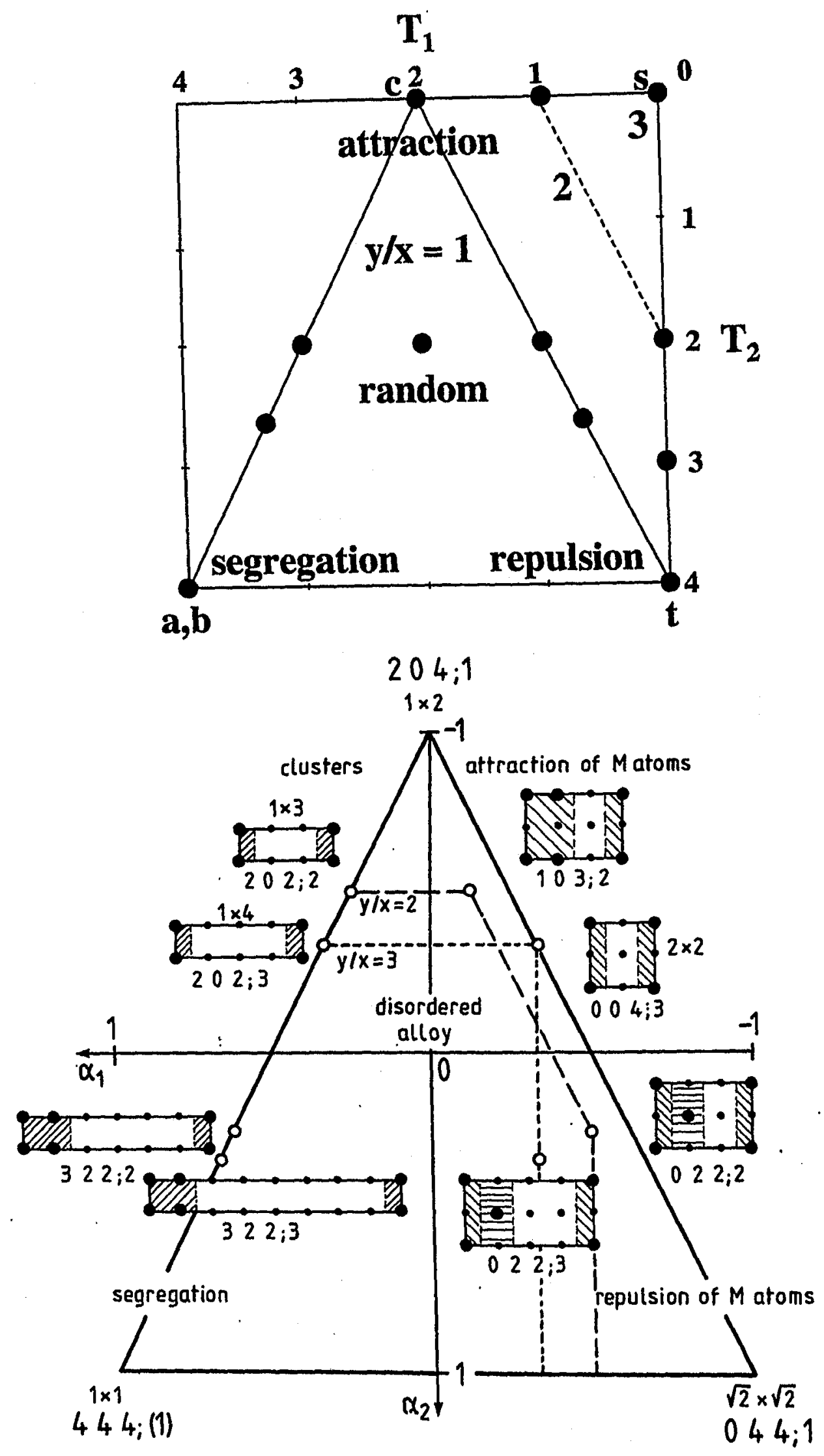

Figure 7: $T_{1}, T_{2}(\mathrm{a})$ and $\alpha_{1}, \alpha_{2}$ (b) structure map of the square net with different borders for $y / x=1,2,3$. The structures of Fig. 8 with structural units $a, b, c, s$ and $t$ (different shading) are at the borderline (•). 

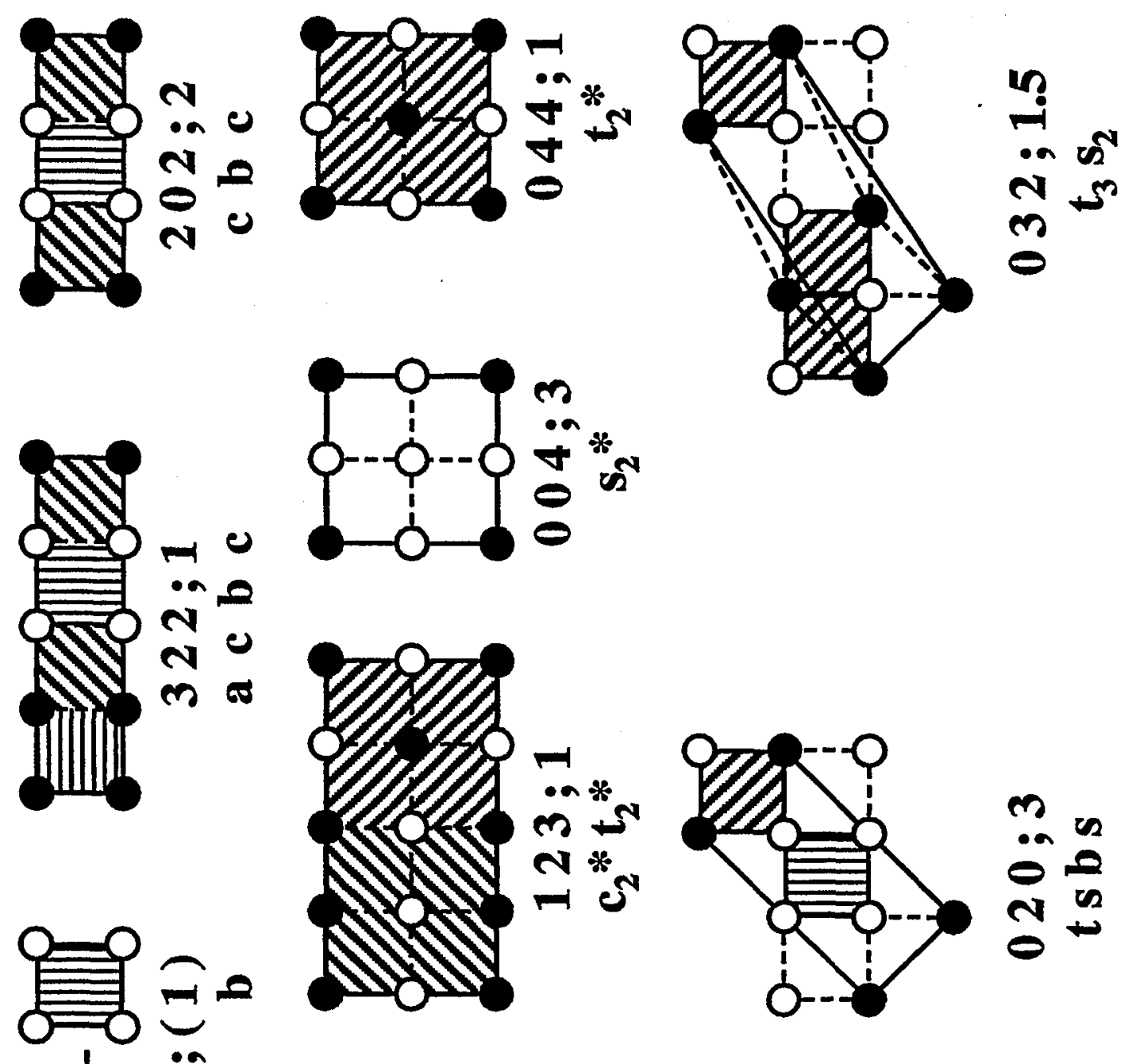
IIII
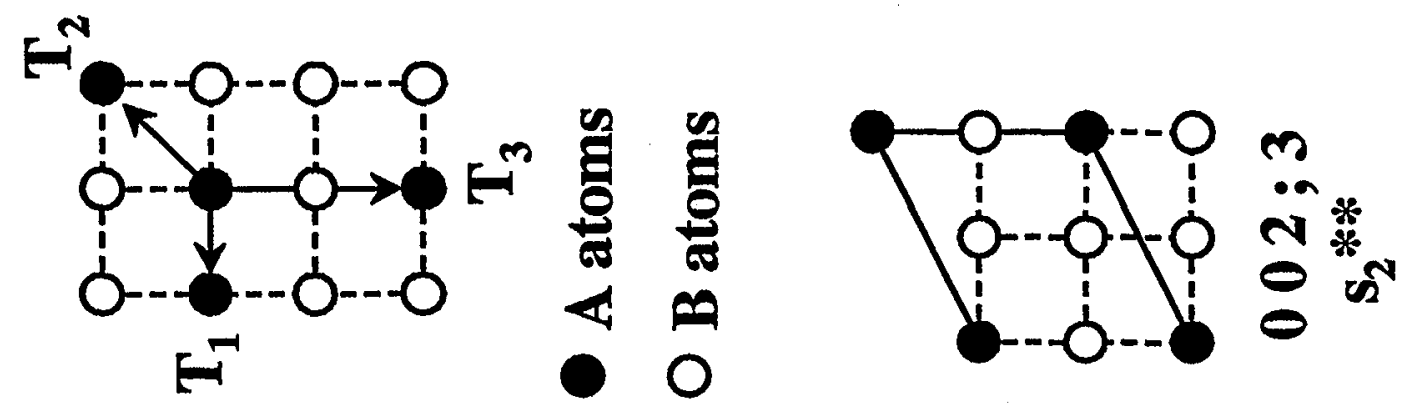

Figure 8: Self-coordination numbers $T_{1} T_{2} T_{3} ; \mathrm{y} / \mathrm{x}$ for the square net of $\mathrm{A}$ atoms in $\mathrm{A}_{\mathrm{x}} \mathrm{B}_{\mathrm{y}}$ compounds $(\mathrm{A}=\bullet, \mathrm{B}=0)$. The different structural units $\mathrm{a}, \mathrm{b}, \mathrm{c}$, etc. are shown by different shading. 
The hexagonal close-packed alloys consist of two hexagonal layers in A and $B$ positions with identical environment of 12 nearest, 6 next-nearest and 2 third neighbors at distances $d, \sqrt{2} d$ and $\sqrt{8 / 3} d$, respectively (Fig.1, Table 2). The maximum $\alpha_{1}, \alpha_{2}$ range of ordered hcp $\mathrm{M}_{\mathrm{x}} \mathrm{N}_{\mathrm{y}}$ alloy structures with the $1262 ;(1), 602 ; 1,442 ; 1,462 ; 1$ (and $062 ; 3$ ) structures at the corners of a tetragon has the same shape as the $\mathrm{y} / \mathrm{x}=$ 1 border of the $T_{1}, T_{2}$ structure map (Figs. 9,10). The $530 ; 1$ and $930 ; 1$ structures are also at the corners in the three-dimensional $\alpha_{1}$, $\alpha_{2}, \alpha_{3}$ space (Mika and Hauck, 1990). Several structures with a single environment of metal atoms are inside of the structure map (Section 33). The $930 ; 1$ structure with two layers of $M$ atoms followed by two layers of $\mathrm{N}$ atoms can be considered as partially segregated sheets of $\mathrm{M}$ and $\mathrm{N}$ atoms (Table 3). The $\mathrm{M}$ atoms of the $602 ; 1 \mathrm{a}$ and $602 ; 1 \mathrm{~b}$ structures are clustered in layers and chains, respectively. The $M$ atoms of the remaining structures of Fig.10 are as far apart as possible. The $462 ; 1$ and $062 ; 3$ structures with identical $\alpha_{i}$ values correspond to the $\mathrm{AuCd}$ and $\mathrm{SnNi}_{3}$ structures. These structures are homologous (Section 9). The $\mathrm{N}$ atom positions of the $062 ; 3 \mathrm{MN}_{3}$ structure can be occupied by $\mathrm{M}$ or $\mathrm{M}^{\prime}$ atoms to $\mathrm{M}_{2} \mathrm{~N}_{2}(=\mathrm{MN})$ or $\mathrm{MM}^{\prime} \mathrm{N}_{2}$ alloys. These structures have $T_{2}=6$. The other experimental structures are found at lower $T_{2}$ values.

The architecture of the different structures built up from structural units is more complicated than for the chain or hexagonal layer. The construction of $\mathrm{MN}_{\mathrm{y}}$ alloys $(\mathrm{y}=1,3,5)$ by combination of structural units a $o$ in [120] direction and of $\mathrm{MN}$ and $\mathrm{MN}_{3}$ structures by combination of $\mathrm{u}, \mathrm{v}, \mathrm{x}$ and $\mathrm{y}$ units in [220] is shown in Fig.10. The $\mathrm{CdAu}_{3}$ structure e.g. can be obtained by combination of these units in the sequence uv (Table 3). The theoretical structures at the left-hand border of Fig.9 and the LiRh structure consist of alternating layers of $\mathrm{M}$ and $\mathrm{N}$ atoms. Most of the other structures have layers with identical composition and environment of the $\mathrm{M}$ atoms in these layers (Table 3). Therefore these structures can also be described by the type and the stacking sequence of hexagonal layers. Layers of $\mathrm{N}=\mathrm{Ag}$ or $\mathrm{Au}$ atoms are alternating in $\mathrm{SbAg}_{3}$ $\left(\mathrm{SbAg} / \mathrm{Ag}_{2}\right)$ and $\mathrm{SnAu}_{5}\left(\mathrm{SnAu}_{2} / \mathrm{Au}_{3}\right)$, to obtain the proper composition. All structures which can be obtained by combination of structural units are considered as a structure family with the name of the pioneers simi- 

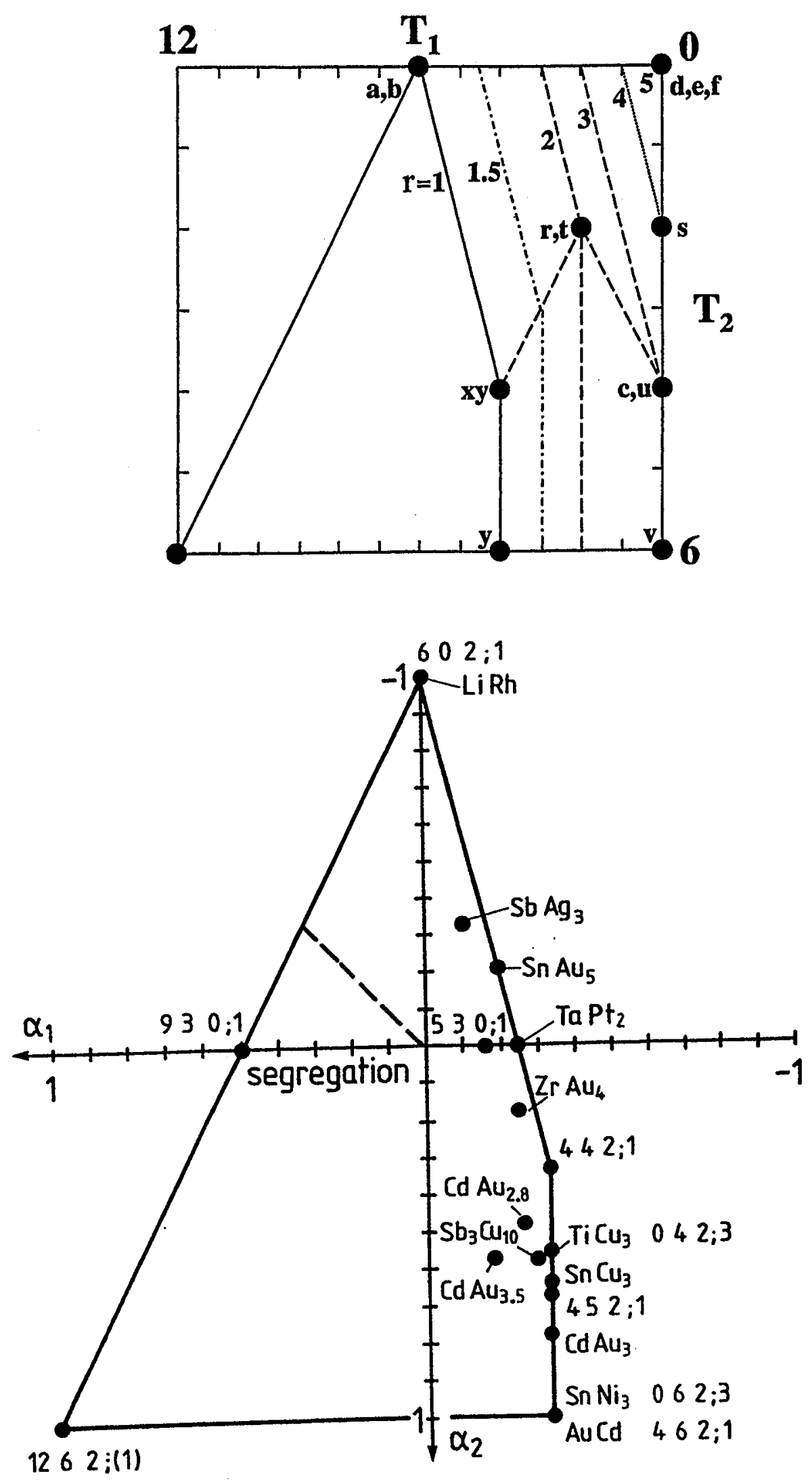

Figure 9: $T_{1}, T_{2}\left(\right.$ a) and $\alpha_{1}, \alpha_{2}$ (b) structure map of ordered hcp structures with structural units $\mathrm{a}-\mathrm{y}$. 


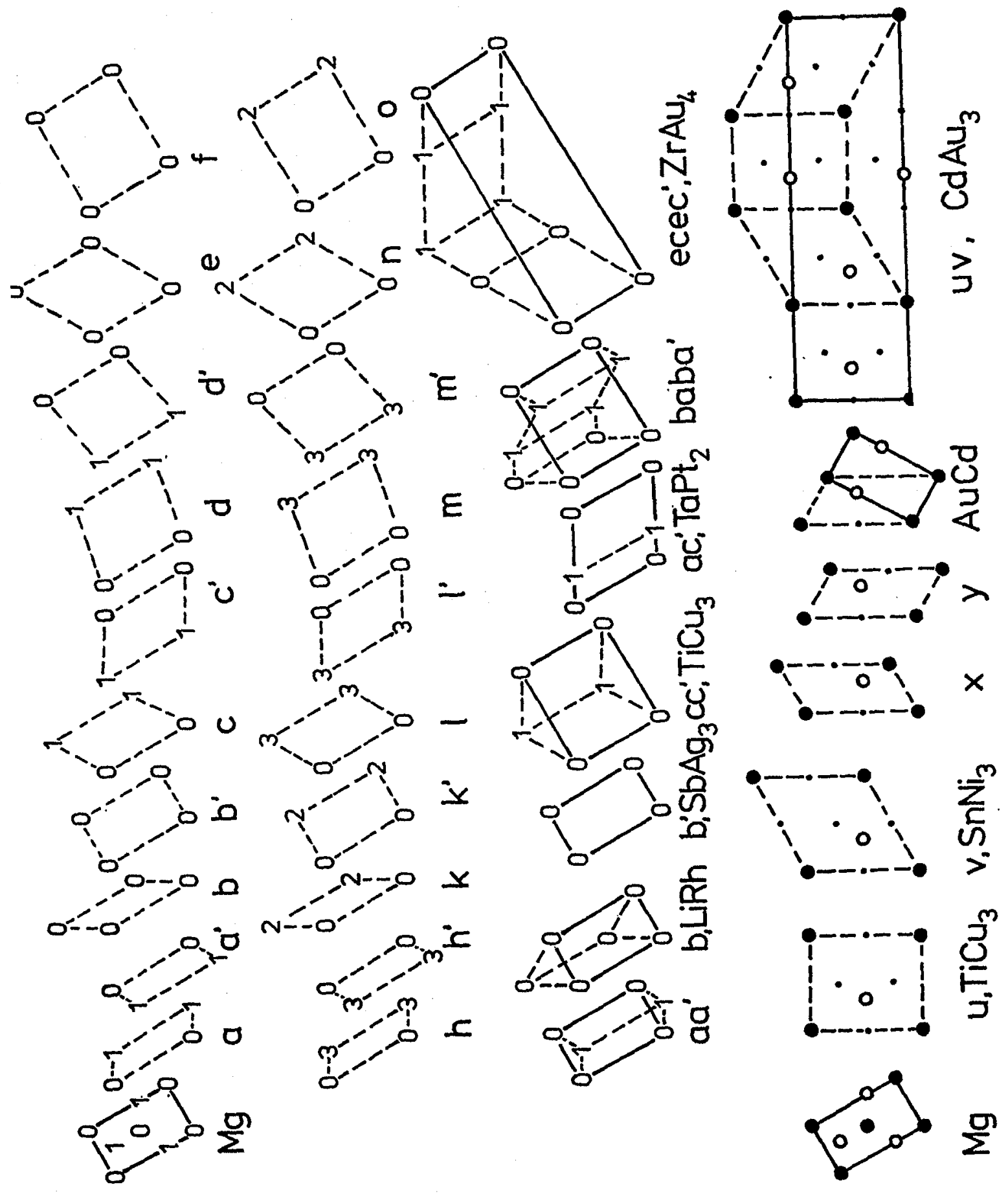

Figure 10: Architecture of hcp structures at the right-hand border of the structure map from structural units a-y. The projection height is given as the layer number for $a-o$. $M$ atoms $(\bullet)$ and $N$ atoms $(\cdot)$ of first, only $M$ atoms (o) of second layer are shown for $\mathrm{u}, \mathrm{v}, \mathrm{x}, \mathrm{y}$ structural units with two layers. 
lar as the Ruddlesden-Popper or Aurivillius phases. The $\mathrm{VAu}_{2}$ or $\mathrm{TaPt}_{2}$ and $\mathrm{ZrAu}_{4}$ structures (Stolz and Schubert, 1962) and the $\mathrm{SnCu}_{3}, \mathrm{CdAu}_{3}$ and (Cd,In)Aus structures (Schubert, 1964) are the first examples for a combination of structural units. The Stolz, Schubert family with structural units $a-o$ and the Schubert family of structures with structural units $\mathrm{u}-\mathrm{y}$ exhibit different $T_{2}$ values $(0-4$ and $4-6$, respectively) (Table 3).

Only few of the possible $\mathrm{MN}_{\mathrm{y}}$ alloy structures are realized as alloys like $\mathrm{LiRh}, \mathrm{SbAg}_{3}, \mathrm{SnAu}_{5}$ (Table 3). Many other theoretical structures have a higher symmetry than the experimental structures (Section 33) (Hauck and Mika, 1999). All alloy structures (except $\mathrm{Cd}_{26} \mathrm{Au}_{72}$ and $\mathrm{Cd}_{12} \mathrm{Au}_{42}$ with $T_{i}$ values in brackets (Table 3)) are at the border of the structure map with maximum interactions between $M$ atoms. The theoretical structures $602 ; 1 \mathrm{~b}$ and $442 ; 1$ (Table 3 ) at corners of the structure map are candidates in a search for new alloy structures. The structures, which are not at the border of the structure map, are supposed to be metastable at low temperatures.

The $602 ; 1 \mathrm{a}(\mathrm{LiRh})$ and $602 ; 1 \mathrm{~b}$ structures are not homometric (identical $T_{i}$ for all atoms for all $i$ ), but differ in the fourth and higher coordination shells (Section 8). These structures at the same location on the structure map with very little differences of the lattice energy are distinguished by $T_{4}, T_{5}$ etc. values or the letters $\mathrm{a}, \mathrm{b}, \mathrm{c}$. The $T_{i}$ values $(i=1-3)$ are usually sufficient to characterize a crystal structure with $\mathrm{y} / \mathrm{x} \leq 5$. The $T_{1}$ and $T_{2}$ values are used for structure maps as a first approximation. Usually the $T_{1}$ and $T_{2}$ values of the structure families vary with the ratio of structural units.

\section{Cubic close-packed structures}

The positions of $\mathrm{Cu}$ atoms in cubic close-packed (ccp) Cu (Table 1) can be substituted by different atoms $M$ and $N$ in $M_{x} N_{y}$ alloys. The reduced rhombohedral cell containing one $\mathrm{Cu}$ atom (instead of four in the face- 
centered unit cell) is increased systematically (Section 33) (Hauck and Mika, 1999), and the metal positions are occupied to an upper limit of $50 \%$ by $\mathrm{M}$ atoms. The structures with a higher percentage of $\mathrm{M}$ atoms are identical at an exchange of $\mathrm{M}$ and $\mathrm{N}$ atoms. The s-CN or averaged $\mathrm{s}-\mathrm{CN}$ values of the minority component $\mathrm{M}$ are determined to characterize the structures by $T_{1} T_{2} T_{3} ; \mathrm{y} / \mathrm{x}$ (Table 4 ). The $T_{1}$ and $T_{2}$ values were plotted in the structure map (Fig.11a) with the same $T_{1}$ and $T_{2}$ values as the hcp structure map (Fig.9a).

Each metal atom of the ccp structure has $T_{1}^{\max }=12, T_{2}^{\max }=6$ and $T_{3}^{\max }=24$ first-, second- and third-nearest neighbors at distances $d, \sqrt{2} d$ and $\sqrt{3} d$ (Fig.1, Table 2). The maximum $T_{1}, T_{2}, T_{3}$ values are reduced in $\mathrm{M}_{\mathrm{x}} \mathrm{N}_{\mathrm{y}}$ alloys where $\mathrm{y} / \mathrm{x} \geq 1$. There are 28 different structures with a single environment of $M$ atoms (Section 33) (Hauck et al., 1988a). The $12624 ;(1), 6012 ; 1 \mathrm{a}, \mathrm{b}, 4416 ; 1$ and $468 ; 1$ (and $060 ; 3$ ) structures are the limiting structures (Figs. 11,12). The $6012 ; 1 \mathrm{a}$ and $6012 ; 1 \mathrm{~b}$ structures are homometric structures - structures with identical $T_{i}$ values, but different symmetry (Section 8 ). The $060 ; 3$ $\mathrm{AuCu}_{3}$ structure and the $468 ; 1 \mathrm{CuAu}$ structure are homologous with identical $\alpha_{i}$ values (Section 9). These structures can be compared with the hcp $062 ; 3 \mathrm{SnNi}_{3}$ and $462 ; 1 \mathrm{AuCd}$ structures for the same $T_{1}$ and $T_{2}$ values (Fig.9). The $4416 ; 1 \mathrm{UPb}$ and the $048 ; 3 \mathrm{TiAl}_{3}$ structures correspond to the hcp $442 ; 1$ and $042 ; 3 \mathrm{TiCu}_{3}$ structures. $\mathrm{PtV}$ crystallizes in the $\mathrm{CuAu}$ or AuCd structure (Villars and Hulliger, 1987). The $4416 ; 1 \mathrm{UPb}$ structure is identical to the NbP structure (Villars and Calvert, 1986). The architecture of structures along the $12624 ;(1)-$ 6012 ; 1a boundary is analogous to that for the hcp structures. The $\mathrm{M}$ and $N$ atoms are completely segregated in 12624 ; (1) with decreasing thickness of hexagonal layers progressing along the boundary (Table 4). Close-packed planes of $\mathrm{M}$ and $\mathrm{N}$ atoms alternate in $6012 ; 1 \mathrm{a} \mathrm{CuPt}$ as in the hcp $602 ; 1$ a LiRh structure. The ccp structures at low $T_{1}$, including most experimental structures, can be assembled from square layers rather than from hexagonal layers alone as is the case for hcp structures (Fig.12). The structures at the upper right-hand border $\left(4 \alpha_{1}\right.$ $\left.+\alpha_{2}+1=0\right)$ can be assembled by two sets of structural units $a-i$. The $\mathrm{NaCl}$ related structure of $\mathrm{Al}_{2} \mathrm{Cl}_{3}\left(\mathrm{ac}^{\prime}\right)$ with two vacancies $\square$ in the ccp metal lattice (Ketelaar, 1935) and $\mathrm{Mo}_{3} \mathrm{Al}_{8}$ (hgh) (Forsyth and Gran, 

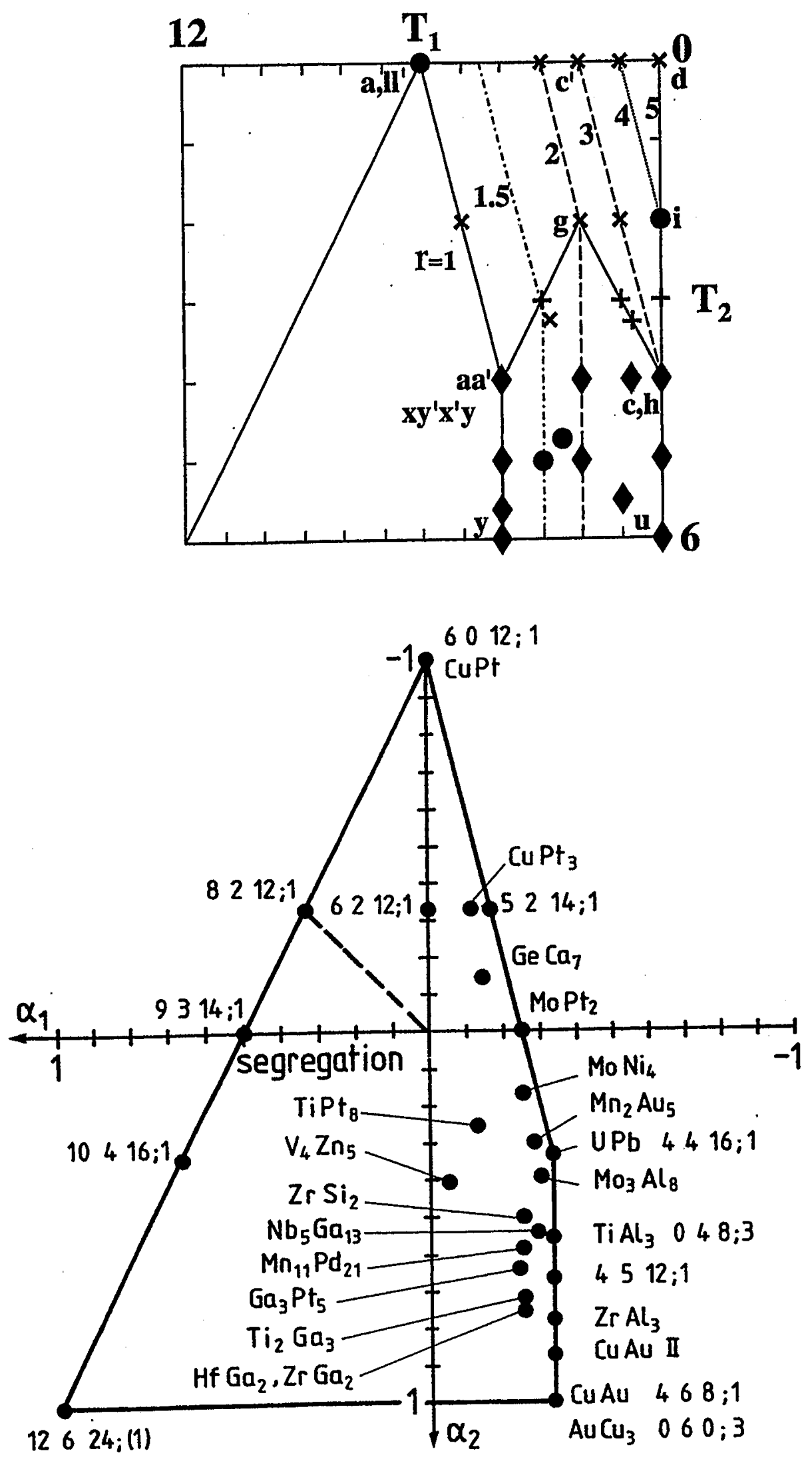

Figure 11: $T_{1}, T_{2}$ (a) and $\alpha_{1}, \alpha_{2}$ (b) structure map of ordered ccp structures of the Brauer $(\bullet)$, Ketelaar $(\times)$, Forsyth, Gran families $(+)$ and others $(\bullet)$. 


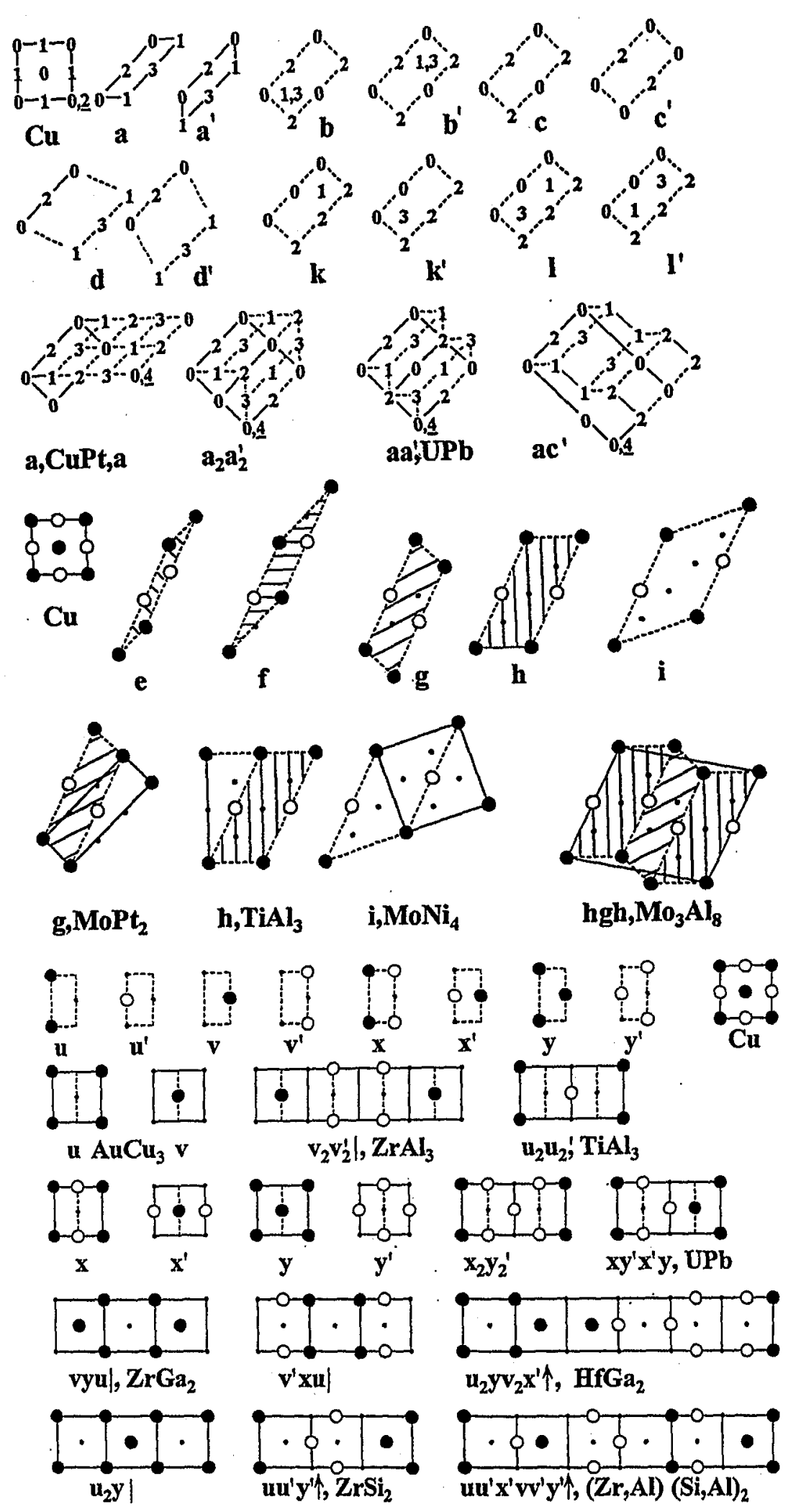

Figure 12: Architecture of $\mathrm{ccp}$ structures from a $-\mathrm{y}$ structural units and structures with two square layers with $M$ atoms at $z=0(\bullet)$ and $z=1 / 2(0)$. Positions of $N$ atoms at $\mathrm{z}=0(\cdot)$ for pattern recognition. The projection height of $\mathrm{M}$ atoms is shown for a 1 units with more than two layers. The asymmetrical part of the string of structural units is given for sequences with mirror plane (|) or glide plane ( $\uparrow)$. 
1962) are the first experimental examples, which can be obtained from combination of a and $c^{\prime}$ or $h$ and $g$ structural units, respectively. The ccp $\mathrm{Na}$ positions of the $\mathrm{NaCl}$ structure are substituted by $\mathrm{M}=\mathrm{Al}$ and $\mathrm{N}=\square$ in $\mathrm{Al}_{2} \mathrm{Cl}_{3}$. Therefore the $\mathrm{NaCl}$ related structures with $\mathrm{M}$ and $\mathrm{N}$ atoms at $\mathrm{Na}$ positions can be characterized by the s-CN values of $\mathrm{M}$ atoms (Section 18). The same applies for $\mathrm{ZnS}$ related structures as e.g. $\mathrm{CuFeS}_{2}$ with the s-CN values $4416 ; 1$ of $\mathrm{Cu}$ and Fe atoms (Hauck and Mika, 1998b). The compositions of the structural units are $\mathrm{M}_{\mathrm{x}} \mathrm{N}_{\mathrm{y}}$ with $\mathrm{y} / \mathrm{x}=1(\mathrm{a}, \mathrm{b}, \mathrm{e}, \mathrm{f}, \mathrm{l}), 5 / 3(\mathrm{k}), 2(\mathrm{~g}), 3(\mathrm{c}, \mathrm{h}), 4(\mathrm{i})$ and $5(\mathrm{~d})$.

The $060 ; 3,048 ; 3,468 ; 1$ and $4416 ; 1$ structures can be split into structural units $\mathrm{u}, \mathrm{v}, \mathrm{x}$ and $\mathrm{y}$, which can be combined like the parts of a puzzle to obtain the crystal structures found at the right-hand border (Figs. 11,12). The $\mathrm{u}^{\prime}, \mathrm{v}^{\prime}, \mathrm{x}^{\prime}$ and $\mathrm{y}^{\prime}$ units are obtained from the $\mathrm{u}, \mathrm{v}, \mathrm{x}$ and $\mathrm{y}$ units by a variation of the origin. The $\mathrm{ZrAl}_{3}$ structure (Brauer, 1939) is obtained from $\mathrm{v}$ and $\mathrm{v}^{\prime}$ units and the CuAu II structure (Johansson and Linde, 1936) from a combination of the $\mathrm{x}$ and $\mathrm{y}$ structural subunits. The $\mathrm{MN}_{2}$ structures $\mathrm{ZrGa}_{2}, \mathrm{HfGa}_{2}, \mathrm{ZrSi}_{2}$, and the $\mathrm{Nb}_{5} \mathrm{Ga}_{13}$ structure are obtained by combination of $\mathrm{u}, \mathrm{v}, \mathrm{x}$ and $\mathrm{y}$ units (Table 4). The sequences of structural units are also observed in $\mathrm{NaCl}$ related structures like $\square \mathrm{Nb}_{3} \square \mathrm{O}_{3}\left(\mathrm{u}_{2}\right), \mathrm{MoO}_{3}\left(\mathrm{uxv}^{\prime} \mathrm{x}^{\prime} \mathrm{u}^{\prime}\right), \mathrm{Nb}_{3} \mathrm{O}_{7} \mathrm{~F}\left(\mathrm{u}_{2} \mathrm{xv}_{4}^{\prime} \mathrm{xu}_{2}\right)$ and $\mathrm{R}-\mathrm{Nb}_{2} \mathrm{O}_{5}$ $\left(\mathrm{xv}_{2}^{\prime} \mathrm{xu}_{2}\right.$ ) (Hyde and Andersson, 1989). Some of these structures are usually described by antiphase boundaries or shearing. The uu' $\mathrm{y}^{\prime}$ structural units e.g. of $\mathrm{ZrSi}_{2}$ with $u^{\prime} \mathrm{y}^{\prime} \mathrm{v}^{\prime}$ vy sequence can be shifted to the $\mathrm{v}^{\prime} \mathrm{vy}$ units as is indicated by an arrow in Fig.12. Other sequences like vyu| in $\mathrm{ZrGa}_{2}$ (vyuuyv) are symmetrical. The symbols | for a mirrorplane or $\uparrow$ for the translation by $a / 2 a / 2$ (shear) are useful in particular for long sequences of structural units.

Most of the experimental structures are at boundary lines for the given composition. The only exception in Table 4 is $\mathrm{V}_{4} \mathrm{Zn}_{5}$, which lies at the boundary line of the structure map belonging to the hexagonal layer with the values $2.51 .52 .5 ; 1.25$ (Fig.5). Structures, which are not at the boundary line of the structure map, are supposed to be unstable at low temperatures. with respect to other structures at the boundary with maximum interactions between $M$ atoms. Sometimes $M$ atoms on one position are disordered, in other cases several positions are disordered 
(Hauck and Mika, 1999) and give rise to decreased $\alpha_{i}$ values.

The structures with a single environment of $\mathrm{M}$ atoms can be characterized by square or hexagonal layers (Section 4 ) with small unit cells:

\begin{tabular}{|l|ll|l|}
\hline & \multicolumn{2}{|c|}{ square layers } & hex. layers \\
\hline $\mathrm{CuAu}$ & $044 ; 1 \quad 444 ;(1)$ & $226 ; 1$ \\
$\mathrm{UPb}$ & $044 ; 1 \quad 220 ; 1$ & $242 ; 1$ \\
$\mathrm{AuCu}_{3}$ & $044 ; 1 \quad 444 ;(1)$ & $006 ; 3$ \\
$\mathrm{TiAl}_{3}$ & $044 ; 1$ & $444 ;(1) \quad 020 ; 3$ & $022 ; 3$ \\
\hline
\end{tabular}

The structures with $M^{i}>2$ (Table 4) of the Johansson, Linde and Brauer family as e.g. the $\mathrm{CuAu} \mathrm{II}$ or $\mathrm{ZrAl}_{3}$ structure at the right-hand boundary of the structure map (Fig.11b) consist of $\mathrm{u}, \mathrm{v}, \mathrm{x}, \mathrm{y}$ structural units, which are connected by $044 ; 1$ square planes. The other layers of the $\mathrm{CuAu}-\mathrm{UPb}$ and $\mathrm{AuCu}_{3}-\mathrm{TiAl}_{3}$ parent structures do not fit together. The structural units of many structures on the right-hand side of the structure map (Fig.11b) are connected by $204 ; 1$ or $044 ; 1$ square planes (Table 4). Most (theoretical) structures on the left-hand side of the structure map are connected by $666 ;(1)$ hexagonal planes.

The $\alpha_{1}, \alpha_{2}$ values of the different layers of a structure are sometimes similar to the $\alpha_{1}, \alpha_{2}$ values of the structure itself (Figs. 5b,7b,11b) and are located in the same area of the structure map. An example is the $204 ; 1$ square and $226 ; 1$ hexagonal layer of the $6012 ; 1 \mathrm{a} \mathrm{CuPt}$ structure. In other structures as e.g. $2212 ; 2 \mathrm{a} \mathrm{MoPt}_{2}$, the $020 ; 2$ and $202 ; 2$ square layers or the $202 ; 2$ and $060 ; 2$ hexagonal layers are at different locations of the structure map, probably because of the directional bonding of Mo atoms (Hauck and Mika, 1994).

Some metals like bcc Mo, which do not form close-packed structures because of directional bonding of the outer electrons, form close-packed structures in alloys as e.g. cubic close-packed $\mathrm{MoNi}_{4}$ or $\mathrm{MoPt}_{2}$. In other 
examples the directional interactions between metal atoms are increased by the formation of an alloy. The CuPd or FeTi alloys e.g. crystallize in an ordered body-centered cubic structure in spite of the close-packed structures of the components. Other pairs of metals like $\mathrm{Cu}$ and $\mathrm{Rh}$ with the ccp structure for each metal do not form compounds at all but segregate as two limited solid solutions. The s-CN values approach $12624 ; 0$ for complete segregation at $y / x=0$.

\section{Ordered structures of complex close-packed al- loys}

The structure maps for hcp and ccp ordered alloys $\mathrm{M}_{\mathrm{x}} \mathrm{N}_{\mathrm{y}}$ are identical with the same numbers of $T_{1}$ and $T_{2}$ values (Figs. 9,11). The comparison of Table 3 and 4 shows many experimental structures with identical $T_{1}$ and $T_{2}$ values. Therefore also the $T_{1}, T_{2}$ structure maps of structures with a complex stacking of $h$ and $c$ layers (Table 1) are identical. The structures with identical $T_{1}$ and $T_{2}$ values exhibit the same kind of hexagonal layers (Table 5). They can be described by the stacking symbol and the ordering of $\mathrm{M}$ and $\mathrm{N}$ atoms in the hexagonal planes. The $T_{3}$ and $T_{4}$ values can be obtained from the fraction $f$ of $h$ layers as was outlined in Section 2.

The complex close-packed metals (Table 1) are similar to hcp stacking with only one direction of packing of the hexagonal layers at occupation with $\mathrm{M}$ atoms only. All structures of Table 1 contain a 3-fold axis in $c$ direction of the stacked layers at occupation with $M$ atoms only. The metal atoms of the complex structures of Table 1 have already two or more different environments. The architecture of many hcp, ccp and complex close-packed $\mathrm{M}_{\mathrm{x}} \mathrm{N}_{\mathrm{y}}$ alloys can be characterized by hexagonal layers with composition $\mathrm{M}_{\mathrm{x}} \mathrm{N}_{\mathrm{y}}$ (instead of $\mathrm{M}$ atoms) and the stacking of these layers similar as in Table 1. These structures can be divided in five major groups corresponding to different $\alpha_{1}, \alpha_{2}$ values and different hexagonal layers (Table 5) (Beck, 1969; Zhao et al., 1991; Parthé et al., 1993). The hcp $\mathrm{CdAu}_{3}$ structure e.g. with $\alpha_{1}=-0.33, \alpha_{2}=0.78$ 
is gradually altered to the ccp $\mathrm{ZrAl}_{3}$ structure with different stacking of $014 ; 3$ hexagonal layers by variation of the Au content or partial substitution of $\mathrm{Au}$ by $\mathrm{In}$ in the series: $\mathrm{CdAu}_{3}, \mathrm{Cd}(\mathrm{Au}, \mathrm{In})_{2.8}=\mathrm{Cd}_{26}(\mathrm{Au}, \mathrm{In})_{72}$ $\left(\mathrm{Cd}_{14} \mathrm{In}_{8} \mathrm{Au}_{78}\right), \mathrm{Cd}(\mathrm{Au}, \mathrm{In})_{3}=\mathrm{Cd}_{144}(\mathrm{Au}, \mathrm{In})_{432}\left(\mathrm{Cd}_{15} \mathrm{In}_{10} \mathrm{Au}_{75}\right)$ (Wegst and Schubert, 1958; Schubert, 1964) (Table 5). The ccp 008 ; 5 structure corresponding to hcp $\mathrm{SnAu}_{5}$ and $(\mathrm{ch})_{2} \mathrm{WAl}_{5}$ is only observed in the $\mathrm{NaCl}$ related $\mathrm{V}_{6} \square \mathrm{C}_{5}\left(\mathrm{~V}_{6} \mathrm{C}_{5}\right)$ structure with $\mathrm{M}=\square$ and $\mathrm{N}=\mathrm{C}$ (Section 18). The $\mathrm{MN}_{5}$ composition is obtained by alternating $\mathrm{MN}_{2}$ und $\mathrm{N}_{3}$ layers.

\section{Structures with identical powder patterns}

Some ccp and hcp structures are homometric with identical coordination $T_{i}$ of all atoms for all $i$ (Fig.13) and some other structures differ in higher coordination spheres (Hauck et al., 1988a). The homometric structures cannot be distinguished by powder diffraction methods, if the lattice is undistorted (Patterson, 1944). Other structures are enantiomorphous, i.e. the crystal structures contain right- or left-hand screw axes which cannot be distinguished by powder diffraction. The homometric, but non-enantiomorphous, structures are characterized by $a, b$, c, .... The hcp 530 ; 1a,b structures (Fig.13) are homometric, but differ from the homometric $530 ; 1 \mathrm{~d}$,e structures in the 4-th and higher coordination shells. The hcp 602 ; 1a,b structures with structural units $a a^{\prime}$ or $b$ (LiRh) (Fig.10) differ from the 4-th coordination onward. The ccp $2212 ; 2 c$ structure (Section 18) is different from the homometric $2212 ; 2 \mathrm{a}, \mathrm{b}$ structures (Fig.13) in the 6-th and higher coordination shells. The crystal structures $a, b, c, \ldots$ with identical positions on the structure maps (Figs. 9,11) are stabilized by about the same amount of lattice energy and are sometimes coexisting as e.g. the $008 ; 5 \mathrm{~b}, \mathrm{e}, \mathrm{f} \mathrm{V}_{6} \mathrm{C}_{5}$ stacking variants (Section 18). The homometric structures (Fig.13) can also be considered as stacking variants, where square or hexagonal layers are stacked perpendicularly to the projection plane with different translations $\mathrm{x}, \mathrm{y}, \mathrm{z}$ (Section 16). 


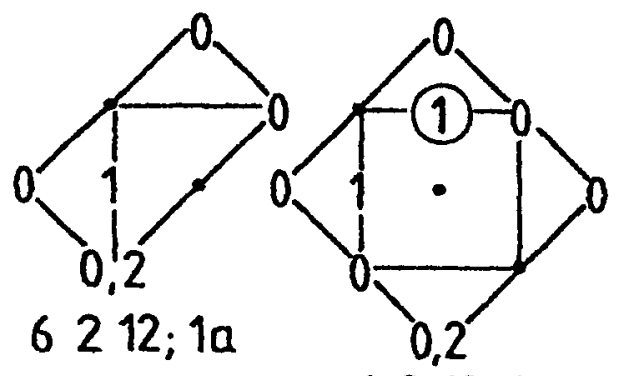

$6212 ; 1 b$
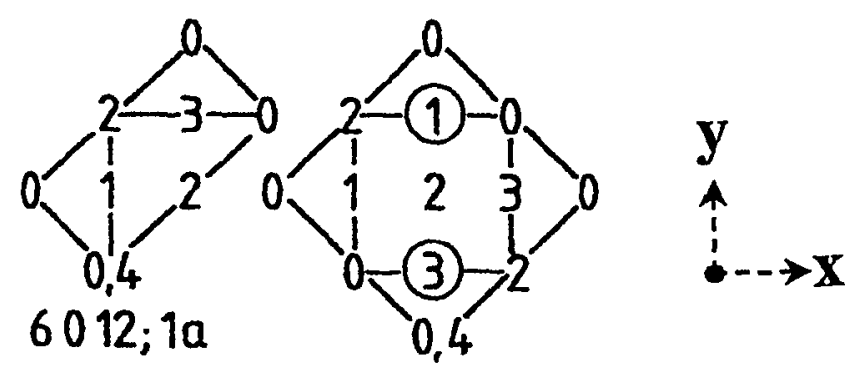

\section{$\mathrm{CuPt}$}

$6012 ; 16$

$y_{r}$

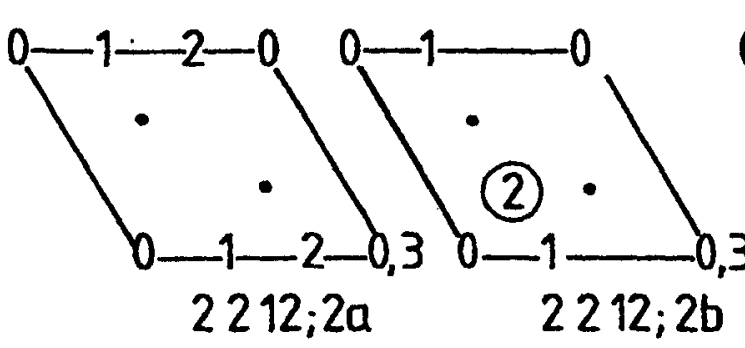

$\mathrm{MoPt}_{2}$

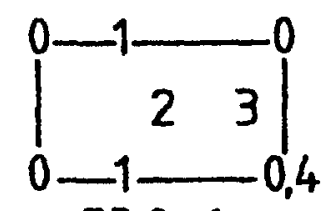

$530 ; 10$

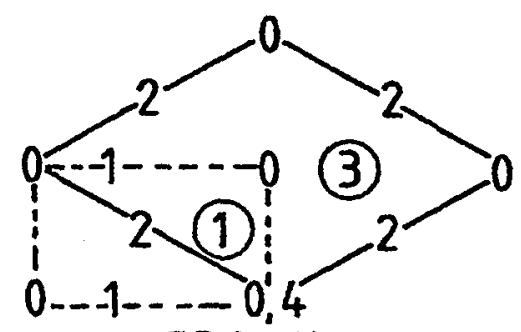

$530 ; 1 b$

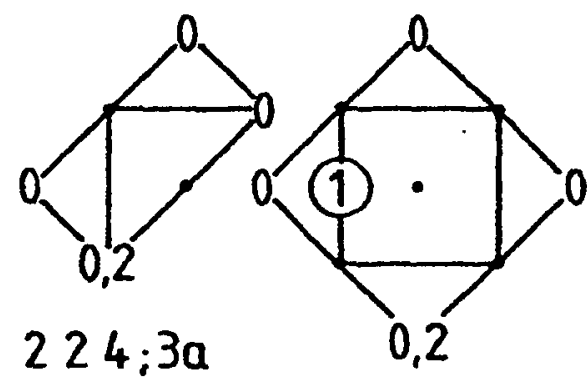

$224 ; 3 b$

Figure 13: Examples of homometric pairs of stuctures with identical self-coordination numbers $T_{i} .6212 ; 1 \mathrm{a}, \mathrm{b}, 6012 ; \mathrm{la}, \mathrm{b}, 2212 ; 2 \mathrm{a}$,b and $224 ; 3 \mathrm{a}$,b are ccp, $220 ; 2 \mathrm{a}$,b, $530 ; 1 \mathrm{a}, \mathrm{b}$ are hcp. The positions of $\mathrm{M}$ atoms are given by the projection height. The different $\mathrm{M}$ positions of homometric pairs are encircled, $\mathrm{N}$ atoms at $\mathrm{z}=0(\cdot), \mathrm{x}, \mathrm{y}, \mathrm{z}$ coordinates for the translation of identical layers. 
There are also homometric structures of close-packed metal atoms with a different stacking sequence but identical $T_{i}$ values (Mardix, 1990), e.g. hcchchcchhchchh,

hochchchhcchhhc,

which are two different structures containing the same unit cell with 15 layers, the same percentage $53.3 \%$ of $h$ layers in the same space group P3m1. Other structures of Table 1, e.g. $9 \mathrm{a}$ and $9 \mathrm{c}$ or $9 \mathrm{~d}$ and $9 \mathrm{e}$ have identical $T_{i}$ for $i=1-8$ but differ in $T_{9}$ and higher coordination shells. They are quasi-homometric with very little difference of the lattice energy as e.g. the Madelung factors of the $\mathrm{NaCl}$ related structures (Hauck et al., 1988b).

\section{Homologous series of structures}

A second type of special structures exhibits identical $\alpha_{i}$ values but at different composition $y / x$. These structures have identical or closely related unit cells and form homologous series of structures which are filled up successively by $M$ atoms until $T_{i}^{\max }$ is reached (Table 6). Each $M$ and each $\mathrm{N}$ atom of these structures must have the same set of numbers $T_{i}$, respectively. The structure with the lowest $M$ content $y / x=r^{*}$, $T_{1}^{*} T_{2}^{*} T_{3}^{*} ; \mathrm{r}^{*}$ is filled up with $\mathrm{M}$ atoms in steps of $\mathrm{k}=\mathrm{r}^{*}, \mathrm{r}^{*}-1, \ldots, 0$ (Hauck et al., 1988a):

$$
\begin{aligned}
T_{i}^{\mathrm{k}} & =T_{i}^{\max }-\left(T_{i}^{\max }-T_{i}^{*}\right) \mathrm{k} / \mathrm{r} \\
\mathrm{y} / \mathrm{x} & =\mathrm{k} /\left(\mathrm{r}^{*}+1-\mathrm{k}\right) .
\end{aligned}
$$

The homologous series of structures contain also structures with $\mathrm{M}$ and $\mathrm{N}$ atoms interchanged as e.g. $\mathrm{AuCu}_{3}$ and $\mathrm{Cu}_{3} \mathrm{Au}$ with $\mathrm{Cu}$ at $\mathrm{Au}$ positions in $\mathrm{CuAu}_{3}$ (Table 6). There are three homologous series for $\operatorname{ccp}\left(\mathrm{r}^{*}=2\right.$, $3,4)$ and two series for hcp $\left(\mathrm{r}^{*}=2,3\right)$ (Table 6$)$. The $\mathrm{r}^{*}=3$ series for the ccp $060 ; 3 \mathrm{AuCu}_{3}$ and $468 ; 1 \mathrm{CuAu}$ structures corresponds to the hcp $062 ; 3 \mathrm{SnNi}_{3}$ and $462 ; 1 \mathrm{AuCd}$ series at identical $\alpha_{1}, \alpha_{2}$ values (Figs. 9b,11b). The ccp $2212 ; 2 a, b$ structures (Fig.14) can be compared with the hcp $220 ; 2 \mathrm{a}, \mathrm{b}$ (Fig.13) or $222 ; 2 \mathrm{TaPt}_{2}$ (Fig.10) structures (Hauck et al., 1988a). The pairs of closely related ccp structures $048 ; 3$ 


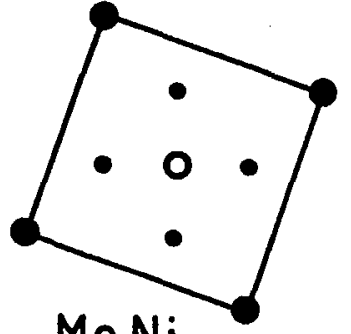

$\mathrm{MoNi}_{4}$

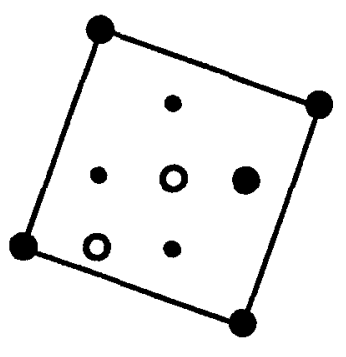

$3312 ; 1.5$

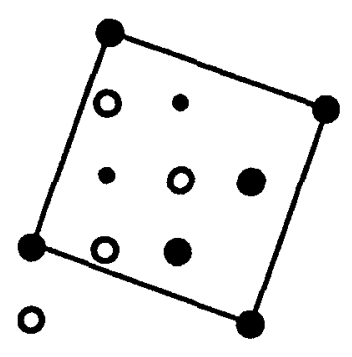

$6416 ; 0.67$

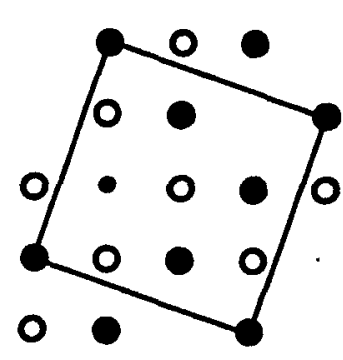

$9520 ; 0.25$

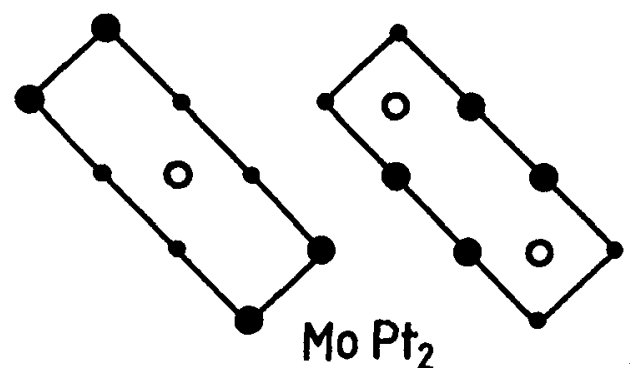

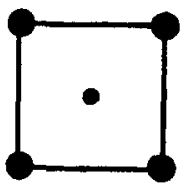

$\mathrm{AuCu}$

$060 ; 3$

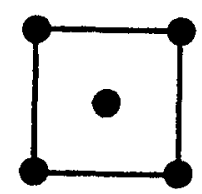

$\mathrm{CuAu}$

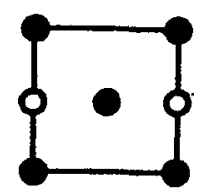

$8616 ; 0.33$

$468 ; 1$

$2212 ; 2 \quad 7418 ; 0.5$

Figure 14: Examples of ccp homologous crystal structures with identical $\alpha_{i}$ values at different concentration $y / x . M$ atoms at $z=0(\bullet)$ and $z=0.5(\circ)$. Positions of $N$ atoms $(\cdot)$ at $\mathrm{z}=\mathbf{0}$ for pattern recognition. 
$\mathrm{TiAl}_{3}$ and $4416 ; 1 \mathrm{UPb}$ (Fig.12) and the corresponding hcp $042 ; 3$ $\mathrm{TiCu}_{3}$ and $442 ; 1 \mathrm{MN}$ structures with structural units baba' or xy (Fig.10) are quasi-homologous because of different $\alpha_{2}$ values. The $T_{i}$ values of quasi-homologous structures are at the border of the $T_{1}, T_{2}$ structure map at the corresponding $\mathrm{y} / \mathrm{x}$ values. A third group of related structures (homologous II series) exhibits identical $\alpha_{i}$ values like the $002 ; 6,(123 ; 5 / 2),(244 ; 4 / 3),(365 ; 3 / 4),(486 ; 2 / 5),(5107 ; 1 / 6)$, $6128 ; 0$ series of the primitive cubic lattice (Section 22). Only the structure with the maximum $\mathrm{r}^{*}=\mathrm{y} / \mathrm{x}$ value $002 ; 6$ is at the border of the $T_{1}, T_{2}$ structure map. The other values in brackets are inside of the structure map. The same applies to the large number of other structure series. The $\mathrm{TiPt}_{8}$ and $\mathrm{V}_{4} \mathrm{Zn}_{5}$ structures with different $\alpha_{i}$ values crystallize even in the same space group at different $y / x$ and can be considered as one structure type (Villars and Calvert, 1986), if the $V_{4} Z_{n_{5}}$ structure is written as $\mathrm{Zn}\left(\mathrm{V}_{4} \mathrm{Zn}_{4}\right)$. The $\mathrm{V}_{4} \mathrm{Zn}_{5}$ structure however is not at the border of the $T_{1}, T_{2}$ structure map.

\section{Symmetry of ordered phases}

The metal atoms of the hcp and ccp lattice have a single environment with a high point symmetry for the metal atoms (International Tables for Crystallography, Hahn, 1987; Villars and Calvert, 1986):

$4 / m \overline{3} 2 / m$ for the $\mathrm{Cu}$ atoms with ccp structure, $\overline{6} m 2$ for the $\mathrm{Mg}$ atoms with hcp structure.

The site symmetry is reduced by distortion (d), e.g. of the Cu lattice in In or $\mathrm{Hg}$ (Table 1), or by different stacking (s), as e.g. the (ch) 2 La or Nd structure, or by formation of ordered structures (o) (Fig.15). Structures with a single environment of all $\mathrm{M}$ and $\mathrm{N}$ atoms at the corners of the structure maps like $\mathrm{AuCu}_{3}$ or $\mathrm{CuAu}$ are usually highly symmetric. Structures containing $M$ atoms with different environments are built from different structural units as e.g. $\mathrm{ZrAl}_{3}, \mathrm{CuAu}$ II or $\mathrm{ZrGa}_{2}$ (Fig.12). The symmetry of these structures is usually lower because of the differ- 

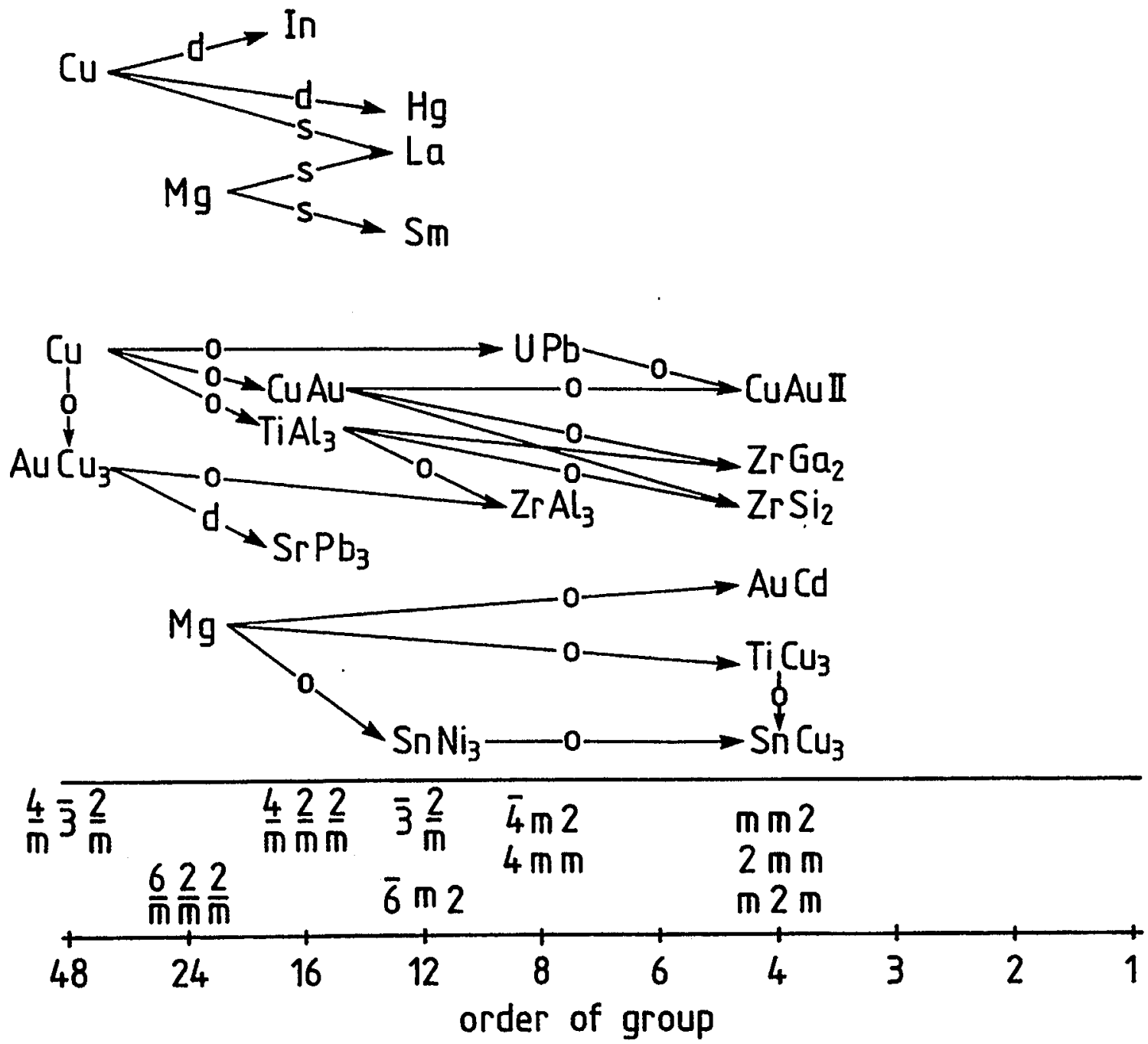

Figure 15: Crystallographic point-group symmetry of $M$ atoms in different ordered alloys $M_{x} N_{y}$. The order of the point symmetry of $M$ atoms is reduced by ordering (o), by stacking (s), e.g. of La atoms in chch layer sequence, or by distortion (d). 
ent symmetry elements of the structural units. The populations of the different structure types obey Pauling's rule of parsimony: The number of essentially different kinds of constituents in a crystal tends to be small (Pauling, 1929). Structure types with a single environment of metal atoms $\mathrm{M}$ and $\mathrm{N}\left(M^{i}=2\right)$ with high symmetry as in the case of hcp alloys with $\mathrm{AuCd}$ or $\mathrm{SnNi}_{3}$ structure (Table 3) or the ccp alloys with $\mathrm{CuAu}, \mathrm{AuCu}_{3}, \mathrm{MoNi}_{4}$ or $\mathrm{MoPt}_{2}$ structure (Table 4) are observed frequently, whereas structures containing metal atoms with different environments as e.g. $\mathrm{CuAu}$ II, $\mathrm{ZrGa}_{2}$ or $\mathrm{Mo}_{3} \mathrm{Al}_{8}$ are rare. The fact, that highly symmetric structures can only be constructed from $\mathrm{M}$ and $\mathrm{N}$ atoms with a single environment shows that the frequent observation of high symmetry (Laves, 1967) is a consequence of Pauling's rule for small numbers of constituents.

In most cases, the experimentally observed symmetry is identical with the symmetry determined for the undistorted lattice. This can be used to find a structural model of the ordered structure from electron diffraction patterns. Alloys with small single crystal domains of ordered structures can be investigated by electron diffraction to determine the unit cell and the symmetry from the diffraction pattern. These values can be compared with the list of theoretical structures to calculate the powder pattern for $\mathrm{x}$-ray or neutron diffraction.

The symmetry of a few ordered alloys as e.g. $\mathrm{NaHg}, \mathrm{CdAu}_{3}$ II, $\mathrm{SiU}_{3}$ or $\mathrm{SrPb}_{3}$ (Table 4) is further decreased due to distortion by directional bonding similar to that in tetragonal In or rhombohedral $\mathrm{Hg}$ compared to cubic $\mathrm{Cu}$ (Table 1 ).

\section{Ising model}

Crystal structures of ordered alloys $\mathrm{M}_{\mathrm{x}} \mathrm{N}_{\mathrm{y}}$ have been calculated for central pairwise interactions $V_{i}, i=1,2,3, \ldots$ with $V_{i}=V_{i}^{\mathrm{MM}}+V_{i}^{\mathrm{NN}}-$ $2 V_{i}^{\mathrm{MN}}$ between nearest, next-nearest, third, etc. metal atoms within the Ising model (Turchi, 1994). The results obtained for the single square 
layer (Kaburagi, 1978), the single hexagonal layer (Kudo and Katsura, 1976; Hiraga and Hirabayashi, 1977), hcp (Kudo and Katsura, 1976) and ccp (Kanamori and Kakehashi, 1977) alloys can be compared with the structures in the structure maps. The present notation $T_{1} T_{2} T_{3} ; \mathrm{y} / \mathrm{x}$ for the self-coordination numbers $T_{i}$ and concentration $\mathrm{y} / \mathrm{x}$ is similar to the notation of the structures used by Kanamori and Kakehashi (1977). The interaction parameters $V_{1}$ and $V_{2}$ between nearest and next-nearest neighbors were varied in the Ising model calculations and the structures obtained by different procedures plotted in a $V_{1}, V_{2}$ coordinate system (de Novion and Landesman, 1985). These plots can be compared with the $T_{1}, T_{2}$ or $\alpha_{1}, \alpha_{2}$ structure maps of Figs. 5, 7, 9 and 11. The parameters $\alpha_{i}$ seem to be almost proportional to $V_{i}$. Only simple structures are obtained by variation of the $V_{1}$ and $V_{2}$ interaction parameters. The complex structures with different environments of $M$ atoms are only found by the additional variation of $V_{3}, V_{4}$, etc. (Ducastelle, 1991). Structures at different positions on the borderline of the structure map, e.g. $\mathrm{CuAu}$ II compared to $\mathrm{CuAu}$, are stabilized by different $V_{3}, V_{4}$, etc., which cause a slightly different $V_{2}$ and $\alpha_{2}$ (Fig.16).

The interaction parameters $V_{i}=0$ for a statistical (random) distribution of $\mathrm{M}$ and $\mathrm{N}$ atoms correspond with $\alpha_{i}=0$. The $\alpha_{i}$ values are positive for attractive interactions between $M$ atoms and negative for repulsive interactions. The structures can be classified within four fields by consideration of $V_{1}, V_{2}$ only: (I) $V_{1}, V_{2}<0$, (II) $V_{1}<0, V_{2}>0$, (III) $V_{1}>0, V_{2}<0$ and (IV) $V_{1}, V_{2}>0$. These areas are subdivided by the diagonals in Ia, Ib, etc. (Fig.16). Most experimental structures are in (I) or (II), with a repulsive interaction $V_{1}$ between nearest-neighbor $M$ atoms. Structures in IIIa and IV, with attractive interactions $V_{1}$ between nearest-neighbor $\mathrm{M}$ atoms, contain already multiple layers of $\mathrm{M}$ and $N$ atoms as a first step towards segregation. Structures with covalent bonding between $M$ atoms are in Ia and IIIb. The structures of IIIb contain clusters, e.g. sheets or rows of $M$ atoms.

The $\mathrm{M}$ atoms of structures in IIb are as far apart as possible, which can be correlated with repulsive interactions $V_{1}$ between nearest $\mathrm{M}$ atoms, e.g. Coulomb repulsion. These structures have the highest Madelung factors (Hauck et al., 1988a). The crystal structures with covalent and 


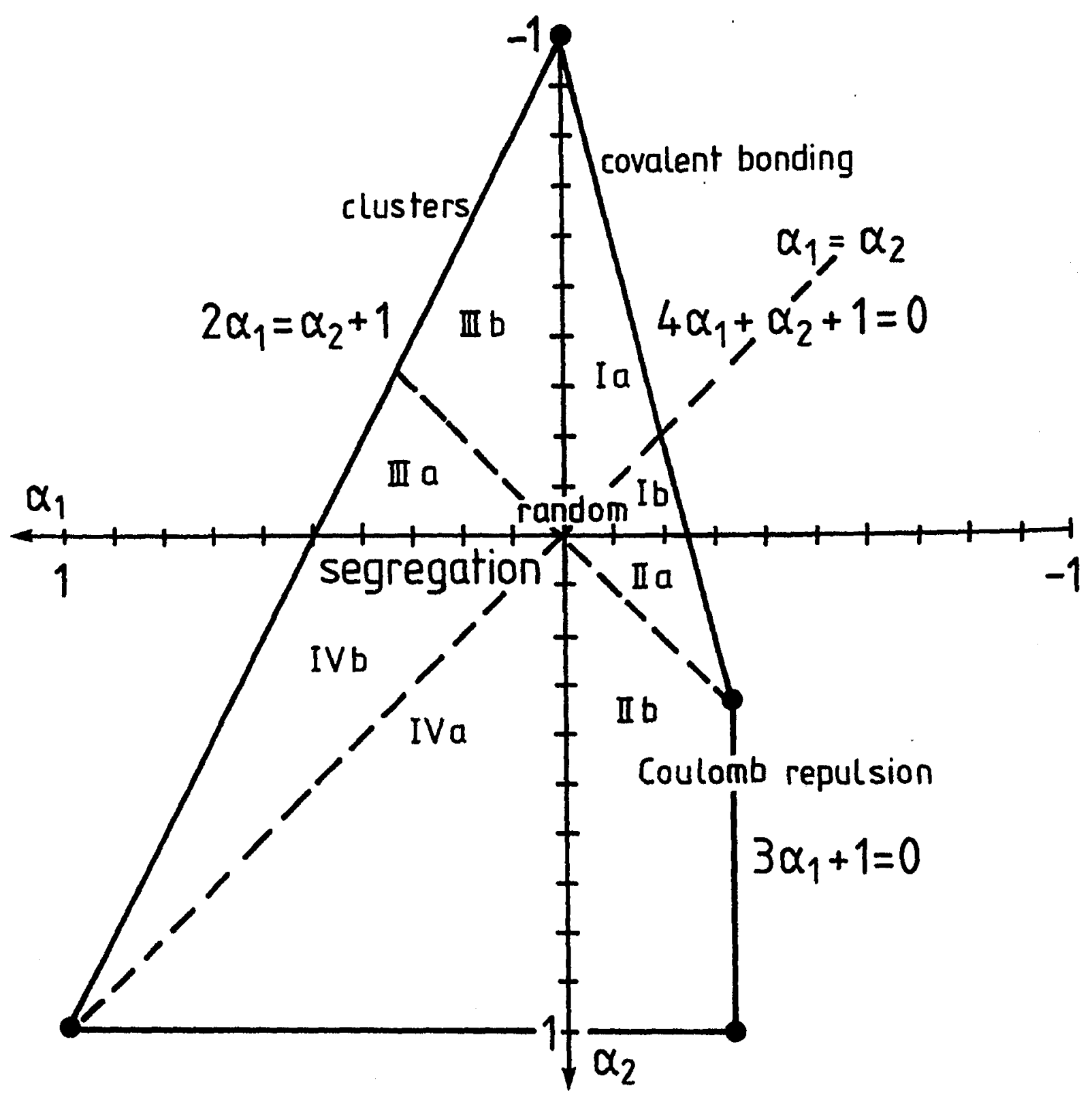

Figure 16: The different areas of the $\alpha_{1}, \alpha_{2}$ structure map of close-packed alloys as derived from the Ising model. 
ionic bonding are separated by the line $\alpha_{1}=\alpha_{2}$. The $\mathrm{M}$ atoms of ionic compounds with low $M$ content are expected at $T_{1}=T_{2}=0$ because of the repulsive interactions. The relation $\alpha_{1}=\alpha_{2}$ however is also valid for covalent compounds with the relation $T_{1} / T_{2}=T_{1}^{\max } / T_{2}^{\max }$ with preferential occupation of the inner coordination shells $T_{1}$ and $T_{2}$. The line $\alpha_{1}=\alpha_{2}$ of dilute alloys intersects the borderline $4 \alpha_{1}+\alpha_{2}+1=0$ of the hcp and ccp structure maps at concentration $y / x=5$. Therefore ionic and covalent compounds with $\mathrm{y} / \mathrm{x} \geq 5$ and $T_{1}=T_{2}=0$ should be on the line $\alpha_{1}=\alpha_{2}$. Ordered ionic compounds with $\mathrm{y} / \mathrm{x}<5$ are expected with increased $T_{2}$, ordered covalent compounds with increased $T_{1}$.

The structures with a maximum interaction are at the outer borderlines. Structures with composition $\mathrm{y} / \mathrm{x}=1,2$ and 5 are on the borderline $4 \alpha_{1}+$ $\alpha_{2}+1=0$ in (I, IIa), structures with $\mathrm{y} / \mathrm{x}=1$ and 3 are on $3 \alpha_{1}+1=$ 0 in (IIb) and structures with all compositions on $2 \alpha_{1}=\alpha_{2}+1$ in (III, IVb). Structures with different composition are in the field, e.g. structures with $\mathrm{y} / \mathrm{x}=2$ are on $4 \alpha_{1}+1=0$ in (II) (Fig.16) or $T_{1}=$ 2 (Fig.11) because of geometrical restrictions of the close-packed metal atoms. Structures with $\mathrm{y} / \mathrm{x}=2$ are less frequent than structures with $y / x=1$ or 3 (Table 4 ). They can be considered as a combination of structural units of composition $\mathrm{y} / \mathrm{x}=1$ and 3 as was outlined before. Other structures inside of the $\alpha_{1}, \alpha_{2}$ field like ccp $6212 ; 1 \mathrm{a}, \mathrm{b}$ have the same composition as structures at the borderline, e.g. $6012 ; 1 \mathrm{a}, \mathrm{b}$, $5214 ; 1$ or $8212 ; 1$ (Fig.11). These structures are supposed to be less favorable than structures at the borderline with maximum interactions between $\mathrm{M}$ atoms.

\section{Characterization of structures by sequences of structural units}

Structures at the left-hand border of the structure map (Figs. 9,11) can be characterized by the sequences of 666 ; (1) hexagonal layers with different metal atoms as e.g. LiRhLiRh for hcp LiRh $(602 ; 1$ a) or CuPtCuPt for ccp CuPt $(6012 ; 1 \mathrm{a})$ (Tables 3,4$)$. 
Structures at the right-hand border can be characterized by sequences of structural units, which are linked by common interfaces as e.g. uvuv $\left(\mathrm{CdAu}_{3}\right), \mathrm{vu}_{4} \mathrm{vu}_{4}\left(\mathrm{SnCu}_{3}\right)(\mathrm{Fig} .10$, Table 3$)$ or $\mathrm{v}_{4} \mathrm{v}_{4}^{\prime}\left(\mathrm{ZrAl}_{3}\right), \mathrm{gh}_{2}\left(\mathrm{Mo}_{3} \mathrm{Al}_{8}\right)$ and $\mathrm{vyu}_{2} \mathrm{yv}\left(\mathrm{ZrGa}_{2}\right)$ (Fig.12, Table 4 ).

The different series of structures allow a characterization of crystal structures by a sequence of structural units as e.g. aa' for the UPb structure, or gh for $\mathrm{Mn}_{2} \mathrm{Au}_{5}$ and hgh for $\mathrm{Mo}_{3} \mathrm{Al}_{8}$, or $\mathrm{v}_{2} \mathrm{v}_{4}^{\prime} \mathrm{v}_{2}$ for $\mathrm{ZrAl}_{3}$ and vyu $2 \mathrm{yv}$ for $\mathrm{ZrGa}_{2}$ (Fig.12). The three-dimensional structures are described by one-dimensional sequences of structural units. These sequences of units can be analyzed by the one-dimensional Ising model (Fig.2) with $T_{1} T_{2} T_{3} ; \mathrm{y} / \mathrm{x}=222 ;(1), 101 ; 1$ and $020 ; 1$ at segregation of $a$ and $b$ structural units, and the sequences aabb or ab for attractive or repulsive interactions, respectively. Chains of more than two structural units like $\mathrm{vyu}_{2} \mathrm{yv}$ for $\mathrm{ZrGa}_{2}$, or $\mathrm{u}_{2} \mathrm{yv}_{4} \mathrm{yu}_{2}$ for $\mathrm{Ce}_{3} \mathrm{Sn}_{7}$ (Table 4) can be analyzed for clusters (C), single (S), double (D), triple $(\mathrm{T})$ and multi $(\mathrm{M})$ rows of structural elements similar as in Fig.2. The one-dimensional Ising model can explain the reason for small numbers of different structural units (Pauling's rule of parsimony) like the examples given before. The longer sequences of structural units like $\mathrm{v}_{2} \mathrm{v}_{4}^{\prime} \mathrm{v}_{2}\left(\mathrm{ZrAl}_{3}\right)$ might segregate in $u_{2}^{\prime} u\left(\mathrm{TiAl}_{3}\right)$ and $\mathrm{u}_{2}$ or $\mathrm{v}_{2}$ structure $\left(\mathrm{AuCu}_{3}\right)$. Most of the longer sequences are symmetrical like $\mathrm{v}_{2} \mathrm{v}_{4}^{\prime} \mathrm{v}_{2}\left(\mathrm{ZrAl}_{3}\right)$ or vyu $\mathrm{yv}$ $\left(\mathrm{ZrGa}_{2}\right)$ and can be characterized by the asymmetric sequence and a vertical bar ( $\mid)$ for the mirror plane like $\mathrm{v}_{2} \mathrm{v}_{2}^{\prime} \mid\left(\mathrm{ZrAl}_{3}\right)$ or vyu $\mid\left(\mathrm{ZrGa}_{2}\right)$.

\section{Disordered alloys}

Many alloys form extensive substitutional solid solutions, in particular alloys of metals with similar radii and electronegativity. Some alloys as e.g. $\mathrm{AuCu}$ and $\mathrm{AuCu}_{3}$ are disordered at high temperatures and become ordered at lower temperatures (Cenedese and Gaspard, 1984). The same applies to interstitial alloys as e.g. $\mathrm{V}_{6} \mathrm{C}_{5}$ or $\mathrm{Ti}_{2} \mathrm{C}$ (Section 18) which have a disordered distribution of $\mathrm{C}$ atoms at high temperatures but an ordering of the $\mathrm{C}$ atoms (and vacancies) at lower temperatures (de Novion, 
1994). The x-ray or neutron diffraction patterns exhibit diffuse intensity which is concentrated on lines or surfaces in reciprocal space. The diffuse intensity can be analyzed for the short-range order parameters $\alpha_{i}$. The experimental values (Schweika, 1985; de Novion and Landesman, 1985) are usually between $\alpha_{i}=0$ for a random distribution of metal or $\mathrm{C}$ atoms with no diffuse intensity and the values of the ordered alloys (Fig.17) and should shift to the values of the ordered alloys as the orderdisorder transition temperature is approached. The relations $4 \alpha_{1}+\alpha_{2}$ $+1=0$ and $\alpha_{1}+\alpha_{3}=0$ were obtained for the $\alpha_{1}, \alpha_{2}, \alpha_{3}$ short-range order parameters of oxides like $\mathrm{LiFeO}_{2}$ and carbides like $\mathrm{V}_{6} \mathrm{C}_{5}$ from the shape of the diffuse intensity patterns in reciprocal space (Sauvage et al., 1974; Ducastelle, 1991). These relations can be explained by a disordered arrangement of carbon atoms in configurations of structural units a -1 allowed by Pauling's electrovalence rule (Section 19) or by the disordered $T_{i}$ configurations $6012 ; 1,3010 ; 2,2212 ; 2,008 ; 5$ or $4416 ; 1$ (Fig.17) (Hauck, 1985). There are many stacking variants of these configurations (Hauck et al., 1988b). The structures of the Ketelaar and Forsyth, Gran families can be obtained by combinations of structural units a - 1 (Table 4). Nonperiodic sequences of these structural units give rise to diffuse intensity at $4 \alpha_{1}+\alpha_{2}+1=0$. Some $\mathrm{ZnS}$ (sphalerite) related structures like $\mathrm{Ga}_{2} \mathrm{Te}_{3}$ or $\mathrm{AsCu}_{3} \mathrm{Se}_{4}$ give rise to diffuse intensities at $3 \alpha_{1}+1=0$ (Fig.16) (Sauvage et al., 1974; Hauck and Mika, 1998b). This corresponds to the Johansson, Linde and Brauer family of structures with a disordered sequence of structural units $u-y$ (Table 4). Other disordered alloys and compounds of Fig. 17 can probably be described as a disordered mixture of several structural families, including clustered structures at the left-hand border of the map.

A special "disorder" is obtained if structural units a and b alternate aperiodically with the sequence of Fibonacci numbers $n=1,2,3,5,8$, $13, \ldots$. The iteration of the Fibonacci substitution $a \rightarrow a b, b \rightarrow a$ creates the nonperiodic sequence $\mathrm{a} \rightarrow \mathrm{ab} \rightarrow \mathrm{aba} \rightarrow \mathrm{abaab} \rightarrow \ldots$ at an infinite number of steps. The structural units $\mathrm{g}(=\mathrm{b})\left(\mathrm{MoPt}_{2}\right)$ and $\mathrm{h}$ (=a) $\left(\mathrm{TiAl}_{3}\right)$ of the Forsyth, Gran series e.g. vary in the sequence gh $(n=2)$ or $\mathrm{gh}_{2}(n=3)$ in $\mathrm{Mn}_{2} \mathrm{Au}_{5}$ or $\mathrm{Mo}_{3} \mathrm{Al}_{8}$ (Table 4). An aperiodic sequence of $\mathrm{g}$ and $\mathrm{h}$ units in the ratio $\tau=(1+\sqrt{5}) / 2 \approx 1.62$ (e.g. hghhg at $n=5$ ) would be possible at the compositions $\mathrm{Mn}_{1.91} \mathrm{Au}_{5}$ or 


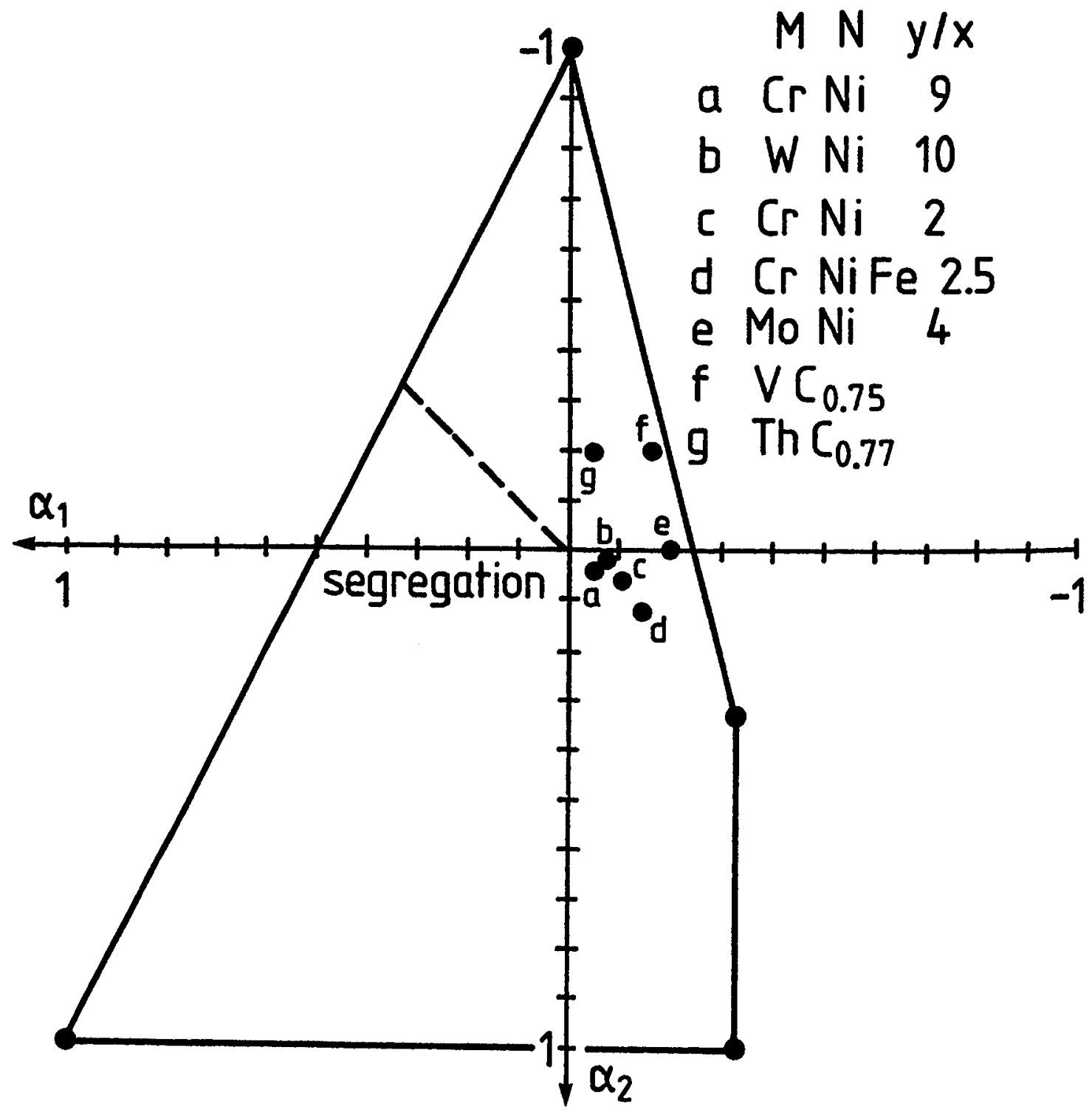

Figure 17: Experimental short-range order parameters $\alpha_{1}, \alpha_{2}$ of disordered alloys $\mathrm{M}_{\mathrm{x}} \mathrm{N}_{\mathrm{y}}$ or $\mathrm{M}_{\mathrm{z}} \square_{\mathrm{x}} \mathrm{C}_{\mathrm{y}}$ carbides within the $\operatorname{ccp} \alpha_{1}, \alpha_{2}$ structure map. 
$\mathrm{Mo}_{3.06} \mathrm{Al}_{8}$. The chain of $\mathrm{g}$ and $\mathrm{h}$ structural units has an infinite number of $T_{i}$ values with an average of $00.761 .24 ; \tau$, which is at the border of the structure map at $\alpha_{2}=0$ (Fig.2). The x-ray diagram would show the reflections of the $\mathrm{g}$ and $\mathrm{h}$ layers similar e.g. to the hexagonal layers of $\mathrm{h}$ and $\mathrm{c}$ stacking in a Fibonacci sequence with $\mathrm{hc}_{2} / \mathrm{hc} \approx 1.62$ (Table 1 ). The aperiodic sequence in $c$ direction can be analyzed as in other onedimensional Fibonacci sequences (Steurer, 1990). The two-dimensional Robinson tiling with a substitution

$$
a \rightarrow \begin{aligned}
& a b \\
& b b
\end{aligned} \quad \text { and } \quad b \rightarrow \quad \begin{aligned}
& b a \\
& a
\end{aligned}
$$

(Allouche and Salon, 1990) or an analogous three-dimensional tiling requires two different structural units, which can be combined twodimensionally or three-dimensionally at common interfaces like the quasihomologous square net structures $004 ; 3$ and $044 ; 1$ for a two-dimensional tiling (Section 28) or the quasi-homologous bcc structures $8612 ;(1)$ (W) and $0612 ; 1(\mathrm{CsCl})$ for a two- or three-dimensional aperiodic tiling. The $T_{i}$ values of the two-dimensional tiling of $004 ; 3$ and $044 ; 1$ approach the corner structure $08 / 310 / 3 ; 5 / 3$ at the right-hand border of the structure map $\left(T_{2}=-2 T_{1}+2(3-\mathrm{r})\right)$ at $T_{1}=0$ (Fig.7a). The three-dimensional Robinson tiling $a \rightarrow\left[\begin{array}{ll}a & b_{7}\end{array}\right]$ and $b \rightarrow\left[\begin{array}{ll}b & a_{7}\end{array}\right]$ with $a=$ $8612 ;(1)$ and $b=0612 ; 1$ was solved up to $n=7$. The density $\mathrm{r}_{\infty}$ $=3$ follows exactly for each starting value $m$ in the limit $n \rightarrow \infty$. For the square net the same recursion relation also holds for $T_{2}^{(n)}$ and $T_{3}^{(n)}$ with $m \geq 2$. Assuming the same behaviour for the bcc series, where the limiting values $T_{i}^{(\infty)}$ follow only approximately from the starting value $m$, we obtained with $m=7$ the values $T_{2}^{(\infty)}=4.0005, T_{3}^{(\infty)}=7.5015$. From this result we conclude, that with $m \rightarrow \infty$ the exact $T_{i}^{(\infty)}$ values are given by $047.5 ; 3$.

Some structures are known, where structural units of different lattices like bcc and fcc lattices with the proper interface are combined. Structural units can probably also be combined in such a way that fivefold or eightfold symmetry is achieved. The $T_{i}$ values of these combinations can not be analyzed in $T_{1}, T_{2}$ structure maps as the theoretical examples outlined before. 


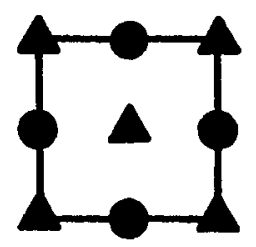

$468 ; 1$

$468 ; 1$

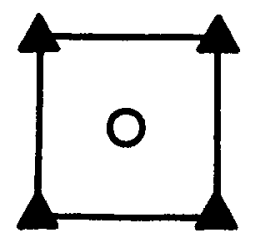

$060 ; 3$

$060 ; 3$

Fe Ni N口

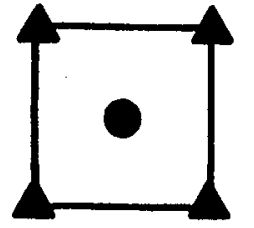

$\begin{array}{llll}0 & 6 & 0 ; 3\end{array}$

$060 ; 3$

$468 ; 1$

$\mathrm{Pd} \mathrm{AuCu}$

$\mathrm{PdCu} \mathrm{Au}_{2}$
$\mathrm{Ca}_{3} \mathrm{Ti}$

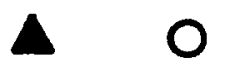

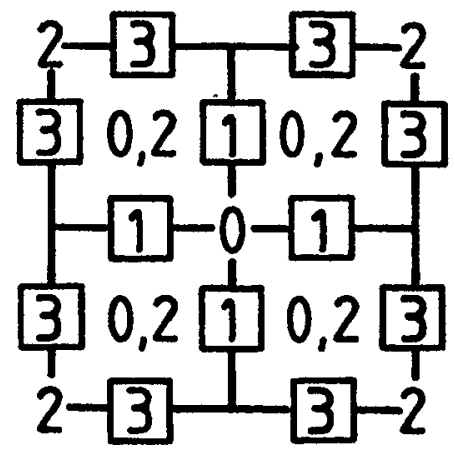

ㅁ $224 ; 3$

Co $1.64 .83 .2 ; 2.2$

Ge $4.62 .99 .1 ; 1.3$

$\square_{4} \mathrm{Co}_{5} \mathrm{Ge}_{7}$

$\square \quad 0,2$

Figure 18: Ternary and quaternary compounds with $T_{1} T_{2} T_{3} ; \mathrm{y} / \mathrm{x}$ of different components with composition $y / x$. Only positions of minority elements are plotted, full and open symbols for elements at projection height $\mathrm{z}=0$ and 0.5 , respectively, or as multiples of $a_{0} / 4$ in cubic $\mathrm{Co}_{5} \mathrm{Ge}_{7}$.

\section{Ordered ternary and quaternary compounds}

Most of the observed binary hcp and ccp $\mathrm{M}_{\mathrm{x}} \mathrm{N}_{\mathrm{y}}$ compounds have two sets of $T_{i}$ values, which are identical at concentration $\mathrm{y} / \mathrm{x}=1$. Other compounds with three or four sets of $T_{i}$ values $\left(M^{i}=3\right.$ or 4 ) (Tables 3,4 ) are potential candidates for ternary or quaternary alloys. Few ternary and quaternary ordered alloys with close-packed metal structures are known (Fig.18). Alloys with the composition $\mathrm{M}_{\mathrm{x}_{1}} \mathrm{M}_{\mathrm{x}_{2}} \mathrm{~N}_{\mathrm{y}}, \mathrm{M}_{\mathrm{x}_{1}} \mathrm{M}_{\mathrm{x}_{2}} \mathrm{M}_{\mathrm{x}_{3}} \mathrm{~N}_{\mathrm{y}}$, $\mathrm{x}_{j} \leq \mathrm{y}$ can be described by the self-coordination numbers $T_{i}$ of the minority components $\mathrm{M}_{\mathbf{x}_{j}}$. There are three sets of $T_{i}$ values for ternary and four sets for quaternary alloys. The $\alpha_{1}, \alpha_{2}$ values are identical for the $468 ; 1$ or $060 ; 3$ derivative structures (homologous structures) or on the border of the structure map as e.g. for $\operatorname{ReAl}(\mathrm{Re}, \mathrm{Al})_{2}$ (Table 8) (quasi-homologous structures). The $T_{i}$ values of all $\mathrm{M}_{\mathrm{x}_{j}}$ can be averaged according to the frequency of their occurrence. The combination of two 
values indicates the interactions between both atoms. The combination of the $060 ; 3$ positions of $\mathrm{Pd}$ and $\mathrm{Au}$ atoms in $\mathrm{PdAuCu}_{2}$ for example yields the same value $468 ; 1$ as the $\mathrm{Cu}$ atoms.

The $T_{i}$ values of the vacancies $\square$ and the Ge atoms of $\square_{4} \mathrm{Co}_{5} \mathrm{Ge}_{7}$ are not on the border of the structure map because of partial disorder. Other alloys forming solid solutions like the binary $\mathrm{CrNi}_{2}$ or the ternary $\mathrm{CrNiFe}_{2.5}$ are listed in the Cu structure type (Villars and Calvert, 1986) with a single environment of metal atoms, though the short-range order parameters are different from $\alpha_{i}=0$ (Fig.17). Metals with similar radii and electronegativity can also substitute for one component of an ordered binary alloy. Such ternary alloys have been investigated frequently to vary the structure of metals of binary alloys in small steps:

(i) The stacking sequences $7 \mathrm{c}$ or 14 instead of $4\left(\mathrm{TiNi}_{3}\right)$ or $9 \mathrm{a}\left(\mathrm{BaPb}_{3}\right)$ were obtained by variation of the $\mathrm{Ti}-\mathrm{Pt}-\mathrm{Ni}$ and $\mathrm{Ba}-\mathrm{Pb}-\mathrm{Tl}$ compositions (Table 5).

(ii) Structures with a different sequence of structural units were obtained e.g. in $(\mathrm{Zr}, \mathrm{Al})(\mathrm{Si}, \mathrm{Al})_{2}$ (Schubert, 1964) with $u^{\prime} \mathrm{x}^{\prime} \mathrm{vv}^{\prime} \mathrm{y}^{\prime} \uparrow$ compared to $\mathrm{ZrSi}_{2}$ (Fig.12) with uu'y' $\uparrow$.

(iii) Homologous structures with identical environment of all atoms at different compositions (Table 6) can be filled up with different atoms. The $\mathrm{Pd}$ and $\mathrm{Au}$ atoms e.g. of $\mathrm{PdAuCu}_{2}$ (Fig.18) have the $060 ; 3$ coordination of the $\mathrm{AuCu}_{3}$ structure. Both $\mathrm{Pd}$ and $\mathrm{Au}$ atoms together and the two $\mathrm{Cu}$ atoms together exhibit the $468 ; 1$ configuration of the $\mathrm{CuAu}$ structure. The $\mathrm{MoNi}_{4}$ structure is a potential candidate for a quaternary compound $\mathrm{MoMNNi}_{2}$ at the proper substitution of two $\mathrm{Ni}$ atoms by $\mathrm{M}$ and $\mathrm{N}$ atoms (Table 6 ).

(iv) Some alloys as e.g. $\mathrm{RhZr}_{3}$ ( $\mathrm{AuCu}_{3}$ structure) become unstable on hydrogen loading and segregate or become amorphous (Bowman, 1988).

(v) Other structures, which are unstable as pure alloys, can be stabilized by interstitial atoms, e.g. $\mathrm{AlFe}_{3}$ with the $\mathrm{AuCu}_{3}$ structure in $\mathrm{AlFe}_{3} \mathrm{C}$ (Fig.18). Among the 139 known compounds with this structure 
(Villars and Calvert, 1986) there are many alloys which are not stable without the presence of interstitial atoms. The atoms of the $\mathrm{AlFe}_{3} \mathrm{C}$ structure have the same positions as the atoms of the $\mathrm{CaO}_{3} \mathrm{Ti}$ perovskite structure with oxygen atoms at the $\mathrm{Fe}$ positions. The sublattice of the metal atoms $\mathrm{Ca}$ and $\mathrm{Ti}$ in $\mathrm{CaTiO}_{3}$, however, is body-centered with oxygen atoms at the octahedral interstices.

(vi) The crystal structures of some alloys can be varied by insertion of interstitial atoms. The ccp $\mathrm{ZrAl}_{3}$ structure e.g. of $\mathrm{MnPd}_{3}$ (Flanagan et al., 1992) changes to the $\mathrm{AuCu}_{3}$ structure in $\mathrm{MnPd}_{3} \mathrm{H}_{3}$, which is isotypic with $\mathrm{AlFe}_{3} \mathrm{C}_{3}$ (Fig.18).

If, however, the properties of the three or more components are too different or the concentration of the substituting component is too high, then the alloys become unstable and favor structures without close-packing. As we pointed out in Section 2, close-packing can be expected for identical atoms or at least similar atoms with weak directional bonding and is not likely to be found in a child's drawer containing a collection of differently sized balls.

\section{Enhanced covalent bonding in layered com- pounds and ordered body-centered alloys}

A well-defined collection of balls with increasing diameter like golf, table tennis and tennis balls $\mathrm{M}, \mathrm{N}$ and $\mathrm{R}$ can be assembled to layered structures, which are similar to close-packed alloys (Fig.19). Many compounds $M_{x} N_{y} R_{z}$ can be described by sequences of $M, N$ and $R$ layers, which are similar to the close-packed metals (Section 2), but with reduced distances between neighboring layers (Parthé et al., 1993). The hexagonal unit cell with lattice constant $a=b=d$ (diameter of $\mathrm{R}$ atoms) has the height $c=n \sqrt{2 / 3} d$ for $n$ close-packed layers. The distance between two neighboring layers, which is $\sqrt{2 / 3} \approx 0.8$ for close-packing with $c / a=n \sqrt{2 / 3}$, decreases to $0.2-0.8$ for atoms $\mathrm{M}$ and $\mathrm{N}$ with smaller size and different bond strength $\mathrm{M}-\mathrm{R}$ or $\mathrm{N}-\mathrm{R}$ between the atoms of neighbor- 

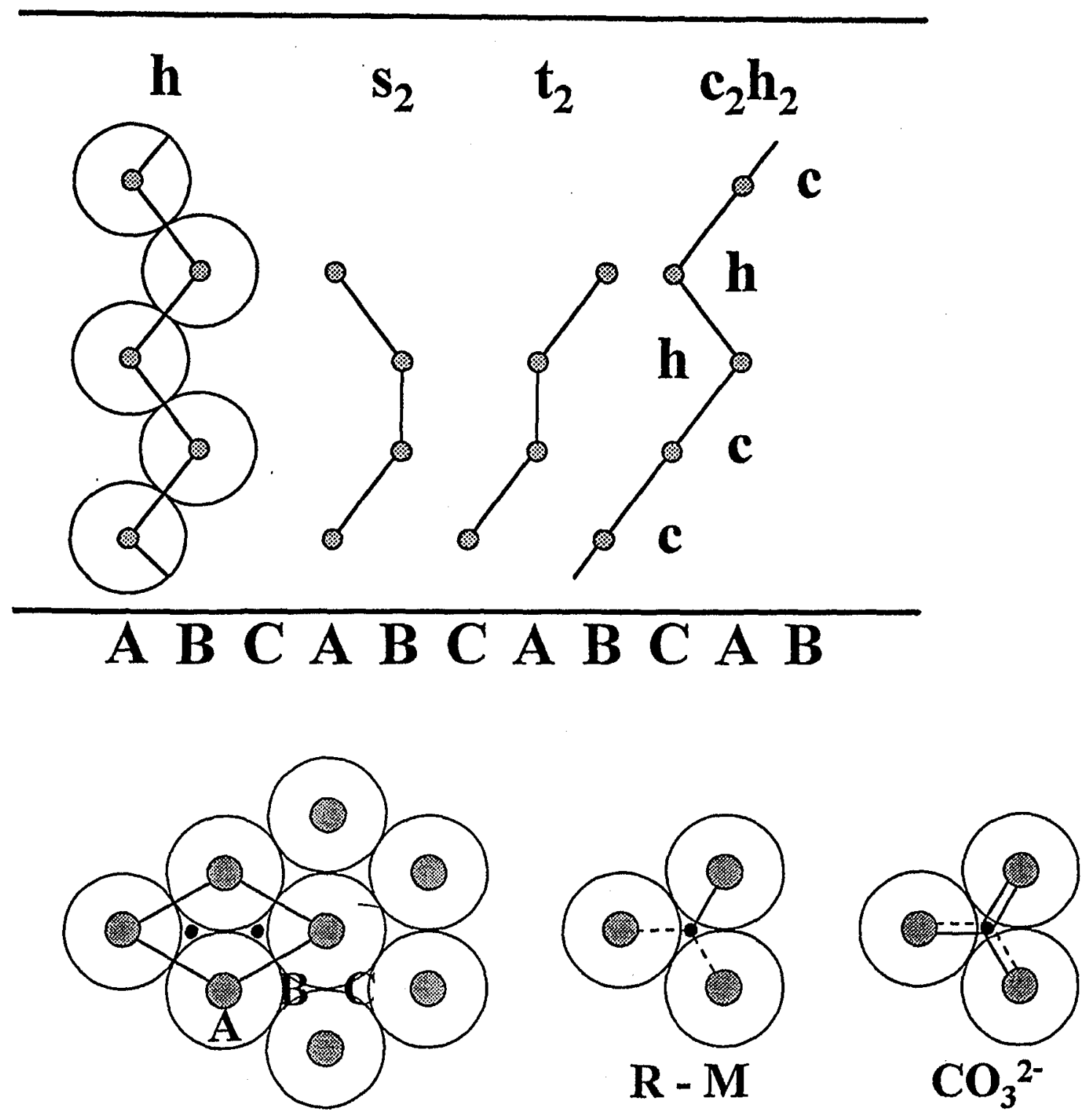

Figure 19: Close-packed hexagonal layer of spheres in A position, unit cell ([001] projection, below) and $B$ or $C$ position of consecutive layers as e.g. $A B A B(h h)$ or $B C A C$ $\left(c_{2} h_{2}\right),(A) B B(A)\left(s_{2}\right)$ and $(C) A A(B)\left(t_{2}\right)$ as explained in the text ([110] projection, above). The single $\mathrm{R}-\mathrm{M}$ bond is delocalized to three neighbors similar to $\mathrm{CO}_{3}^{2-}$ (below). 


\section{$\operatorname{ccp} M I^{0} I^{\dagger}$}

\section{hep $M I^{0} I^{\dagger}$}

123

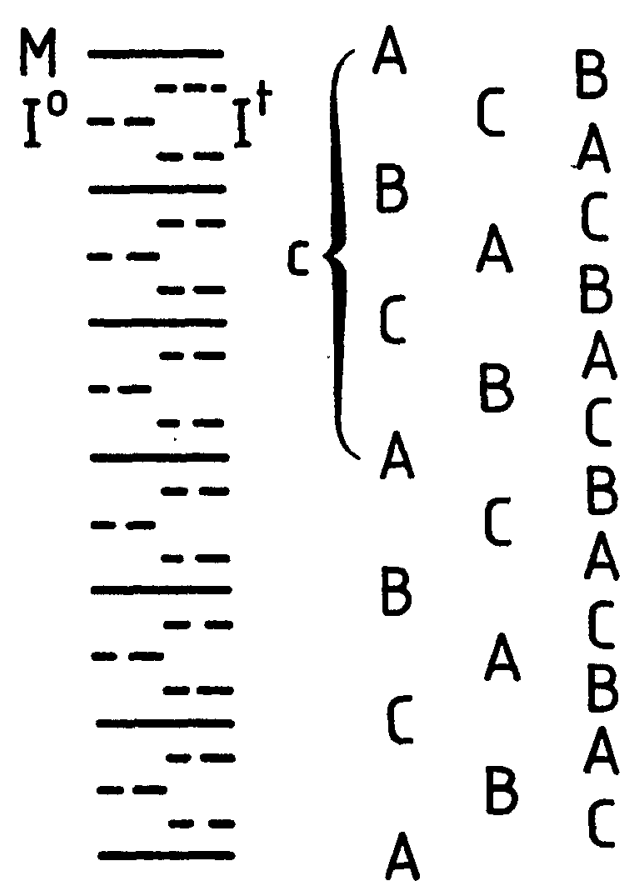

$c\left\{\begin{array}{lll}A & & B \\ & C & A \\ B & & A \\ & C & B \\ A & & B \\ & C & A \\ B & & A \\ & C & B \\ A & & B \\ & C & A \\ B & & A \\ & C & B \\ A & & B\end{array}\right.$

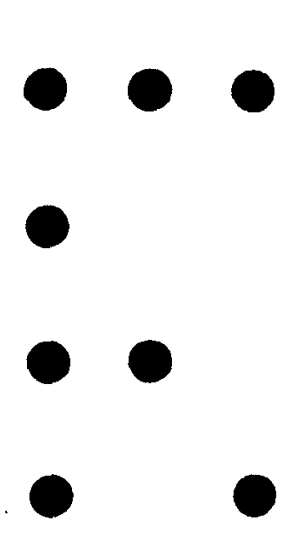

Figure 20: Position of interstitial atoms $\mathrm{I}^{\circ}$ and $\mathrm{I}^{\mathrm{t}}$ in octahedral and tetrahedral sites of the $\mathrm{ABC}$ ccp or $\mathrm{AB}$ hcp M lattice. Only $1 / 2$ or $1 / 3$ (for $\varepsilon-\mathrm{Ni}_{3} \mathrm{C}$ ) of the octahedral sites $I^{0}$ at identical positions in $c$ direction of the hcp $M$ lattice are occupied at $z / y=$ 2 or 3 , respectively.

ing layers (Table 7). Some compounds like $\mathrm{SnSb}_{2} \mathrm{Te}_{4}$ can be described by a sequence TeSbTeSnTeSbTe of large atoms $R=T e$, which are approximately close-packed, and smaller atoms $\mathrm{M}=\mathrm{Sn}$ and $\mathrm{N}=\mathrm{Sb}$ at octahedral interstices. The hexagonal unit cell with $n=m \cdot p(7 \cdot 3=21)$ layers contains $p=3$ formula units $\left(\mathrm{SnSb}_{2} \mathrm{Te}_{4}\right)$ with $m=7$ atoms. The distance between $\mathrm{Sb}$ and Te or $\mathrm{Sn}$ and Te layers $\Delta \mathrm{z} c / a$ is reduced to $\approx$ 0.4. These compounds are usually described by the stacking of $\mathrm{R}$ atoms and by sequences of Greek or lower case letters for $M$ and $N$ atoms at tetrahedral or octahedral positions (Hulliger, 1976; Parthé et al., 1993) (Fig.20). The approximate structure can be characterized by two strings in Table 7, if the locations of all $\mathrm{M}, \mathrm{N}$ and $\mathrm{R}$ atoms are characterized by $h, c, s$ or $t$ symbols. The $s_{2}$ and $t_{2}$ groups with two atoms at the same positions (at different projection height) are defined similar to the $\mathrm{h}$ and $\mathrm{c}$ symbols (Section 2): The trans (or $t_{2}$ ) configuration for different 
positions of the neighbors next to $t_{2}$ and the cis (or $\mathrm{s}_{2}$ ) configuration at identical positions (Fig.19). In that case the different coordinations of $\mathrm{M}$ in a sequence of RMR atoms can be obtained by the symbol ( $\mathrm{c}=$ octahedral, $\mathrm{h}=$ trigonal prismatic, $\mathrm{t}$ or $\mathrm{s}=$ tetrahedral) and structures with the same sequence of $R$ and $M$ atoms can be compared by the different coordinations of $\mathrm{M}$ atoms or - with other words - the different configurations of the chain (Table 7). The alternative description by sequences of $\mathrm{A}, \mathrm{B}$ and $\mathrm{C}$ positions is not specific and in many cases longer by a factor $p=2$ or 3 than the present notation as was shown before for the (ch) $)_{2} \alpha-\mathrm{Nd},\left(\mathrm{hc}_{2}\right)_{2} \mathrm{~Tb} \mathrm{HP}$ and $\left(\mathrm{ch}_{2}\right)_{3} \alpha$-Sm structures (Table 1). These stackings with $M^{i}=2$ sets of $T_{i}$ values (Section 3 ) are observed in $\mathrm{NiAs}(\mathrm{ch}), \mathrm{NbS}_{2}\left(\mathrm{hc}_{2}\right)$ and $\mathrm{CdCl}_{2}\left(\mathrm{ch}_{2}\right)$ structures at decreased $\Delta \mathrm{z} c / a$ (I form). Other more complex stackings like hchc $c_{4}, c_{3} h_{4}$ or $\operatorname{ch}_{2} c_{2} h_{2}$ for $\mathrm{MN}_{2} \mathrm{R}_{4}$ composition, $\operatorname{chch}_{3}\left(\mathrm{MN}_{2} \mathrm{R}_{3}\right), \mathrm{hc}_{3}, \mathrm{~h}_{2} \mathrm{c}_{6}$ or $\mathrm{ch}_{2} \mathrm{chc}_{2} \mathrm{~h}\left(\mathrm{MNR}_{2}\right)$ are not observed for these compositions. Most stackings and layer sequences like TeSbTeSnTeSbTe are symmetric. Some structures like $\mathrm{TaS}_{2} 6 \mathrm{~s}$ with $\mathrm{ch}_{2} \mathrm{c}_{3}$ stacking can be considered as a combination of $\mathrm{ch}_{2}\left(\mathrm{MoS}_{2} 3 \mathrm{R}\right)$ and $\mathrm{c}_{3}\left(\mathrm{CdI}_{2} \mathrm{C6}\right)$. Few compounds like $\mathrm{ZnS}$ or $\mathrm{SiC}$ can occur in many different structures (polytypes), which can be described in series of stackings like $\left(\mathrm{hc}_{2}\right)_{2 n+1} \mathrm{hc}$ or $\left(\mathrm{hc}_{2}\right)_{2 n+1} \mathrm{hc}_{3}$ at consideration of $\mathrm{R}=\mathrm{S}, \mathrm{C}$ atom packing (Parthé et al., 1993) or $\left(\mathrm{s}_{2} \mathrm{t}_{4}\right)_{2 n+1} \mathrm{~s}_{2} \mathrm{t}_{2}$ and $\left(\mathrm{s}_{2} \mathrm{t}_{4}\right)_{2 n+1} \mathrm{~s}_{2} \mathrm{t}_{6}$ in the present notation $\left(h=s_{2}, c=t_{2}\right)$. The combinations of $a b_{2}$ and $a b$ or $a b_{3}(h=$ $a, b=c)$ structural units are approaching point $a_{2} b_{4}$ for $s_{2} t_{4}$ or $a b_{2}$ for the $\mathrm{hc}_{2}$ composition (S) of the structure map (Fig.2b) at large $n$.

Some structures of ordered ccp alloys like $\mathrm{CuAu}$ can be described by sequences of $\mathrm{Cu}$ and $\mathrm{Au}$ square layers, which are packed in [001] direction (Figs. 3,21). The distance between neighboring layers of spheres with diameter $d, \Delta \mathrm{z} c / d=\sqrt{2} / 2 \approx 0.71$ is reduced to $1 / \sqrt{3} \approx 0.58$ in some ordered bcc alloys, or increased to $\Delta \mathrm{z} c / d=1$ in the primitive cubic lattice of $\mathrm{Po}$, disordered alloys like $\mathrm{AuBi}_{4}$ or $\mathrm{AuTe}_{3}$ (Villars and Calvert, 1986) and $\mathrm{CaF}_{2}$ related structures (Section 18, Table 8). The sequences of layers are characterized by the letters $\mathrm{V}, \mathrm{v}$ or I according to the configurations with the shape of the letter $V$ or $v$ in fcc or bcc and $I$ in the primitive cubic lattice of Po (Galasso, 1970) and the related structures of $\mathrm{As}, \mathrm{Sb}, \mathrm{Bi}$, Se and Te (Hyde and Andersson, 1989; Burdett, 1995). The $I^{\prime}, i^{\prime}$ or $v^{\prime}$ configurations are important for the occupation of octahedral 

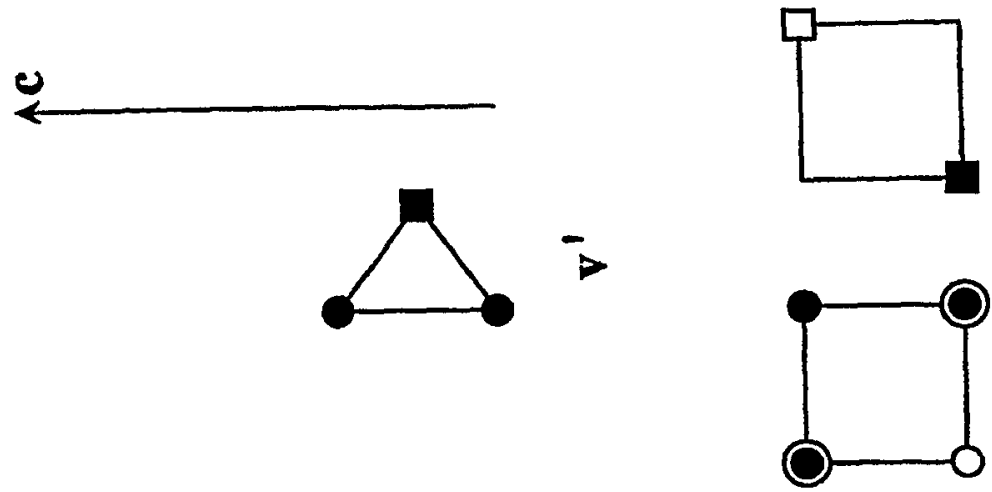

m ह-
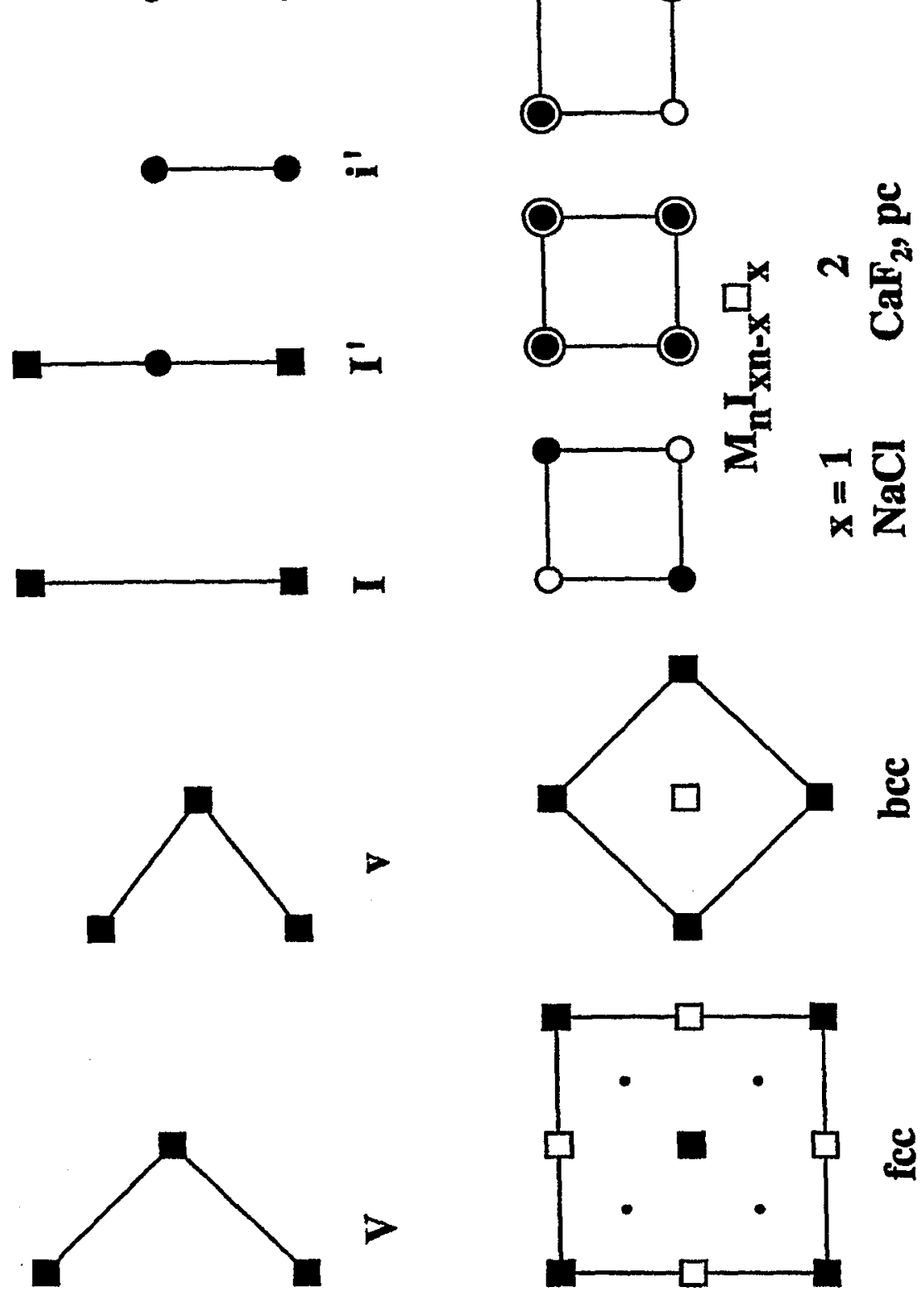

Figure 21: [001] projection of fcc, bcc metal lattices and interstitial alloys $M_{n} I_{x n-x} \square_{x}$ with metal atoms at $0(\square)$ or $0.5(\square)$ and I atoms at $0(\bullet)$ or $0.5(0)$. The $F$ atoms of $\mathrm{CaF}_{2}$ form a primitive cubic (pc) lattice, which are at projection height 0.25 and $0.75(\odot)$ of the fcc $\mathrm{Ca}$ lattice at the positions $(\cdot)$. The square layers are stacked in $c$ direction in $\mathrm{V}(\mathrm{fcc}), \mathrm{v}(\mathrm{bcc}), \mathrm{I}(\mathrm{pc}), \mathrm{I}^{\prime}(\mathrm{NaCl}), \mathrm{i}^{\prime}$ and $\mathrm{v}^{\prime}(\mathrm{pc}$ and $\mathrm{PER})$ configurations. 
or tetrahedral sites with interstitial I atoms in compounds $M_{n} I_{x n-x} \square_{x}$ (Section 18).

Most ordered bcc alloys can be characterized by sequences of layers in [001] or [111] direction (Tables 7,8), which are named by the pioneers, who investigated the structures of the first examples: $\mathrm{Au}_{2} \mathrm{Nb}_{3}, \mathrm{Ti}_{2} \mathrm{Cu}_{3}$ and $\mathrm{Ti}_{3} \mathrm{Cu}_{4}$ of the Schubert series (Schubert et al., 1960), and $\mathrm{Pb}_{2} \mathrm{Li}_{7}$ and $\mathrm{Pb}_{3} \mathrm{Li}_{8}$ of the Zalkin, Ramsey (1956) series. The structures of the Schubert series can be described by the sequences of metal atoms in [001] direction like $\mathrm{MoSiSi}(=\mathrm{b})$ for $\mathrm{MoSi}_{2}, \mathrm{Ti}_{2} \mathrm{Cu}_{2}(=\mathrm{d})$ for the $\mathrm{TiCu}$ and $\mathrm{TiCu}_{2} \mathrm{Ti}_{2} \mathrm{Cu}_{2}(=\mathrm{bd})$ for $\mathrm{Ti}_{3} \mathrm{Cu}_{4}$. The structures of the Zalkin, Ramsey series can be described by sequences of metal atoms in [111] direction like $\mathrm{CeCdCd}(=\mathrm{k})$ for $\mathrm{CeCd}_{2}, \mathrm{AlFeFeFe}(=1)$ for $\mathrm{AlFe}_{3}$ and $\mathrm{PbLi}_{3} \mathrm{PbLi}_{2} \mathrm{PbLi}_{3}\left(=1 \mathrm{kl}\right.$ ) for $\mathrm{Pb}_{3} \mathrm{Li}_{8}$. Structures with long sequences of structural units like $\mathrm{Pb}_{3} \mathrm{Li}_{8}(\mathrm{kl})$ or clustering like $\mathrm{TiCu}\left(\mathrm{Ti}_{2} \mathrm{Cu}_{2}\right)$ can segregate to $2 \mathrm{PbLi}_{3}(\mathrm{l})+\mathrm{PbLi}_{2}(\mathrm{k})$ or $\mathrm{Ti}+\mathrm{Cu}$ (Section 19).

\section{Enhanced repulsive interactions}

The following structures with minimum $T_{1}$ and maximum $T_{2}$ values are obtained at repulsive interactions: $468 ; 1$ of ccp CuAu and $0612 ; 1$ of bcc $\mathrm{CsCl}$. The compounds contain alternating square layers of $\mathrm{Cu}$ and $\mathrm{Au}$ or $\mathrm{Cs}$ and $\mathrm{Cl}$ atoms, respectively. Vacancies or atoms of neighboring layers are inserted at repulsive interactions between $M$ and $N$ atoms. Part of the $\mathrm{M}$ atoms of $\mathrm{CsCl}(\mathrm{MN})$ for example are substituted by $\mathrm{N}$ atoms in [001] layers with composition $\mathrm{MN}$ and $\mathrm{N}_{2}$ of neighboring layers of the $(\mathrm{Mo}, \mathrm{U}) \mathrm{U}_{3}$ structure or $\mathrm{M}_{2} \mathrm{~N}_{2}$ and $\mathrm{N}_{4}$ layers of the $\mathrm{Au}(\mathrm{Zn}, \mathrm{Au})_{3}$ structure (Table 9a). Other $\mathrm{CsCl}$ related structures like $\mathrm{Pu}_{3} \mathrm{Pd}_{4}$ or $\mathrm{Zn}_{3} \mathrm{Ga}_{4} \mathrm{Pd}_{7}$ can be described by different [111] layers with composition $\mathrm{Pu}_{6} \mathrm{Pd}$ and $\mathrm{Pd}_{7}$ or $\mathrm{Zn}_{3} \mathrm{Ga}_{4}$ and $\mathrm{Pd}_{7}$ (Table 9b). Examples of ordered ccp alloys are $\mathrm{GeCa}_{7}$ with $\mathrm{GeCa}_{3}$ and $\mathrm{Ca}_{4}$ layers or $\mathrm{Ga}_{3} \mathrm{Pt}_{5}$ with $\mathrm{PtGa}_{3}$ and $\mathrm{Pt}_{4}$ layers (Table $9 \mathrm{a}$ ). The hcp structures $\mathrm{SbAg}_{3}$ or $\mathrm{SnAu} \mathrm{u}_{5}$ contain layers of $\mathrm{SbAg}$ and $\mathrm{Ag}_{2}$ or $\mathrm{SnAu}_{2}$ and $\mathrm{Au}_{3}$. These structures indicate little repulsive interactions for $\mathrm{Pd}, \mathrm{Pt}, \mathrm{Ag}, \mathrm{Au}$ or $\mathrm{Ca}$ atoms. 
All other structures of Table 9 are characterized by identical composition $y / x$ of the layers and the three-dimensional alloy structure. The stacking of the layers can be described by the translation $\mathrm{x}, \mathrm{y}$ and $\mathrm{z}$ of the minority component like the translation in $\mathrm{x}$ direction in the $2212 ; 2 \mathrm{a}$ $\left(\mathrm{MoPt}_{2}\right)$ structure or the three different translations $\mathrm{x}, \mathrm{y}$ and $\mathrm{z}$ in the homometric $2212 ; 2 \mathrm{~b}$ structure (Fig.13). The homometric hcp $220 ; 2 \mathrm{a}, \mathrm{b}$ structures can be described by the stacking of the $060 ; 2 \mathrm{MN}_{2}$ layers with translations $x \bar{y}(220 ; 2 \mathrm{a})$ or $x \bar{x} \bar{y} \bar{x} z \bar{y}(220 ; 2 b)$. The stacking of the $204 ; 1$ square layers of homometric ccp CuPt,a and b can be described in a similar way by the translations yyxx in $6012 ; 1 \mathrm{a}$ and yyyy in $6012 ; 1 \mathrm{~b}$. The number of nearest and next-nearest neighbors in the layers can be visualized by the density of lines in different patterns (Section 28).

\section{Valence compounds}

In non-metallic valence compounds $\mathrm{M}_{\mathrm{x}} \mathrm{N}_{\mathrm{y}} \mathrm{R}_{\mathrm{z}}$ the number of electrons at the atoms can be completed to octets, if the sum of electrons of $\mathrm{M}, \mathrm{N}$ and $R$ atoms is eight or a multiple of eight (Parthé et al., 1993; Parthé, 1994; Ellner and Predel, 1994; Villars, 1994):

$$
x e_{M}+y e_{N}+z e_{R}=8 z \text {. }
$$

This equation satisfies the requirement of electroneutrality and can be applied for different distributions of valence electrons e.g. in $\mathrm{ZnS}$ (Table 10):

(a) Formation of $\mathrm{Zn}^{2+}$ and $\mathrm{S}^{2-}$ ions (ionic chain).

(b) The eight electrons (four pairs) form four bonds to $\mathrm{Zn}$ atoms in tetrahedral coordination (Parthé et al., 1993; Hauck and Mika, 1998b).

(c) The electrons are shared between neutral $\mathrm{S}$ and $\mathrm{Zn}$ atoms with two covalent bonds (covalent chain). 
Each atom in a close-packed layered compound has three neighbors in the next layer $\left(1+3\right.$ for tetrahedral $t_{2}$ or $s_{2}$ configuration) (Fig.19). One bond of $-\mathrm{S}-\mathrm{Zn}$ - with $\mathrm{S}$ in $\mathrm{t}$ or s position is delocalized to three $\mathrm{Zn}$ atoms. Therefore these bonds are weaker than the remaining bond. The delocalization of the double bond of the $\mathrm{S}=\mathrm{Zn}$ group enhances the bond strength to three neighbors $(=2 / 3)$ compared to the fourth neighbor without bond. The same bond strength $1 / 3+2 / 3$ or $1+0$ for all $\mathrm{Zn}$ atoms is achieved at a consideration of both formulas $-\mathrm{S}-\mathrm{Zn}-\mathrm{S}$ - and $\mathrm{S}=\mathrm{Zn}$. The structural data show positive or negative deviations. Equal bonding to all neighboring atoms as in (b) is also achieved for the three $\mathrm{M}$ atoms in octahedral or trigonal prismatic coordination, if the chain is symmetric like in $-\mathrm{S}-\mathrm{Mg}$ - or at consideration of resonance hybrids e.g. of $\mathrm{Cu} \mathrm{I-Cu}$ and $\mathrm{Cu}-\mathrm{I} \mathrm{Cu}$ or $\mathrm{Mg} \mathrm{S}=\mathrm{Mg}$ and $\mathrm{Mg}=\mathrm{S} \mathrm{Mg}$. The covalent chain (c) allows close-packed layers of neutral atoms in the [001] plane and different sequences of $\mathrm{M}, \mathrm{N}$ and $\mathrm{R}$ atoms in [110], that can explain the ordering of these atoms in most structures.

Table 10 shows the different combinations for binary and ternary compounds. The $R$ and $M$ atoms usually alternate until an $M$ atom is missing, which is indicated by $\mathrm{e}_{\mathrm{M}}=0$ (Hauck and Mika, 1998b). Therefore chains of RMR occur in $027_{2},\left(061_{2}\right), 046_{2}$ and $\left(042_{2}\right)$ compounds and chains with three or four $R$ atoms in the remaining structures containing 0 . The $\left(\begin{array}{lll}0 & 6 & 1_{2}\end{array}\right)$ or $\left(042_{2}\right)$ structures are antistructures to $027_{2}$ or $046_{2}$ with $\mathrm{e}_{\mathrm{N}}+\mathrm{e}_{\mathrm{N}^{\prime}}=8 n$ or $\mathrm{e}_{\mathrm{R}}+\mathrm{e}_{\mathrm{R}^{\prime}}=8 n$ (Parthé et al., 1993). In normal valence compounds $M_{x} R_{z}$ without $M-M$ or $R-R$ bonding the number of bonds pointing to each $M$ or $R$ atom is proportional to the number of $R$ or $M$ atoms ( $\mathrm{z}$ or $\mathrm{x}$ ). The $\mathrm{R}=\mathrm{M}-\mathrm{R}-\mathrm{M}=\mathrm{R}$ chain of $\mathrm{M}_{2} \mathrm{R}_{3}$ compositions is obtained for divalent $R$ and trivalent $M$ (tetravalent $R$ and hexavalent $M$ must not be considered). The $\mathrm{M}_{3} \mathrm{R}_{4}$ composition yields the largest chain with three tetravalent $M$ atoms. In ternary compounds the length of the chains is extended, if 26 groups are introduced in the $03_{2} 6_{3}$ chain or 35 groups in the $04_{3} 5_{4}$ chain at the single bond position. Table 7 shows many experimental examples of $\mathrm{RN}(\mathrm{RM})_{n} \mathrm{RNR}$ and $\mathrm{RN}(\mathrm{RM})_{n}$ RNR(MR $)_{m}$ NR chains. The last example with two different possibilities for the introduction of $-\mathrm{N}=\mathrm{Al}-$ groups was only observed for the antistructures with composition $\mathrm{N}_{\mathrm{x}} \mathrm{C}_{3} \mathrm{Al}_{4+\mathrm{x}}(\mathrm{x}=0-4)$. The chains are symmetrical for $\mathrm{x}=2$ and 4 . 
Another theoretical approach is the combination of different chains like $\mathrm{S}=\mathrm{Zn} \mathrm{S}=\mathrm{In}-\mathrm{S}-\mathrm{In}=\mathrm{S}$ in $\mathrm{ZnIn}_{2} \mathrm{~S}_{4}$ modifications or $\mathrm{Te}=\mathrm{Bi}-\mathrm{Te}-\mathrm{Ge}-\mathrm{Te}-\mathrm{Bi}=$ $\mathrm{Te} \mathrm{Te}=\mathrm{Bi}-\mathrm{Te}-\mathrm{Bi}=\mathrm{Te}$ in $\mathrm{GeBi}_{4} \mathrm{Te}_{7}$ with divalent Ge (Parthé et al., 1993) (Table 7). Layered compounds containing monovalent elements in the composition $14_{2} 5_{3}, 1_{2} 46_{3}$ or $136_{2}$ like $\alpha-\mathrm{NaFeO}_{2}$ with a shared single bond $\mathrm{Na}-\mathrm{O}-\mathrm{Fe}=\mathrm{O} \cdots \mathrm{Na}-\mathrm{O}-\mathrm{Fe}=\mathrm{O}$ similar to $\mathrm{a}=\mathrm{O} \cdots \mathrm{H}-\mathrm{O}-$ hydrogen bond are rare.

Some compounds with trivalent $\mathrm{Ga}$ and In contain $\approx 30 \%$ vacancies at the $\mathrm{M}^{\prime}$ or $\mathrm{N}^{\prime}$ positions (Table 7). In that case about one of the three $\mathrm{M}$ or $\mathrm{N}$ atoms in the neighboring layers is missing and the average of charge of $\mathrm{M}^{\prime}$ or $\mathrm{N}^{\prime}$ atoms is reduced to $\approx 2$. This can explain the occurrence of vacancies in some cases as e.g. the first $\mathrm{Ga}^{\prime}$ and the $\mathrm{Ga}^{\prime \prime}$ position in $(\mathrm{Ga}, \mathrm{In})_{3-\mathrm{x}} \mathrm{InS}_{5}$ with $\mathrm{S}=\mathrm{Ga}^{\prime} \mathrm{S}=\mathrm{In}-\mathrm{S}-\mathrm{Ga}^{\prime \prime}-\mathrm{S}-\mathrm{Ga}^{\prime}=\mathrm{S}\left(\mathrm{Ga}^{\prime}=\mathrm{Ga}_{0.50} \mathrm{In}_{0.17}\right.$, $\left.\mathrm{Ga}^{\prime \prime}=\mathrm{Ga}_{0.67}\right)$. A second formulation $\mathrm{S}=\mathrm{Ga}^{\prime} \mathrm{S}=\mathrm{In}-\mathrm{S}-\mathrm{Ga}^{\prime \prime}=\mathrm{S} \mathrm{Ga}^{\prime}=\mathrm{S}$ with equivalent $\mathrm{Ga}^{\prime}$ positions requires no vacancies in $\mathrm{Ga}^{\prime \prime}$ position. The $(\mathrm{In}, \mathrm{Ga})_{4.67} \mathrm{~S}_{7}$ and $(\mathrm{In}, \mathrm{Ga})_{7.33} \mathrm{~S}_{11}$ compounds contain $\mathrm{R}-\mathrm{M}-\mathrm{R}-\mathrm{M}-\mathrm{R}$ units of the $\operatorname{In}_{2} \mathrm{~S}_{3}$ structure with $\mathrm{hc}_{3} \mathrm{~h}$ stacking, but $\approx 0.2$ additional $\mathrm{M}$ atoms next to the $R$ atoms at the end of the chain (Parthé et al., 1993) (the Pearson symbols are $h P 14$ and $h R 72$ instead of $h P 12$ and $h R 57$ ). The vacancies are probably disordered within the layers. Additional reflections would be observed for ordered layers similar as for structures discussed in Section 16.

The last group of Table 7 considers general valence compounds. These are valence compounds where either the cations do not transfer all their valence electrons - they are used for bonds between them or for nonbonding orbitals - or where the anions due to bonds between themselves do not need as many electrons to complete their octet shell (Parthé et al., 1993). For a compound $\mathrm{M}_{\mathrm{x}} \mathrm{R}_{\mathrm{z}}$ with

$$
x\left(e_{M}-e_{M M}\right)+z\left(e_{R}+e_{R R}\right)=8 z
$$

the average number of remaining valence electrons $e_{M M}$ and $e_{R R}$ at $M$ or $\mathrm{R}$ atoms can be used to $\mathrm{M}-\mathrm{M}$ or $\mathrm{R}-\mathrm{R}$ bonds, respectively as for $\mathrm{Cl}-\mathrm{Zr} \equiv \mathrm{Zr}-\mathrm{Cl}, \mathrm{Te}=\mathrm{Pt}=\mathrm{Pt}=\mathrm{Te}$ or $\mathrm{S}=\mathrm{Ga}-\mathrm{Ga}=\mathrm{S}$ in $\mathrm{ZrCl}, \mathrm{PtTe}$ or $\mathrm{GaS}$ and $\mathrm{GaSe}$ structures. $\mathrm{Bi}_{2} \mathrm{Te}_{3}$ II $(-\mathrm{Bi}=\mathrm{Bi}-\mathrm{Te}-\mathrm{Te}-\mathrm{Te}-)$ and the hexagonal structures of $\mathrm{Se}$ and $\mathrm{Te}$ mentioned before are examples for $-\mathrm{Se}-\mathrm{Se}-$ or 
- Te-Te- bonding. The $\mathrm{Pt}_{3} \mathrm{Te}_{4}$ and $\mathrm{Pt}_{2} \mathrm{Te}_{3}$ structures can be considered as combinations of $\mathrm{Te}=\mathrm{Pt}=\mathrm{Pt}=\mathrm{Te}$ and $\mathrm{Te}=\mathrm{Pt}=\mathrm{Te}$ chains. $\mathrm{Pt}$ and $\mathrm{Zr}$ are supposed to be tetravalent, though they do not belong to the elements considered for valence compounds (Parthé et al., 1993). These atoms with lone electron pairs or low electronegativity $(\mathrm{Mg}, \mathrm{Zr})$ given in brackets (Table 10b) are ordered for increasing size (Schubert, 1967). The $\mathrm{Zn}, \mathrm{Al}, \mathrm{Ga}$ and $\mathrm{N}$ atoms underlined in Table 10b prefer tetrahedral sites. Divalent $\mathrm{Mn}$, trivalent $\mathrm{Fe}$ or In and tetravalent $\mathrm{C}$ (bold face) are observed in tetrahedral or octahedral coordination. Besides this some ordered alloys like $\mathrm{LiRh}, \mathrm{CuPt}, \mathrm{Pt}_{2} \mathrm{Sn}_{3}$ and some metallic carbides or nitrides like $\mathrm{Ta}_{2} \mathrm{C}$ or $\mathrm{Th}_{3} \mathrm{~N}_{4}$ (which are not valence compounds) are also listed in Table 7 because of related structures. The distance between layers $\Delta \mathrm{z} c / a \approx 0.8$ is normal ( $\mathrm{N}$ form) in the close-packed metals, decreased to $0.2-0.8$ at occupation of interstitial sites (I form) or to $\approx$ 0.2 in ordered body-centered alloys ( $\mathrm{B}$ form). $\mathrm{B}_{4} \mathrm{Rh}_{5}$ with the sequence RMRMRMRMR (Parthé et al., 1993) not allowed in the present scheme is omitted in Table 7. The distance $\Delta z c / a$ (at $p=1, \mathrm{z}=1)$ between neighboring layers usually corresponds with the bond strength. The distances between $\mathrm{Th}$ and $\mathrm{N}$ layers e.g. of $\mathrm{Th}_{3} \mathrm{~N}_{4}(\mathrm{~N} \equiv \mathrm{Th}-\mathrm{N}=\mathrm{Th}=\mathrm{N}-\mathrm{Th} \equiv \mathrm{N}$ sequence) are $0.51(\mathrm{Th}-\mathrm{N}), 0.31(\mathrm{Th}=\mathrm{N}), 0.12(\mathrm{Th} \equiv \mathrm{N})$ and $0.49(\mathrm{~N}$ to $\mathrm{N}$ of next chain).

\section{Occupation of interstitial sites}

Compounds with the composition $\mathrm{M}_{n} \mathrm{I}_{\mathrm{x} n-\mathrm{x}} \square_{\mathrm{x}}, \mathrm{x}=1,2,3$, can be considered as interstitial alloys, where $\mathrm{I}=\mathrm{H}, \mathrm{C}, \mathrm{N}, \mathrm{Cl}, \mathrm{O}, \ldots$ atoms occupy interstitial sites like the octahedral or tetrahedral interstices of the ccp lattice of $\mathrm{M}$ atoms (Fig.20). All octahedral interstices are filled in $\mathrm{NaCl}$, all tetrahedral interstices in the $\mathrm{CaF}_{2}$ structure. The $\mathrm{NaCl}$ structure is also obtained from the primitive cubic structure (pc), if four corners of the cube are occupied by $\mathrm{Na}$ atoms and the remaining four by $\mathrm{Cl}$ atoms (Fig.21). The occupation of two positions of the pc lattice by $M$ atoms and the remaining six positions by $I$ atoms yields $M_{2} I_{6}$ or $M_{n} I_{3 n-3} \square_{3}$ like the perovskite structure $\mathrm{CaTiO}_{3} \square_{3}$ with vacancies at I positions (PER group). Most superconducting oxides are in this group (Hauck and Mika, 
1997).

The undistorted $\mathrm{M}_{n} \mathrm{I}_{x n-\mathrm{x}} \square_{\mathrm{x}}$ structures can be characterized by the coordination numbers $\mathrm{CN}$ of $\mathrm{M}$ atoms with I atoms (Fig.22). The $\mathrm{CN}=6$ (octahedral) coordination of $6 \mathrm{Cl}$ atoms next to each $\mathrm{Na}$ atom is reduced, if some I positions are vacant like $\mathrm{CN}=3$ for $\mathrm{Pd}$ in $\mathrm{Pd}_{2} \mathrm{H} \square$ (Fig.23a) (Table 11a). The $\mathrm{CN}=8$ (cubic) coordination of $\mathrm{Ca}$ in $\mathrm{CaF}_{2}$ (pc lattice of $\mathrm{F}$ atoms) and the $\mathrm{CN}=18$ coordination of the $\mathrm{M}$ atoms in the PER group are reduced in a similar way. Most compounds can be described by sequences of $C N$ values for sequences of $M$ atoms in [001] (PER group) or [110] direction ( $\mathrm{NaCl}$ or $\mathrm{CaF}_{2}$ group). Most sequences of the PER group are symmetrical like $\mathrm{CN}=41058510,4$ for the sequence $\mathrm{Cu}^{\prime} \mathrm{BaCuY} \mathrm{Y}^{\prime} \mathrm{CuBa}, \mathrm{Cu}^{\prime}$ in $\mathrm{YBa}_{2} \mathrm{Cu}_{3} \mathrm{O}_{7}$. The formula $\mathrm{Cu}^{\prime} \mathrm{Ba}_{2} \mathrm{Cu}_{2} \mathrm{Y}^{\prime} \mathrm{O}_{7}$ or $\mathrm{CN}=410_{2} 5_{2} 8$ used in the present investigation indicates the sequence of metal atoms with single $\mathrm{Cu}^{\prime}$ and $\mathrm{Y}^{\prime}$ atoms at mirror planes and two $\mathrm{Ba}$ and $\mathrm{Cu}$ atoms between the mirror planes. The ordering of metal atoms in superconducting oxides corresponds to the [001] series of bcc alloys like $\mathrm{CuLa}_{2} \mathrm{O}_{4}$ or $\mathrm{Cu}_{2} \mathrm{La}_{3} \mathrm{O}_{7}$ with $\mathrm{MoSi}_{2}$ or $\mathrm{Os}_{2} \mathrm{Al}_{3}$ structure (Table 8) of $\mathrm{Cu}$ and $\mathrm{La}$ atoms (Fig.23b). The sum of all $\mathrm{CN}$ values corresponds to $6 \mathrm{y}$ of the total oxygen content like $6 \cdot 7=42$ in $\mathrm{Cu}^{\prime} \mathrm{Ba}_{2} \mathrm{Cu}_{2} \mathrm{Y}^{\prime} \mathrm{O}_{7}(\mathrm{y}=7)$ because of the six $\mathrm{M}-\mathrm{O}$ bonds of each oxygen atom. The same applies for $\Sigma \mathrm{CN}=6 \mathrm{y}$ in the $\mathrm{NaCl}$ group and $\Sigma \mathrm{CN}=4 \mathrm{y}$ for the $\mathrm{CaF}_{2}$ group.

The three series $\mathrm{M}_{n} \mathrm{I}_{\mathrm{x} n-\mathrm{x}} \square_{\mathrm{x}}, \mathrm{x}=1,2,3$, can be compared for example for $\mathrm{I}=$ oxygen atoms. The composition of the compounds can be obtained by the proper mixture of $\mathrm{MO}, \mathrm{MO}_{1.5}$ and $\mathrm{MO}_{0.5}(\mathrm{NaCl}$ group) or $\mathrm{MO}_{2}$ (pc and PER group) (Fig.24). There are closely related compounds with composition $\mathrm{M}_{n} \mathrm{O}_{\mathrm{x}} \square_{\mathrm{x} n-\mathrm{x}}$ of the $\mathrm{PER}^{\prime}$ and $\mathrm{pc}^{\prime}$ group with the oxygen atoms as minority component like $\mathrm{CuSr}_{2} \mathrm{O}_{3} \square_{6} \cdot\left(\mathrm{PER}^{\prime}\right)$ and the Aurivillius phase $\mathrm{WBi}_{2} \mathrm{O}_{6} \square_{3}\left(3 \mathrm{MO}_{2}\right)$ of the PER group or $\mathrm{Cu}_{2} \mathrm{O}_{3}$ $\left(\mathrm{pc}^{\prime}\right)$ and $(\mathrm{Fe}, \mathrm{Mn})_{2} \mathrm{O}_{3} \square$ of the pc group with vacancies as minority component. Less than half of the $\mathrm{M}$ atoms of the PER group should have $\mathrm{CN} \leq 6$ like the $\mathrm{Cu}$ and $\mathrm{Cu}^{\prime}$ atoms in $\mathrm{Cu}^{\prime} \mathrm{Ba}_{2} \mathrm{Cu}_{2} \mathrm{Y}^{\prime} \mathrm{O}_{7}\left(\mathrm{CN} 410_{2} 5_{2} 8\right)$ or the $\mathrm{W}$ atom in $\mathrm{WBi}_{2} \mathrm{O}_{6}\left(\mathrm{CN} 612_{2}^{\prime}\right)$. The valency of the atoms with $\mathrm{CN}$ $\leq 6$ is $q=4-6$ in the Aurivillius phases and $q=2-3$ (formal valency) in compounds of the PER' group because of the formation of stable compounds with perovskite structure like $\mathrm{CaTiO}_{3}$ (CN 12 6) instead of 


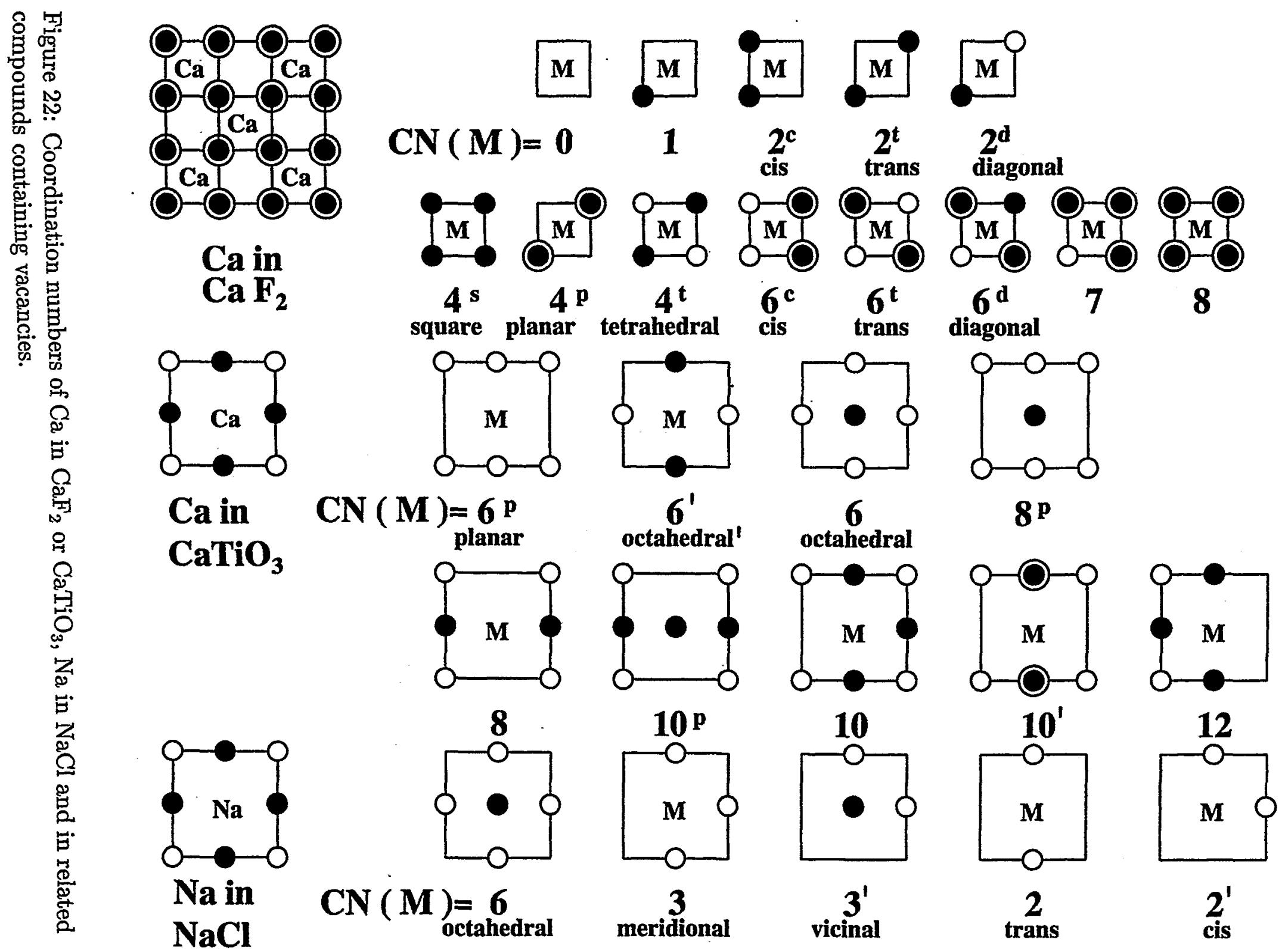




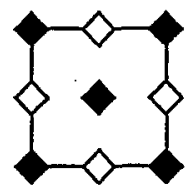

$\mathbf{M}_{4}$

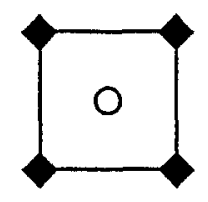

$\square \mathbf{N b}_{3} \square \mathbf{O}_{3}, \mathbf{h}$

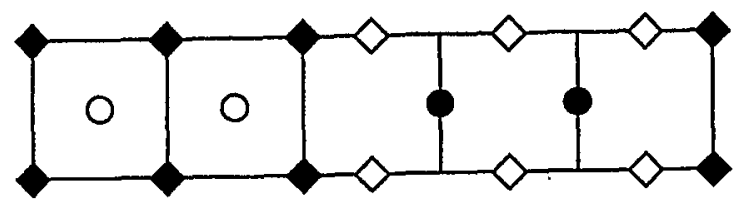

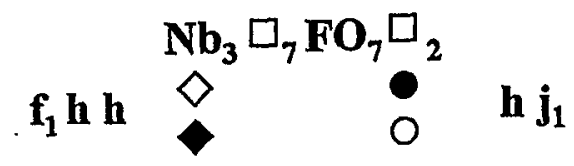

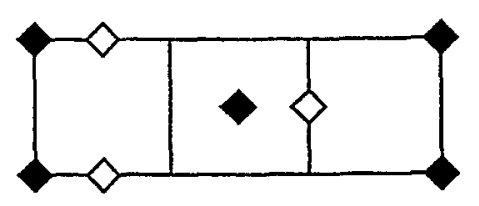

$\mathrm{Mo}_{2} \mathrm{O}_{3}, \mathbf{f}_{1} \mathrm{i}_{1}$

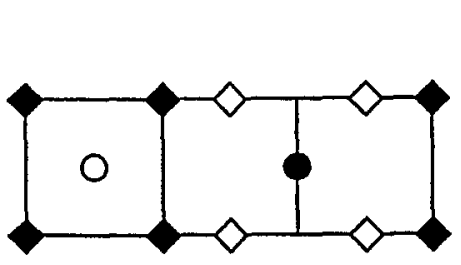

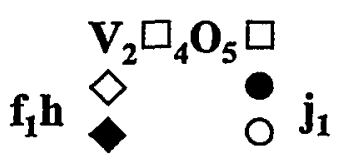

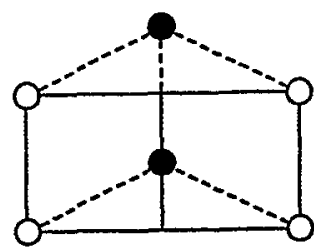

$\mathbf{N b}_{\mathbf{4}} \mathbf{N}_{\mathbf{3}} \square, \mathbf{i}_{\mathbf{1}}$
0
0

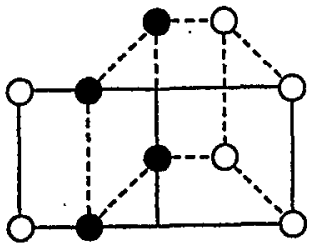

$\mathbf{P d}_{\mathbf{2}} \mathbf{H} \square, \mathbf{f}_{\mathbf{1}} \mathbf{g}$

Figure 23: Projection of $\mathrm{NaCl}(\mathrm{MI})$ related structures with $\mathrm{M}$ atoms at $0(\diamond), 0.5$ $(\diamond)$ and I atoms or vacancies $\square$ at $0(\bullet)$ or 0.5 (o) (a) and $\mathrm{CaTiO}_{3}$ related structures (b) (next page) with different structural units and different metal atoms (ordered bcc lattice).

layered compounds. Structures with more than $8 \mathrm{M}$ atoms/formula unit usually contain sequences of $\mathrm{M}$ atoms with $\mathrm{CN}=126,6^{\prime}$ or 84 (underlined in Table 12), which can form compounds with $\mathrm{CaTiO}_{3}, \mathrm{SrO}$ or $(\mathrm{Ca}, \mathrm{Sr}) \mathrm{CuO}_{2}$ structure. Many compounds with more than $8 \mathrm{M}$ atoms are difficult to synthesize because of the stability of the $\mathrm{CaTiO}_{3}, \mathrm{SrO}$ and $(\mathrm{Ca}, \mathrm{Sr}) \mathrm{CuO}_{2}$ structure. The other compounds are obtained from 8 different mixtures of $\mathrm{M}^{3+}=\mathrm{Y}, \mathrm{La}, \mathrm{Tl}, \mathrm{Bi}, \ldots, \mathrm{N}^{2+}=\mathrm{Ca}, \mathrm{Sr}, \mathrm{Ba}$ and Cu: $\mathrm{M}_{n^{\prime}} \mathrm{O}_{\mathrm{x}}, n^{\prime}+1 \leq \mathrm{x} \leq 4 / 3 n^{\prime}, 3 \leq n^{\prime} \leq 8$ with $50 \%$ or less $\mathrm{Cu}$ atoms. The number $n^{\prime}$ of $\mathrm{M}$ atoms/formula unit $\mathrm{M}_{n^{\prime}} \mathrm{O}_{n^{\prime}+1}$ or $\mathrm{M}_{n^{\prime}} \mathrm{O}_{n^{\prime}+2}$ can be obtained from $n^{\prime}=n /(3-n)$.

The I atoms and vacancy positions of the $\mathrm{NaCl}$ group of structures exhibit a ccp lattice. Therefore the undistorted structures can be compared with the alloy structures (Tables 4,11a). The ordering of $\mathrm{C}$ atoms in $\mathrm{Gd}_{2} \mathrm{C} \square$ or $\mathrm{Ti}_{2} \mathrm{C} \square$ corresponds to the ordering of $\mathrm{Cu}$ atoms in $\mathrm{CuPt}$,a or b, respectively. The metal atoms of $\alpha-\mathrm{NaFeO}_{2}$ and $\mathrm{LiTbS}_{2}$ occupy the same positions as the $\mathrm{Cu}$ and $\mathrm{Pt}$ atoms (Table 12). The same applies for $\mathrm{SrCrF}_{4}(\mathrm{CuAu}), \mathrm{CuSr}_{2} \mathrm{~F}_{6}\left(\mathrm{CuZr}_{2}\right), \mathrm{TiTe}_{3} \mathrm{O}_{8}\left(\mathrm{AuCu}_{3}\right)$ and $\mathrm{UNa}_{3} \mathrm{~F}_{8}$ 

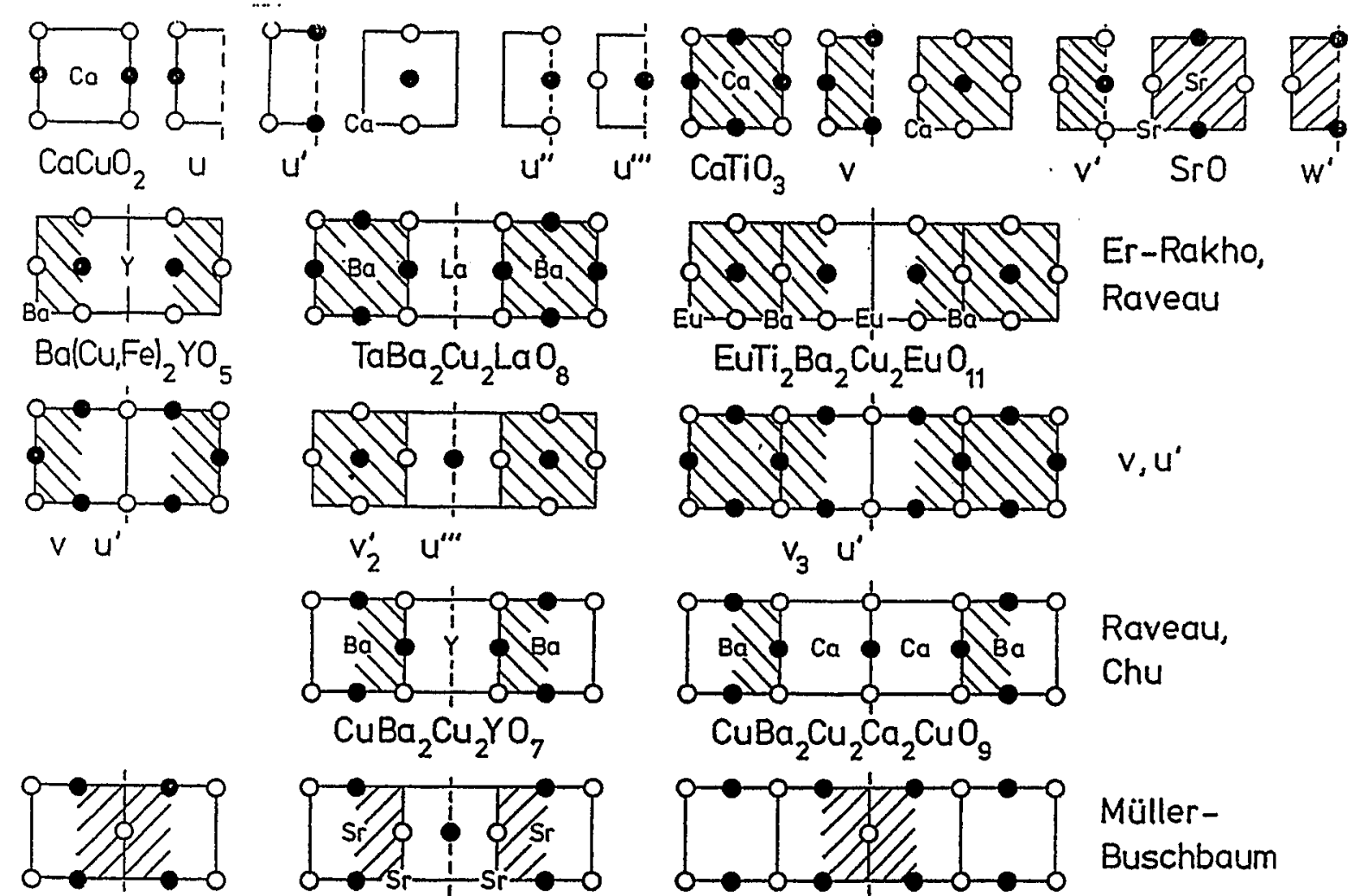

$u^{\prime} w^{\prime}$
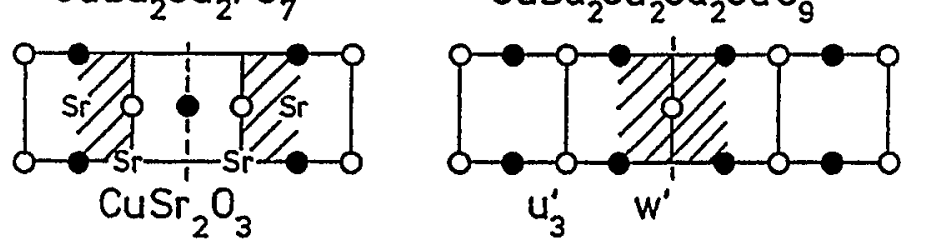

Müller-

Buschbaum
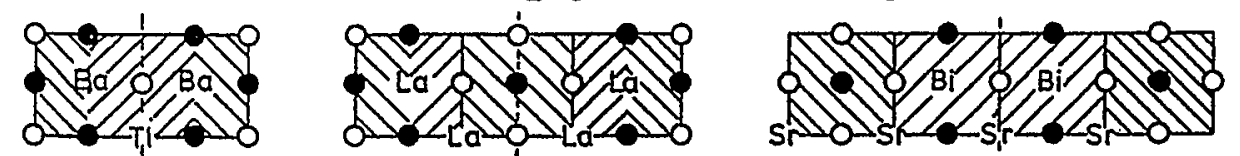

Ruddlesden,

Popper

Müller.
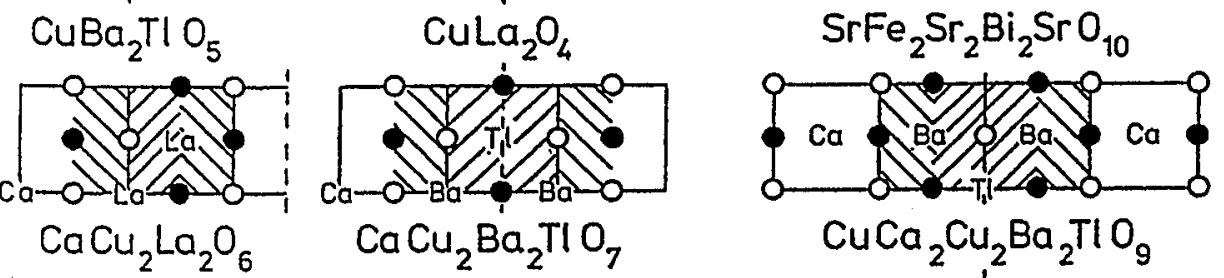

Bednorz
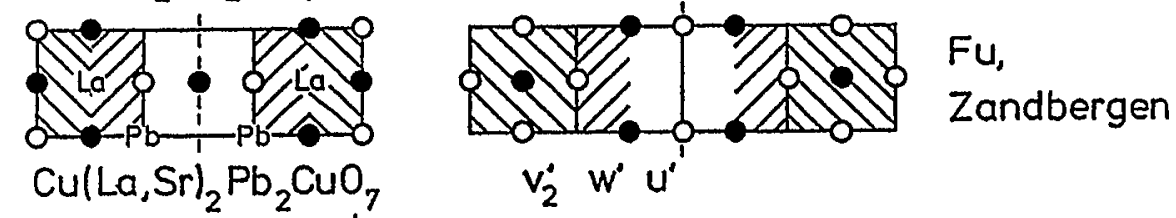

$$
v_{2}^{\prime} w^{\prime} u^{\prime}
$$
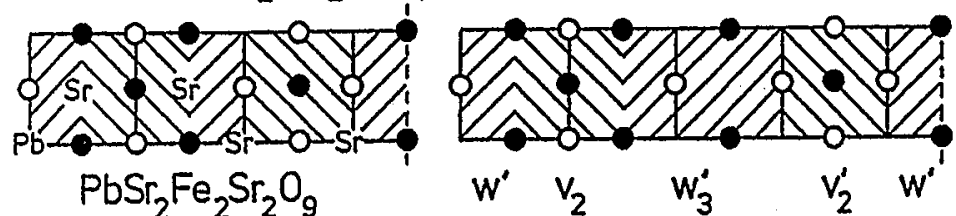

Lucas,

Raveau

$\mathrm{PbSr}_{2} \mathrm{Fe}_{2} \mathrm{Sr}_{2} \mathrm{O}_{9}$

$\begin{array}{lllll}w^{\prime} & v_{2} & w_{3}^{\prime} & v_{2}^{\prime} & w^{\prime}\end{array}$
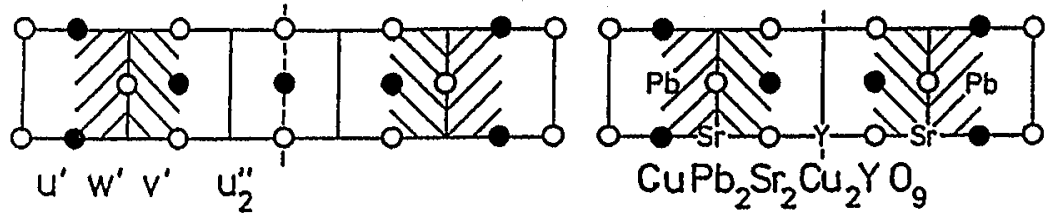

Subramanian, Cava 


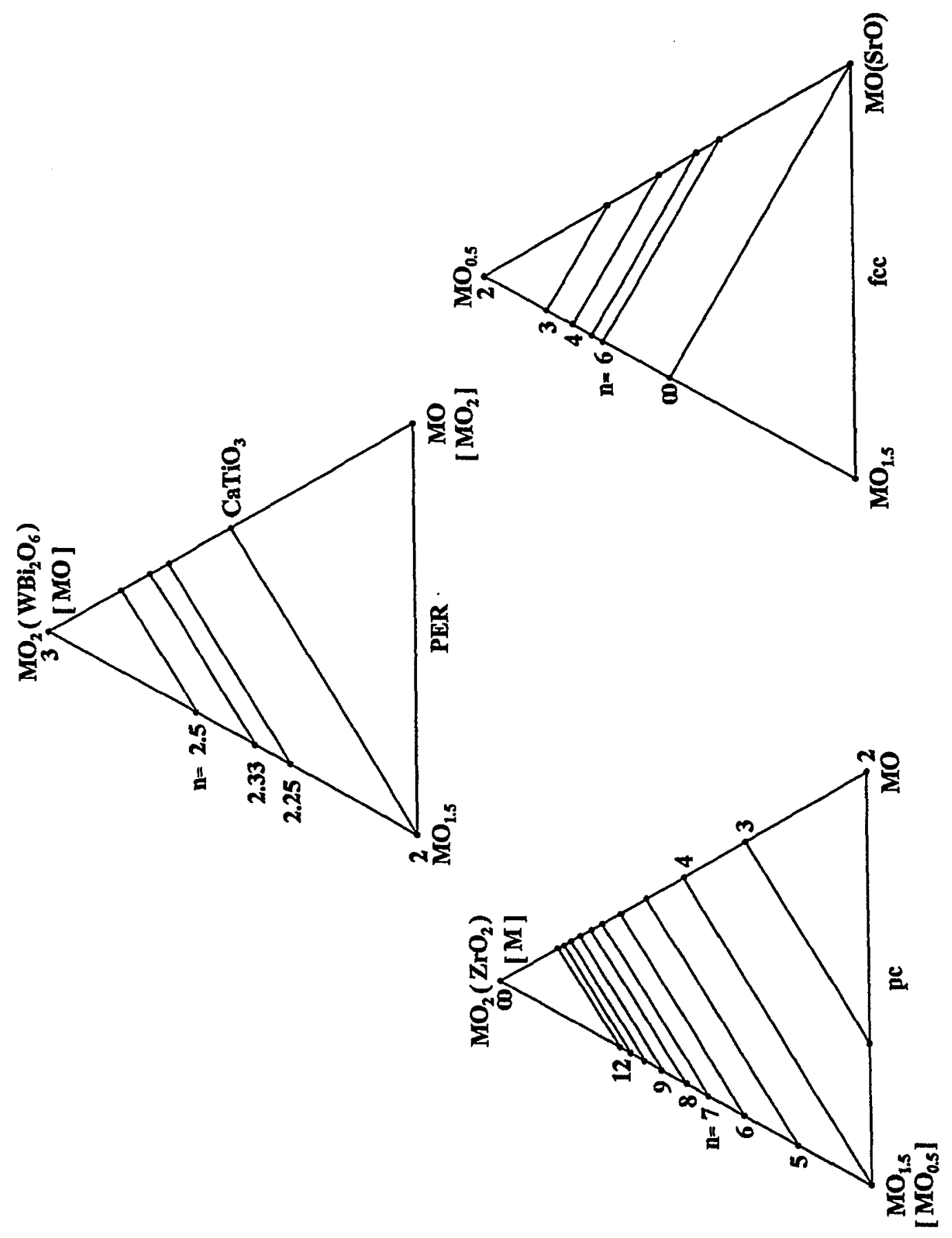

Figure 24: Pseudoternary phase diagrams of $\mathrm{M}_{n} I_{\mathrm{x} n-\mathrm{x}} \square_{\mathrm{x}}$ structures of the $\mathrm{NaCl}$ ( $\mathrm{x}=$ 1), $\mathrm{CaF}_{2}(\mathrm{x}=2)$ and PER group $(\mathrm{x}=3)$ and different $n$ values and related $\mathrm{M}_{n} \mathrm{I}_{\mathrm{x}} \square_{\mathrm{x} n-\mathrm{x}}$ structures in brackets. The $n^{\prime}=n /(3-n)$ values of superconducting oxides $\mathrm{M}_{n^{\prime}} \mathrm{O}_{n^{\prime}+1}$ and $\mathrm{M}_{n^{\prime}} \mathrm{O}_{n^{\prime}+2}$ can be obtained from the $n$ values. 
$\left(\mathrm{TiAl}_{3}\right)$ (Wells, 1984). The $\mathrm{F}$ or $\mathrm{O}$ atoms at tetrahedral interstices have the pc lattice of the $\mathrm{CaF}_{2}$ structure.

The three-dimensional structure maps for the PER group (Hauck and Mika, 1993) and the pc group contain different polyhedra at different $\mathrm{r}=\mathrm{y} / \mathrm{x}$ values. The four structures $6128 ;(1), 440 ; 1,248 ; 1$ and $0120 ; 1$ of the pc group are at the corners of a tetrahedron containing 4 planes and 6 edges. The points $6128 ;(1), 440 ; 1$ and $0120 ; 1$ remain in the $T_{1} T_{2} T_{3} ; \mathrm{r}$ structure map at increased $\mathrm{r}=\mathrm{y} / \mathrm{x}$ values, while new corners occur forming different polyhedra:

\begin{tabular}{|c|c|c|c|c|c|c|c|c|c|}
\hline range & $\mathrm{C}$ & $\mathrm{P}$ & $\mathrm{C}_{4}$ & $\mathrm{C}_{5}$ & $\mathrm{C}_{6}$ & $\mathrm{C}_{7}$ & $\mathrm{C}_{8}$ & $\mathrm{C}_{9}$ & $\mathrm{C}_{10}$ \\
\hline $1<\mathrm{r} \leq 5 / 3$ & 9 & 8 & $7 / 230$ & 330 & $3 / 236$ & $3 / 238$ & 090 & 092 & \\
\hline $5 / 3<r<3$ & 10 & 8 & 320 & $5 / 220$ & 126 & 128 & 062 & 064 & 070 \\
\hline $\mathrm{r}=3$ & 6 & 6 & 200 & 040 & 008 & & & & \\
\hline $3<\mathrm{r}<7$ & 8 & 7 & 200 & 100 & 004 & 008 & 020 & & \\
\hline $\mathrm{r} \geq 7$ & 6 & 6 & 200 & $\begin{array}{llll}0 & 0 & 0\end{array}$ & 008 & & & & \\
\hline
\end{tabular}

The corners $\mathrm{C}_{i}$ at intermediate $\mathrm{r}$ values are chosen for $\mathrm{r}=1.5,2$ and 5 . The variations of the corners at intermediate $\mathrm{r}$ values can be expressed by the series

\begin{tabular}{|c|c|c|c|c|c|c|}
\hline 440 & $\rightarrow$ & $3.32 .70 ; 1.7$ & & $200 ; \mathrm{r}(\mathrm{r} \geq 3)$ & & \\
\hline & $\rightarrow$ & $2.72 .70 ; 1.7$ & $\rightarrow$ & $200 ; \mathrm{r}(\mathrm{r} \geq 3)$ & $\rightarrow$ & $000 ; \mathrm{r}(\mathrm{r} \geq 7)$ \\
\hline & $\rightarrow$ & $1.32 .75 .3 ; 1.7$ & $\rightarrow$ & $008 ; \mathrm{r}(\mathrm{r} \geq 3)$ & & \\
\hline & $\rightarrow$ & $1.32 .78 ; 1.7$ & & $008 ; \mathrm{r}(\mathrm{r} \geq 3)$ & $\rightarrow$ & 000 \\
\hline & $\rightarrow$ & $080 ; 1.7$ & & $040 ; 3$ & $\rightarrow$ & $000 ; \mathrm{r}$ \\
\hline & & $082.7 ; 1.7$ & & $008 ; r(r \geq 3$ & $\rightarrow$ & $000 ; \mathrm{r}$ \\
\hline
\end{tabular}

The intermediate points depend linearly on $r$. The $008 ; 3$ structure forms a homologous series with the $248 ; 1$ and $488 ; 0.33$ structures at constant $\alpha_{1}=\alpha_{2}=-0.33$ and $\alpha_{3}=1$. The structure maps and structural units of the pc and PER group of structures are very similar (Figs. 25,26). Some structural units like $\alpha$ and $\beta$ are not possible in the PER group because of the metal atoms at I positions. Other structural 


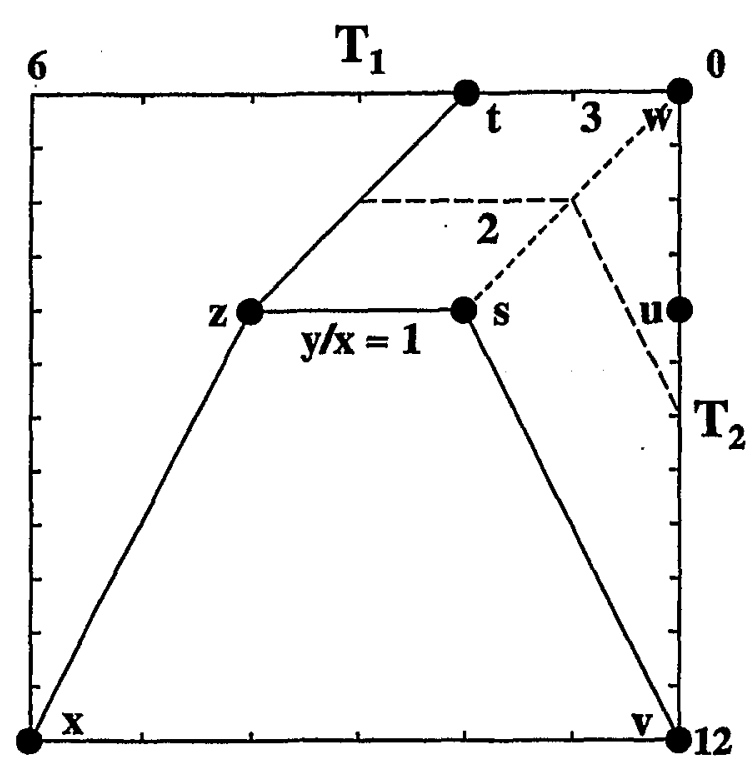

pc

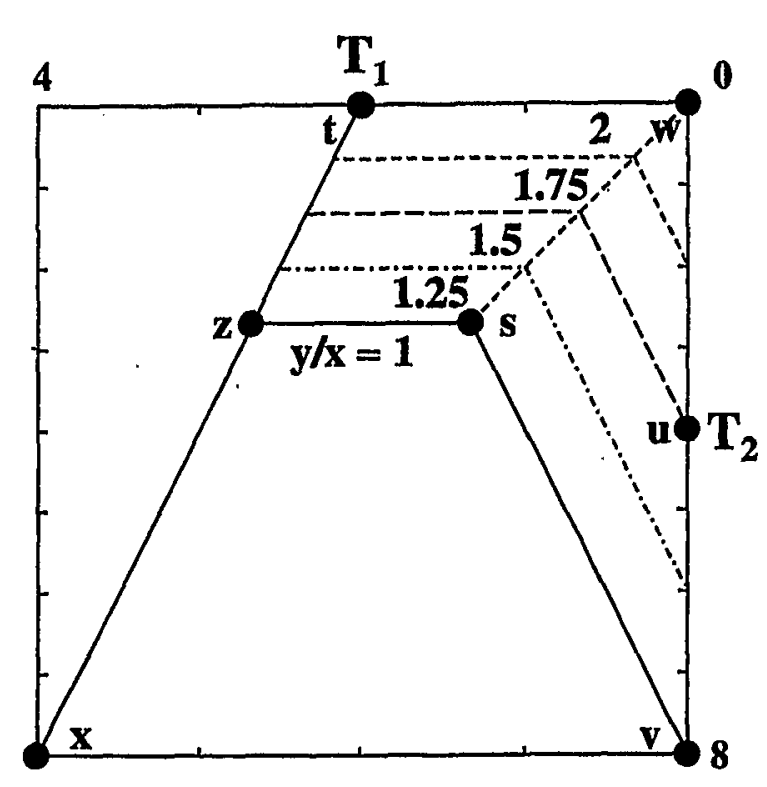

PER

Figure 25: $T_{1}, T_{2}$ structure maps of $\mathrm{CaF}_{2}(\mathrm{pc})$ and $\mathrm{CaTiO}_{3}$ (PER) related structures with structural units $\mathrm{u}-\mathrm{z}$.

units of Fig.26 are identical to structural units $\mathrm{u}-\mathrm{y}$ of the ccp group (Fig.12). The occupation of $50 \%$ of the tetrahedral interstices of the ccp or hcp lattice yields $\mathrm{ZnS} 3 \mathrm{C}$ (sphalerite) or $\mathrm{ZnS} 2 \mathrm{H}$ (wurtzite) (Table 11). The $\mathrm{Zn}$ atoms of sphalerite or wurtzite at the second, fourth, sixth $\mathrm{I}^{\mathrm{t}}$ layer (Fig.20) form a ccp (ABC) or hcp (AB) lattice. The divalent $\mathrm{Zn}$ atoms can be substituted by two or three atoms with different valencies like $\mathrm{SbCu}_{3} \mathrm{~S}_{4}$ or $\mathrm{AsCu}_{3} \mathrm{~S}_{4}$. The ordering of metal atoms can be compared with ordered alloy structures $\mathrm{TiAl}_{3}$ or $\mathrm{TiCu}_{3}$, respectively (Table 11). The $\mathrm{ZnS}$ related adamantane structures however are non-metallic valence compounds (Section 17). Table 11 shows also some structures of the $\mathrm{Ni}_{2} \mathrm{In}$ (Lidin and Larsson, 1995) and $\mathrm{BiF}_{3} / \mathrm{LaH}_{3}$ (Parthé et al., 1993) families of structures, where part of the $\mathrm{Ni}$ or $\mathrm{F}$ atoms, $\mathrm{A}=\mathrm{Ni}^{\prime}$ or $\mathrm{F}^{\prime}$, occupy a hcp or ccp lattice. The structures with vacancies $\square$ at the $\mathrm{Ni}^{\prime}$ or $\mathrm{F}^{\prime}$ positions can be formulated as $\mathrm{Ni}_{n} \mathrm{In}_{n} \square_{\mathrm{x}} \mathrm{Ni}_{n-\mathrm{x}}^{\prime}$ and $\left(\mathrm{BiF}_{2}\right)_{n} \mathrm{~F}_{n-\mathrm{x}}^{\prime} \square_{\mathrm{x}}$ (Table 11).

The occupation of tetrahedral interstices in ccp metals like $\mathrm{CaF}_{2}$ with $\mathrm{M}=\mathrm{Ca}$ at $\mathrm{A}, \mathrm{B}$ and $\mathrm{C}$ positions (Fig.20) yields the sequence ABABCB$\mathrm{CAC}, \mathrm{A} \hat{=} \mathrm{ch}_{2}$ of $\mathrm{Ca}$ and $\mathrm{F}$ atoms with the sequence $\mathrm{CaFF}, \mathrm{Ca}$ of $\mathrm{Ca}$ 

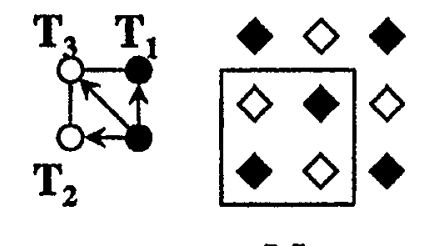

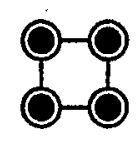

$\mathbf{x}$

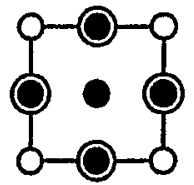

$\mathbf{x} \mathrm{x}$

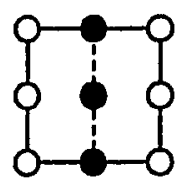

$s^{\prime \prime} s^{\prime \prime}$

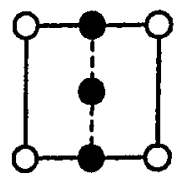

s" s"

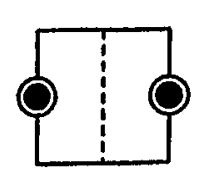

$t^{\prime} \mathbf{t}^{\prime}$

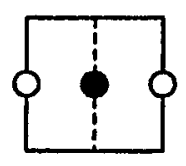

$u^{\prime \prime \prime} u^{\prime \prime \prime}$.

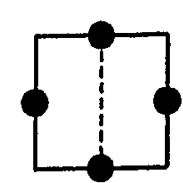

$\overline{\mathbf{u}} \overline{\mathbf{u}}$

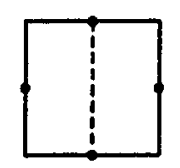

y $\mathbf{y}$

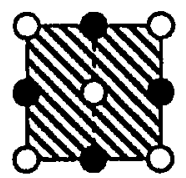

v v

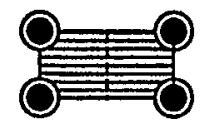

z $\mathbf{z}$

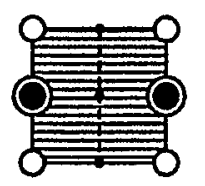

z $\mathbf{z}$

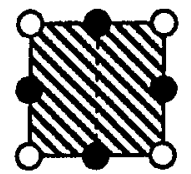

$\mathbf{v}$

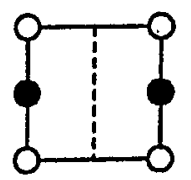

u u

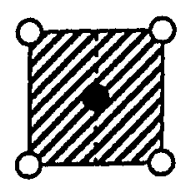

$\mathbf{w} \mathbf{w}$

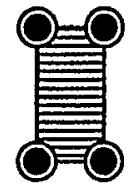

$\mathbf{z}^{\prime}$

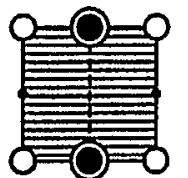

$\mathbf{z}^{\prime} \mathbf{z}^{\prime}$

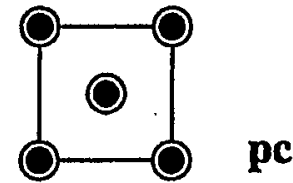

s $\mathbf{s}$

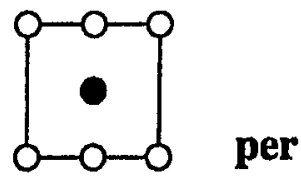

s $\mathbf{s}$

pe

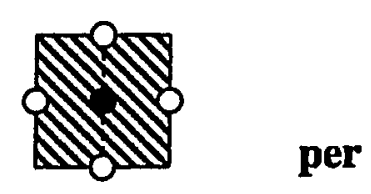

$\begin{array}{ll}\mathbf{v}^{\prime} & \mathbf{v}^{\prime}\end{array}$

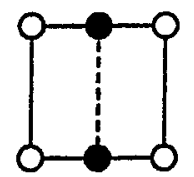

$\mathbf{u}^{\prime} \mathbf{u}^{\prime}$
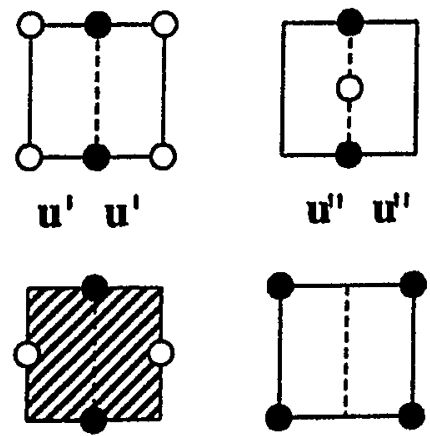

$\mathbf{w}^{\prime} \mathbf{w}^{\prime}$ $\mathbf{u}^{\prime \prime} \mathbf{u}^{\prime \prime}$

pe, per

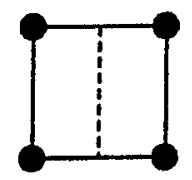

pe, per

h $\mathbf{h}$

Figure 26: Structural units of pc and PER group with $\mathrm{M}$ atoms at projection height 0 $(\square), 0.5(\diamond), 1(\square), 1.5(\diamond)$ and I atoms at $0(\bullet), 1(\circ)$ or 0 and $1(\odot)$ (to be continued on next page). 

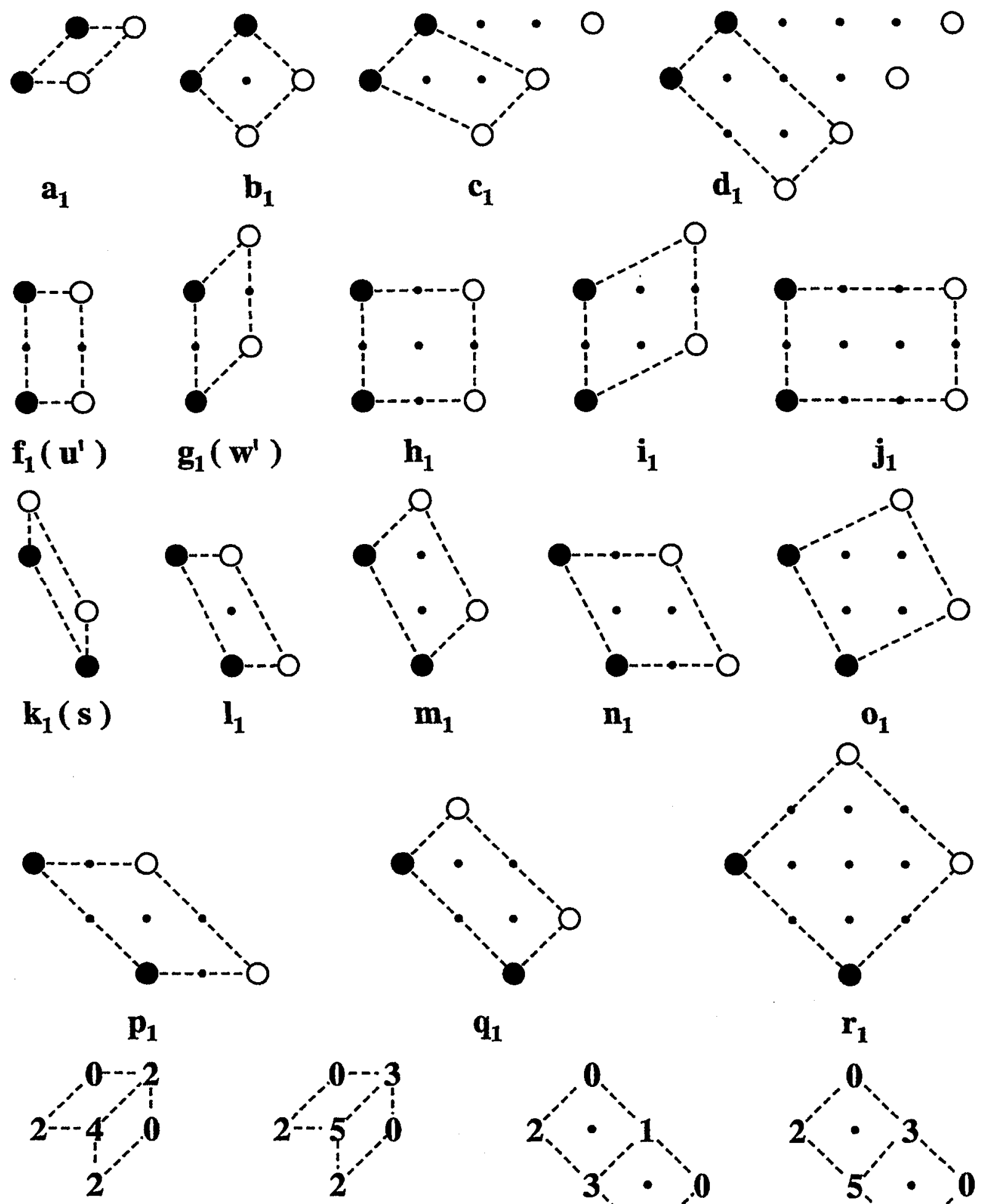

$\mathbf{q}_{1}$

$\mathbf{n}_{1}$

$\alpha \quad \alpha^{\prime}$

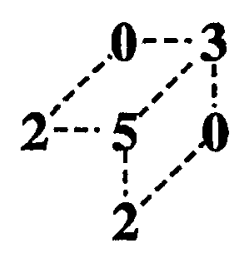

$\beta \quad \beta^{\prime}$

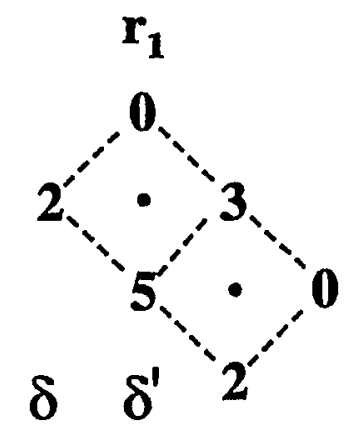


and $\mathrm{F}$ atoms at a distance $\Delta \mathrm{z} c / a \approx 0.2$ between $\mathrm{Ca}$ and $\mathrm{F}$ atoms and $\approx 0.4$ between $\mathrm{F}$ atoms. Many compounds with different properties like $\mathrm{SnMg}_{2}$ (intermetallic), $\mathrm{CeH}_{2}$ (interstitial metallic hydride), $\mathrm{CeO}_{2}$ (ionic conducting oxide) and $\mathrm{Li}_{2} \mathrm{O}$ ( $\mathrm{Li}$ conducting compound with antifluorite structure) are isotypic to $\mathrm{CaF}_{2}$ (Wyckoff, 1982). The $\mathrm{CdCl}_{2} \mathrm{C19}$ structure with the same sequences RMR and hch (Table 7) contains $\mathrm{Cd}$ atoms at octahedral interstices of $\operatorname{ccp~Cl}$ atoms with $\Delta \mathrm{z} c / a \approx 0.4$ or 0.8 between $\mathrm{Cd}$ and $\mathrm{Cl}$ or $\mathrm{Cl}$ and $\mathrm{Cl}$ atoms, respectively. The $\mathrm{Ni}_{2} \square \mathrm{Al}_{3}$ structure, which is usually considered as a bcc alloy with $\mathrm{c}_{6}$ stacking and $\Delta \mathrm{z} c / a \approx 0.2$ (Table 7 ), changes to the hc ${ }_{3} \mathrm{~h}$ stacking of $\gamma-\operatorname{In}_{2} \mathrm{~S}_{3}$ with the same space group but $\Delta \mathrm{z} c / a \approx 0.4$ between In and $\mathrm{S}$ atoms. The distances of all layers are $\Delta z c / a \approx 0.2$ in the $\mathrm{BiF}_{3}$ or $\mathrm{LaH}_{3}$ structure at the occupation of all tetrahedral and octahedral interstices with the sequences $\mathrm{ABC}, \mathrm{A} \cong \mathrm{c}$ of $\mathrm{BiFFF}, \mathrm{Bi}$ (Fig.20).

The coordination of the $\mathrm{Bi}$ atoms corresponds to $\mathrm{CN}=14$ of the ccp dodecahedron (Fig.27), if the eight $\mathrm{F}$ atoms at tetrahedral sites $\mathrm{a}-\mathrm{h}$ are added to the six $\mathrm{F}$ atoms at $1-6$. The structure of the interstitial (non-metallic) compound $\mathrm{BiF}_{3}$ is identical to the structure of the ordered bcc alloy $\mathrm{AlFe}_{3}$ (Table 7, B form).

The $74.1 \%$ density of cubic close-packed $\mathrm{M}$ atoms is increased by $1.7 \%$ and $5.3 \%$ at complete occupation of all tetrahedral and octahedral interstices by $\mathrm{I}$ atoms with $0.225 d$ and $0.414 d$ of the $\mathrm{M}$ atom radii. Therefore the density of ordered bcc alloys like $\mathrm{AlFe}_{3} \widehat{=} \mathrm{BiF}_{3}$ is not $68.0 \%$ as for bcc W with equally sized $M$, but increased to a maximum of $81 \%$ for fcc $\mathrm{Bi}$ with an occupation of all interstitial sites. Another example is the $52.4 \%$ density of a pc lattice which is increased to $72.9 \%$ in the $\mathrm{CsCl}$ structure, if the interstitial atom radius is $0.732 d$.

The occupation of all tetrahedral interstices $\mathrm{I}^{\mathrm{t}}$ in hcp metals (Fig.20) yields the sequence $A B, A \cong h$ of atoms $M I I, M$ and $A B C A B A C B, A$ $\widehat{=} \mathrm{hc}_{3}$ of atoms MIII,M at occupation of tetrahedral and octahedral interstices of the non-existing compounds $\mathrm{MI}_{2}$ and $\mathrm{MI}_{3}$, respectively. The distances between the layers are identical to the $\mathrm{CaF}_{2}$ and $\mathrm{BiF}_{3}$ structures. The $\mathrm{MI}_{2}$ and $\mathrm{MI}_{3}$ compounds are supposed to be unfavorable compared to the $\mathrm{CaF}_{2}$ and $\mathrm{BiF}_{3}$ structures because of the short distance 

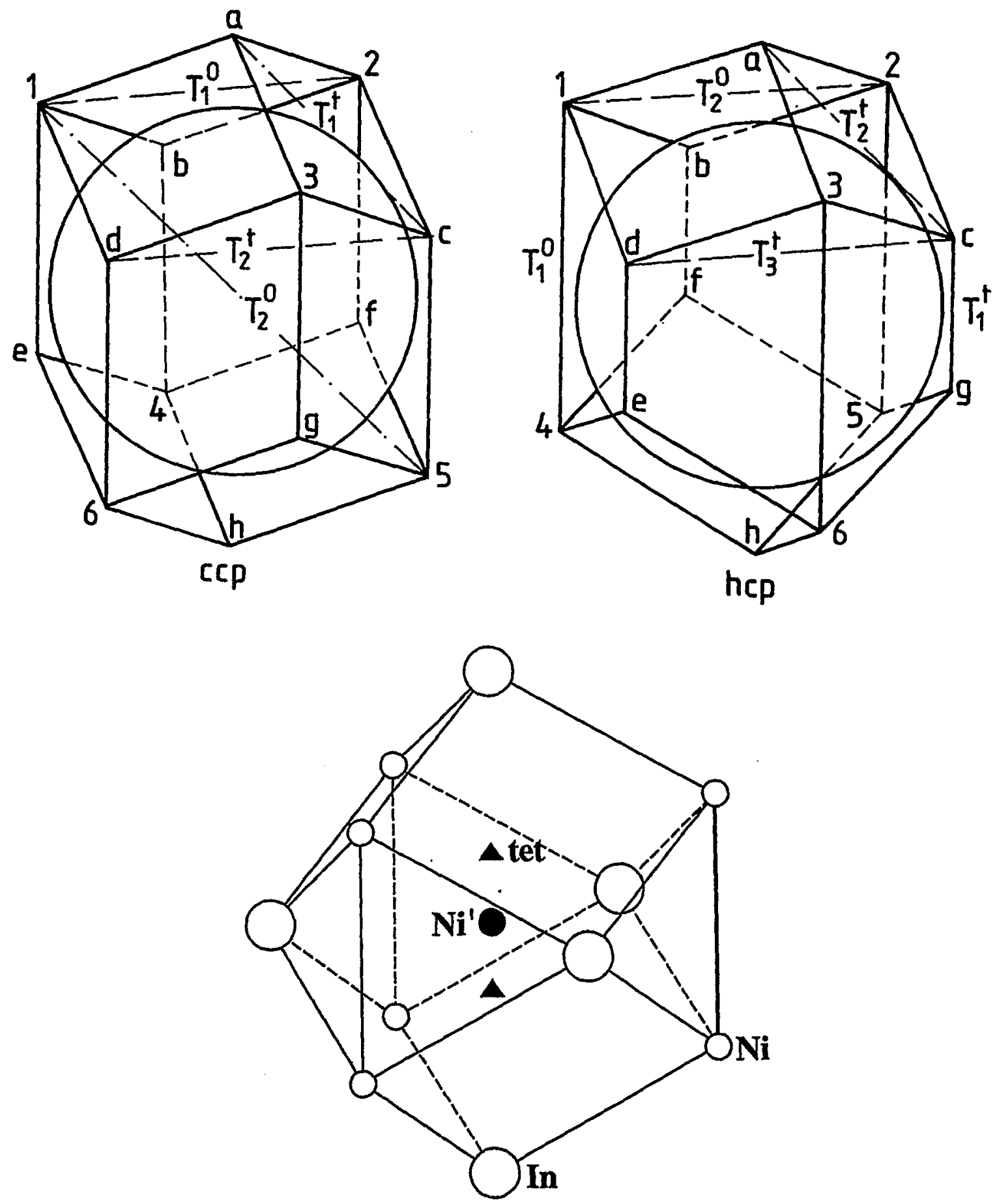

Edshammar polyhedron

Figure 27: Positions $1-6$ of octahedral and $\mathrm{a}-\mathrm{h}$ of tetrahedral sites in the neighborhood of a ccp or hcp M atom with some self-coordination numbers $T_{i}^{o}$ and $T_{i}^{t}$ of octahedral and tetrahedral interstices and the Edshammar polyhedron of $\mathrm{Ni}^{\prime}$ of $\mathrm{Ni}_{2} \mathrm{In}$ with five In and six $\mathrm{Ni}$ neighbors. The $\mathrm{Ni}^{\prime}$ atoms are between two tetrahedral sites $\mathrm{c}$ and $g$ in the hcp In lattice. 

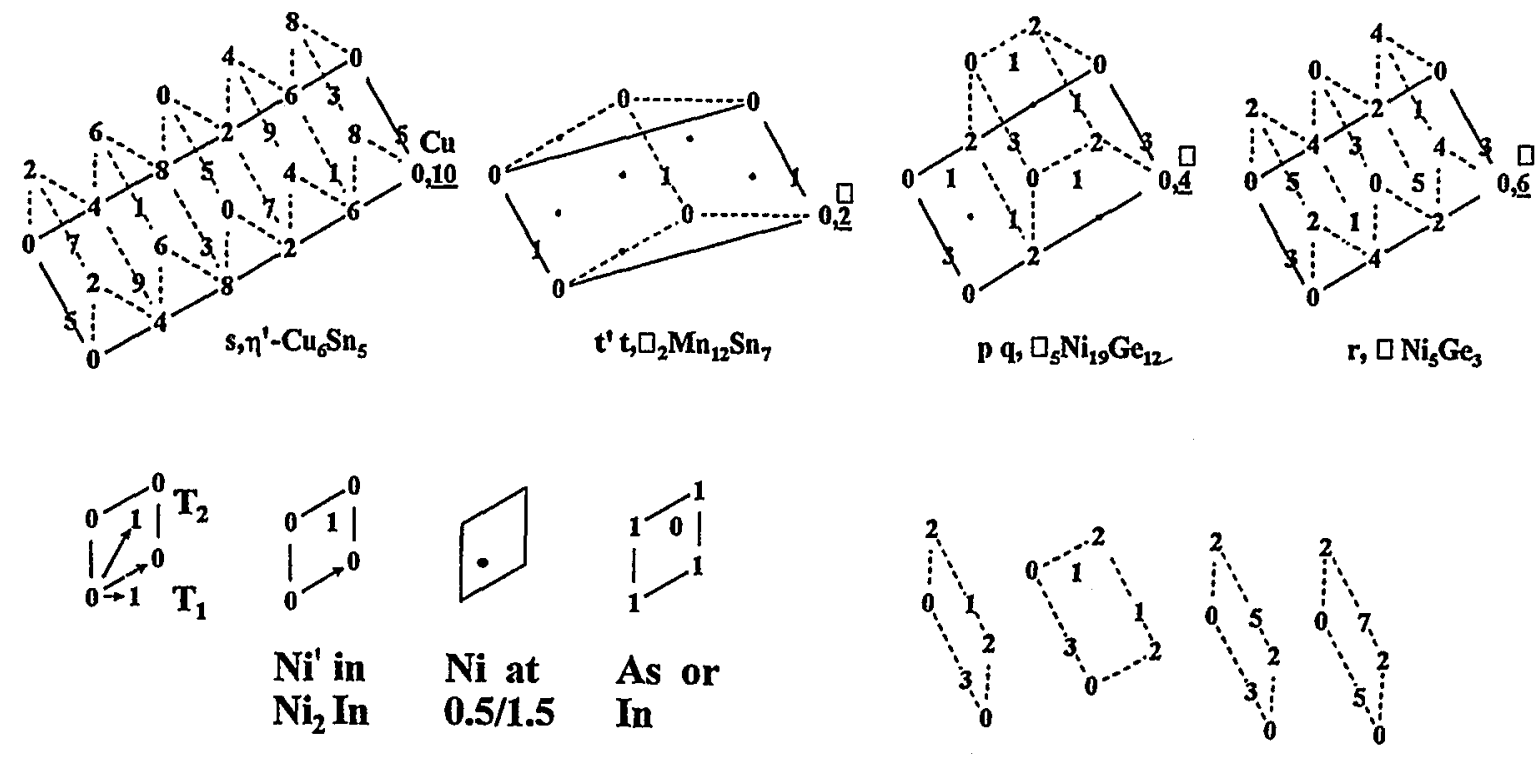

$\mathbf{p} \quad \mathbf{r} \quad \mathbf{s}$

Figure 28: Projection of $\mathrm{Ni}_{2}$ In structure with hcp ordering of $\mathrm{Ni}^{\prime}$ atoms or vacant $\mathrm{Ni}^{\prime}$ positions $\square$ in different compounds with structural units $p-t$.

of $d / \sqrt{6}$ between nearest I atoms compared to $d / \sqrt{2}$ in ccp metals with diameter $d$ of $\mathrm{M}$ atoms (like $c$ and $\mathrm{g}$ sites in the hcp and ccp M lattice, Fig.27).

The $\mathrm{Ni}^{\prime}$ atoms of the $\mathrm{Ni}_{2} \mathrm{In}$ related series occupy a single position between $\mathrm{c}$ and $\mathrm{g}$ of the hcp lattice. The $\mathrm{Ni}^{\prime}$ atom has three $\mathrm{M}=\mathrm{In}$ atoms at the same projection height and two other $M$ neighbors in the same position $\mathrm{A}$ (or $\mathrm{B}$ ) as $\mathrm{Ni}^{\prime}$ in the two neighboring layers. The $\mathrm{CN}=5$ coordination of the trigonal bipyramidal site is also known as Edshammar polyhedron (Larsson et al., 1994), if the six nearest $\mathrm{Ni}$ atoms at $\mathrm{C}$ positions (octahedral site) are included (Fig.27) (Lidin and Larsson, 1995). Therefore the $\mathrm{Ni}_{2}$ In structure can also be described as a hexagonal close-packed array of $\mathrm{Ni}^{\prime}$ centered Edshammar, $\mathrm{CN}=11$ polyhedra, which are empty in the NiAs structure. The $\mathrm{Ni}_{2} \mathrm{In}$ related structures contain many ordered phases, which are not known for ordered hcp alloys including a different family of structural units $\mathrm{p}-\mathrm{t}$ in $\mathrm{Ni}_{12} \mathrm{Ge}_{12} \square_{5} \mathrm{Ni}_{7}$ (pq) and $\mathrm{Ni}_{3} \mathrm{Ge}_{3} \square \mathrm{Ni}_{2}$ (r) (Ellner et al., 1971) (Fig.28). 
Octahedral interstices of hcp $\mathrm{Nb}$ are occupied by $\mathrm{I}=\mathrm{N}$ atoms in the $\delta$-NbN structure (or the antistructure NiAs with $\mathrm{As}$ at $\mathrm{Nb}$ positions, Table 7, I form). The interstitial $\mathrm{N}$ atoms of $\mathrm{NbN}$ are all on $\mathrm{C}$ positions (Fig.20) with a primitive hexagonal (ph) lattice with the same distance $\Delta \mathrm{z} c / a \approx 0.4$ between neighboring layers as in $\mathrm{NaCl}$. The ph lattice of $\mathrm{N}$ atoms has the lattice constants $a_{\text {hex }}$ of the hexagonal layer and $c=\sqrt{2 / 3} a_{\text {hex }}$. The distance between two layers is only $82 \%$ of the shortest $\mathrm{M}-\mathrm{M}$ distance in undistorted structures. This can explain why interstitial atom positions at identical projection sites are not occupied in adjacent layers because of the size of interstitial atoms or the Coulomb repulsion between these atoms. Therefore, the ordered distribution of I atoms in hcp metals can be treated as single hexagonal layers (Hiraga and Hirabayashi, 1977) (Table 13). The coordinates of the structure map (Fig.29a,b) are identical with the $\alpha_{2}, \alpha_{5}, \alpha_{7}$ coordinates of the three-dimensional case with $T_{i}^{\max }=2,6,12,2,6,24,6$ for $i=1-$ 7. The $T_{1}, T_{2}, T_{3}$ values of the $\mathrm{ph}$ lattice contribute less information because of the $T_{1}=0$ values of the right-hand border of the structure map (Fig.29b). The formula $\mathrm{M}_{n} I \square_{n-1}$ is identical to the $\mathrm{NaCl}$ group. The Nb atoms of $\mathrm{NbN}$ however have a prismatic coordination. The CN $=6$ value of $\mathrm{N}$ in $\mathrm{NbN}$ is reduced to $\mathrm{CN}=1-5$ in the other compounds containing vacancies. The configurations can be obtained from the type and stacking of hexagonal layers.

Structures with a primitive hexagonal lattice and occupation of most positions like the ionic conductor $\mathrm{Li}_{3} \mathrm{~N}_{2}$ with s-CN values $0282642 ; 1$ of $\mathrm{Li}$ atoms and $0002600 ; 5$ of $\mathrm{N}$ atoms are very rare. Hexagonal and rhombohedral graphite or the corresponding $\mathrm{BN}$ modifications can be described by distorted ph lattices containing vacant positions $\square$ in the center of the honeycomb net with composition $\square \mathrm{C}_{2}$ or $\square \mathrm{BN}$ and $T_{i}(\square)$ values $0062600 ; 2$ or $0060660 ; 2$, respectively. The sequences of vacancies in the hexagonal unit cells correspond to the $\mathrm{Mg}$ and $\mathrm{Cu}$ structures. The $\mathrm{N}$ or $\mathrm{C}$ atoms in $\varepsilon-\mathrm{Fe}_{3} \mathrm{~N}$ or $\varepsilon-\mathrm{Ni}_{3} \mathrm{C}$ are ordered in the same way (Table 13). Different ratios $c / a_{\text {hex }}$ in these compounds give rise to different sequences of $T_{i}$ values. At $c / a_{\text {hex }}=1$ for example the spheres with diameter $d$ of a primitive hexagonal packing ( $\mathrm{ph}^{\prime}$ system) have $2+$ $6=8$ nearest neighbors at distance $d, 12$ next-nearest neighbors at $\sqrt{2} d$ and 6 third neighbors at $\sqrt{3} d$ (corresponding to $T_{3}$ and $T_{5}$ values of the ph 

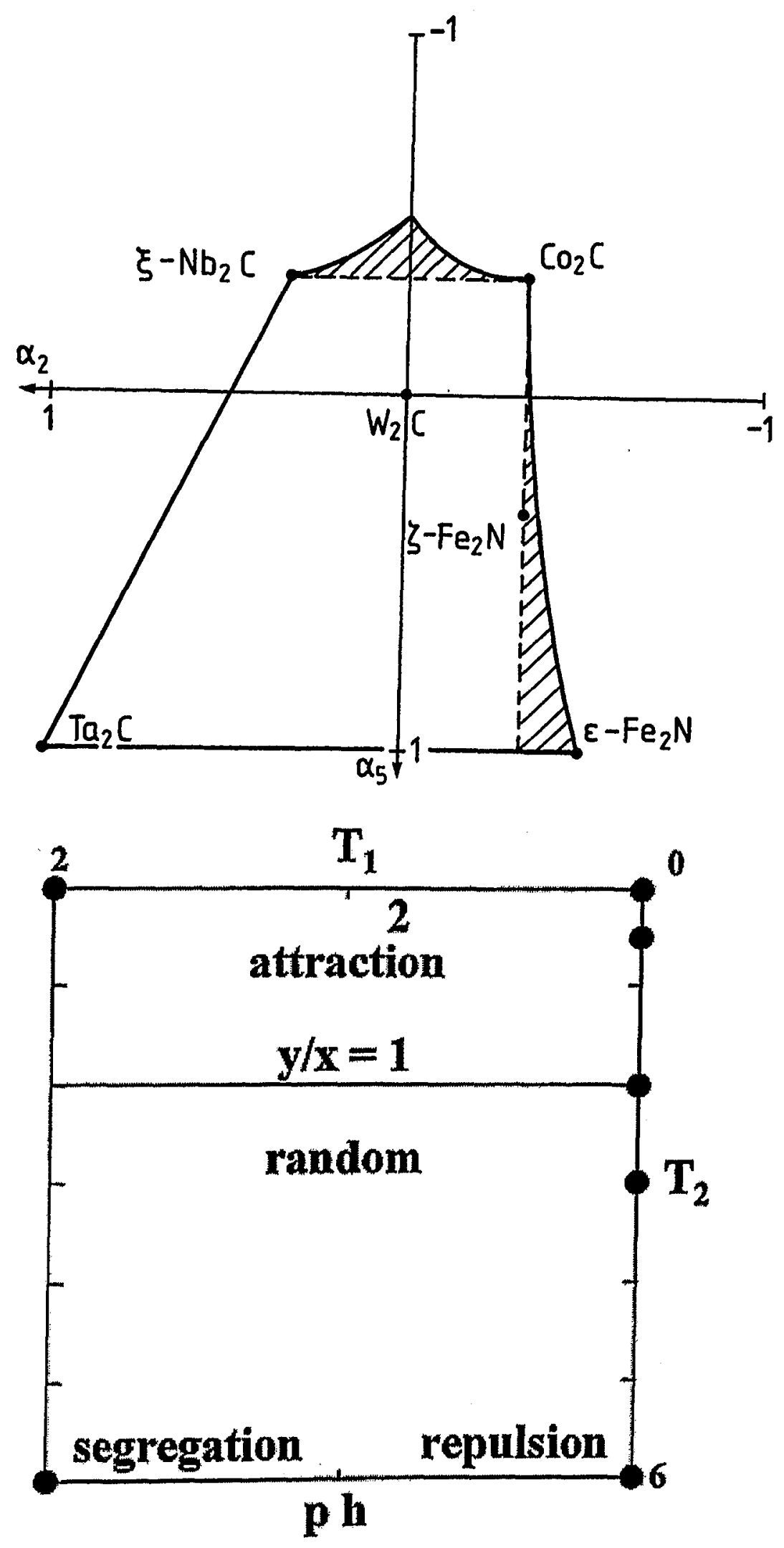


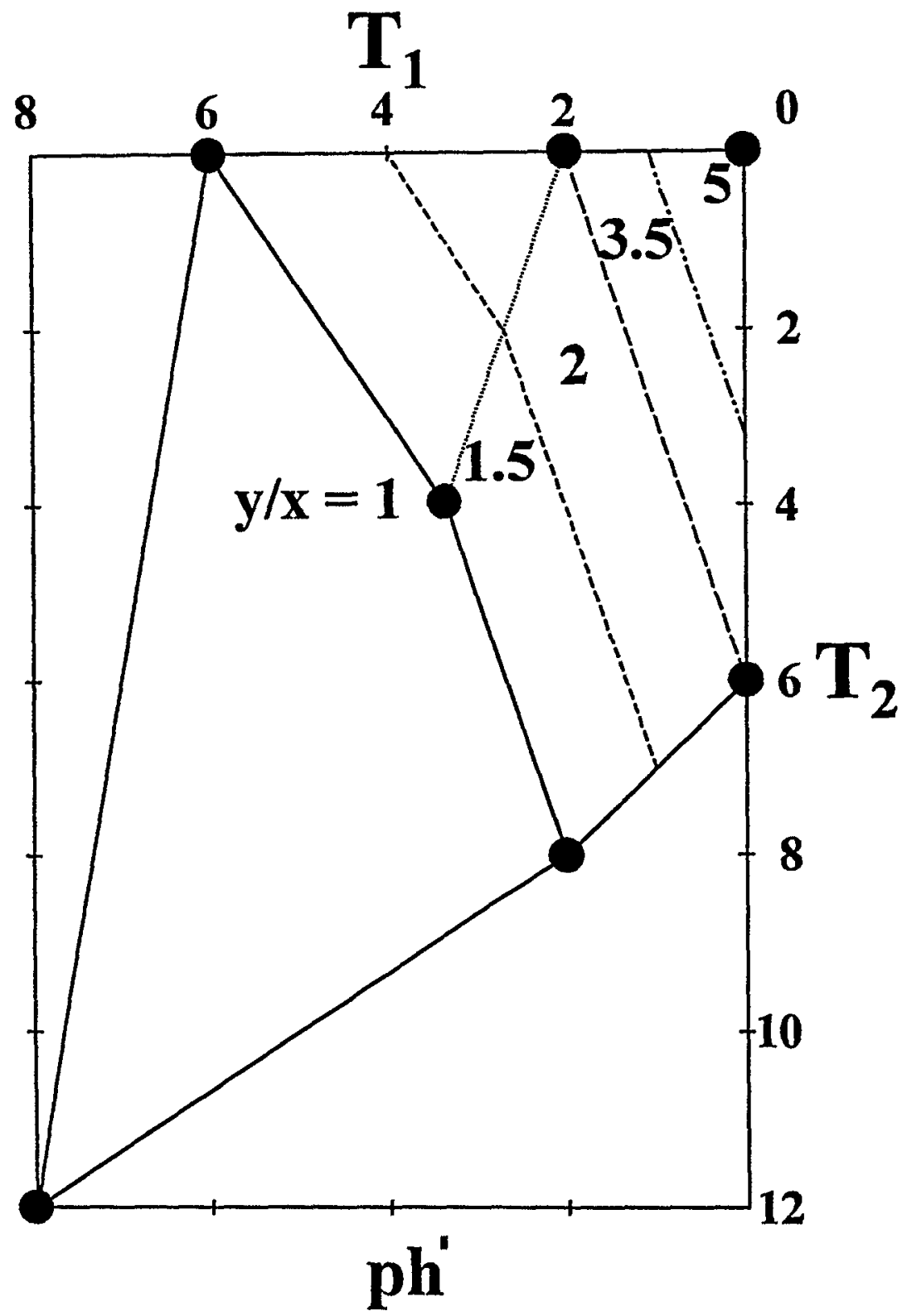

Figure 29: $\alpha_{2}, \alpha_{5}$ structure map of hcp $\mathrm{M}_{z} \square_{\mathrm{x}} \mathrm{I}_{\mathrm{y}}$ interstitial alloys, corresponding to $\alpha_{1}, \alpha_{2}$ of hexagonal plane (Fig.6) (a) and $T_{1}, T_{2}$ structure map of primitive hexagonal lattices $\mathrm{ph}(\mathrm{b})$ and $\mathrm{ph}^{\prime}(\mathrm{c})$. 
system). The distorted structures with increased distances between the layers $c / a_{\text {hex }}>1$ can be derived from the $T_{i}$ values of the undistorted $\mathrm{ph}$ and $\mathrm{ph}^{\prime}$ systems (Table 13). The neighboring hexagonal layers of most compounds with $T_{1}=0$ are shifted by translations $\mathrm{x}, \mathrm{y}, \mathrm{z}$ to avoid $T_{1}$ neighbors of the ph lattice. Structures with identical projections in [0001] direction are characterized by the same set of $T_{i}$ values. The $T_{i}$ values of hexagonal or trigonal structures with fixed $x, y, z$ parameters and structure types containing $M$ atoms in these positions are listed in Section 33 (Table 25). The Kiessling and Ganglberger structure families containing $\mathrm{Ta}_{3} \mathrm{~B}_{4}$ (Kiessling, 1949) and $\mathrm{Zr}_{2} \mathrm{Fe}_{12} \mathrm{P}_{7}$ (Ganglberger, 1968) (Table 13) can be identified by different patterns of the hexagonal layers (Section 28).

\section{Bonding in interstitial alloys}

The interstitial atoms $\mathrm{I}=\mathrm{H}, \mathrm{C}, \mathrm{N}, \ldots$ can occupy the tetrahedral or octahedral sites of the ccp $M$ structure at the composition $\mathrm{M}_{\mathrm{z}} \mathrm{I}_{\mathrm{y}}$ with $0.5 \leq \mathrm{y} / \mathrm{z} \leq 1$. The remaining $(\mathrm{z}-\mathrm{y})$ octahedral sites are empty. The larger number of metal atoms and interstitial atoms can be neglected in a description of the ordered structures, if the positions of the smaller number of vacant sites $\square$ are considered in the formula $M_{z} \square_{x} I_{y}, x+$ $y=z$. The positions of the $M$ and I atoms must only be considered for the effect of lattice distortion caused by vacancy ordering and for the determination of the space group. The sublattice of vacancies plus interstitial atoms $\square_{x} I_{y}$ can be compared with the lattice of ordered $M_{x} N_{y}$ alloys (Hauck et al., 1988b). The vacancies and I atoms exhibit the same $\mathrm{ABC}$ layer sequence as the metal atoms of ccp M (Fig.20). Therefore the distribution of vacancies of the $\square_{\mathrm{x}} \mathrm{I}_{\mathrm{y}}$ sublattice can be described by the same coordination numbers $T_{1}, T_{2}, T_{3}$ and concentration $\mathrm{y} / \mathrm{x}$ as for ordered ccp alloys $\mathrm{M}_{\mathrm{x}} \mathrm{N}_{\mathrm{y}}$ (Table 11a). The carbides $\mathrm{Ti}_{2} \mathrm{C}, \mathrm{Gd}_{2} \mathrm{C}, \mathrm{Ti}_{8} \mathrm{C}_{5}$, $\mathrm{V}_{6} \mathrm{C}_{5}$ and $\mathrm{V}_{8} \mathrm{C}_{7}$ are located in area Ia of the structure map indicating covalent-type bonding, while the nitrides $\mathrm{Ti}_{2} \mathrm{~N}, \mathrm{Nb}_{4} \mathrm{~N}_{3}$ and the hydrides $\mathrm{Pd}_{2} \mathrm{H}$ and $\mathrm{PdD}_{0.8}$ are located at low $\alpha_{1}$ indicating Coulomb-type interactions (Fig.30). 


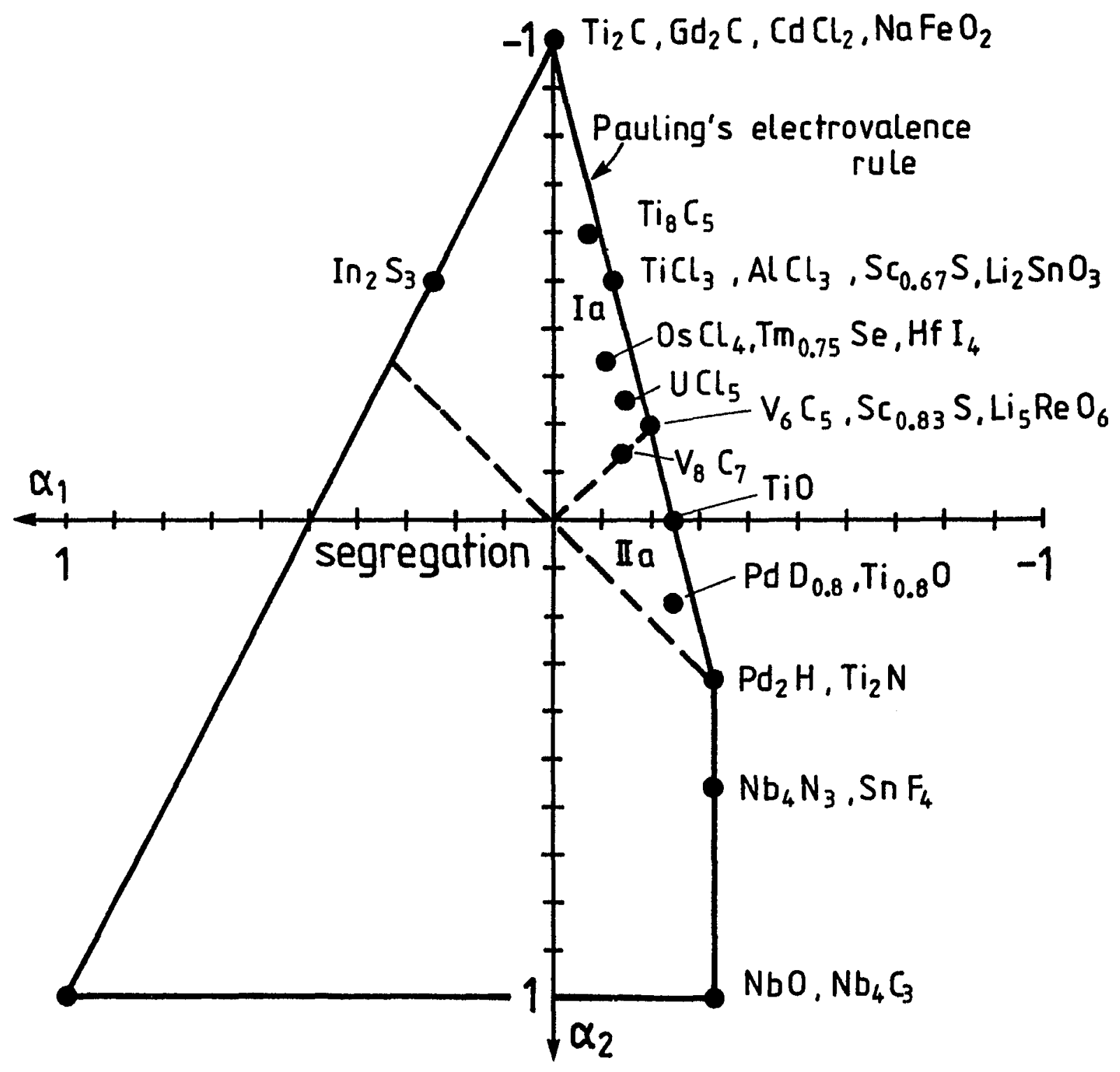

Figure 30: $\alpha_{1}, \alpha_{2}$ structure map of ccp interstitial alloys $M_{z} \square_{x} I_{y}$. Some halides $\square_{x} M_{y} I_{z}$ with metal atoms at octahedral interstices of the ccp I lattice (antitype structure) are also included. 
Two other types of interstitial alloys can be compared if the composition is formulated in a similar way to that used for the $\mathrm{M}_{\mathrm{z}} \square_{\mathrm{x}} \mathrm{I}_{\mathrm{y}}$ compounds with the convention that $\mathrm{z} \geq \mathrm{y} \geq \mathrm{x}: \square_{\mathrm{x}} \mathrm{M}_{\mathrm{y}} \mathrm{I}_{\mathrm{z}}$ with $\mathrm{I}=\mathrm{O}, \mathrm{S}, \mathrm{Se}, \mathrm{F}, \mathrm{Cl}$, I as in $\mathrm{Ti}_{0.8} \mathrm{O}, \mathrm{Lu}_{0.83} \mathrm{~S}, \mathrm{Tm}_{0.75} \mathrm{Se}_{\mathrm{SnF}}, \mathrm{SnCl}_{4}$ or $\mathrm{HfI}_{4}$ (Table 11a) and $\square_{\mathrm{x}} \mathrm{M}_{\mathrm{y}} \square_{\mathrm{x}}^{\prime} \mathrm{O}_{\mathrm{y}}^{\prime}$ as in $\square \mathrm{Ti}_{2} \square \mathrm{O}_{2}$ or $\square \mathrm{Nb}_{3} \square \mathrm{O}_{3}$ (Table 11a) with vacancies in both sublattices (Hauck and Mika, 1988b).

The $\mathrm{I}=\mathrm{O}, \mathrm{S}, \mathrm{Se}, \mathrm{F}, \mathrm{Cl}$ or I atoms are bigger than the $\mathrm{M}$ atoms. Therefore the $\mathrm{M}$ atoms fill part of the octahedral interstices of the close-packed I lattice. The structures are the antitype of $\mathrm{M}_{\mathrm{z}} \square_{\mathrm{x}} \mathrm{I}_{\mathrm{y}}$ (Lima-de-Faria et al., 1990). The sublattices of $\mathrm{M}$ and $\mathrm{I}=\mathrm{O}$ atoms of $\mathrm{NbO}$ and $\mathrm{TiO}$ are only partly filled. The vacancies of the $M$ and the I sublattice form the same structure: $2212 ; 2 \mathrm{a}$ for $\mathrm{TiO}$ and $060 ; 3$ for $\mathrm{NbO}$ (Fig.18).

Most interstitial alloys are metallic, some are superconducting like PdD, $\mathrm{TiC}, \mathrm{NbN}, \mathrm{TiO}, \mathrm{NbO}$ or $\mathrm{Sc}_{0.83} \mathrm{~S}$ (Toth, 1971; Moodenbaugh et al., 1978). The halides are non-metallic. Other non-metallic compounds which can be compared with the antitype structure $\square_{\mathrm{x}} \mathrm{M}_{\mathrm{y}} \mathrm{I}_{\mathrm{z}}$ are the ternary oxides $\mathrm{M}_{\mathrm{x}} \mathrm{N}_{\mathrm{y}} \mathrm{I}_{\mathrm{z}}$, e.g. $\mathrm{Li}_{5} \mathrm{ReO}_{6}, \mathrm{Li}_{2} \mathrm{SnO}_{3}$ or $\alpha-\mathrm{NaFeO}_{2}$ (Tables 11a,12, Fig.30), with $\mathrm{Re}, \mathrm{Sn}$ or $\mathrm{Fe}$ atoms at the vacancy positions.

The compositional parameters $\mathrm{x}, \mathrm{y}$ and $\mathrm{z}=\mathrm{x}+\mathrm{y}$ of the non-metallic compounds can not vary independently. The electroneutrality rule requires compensation of the negative charge of the oxygen or halide atoms. The charge $q(I)=-2$ or -1 , respectively, of these anions should be compensated by the electrovalence $\mathrm{q}_{i} /(\mathrm{CN})_{i}$ of the $\mathrm{p}_{i}$ neighboring metal atoms with coordination number $(\mathrm{CN})_{i}$ and charge $\mathrm{q}_{i}$ as defined by Pauling (1929 and 1945/1960; Burdett, 1995):

$$
\sum \frac{\mathrm{p}_{i} \mathrm{q}_{i}}{(\mathrm{CN})_{i}}=-\mathrm{q}(\mathrm{I})
$$

The $\mathrm{I}=\mathrm{O}$ atoms of the $\mathrm{N}_{\mathrm{y}} \mathrm{M}_{\mathrm{x}} \mathrm{I}_{\mathrm{z}}$ compounds, e.g. $\mathrm{Li}_{5} \mathrm{ReO}_{6}, \mathrm{Li}_{2} \mathrm{SnO}_{3}$ and $\alpha-\mathrm{NaFeO}_{2}$, are coordinated by $\mathrm{p}_{\mathrm{M}}=6 \mathrm{x} / \mathrm{z}=1,2$ or $3 \mathrm{M}$ atoms, respectively, $\mathrm{M}=\mathrm{Re}^{7+}, \mathrm{Sn}^{4+}$ and $\mathrm{Fe}^{3+}$, and $\mathrm{p}_{\mathrm{N}}=6 \mathrm{y} / \mathrm{z}=5,4$ or 3 $\mathrm{N}$ atoms, respectively ( $\mathrm{N}=\mathrm{Li}^{+}$or $\mathrm{Na}^{+}$atoms), which compensate the charge -2 of the oxygen atoms according to Pauling's electrovalence rule. The structures of these compounds correspond with the $\mathrm{V}_{6} \mathrm{C}_{5}, \mathrm{TiCl}_{3}$ and 
$\mathrm{Gd}_{2} \mathrm{C}$ structures (Table 11a) and are at the borderline of the (I, IIa) field of the structure map (Fig.30) with the correlations $4 \alpha_{1}+\alpha_{2}+1=0$ and $\alpha_{1}+\alpha_{3}=0$ between $\alpha_{1}, \alpha_{2}$ and $\alpha_{3}$ values (Hauck et al., 1989).

The metal atoms of the interstitial alloys $\mathrm{M}_{\mathrm{z}} \mathrm{I}_{\mathrm{y}}, \mathrm{I}=\mathrm{H}, \mathrm{C}, \mathrm{N}$ are surrounded by $\mathrm{CN}=6 \mathrm{y} / \mathrm{z}$ I atoms: $\mathrm{CN}=3$ at $\mathrm{y} / \mathrm{z}=0.5$, e.g. in $\mathrm{TiC}_{0.5}$ or $\mathrm{TiN}_{0.5} ; \mathrm{CN}=4$ at $\mathrm{y} / \mathrm{z}=0.67$ and $\mathrm{CN}=5$ at $\mathrm{y} / \mathrm{z}=0.83$, e.g. in $\mathrm{V}_{6} \mathrm{C}_{5}$. The three I atoms of $\mathrm{MI}_{0.5}$ are in the neighboring positions 1,2 , 3 (Fig.27) of facial configuration as in $\mathrm{TiC}_{0.5}$ or $\mathrm{GdC}_{0.5}$ or further apart at 1,2,5 in meridional configuration as in $\mathrm{PdH}_{0.5}, \mathrm{TiN}_{0.5}$ (Table 14) (Hauck, 1981). The self-coordination number $T_{1}$ of the $6012 ; 1 \mathrm{~b} \mathrm{TiC} \mathrm{T}_{0.5}$ structure is increased compared with the $4416 ; 1 \mathrm{TiN}_{0.5}$ structure, because of the close distance between I atoms in facial configuration. The $\mathrm{M}$ atoms of the facial configuration can be shifted to the $\mathrm{C}$ atoms to decrease the $\mathrm{M}-\mathrm{C}$ bonds by covalent bonding (Fig.27). A reduction of all Ti-N bonds with nitrogen atoms in meridional configuration $1,2,5$ is not possible. The $\mathrm{N}$ atoms of the $4416 ; 1 \mathrm{TiN}_{0.5}$ structure, however, are further apart and are stabilized by a $1.9 \%$ increased Madelung factor for the Coulomb interactions.

The other compounds with $\mathrm{y} / \mathrm{z} \neq 0.5,0.67$ or 0.83 as e.g. $\mathrm{Ti}_{8} \mathrm{C}_{5}, \mathrm{~V}_{8} \mathrm{C}_{7}$ or $\mathrm{PdD}_{0.8}$ contain two different configurations of $\mathrm{M}$ atoms (Hauck et al., 1988b). In $\mathrm{Ti}_{8} \mathrm{C}_{5}$ e.g. with $6 \mathrm{y} / \mathrm{z}=3.75$, two $\mathrm{Ti}$ atoms have three $\mathrm{C}$ atoms, and six $\mathrm{Ti}$ atoms four $\mathrm{C}$ atoms in the same configuration as the compounds $\mathrm{Ti}_{2} \mathrm{C}$ and $\mathrm{AlCl}_{3}$ (Table 11a). $\mathrm{Ti}_{8} \mathrm{C}_{5}$ is located in the vicinity of $\mathrm{Ti}_{2} \mathrm{C}$ and $\mathrm{AlCl}_{3}$ of the structure map (Fig.30). The structures close to the borderline with only two different configurations obey Pauling's rule of parsimony (Pauling, 1929). The structures at or close to the borderline $3 \alpha_{1}+1=0$, e.g. $060 ; 3$ and $468 ; 1$, which are observed as ordered alloys, are unfavorable for interstitial compounds because of two or more configurations. The $\mathrm{Nb}$ atoms e.g. of $060 ; 3 \mathrm{Nb}_{4} \mathrm{C}_{3}$ are surrounded either by four or six $\mathrm{C}$ atoms. The number of configurations, however, is reduced to a single configuration in $\mathrm{NbO}\left(=\square \mathrm{Nb}_{3} \square \mathrm{O}_{3}\right)$ and in ternary interstitial alloys $\mathrm{AlFe}_{3} \mathrm{C}$ and FeNiN (Fig.18).

The $T_{1}$ and $T_{2}$ values in the $T_{1} T_{2} T_{3} ; \mathrm{y} / \mathrm{x}$ notation of interstitial compounds describe the connection of octahedra by edges and corners, re- 

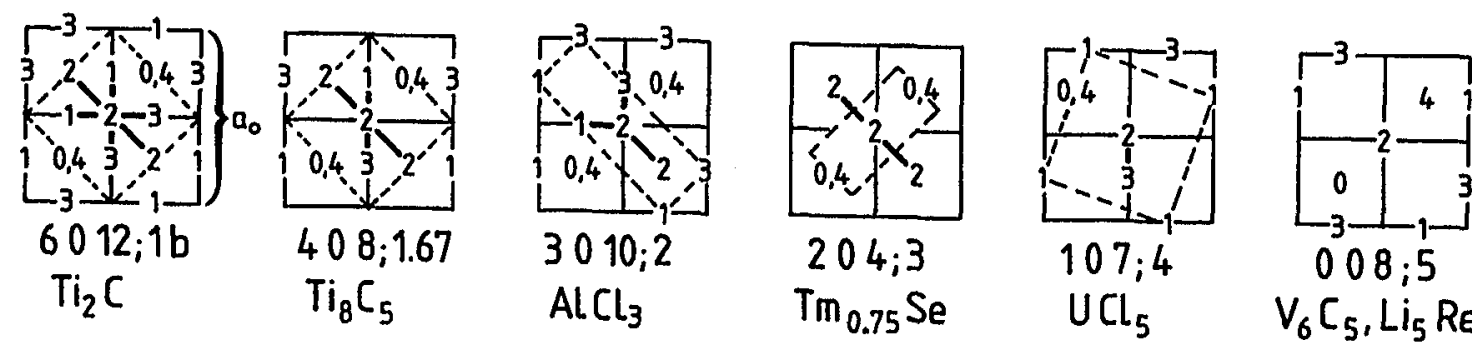

$\mathrm{Tm}_{0.75} \mathrm{Se}$

$008 ; 5$
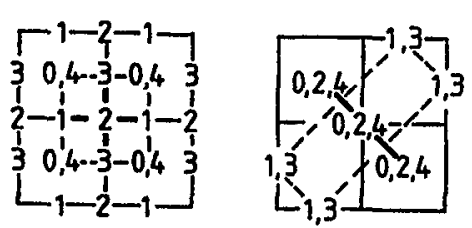

4416;1

$2212 ; 2$

$\mathrm{Pd}_{2} \mathrm{H}, \mathrm{Ti}_{2} \mathrm{~N}$

$\square \mathrm{Ti}_{2} \square \mathrm{O}_{2}$
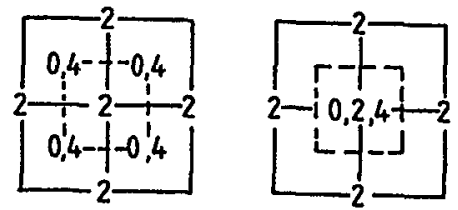

$060 ; 3$

$048 ; 3$

$\mathrm{Nb}_{4} \mathrm{~N}_{3}$

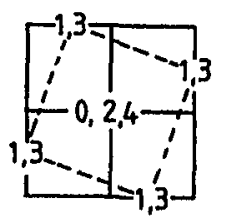

$028 ; 4$

$\mathrm{PdH}_{0.8}$
$\mathrm{V}_{6} \mathrm{C}_{5}, \mathrm{Li}_{5} \mathrm{ReO}_{6}$

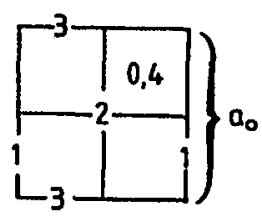

$006 ; 7$

$\mathrm{V}_{8} \mathrm{C}_{7}$

Figure 31: Self-coordination numbers $T_{1}, T_{2}, T_{3}$ of interstitial atoms in covalent (upper row) or ionic (lower row) compounds. An interstitial atom is placed in the middle of eight face-centered cubic cells with lattice constant $a_{\mathrm{c}}$. The numbers $\mathrm{z}$ give the position of neighboring interstitial atoms at height $\mathrm{z} a_{\mathrm{c}} / 2$. Nearest neighbors are connected by bold lines. The broken lines indicate the unit cells. $\mathrm{Ti}_{2} \mathrm{C}$ and $\mathrm{V}_{8} \mathrm{C}_{7}$ are cubic with lattice constant $a_{0}=2 a_{\mathrm{c}}$.

spectively. The $107 ; 4 \mathrm{UCl}_{5}$ structure contains $\mathrm{UCl}_{6}$ octahedra connected by an edge to $\left(\mathrm{UCl}_{5}\right)_{2}$, while the $\mathrm{UF}_{6}$ octahedra of the $028 ; 4$ $\mathrm{UF}_{5}$ structure are connected by two corners in a one-dimensional row. The $\mathrm{CTi}_{6}$ octahedra of the $6012 ; 1 \mathrm{~b} \mathrm{Ti} 2 \mathrm{C}$ structure are linked by six edges, the $\mathrm{NTi}_{6}$ octahedra of the $4416 ; 1 \mathrm{TiN}_{0.5}$ structure by 4 corners and 4 edges. The vacancies of $008 ; 5 \mathrm{~V}_{6} \mathrm{C}_{5} \square$ or the Re atoms of $008 ; 5$ $\mathrm{Li}_{5} \mathrm{ReO}_{6}$ form isolated octahedra. Similar relations between $T_{1}, T_{2}, T_{3}$ values and the number of shared faces, edges or corners of the polyhedra of other interstitial alloys are listed in Table 15.

The covalent and ionic compounds can be compared by two series of interstitial compounds $M_{z} \square_{x} I_{y}$, the $T_{2} C$ series with covalent bonding and the $\mathrm{Ti}_{2} \mathrm{~N}$ series with ionic bonding (Fig.31).

The vacancies of the $4416 ; 1 \mathrm{Ti}_{2} \square \mathrm{N}$ structure are filled up with interstitial atoms in such a way that they are as far apart as possible. These structures with $50,33,25,20$ and $12.5 \%$ vacancies, shown in the lower row of Fig.31, have a minimum of $T_{1}$ coordination. The vacan- 


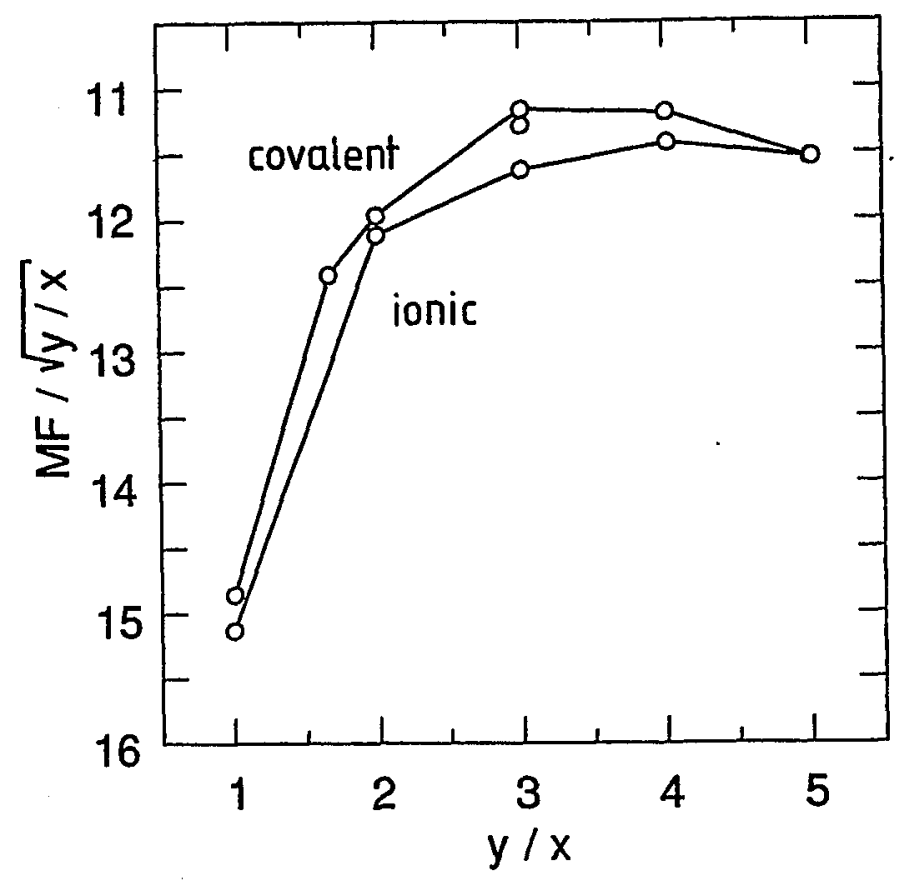

Figure 32: Madelung factors $M F / \sqrt{y / x}$ versus concentration $y / x$ for the undistorted covalent and ionic compounds of Fig.31.

cies of the $6012 ; 1 \mathrm{~b} \mathrm{Ti}{ }_{2} \square \mathrm{C}$ structure in the upper structural series are successively occupied at increased $T_{1}$ and $T_{2}=0$. The increased $T_{1}$ coordination numbers of this series allow covalent bonding to interstitial atoms in neighboring facial or cis configuration. The Madelung factors of these structures are decreased by $1 \%-3 \%$ (Fig.32). This difference of the lattice energy must be compensated by covalent bonding to stabilize the structure. The $\square \mathrm{V}_{6}$ octahedra of $\mathrm{V}_{6} \mathrm{C}_{5} \square$ or $\mathrm{ReO}_{6}$ octahedra of $\mathrm{Li}_{5} \mathrm{ReO}_{6}$ are isolated at the composition $\mathrm{y} / \mathrm{x} \geq 5$. The vacancies or $\mathrm{Re}^{7+}$ atoms are as far apart as possible for a maximum Coulomb energy. Covalent bonding is also favorable at this composition, because of the possibility of decreasing all $\mathrm{Re}-\mathrm{O}$ bonds of $\mathrm{ReO}_{6}$ or increasing all $\square-\mathrm{V}$ distances of the $\square V_{6}$ octahedra for covalent $V-C$ bonding. Therefore the $008 ; 5$ structure is favorable for both systems. This structure lies at the intersection of the $4 \alpha_{1}+\alpha_{2}+1=0$ boundary line of the structure map (Fig.16) and the $\alpha_{1}=\alpha_{2}$ line for diluted alloys as e.g. $\mathrm{V}_{8} \mathrm{C}_{7}$ with $T_{1}=$ $T_{2}=0$. The $008 ; 5 \mathrm{a}-\mathrm{i}$ structures are different stacking variants, which can occur in the same sample like $\mathrm{V}_{6} \mathrm{C}_{5}$ I, II and III or as disordered structures. Some structures like $008 ; 5 \mathrm{e}, \mathrm{f}$ are homometric analogous to 
$6012 ; 1 \mathrm{a}, \mathrm{b} \mathrm{Ti}_{2} \mathrm{C}$ and $\mathrm{Gd}_{2} \mathrm{C}$ (Section 8, Fig.13) (Parthé and Yvon, 1970; Hauck et al., 1988b).

The $\mathrm{Zn}$ atoms of $\mathrm{ZnS}$ are at tetrahedral sites of the ccp lattice in the sphalerite modification or the hcp lattice in the wurtzite modification. The $\mathrm{Zn}$ atoms forming a ccp or hcp lattice as the S atoms can be substituted by other atoms like $\mathrm{Cu}, \mathrm{Sb}$ or $\mathrm{As}$ in $\mathrm{SbCu}_{3} \mathrm{~S}_{4}$ (Table 11a) or $\mathrm{AsCu}_{3} \mathrm{~S}_{4}$ (Table 11b). The structure of the metal atoms corresponds to the $\mathrm{TiAl}_{3}$ and $\mathrm{TiCu}_{3}$ structures. The $T_{1}$ values indicate the number of corner shared tetrahedra (Table 15). Most structures are in the Johansson, Linde and Brauer family of structures with $3 \alpha_{1}+1=0$ (Fig.16) and satisfy Pauling's electrovalence rule (Hauck and Mika, 1998b).

The coordination numbers $\mathrm{CN}$ and the bonding geometries of the metal atoms depend on the size and the hybridization of $s, p$ and $d$ orbitals (Sutton, 1993). The $\mathrm{CN}=4$ planar coordination is favored for $d s p^{2}$ hybridization compared to tetrahedral coordination for $s p^{3}$ or $d^{3} s$ hybridization. The $\mathrm{CN}=6$ trigonal prismatic coordination occurs for $d^{4} s p$ instead of $d^{2} s p^{3}$ of octahedral coordination. The $\mathrm{CN}$ values vary with the size and number of valence electrons of the elements (Section 29).

\section{Magnetic interactions}

Transition, rare earth or actinide metal atoms with $d$ or $f$ electrons exhibit a magnetic moment, which gives rise to ferromagnetic or antiferromagnetic ordering below the Curie or Néel temperature, respectively and a small lattice distortion. The spins of different atoms are oriented parallel in ferromagnetic and antiparallel in antiferromagnetic compounds (Oleś et al., 1976). The relation between interaction energies $V_{i}$ and ordering was investigated by Ising (1925), and a similar treatment applied to the ordering of alloys by different investigators (Section 11). These investigations had shown that the magnetic interactions are less than $1 \%$ of the lattice energies of ordered alloys. The arrangement of the spins depends on the distances between atoms, geometry of neighboring 
atoms and bonding to other atoms like in the superexchange coupling (Goodenough, 1982). The situation seems to be very similar to interstitial compounds $M_{n} I_{x} \square_{y}$ like the hydrides, where the occupation of tetrahedral or octahedral interstices is supposed to be influenced by the $d$ electrons of $\mathrm{M}$ atoms, the distances and interactions between $\mathrm{H}$ atoms (Section 24). The magnetic metal atoms however are fixed on lattice sites, which are given by the large lattice energy, and the spins must find an optimum arrangement for this situation.

The ordering of the spins can be analyzed for attractive or repulsive interactions in different families of structures with composition $R_{n} M_{x} M_{y}^{\prime}$, if the metal atoms $M$ with a magnetic moment $\oplus$ and other $M$ atoms $M^{\prime}$ with $\ominus$ spin direction or vacancies $\square$ are forming one of the lattices of the present investigation. The other atoms, the rest $R$, can be a single atom like $\mathrm{P}$ in $\mathrm{PHo}$ with fcc $\mathrm{Ho}$ lattice or several atoms like $\mathrm{O}_{3} \mathrm{Al}$ in the $\mathrm{O}_{3}$ AlDy structure (Table 16). Ferromagnetic compounds with a single orientation of the spins can be treated as pseudobinary interstitial compounds with $T_{i}(\square)$ of vacancies or non-magnetic $R$ atoms and $T_{i}(\mathrm{M})$ of the $M$ atoms. The interactions in antiferromagnetic compounds with a $\oplus$ and $\ominus$ orientation of antiparallel spins are treated as pseudoternary compounds with equal self-coordination numbers for atoms with positive and negative spin direction $\left(T_{i}(\oplus)=T_{i}(\ominus)\right)$ with few exceptions like $\mathrm{S}_{2} \mathrm{Cr}_{2}$ (0 $060 ; 2$ and $030 ; 2$ for $\oplus$ and $\ominus$ spin directions). The selfcoordination numbers $T_{i}(\mathrm{M})$ of all magnetic atoms with antiparallel spin directions usually show a different point in the structure map because of the different $y / x$ values $(2 y / x(M)=y / x(\oplus)-1)$. Compounds with different spin directions like $\mathrm{FePd} \mathrm{d}_{1.6} \mathrm{Pt}_{1.4}$ are treated as multinary compounds with different sets of $T_{i}(\oplus)$ and $T_{i}(\mathrm{M})$ values for each $\mathrm{M}$ atom (Table 16). This alloy is listed also as $\mathrm{Pd}_{1.6} \mathrm{Pt}_{1.4} \mathrm{Fe}$ for the magnetic moments of $\mathrm{Fe}$ atoms. The four or five different directions of magnetic moments of Dy atoms in $\mathrm{DyAlO}_{3}$ or $\mathrm{Mn}$ atoms in $\mathrm{Mn}(\mathrm{OH})_{2}$ can be combined in different ways. The combinations can be homologous structures (identical $\alpha_{i}$ values) or quasi-homologous structures at the same border indicating the same kind of interactions for combinations or at different areas of the structure maps. The $T_{i}(\mathrm{M})$ values correspond to all values of Dy or $\mathrm{Mn}$ atoms, respectively. The concentration of magnetic $\mathrm{Mn}$ or $\mathrm{Cr}$ atoms in $\mathrm{Ga}_{1.15} \mathrm{Mn}_{2.85}, \mathrm{PtMn}_{3}, \mathrm{GePd}_{2} \mathrm{Mn},(\mathrm{OH})_{2} \mathrm{Mn}$ and $\mathrm{S}_{3} \mathrm{Cr}_{2}$ 
is increased to $\mathrm{y} / \mathrm{x}<1$. The underlined $T_{i}(\mathrm{~N})$ or $T_{i}(\square)$ values of the non-magnetic minority component or vacancy for the structure map are different (Sections 2,4). Most $T_{i}$ values are at the boundaries of the structure maps and can be analyzed for repulsive or attractive interactions. Usually the lattice with the highest density (lowest $r$ values) of magnetic $\mathrm{M}$ atoms is preferred like the pc lattice of $\mathrm{Dy}$ atoms in $\mathrm{DyAlO}_{3}$ instead of the bcc lattice of $\mathrm{Dy}$ and $\mathrm{Al}$ atoms. The ordering of different structures like $\mathrm{Cu}_{2} \mathrm{AlMn}$ (Heusler alloy) and $\mathrm{OEu}(\mathrm{NaCl})$ or $\mathrm{O}_{4} \mathrm{ZnCr}_{2}\left(\mathrm{O}_{4} \mathrm{MgAl}_{2}\right.$ spinel structure) and $\mathrm{TbCo}_{2}\left(\mathrm{MgCu}_{2}\right.$ Laves phase) can be compared for example in the fcc lattice of $M$ atoms. The magnetic atoms of many cubic, hexagonal, trigonal or tetragonal structure types like the $\mathrm{Mn}$ and $\mathrm{Eu}$ atoms of $\mathrm{Cu}_{2} \mathrm{AlMn} / \mathrm{OEu}$ or the $\mathrm{Cr}$ and Co atoms of $\mathrm{O}_{4} \mathrm{ZnCr}_{2} / \mathrm{TbCo}_{2}$ occupy positions with fixed $x, y, z$ parameters (Section 33, Table 25). The $T_{i}(\mathrm{M})$ values of cubic compounds projected in [001] or [111] direction are analyzed for the $T_{i}(\mathrm{M})$ values of the square or hexagonal layers. The characterization by square layers and translational vectors of these layers is preferred in most cases because of the higher densities (lower $r$ values) of $M$ atoms $r($ hex $)=3 r$ (square) +2 . Some structure types like fcc 3,5 and 8 containing different square layers at different projection heights or an overlap of $M$ positions in [001] projection should be projected in [111] direction. Many hexagonal, trigonal or tetragonal structures with fixed $x, y, z$ parameters can be considered as distorted cubic structures with identical projections in [111] or [001] direction. The tetragonally distorted bcc and fcc structures with identical projection patterns are characterized by a slightly distorted bcc' lattice with $T_{i}$ values 84248168 . The $T_{i}$ values of the cubic bcc (8612 $\cong T_{1}$, $\left.T_{2}+T_{3}, T_{4}+T_{5}\right)$ or fcc $\left(12624 \hat{=} T_{1}+T_{2}, T_{3}+T_{4}, T_{5}+T_{6}\right)$ structure or of the square plane $\left(444 \hat{=} T_{2}, T_{4}\right)$ can be derived from the $b_{c c}^{\prime}$ values in a similar way as was discussed for distorted ph lattices (Section 18). The $T_{i}$ values of the cubic pc lattice (6128 $\left.\widehat{=} T_{1}+T_{2}, T_{3}+T_{4}, T_{5}\right)$ can be derived from tetragonally distorted $\mathrm{pc}^{\prime}\left(T_{i}=42488\right)$. Other invariant lattice complexes including orthorhombic distortions were derived by Hellner and Schwarz (1994). Different symbols like D (= bcc 1$)$ or $\mathrm{W}(=\mathrm{fcc} 5)$ are used for the diamond and $\beta$-W structures. The fcc $7 \mathrm{a}$ and $b$ structures with the Hellner and Schwarz symbols $V^{*}$ and $S^{*}$ are homometric for the $\mathrm{M}$ and in the average homometric for the $\mathrm{N}$ atoms. 


\section{Ordering of I atoms on metal surfaces}

The compilation of different structures of the primitive cubic (pc) lattice had shown, that the ccp or bcc lattices can be considered as ordered pc lattice, where $50 \%$ (ccp) or $75 \%$ (bcc) of the metal atom positions are vacant and can be occupied by $\mathrm{I}=\mathrm{O}, \mathrm{Cl}$, etc. atoms in the $\mathrm{NaCl}$ or perovskite group. A similar situation occurs for the two-dimensional ordering of adatoms $\mathrm{A}$ on metal surfaces like the square or hexagonal net of structures, if $\mathrm{A}=\mathrm{H}, \mathrm{Xe}, \mathrm{Cl}$ atoms or $\mathrm{CO}, \mathrm{C}_{6} \mathrm{H}_{6}$ molecules are adsorbed on the top positions of [001] or [111] ccp metal surfaces (Table 12) (Watson et al., 1994). The composition of the metal surface $\mathrm{M}_{n} \mathrm{~A} \square_{n-1}$ with a maximum of one $\mathrm{A}$ atom/M atom is similar to the $\mathrm{NaCl}$ or NiAs group of interstitial alloys. The ratio A/M can be varied at the occupation of hollow or bridge positions. A honeycomb net of A atoms is obtained at occupation of all hollow positions, a kagomé net at occupation of all bridge positions (Fig.33). The compositions can be formulated as $M_{n} A_{x n-x} \square_{x}$, with $x=2$ (honeycomb net) or $x=3$ (kagomé net) similar to the pc and PER groups of structures. The $\mathrm{x}=$ 1 or 2 series is obtained for the occupation of hollow or bridge positions on [001] surfaces of ccp or bcc metals (Table 12). Each M atom of the honeycomb net exhibits a maximum coordination number $\mathrm{CN}=6$, if all hollow positions are occupied. The coordination in the kagomé net includes two additional adatoms with larger $\mathrm{M}-\mathrm{A}$ bond distances, which are not included for the $\mathrm{CN}$ values. The $\mathrm{M}$ atoms of [001] surfaces of ccp or bcc metals can have a maximum of $\mathrm{CN}=4 \mathrm{~A}$ atoms at hollow or bridge positions, if the size of A atoms is smaller than the size $d$ of $\mathrm{M}$ atoms (hollow positions) or smaller than $0.7 d$ (bridge positions). The occupation of $l=\mathrm{CN}$ A atom positions in the hexagon next to each $\mathrm{M}$ atom in [111] ccp or [0001] hcp is given by letters a $(\mathrm{CN}=6), \mathrm{b}(\mathrm{CN}=$ $0), \mathrm{c}(\mathrm{CN}=1), \mathrm{o}, \mathrm{m}$ or $\mathrm{p}(\mathrm{CN}=2), \mathrm{v}, \mathrm{s}$ or y $(\mathrm{CN}=3), \mathrm{o}^{\prime}, \mathrm{m}^{\prime}$ or $\mathrm{p}^{\prime}(\mathrm{CN}$ $=4)$ and $\mathrm{c}^{\prime}(\mathrm{CN}=5)$ (Fig.34). The different configurations o (ortho), $\mathrm{m}$ (meta), p (para), v (vicinal), s (symmetrical) and y (asymmetrical with the shape of the letter y) are similar to those used for benzene derivatives in organic chemistry (Latscha and Klein, 1982). The same applies for the occupation of neighboring sites (cis positions) or diagonal sites (trans positions) in the square net (Fig.8). The t (trans) configuration 


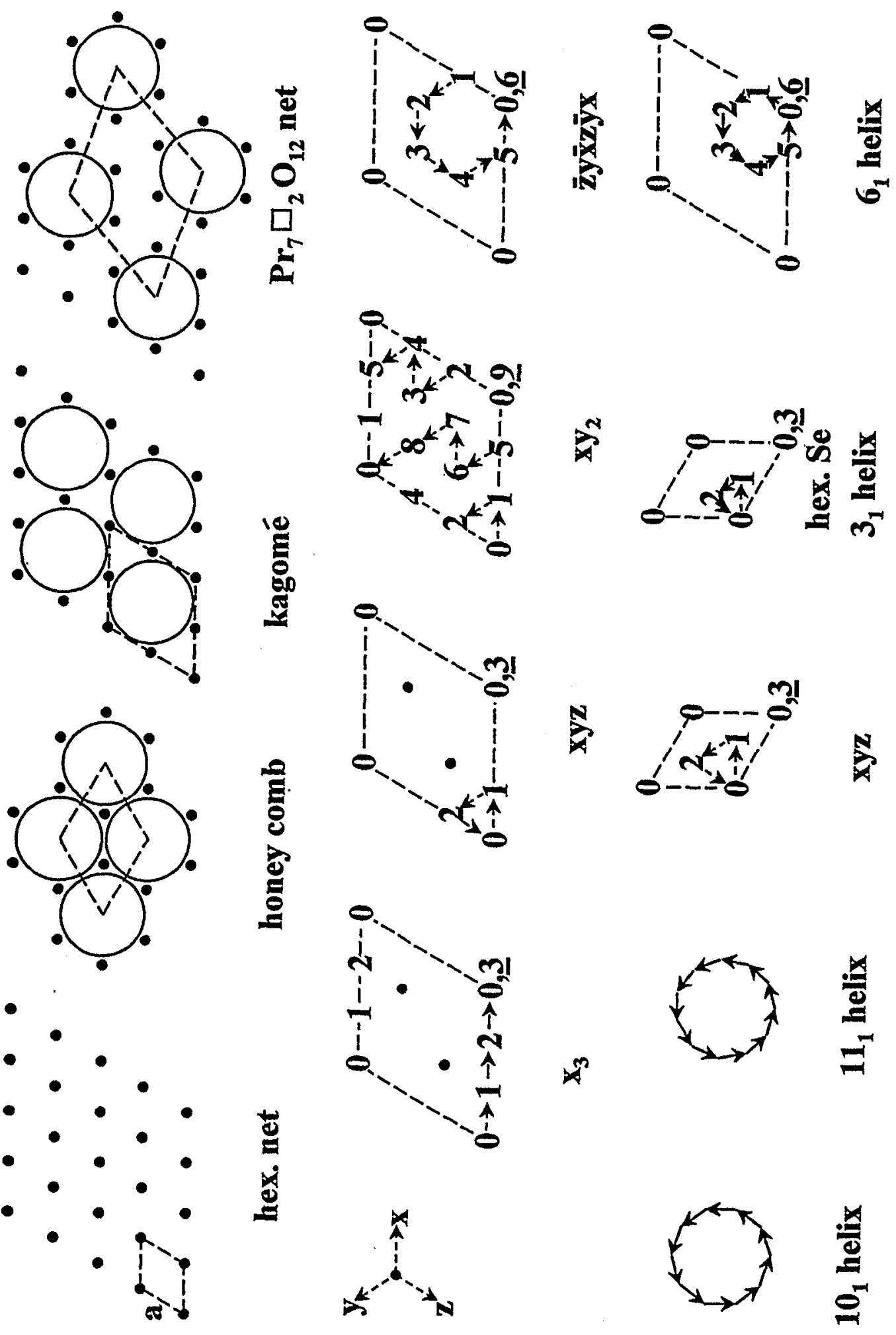

Figure 33: Projection of the hexagonal, honeycomb, kagomé and $\operatorname{Pr}_{7} \square_{2} \mathrm{O}_{12}$ net with composition $\mathrm{AB}_{2}, \mathrm{AB}_{3}$ and $\mathrm{AB}_{6}$ of vacancies $\mathrm{A}=\square$ at the positions of $\mathrm{M}$ atoms (O) and occupied sites $B(\bullet)$. Honeycomb and kagomé net in surface structures at the occupation of hollow or bridge positions, respectively. Layered ccp compounds with translation of $\mathrm{A}$ atoms in $\mathrm{x}, \mathrm{y}$ or $\mathrm{z}$ direction, $3_{1}$ or $6_{1}$ helical structures at distortion of close-packed compounds and $10_{1}$ or $11_{1}$ helical structure of DNA. 

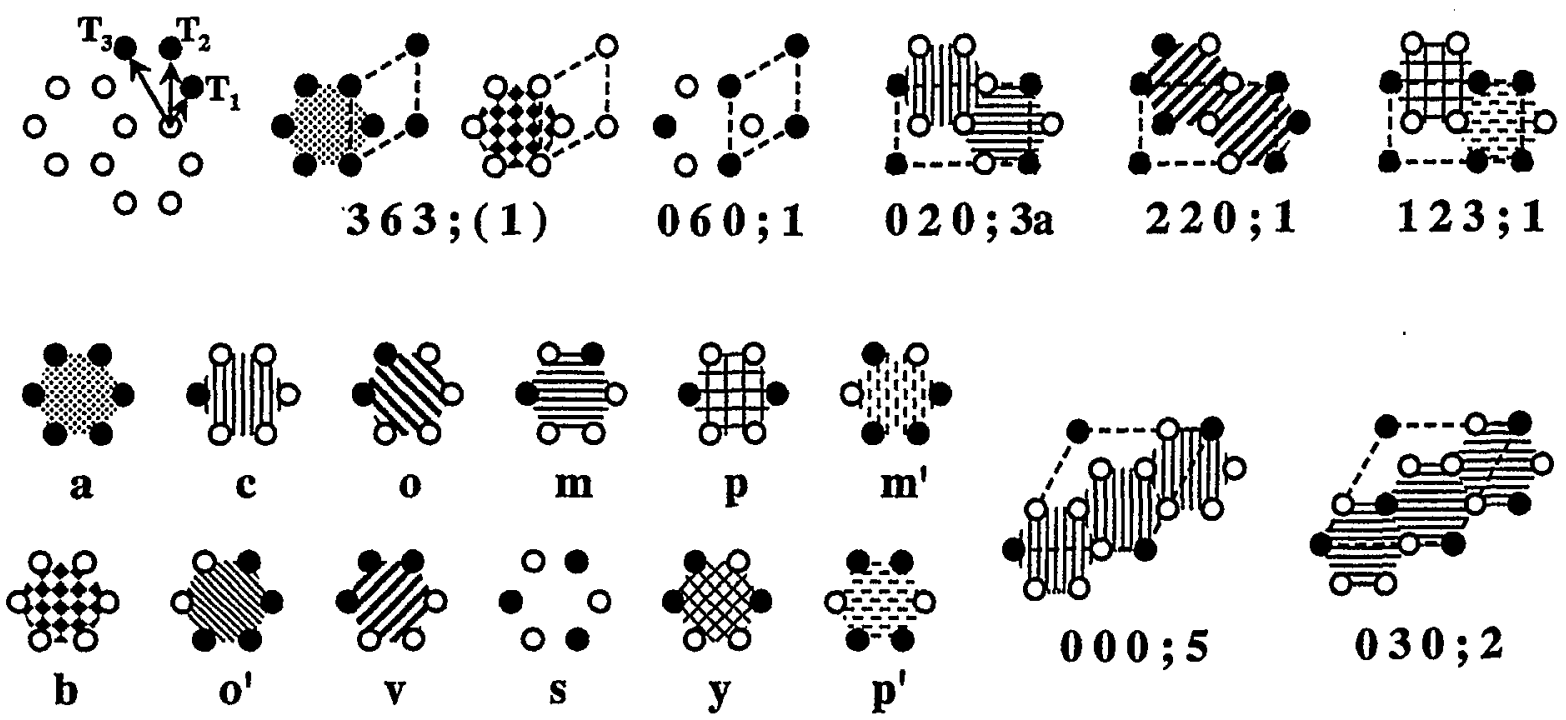

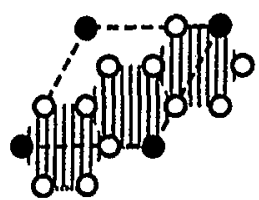

$000 ; 5$

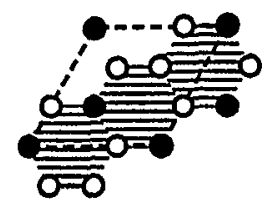

$030 ; 2$
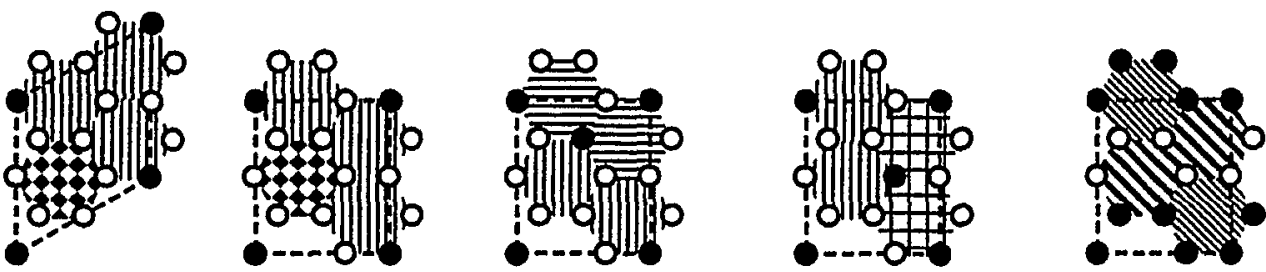

22 1; 1

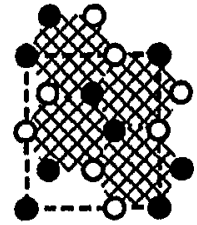

$002 ; 3$

$122 ; 1$
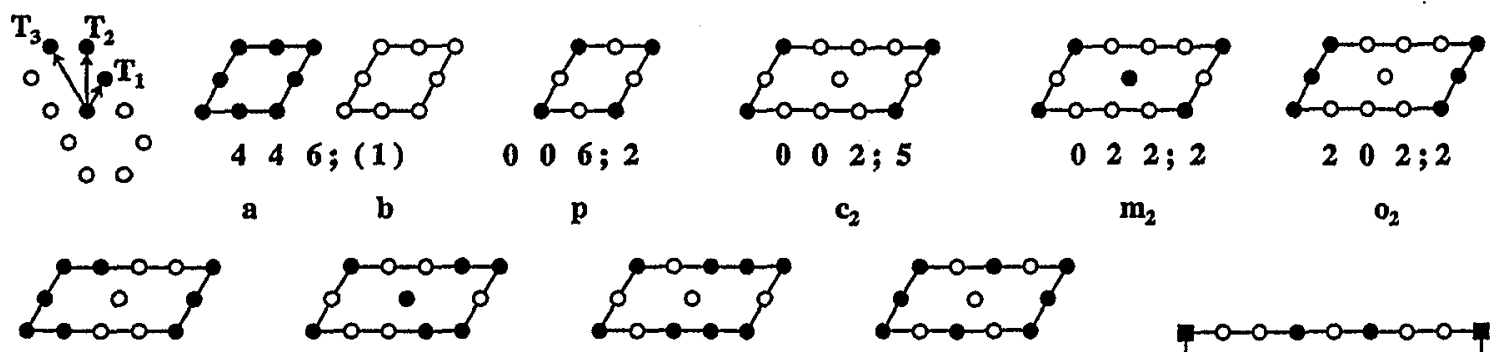

p

$c_{2}$

$\mathbf{m}_{2}$

$\mathrm{O}_{2}$

2.71 .32 ; $1 \mathrm{a}$

- $0^{\prime}$

\section{$1.32 .72 ; 1$}

$\mathbf{m} \mathbf{m}^{\prime}$

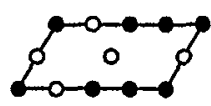

1.31 .34 .7 ; $1 \mathrm{a}$

p p'

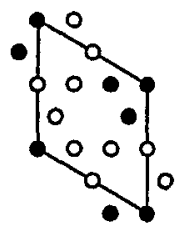

$200 ; 2$

$\mathbf{O}_{3}$

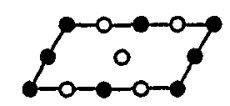

$1.31 .34 .7 ; 1 b$

$\mathbf{y}_{2}$
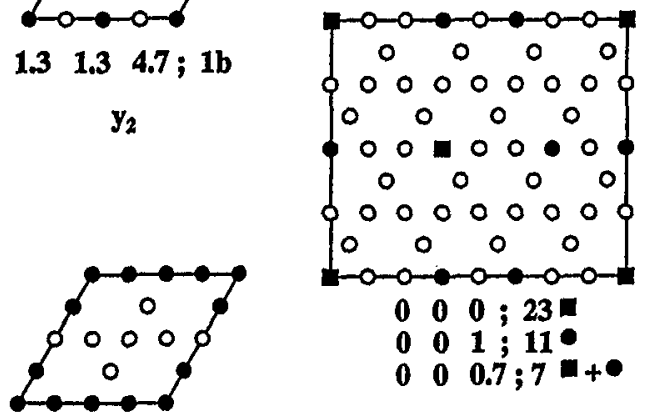

$2.71 .32 ; 1 \mathrm{~b}$

$v_{4}$

Figure 34: Structures of the honeycomb net (a) and kagomé net (b) with structural units $a, c, \ldots$ (different shading in honeycomb net). 
in the square lattice and s (symmetrical A atom) configuration in the honeycomb or kagomé net are obtained at repulsive interactions between A atoms. The $\mathrm{c}$ (cis) configuration in the square lattice, $\mathrm{v}$ (vicinal) and o (ortho) configuration in the honeycomb or kagomé net are obtained for attractive interactions (Figs. 7,35). Structures with o or p configuration are not possible in the honeycomb system, while a structure with s configuration is not possible in the kagomé net.

\section{Related groups of structures}

The maximum possible range of $T_{1}, T_{2}$ values of possible structures is reduced in all systems of the present investigation. The different borders can be described by the relations given in the upper part of Table 17. The different constants $\mathrm{a}_{i}, \mathrm{~b}_{i}, \mathrm{c}_{i}$, which depend on $T_{1}^{\max }, T_{2}^{\max }$ and geometrical constraints, are listed in Table 17. The geometrical constraints are given by the minimum range of $T_{1}$ and $T_{2}$ values at $\mathrm{r}=1$ and the maximum range for structures with $T_{1}=T_{2}=0$ at increased $r$. Each border can be correlated to a structure series with different structural units. Several series of structures containing similar structural units are listed as structure family. Structures at the left-hand border of the structure maps (B1, B2) can be described by sequences of atoms or layers of atoms. Structures at the right-hand borders of the structure maps (B5 - B7) usually contain double rows of structural units with different atoms like a combination of two squares or rhombs with cis occupation in $\mathrm{c}^{*}$ (Fig.8) or $c^{\prime}$ (Fig.6).

The different groups of structures can be classified in a similar way as the homologous and homometric structures (Sections 8,9).

(1) Quasi-homologous structure groups

The bcc, ccp, perovskite (PER) and square net structures can be obtained from the primitive cubic (pc) lattice, if some sites are excluded or occupied with other atoms. Part of the $M$ atoms of a pc $M$ lattice can be substituted by vacancies or I atoms in the 

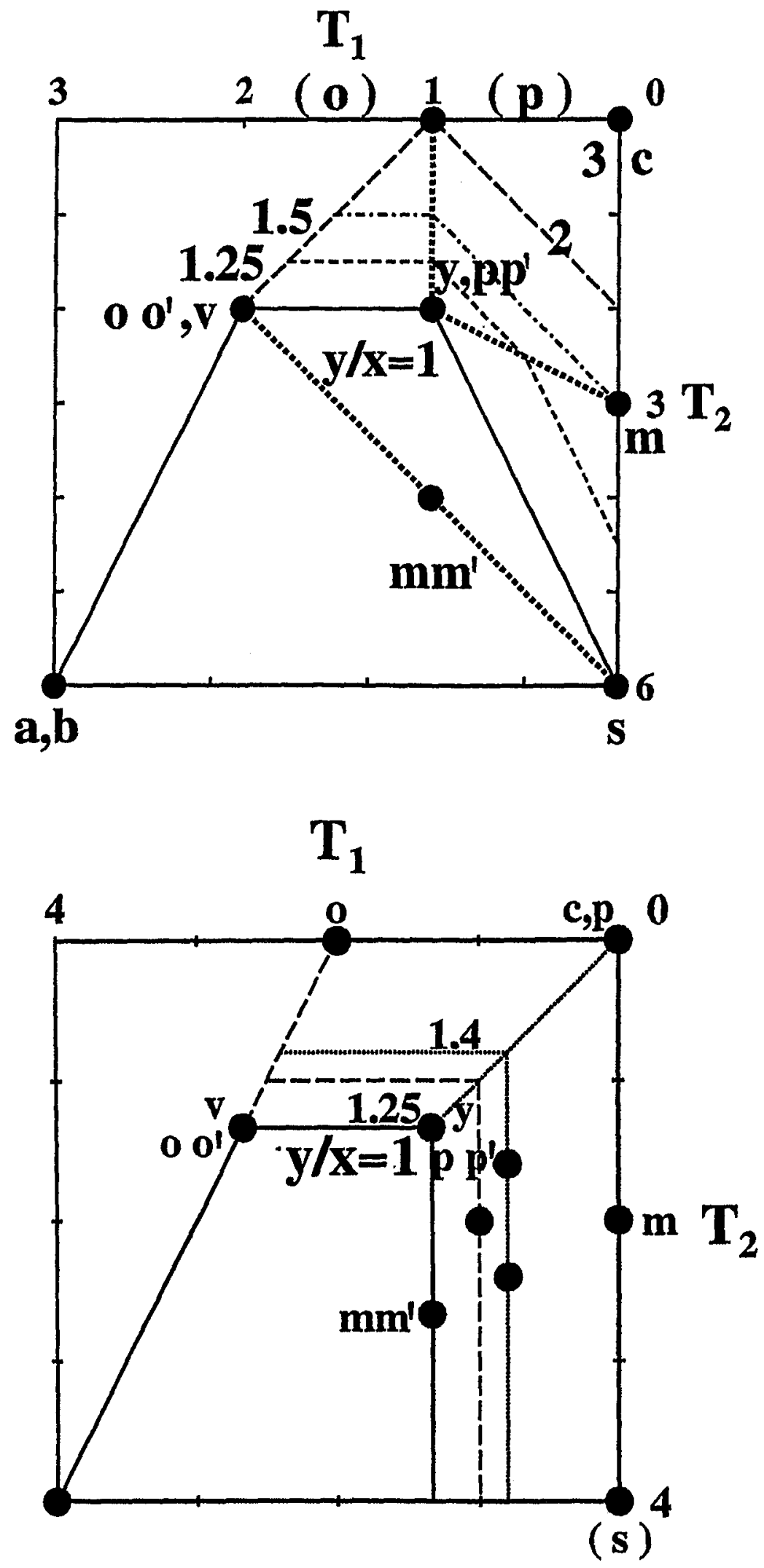

Figure 35: Structure maps of the honeycomb net (a) and kagomé net (b) with $T_{i}$ values of structural units. Structures with structural units in brackets are impossible. 
sequence $\mathrm{M}_{8}$ (pc), $\mathrm{M}_{6} \mathrm{I}_{2}$ (anti-perovskite), $\mathrm{M}_{4} \mathrm{I}_{4}(\mathrm{NaCl}), \mathrm{M}_{2} \mathrm{I}_{6}$ (perovskite, $\mathrm{PER}$ group) and $\mathrm{I}_{8}\left(\mathrm{CaF}_{2}\right.$ group). The $\mathrm{M}_{4} \square_{4}$ and $\mathrm{M}_{2} \square_{6}$ structures correspond to ccp and bcc metal structure groups. These structures are at the border of the pc structure map with a single set of $T_{i}(\mathrm{M})$ and $T_{i}(\mathrm{I})$ values $\left(M^{i}=1\right)$ (Section 33$)$. The bcc system with two $M$ atoms and the PER group with six I atom positions are conjugated systems similar as the combination of two ccp lattices to the $\mathrm{NaCl}$ group with the $T_{i}(\mathrm{Na})$ and $T_{i}(\mathrm{Cl})$ values $0120 ; 1$. Other structures at the border of the pc structure map with $M^{i}=1$ are $248 ; 1$ (rectangle), $440 ; 1$ or $440 ; 2$ (square net), $184 ; 1$ (honeycomb related net) and $002 ; 6\left(\operatorname{Pr}_{7} \square_{2} \mathrm{O}_{12}\right.$ structure net, Fig.33). The vacancies and oxygen atoms of $\operatorname{Pr}_{7} \square_{2} \mathrm{O}_{12}$ form a conjugated system. The ccp lattice of $\mathrm{Pr}$ atoms is obtained from the positions of $\square$ or $\mathrm{O}$ by the translation $a_{\mathrm{c}} / 4 a_{\mathrm{c}} / 4 a_{\mathrm{c}} / 4\left(a_{\mathrm{c}}=\right.$ cubic ccp lattice constant of Pr atoms).

The honeycomb and kagomé nets are quasi-homologous groups of the hexagonal layer with self-coordination numbers $060 ; 2,006 ; 3$ and $\mathrm{MI}_{2}$ or $\mathrm{MI}_{3}$ composition. Usually the structures of quasihomologous subgroups are inside of the structure map of the original group (Fig.36). The maximum interactions between I atoms are impossible because of the hindrance of $\mathrm{M}$ atoms at I positions (Fig.33).

(2) Quasi-homologous structure groups with similar structure maps Some groups of structures exhibit similar structure maps with a relation between the $T_{i}(\mathrm{M})$ values of both maps like the maps of ordered bcc alloys, the square net and the one-dimensional chain (Figs. 37a,7a,2a). The three corners of the bcc structure map (Hauck and Mika, 1997) at repulsion ( $\mathrm{CsCl}$ structure), attraction (NaTl structure) and segregation can be characterized by the layer sequences $\mathrm{MN}(\mathrm{CsCl}), \mathrm{M}_{2} \mathrm{~N}_{2}(\mathrm{NaTl})$ and $\mathrm{M}_{\infty} \mathrm{N}_{\infty}$ in the same way as for the chain of $\mathrm{M}$ and $\mathrm{N}$ atoms (Table 7, $\mathrm{B}$ form) or the sequences of $\mathrm{M}$ and $\mathrm{N}$ atoms in the square net (Fig.8). The structure with sequences $\mathrm{M}_{2} \mathrm{~N}_{2}$ of $\mathrm{M}$ and $\mathrm{N}$ atoms $(220 ; 1)$ (Section 28) however is not at the corner of the structure map of the square net. Another example are the structure maps of the pc and PER groups (Fig.25). Many structures can be described by the same or similar structural 


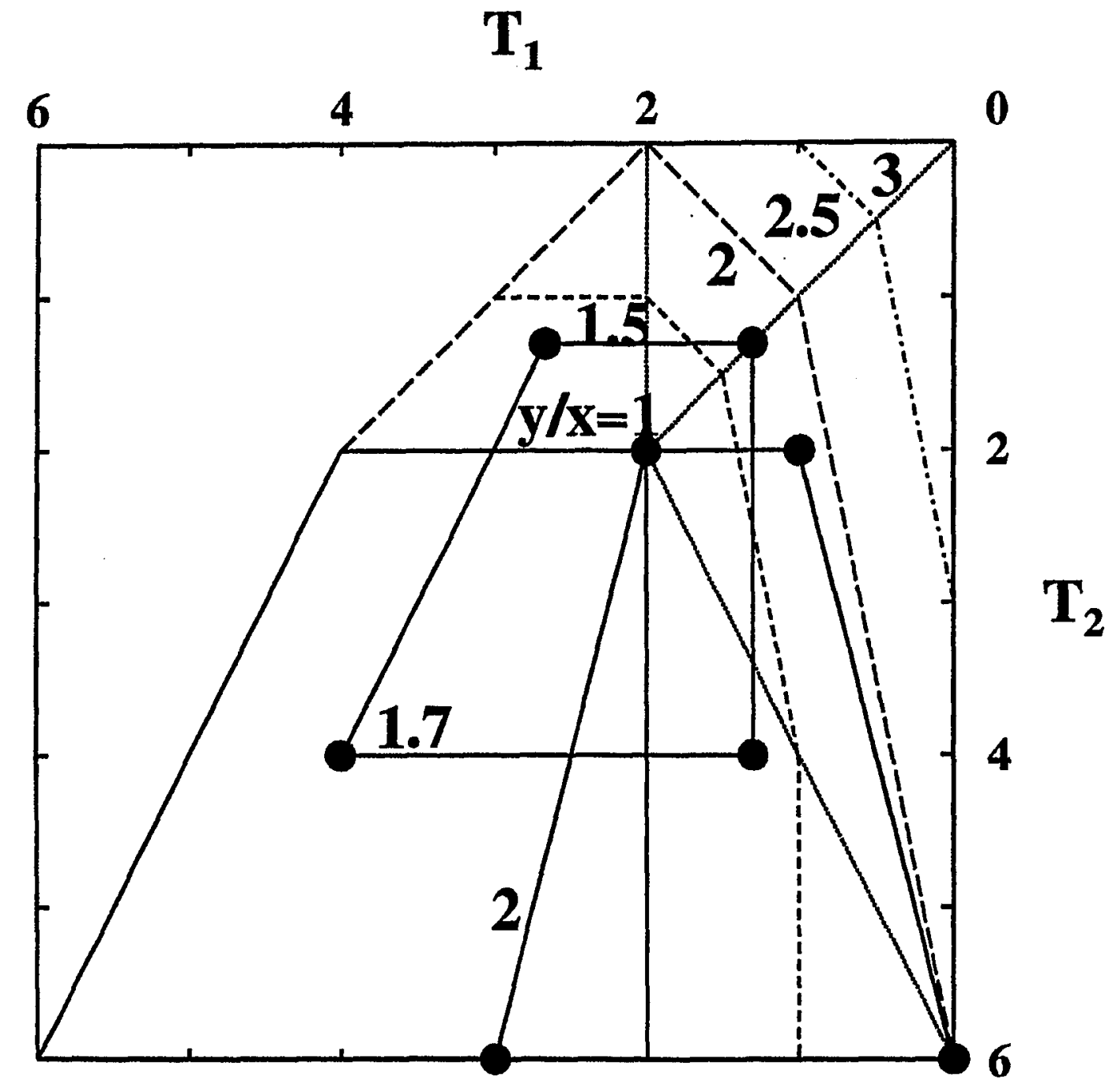

Figure 36: The structures of the honeycomb and kagomé net in the structure map of the hexagonal net. The structures at $y / x=1$ correspond to the $y / x=1.7$ (honeycomb) or $y / x=2$ (kagomé) structures in the hexagonal net and are inside of the hexagonal structure map. 

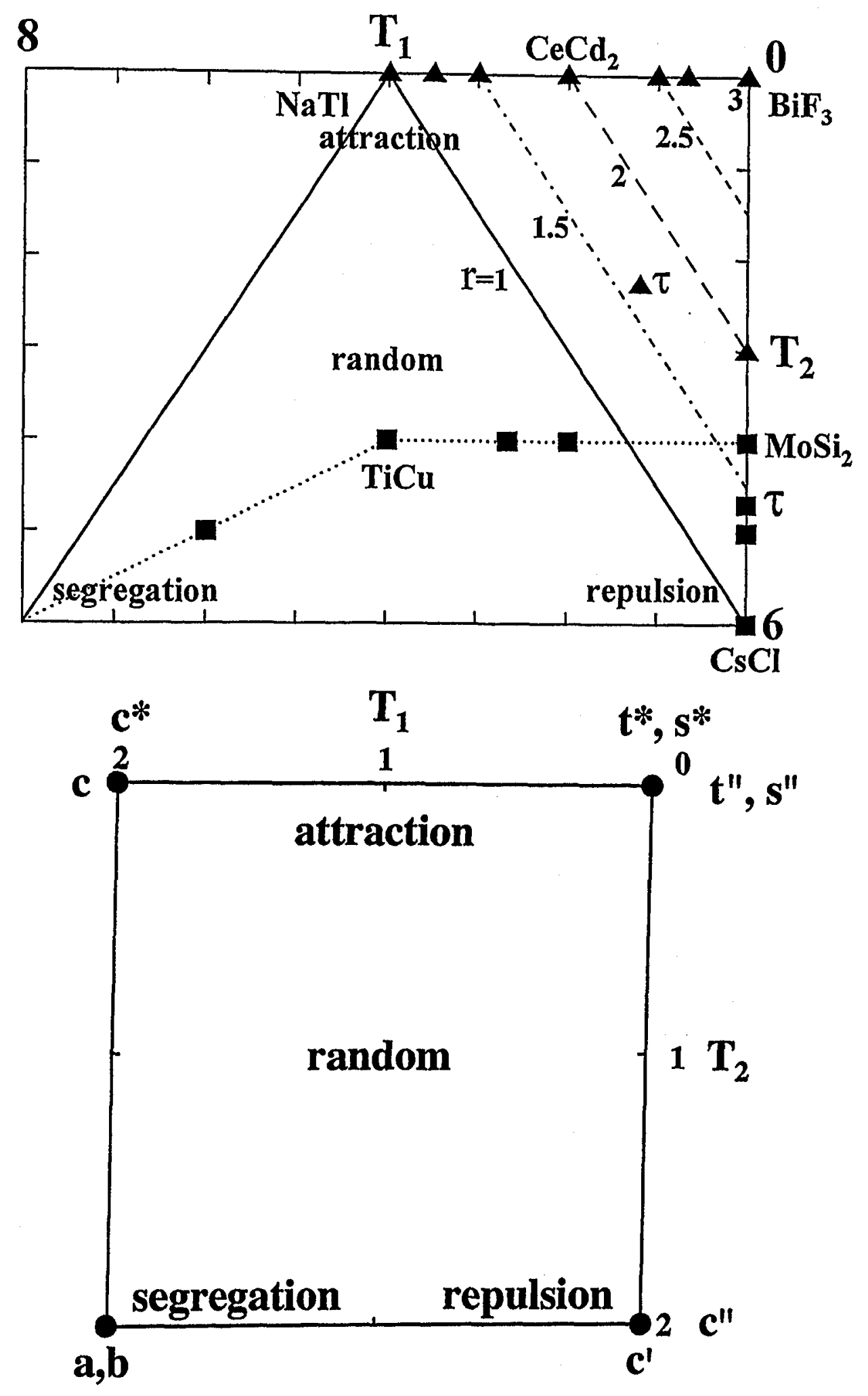

Figure 37: $T_{1}, T_{2}$ structure map for the bcc (a) and rectangle net (b). The bcc structures with layers in [111] $(\boldsymbol{\Lambda})$ or [001] $(\mathbf{\square})$ direction (Tables 7,8) are in different areas. The structures of Fig. 38 are combinations of different structural units $a, b, s$ and $\mathrm{t}$. 


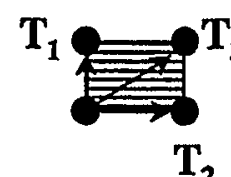

$T_{2}$

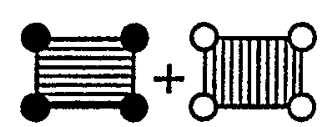

224 ; (1)

a

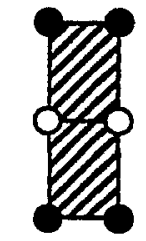

$200 ; 1$

**

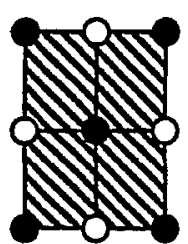

$004 ; 1$

$t_{2}^{*}, t_{2}^{\prime \prime}$ b

$200 ; 1$

$c_{2}$

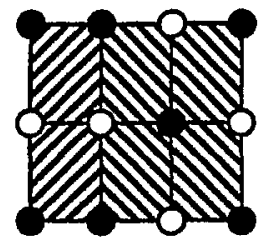

$0.702 .7 ; 1$

$c^{*} t_{2}{ }^{*}$

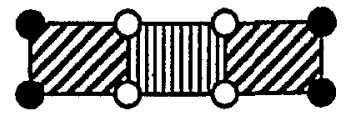

$200 ; 2$

c b c

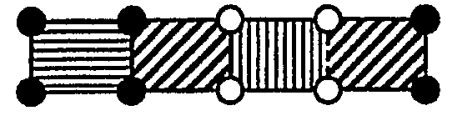

212; 1

a c b c

Figure 38: Self-coordination numbers $T_{1} T_{2} T_{3} ; \mathrm{y} / \mathrm{x}$ of $\mathrm{A}$ atoms in $\mathrm{A}_{\mathrm{x}} \mathrm{B}_{\mathrm{y}}$ compounds $(\mathrm{A}=\bullet, \mathrm{B}=0)$ for the rectangle net. A atoms with concentration $\mathrm{y} / \mathrm{x} \geq 1$ are the minority component. The different structural units $a, b, c$, etc. are shown by different shading.

units (Fig.26).

(3) Quasi-homometric groups

Groups of structures with identical $T_{1}, T_{2}$ structure maps like hcp, ccp and complex close-packed ordered alloys are closely related with slightly different lattice energies because of different $T_{3}$ and higher $T_{i}$ values. Structures of $\mathrm{M}_{\mathrm{x}} \mathrm{N}_{\mathrm{y}}$ compounds are formed by sequences of $M$ and $N$ layers or sequences of $M_{x} N_{y}$ layers (Tables 3-5,9b) with different stacking. The stacking of $\mathrm{M}$ and $\mathrm{N}$ layers can be described by the letters $c$ and $h$ (Section 2). The stacking of $M_{x} N_{y}$ layers is obtained by the translations of $\mathrm{M}$ atoms in $\mathrm{x}, \mathrm{y}$ or $\mathrm{z}$ direction in the next layer (Figs. 13,33).

\section{Distorted structures}

The hexagonal net with s-CN values 666 (Fig.39) can be distorted in such a way that the angle $\alpha=120^{\circ}$ decreases to $\alpha=90^{\circ}$ of the square net with s-CN values 444 . The intermediate net with $\alpha \approx 103^{\circ}$ and s-CN values $4(2+2) 4$ shows the relation to the square net, if the $2+$ $2^{\prime}$ atoms are added to 444 . Adding the $1+2$ atoms to $6 T_{1}$ and $2^{\prime}+$ 
3 atoms to $6 T_{2}$ yields the s-CN values of the hexagonal net. A threedimensional $T_{1}, T_{2}, T_{3}$ structure map of the rhomb net gives the same information as the $T_{1}, T_{2}$ structure maps of the square or hexagonal net. The structural units of the different structure families are distorted $a$, $b, c, s, t$ units of the square net (with different $s, s^{\prime}$ and $t, t^{\prime}$ for the occupation of different single sites $s$ and trans positions $t$ in $a$ rhomb).

The rectangle net with s-CN values $(2+2) 4(2+2)$ (Figs. $37 \mathrm{~b}, 38)$ is another distortion of the 444 square net, if the $1+1^{\prime}$ and $3+3^{\prime}$ atoms are added to 444 of the square net at small distortions $(b / a \leq \sqrt{3})$. The different families of structures are the same as for the square net with the same structural units. The $T_{1}, T_{2}$ structure map of the rectangle net however has only the information of the 1 and $1^{\prime}$ atoms. The threedimensional $T_{1}, T_{2}, T_{3}$ atlas yields the same information as the $T_{1}, T_{2}$ structure map of the square net.

The different structural units of rectangles or rhombs can be added only in one orientation for a two-dimensional tiling. The situation is similar as for chains of $A$ and $B$ atoms in the direction of nearest neighbors. The $212 ; 1$ structure (Fig.38) for example can be characterized by sequences of $\mathrm{A}$ and $\mathrm{B}$ atoms $\mathrm{AABB}$ or structural units acbc. Both sequences can be analyzed by the structure map of a chain of $A$ and $B$ atoms or $a, b$ and $c$ structural units (Fig.2b), where the s-CN values of AABB $\left(\begin{array}{llll}1 & 0 & 1 & ; 1)\end{array}\right.$ or of the units a $(000 ; 3), \mathrm{b}(000 ; 3)$ and $c(020 ; 1)$ of the acbc chain correspond to different points of the structure map (Fig.2b). An alternation of $a b a b$ of a units with other units like $b$ yields $M$. The single unit $\mathrm{ab}_{n}, n \geq 2$, where a units are separated by two or more other units, yields $\mathrm{S}$. The double row (D) in a sequence $\mathrm{abab}_{n}$ or triple row (T) of three alternating $a$ in $a_{b a b a b}$ can be considered as a combination of ab and $a b_{n}$.

Molecules with low symmetry are frequently packed with two different orientations $a$ and $b$. Rows of molecules staggered by a glide lattice operation can produce a very efficient close packing by repeating a molecule of arbitrary form in the space groups $1,4,14,19,29$ or $33(2,14,15$ or 16 for centrosymmetric molecules). These space groups are actually the most frequently observed for molecular crystals (Kitaigorodski, 1973). 


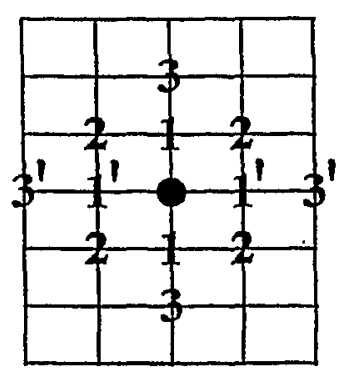

$(2+2) 4(2+2)$

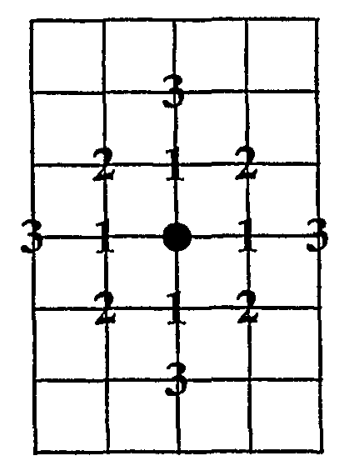

$\begin{array}{lll}4 & 4 & 4\end{array}$

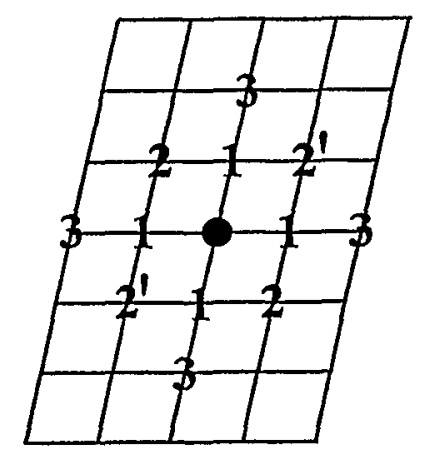

$4(2+2) 4$

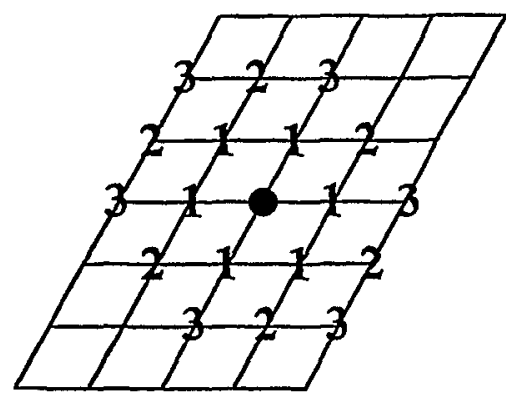

666

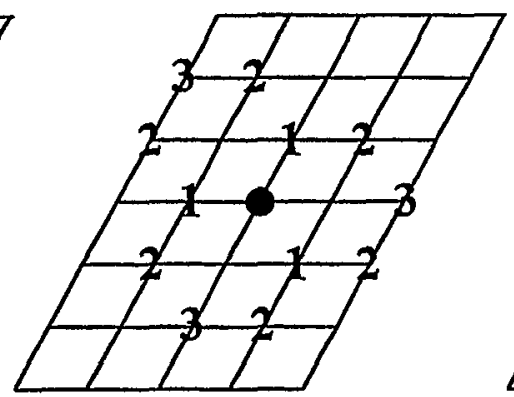

$\begin{array}{lll}3 & 6 & 3\end{array}$

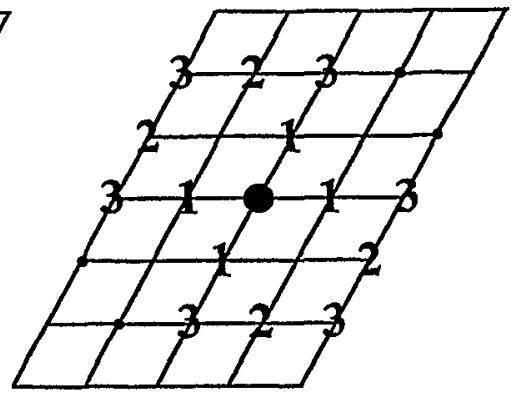

$4 \quad 4 \quad 6$

(6-2) (6-2) 6

Figure 39: All nearest (1), second (2) and third (3) neighbors $T_{1}, T_{2}, T_{3}$ of a central atom $(\bullet)$ in the rectangle $((2+2) 4(2+2))$, square $(444)$, distorted square $(4(2+2)$ 4), hexagonal ( 666 6), honeycomb (3 63 ) or kagomé (4 4 6) net. The distances from the central atom to $1^{\prime}, 2^{\prime}$ or $3^{\prime}$ positions are larger than for 1,2 or 3 . 
The numbers of molecules with the same orientation like aabb and a two-dimensional tiling in some cases like the $\beta$-sheet structure of amino acids depend on the shape and the interactions of the molecules.

Chains of identical atoms $\mathrm{Se}_{3}, \mathrm{Se}_{7}$ or $\mathrm{Se}_{\infty}$ in $\mathrm{RbDy}_{3} \mathrm{Se}_{8}$ or dimers $\mathrm{Se}_{2}$ in $\mathrm{La}_{10} \mathrm{Se}_{19}$ or $\mathrm{LaSe}_{2}$ with Se atoms on a square net (Lee and Foran, 1994) can be characterized by the orientation of neighboring atoms like $\ominus$ for horizontal and $\oplus$ for vertical orientation of two neighboring atoms. Other chains with an increased number of orientations can be characterized by an increased number of symbols similar to magnetic structures (Section 20).

Three-dimensional layered structures can be distorted by variation of the distance $\Delta \mathrm{z} c / a$ between layers like for ccp and bcc ordered structures (Section 15). The symmetry of most structures is not reduced by this procedure. The variation of $x, y, z$ parameters within the plane is another possibility for distortion. The structures of $\mathrm{As}, \mathrm{Sb}$ and $\mathrm{Bi}$ with $z \approx 0.23$ (Wyckoff, 1982) and $\Delta \mathrm{z} c / a \approx 0.47$ can be considered as a distortion of the pc lattice $(z=0.25, \Delta \mathrm{z} c / a \approx 0.41)$ or a distortion of trigonal graphite $(z=1 / 6, \Delta \mathrm{z} c / a=1.35)$. The symmetry of the crystal remains unchanged for the helix structure of Se or Te with $3_{1}$ symmetry (Fig.33). The bonding between Te atoms in a chiral chain is enhanced. The distance to neighboring Te atoms is increased, if the $x$ parameter of a close-packed layer $(x=0.33)$ is decreased to $x=0.269$ in Te or 0.217 in Se (Wyckoff, 1982). The distance to neighboring atoms $a$ $\approx 0.45 \mathrm{~nm}$ and the height of the helix $c=0.59 \mathrm{~nm}$ in Te or $0.49 \mathrm{~nm}$ in Se is similar as the distance $c / 3 a \approx 0.4$ between the three layers in the pc lattice (Burdett, 1995). The helices with $3_{1}$ or $6_{1}$ symmetry contain 3 or 6 atoms/turn like the $2212 ; 2 \mathrm{~b}$ and $220 ; 2 \mathrm{~b}$ structures (Fig.13). The helices of DNA contain $10-11$ base pairs/turn (Fig.33) with different distances $0.29-0.34 \mathrm{~nm}$ in the direction of the helix similar to the different $\Delta z c / a$ in layered compounds (Table 7) (Section 26). The $\alpha$-helix of proteins containing 3.6 amino acids/turn with a distance of $0.15 \mathrm{~nm}$ between amino acids is intermediate to the $3_{1}$ or $4_{1}$ helices of inorganic compounds. A two- or three-dimensional periodic combination is avoided in protein and DNA helices. 
The loss of the 3-fold axis in [111] layered compounds implies, that the bonding to the three atoms of the neighboring layer is different. The same applies if the 4-fold axis is lost in [001] layered compounds.

\section{Physical and chemical properties of structural units}

The relation between structural units and properties is known to a large extent in the nucleic acids DNA and RNA with the four bases adenine $(\mathbf{A})$, thymine $(\mathbf{T})$, cytosine $(\mathbf{C})$ or guanine $(\mathbf{G})$ which are forming chains in the helix or double helix structure (Glusker et al., 1994). The sequences of three bases (triplets or codons) convey the information for one of the 20 amino acids or in some cases a stop signal to indicate the end of the protein, which is a sequence of different amino acids (Section 26).

Some physical properties can also be related to the structural units $\mathrm{u}, \mathrm{u}^{\prime}$, $\mathrm{v}$ and $\mathrm{w}^{\prime}$ of superconducting oxides (Hauck and Mika, 1997 and 1998a). All superconducting oxides contain $\mathrm{v}$ units with the $\mathrm{CuO}_{2}$ planes and neighboring $\mathrm{u}^{\prime}$ or $\mathrm{w}^{\prime}$ units like $\mathrm{Cu}^{\prime} \mathrm{O}$ in $\mathrm{Cu}^{\prime} \mathrm{Ba}_{2} \mathrm{Cu}_{2} \mathrm{YO}_{7}\left(\mathrm{u}^{\prime} v \mathrm{u} \mid\right.$ ) or $\mathrm{TlO}$ in $\mathrm{CaCu}_{2} \mathrm{Ba}_{2} \mathrm{TlO}_{7}\left(\mathrm{uvw}^{\prime} \mid\right)$, to transfer $\mathrm{e}^{+}$charges to the superconducting $\mathrm{CuO}_{2}$ planes. In high $T_{\mathrm{c}}$ compounds with $T_{\mathrm{c}}>40 \mathrm{~K}$ the $\mathrm{CuO}_{2}$ planes are next to insulating $\mathrm{u}_{2}$ units like in $\mathrm{u}^{\prime} \mathrm{vu} \mid=\mathrm{u}^{\prime} \mathrm{vu}_{2} \mathrm{vu}^{\prime}$ or $\mathrm{uvw}^{\prime} \mid=$ $\mathrm{uvw}_{2}^{\prime} \mathrm{vu}_{2} \mathrm{vw}_{2}^{\prime} \ldots$.

The sequences of coordination numbers of the metal atoms $\mathrm{Cu}^{\prime} \mathrm{BaCuYCu}$ $\mathrm{Ba}, \mathrm{Cu}^{\prime} \mathrm{CN}=41058510,4$ in $\mathrm{Cu}^{\prime} \mathrm{Ba}_{2} \mathrm{Cu}_{2} \mathrm{YO}_{7}$ or $\mathrm{CN}=8596^{\prime} 95,8$ for $\mathrm{CaCuBaTlBaCu}, \mathrm{Ca}$ in $\mathrm{CaCu}_{2} \mathrm{Ba}_{2} \mathrm{TlO}_{7}$ (Table 12) shows an asymmetric arrangement of the atoms next to the $\mathrm{Cu}$ atoms with $\mathrm{CN}=5$. The $\mathrm{CuO}_{2}$ layers are bent towards the $\mathrm{Ca}, \mathrm{Sr}$ or $\mathrm{Y}$ atoms, because of the stronger bonding of these atoms to the oxygen atoms compared to the $\mathrm{BaO}$ bonding of $\mathrm{Ba}$ atoms with $\mathrm{CN}=10$ or 9 . The bond strength is increased by the inductive effect of $\mathrm{M}$ atoms in the sequence $\mathrm{Ba}, \mathrm{Na}<$ $\mathrm{Sr}<\mathrm{Ca}, \mathrm{Li}<\mathrm{Mg}<\mathrm{M}^{3+}<\mathrm{M}^{4+}$ (Hauck and Mika, 1997) similar to the 
inductive effect in organic compounds (Glusker et al., 1994; Latscha and Klein, 1982). The $\mathrm{Cu}^{\prime} \mathrm{O}$ layers of $\mathrm{Cu}^{\prime} \mathrm{Ba}_{2} \mathrm{Cu}_{2} \mathrm{YO}_{7}$ can not be influenced by the symmetrical neighborhood of $\mathrm{Ba}$ atoms.

The principle of a central structural unit with a physical property that can be modified by one or two (different) neighboring units is quite frequent in organic systems like drugs, pigments or pesticides. In diphenylazo compounds for example the yellow color can be modified to orange or red by the inductive effect of $\mathrm{OH}, \mathrm{NH}_{2}$, etc. groups on one phenyl group. One or two $\mathrm{H}$ atoms of the other phenyl group are substituted by $\mathrm{COOH}$ or $\mathrm{SO}_{3} \mathrm{H}$ groups which can react with the amino groups of wool for example for a stable coloring of wool. The same applies for organic acids like

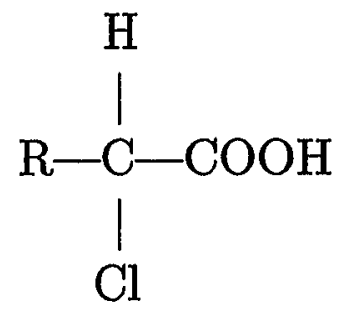

which can be varied by the inductive effect of $\mathrm{F}>\mathrm{Cl}>\mathrm{Br}>\mathrm{I}$, etc. atoms and the different hydrophobic character of short or long chains of $\mathrm{R} \cong\left(\mathrm{CH}_{2}\right)_{n}-\mathrm{CH}_{3}$ groups.

The layered compounds are non-metallic, if the valence rule (Section 17) or Pauling's electrovalence rule (Section 19) are satisfied. Pauling's electrovalence rule is obeyed for the Ketelaar and Forsyth, Gran families of $\mathrm{NaCl}$ related compounds and for the Johansson, Linde and Brauer or Schubert families of $\mathrm{ZnS}$ (sphalerite and wurtzite) related structures (adamantane structures) (Tables 11,12). The band gap of the semiconducting compounds varies with the lattice constant and the ordering of metal atoms in adamantane structures (Waag et al., 1998). A physical property of a compound is varied by homologous substitution. The physical properties of semiconducting $\mathrm{Si}$ for example are varied in $\mathrm{GaP}$ or $\operatorname{In}(\mathrm{Ga}, \mathrm{Al}) \mathrm{P}_{2}$ (Table 11a).

The comparison of $\mathrm{NaCl}$ related compounds shows attractive interactions in carbides $\mathrm{Ti}_{2} \mathrm{C}, \mathrm{Gd}_{2} \mathrm{C}(6012 ; 1 \mathrm{a}, \mathrm{b})$ with facial configuration of $\mathrm{C}$ atoms and repulsive interactions in hydrides or nitrides like $\mathrm{Pd}_{2} \mathrm{H}$, 
$\mathrm{Ti}_{2} \mathrm{~N}(4416 ; 1)$ with meridional configurations of $\mathrm{H}$ or $\mathrm{N}$ atoms. Carbides like $\mathrm{TaC}$ or HfC exhibit very high melting points (Toth, 1971). The elastic constants are supposed to be related with bonding properties. Compounds with directional covalent bonding like diamond, $\alpha$ $\mathrm{Al}_{2} \mathrm{O}_{3}$ (Tables 13,14 ) or $\mathrm{TiC}$ are very hard. These materials are used as abrasives or for coating of tools and other purposes (Toth, 1971).

The compounds of the Thornber, Bevan (1970) family (Table 12) like $\mathrm{Zr}_{7} \mathrm{Ca}_{2} \square_{2} \mathrm{O}_{16}(\alpha \gamma)$ or $\mathrm{Zr}_{3} \mathrm{Sc}_{4} \square_{2} \mathrm{O}_{12}\left(\mathrm{Pr}_{7} \square_{2} \mathrm{O}_{12}\right.$ structure, $\left.\beta(\bmod 7)\right)$ are interesting for the SOFC (solid oxide fuel cell) because of the high oxygen mobility in the disordered phase. The perovskites ( $\mathrm{La}, \mathrm{Sr}) \mathrm{MnO}_{3}$ and $(\mathrm{La}, \mathrm{Sr}) \mathrm{CrO}_{3}$ are used as cathode and interconnector materials in the SOFC (Hammou and Guindet, 1997). The compounds of the Raveau, Chu family like $\mathrm{CuBa}_{2} \mathrm{Cu}_{2} \mathrm{YO}_{7}$ and $(\mathrm{Ca}, \mathrm{Sr}) \mathrm{CuO}_{2}$ exhibit also a high oxygen mobility and some catalytic properties (Schoonman, 1997; Hauck et al., 1995).

Hydrides like $\mathrm{VH}_{\mathrm{x}}, \mathrm{Li}_{2} \mathrm{PdH}_{\mathrm{x}}$ or $\mathrm{FeTiH} \mathrm{x}_{\mathrm{x}}$ with structural units $\mathrm{g}_{1}, \mathrm{~h}, \mathrm{q}_{1}$ are used for storage of hydrogen (Parthé et al., 1993; Schlapbach et al., 1994). Hydrides like $\mathrm{Pd}_{2} \mathrm{H}, \mathrm{Pd}_{5} \mathrm{H}_{4}$ are interesting because of the high mobility of hydrogen atoms. The preference of $\mathrm{H}$ atoms for tetrahedral or octahedral interstices has been explained by the different size of the interstitial sites (Westlake, 1983) or the different mechanisms of $\mathrm{MH}$ bonding (Hauck, 1983). The first type of model considers a minimum hole size of $40 \mathrm{pm}$ (or a critical metal atom radius of $139 \mathrm{pm}$ ) and a minimum distance of $210 \mathrm{pm}$ between $\mathrm{H}$ atoms to be necessary. The larger octahedral site should be occupied in metals with small atomic radii, the smaller tetrahedral site in metals with large atomic radii.

The second type of model is based on a charge transfer between $\mathrm{H}$ and metal atoms with different electronegativities $\phi^{*}$ :

$\mathrm{M}^{\delta-} \mathrm{H}^{\delta+}$ for metals with high $\phi^{*}$, e.g. $\mathrm{M}=\mathrm{Cr}, \mathrm{Mn}, \mathrm{Fe}, \mathrm{Co}, \mathrm{Ni}, \mathrm{Pd}$, $\mathrm{M}^{\delta+} \mathrm{H}^{\delta-}$ for metals with low $\phi^{*}$, e.g. $\mathrm{M}=\mathrm{Li}, \mathrm{Na}, \mathrm{Ca}, \mathrm{Sc}, \mathrm{Y}, \mathrm{Ti}$.

The $\mathrm{H}^{\delta+}$ atoms prefer the octahedral site with a bonding to the transition metal $d$ electrons. The $\mathrm{H}^{\delta-}$ occupies octahedral interstices of metals with no $d$ electrons, otherwise tetrahedral interstices. Both sites can be 


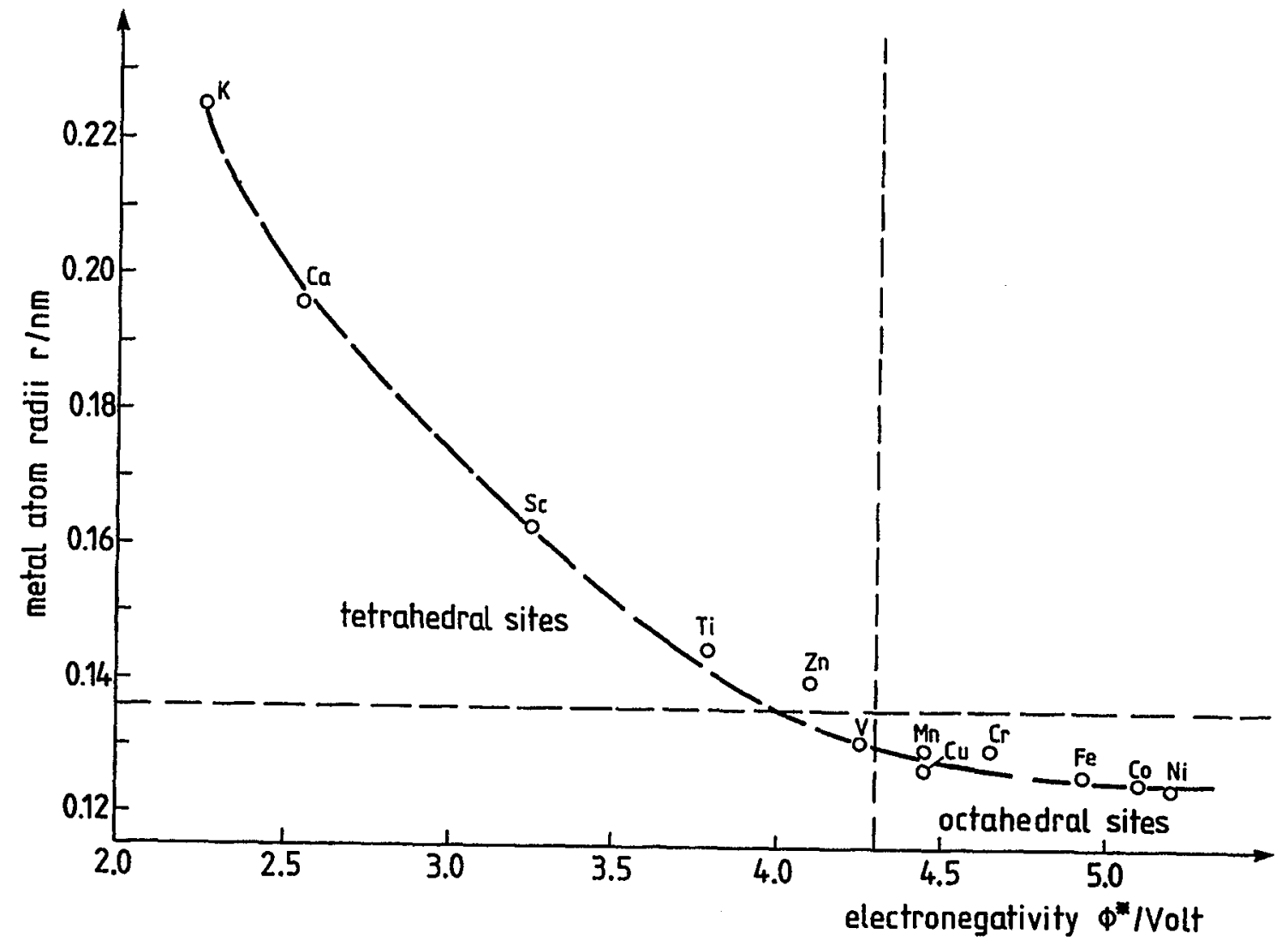

Figure 40: Hydrogen occupancy of octahedral sites in metals with small radii and high electronegativity, otherwise tetrahedral sites.

occupied in vanadium depending on temperature, hydrogen concentration and isotope (Hauck, 1983).

The two models give the same prediction for most metals because of the correlation between small metal atom radii and high electronegativity (Fig.40). The critical radius of metal atoms, $139 \mathrm{pm}$, corresponds with the cubic lattice constant of $393 \mathrm{pm}$ and can explain the variation from octahedral coordination in $\mathrm{PdD}_{0.7}$ and $\mathrm{LiH}$ to the tetrahedral coordination in $\mathrm{VH}_{2}$, etc. (Fig.41). Octahedral sites, however, are also occupied in $\mathrm{NaH}, \mathrm{KH}, \mathrm{RbH}$ or $\mathrm{CsH}$ which have larger lattice constants. In rare earth hydrides the smaller tetrahedral sites are occupied first, while octahedral sites are occupied at $H / M \geq 1.95$. This behaviour can be explained by the second type of model with $\mathrm{H}$ atoms as a sensitive probe for $d$ electrons. The $d$ electrons of the rare earth metals are depleted at increasing $\mathrm{H}$ content because of the charge transfer to $\mathrm{H}^{\delta-}$. The loss 


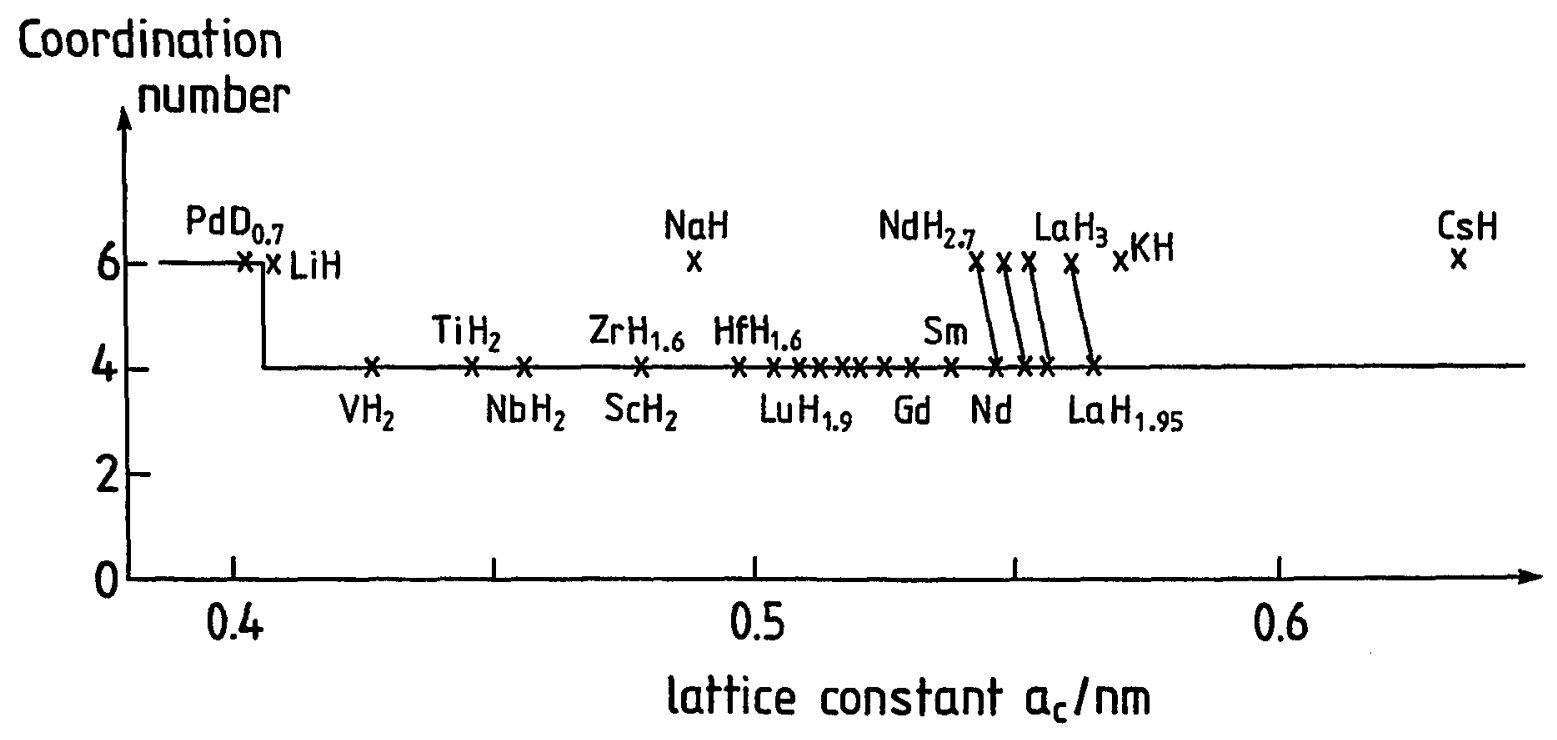

Figure 41: Coordination of hydrogen atoms in ccp $M$ structures with cubic lattice constant $a_{\mathrm{c}}$.

of $d$ electrons gives rise to a metal-semiconductor transition, different magnetic properties and smaller lattice constants (Hauck, 1983).

The other interstitial atoms $\mathrm{I}=\mathrm{C}, \mathrm{N}, \mathrm{O}, \ldots$ are too big for the occupation of tetrahedral sites. The bonding geometries of the metal atoms however can be related to the hybridization of $s, p$ and $d$ orbitals like the $\mathrm{CN}=4$ planar $\left(d s p^{2}\right)$ and tetrahedral coordination $\left(s p^{3}\right.$ or $\left.d^{3} s\right)$ or the $\mathrm{CN}$ $=6$ trigonal prismatic $\left(d^{4} s p\right)$ or octahedral coordination $\left(d^{2} s p^{3}\right)$ (Sutton, 1993). P, S and Se atoms usually prefer tetrahedral coordination, $\mathrm{O}$ atoms octahedral coordination as can be seen from the pairs of structures with identical ordering of metal atoms $\alpha-\mathrm{NaFeO}_{2}-\mathrm{In}(\mathrm{Ga}, \mathrm{Al}) \mathrm{P}_{2}$, $\gamma-\mathrm{LiFeO}_{2}-\mathrm{CuFeS}_{2}, \mathrm{Li}_{2} \mathrm{ZrO}_{3}-\mathrm{GeCu}_{2} \mathrm{Se}_{3}$. The $\mathrm{O}$ atoms however can also be in tetrahedral coordination in $\beta-\mathrm{NaFeO}_{2}$ or $\mathrm{SiLi}_{2} \mathrm{O}_{3}$ (Tables 11,12).

\section{Homogeneous sphere packings}

A sphere packing is called homogeneous, if all spheres are symmetryequivalent, otherwise it is called heterogeneous (Koch and Fischer, 1992). 
The same applies to the two-dimensional packing of circles. The different packings are characterized by the number of contacts $k$ between the spheres (circles), which is $3 \leq k \leq 12$ ( $3 \leq k \leq 6$ for circles). A sphere (circle) packing is called stable, if no sphere (circle) can be moved without moving neighboring spheres (circles) at the same time. A minimum of four (three) contacts per sphere (circle) is required. Not all contacts must fall in one hemisphere (semicircle) (Koch and Fischer, 1992). Stable homogeneous sphere packings are interesting in searching for materials with low density. They are classified in types with constant $k$ at different distortions. Two sphere packings belong to the same type, if there exists a biunique mapping that brings the spheres of one packing onto the spheres of the other packing. The number of types of homogeneous sphere packing is finite. The types of homogeneous circle and sphere packings with cubic or tetragonal symmetry are known (Koch and Fischer, 1992). They can be compared with the two- or three-dimensional structures of Section 4 or Table 21a-d with a single set of $T_{i}$ values (environment-equivalent) for $\mathrm{A}$ and $\mathrm{B}$ atoms in $\mathrm{A}_{\mathrm{x}} \mathrm{B}_{\mathrm{y}}$. The number of contacts $k$ corresponds to the $T_{1}$ values for spheres (circles) with diameter $d$. The density of the hexagonal lattice $(\rho=0.907)$, square lattice $(\rho=0.785)$, hcp or ccp $(\rho=0.741)$, bcc $(\rho=0.680)$ and pc $(\rho=0.524)$ of $A_{x} B_{y}$ compounds is reduced by the factor $x /(x+y)$ (or $y /(x+y)$ ), if the $\mathrm{A}$ (or B) atom positions are vacant. The $k=T_{2}$ (or $T_{3}$ ) values, increased diameters (Table 2) and different densities are derived for structures with $T_{1}=0$ (or $T_{1}=T_{2}=0$ ). The two-dimensional $666 ;$ (1), $060 ; 2,006 ; 3$ (Fig.6), $444 ;$;), $044 ; 1,004 ; 3$ (Fig.8) and $000 ; 6$ structures $\left(\operatorname{Pr}_{7} \square_{2} \mathrm{O}_{12}\right.$ net, Fig.33) are homogeneous circle packings (Koch and Fischer, 1992). The number of contacts $k=5\left(\operatorname{Pr}_{7} \square_{2} \mathrm{O}_{12}\right.$ net), 4 (0 $06 ; 3$, kagomé net) or $3(060 ; 2$, honeycomb net) between $\mathrm{B}$ atoms is obtained for vacant A positions (Section 28). The three-dimensional structures of the pc lattice (Table 21d) $6812 ;(1), 008 ; 3$ (bcc) and $0120 ; 1$ (fcc) are stable homogeneous sphere packings, if the $B$ atoms are removed. They correspond to the same type of sphere packing with $k=6,8$ and 12 contacts in $0612 ; 1,8612 ;(1)$ and $0012 ; 3$ in the bcc lattice (Table 21c) or $060 ; 3$ and $12624 ;$ (1) in the ccp lattice (Table 21b). The hcp, ccp or bcc systems contain other stable sphere packings, which are underlined in Table 22 (or underlined with square brackets for stable packings of $B$ atoms at $y / x>1$ ). The structures at 
the left-hand borders of the structure maps containing alternating layers of $\mathrm{A}$ and $\mathrm{B}$ atoms are not stable, if the $\mathrm{A}$ or $\mathrm{B}$ atoms are removed. Many homogeneous sphere packings of the bcc, fcc and pc lattice belong to the same type with the same space group like $0612 ; 1$ (bcc), $060 ; 3$ (fcc) and $6128 ;(1)$ (pc) with $k=6$ (Table 22) or the same space group and Pearson symbol like $424 ; 1.33 a, b$ (bcc) and $4212 ; 1.33 a, b$ (fcc) with $k$ $=4$ contacts.

The $\mathrm{CsCl}, \mathrm{NaCl}$ or $\mathrm{CaF}_{2}$ structures are examples of heterogeneous sphere packings with the ideal ratios of diameters of $\mathrm{A}$ and $\mathrm{B}$ atoms $d_{\mathrm{B}} / d_{\mathrm{A}}=$ $0.732,0.414$ or 0.225 , respectively.

\section{Colloids, clusters and DNA}

An ordering of hard-sphere colloidal crystals was first observed for $\mathrm{AB}_{2}$ and $\mathrm{AB}_{13}$ compositions in native gem opals. They consist of dried arrays of colloidal silica spheres with diameters $d_{\mathrm{A}}=362 \mathrm{~nm}$ and $d_{\mathrm{B}}=210 \mathrm{~nm}$ (Sanders, 1980). Colloidal particles like amorphous $\mathrm{SiO}_{2}$, polymers or inorganic clusters with a particle size of $10-2000 \mathrm{~nm}$ can be obtained by chemical synthesis and are stabilized sterically by thin $(\approx 10 \mathrm{~nm})$ coatings. They are suspended in polar or apolar mixtures of liquids with the same refractive index for transparent suspensions which are suitable for quantitative light scattering. For the proper composition like 50\% solvent a process similar to crystallization is observed after several days of sedimentation (Pusey and van Megen, 1986). The particles interact via short-ranged repulsive forces because of the stabilizing layer which mitigates the attractive van der Waals forces of the polymer cores, for example. The interaction between the particles can be tuned by different charges of the coatings and different solvents. The interactions between $A$ atoms as predicted by the structure maps can probably be tested by mixtures $A_{x} B_{y}$ of spherical particles $A$ and $B$ with the proper ratio of diameters and suitable coatings. The properties of suspended particles however can differ from those for purely atomic systems due to the presence of the solvent (Pusey and van Megen, 1986). A ccp structure with 
disordered stacking in [111] direction with a fraction $f=0.42-0.5$ of $\mathrm{h}$ layers depending on time, temperature and composition was observed for a one-component system (Pusey et al., 1989). The analysis of $T_{i}$ values in close-packed structures had shown a single set $\left(M^{i}=1\right)$ for hcp or ccp structures (Table 1, Section 2). The repulsive interactions are increased slightly in ccp, because of the ordering of the second-nearest neighbors on a straight line in [110] direction (Fig.1).

Spherical clusters of non-metallic compounds might be obtained only for few diameters because of the requirement for electroneutrality. This is demonstrated for the $i=1-3$ shell of clusters with $\mathrm{NaCl}$ structure. $\mathrm{Na}$ and $\mathrm{Cl}$ atoms form a pc lattice with $T_{i}$ values $0120 ; 1$ compared to the maximum values 6128 of closed shells (Table 23d). A Na atom in the center is coordinated by $0 \mathrm{Na}$ and $6 \mathrm{Cl}$ atoms at $T_{1}, 12 \mathrm{Na}$ and $0 \mathrm{Cl}$ atoms at $T_{2}$ and $0 \mathrm{Na}$ and $8 \mathrm{Cl}$ atoms at $T_{3}$. The compositions $\mathrm{NaCl}_{6}$, $\mathrm{Na}_{13} \mathrm{Cl}_{6}$ and $\mathrm{Na}_{13} \mathrm{Cl}_{14}$ for spheres with complete occupation of the first, second or third shell have an excess of positive or negative charges. The $T_{i}$ values for larger clusters containing up to $\approx 200$ atoms are given in Table 23 for hcp, ccp, bcc and pc compounds with single sets of $T_{i}$ values for $\mathrm{A}$ and $\mathrm{B}$ atoms. The rare gases $\mathrm{Ar}$ and $\mathrm{Xe}$, which crystallize in the ccp lattice at low temperatures, form clusters with $\mathrm{x}=13,19,23,25,55$, 71, 87, 135 and 147 (Sugano and Koizumi, 1998). The magic numbers $\mathrm{x}=7,10,14,23$ and $30\left({ }^{4} \mathrm{He}\right)$ and $\mathrm{x}=7,10,14,21$ and $30\left({ }^{3} \mathrm{He}\right)$ were found for the different He isotopes, which crystallize in the hcp or ccp structure at low temperatures (Wyckoff, 1982). The $T_{i}$ values 1262 of a central atom of the hcp packing yields $\mathrm{x}=13,19,21,39,51,57$, $69,81,87,93,105,129,135,147, \ldots$ compared to $\mathrm{x}=13,19,43,55$, $79,87,135,141,147,201$ for a ccp cluster. The $\mathrm{x}=23,25,71$ values of $\mathrm{Xe}$ and most magic numbers of the He isotopes are not in the list for stable low temperature structures. A similar situation occurs for the magic numbers $\mathrm{x}=8,20,40,58,92$ of Na clusters compared with the closed shells of the bcc lattice $\mathrm{x}=9,15,27,51,59,65,89,113$ (Table 23c) and for the $\mathrm{Si}$ clusters. The diamond structure of crystalline $\mathrm{Si}$ is obtained as an ordered bcc structure $\mathrm{Si} \square$ with $T_{i}$ values $4012 ; 1$, where half of the positions of the bcc lattice are vacant $(\square)$. The cluster sizes $\mathrm{x}=5,17,29,35$, which are obtained for the occupied shells at $i=1,3$, $4,6, \ldots$ (Table $23 \mathrm{c}$ ), do not coincide with the observed magic numbers 
$\mathrm{x}=6,10$. Other clusters with reduced density for vacant $\mathrm{B}$ positions like the ccp $7210 ; 1$ structure exhibit the proper $\mathrm{x}=8,10,20,26,36$, $40,68,70,91$ values of $\mathrm{A}=\mathrm{Si}$ or $\mathrm{Na}$ atoms. Both positions of $\mathrm{A}$ and $\mathrm{B}$ atoms are occupied in the $\mathrm{CsCl}$ structure $(0612 ; 1$ of the bcc lattice, Table 23c) and $0120 ; 1$ of the pc lattice (Table 23d). Most clusters of the bcc lattice with closed shells at $\mathrm{x}=9,15,27$, etc. and of the pc lattice at $x=7,19,27,33,51,75$, etc. however are not neutral. All $\mathrm{NaCl}$ or $\mathrm{CsCl}$ clusters can only be neutral, if one atom like the central atom is missing. Only few magic numbers for clusters coincide with the cluster sizes of closed shells, if the same structure as in the crystalline phase is assumed. Clusters of other metastable phases could be formed by the rapid quenching process. The present compilation shows all spherical clusters with the same surrounding of each atom except the surface atoms, which are different in all kinds of theoretical approaches. Therefore the numbers $\mathrm{x}$ of the present investigation might be useful to discuss magic numbers $\mathrm{x} \gtrsim 10$ for clusters of $\mathrm{A}$ and $\mathrm{B}$ atoms.

Microclusters like $\mathrm{NaCl}$ are generated by quenching the metal halide vapor of typically $20 \mathrm{~Pa}$ at $1000 \mathrm{~K}$ within less than 100 microseconds in a helium atmosphere of about $100 \mathrm{~Pa}$ at $70-300 \mathrm{~K}$ (Sattler, 1987). The observation of magic numbers $\mathrm{x}$ and $\mathrm{y}$ of stable clusters depending on the temperatures of quenching and the methods of analysis are explained by the assumption of neutral spherical clusters, which crystallize from the gas phase. Several atoms of the original clusters evaporate in such a way that charged $\mathrm{Na}_{\mathrm{x}} \mathrm{Cl}_{\mathrm{x}-1}^{+}$cuboids containing $3 \times 3 \times 3(\mathrm{x}=14), 3 \times 3 \times 5$ $(\mathrm{x}=23), 3 \times 5 \times 5(\mathrm{x}=38)$ atoms or neutral $\mathrm{Na}_{\mathrm{x}} \mathrm{Cl}_{\mathrm{x}}$ clusters containing $3 \times 3 \times 4(\mathrm{x}=18), 2 \times 4 \times 5(\mathrm{x}=20), 3 \times 4 \times 4(\mathrm{x}=24)$ atoms are left at increased temperatures. This process is similar to the crystal growth process with a formation of stable external forms and habits of crystals (Wulff, 1901). The present compilation (Table 23) shows all clusters with closed shells. Some special clusters can be obtained by $T_{j}$ $=a j^{3}+b j^{2}+c j+1$. The shells $j=1,2, \ldots$ are a selection of the $i=$ $1,2, \ldots$ shells, where clusters with the shape of crystals are obtained. This can be demonstrated for the 444 ; (1) structure of the square net. The coefficients $a=0, b=c=2$ yield square clusters with $\mathrm{x}=\underline{5}, \underline{13}, \underline{25}$, 41 atoms for $j=1,2,3,4$, which coincide with the underlined $\mathrm{x}$ values of the $444 ;(1)$ structure $(\mathrm{x}=\underline{5}, 9, \underline{13}, 21, \underline{25}, 29,37,45, \ldots)$ for $i=$ 
$1-8$ (Table 2). The cluster containing 41 atoms does not have a closed shell like the $\mathrm{x}=37$ and $\mathrm{x}=45$ clusters. The coefficients are $a=0, b=$ $c=3$ for hexagonal clusters with $\mathrm{x}=7,19,37,61$, etc. particles with the 666 ; (1) structure of the hexagonal net. The situation is similar for the three-dimensional structures. The constants $a=10 / 3, b=5$ and $c$ $=11 / 3$ were obtained for a packing of cubo-octahedra in the $12624 ;(1)$ structure of the ccp lattice (Urban, 1998; Wulff, 1901). The underlined magic numbers $\mathrm{x}=\underline{13}, \underline{55}, 147$ coincide with closed shells for $\mathrm{x}=\underline{13}$, $19,43, \underline{55}, 79,87,135,141,177,201$ (Table 23b). The missing central atom is suggested for the stabilization of clusters (Urban, 1998).

DNA or long chains of proteins can have the size of colloids $\left(\approx 10^{2}-\right.$ $10^{4} \mathrm{~nm}$ ). Their structures can be analyzed like a one-dimensional cluster with four to twenty different structural units. The basic components of DNA are alternating units of 2 -deoxyribose (D) and phosphate groups (P) forming a DP chain with phosphodiester bonds between the $5^{\prime}$ position of one deoxyribose and the $3^{\prime}$ position of the next deoxyribose (Dale, 1996; Glusker et al., 1994; Diekerson, 1989). One of the four bases adenine $(\mathbf{A})$, thymine $(\mathbf{T})$, cytosine $(\mathbf{C})$ or guanine $(\mathbf{G})$ is attached to the $1^{\prime}$ position of each deoxyribose. Each $\mathbf{A}$ is linked to a $\mathbf{T}$ of the neighboring chain by two hydrogen bonds, and each $\mathbf{G}$ is linked to a $\mathbf{C}$ by three hydrogen bonds. The $\mathbf{A}$ and $\mathbf{G}$ units are larger than the $\mathbf{T}$ and $\mathbf{C}$ units. The complementary strands are twisted around each other in the now familiar "double helix", with the bases in the center and the hydrophilic deoxyribose (or ribose in RNA) and phosphate residues on the outside. The exact DNA structure is influenced by several factors such as the sequence of bases and the interaction with the surrounding water. The Watson and Crick structure (B form) is a right-handed helix with 10 base pairs (bp) per turn and a distance $d=0.34 \mathrm{~nm}$ between the base pairs. A more compact form (A form) with about $11 \mathrm{bp}$ per turn and $d=$ $0.29 \mathrm{~nm}$ occurs at reduced water content. Certain DNA sequences with alternating $\mathbf{G}$ and $\mathbf{C}$ bases tend to form a left-handed helix ( $\mathrm{Z}$ form), at least over a short distance, with $12 \mathrm{bp}$ per turn and $d=0.38 \mathrm{~nm}$. The different rotations give rise to a winding of the helix into coils (Dale, 1996). The in vitro structures of the DNA's, however, are not known in detail, and the number of different structures is probably larger than the number of polytypes in layered compounds. The interactions be- 
tween the bases of one strand are assumed to be very weak because of the absence of direct bonding (in helices without defects). Dipole-dipole interactions are considered besides the van der Waals interactions because of partial positive or negative charges in the neighborhood of the $\mathrm{N}-\mathrm{H} \cdot \cdots \mathrm{O}$ or $\mathrm{N}-\mathrm{H} \cdots \mathrm{N}$ hydrogen bonds. The sequences of bases deviate from a random distribution $\left(\alpha_{i}=0\right)$ in such a way that attractive or repulsive interactions are preferred in some portions of the DNA.

The ratios $r=1.5-7.5$ of the nucleic acids $\mathbf{A}, \mathbf{T}, \mathbf{C}, \mathbf{G}$ like $\mathrm{r}=(\mathbf{T}+\mathbf{C}+$ $\mathbf{G}) / \mathbf{A}$ for the base $\mathbf{A}$ are similar to those of the layered compounds ( $r=1$ - 11) (Table 7). The $\alpha_{1}, \alpha_{2}$ values which were obtained from the averaged $T_{i}$ values are similar to the values of disordered alloys (Fig.17) (Hauck et al., 1999). The $\alpha_{i}$ values of the bases in different sections of the structure map Ia - IVb (Fig.16) can be correlated with a distinct occurrence of clusters like AAA (in IIIb - IVa), isolated bases A like TCG ACT (in Ia,b) or bases $\mathbf{A}$ alternating with other bases like ATA CAG ..., etc. (in IIa,b). Eighteen different DNA or RNA sections with the same kind of functions (enolase and secA), which occur in bacterias (like secA 15), rats (enolase 15) or human being (enolase 8), were classified in different groups (Table 24). Most DNA's contain clusters, which are avoided in disordered alloys (Section 13).

The mRNA carries the information for the sequence of amino acids in a protein in the form of the genetic code in which each occurrence of one of the 64 groups of three nucleotides (triplets or codons) conveys the information for a specific amino acid (or in some cases a stop signal to indicate the end of the protein) (Dale, 1996). Fifteen amino acids are obtained by symmetrical triplets AAA (Lys), ATA (Ile), ACA (Thr), AGA (Arg), TTT (Phe), TCT (Ser), TGT (Cys), TAT (Tyr), CCC (Pro), CGC (Arg), CAC (His), CTC (Leu), GGG (Gly), GAG (Glu), GTG (Val) and GCG (Ala). Arginine (Arg) is obtained by AGA or CGC.

The remaining five amino acids Asn, Asp, Glu, Met and Trp can be obtained only by non-symmetrical triplets. This is only possible because of a given direction from $5^{\prime}$ to $3^{\prime}$ on each strand (Dale, 1996) and of stop signals (TAA, TAG or TGA). 
The increased fraction of $\mathbf{A}$ and $\mathbf{T}$ clusters in the first group would be obtained, e.g., at an increased number of AAA (Lys) and TTT (Phe) clusters, while the increased percentage of double units for $\mathbf{T}$ in the second group could be possible at an increased number of TCT (Ser), TGT (Cys) and TAT (Tyr).

The different groups can probably be related with a variation of the number of structural units in evolution. The 16 different combinations of two bases (A, C, G, T) like AA, AC, CA, AG, etc. yield 15 different amino acids. The information of the 16 symmetrical triplets mentioned before is maintained, if the first or last letter is missing. At least one combination like GA is required for a stop signal for the production of proteins. 63 instead of 20 amino acids could occur for the combination of three bases (codons) like AAA (Lys), AAC (Asn), CAA (Glu), etc. . The same amino acid is synthesized by several combinations like Gly by the four combinations GGT, GGC, GGA and GGG. Usually all combinations are used. The restriction to few combinations in bacterias is probably related with the early stage of evolution and is maintained in few species like the silkworm B.mori producing silk with a speed of $\approx 100$ amino acids/sec (Glusker et al., 1994). The polypeptide of silk contains mainly alternating units of Ser and Gly or Ala and Gly in the sequence Gly-(Ala-Gly) ${ }_{2}-\left[\mathrm{Ser}-\mathrm{Gly}-(\mathrm{Ala}-\mathrm{Gly})_{n}\right]_{8^{-}}$(Ser-Gly)-Ala-AlaGly-Tyr. The codons GGT and GGA for Gly, TCA for Ser and GCT for Ala are used. Four chains are combined in the $\beta$-sheet structure with a connection of the amino acids similar to a zipper. The periodic sequences and the nonperiodic end of the chain Ala-Ala-Gly-Tyr are related with the high strength and the elasticity of silk, respectively. The $T_{i}$ values of the sequence of amino acids in silk are close to the border of the structure map. How would it look like, if evolution would have selected periodic sequences of bases in DNA and amino acids in proteins, which are on the border of the structure map? The number of different species would have been reduced.

The numbers of different structures $N_{\mathrm{H}}$ at the borders of the structure map, which are stabilized by the enthalpy $\mathrm{H}$, and other entropy stabilized structures $N_{\mathrm{S}}$ increase by $N_{\mathrm{H}}(n) \approx \mathrm{C}_{\mathrm{H}} \times 10^{0.16 n}$ and $N_{\mathrm{S}}(n) \approx \mathrm{C}_{\mathrm{S}} \times 10^{0.26 n}$, respectively, where $\mathrm{C}_{\mathrm{H}}, \mathrm{C}_{\mathrm{S}}$ depend on $\mathrm{r}\left(\mathrm{C}_{\mathrm{H}} \approx 0.1\right.$ for $\left.\mathrm{r}=1\right)$ and, for 
border structures, on the particular border, for an increased length of the chain $n .1504$ structures with different $T_{i}$ values were selected from 5200300 structures at $n=26$ and $\mathrm{r}=1$, for example. The natural language of DNA may be compared with the man made language with 26 letters and several thousand words, or with chains of music notes or numbers with 7 or 10 structural units, respectively. The different codons for the same protein can probably be compared with different words with the same meaning like the Roman or Italian VIA, the English WAY and the German WEG. The "evolution" of languages or music within centuries can probably also be analyzed by typical deviations from randomness. Periodic sequences like certain poems (hexameter for example), the ringing of church clocks or the decades 10, 100, 1000, etc. are either nice or useful. The different languages allow a large variety, while identical numbers and music notes are practical for a fast communication with a minimum of errors.

Two types of right-handed helices (A or B form) with 11 or $10 \mathrm{bp}$ per turn and a left-handed helix ( $\mathrm{Z}$ form) are known (Glusker et al., 1994). Within the cell the DNA helix is wound up into coils (Dale, 1996). For example, bacterial DNA is normally negatively supercoiled to the extent of about one negative turn per $200 \mathrm{bp}$. The density is increased by the supercoiling. The Escherichia coli chromosome, in its expanded state, for example, would be several hundred times longer than the bacterial cell itself (Dale, 1996).

There are six parameters for the exact analysis of the translation and rotation from one base pair to the next, which are correlated and depend to a certain extent upon the choice of the overall helix axis (Diekerson et al., 1989). An approximate description with A, B or $\mathrm{Z}$ indices for each base pair in the $\mathrm{A}, \mathrm{B}$ or $\mathrm{Z}$ helix and $\mathrm{X}$ values for deviations from the ideal helix by about $10 \%$ would show whether there is a correlation between the sequence of bases with, e.g., clustering in certain areas and the approximated configuration of the helix. The sections of the helix containing single bases $(\mathbf{A}+\mathbf{G} \neq \mathbf{C}+\mathbf{T})$ could be indicated by $\mathrm{A}^{\prime}, \mathrm{B}^{\prime}$, $\mathrm{Z}^{\prime}$ and $\mathrm{X}^{\prime}$, respectively. 


\section{Structure and interactions of atomic nuclei}

The atomic nuclei contain $\mathrm{x}$ protons $\mathrm{M}$ and y neutrons $\mathrm{N}$ with slightly different mass and different interactions. They probably can be treated like ordered alloys $M_{x} N_{y}$ with large thermal vibrations of the nuclei at temperatures close to the melting point. The ordering of protons and neutrons is compared with small grains of $\mathrm{M}_{\mathrm{x}} \mathrm{N}_{\mathrm{y}}$ alloys with $\mathrm{x}+\mathrm{y} \lesssim$ 208 in the present investigation. Small grains of $\mathrm{M}_{\mathrm{x}} \mathrm{N}_{\mathrm{y}}$ alloys usually have the same structure as large single crystals because of the major contribution of nearest and next-nearest neighbors $T_{1}$ and $T_{2}$ to the lattice energy. The bonding of the surface atoms is different because of the different numbers $T_{i}$ at the surface. $11 \mathrm{hcp}, 19 \mathrm{ccp}, 8 \mathrm{bcc}$ and 17 pc structures with identical $T_{i}$ values were obtained for the borders of the $T_{1}, T_{2}$ structure maps (Tables 21,23). The composition of structures with identical $T_{1}$ and $T_{2}$ values of the $\mathrm{M}$ and $\mathrm{N}$ particles (except the particles at the surface) can be obtained from the $T_{i}^{\mathrm{M}}$ values of the protons, which are the minority component in most cases (Zeldes, 1996). The numbers of all particles including the $i$-th sphere are obtained from Table 2. The numbers $\mathrm{x}$ of protons are the values of Table 23 plus the central position. (The symmetrical configuration is obtained for $\mathrm{M}_{\mathrm{y}} \mathrm{N}_{\mathrm{x}}$ composition). The ratio $\mathrm{r}=\mathrm{y} / \mathrm{x}$ is $\approx 1$ for $\mathrm{x} \lesssim 20$ and increases to $\mathrm{r}=126 / 82 \approx 1.5$ at the upper limit of stable isotopes. The numbers $\mathrm{x}$ or $\mathrm{y}=2,8,20,28$, 50,82 and 126 are magic numbers with an increased stability of isotopes with $\mathrm{x}=2(\mathrm{He}), 8(\mathrm{O}), 20(\mathrm{Ca}), 28(\mathrm{Ni}), 50(\mathrm{Sn})$ and $82(\mathrm{~Pb})$ compared to neighboring elements. Magic numbers of neutrons are obtained for $\mathrm{M}_{\mathrm{x}} \mathrm{N}_{\mathrm{y}}, \mathrm{x}=\mathrm{y}=2,8,20, \mathrm{M}_{20} \mathrm{~N}_{28}, \mathrm{M}_{28} \mathrm{~N}_{50}, \mathrm{M}_{50} \mathrm{~N}_{82}$ and $\mathrm{M}_{82} \mathrm{~N}_{126}$ (Zeldes, 1996).

Two protons and two neutrons are supposed to order in a tetrahedral cluster. The larger values $\mathrm{x}+\mathrm{y}=16,40,48,56,78,100,132,164$ and 208 can be compared with the $S_{i}=\sum_{j=1}^{i} T_{j}^{\max }$ values for $i=1-I$, where $I$ is defined by $S_{I} \leq 208$ (Table 2). They are close to the $i=1-$ 11 sequence of values $13,19,43,55,79,87,135,141,177,201$ and 225 of the ccp lattice. The numbers of approximate values $13(i=1), 19(i$ $=2), 39(i=4), 51(i=5), 57(i=6), 81(i=8), 105(i=11), 135(i=$ $13), 207(i=18)$ in hcp, $15(i=2), 51(i=4), 59(i=5), 137(i=9)$, 
$169(i=10)$ in bcc and $19(i=2), 57(i=5), 81(i=6), 203(i=12)$ in pc are smaller. The total numbers $x+y$ of $M$ and $N$ atoms at each shell are increased by $1-5$ compared to the $\mathrm{x}$ and $\mathrm{y}$ values of isobars with magic numbers $x$ and $y$. The removal of these extra protons and neutrons at the surface with decreased bonding energy gives rise to non-spherical shape of atomic nuclei. The symmetrical clusters with closed shells of the present investigation however contain an odd number of $x+y$ values, while nuclei with even $x$ and even $y$ values exhibit an increased stability (Zeldes, 1996). Clusters with even $\mathrm{x}$ and $\mathrm{y}$ values can only be obtained for vacancies in the inner shells or at the central position like the $\mathrm{x}+\mathrm{y}$ $=4$ tetrahedron, the $\mathrm{x}+\mathrm{y}=8$ cube or the $\mathrm{x}+\mathrm{y}=12$ cubo-octahedron or icosahedron. The sequence of $T_{i}^{\mathrm{M}}$ values $1612080220320 ; 1.5$ and $T_{i}^{\max }$ values $3,12,24,8,8,22,22,32,32,44$ are required for stable isotopes with magic $\mathrm{x}$ (and $\mathrm{y}$ ) values. The pairwise interactions between protons are obtained for $T_{1}=1$. We suggest vacant positions in the central part. The sequence of $T_{i}^{\max }$ values $2,1,12,24,8,6,24,24,24$, $32,12,48$ is obtained for a bcc lattice with 6 and 5 vacancies in the $T_{1}$ and $T_{2}$ shell, respectively. The proton in the center and the $T_{2}=2$ and $T_{3}=1$ nucleons are forming a tetrahedron. Structures with $T_{1}=$ 1 nearest proton for pairwise interactions can not be obtained in closepacked lattices at $\mathrm{r}=1$ to 2.5 . The minimum number $T_{1}=4$ at $\mathrm{r}=1$ is decreased at increased $\mathrm{r}$ values $\left(T_{1}=6-2 \mathrm{r}\right.$ ) (Sections 5,6$)$.

\section{Classification of symmetrical patterns}

The chemist and Nobel prize winner of $1909 \mathrm{~W}$. Ostwald published three collections of symmetrical patterns based on the square, hexagonal and honeycomb nets (Ostwald, 1922). The origin of a unit cell containing $p$ $\times p$ points $(p=1-4$ or 5 ) is connected to nearest, second, third, etc. neighbors in a systematic way and symmetrical lines obtained by the different mirror planes. The 190 patterns on single sheets of transparent paper can be combined to a large number of new patterns. They can be characterized by the numbers $T_{i}$ of $i=1,2, \ldots$ nearest, next-nearest, ... neighbors and the ratio $\mathrm{y} / \mathrm{x}$ of $\mathrm{x}$ points, which are connected by lines, and $y$ other points of the unit cell. 
The structures of the two-dimensional nets were investigated in a systematic way in Sections 4 and 21. Figure 42 shows a selection of these structures in a similar way as given by Ostwald. The unit cell containing $\mathrm{x}+\mathrm{y}$ positions for $\mathrm{A}_{\mathrm{x}} \mathrm{B}_{\mathrm{y}}$ compositions is shown by dots. (The unit cell is defined by two non-collinear lines from one dot, the origin, and the two parallel lines, which are not shown in Fig.42. All points at the corners (origin) of the unit cell and all points at the edges of the unit cell, which are shared with neighboring unit cells, are counted as single points.) The structures are characterized by the $T_{i} ; \mathrm{r}=\mathrm{y} / \mathrm{x}$ values of the $\mathrm{x}$ A positions. The $T_{i}$ values of $\mathrm{A}$ positions, which are connected by lines, are underlined. The A positions are connected by solid lines to $T_{1}$ nearest neighbors and by dashed lines to $T_{2}$ next-nearest neighbors in most cases. The $T_{2}$ lines are omitted for some structures containing clusters of A positions to show the clustering more clearly. All A and all $\mathrm{B}$ positions of the hexagonal net with $T_{i}$ of $\mathrm{A}$ positions $060 ; 2$, $006 ; 3,0006 ; 6$ and $044 ; 1,004 ; 3,0004 ; 4,220 ; 1$ of the square net have identical sets of $T_{i}$ values $\left(M^{i}=2\right)$. The patterns of these structures (except $220 ; 1$ ) at different corners of the structure maps are identical, if the $T_{3}$ or $T_{4}$ neighbors of $006 ; 3$ and $004 ; 3$ or $0006 ; 6$ and $0004 ; 4$ structures are connected by lines. The A positions can not be occupied in many surface structures because of the occupation by metal atoms (Section 21). The forbidden positions are indicated by circles in Figure 42 and the remaining positions of the honeycomb, kagomé, $\operatorname{Pr}_{7} \square_{2} \mathrm{O}_{12}$, etc. nets divided in $A$ and $B$ positions in the usual way. Crystal structures like $\operatorname{Pr}_{7} \square_{2} \mathrm{O}_{12}$ containing hexagonal or square layers of atoms can be visualized by these patterns and a translational vector to neighboring layers. Figure 42 shows also the hexagonal layers of the $\mathrm{Ta}_{2} \mathrm{C} \cong \mathrm{I}_{2} \mathrm{Cd}, \mathrm{Ta}_{3} \mathrm{~B}_{4}$ and $\mathrm{Zr}_{2} \mathrm{Fe}_{12} \mathrm{P}_{7}$ structures of the Bozorth, Kiessling and Ganglberger families of the $\mathrm{ph} / \mathrm{ph}^{\prime}$ lattice (Section 18) with solid and dashed lines for $T_{1}$ and $T_{2}$ translations. The $\mathrm{Zr}$ atoms of the Ganglberger family are indicated by circles. These examples might demonstrate the different kinds of combinations of hexagonal layers.

The metal atoms of the different modifications of $\mathrm{Ta}_{2} \mathrm{C} \square, \mathrm{Nb}_{2} \mathrm{C} \square, \mathrm{Co}_{2} \mathrm{C} \square$, $\mathrm{Fe}_{2} \mathrm{~N} \square$ and $\mathrm{Ni}_{3} \mathrm{C}_{2}$ listed in Table 13 (Bozorth family) form a hcp packing on $A$ and $B$ sites at $z=0$ and 1 , which are not shown in Fig.42. The $\mathrm{C}$ or $\mathrm{N}$ atoms of these compounds occupy $50 \%$ of the $\mathrm{C}$ sites (octahedral 

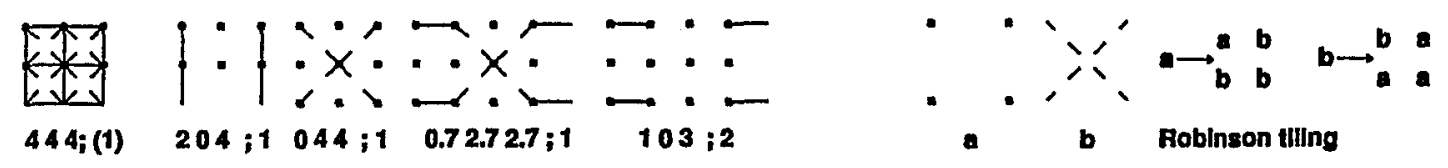

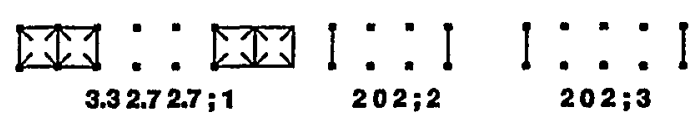

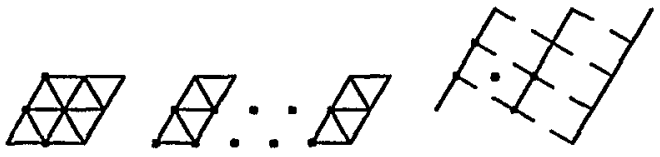
$666 ;(1) . \quad 422 ; 1 \quad 226 ; 1$

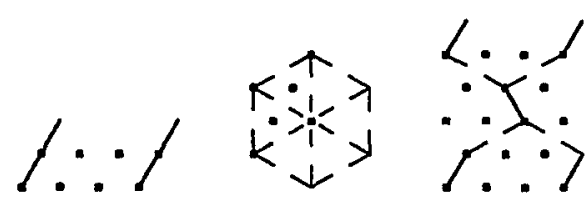

202;2 $060 ; 2 \quad 122 ; 2$
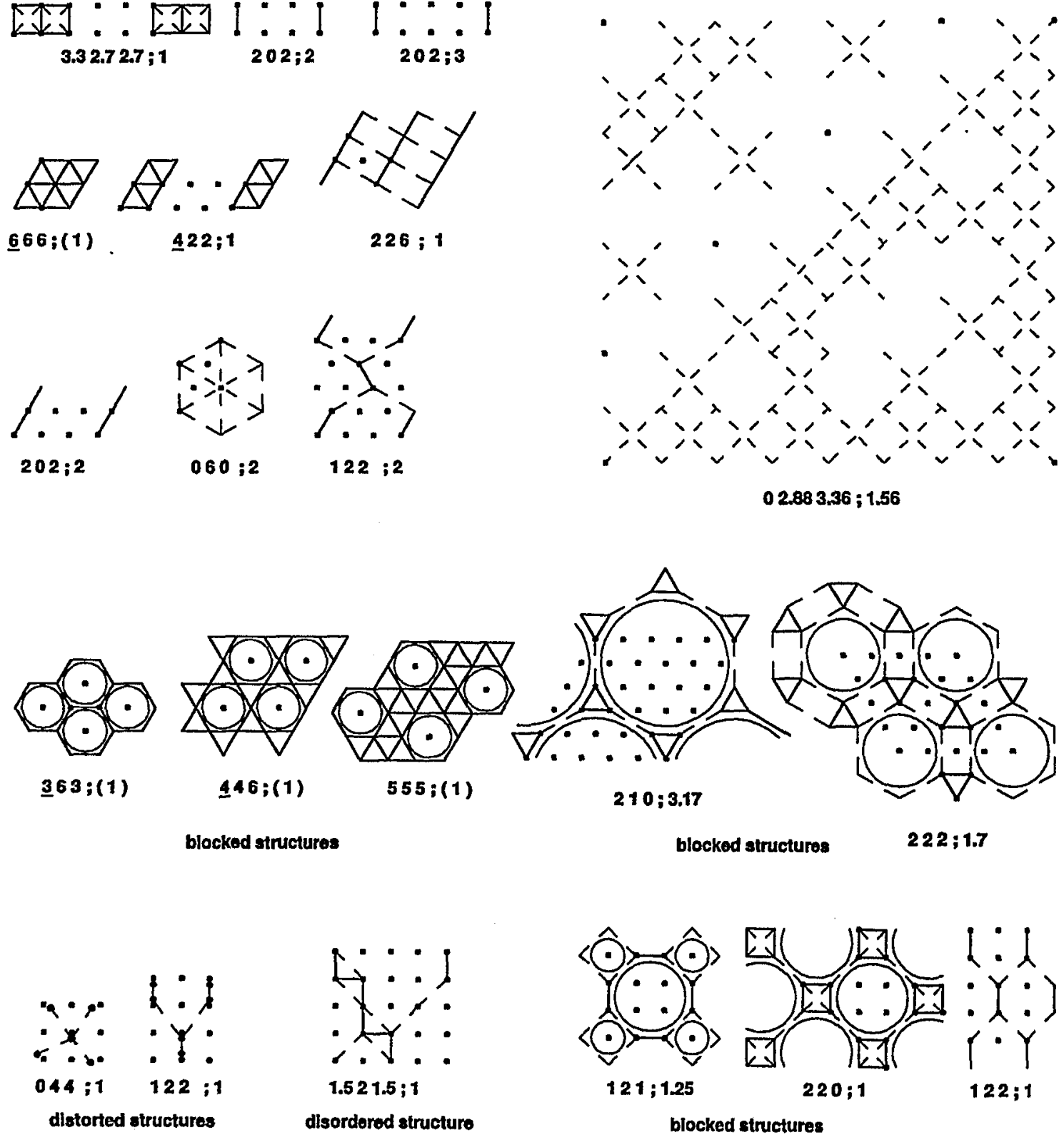


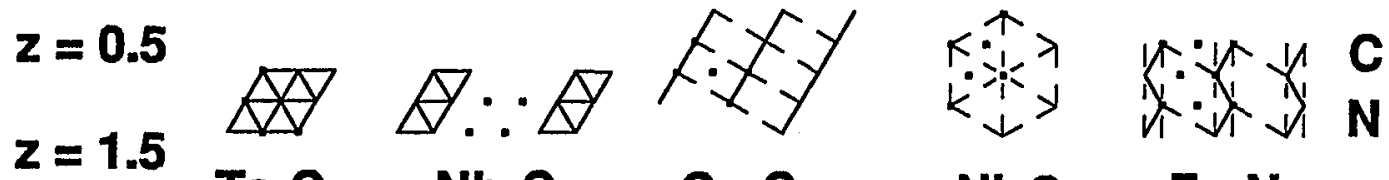

$$
\begin{aligned}
& \begin{array}{lllll}
\mathrm{Ta}_{2} \mathrm{C} & \mathrm{Nb}_{2} \mathrm{C} & \mathrm{Co}_{2} \mathrm{C} & \mathrm{Ni}_{3} \mathrm{C} & \mathrm{Fe}_{2} \mathrm{~N}
\end{array}
\end{aligned}
$$

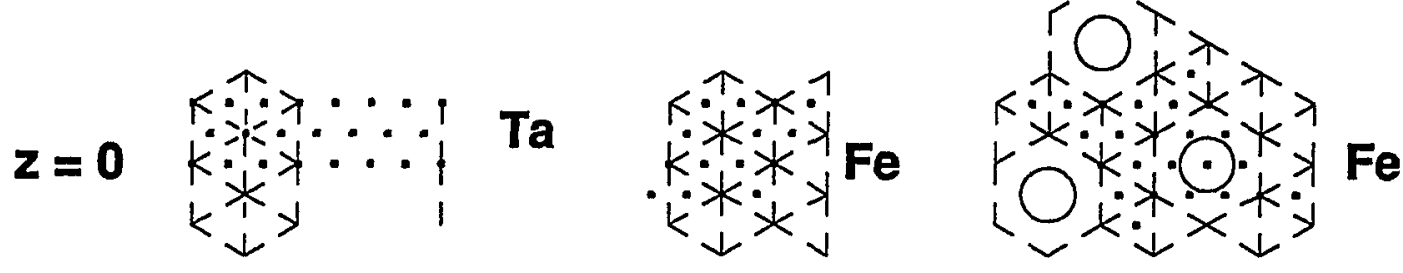

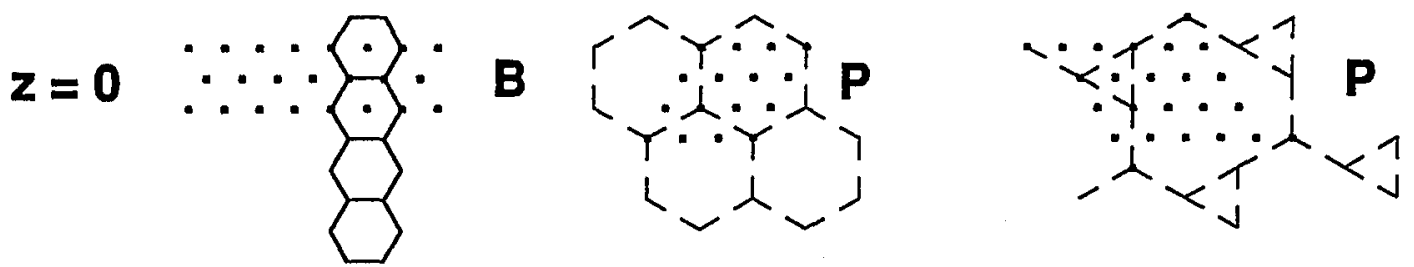

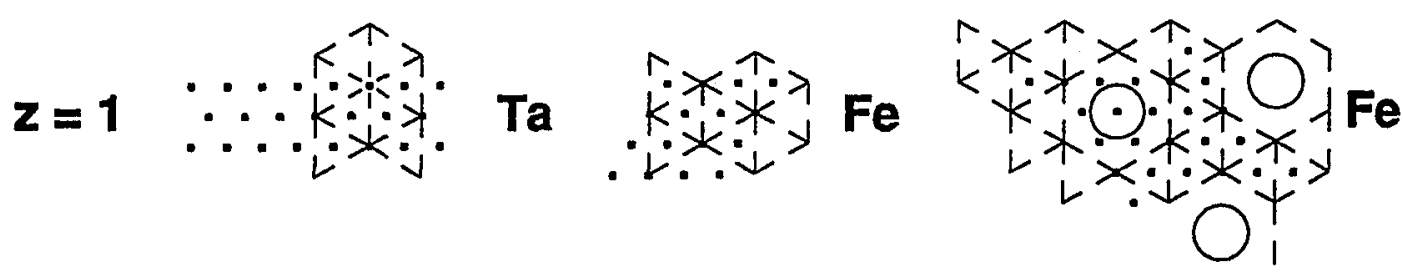

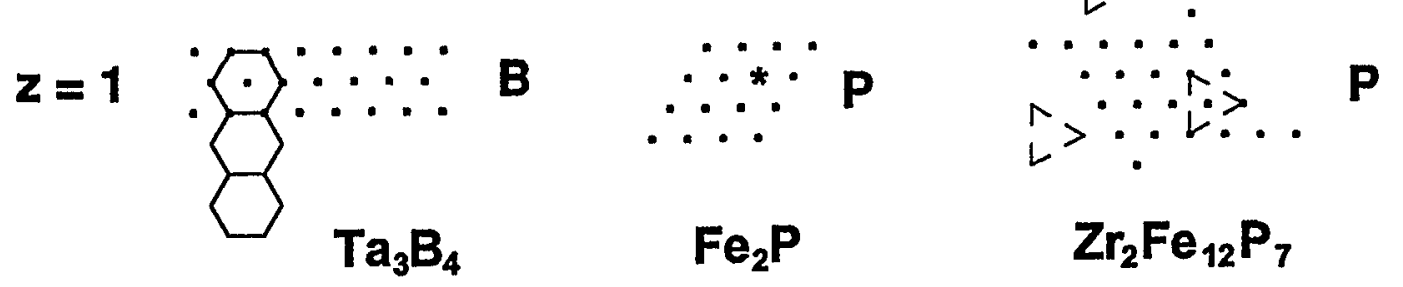



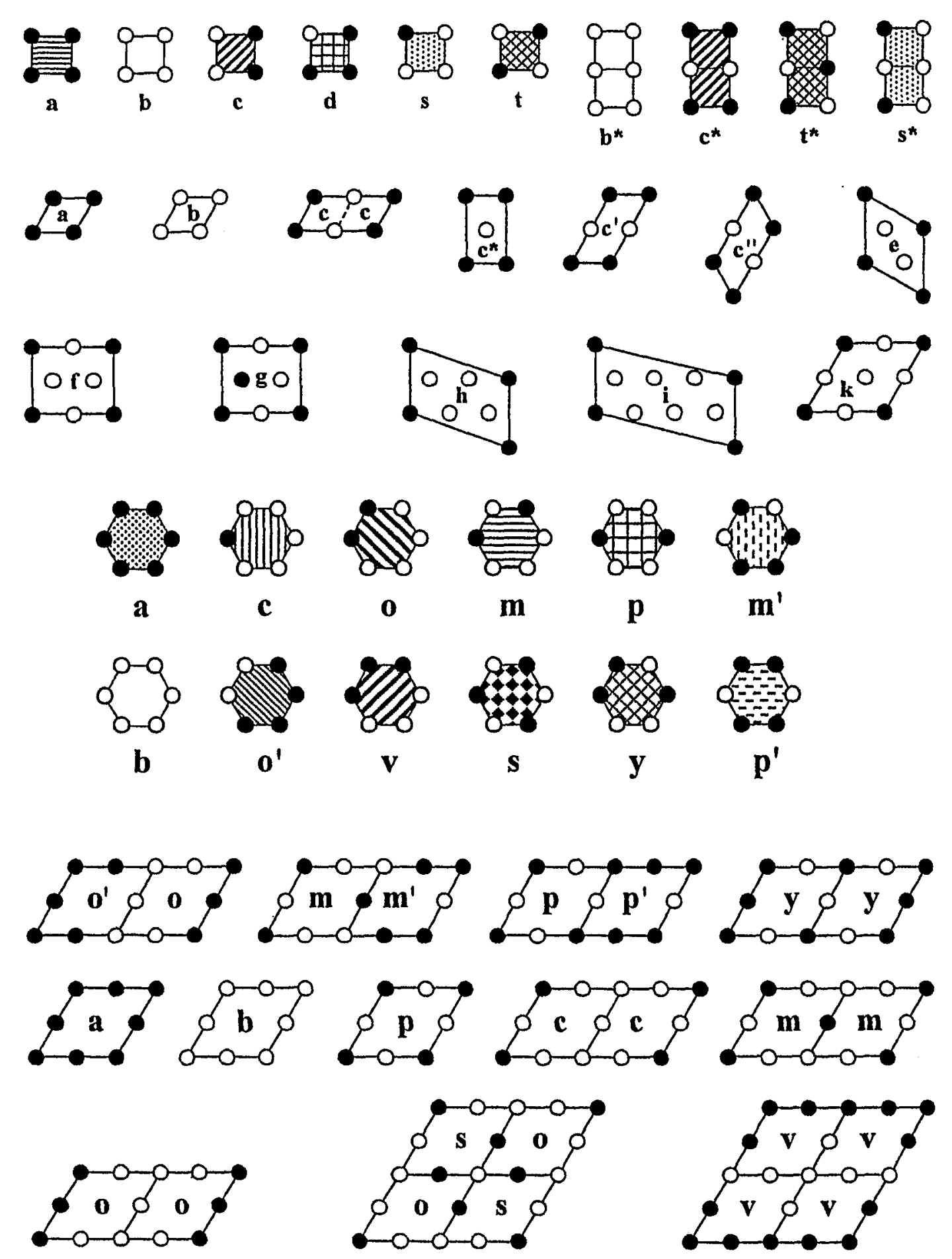

Figure 42: Periodic patterns $A_{x} B_{y}$, which are based on the square or hexagonal net and related nets like honeycomb or kagomé net with $T_{1} T_{2} T_{3} ; \mathrm{y} / \mathrm{x}$ values. The unit cell with $\times$ A and y B atom positions are indicated by points. The $T_{1}$ and $T_{2}$ neighbors of A positions are linked by solid or dashed lines, respectively (in $\underline{6} 66 ;(1)$ etc. only $T_{1}$ neighbors). The structural units can be enlarged and photocopied to obtain a two-dimensional jig-saw puzzle. 
interstices) by alternation of occupied and vacant $(\square)$ hexagonal layers at $\mathrm{z}=0.5$ and 1.5 in $\mathrm{Ta}_{2} \mathrm{C} \square \widehat{=} \mathrm{I}_{2} \mathrm{Cd} \square$ or identical layers $\mathrm{C} \square$ or $\mathrm{N} \square$ in $\mathrm{Nb}_{2} \mathrm{C} \square, \mathrm{Co}_{2} \mathrm{C} \square, \mathrm{Fe}_{2} \mathrm{~N} \square$ (or $\mathrm{C}_{2}$ in $\mathrm{Ni}_{3} \mathrm{C}$ ). The antistructure $\mathrm{CdI}_{2}$ (Bozorth, 1922) is the first compound of this family (Table 13).

The Fe atoms of "undistorted" $\mathrm{Fe}_{2} \mathrm{P}(x(\mathrm{Fe})=0.33$ and 0.67 instead of 0.26 and 0.59 ) occupy only $1 / 3$ of the $A$ and $B$ positions at $\mathrm{z}=0$ and 1 , respectively (Fig.42). The distance between $\mathrm{Fe}$ atoms is increased from $a_{\text {hex }}=0.271 \mathrm{~nm}$ in $\mathrm{Fe}_{2} \mathrm{~N}$ to $a_{\text {hex }}=0.587 \mathrm{~nm}$ in $\mathrm{Fe}_{2} \mathrm{P}$ to intercalate two $\mathrm{P}$ atoms on $\mathrm{C}$ positions at $\mathrm{z}=0$ and one $\mathrm{P}$ atom on $\mathrm{C}$ positions at $\mathrm{z}=$ 1. The projection of the unit cell of $\mathrm{Ni}_{3} \mathrm{C}$ for example containing three points for the $\mathrm{C}_{2}$ layers in $C$ positions is increased to nine points in $\mathrm{Fe}_{2} \mathrm{P}$ with three $\mathrm{A}, \mathrm{B}$ and $\mathrm{C}$ positions in each case at the same projection height. The composition $\mathrm{Fe}_{3} \mathrm{P}_{2} \square_{4}$ at $\mathrm{z}=0$ and $\mathrm{Fe}_{3} \mathrm{P}_{5}$ at $\mathrm{z}=1$ add to a total of $\mathrm{Fe}_{6} \mathrm{P}_{3} \square_{9}$ or $\mathrm{Fe}_{2} \mathrm{P}_{3}$. The composition of the two layers is varied in $\mathrm{Zr}_{2} \mathrm{Fe}_{12} \mathrm{P}_{7} \square_{21}$ (Ganglberger, 1968) with 21 positions in each layer of the unit cell by insertion of $\mathrm{Zr}$ atoms ( $\mathrm{O}$ in Fig.42). The neighboring positions of $\mathrm{Zr}$ atoms are not occupied because of the increased size and repulsive interactions. Other compounds of this family contain clusters of three $\left(\mathrm{Zr}_{6} \mathrm{Ni}_{20} \mathrm{P}_{13} \square_{39}\right)$ or six atoms ((La, Ce) $\left.{ }_{12} \mathrm{Rh}_{30} \mathrm{P}_{21} \square_{63}\right)$ or mixtures of single atoms and clusters in different configurations (Madar et al., 1987; Pivan et al., 1987; Parthé et al., 1993). An even number of A, B

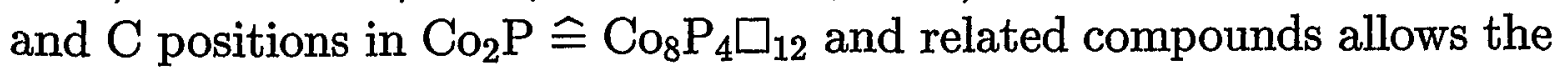
occupation with two $\mathrm{P}$ atoms in each layer.

Different areas of the two layers are occupied in $\mathrm{Ta}_{3} \mathrm{~B}_{4}$ (Kiessling, 1949) and related compounds (Table 13). The 14 positions of the projected structure (Fig.42) are occupied by three Ta atoms on A positions and four Bor atoms on $\mathrm{A}$ and $\mathrm{C}$ positions at $\mathrm{z}=0$ or three $\mathrm{Ta}$ atoms on $\mathrm{C}$ positions and four Bor atoms on $\mathrm{B}$ and $\mathrm{C}$ positions at $\mathrm{z}=1$. The occupation of the different $A, B$ and $C$ positions varies also within the layer because of 14 points instead of 12 points. The Ta atoms of the $z$ $=0$ layer for example occupy $\mathrm{B}$ positions instead of $\mathrm{A}$ in the next area on the right-hand side of the unit cell (Fig.42).

Symmetrical structures at the corners of the structure maps with a single set of $T_{i}$ values for A (or A and B atoms) are usually more pleasant com- 
pared to structures at the borders with reduced symmetry. Ostwald cites J.W. Goethe: Beauty is a matter of regularity. The blocked structures of the Ganglberger family and some complex structures which are related to the patterns of homogeneous sphere packing (Section 25) (Koch and Fischer, 1992) are attractive. (The identical length of solid and dashed lines for homogeneous sphere packing is impossible in the hexagonal or square net. The B positions of these structures with different sets of $T_{i}$ values are encircled.)

The patterns of the hexagonal and square net are related, if the rhombs, which are obtained from two neighboring triangles of the hexagonal net, are compared with the squares. The rhombs with $\alpha=120^{\circ}$ can be distorted to the squares with $\alpha=90^{\circ}$ in a continuous way with similar stuctural units. An increased number of structural units is obtained for orthorhombic (rectangular) or monoclinic (oblique) distortions (general parallelogram). In that case the structural units can be assembled only in one direction. The patterns at the border of the structure maps can be characterized by sequences of structural units (Section 21).

All kinds of patterns with reduced number of symmetry elements are usually less attractive. This can be demonstrated in an educational program for teaching symmetry in arts and nature. A typical example would be the creation of a unit cell containing eight positions $\mathrm{x}+\mathrm{y}$ on a squared paper or a graphic computer program. The choice of four A positions for $r=1$ structures, the extension to neighboring unit cells and the connection of nearest (and next-nearest) A positions by solid (and dashed) lines yield many different patterns. The individual result might indicate the creativity of the artist. Some will put all A positions in one half of the unit cell like $322 ; 1$ or patterns with low symmetry like $1.522 ; 1$ or $122.5 ; 1$. Others will occupy alternating lines (20 $0 ; 1$ ) or create symmetrical patterns with a single set of $T_{i}$ values for all positions like $044 ; 1$ and $220 ; 1$. They have to think about the proper distribution, but avoid the averaging of different $T_{i}$ values in other structures. Those who have analyzed some solutions might be able to search for a specific pattern like $032 ; 1.5$ of the square net or $030 ; 2$ of the honeycomb net, if they are supported by the structure maps with the structural units. (The solutions are shown in Figs. 8,34.) The next 
more sophisticated step could be the analysis of patterns containing figures like stars or leaves instead of points (mandalas) or the analysis of the three-dimensional case in architecture. Also the discussion of practical aspects like shorter walking distances or smaller densities for the arrangement of houses in cities or trees in forests like the $\operatorname{Pr}_{7} \square_{2} \mathrm{O}_{12}$ net instead of $044 ; 1$ (square net) for houses or $060 ; 2$ instead of $226 ; 1$ (hexagonal net) for trees might be useful. Solutions with $T_{1}$ or $T_{2}=$ 1 or 2 are interesting for folklore dances with a variation of $T_{1}$ nearest and $T_{2}$ next-nearest partners. The direction of the dancing people (or of adatoms moving on surfaces, etc.) can be shown by arrows instead of lines. Those who prefer jig-saw puzzles might enlarge and photocopy the structural units of Fig.42. Some informations will also be available on the internet (http://www.fz-juelich.de/iff/personen/J.Hauck) for an analysis of a pattern by computer graphics.

\section{Conclusions}

Close-packed alloys are obtained for weak interactions between metal atoms like $\mathrm{Mg}$ or $\mathrm{Cu}$. The bonding between metal atoms is enhanced in $\alpha-\mathrm{Nd}, \alpha-\mathrm{Sm}$ or Tb HP structures with different stackings (ch), $\left(\mathrm{ch}_{2}\right)$ or $\left(\mathrm{hc}_{2}\right.$ ) (Table 1), which are also observed in $\mathrm{NiAs}, \mathrm{CdCl}_{2}$ and $\mathrm{NbS}_{2}$ structures with decreased $\Delta \mathrm{z} c / a$ values (Table 7 ). The distances between hexagonal or tetragonal layers are varied in $\mathrm{Hg}$ or In (Table 1) and to a larger extent in ordered body-centered alloys of the Zalkin, Ramsey family with hexagonal layers or the Schubert family with square layers (Tables 7,8). Interstitial atoms $\mathrm{I}=\mathrm{O}, \mathrm{Cl}, \mathrm{H}$ can be inserted in different octahedral, tetrahedral, prismatic and other sites like $\mathrm{Ni}^{\prime}$ in $\mathrm{Ni}_{2} \mathrm{In}$ related structures (Table 11b). These structures can be characterized by the selfcoordination numbers $T_{i}(\mathrm{I})$ of I atoms with I atoms similar to $T_{i}(\mathrm{M})$ of ordered alloys (Table 11a,b). The I atoms can also be adsorbed on metal surfaces like [001] or [111] surfaces of ccp alloys. The composition of the interstitial alloys and surface structures $M_{n} \square_{\mathrm{x}} \mathrm{I}_{n \mathrm{x}-\mathrm{x}}$ varies with the ratio $\mathrm{I} / \mathrm{M}=\mathrm{x}(n-1) / n$ with $\mathrm{x}=1$ for the $\mathrm{NaCl}$ group, $\mathrm{x}=2$ for the $\mathrm{CaF}_{2}$ related structures and $\mathrm{x}=3$ for the superconducting oxides, which are related to the $\mathrm{CaTiO}_{3}$ structure (PER group). The occupation of hollow 
or bridge positions on [001] ccp metal surfaces corresponds to $\mathrm{x}=1$ or $\mathrm{x}=2$, respectively, the occupation of hollow or bridge positions on [111] surfaces of ccp alloys to $\mathrm{x}=2$ or $\mathrm{x}=3$, respectively. The interstitial compounds and surface structures can be characterized by sequences of coordination numbers CN (Table 12) with different configurations like $4^{\mathrm{t}}$ (tetrahedral), $4^{\mathrm{p}}$ (planar) or $\mathrm{o}, \mathrm{m}, \mathrm{p}(\mathrm{CN}=2$ with ortho-, meta- or para-configurations) (Figs. 34a,b,42). The interactions between I atoms are reduced because of the $M$ atoms at $I$ atom positions. The structures are inside of the corresponding structure maps for $M_{n} \square_{x} I_{n x-x}$ with $M=$ I atoms (Fig.36).

The ordering of interstitial atoms or magnetic moments in the same structure families as close-packed metal atoms shows the coexistence of different views of sphere packing, which were discussed for many centuries (Brunner, 1971) like the statement of Boscovich (1758): "Atoms are centres of interaction whose diameter are negligible or of minor interest as compared to their separations", or the Wiener-Sohncke principle: "points are disposed around each point in the same way as around every other" (Wiener, 1863; Sohncke, 1879). The last statement is correlated with the problem of homogeneous sphere packing (Koch and Fischer, 1992). Most structures at the corners of the structure maps and some additional structures listed in Section 33 have a single set of $T_{i}$ values $\left(M_{i}=1\right)$.

The present compilation of structures is a general crystallographic approach similar to W. Ostwald's two-dimensional patterns and his last chemical textbook "Principles of Chemistry" with the subtitle "A Chemistry without Substances" covering general concepts and relationships (laws of nature) which can be applied on all substances without dependence on their nature (Ostwald, 1907).

The numbers of shells $i$ and the self-coordination numbers $T_{i}$ of $\mathrm{M}$ atoms in these shells were extended to $i=11-18$ (Table 23), to compare the magic numbers $x$ and $y$ of stable isotopes $\mathrm{M}_{\mathrm{x}} \mathrm{N}_{\mathrm{y}}$ with $\mathrm{x}$ protons and $\mathrm{y}$ neutrons or small clusters of atoms with $\mathrm{x}+\mathrm{y} \lesssim 208$ with the $\mathrm{x}$ and $y$ values of closed shells. The enhanced stability of these isotopes and deviations from spherical shape for other isotopes (Zeldes, 1996) are in- 
dications for an ordering process. The relation between ordering and interactions however can be difficult for very small particles which can convert to energy. Heisenberg points out that a qualitative distinction between elementary particles and larger compound particles is impossible, while other scientists suggest a different treatise (Heisenberg, 1971). A proton for example can convert to a neutron and pion, a $\Lambda$-hyperon and kaon or three quarks. Particles with very strong attractive or repulsive interactions can not order in different areas of the structure maps. A strong deviation from balanced interactions will give rise to clusters, which can not be separated for attractive interactions, and to a continuous separation of the particles for repulsive interactions. Different energies give rise to a variation of the distances between elementary particles or compound particles.

The lattice constants of different elements like $\mathrm{C}, \mathrm{Si}, \mathrm{Ge}$ and $\mathrm{Sn}$ are increased because of the increased distances between $2,3,4$ and $5 s$ and $p$ electrons. The $(2 s)$ and $(2 p)^{3}$ electrons of carbon atoms for example are forming a tetrahedral "cluster", where electrons with identical spin are as far apart as possible (Kettle, 1992). The atoms are "blocked structures" (Section 28) like the bcc $4012 ; 1$ structure with an occupation of the $T_{1}$ shell by electrons. Different "clusters" are formed in graphite with an occupation of the $T_{1}=2$ and $T_{2}=3$ shells of the ph lattice by electrons or in lead with $T_{1}=12$ of the fcc lattice. The $T_{1}=4$ configuration of the square net or the $T_{1}=2$ configuration of the chain are preferred by atoms with increased numbers of electrons like Se, Te or I, which are forming polyanions (Parthé et al., 1993).

Large particles are either not ordered because of the lack of mobility (like balls in a drawer) or they form inhomogeneous clusters without other particles of the same kind (like planets with $T_{i}=0$ ). Identical forces on the same kind of particles with a minimum of deviations are required for ordering as is demonstrated by ocean waves or lattice defects.

The structures of ordered alloys and interstitial compounds can be characterized by sequences of structural units or by sequences of layers and the $\mathrm{x}, \mathrm{y}, \mathrm{z}$ translations of neighboring layers. The $T_{i}(\mathrm{M})$ or $T_{i}(\mathrm{I})$ values of $\mathrm{M}$ or I atoms are usually in the same area of the structure maps of 
three-dimensional and two-dimensional structures. Different locations like the $202 ; 2,060 ; 2$ hexagonal layers or $020 ; 2,202 ; 2$ square layers of the ccp $2212 ; 2 \mathrm{a} \mathrm{MoPt}_{2}$ structure indicate a directional bonding of Mo atoms with a distortion of the structure. A stronger distortion is obtained in the $\mathrm{MoSi}_{2}$ structure, which is usually considered as ordered bcc structure. Many ordered bcc alloys $M_{x} N_{y} R_{z}$ can be described by sequences of $\mathrm{x} \mathrm{M}, \mathrm{y} \mathrm{N}$ and $\mathrm{z} R$ layers similar to some ordered hcp or ccp alloys (Tables 7,8). The reduced distances between layers are related to an increased directional bonding in $c$ direction (or decreased bonding in the $a, b$ plane). The consideration of bcc and pc metals ( $\mathrm{Po}$ ) as a chain similar to hcp and ccp suggests an increased directional bonding at an increased percentage of $\mathrm{h}$ layers $\left(\mathrm{c}_{3}(\mathrm{Cu}), \mathrm{hc} \mathrm{c}_{2}(\mathrm{~Tb} \mathrm{HP}), \operatorname{ch}(\alpha-\mathrm{Nd})\right.$, $\left.\mathrm{ch}_{2}(\alpha-\mathrm{Sm}), \mathrm{h}_{2}(\mathrm{Mg})\right)$ and reduced distances between layers $\left(\mathrm{c}_{3}(\mathrm{Cu}, \mathrm{Hg}\right.$, In, Po, W), hc 2 (Tb HP, CdI $)$, ch ( $\alpha-\mathrm{Nd}, \mathrm{NiAs}), \mathrm{ch}_{2}\left(\alpha-\mathrm{Sm}, \mathrm{MoS}_{2}\right)$ ) (Table 7). Next-nearest neighbors of the $c_{3}$ chain are further apart than in the $h_{2}$ chain (Fig.19). The periodic system with the low-temperature modifications of the elements (Fig.43) suggests a periodic sequence of bcc, hcp, ccp crystal structures with some deviations like the diamond $(\mathrm{ZnS})$ structure of $\mathrm{C}, \mathrm{Ge}$ and $\mathrm{Sn}$, or the helical structure of Se and $\mathrm{Te}$ with increased directional bonding instead of the bcc structure (Sutton, 1993). The $\mathrm{C}$ atoms of diamond exhibit the same structure as the $\mathrm{Tl}$ atoms in the ordered bcc alloy $\mathrm{NaTl}$. The $\mathrm{Na}$ positions are vacant in the diamond structure. The structures of As, Se and Po can be obtained in a similar way from distorted $\mathrm{CsCl}$ with vacant $\mathrm{Cs}$ positions. The number of vacancies is increased in an idealized $\alpha$-Mn $\left(\begin{array}{lll}0 & 0 & 1.24 ; 6.45\end{array}\right)$ with the $x, y, z$ parameters of the $58 \mathrm{Mn}$ atoms $1 / 3(w=0.317, u=0.356), 1 / 4$ $\left(v^{\prime}=0.278\right), 1 / 12\left(u^{\prime}=0.089\right)$ and $0(v=0.042)$ instead of the observed values in brackets (Wyckoff, 1982). Usually the directional bonding decreases at increased atomic radii (Buchanan and Park, 1997) like in ccp $\mathrm{Ca}$ and $\mathrm{Sr}$, hcp Tc and Re or ccp Pb (bcc Ba seems to be an exception).

A periodic variation of the crystal structures with a periodic variation of directional bonding in the sequence ccp hcp bcc hcp, ccp ... (Fig.43) is obtained for the elements $\mathrm{Ne} \mathrm{He} \mathrm{Li} \mathrm{Mg,} \mathrm{Ca} \mathrm{Y} \mathrm{Ta} \mathrm{Re,} \mathrm{Pt} \mathrm{Cd} \mathrm{Sn} \mathrm{M} \mathrm{and}$ $\mathrm{Pb} \mathrm{N} \mathrm{Po}$. The sequence of these and neighboring elements is similar to the relative ordering number $\mathcal{M}$ (Table 18). The bcc crystal structure with directional bonding is replaced by other structures like $\alpha-\mathrm{Mn}, \mathrm{Ga}$, 


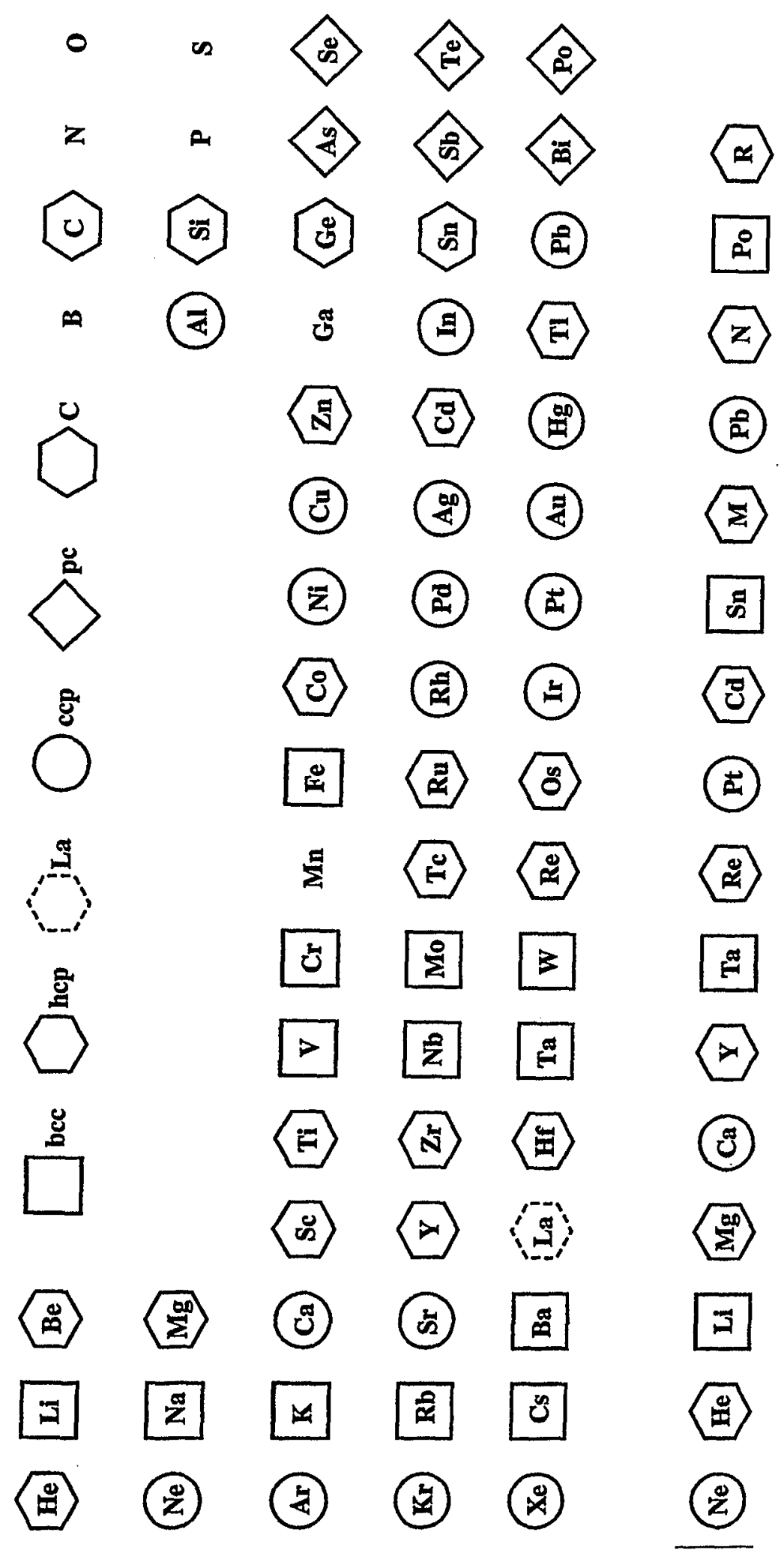

Figure 43: Crystal structures of metal atoms at room temperature and of noble gases at decreased temperatures. Comparison with a periodic variation of ccp hcp bcc hcp sequence of structures in the last row. 
C, As, Se and Po. The hcp elements M, N and R with weak directional bonding are not observed in the right hand area of the periodic system.

Some exceptions of crystal structures, which are not at the border of the structure maps, are also found in ordered alloys. Most of the ordered bcc alloys are at the surface of the three-dimensional $T_{1}, T_{2}, T_{3}$ structure map (Hauck and Mika, 1997). The layered [111] structure like $\mathrm{MNM}_{2} \mathrm{~N}_{2}$, which can be obtained by a combination of the sequences $\mathrm{MN}(\mathrm{CsCl}$ structure) and $\mathrm{M}_{2} \mathrm{~N}_{2}$ (NaTl structure) (Table 7 ), yields the s-CN values $2.9210 .7 ; 1$ instead of $2.91 .7 T_{3} ;(1)$, which can be obtained by adding other structural units in very large unit cells (suprema) (Fig.37a). Other combinations of both structures are impossible because of geometrical constraints. Some structures of the bcc system are at the border of the three-dimensional $T_{1} T_{2} T_{3} ; \mathrm{y} / \mathrm{x}$ structure map, but inside of the $T_{1}, T_{2}$ projection (Fig.37a). The same applies to the hcp ZnS related structure of $\alpha$-LiSiNO with s-CN values $622 ; 1$ (Hauck and Mika, 1998b).

Few structures like $\mathrm{Cd}_{26} \mathrm{Au}_{72}$ (Table 3) or $\mathrm{ZnAu}_{3}$ (Teuho et al., 1987) (Fig.44) can be considered as a combination of structural units $\mathrm{u}, \mathrm{v}$ and the $222 ; 3$ structure for $\mathrm{Cd}_{26} \mathrm{Au}_{72}$ or $\mathrm{u}-\mathrm{y}$ (Fig.12) for $\mathrm{ZnAu}_{3}$ with some other (dashed) additional units. The $T_{1}, T_{2}$ values of these structures are close to the border of the structure maps. Other structures can be considered as a combination of structural units from different systems like $\mathrm{CaF}_{2}$ and $\mathrm{W}$ (Parthé et al., 1993). Quasicrystals are probably nonperiodic combinations of such units. If the size and bonding of the components is too different, then the close-packed alloys become unstable and favor structures without close-packing.

Binary compounds $\mathrm{AB}, \mathrm{AB}_{2}$ and $\mathrm{AB}_{3}$ from the different groups of the present investigation are plotted in structure maps with the relative ordering numbers $\mathcal{M}_{\mathrm{A}}$ and $\mathcal{M}_{\mathrm{B}}$ as parameters (Pettifor, 1994) (Table 18, Fig.45). Most of the structures are in different areas of the field with $\mathcal{M}_{\mathrm{A}}$ $+\mathcal{M}_{\mathrm{B}}>103$ (right of the dashed line in Fig.45). The proper composition of new compounds can be selected from this structure map, the relative ordering numbers, electronegativities, pseudopotential radii sum (Table 18) or coordination numbers for interstitial compounds (Table 19). 

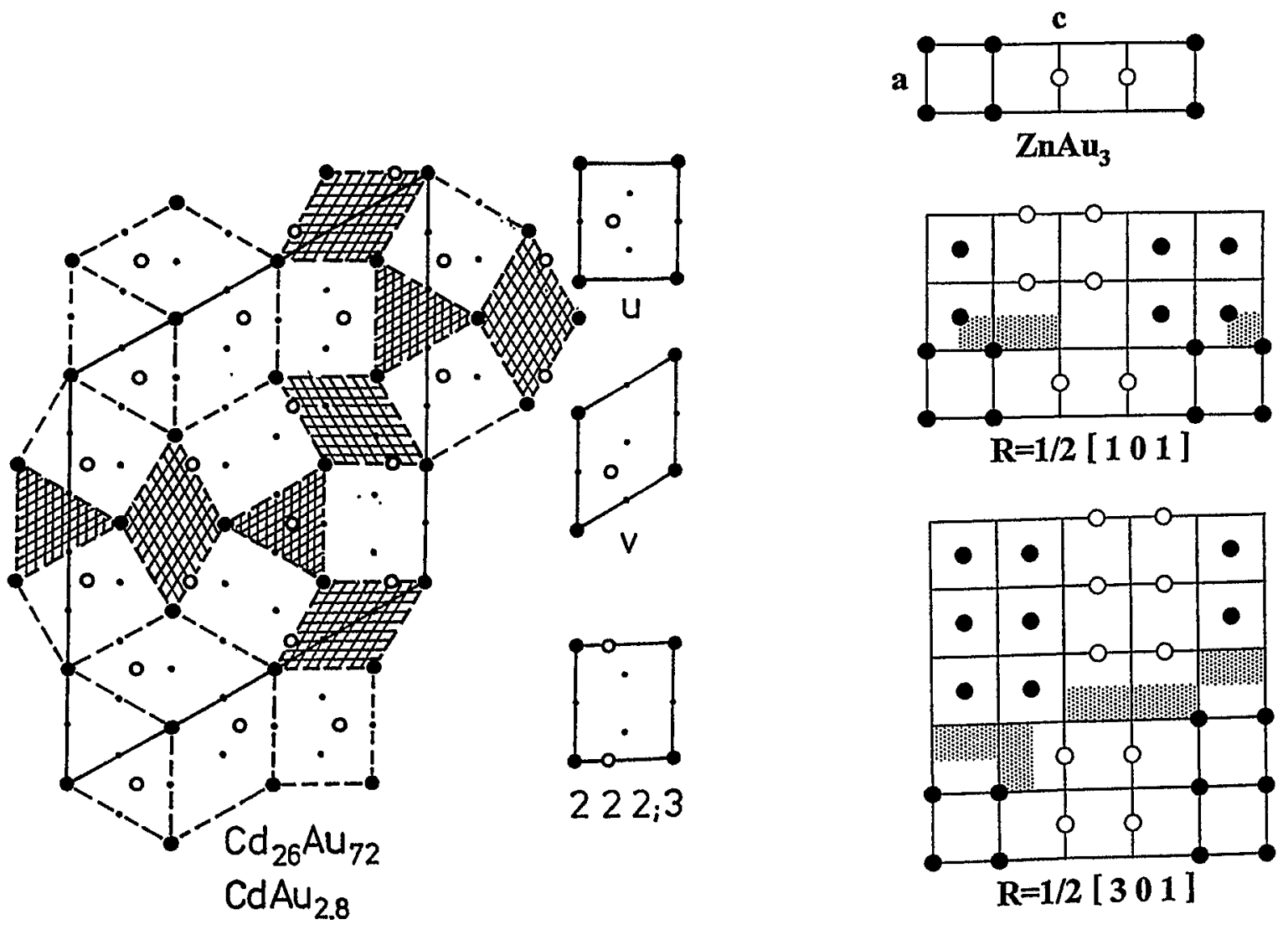

Figure 44: Projection of the $\mathrm{Cd}_{26} \mathrm{Au}_{72}$ structure (Table 3) and different nonstoichiometric $\mathrm{ZnAu}_{3-\mathrm{x}}$ structure variants (Table 4) with non-periodic shear planes (Hyde and Andersson, 1989). Cd or Zn atoms at $0(\bullet)$ or 0.5 (o). The dashed areas are structural units, which are not at the boundary of the structure maps. 
New structures of ordered alloys or interstitial compounds can be analyzed, if the unit cell or reduced unit cell (Table 20) is determined by electron diffraction. The structural units and $T_{1}, T_{2}$ structure maps of the present investigation can help to find the proper structure model for the refinement of weak superstructure reflections of samples, which are twinned by the ordering process. The large number of theoretical structures is reduced to a small number, if the size of the jig-saw puzzle is known. 


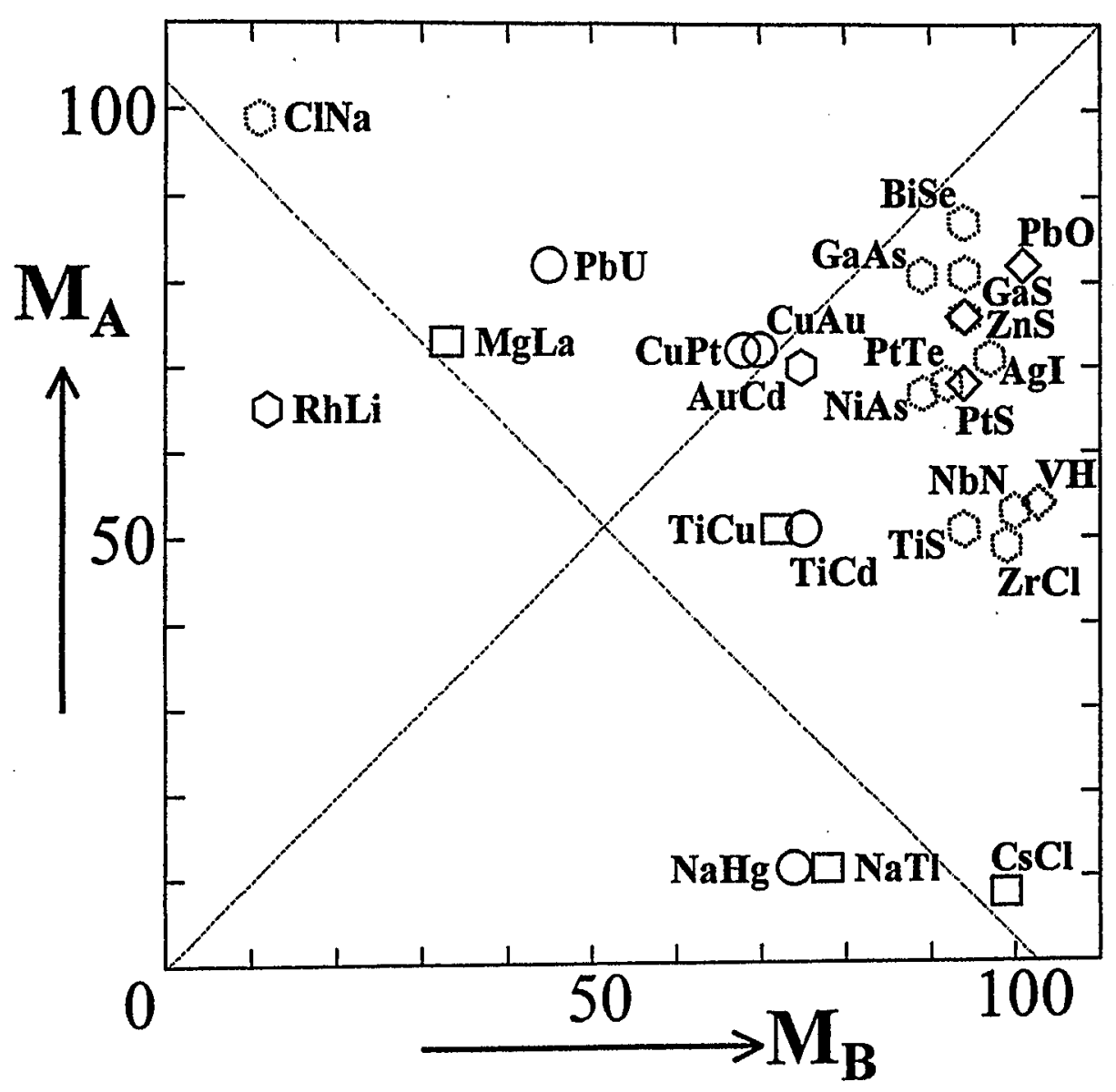

Pettifor structure map for AB compounds

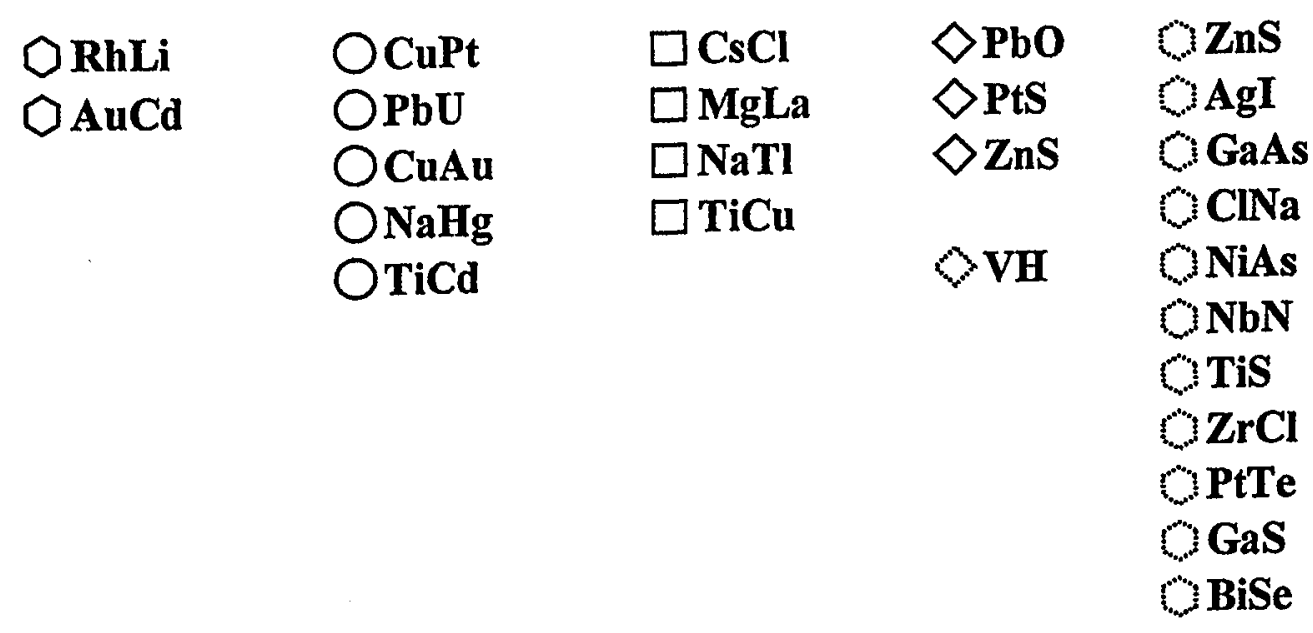

Figure 45: Structure maps for $\mathrm{AB}$ (a), $\mathrm{AB}_{2}$ (b) and $\mathrm{AB}_{3}$ (c) compounds of Table 3 (hexagon), 4 ( $\bigcirc), 5$ (dashed hexagon), 7, 8, 13 (dashed hexagon, $\square$ or $\diamond$ ) with the relative ordering numbers $\mathcal{M}_{\mathrm{A}}$ and $\mathcal{M}_{\mathrm{B}}$ of $\mathrm{A}$ and $\mathrm{B}$ atoms (Table 18) (to be continued on next pages). 


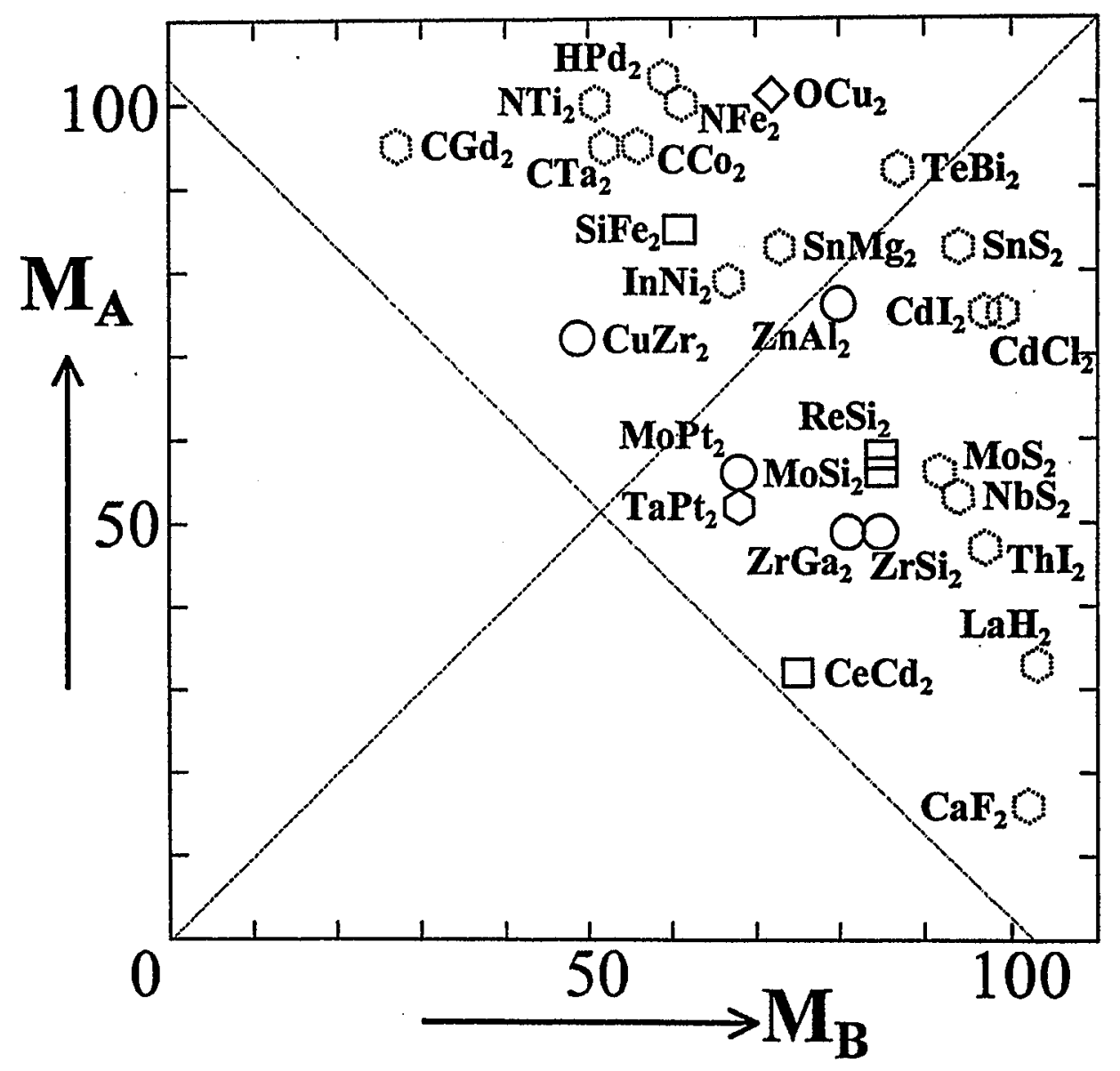

Pettifor structure map for $\mathbf{A B}_{2}$ compounds

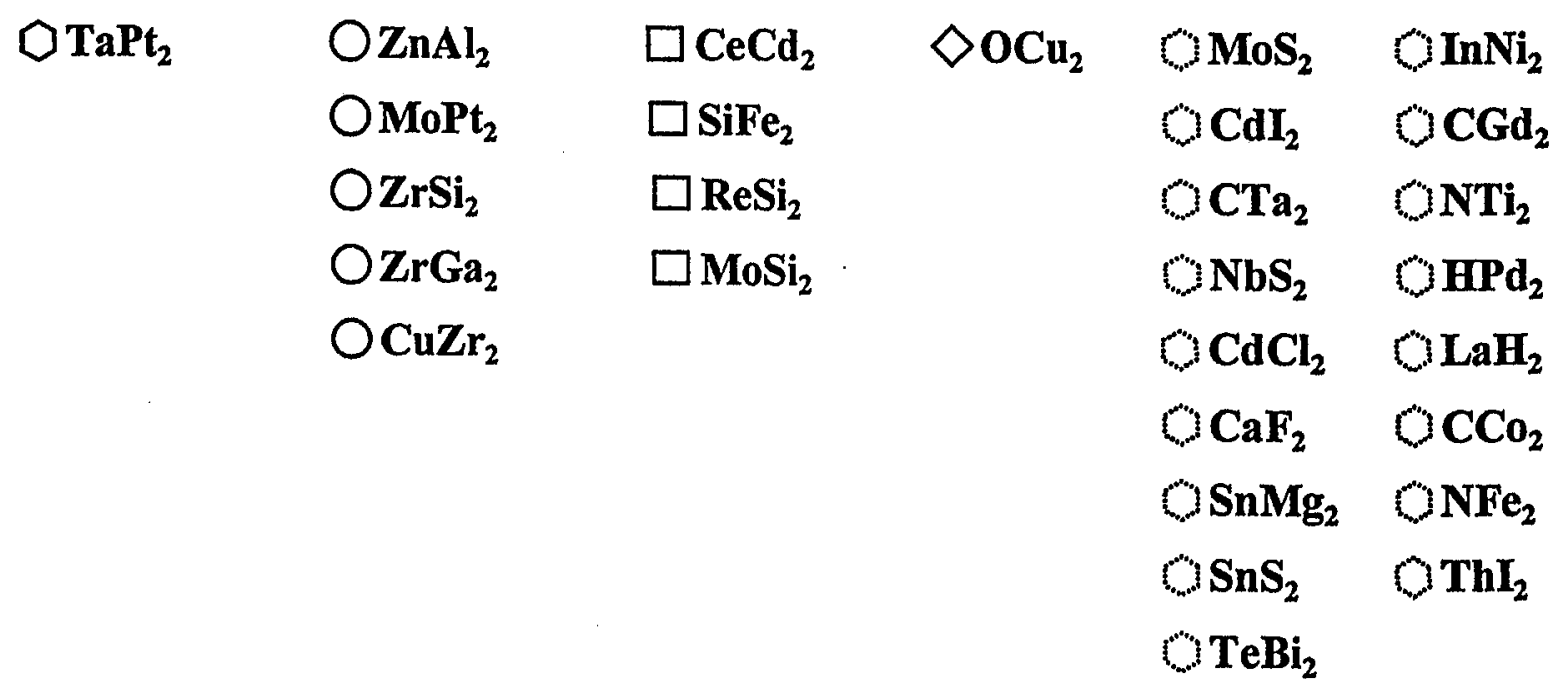




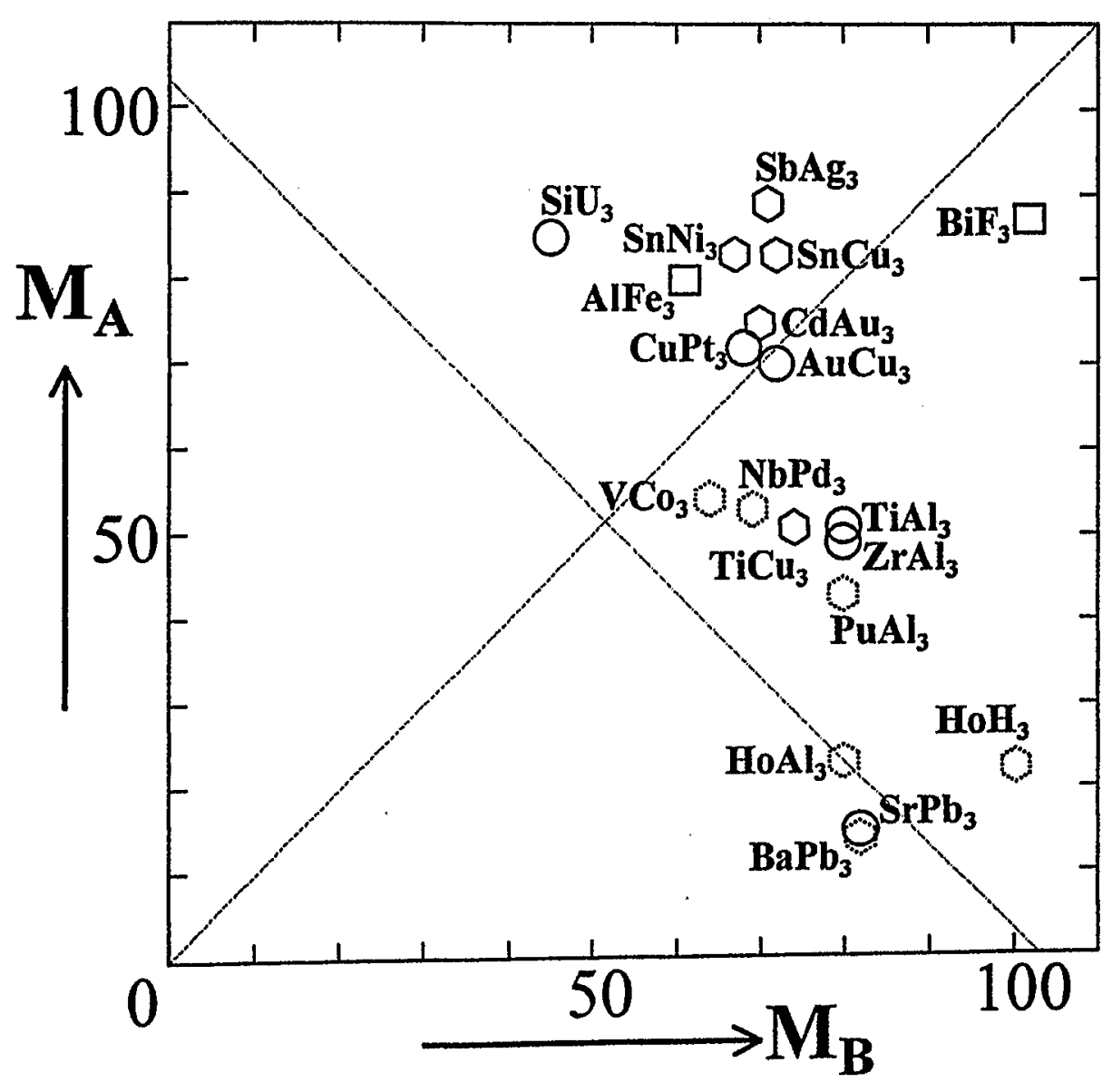

Pettifor structure map for $\mathbf{A B}_{3}$ compounds

\begin{tabular}{|c|c|c|c|}
\hline $\mathrm{OSbAg}$ & $\mathrm{CuPt}_{3}$ & $\square \mathrm{BiF}_{3}$ & $\mathrm{PuAl}_{3}$ \\
\hline $\mathrm{O} \mathrm{TiCu}_{3}$ & $\mathrm{OTiAl}_{3}$ & $\square \mathrm{AlFe}_{3}$ & $\mathrm{VVCo}_{3}$ \\
\hline $\mathrm{OSnCu}$ & $\bigcirc \mathbf{Z r A l}_{3}$ & & $\mathrm{BaPb}_{3}$ \\
\hline $\mathrm{OCdAu}_{3}$ & $\bigcirc \mathrm{AuCu}_{3}$ & & $\mathrm{HoAl}_{3}$ \\
\hline$\bigcirc \mathrm{SnNi}_{3}$ & $\mathrm{OSiU}_{3}$ & & $\mathrm{NbPd}_{3}$ \\
\hline & $\bigcirc \mathrm{SrPb}_{3}$ & & $\mathrm{HoH}_{3}$ \\
\hline
\end{tabular}




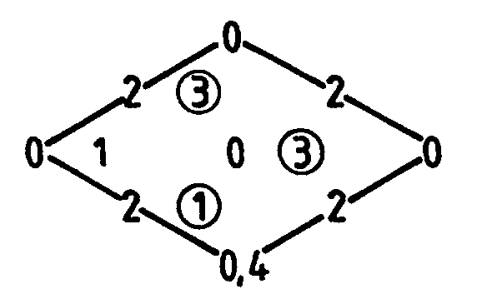

$530 ; 16$

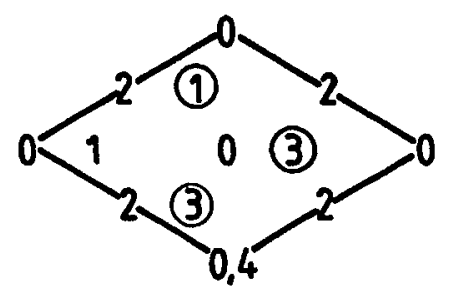

$530 ; 16$

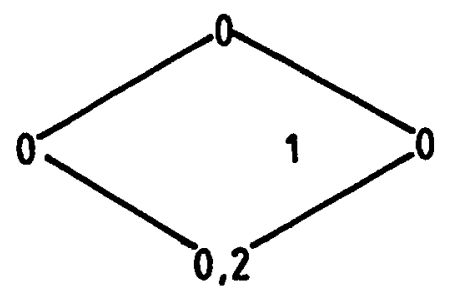

$062 ; 3$

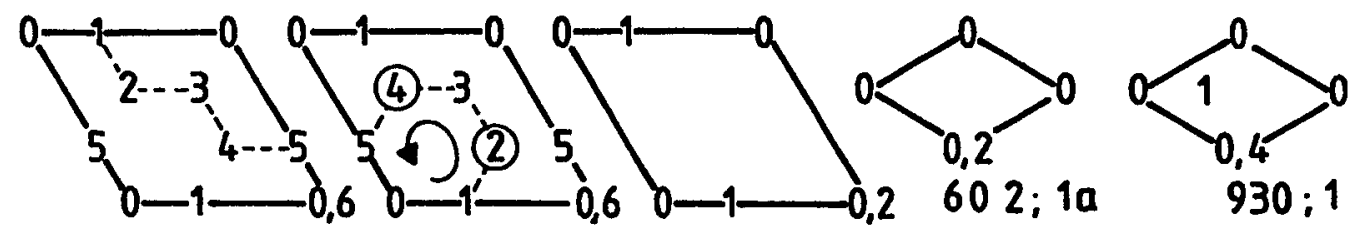

$220 ; 2 a \quad 220 ; 2 b \quad 222 ; 2$

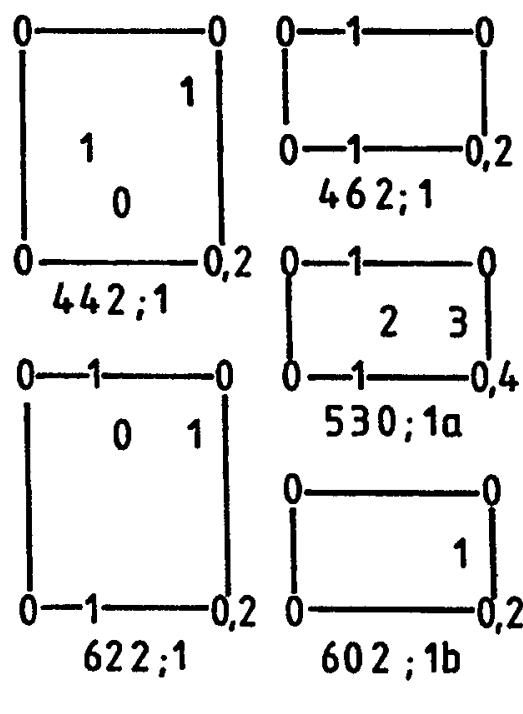

$\left|\begin{array}{rr|r}0 & 3\end{array}\right| \begin{array}{rr}0-(3)-0 \\ 0 & 3\end{array}$
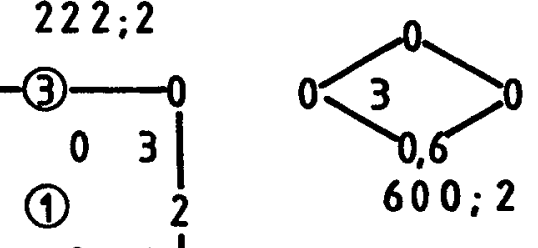

$\left.\left.\left.\right|_{0-1} ^{3} 21\right|_{530 ; 10} ^{0,4} \int_{0-(3)}^{2} 1\right|_{530 ; 10} ^{2}$
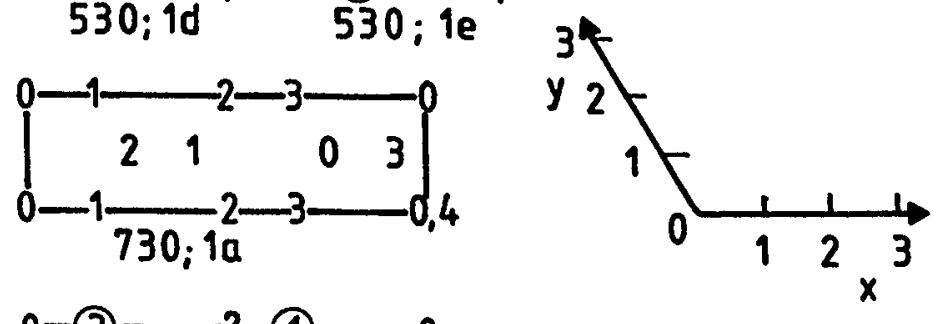

Figure 46: Projections of hcp (a), ccp (b), bcc (c) and pc structures (d) of Table 21a-d (to be continued). 


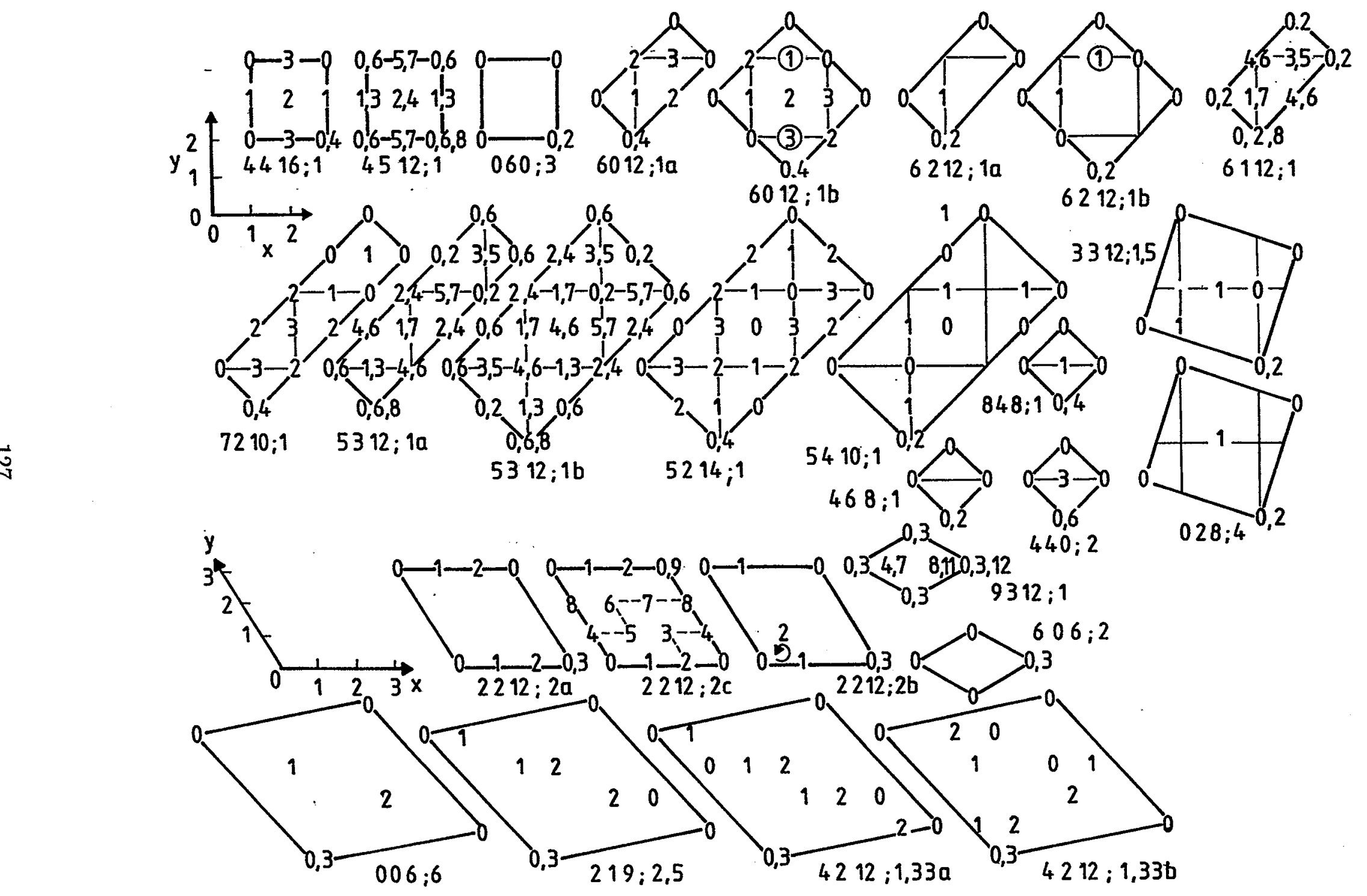

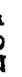




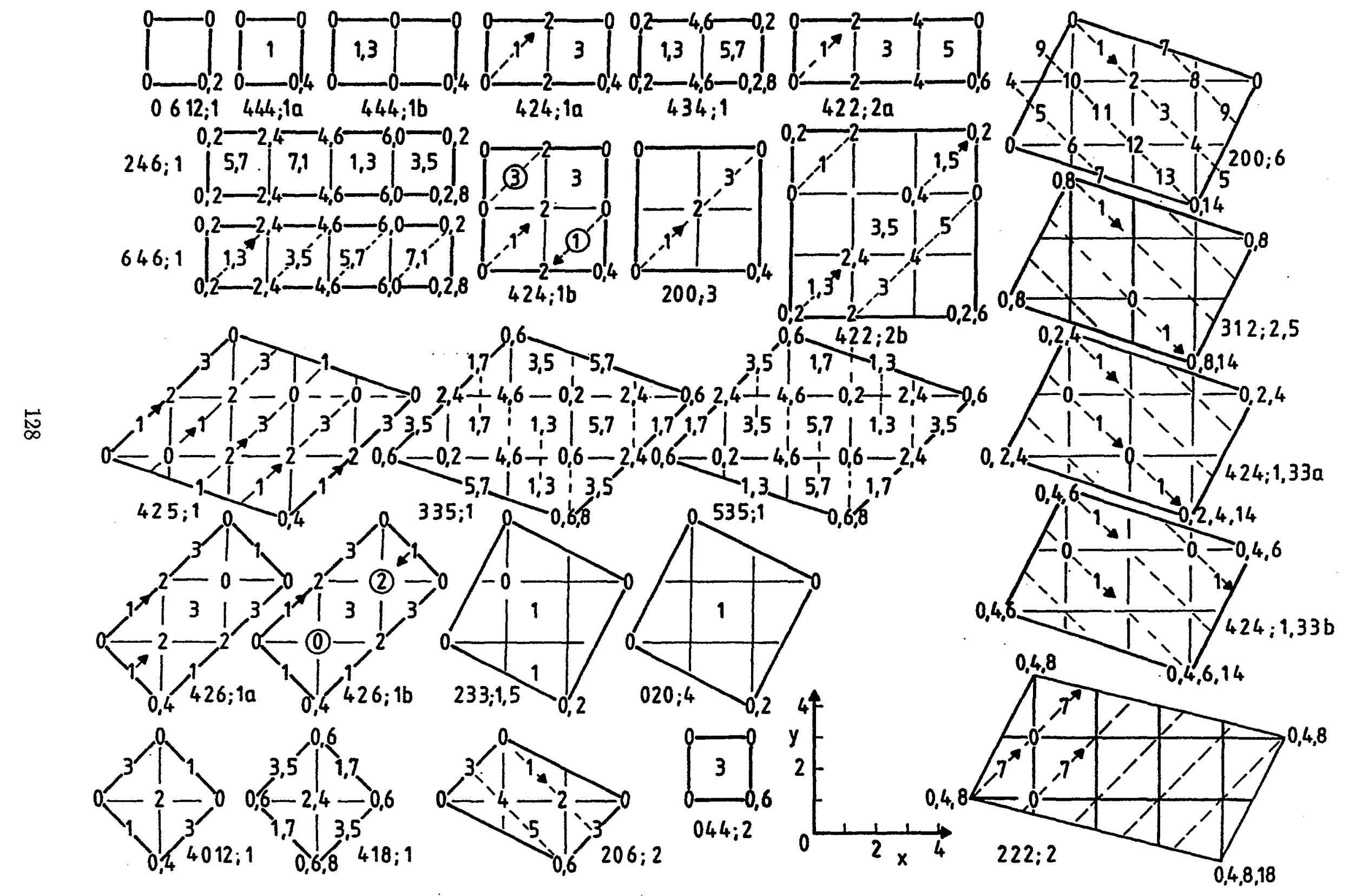




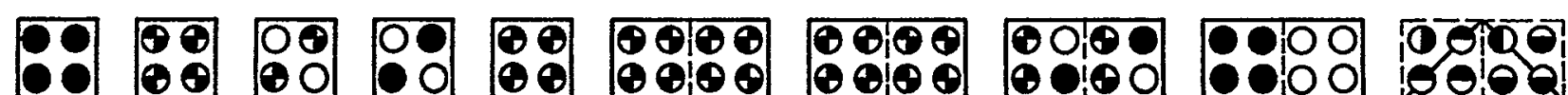
6128.110120.1 008.3 248.1 440.1 184.1 344.10

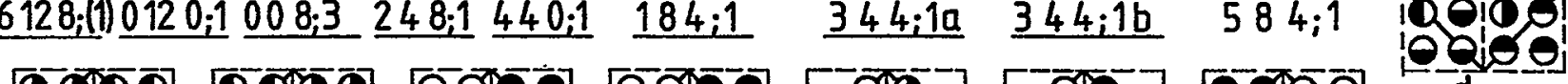

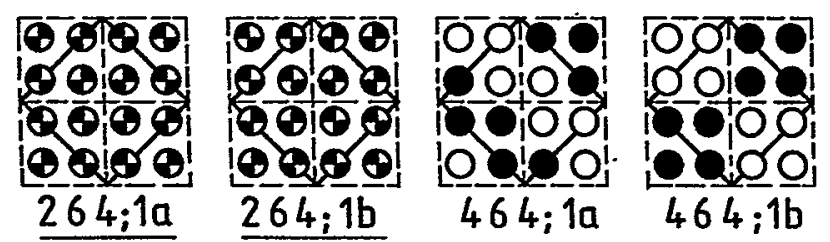

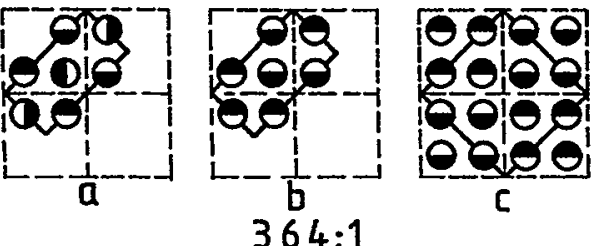

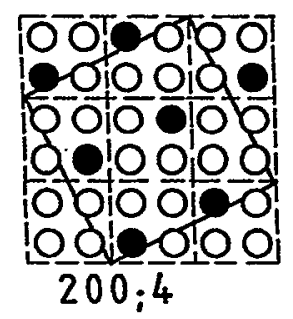
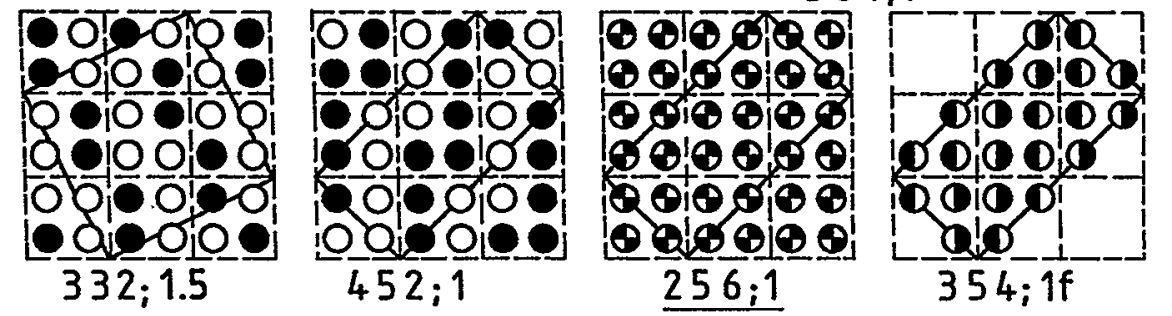

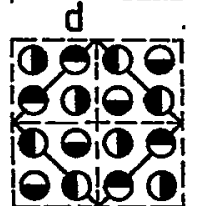
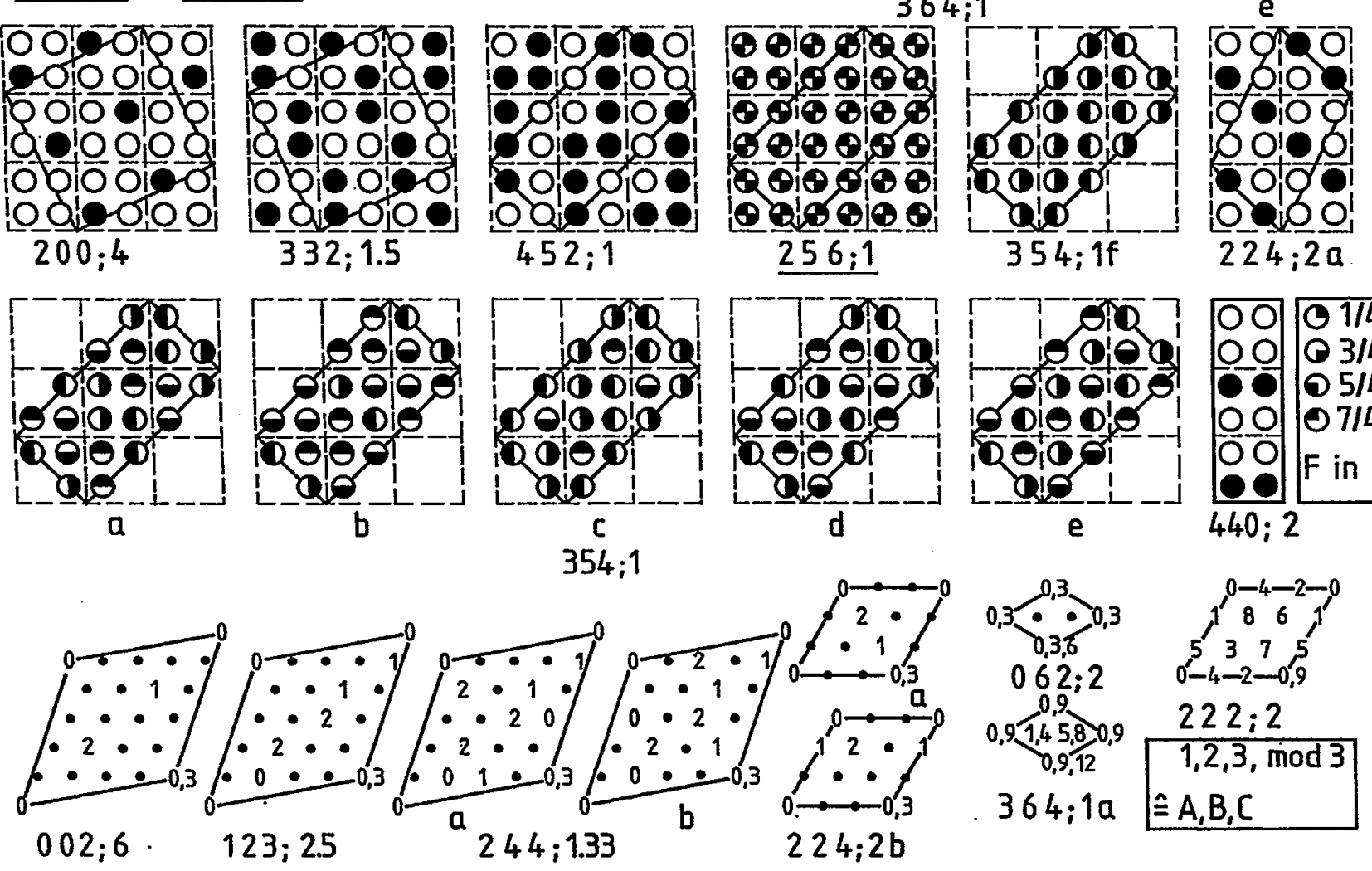


\section{Notation}

$\mathrm{A}, \mathrm{B}, \mathrm{C}$
$\mathrm{K}, \mathrm{L}$
$m$
$n$
$n=2$
$n=3$
$p$
$d$

$a=d, c=n \sqrt{2 / 3} d$

$a_{\mathrm{c}}=\sqrt{2} d$

$\mathrm{f}=\mathrm{h} /(\mathrm{h}+\mathrm{c})$

$\mathrm{M}_{\mathrm{x}} \mathrm{N}_{\mathrm{y}}, \mathrm{r}=\mathrm{y} / \mathrm{x} \geq 1$ $T_{i}=T_{i}(\mathrm{M})$ or $T_{i}(\mathrm{I})$

$k=T_{i}^{\mathrm{A}}$ or $T_{1}^{\mathrm{B}}$

$T_{i}(\mathrm{M}, \mathrm{N})$

$\mathrm{CN}$

$\mathrm{p}_{i}$

$\mathrm{q}_{i}$

$T_{i}^{\max }$

$T_{1} T_{2} T_{3} ; \mathrm{y} / \mathrm{x}$ positions of metal atoms in hexagonal layers positions of metal atoms in square layers number of atoms/formula unit number of hexagonal layers layers in A, B positions of hcp or $h$ hexagonal closepacked structures

layers in A, B, C positions of ccp or c cubic closepacked structures

number of sequences/unit cell

diameter of atoms $=$ distance between the nearest $\mathrm{M}$ atoms

hexagonal lattice constants of structures with $n$ layers

lattice constant of cubic fcc unit cell (Fig.3)

fraction of $\mathrm{h}$ layers in complex close-packed structures

alloy composition with minority component $M$

self-coordination numbers of $M$ atoms with $M$ atoms or I atoms with I atoms for $i=1,2, \ldots$ coordination shell

number of contacts between the spheres of $\mathrm{A}$ or $\mathrm{B}$ atoms

coordination numbers of $\mathrm{M}$ atoms with $\mathrm{N}$ atoms coordination number of $\mathrm{M}$ atoms with other atoms $i=\mathrm{M}$ or $\mathrm{N}$, number of metal atoms $\mathrm{M}$ or $\mathrm{N}$ next to each interstitial atom $\mathrm{I}=\mathrm{O}, \mathrm{Cl}$, etc. in antistructures

charge of $\mathrm{M}$ or $\mathrm{N}$ atoms

maximum self-coordination number in pure metals characterization of crystal structures by the $M$ atom environment, where $T_{i}$ is the number of $i$ th nearest neighbors and $y / x$ the ratio of the components 
$\left(T_{1} T_{2} T_{3} ; \mathrm{y} / \mathrm{x}\right)$ s-CN values, which are not on the boundary of the structure map

$\alpha_{i},-1 \leq \alpha_{i} \leq 1 \quad$ Cowley's short-range order parameter, $\alpha_{i} T_{i}^{\max }=T_{i^{-}}$ $\left(T_{i}^{\max }-T_{i}\right) / \mathrm{r}$, the $\alpha_{1}, \alpha_{2}$ parameters are used for structure maps

$\pm V_{i} \quad$ repulsive $(-)$ or attractive $(+)$ interactions between $\mathrm{M}$

$M^{i}$ atoms, e.g. Coulomb repulsion or covalent bonding number of $\mathrm{M}$ and $\mathrm{N}$ atoms with different environment (= different $T_{i}$ values)

$\mathrm{c}, \mathrm{h}, \mathrm{t}, \mathrm{s}$

$\Delta \mathrm{z} c / a$ notation for the stacking of hexagonal layers (Fig.19)

$\Delta \mathrm{z} c / d$ abc ... distance between hexagonal layers distance between square layers sequence of structural units mirror plane, the asymmetric sequence (Fig.12) of structural units $a b c \mid$ should be completed to the periodic sequence abccba

$\uparrow$ translation $a_{\mathrm{c}} / 2 a_{\mathrm{c}} / 2$ at the interface of structural units to continue a sequence of structural units abc $\uparrow$ in the periodic sequence $a b c a^{\prime} b^{\prime} c^{\prime}$ of shear structures with antiphase boundaries

[abc ] two- or three-dimensional tiling of structural units. 


\section{References}

Allouche, J.-P., and Salon, O. (1990). In Quasicrystals, Networks, and Molecules of Fivefold Symmetry (ed. J. Hargittai). VCH Publishers, New York, p.97.

Beattie, H.J. Jr. (1967). In Intermetallic Compounds (ed. J.H. Westbrook). Wiley, New York, p.144.

Beck, P.A. (1969). In Advances in X-ray Analysis, 12 (eds. C.S. Barrett, J.B. Newkirk and G.R. Mallett). Plenum Press, New York, p.1.

de Boer, F.R., Boom, R., Mattens, W.C.M., Miedema, A.R., and Niessen, A.K. (1988). Cohesion in Metals: Transition Metal Alloys (Cohesion and Structure, Vol. 1) (eds. F.R. de Boer and D.G. Pettifor). North-Holland, Amsterdam.

Boscovich, R.J. (1758). Theoria Philosophiae Naturalis Redacta ad Unicam Legem Virium in Natura Existentium. For a review, see Roger Joseph Boscovich, Studies of his Life and Work (1961) (ed. L.L. White). George Allen \& Unwin Ltd., London, pp.106, 120, 121.

Bowman, R.C. Jr. (1988). In Hydrogen Storage Materials (ed. R.G. Barnes). Mater. Sci. Forum, 31, 197.

Bozorth, R.M. (1922). J. Amer. Chem. Soc., 44, 2232.

Brauer, G. (1939). Z. anorg. allg. Chem., 242, 1.

Brunner, G.O. (1971). Acta Crystallogr., A27, 388.

Buchanan, R.C., and Park, T. (1997). Materials Crystal Chemistry, Marcel Dekker, New York.

Burdett, J.K. (1995). Chemical Bonding in Solids. Oxford University Press, Oxford.

Carlsson, A.E., and Meschter, P.J. (1994). In Intermetallic Compounds, 1 (eds. J.H. Westbrook and R.L. Fleischer). Wiley, Chichester, p.55.

Cenedese, P., and Gaspard, J.P. (1984). Mat. Res. Soc. Symp. Proc., 21, 51.

Dale, J.W. (1996). Molecular Genetics of Bacteria. Wiley, Chichester.

Diekerson, R.E. et al. (1989). J. Mol. Biol., 205, 787.

Ducastelle, F. (1991). In Cohesion and Structure, 3 (eds. F.R. de Boer and D.G. Pettifor). North-Holland, Amsterdam.

Ellner, M., Gödecke, T., and Schubert, K. (1971). J. Less-Common Metals, 24, 23.

Ellner, M., and Predel, B. (1994). In Intermetallic Compounds, 1. (eds. J.H. Westbrook and R.L. Fleischer). Wiley, Chichester, p.91. 
Flanagan, T.B., Craft, A.P., Niki, Y., Baba, K., and Sakamoto, Y. (1992). J. Alloys and Compounds, 184, 69.

Forsyth, J.B., and Gran, G. (1962). Acta Crystallogr., 15, 100.

Galasso, F.S. (1970). Structure and Properties of Inorganic Solids. Pergamon Press, Oxford.

Ganglberger, E. (1968). Monatshefte für Chemie, 99, 557.

Glusker, J.P., Mitchell, L., and Rossi, M. (1994). Crystal Structure Analysis for Chemists and Biologists. VCH, Weinheim.

Goodenough, J.B. (1982). In Handbook on the Physics and Chemistry of the Rare Earths, 5 (eds. K.A. Gschneidner, Jr. and L. Eyring). North-Holland, Amsterdam, p.215.

Hahn, Th. (ed.) (1987). International Tables for Crystallography, Vol.A. D. Reidel, Dordrecht.

Hammou, A., and Guindet, J. (1997). In The CRC Handbook of Solid State Electrochemistry (eds. P.J. Gellings and H.J.M. Bouwmeester). CRC Press, Boca Raton, p.407.

Hauck, J. (1981). J. Less-Common Met., 77, 99.

Hauck, J. (1983). J. Less-Common Met., 94, 123.

Hauck, J. (1985). J. Less-Common Met., 105, 283.

Hauck, J., Henkel, D., and Mika, K. (1988a). Z. Phys. B - Cond. Matt., 71, 187.

Hauck, J., Henkel, D., and Mika, K. (1988b). Z. Kristallogr., 182, 297.

Hauck, J., Henkel, D., and Mika, K. (1989). Int. J. Mod. Phys., B3, 1425.

Hauck, J., and Mika, K. (1993). Int. J. Mod. Phys., B7, 3423.

Hauck, J., and Mika, K. (1994). In Intermetallic Compounds, 1 (eds. J.H. Westbrook and R.L. Fleischer). Wiley, Chichester, p.277.

Hauck, J., Bickmann, K., Bischof, B., Janning, E., and Mika, K. (1995). Materials Letters, 22, 221.

Hauck, J., and Mika, K. (1997). In Studies of High Temperature Superconductors, 25 (ed. A. Narlikar). Nova Science Publishers, Commack NY, p. 1.

Hauck, J., and Mika, K. (1998a). Supercond. Sci. Technol., 11, 614.

Hauck, J., and Mika, K. (1998b). J. Solid State Chem., 138, 334.

Hauck, J., and Mika, K. (1999). Z. Kristallogr., 214, 443. 
Hauck, J., Henkel, D., Klein, M., and Mika, K. (1999). J. Solid State Chem., 145, 150.

Heisenberg, W. (1971). Schritte über Grenzen - Gesammelte Reden und Aufsätze. Piper, München.

Hellner, E., and Schwarz, R. (1994). In Intermetallic Compounds, 1 (eds. J.H. Westbrook and R.L. Fleischer). Wiley, Chichester, p.309.

Hiraga, K., and Hirabayashi, M. (1977). J. Physique, 38, C7: 224.

Ho, S.-M., and Douglas, B.E. (1968). J. Chem. Educ., 45, 474.

Hulliger, F. (1976). In Physics and Chemistry of Materials with Layered Structures (ed. F. Lévy), Vol.5. D. Reidel, Dordrecht.

Hyde, B.G., and Andersson, S. (1989). Inorganic Crystal Structures. Wiley \& sons, New York.

Ising, E. (1925). Z. Phys., 31, 253.

Johansson, C.H., and Linde, J.O. (1936). Ann. d. Physik, 25, 1.

Kaburagi, M. (1978). J. Phys. Soc. Japan, 44, 54.

Kanamori, J., and Kakehashi, Y. (1977). J. Physique, 38, C7: 274.

Ketelaar, J.A.A. (1935). Z. Kristallogr. (A), 90, 237.

Kettle, S.F.A. (1992). Symmetry and Structure. Wiley, Chichester.

Kiessling, R. (1949). Acta Chem. Scand., 3, 603.

Kitaigorodski, A.I. (1973). Molecular Crystals and Molecules. Academic Press, New York.

Koch, E., and Fischer, W. (1992). In International Tables for Crystallography (ed. A.J.C. Wilson), Vol.C. Kluwer Academic Publishers, Dordrecht, p.654.

Kudo, T., and Katsura, S. (1976). Progr. Theor. Phys., 56, 435.

Larsson, A.-K., Stenberg, L., and Lidin, S. (1994). Acta Crystallogr., B50, 636.

Latscha, H.P., and Klein, H.A. (1982). Organische Chemie. Springer, Berlin, p.88.

Laves, F. (1967). In Intermetallic Compounds (ed. J.H. Westbrook). Wiley, New York, p.129.

Lee, S., and Foran, B. (1994). J. Am. Chem. Soc., 116, 154.

Lidin, S., and Larsson, A.-K. (1995). J. Solid State Chem., 118, 313.

Lima-de-Faria, J., and Figueiredo, M.O. (1969). Z. Kristallogr., 130, 41 and 54. 
Lima-de-Faria, J., Hellner, E., Liebau, F., Makovicky, E., and Parthé, E. (1990). Acta Crystallogr., A46, 1.

Madar, R., Ghetta, V., Dhahri, E., Chaudouet, P., and Senateur, J.P. (1987). J. Solid State Chem., 66, 73.

Mardix, S. (1990). Acta Crystallogr., A46, 133.

Mika, K., and Hauck, J. (1990). Acta Crystallogr., 46, Suppl. C-269.

Mika, K., Hauck, J., and Funk-Kath, U. (1994). J. Appl. Crystallogr., 27, 1052.

Moodenbaugh, A.R., Johnston, D.C., Viswanathan, R., Shelton, R.N., Delong, L.E., and Fertig, W.A. (1978). J. Low Temp. Phys., 33, 175.

de Novion, Ch. (1994). In Intermetallic Compounds, 1 (eds. J.H. Westbrook and R.L. Fleischer). Wiley \& sons, Chichester, p.559.

de Novion, Ch., and Landesman, J.P. (1985). Pure \& Appl. Chem., 57, 1391.

Ogawa, S. (1974). In Order-Disorder Transformations in Alloys (ed. H. Warlimont). Springer, Berlin, p.240.

Oleś, A., Kajzar, F., Kucab, M., and Sikora, W. (1976). Magnetic Structures by Neutron Diffraction. Państwowe Wydawnictwo Nakowe, Warszawa.

Ostwald, W. (1922). Die Welt der Formen - Entwicklung und Ordnung der gesetzlich=schönen Gebilde (The World of Patterns - Development and Order of the Legal=beautiful Figures). Unesma, Leipzig.

Ostwald, W. (1907). Principles of Chemistry - A chemistry without Substances. Steinkopff, Leipzig.

Parthé, E., and Yvon, K. (1970). Acta Crystallogr., B26, 153.

Parthé, E., Gelato, L., Chabot, B., Penzo, M., Cenzual, K., and Gladyshevskii, R. (1993). TYPIX: Standardized Data and Crystal Chemical Characterization of Inorganic Structure Types. In Gmelin Handbook of Inorganic and Organometallic Chemistry, 1. Springer, Berlin.

Parthé, E., (1994). In Intermetallic Compounds, 1, (eds. J.H. Westbrook and R.L. Fleischer). Wiley \& sons Ltd. Chichester, p.343.

Patterson, A.L. (1944). Phys. Rev., 65, 195.

Patterson, A.L., and Kasper, J.S. (1959/1967). In International Tables for X-ray Crystallography, II (eds. J.S. Kasper, and K. Lonsdale). The Kynoch Press, Birmingham, p.342.

Pauling, L. (1929). J. Am. Chem. Soc., 51, 1010. 
Pauling, L. (1945/1960). The Nature of the Chemical Bond. Cornell University Press, Ithaca NY.

Pearson, W.B. (1967). In Metal Physics and Physical Metallurgy, 8 (ed. G.V. Raynor). Pergamon Press, Oxford.

Pearson, W.B. (1972). The Crystal Chemistry and Physics of Metals and Alloys. Wiley-Interscience, New York.

Pettifor, D.G. (1994). In Intermetallic Compounds, 1, (eds. J.H. Westbrook and R.L. Fleischer). Wiley \& sons Ltd. Chichester, p.419.

Pivan, J.-Y., Guérin, R., and Sergent, M. (1987). J. Solid State Chem., 68, 11.

Pusey, P.N., and van Megen, W. (1986). Nature, 320, 340.

Pusey, P.N., van Megen, W., Bartlett, P., Ackerson, B.J., Rarity, J.G., and Underwood, S.M. (1989). Phys. Rev. Lett., 63, 2753.

Sanders, J.V. (1980). Phil. Mag., 42, 705.

Sato, H., and Toth, R.S. (1965). In Alloying Behavior and Effects in Concentrated Solid Solutions, (ed. T.B. Massalski), Metallur. Soc. Conf., 29, 295.

Sattler, K. (1987). In Microclusters (eds. S. Sugano, Y. Nishina and S. Ohnishi). Springer, Berlin, p.107.

Sauvage, M., Parthé, E., and Yelon, W.B. (1974). Acta Crystallogr. A30 597.

Schlapbach, L., Meli, F., and Züttel, A. (1994). In Intermetallic Compounds, 2, (eds. J.H. Westbrook and R.L. Fleischer). Wiley \& sons Ltd. Chichester, p.475.

Schoonman, (1997). In The CRC Handbook of Solid State Electrochemistry (eds. P.J. Gellings and H.J.M. Bouwmeester). CRC Press, Boca Raton, p.161.

Schubert, K. (1964). Kristallstrukturen zweikomponentiger Phasen (Crystal Structures of Binary Phases). Springer, Berlin.

Schubert, K. (1967). In Intermetallic Compounds (ed. J.H. Westbrook). Wiley, New York, p.100.

Schubert, K., Anantharaman, T.R., Ata, H.O.K., Meissner, H.G., Pötzschke, M., Rossteutscher, W., and Stolz, E. (1960). Die Naturw., 47, 512.

Schweika, W. (1985). Jül. Report 2004, Forschungszentrum Jülich.

Shannon, R.D. (1976). Acta Crystallogr., A32, 751.

Sohncke, L. (1879). Entwickelung einer Theorie der Kristallstruktur. B.G. Teubner, Leipzig, p23.

Steurer, W. (1990). Z. Kristallogr., 190, 179. 
Stolz, E., and Schubert, K. (1962). Z. Metallkunde, 53, 433.

Sugano, S., and Koizumi, H. (1998). Microcluster Physics. Springer, Berlin.

Sutton, A.P. (1993). Electronic Structure of Materials. Clarendon Pr., Oxford.

Teuho, J., Mäki, J., and Hiraga, K. (1987). Acta Metall., 35, 721.

Thornber, M.R., and Bevan, D.J.M. (1970). J. Solid State Chem.; 1, 536.

Toth, L.E. (1971). Transition Metal Carbides and Nitrides. Academic Press, New York.

Turchi, P.E.A. (1994). In Intermetallic Compounds, 1 , (eds. J.H. Westbrook and R.L. Fleischer). Wiley \& sons Ltd., Chichester, p.21.

Urban, J. (1998). Cryst. Res. Technol., 33, 1009.

Villars, P. (1994). In Intermetallic Compounds, 1, (eds. J.H. Westbrook and R.L. Fleischer). Wiley \& sons Ltd., Chichester, p.227.

Villars, P., and Calvert, L.D. (1986). Pearson's Handbook of Crystallographic Data for Intermetallic Phases, Volumes 1-3. Am. Soc. for Metals, Metals Park OH.

Villars, P., and Hulliger, F. (1987). J. Less-Common Met., 132, 289.

Villars, P., Mathis K., and Hulliger, F. (1989). In Cohesion and Structure. The Structures of Binary Compounds, 2 (eds. F.R. de Boer and D.G. Pettifor). North-Holland, Amsterdam, p.1.

Waag, A., Litz, Th., Fischer, F., Lugauer, H.-J., Baron, T., Schüll, K., Zehnder, U., Gerhard, T., Lunz, U., Keim, M., Reuscher, G., and Landwehr, G. (1998). J. Crystal Growth, 184/185, 1.

Watson, P.R., Van Hove, M.A., and Hermann, K. (1994). Atlas of Surface Structures: Volume 1. J. Phys. and Chem. Reference Data. Monograph No 5

Wegst, J., and Schubert, K. (1958). Z. Metallkunde, 49, 533.

Wells, A.F. (1984). Structural Inorganic Chemistry. Clarendon Press, Oxford.

Westlake, D.G. (1983). J. Less-Common Met., 91, 1.

Wiener, C. (1863). Die Grundzüge der Weltordnung. I. Atomlehre. Winter, Leipzig, p.82.

Wulff, G. (1901). Z. Krystallogr., 34, 449.

Wyckoff, R.W.G. (1982). Crystal structures Volumes 1-3. Krieger, Malabar FL.

Zalkin, A., and Ramsey, W.J. (1956). J. Phys. Chem., 60, 234.

Zalkin, A., Ramsey, W.J., and Templeton, D.H. (1956). J. Phys. Chem., 60, 1275. 
Zeldes, N. (1996). In Handbook of Nuclear Properties, (eds. D. Poenaru and W. Greiner). Clarendon Press, Oxford, p.13.

Zhao, J.-T., Gelato L., and Parthé, E. (1991). Acta Crystallogr., C47, 479. 
Table 1: Stacking sequences of all $n \leq 9$ hexagonal layers and some experimental structures with $n>9$ with stacking symbols and number $M^{i}$ of metal atoms with different coordination (Fig.1), $T_{i}(\mathrm{~h})$ or $T_{i}$ (c) values of the chain of $\mathrm{hc}_{\mathrm{x}}, \mathrm{ch}_{\mathrm{x}}$, etc. stacking sequences, prototype, space group (here and in the following determined by a program (Mika et al., 1994)) and Pearson symbol (PS).

\begin{tabular}{|c|c|c|c|c|c|c|c|}
\hline $\begin{array}{c}\text { layers } \\
n\end{array}$ & $\begin{array}{c}T_{i}(\mathrm{~h}) \text { or } T_{i}(\mathrm{c}) ; \mathrm{r} \\
\text { chain }\end{array}$ & $\begin{array}{l}\text { stacking } \\
\text { symbols }\end{array}$ & $M^{i}$ & $\begin{array}{c}\text { proto- } \\
\text { type }\end{array}$ & PS & $\begin{array}{l}\text { space } \\
\text { group }\end{array}$ & $\begin{array}{l}\text { popul- } \\
\text { ation }\end{array}$ \\
\hline \multicolumn{8}{|c|}{ segregation or clustering of $h$ and $c$ at left border of the map } \\
\hline $\begin{array}{l}5 \\
7 \mathrm{a} \\
8 \mathrm{e} \\
9 \mathrm{~b}\end{array}$ & $\begin{aligned} 222 ;(1) \\
222 ;(1) \\
\\
\\
100 ; 1.5 \\
1.30 .70 ; 1.3 \\
100 ; 3 \\
1.30 .70 ; 2\end{aligned}$ & $\begin{array}{l}\mathrm{h}_{2} \mathrm{c}_{3} \\
\mathrm{c}_{3} \mathrm{~h}_{4} \\
\mathrm{~h}_{2} \mathrm{c}_{6} \\
\mathrm{c}_{3} \mathrm{~h}_{6}\end{array}$ & $\begin{array}{l}2 \\
3 \\
3 \\
3\end{array}$ & $\begin{array}{l}\mathrm{Mg} \\
\mathrm{Cu} \\
\mathrm{Hg} \\
\mathrm{In}\end{array}$ & $\begin{array}{l}h P 2 \\
c F 4 \\
h R 1 \\
t I 2\end{array}$ & $\begin{array}{l}P 6_{3} / m m c \\
F m \overline{3} m \\
R \overline{3} m \\
I 4 / m m m \\
P \overline{3} m 1 \\
P \overline{3} m 1 \\
P \overline{3} m 1 \\
P \overline{3} m 1\end{array}$ & $\begin{array}{r}271 \\
369 \\
5 \\
28\end{array}$ \\
\hline \multicolumn{8}{|c|}{ combination of $c_{2} h_{2}$, ch and $c_{x}$ or $h c_{x}$} \\
\hline $\begin{array}{l}4 \\
6 \mathrm{~b} \\
8 \mathrm{a} \\
9 \mathrm{a} \\
12 \\
14 \\
21 \\
6 \mathrm{a} \\
7 \mathrm{~b} \\
7 \mathrm{c} \\
8 \mathrm{~b} \\
8 \mathrm{c} \\
8 \mathrm{f} \\
9 \mathrm{c} \\
10 \\
15 \\
9 \mathrm{f} \\
9 \mathrm{~g} \\
\infty\end{array}$ & 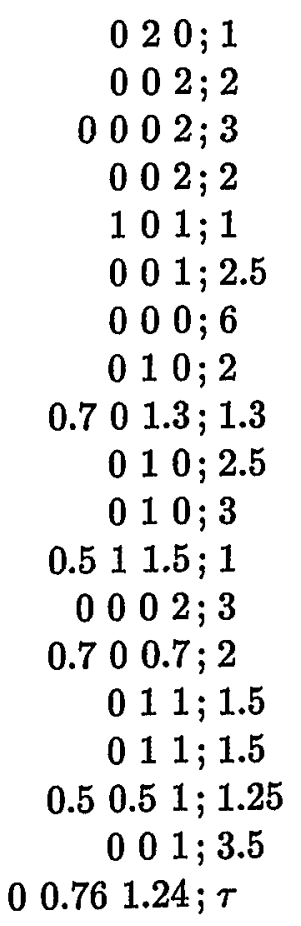 & $\begin{array}{l}\mathrm{ch} \\
\mathrm{hc}_{2} \\
\mathrm{ch}_{3} \\
\mathrm{ch}_{2} \\
\mathrm{~h}_{2} \mathrm{c}_{2} \\
\mathrm{ch}_{2} \mathrm{ch}_{3} \\
\mathrm{ch}_{6} \\
\operatorname{chch}_{3} \\
\mathrm{ch}_{2} \mathrm{c}_{2} \mathrm{~h}_{2} \\
\mathrm{hch}_{4} \\
\mathrm{chch}_{5} \\
\mathrm{ch}_{2} \mathrm{ch}_{2} \mathrm{~h} \\
\mathrm{hc}_{3} \\
\mathrm{ch}_{4} \mathrm{c}_{2} \mathrm{~h}_{2} \\
\mathrm{chch}_{2} \\
\mathrm{hch}_{2} \\
\mathrm{hch}_{2} \mathrm{~h}_{2} \mathrm{c}_{2} \\
\mathrm{hc}_{2} \mathrm{~h}_{5} \\
\text { Fibonacci }\end{array}$ & $\begin{array}{l}2 \\
2 \\
2 \\
2 \\
2 \\
2 \\
2 \\
3 \\
3 \\
3 \\
3 \\
3 \\
3 \\
3 \\
3 \\
3 \\
4 \\
4 \\
\infty\end{array}$ & $\begin{array}{c}\alpha-\mathrm{Nd} \\
\mathrm{Tb} \mathrm{HP} \\
\mathrm{M}_{\mathrm{x}} \mathrm{N}_{\mathrm{y}} \\
\alpha-\mathrm{Sm} \\
\mathrm{M}_{\mathrm{x}} \mathrm{N}_{\mathrm{y}} \\
\mathrm{M}_{\mathrm{x}} \mathrm{N}_{\mathrm{y}} \\
\mathrm{M}_{\mathrm{x}} \mathrm{N}_{\mathrm{y}} \\
\mathrm{M}_{\mathrm{x}} \mathrm{N}_{\mathrm{y}} \\
\mathrm{M}_{\mathrm{x}} \mathrm{N}_{\mathrm{y}}\end{array}$ & $\begin{array}{l}h P 4 \\
h P 6 \\
h R 3\end{array}$ & $\begin{array}{l}P 6_{3} / m m c \\
P 6_{3} / m m c \\
P 6_{3} / m m c \\
R \overline{3} m \\
R \overline{3} m \\
P 6_{3} / m m c \\
R \overline{3} m \\
P \overline{6} m 2 \\
P \overline{3} m 1 \\
P \overline{3} m 1 \\
P \overline{6} m 2 \\
P \overline{3} m 1 \\
P 6_{3} / m m c \\
P 3 m 1 \\
P 6_{3} / m m c \\
R \overline{3} m \\
P \overline{3} m 1 \\
P \overline{3} m 1\end{array}$ & $\begin{array}{r}33 \\
1\end{array}$ \\
\hline \multicolumn{8}{|c|}{ combinations inside of the map } \\
\hline $\begin{array}{l}8 d \\
9 d \\
9 e\end{array}$ & $\begin{array}{rl}1 & 0.51 ; 1 \\
1 & 10.5 ; 1.25 \\
0.5 & 10.5 ; 1.25\end{array}$ & $\begin{array}{l}\mathrm{c}_{2} \mathrm{hc}_{2} \mathrm{~h}_{3} \\
\mathrm{c}_{4} \mathrm{hch}_{3} \\
\mathrm{~h}_{2} \mathrm{c}_{3} \mathrm{hchc}\end{array}$ & $\begin{array}{l}4 \\
4 \\
5\end{array}$ & & & $\begin{array}{l}P \overline{6} m 2 \\
P 3 m 1 \\
P 3 m 1\end{array}$ & \\
\hline
\end{tabular}


Table 2: Self-coordination numbers $T_{i}=T_{i}^{\max }$ of pure metals in hexagonal and square plane, hcp, ccp, bcc, pc and $R_{i}^{2} / d^{2}$ values, $R_{i}=$ radius of $i$-th shell, $d=$ diameter of spheres.

\begin{tabular}{|c|c|c|c|c|c|c|c|c|c|c|c|c|}
\hline \multirow{2}{*}{$\frac{i}{1}$} & \multicolumn{2}{|c|}{$\begin{array}{cc}T_{i}^{\max } & R_{i}^{2} / d^{2} \\
\text { hex. } & \text { plane }\end{array}$} & \multicolumn{2}{|c|}{$\begin{array}{c}T_{i}^{\max } R_{i}^{2} / d^{2} \\
\text { square }\end{array}$} & \multicolumn{2}{|c|}{$\begin{array}{c}T_{i}^{\max } R_{i}^{2} / d^{2} \\
\text { hcp }\end{array}$} & \multicolumn{2}{|c|}{$\begin{array}{c}T_{i}^{\max } R_{i}^{2} / d^{2} \\
\operatorname{ccp}\end{array}$} & \multicolumn{2}{|c|}{$\begin{array}{c}T_{i}^{\max } R_{i}^{2} / d^{2} \\
\text { bcc }\end{array}$} & \multicolumn{2}{|c|}{$\begin{array}{c}T_{i}^{\max } R_{i}^{2} / d^{2} \\
\mathrm{pc}\end{array}$} \\
\hline & 6 & 1 & 4 & 1 & 12 & 1 & 12 & 1 & 8 & 1 & 6 & 1 \\
\hline 2 & 6 & 3 & 4 & 2 & 6 & 2 & 0 & 2 & 6 & $4 / 3$ & 12 & 2 \\
\hline 3 & 6 & 4 & 4 & 4 & 2 & $8 / 3$ & 24 & 3 & 12 & $8 / 3$ & 8 & 3 \\
\hline 4 & 12 & 7 & 8 & 5 & 18 & 3 & 12 & 4 & 24 & $11 / 3$ & 6 & 4 \\
\hline 5 & 6 & 9 & 4 & 8 & 12 & $11 / 3$ & 24 & 5 & 8 & 4 & 24 & 5 \\
\hline 6 & 6 & 12 & 4 & 9 & 6 & 4 & 8 & 6 & 6 & $16 / 3$ & 24 & 6 \\
\hline 7 & 12 & 13 & 8 & 10 & 12 & 5 & 48 & 7 & 24 & $19 / 3$ & 12 & 8 \\
\hline 8 & 6 & 16 & 8 & 13 & 12 & $17 / 3$ & 6 & 8 & 24 & $20 / 3$ & 30 & 9 \\
\hline 9 & 12 & 19 & 4 & 16 & 6 & 6 & 36 & 9 & 24 & 8 & 24 & 10 \\
\hline 10 & 12 & 21 & 8 & 17 & 6 & $19 / 3$ & 24 & 10 & 32 & 9 & 24 & 11 \\
\hline 11 & 6 & 25 & 4 & 18 & 12 & $20 / 3$ & 24 & 11 & 12 & $32 / 3$ & 8 & 12 \\
\hline 12 & 6 & 27 & 8 & 20 & 24 & 7 & 24 & 12 & 48 & $35 / 3$ & 24 & 13 \\
\hline 13 & 12 & 28 & 12 & 25 & 6 & $22 / 3$ & 72 & 13 & 30 & 12 & 48 & 14 \\
\hline 14 & 12 & 31 & 8 & 26 & 12 & $25 / 3$ & 48 & 15 & 24 & $40 / 3$ & 6 & 16 \\
\hline 15 & 6 & 36 & 8 & 29 & 12 & 9 & 12 & 16 & 24 & $43 / 3$ & 48 & 17 \\
\hline 16 & 12 & 37 & 4 & 32 & 24 & $29 / 3$ & 48 & 17 & 24 & $44 / 3$ & 36 & 18 \\
\hline 17 & 12 & 39 & 8 & 34 & 12 & 10 & 30 & 18 & 8 & 16 & 24 & 19 \\
\hline 18 & 12 & 43 & 4 & 36 & 12 & $31 / 3$ & 72 & 19 & 48 & $51 / 3$ & 24 & 20 \\
\hline 19 & 6 & 48 & 8 & 37 & 2 & $32 / 3$ & 24 & 20 & 24 & $52 / 3$ & 48 & 21 \\
\hline 20 & 18 & 49 & 8 & 40 & 12 & 11 & 48 & 21 & 48 & $56 / 3$ & 24 & 22 \\
\hline
\end{tabular}


Table 3: Hexagonal close-packed alloys $\mathrm{M}_{\mathrm{x}} \mathrm{N}_{\mathrm{y}}$ characterized by the self-coordination numbers $T_{i}$ of $\mathrm{M}$ atoms in the hcp structure and in each hexagonal plane, number $M^{i}$ of metal atoms with different sets of hcp $T_{i}$ values, sequences of structural units (Fig.10), space group and Pearson symbol (PS).

\begin{tabular}{|c|c|c|c|c|c|c|}
\hline $\begin{array}{c}T_{i}(\mathrm{M}) ; \mathrm{r} \\
\mathrm{hcp}\end{array}$ & $\begin{array}{c}T_{i}(\mathrm{M}) ; \mathrm{r} \\
\text { hex. plan. }\end{array}$ & $M^{i}$ & $\begin{array}{l}\text { proto- } \\
\text { type }\end{array}$ & PS & $\begin{array}{l}\text { space } \\
\text { group }\end{array}$ & $\begin{array}{c}\text { struct. } \\
\text { units }\end{array}$ \\
\hline \multicolumn{7}{|c|}{ layers of $M$ and $N$ atoms in [001] direction } \\
\hline 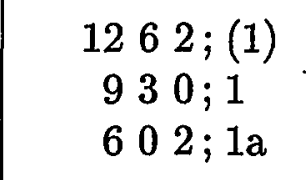 & 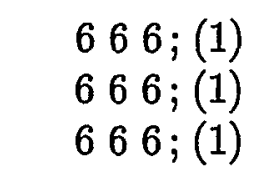 & $\begin{array}{l}1 \\
2 \\
2\end{array}$ & $\begin{array}{l}\mathrm{Mg} \\
\mathrm{LiRh}\end{array}$ & $\begin{array}{l}h P 2 \\
h P 2\end{array}$ & $\begin{array}{l}P 6_{3} / m m c \\
P \overline{3} m 1 \\
P \overline{6} m 2\end{array}$ & $\begin{array}{l}\mathrm{M}_{\infty} \mathrm{N}_{\infty} \\
\mathrm{M}_{2} \mathrm{~N}_{2} \\
\mathrm{MN}\end{array}$ \\
\hline \multicolumn{7}{|c|}{ [120] structural units a - o (Stolz, Schubert, 1962) } \\
\hline $\begin{array}{lll}6 & 0 & 2 ; 1 \mathrm{a} \\
6 & 0 & 2 ; 1 \mathrm{~b} \\
4 & 4 & 2 ; 1 \\
2 & 2 & 2 ; 2 \\
2 & 0 & 2 ; 3 \\
0 & 4 & 2 ; 3 \\
0 & 2 & 2 ; 4 \\
0 & 0 & 0 ; 5\end{array}$ & 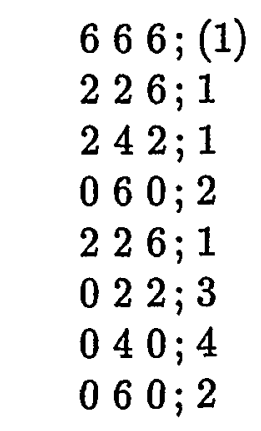 & $\begin{array}{l}2 \\
2 \\
2 \\
2 \\
4 \\
3 \\
5 \\
5 \\
3\end{array}$ & $\begin{array}{l}\mathrm{LiRh} \\
\mathrm{MN} \\
\mathrm{MN} \\
\mathrm{TaPt}_{2} \\
\mathrm{SbAg}_{3} \\
\mathrm{TiCu}_{3} \\
\mathrm{ZrAu}_{4} \\
\mathrm{SnAu}_{5}\end{array}$ & $\begin{array}{l}h P 2 \\
\\
o P 20 \\
o C 12 \\
o P 4 \\
o P 8 \\
o P 20 \\
h R 6\end{array}$ & $\begin{array}{l}P \overline{6} m 2 \\
P m m n \\
P n m a \\
C m c m \\
P m m 2 \\
P m m n \\
P n m a \\
R 32\end{array}$ & $\begin{array}{l}\mathrm{b} \\
\mathrm{aa}^{\prime} \\
\mathrm{aba} \mathrm{a}^{\prime} \mathrm{b} \\
\mathrm{ac}{ }^{\prime} \\
\mathrm{b}^{\prime} \\
\mathrm{cc}^{\prime} \\
\mathrm{ecec}^{\prime} \\
\mathrm{e}\end{array}$ \\
\hline \multicolumn{7}{|c|}{ [220] structural units $\mathrm{u}-\mathrm{y}$ (Schubert, 1964) } \\
\hline $\begin{array}{r}442 ; 1 \\
462 ; 1 \\
042 ; 3 \\
04.42 ; 3 \\
04.72 ; 3 \\
052 ; 3 \\
062 ; 3\end{array}$ & $\begin{array}{r}242 ; 1 \\
226 ; 1 \\
022 ; 3 \\
01.62 .8 ; 3 \\
01.33 .3 ; 3 \\
014 ; 3 \\
006 ; 3\end{array}$ & $\begin{array}{l}2 \\
2 \\
3 \\
7 \\
7 \\
5 \\
2\end{array}$ & $\begin{array}{l}\mathrm{MN} \\
\mathrm{AuCd} \\
\mathrm{TiCu}_{3} \\
\mathrm{SnCu}_{3} \\
(\mathrm{Cd}, \mathrm{In}) \mathrm{Au}_{3} \\
\mathrm{CdAu}_{3} \\
\mathrm{SnNi}_{3}\end{array}$ & $\begin{array}{l}o P 20 \\
o P 4 \\
o P 8 \\
o C 80 \\
o C 48 \\
o C 32 \\
h P 8\end{array}$ & $\begin{array}{l}\text { Pnma } \\
\text { Pmma } \\
P m m n \\
C m c m \\
C m c m \\
C m c m \\
P 6_{3} / m m c\end{array}$ & $\begin{array}{l}\mathrm{xy} \\
\mathrm{y} \\
\mathrm{u} \\
\mathrm{vu}_{4} \\
\mathrm{vu}_{2} \\
\mathrm{vu} \\
\mathrm{v}\end{array}$ \\
\hline \multicolumn{7}{|c|}{ other structures } \\
\hline $\begin{array}{r}(0.93 .72 ; 2.8) \\
042 ; 3.3 \\
(142 ; 3.5)\end{array}$ & $\begin{array}{r}02.32 .8 ; 2.8 \\
022 ; 3.3 \\
013 ; 3.5\end{array}$ & $\begin{array}{l}12 \\
5 \\
6\end{array}$ & $\begin{array}{l}\mathrm{Cd}_{26} \mathrm{Au}_{72} \\
\mathrm{Sb}_{3} \mathrm{Cu}_{10} \\
\mathrm{Cd}_{12} \mathrm{Au}_{42}\end{array}$ & $\begin{array}{l}h P 98 \\
h P 26 \\
h P 54\end{array}$ & $\begin{array}{l}P 6_{3} / m m c \\
P 6_{3} / m \\
P 6_{3} / m c m\end{array}$ & \\
\hline
\end{tabular}


Table 4: Cubic close-packed alloys $M_{x} N_{y}$ characterized by the self-coordination numbers $T_{i}$ of $\mathrm{M}$ atoms in ccp structures and in square planes (only occupied planes perpendicular to the direction with highest symmetry are included), number $M^{i}$ of metal atoms with different sets of $T_{i}$ values, sequences of structural units (Fig.12), space group and Pearson symbol (PS).

\begin{tabular}{|c|c|c|c|c|c|c|}
\hline $\begin{array}{l}T_{i}(\mathrm{M}) ; \mathrm{r} \\
\quad \mathrm{ccp}\end{array}$ & $\begin{array}{c}T_{i}(\mathrm{M}) ; \mathrm{r} \\
\text { square }\end{array}$ & $M^{i}$ & $\begin{array}{l}\text { proto- } \\
\text { type }\end{array}$ & PS & $\begin{array}{l}\text { space } \\
\text { group }\end{array}$ & $\begin{array}{c}\text { struct. } \\
\text { units }\end{array}$ \\
\hline \multicolumn{7}{|c|}{ layers of $\mathrm{M}$ and $\mathrm{N}$ atoms in [111] direction } \\
\hline $\begin{array}{c}12624 ;(1) \\
9312 ; 1 \\
606 ; 2 \\
6012 ; 1 \mathrm{a}\end{array}$ & $444 ;(1)$ & $\begin{array}{l}1 \\
2 \\
2 \\
2\end{array}$ & $\begin{array}{l}\mathrm{Cu} \\
\mathrm{MN} \\
\mathrm{ZnAl}_{2} \\
\mathrm{CuPt}, \mathrm{a}\end{array}$ & $\begin{array}{l}c F 4 \\
h R 4 \\
h P 3 \\
h R 2\end{array}$ & $\begin{array}{l}F m \overline{3} m \\
R \overline{3} m \\
P \overline{3} m 1 \\
R \overline{3} m\end{array}$ & $\begin{array}{l}\mathrm{M}_{\infty} \mathrm{N}_{\infty} \\
\mathrm{M}_{2} \mathrm{~N}_{2} \\
\mathrm{MN}_{2} \\
\mathrm{MN}\end{array}$ \\
\hline \multicolumn{7}{|c|}{ [110] structural units a - d, k, l (Ketelaar, 1935) } \\
\hline $\begin{array}{rl}6 & 012 ; 1 \mathrm{a} \\
6 & 012 ; 1 \mathrm{~b} \\
4 & 416 ; 1 \\
2.73 .3 & 10.7 ; 1.7 \\
3 & 010 ; 2 \mathrm{~b} \\
2 & 04 ; 3 \\
& 048 ; 3\end{array}$ & $\begin{array}{lll}2 & 0 & 4 ; 1 \\
2 & 0 & 4 ; 1 \\
0 & 4 & 4 ; 1 \\
2 & 0 & 4 ; 1 \\
2 & 0 & 4 ; 1 \\
2 & 0 & 4 ; 1 \\
0 & 4 & 4 ; 1\end{array}$ & $\begin{array}{l}2 \\
2 \\
2 \\
5 \\
3 \\
3 \\
3\end{array}$ & $\begin{array}{l}\mathrm{CuPt}, \mathrm{a} \\
\mathrm{CuPt}, \mathrm{b} \\
\mathrm{UPb} \\
(\mathrm{Zn}, \mathrm{Ga})_{3} \mathrm{Au}_{5} \\
\mathrm{Al}_{2} \mathrm{Cl}_{3} \\
\mathrm{CuPt}_{3} \\
\mathrm{TiAl}_{3}\end{array}$ & $\begin{array}{l}h R 2 \\
c F 32 \\
t I 8 \\
o I 32 \\
m C 12 \\
o C 8 \\
t I 8\end{array}$ & $\begin{array}{l}R \overline{3} m \\
F d \overline{3} m \\
I 4_{1} / a m d \\
I b a m \\
C 2 / m \\
C m m m \\
I 4 / m m m\end{array}$ & $\begin{array}{l}\mathrm{a} \\
11^{\prime} \\
\mathrm{aa}^{\prime} \\
\mathrm{bcb}^{\prime} \mathrm{c} \\
\mathrm{ac}^{\prime} \\
\mathrm{c}^{\prime} \\
\mathrm{c}\end{array}$ \\
\hline \multicolumn{7}{|c|}{ [210] structural units e - i (Forsyth, Gran, 1962) } \\
\hline $\begin{array}{c}4416 ; 1 \\
468 ; 1 \\
\\
2212 ; 2 \mathrm{a} \\
1310 ; 2.5 \\
0.73 .39 .3 ; 2.7 \\
048 ; 3 \\
028 ; 4 a\end{array}$ & 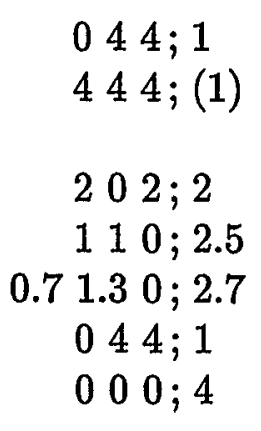 & $\begin{array}{l}2 \\
4 \\
6 \\
3 \\
2\end{array}$ & $\begin{array}{l}\mathrm{UPb} \\
\mathrm{CuAu} \\
\mathrm{NaHg} \\
\mathrm{MoPt} \\
\mathrm{Mn}_{2} \mathrm{Au}_{5} \\
\mathrm{Mo}_{3} \mathrm{Al}_{8} \\
\mathrm{TiAl}_{3} \\
\mathrm{MoNi}_{4}\end{array}$ & $\begin{array}{l}t I 8 \\
t P 4 \\
o C 16 \\
o I 6 \\
m C 14 \\
m C 22 \\
t I 8 \\
t I 10\end{array}$ & $\begin{array}{l}I 4_{1} / a m d \\
P 4 / m m m \\
C m c m \\
I m m m \\
C 2 / m \\
C 2 / m \\
I 4 / m m m \\
I 4 / m\end{array}$ & $\begin{array}{l}\mathrm{g} \\
\mathrm{gh} \\
\mathrm{gh}_{2} \\
\mathrm{~h} \\
\mathrm{i}\end{array}$ \\
\hline
\end{tabular}


Table 4 (continued)

\begin{tabular}{|c|c|c|c|c|c|c|}
\hline $\begin{array}{l}T_{i}(\mathrm{M}) ; \mathrm{r} \\
\quad \mathrm{ccp}\end{array}$ & $\begin{array}{c}T_{i}(\mathrm{M}) ; \mathrm{r} \\
\text { square }\end{array}$ & $M^{i}$ & $\begin{array}{c}\text { proto- } \\
\text { type }\end{array}$ & PS & $\begin{array}{l}\text { space } \\
\text { group }\end{array}$ & $\begin{array}{c}\text { struct. } \\
\text { units }\end{array}$ \\
\hline \multicolumn{7}{|c|}{ [001] structural units u - y (Johansson, Linde, 1936; Brauer, 1939) } \\
\hline \multirow{5}{*}{$\begin{array}{r}4416 ; 1 \\
45.69 .6 ; 1 \\
468 ; 1 \\
248 ; 2 \mathrm{a} \\
248 ; 2 \mathrm{~b}\end{array}$} & $044 ; 1$ & 2 & $\mathrm{UPb}$ & $t I 8$ & $I 4_{1} /$ amd & \multirow{5}{*}{$\begin{array}{l}x y^{\prime} x^{\prime} y \\
y x_{9} y^{\prime} x_{9}^{\prime} \\
x \\
u u^{\prime} y^{\prime} v^{\prime} v y \\
u u^{\prime} x^{\prime} v v^{\prime} y^{\prime} \\
u^{\prime} u x v^{\prime} v y\end{array}$} \\
\hline & $044 ; 1$ & 6 & CuAu II & oI 40 & Imma & \\
\hline & $444 ;(1)$ & 2 & $\mathrm{CuAu}$ & $t P 4$ & $P 4 / m m m$ & \\
\hline & $044 ; 1$ & 3 & $\mathrm{ZrSi}_{2}$ & $o C 12$ & $\mathrm{Cmcm}$ & \\
\hline & $044 ; 1$ & 4 & $(\mathrm{Zr}, \mathrm{Al})(\mathrm{Si}, \mathrm{Al})_{2}$ & $o C 24$ & $C m m m$ & \\
\hline $254 ; 2 \mathrm{a}$ & $044 ; 1$ & 4 & $\mathrm{ZrGa}_{2}$ & oC12 & $C m m m$ & $\mathrm{vyu}_{2} \mathrm{yv}$ \\
\hline $254 ; 2 b$ & $044 ; 1$ & 3 & $\mathrm{HfGa}_{2}$ & $t I 24$ & $I 4_{1} /$ amd & $\begin{array}{l}\mathrm{u}_{2} \mathrm{yv}_{2} \mathrm{x}^{\prime} \\
\mathrm{u}_{0}^{\prime} \mathrm{y}^{\prime} \mathrm{v}_{2}^{\prime} \mathrm{x}\end{array}$ \\
\hline $1.348 ; 2.3$ & $044 ; 1$ & 6 & $\mathrm{Gd}_{3} \mathrm{Sn}_{7}$ & $o C 20$ & $C m m m$ & $\begin{array}{l}u^{\prime} u y v \\
v_{2}^{\prime} \text { vyuu }^{\prime}\end{array}$ \\
\hline \multirow{3}{*}{$\begin{array}{r}1.35 .32 .7 ; 2.3 \\
15.52 ; 2.5 \\
0.848 ; 2.6\end{array}$} & $044 ; 1$ & 6 & $\mathrm{Ce}_{3} \mathrm{Sn}_{7}$ & $o C 20$ & Cmmm & \multirow{7}{*}{$\begin{array}{l}\mathrm{u}_{2} \mathrm{yv}_{4} \mathrm{yu}_{2} \\
\mathrm{v}_{3}^{\prime} \mathrm{y}^{\prime} \mathrm{u}_{6}^{\prime} \mathrm{y}^{\prime} \mathrm{v}_{3}^{\prime} \\
\mathrm{uu}_{2}^{\prime} \mathrm{uyvv}_{2}^{\prime} \mathrm{v}_{2} \\
\mathrm{v}_{2}^{\prime} \mathrm{vyuu}_{2}^{\prime} \mathrm{u} \\
\mathrm{u}_{2} \mathrm{u}_{2}^{\prime} \\
\mathrm{v}_{4} \mathrm{v}_{4}^{\prime} \\
\mathrm{u}\end{array}$} \\
\hline & $044 ; 1$ & 7 & $\mathrm{Ce}_{2} \mathrm{Sn}_{5}$ & oC28 & $C m m m$ & \\
\hline & $044 ; 1$ & 10 & $\mathrm{Nb}_{5} \mathrm{Ga}_{13}$ & oC36 & $C m m m$ & \\
\hline $048 ; 3$ & $044 ; 1$ & 3 & $\mathrm{TiAl}_{3}$ & $t I 8$ & $I 4 / m m m$ & \\
\hline $054 ; 3$ & $044 ; 1$ & 3 & $\begin{array}{l}\mathrm{ZrAl}_{3} \\
\mathrm{CdAu}_{3} \text { II }\end{array}$ & $\begin{array}{l}t I 16 \\
t I 16\end{array}$ & $\begin{array}{l}I 4 / \mathrm{mmm} \\
I 4 m m\end{array}$ & \\
\hline \multirow[t]{2}{*}{$060 ; 3$} & $044 ; 1$ & 2 & $\mathrm{AuCu}_{3}$ & $c P 4$ & $P m \overline{3} m$ & \\
\hline & & & $\begin{array}{l}\mathrm{SiU}_{3} \\
\mathrm{SrPb}_{3}\end{array}$ & $\begin{array}{l}t I 16 \\
t P 4\end{array}$ & $\begin{array}{l}I 4 / \mathrm{mcm} \\
P 4 / \mathrm{mmm}\end{array}$ & \\
\hline \multicolumn{7}{|c|}{ other structures } \\
\hline$(546 ; 1.25)$ & $(121 ; 1.25)$ & 3 & $\mathrm{~V}_{4} \mathrm{Zn}_{5}$ & $t I 18$ & $I 4 / m m m$ & \\
\hline $356 ; 1.5$ & $333 ; 0.25$ & 4 & $\mathrm{Ti}_{2} \mathrm{Ga}_{3}$ & $t P 10$ & $P 4 / m$ & \\
\hline $2.74 .75 .3 ; 1.7$ & $004 ; 3$ & 4 & $\mathrm{Ga}_{3} \mathrm{Pt}_{5}$ & $o C 16$ & $C m m m$ & \\
\hline & $044 ; 1$ & & & & & \\
\hline $000 ; 7$ & $004 ; 3$ & 3 & $\mathrm{GeCa}_{7}$ & $c F 32$ & $F m \overline{3} m$ & \\
\hline $020 ; 8$ & $000 ; 8$ & 3 & $\mathrm{TiPt}_{8}$ & $t I 18$ & $I 4 / \mathrm{mmm}$ & \\
\hline
\end{tabular}


Table 5: Complex close-packed ordered alloys $M_{x} N_{y}$ with the same hexagonal planes as hcp or ccp structures, space group and Pearson symbol (PS).

\begin{tabular}{|c|c|c|c|c|c|}
\hline layers & $\begin{array}{l}\text { stacking } \\
\text { symbol }\end{array}$ & $\begin{array}{c}T_{i}(\mathrm{M}) ; \mathrm{r} \\
\text { hex. plan. }\end{array}$ & prototype & PS & $\begin{array}{l}\text { space } \\
\text { group }\end{array}$ \\
\hline 2 & $\mathrm{hh}$ & $226 ; 1$ & $\mathrm{AuCd}$ & $o P 4$ & Pmma \\
\hline 3 & $\operatorname{ccc}$ & $226 ; 1$ & $\mathrm{CuAu}$ & $t P 2$ & $P 4 / m m m$ \\
\hline $6 \mathrm{~b}$ & $\left(h c_{2}\right)_{2}$ & $226 ; 1$ & IrTa & $o P 12$ & Pmma \\
\hline $9 a$ & $\left(\operatorname{ch}_{2}\right)_{3}$ & $226 ; 1$ & $\operatorname{LiSn}\left(\mathrm{Nb}_{2} \mathrm{Rh}_{3}\right)$ & $m P 6$ & $P 2 / m$ \\
\hline 2 & $\mathrm{hh}$ & $060 ; 2$ & $\mathrm{SnAu}_{5}$ & $h R 6$ & $R 32$ \\
\hline 3 & $\mathrm{ccc}$ & $060 ; 2$ & $008 ; 5$ & & $P 3_{1} 12$ \\
\hline 4 & $(\mathrm{ch})_{2}$ & $060 ; 2$ & $\mathrm{WAI}_{5}$ & $h P 12$ & $P 6_{3} 22$ \\
\hline 2 & $\mathrm{hh}$ & $022 ; 3$ & $\mathrm{TiCu}_{3}$ & $o P 8$ & $P m m n$ \\
\hline 3 & $\operatorname{ccc}$ & $022 ; 3$ & $\mathrm{TiAl}_{3}$ & $t I 8$ & $I 4 / \mathrm{mmm}$ \\
\hline $6 b$ & $\left(\mathrm{hc}_{2}\right)_{2}$ & $022 ; 3$ & $\beta-\mathrm{NbPd}_{3}$ & $o P 24$ & $P m m n$ \\
\hline 12 & $\left(h_{2} c_{2}\right)_{3}$ & $022 ; 3$ & $\beta-\mathrm{NbPt}_{3}$ & $m P 48$ & $P 2_{1} / m$ \\
\hline 2 & $\mathrm{hh}$ & $014 ; 3$ & $\mathrm{CdAu}_{3}$ & $o C 32$ & $C m c m$ \\
\hline 3 & $\operatorname{ccc}$ & $014 ; 3$ & $\mathrm{ZrAl}_{3}$ & $t I 16$ & $I 4 / \mathrm{mmm}$ \\
\hline 4 & $(\mathrm{ch})_{2}$ & $014 ; 3$ & $\mathrm{MgAu}_{3}$ & $o C 64$ & $\mathrm{Cmcm}$ \\
\hline $6 a$ & $\mathrm{chch}_{3}$ & $014 ; 3$ & $\mathrm{Cd}(\mathrm{Au}, \mathrm{In})_{2.8}$ & $o C 96$ & $A m m 2$ \\
\hline $9 a$ & $\left(\operatorname{ch}_{2}\right)_{3}$ & $014 ; 3$ & $\mathrm{Cd}(\mathrm{Au}, \mathrm{In})_{3}$ & $m C 576$ & $C 2 / m$ \\
\hline 10 & $\left(\operatorname{chch}_{2}\right)_{2}$ & $014 ; 3$ & $\mathrm{MgAu}_{3}$ & $o C 160$ & $\mathrm{Cmcm}$ \\
\hline 2 & $\mathrm{hh}$ & $006 ; 3$ & $\mathrm{SnNi}_{3}$ & $h P 8$ & $P 6_{3} / m m c$ \\
\hline 3 & $\operatorname{ccc}$ & $006 ; 3$ & $\mathrm{AuCu}_{3}$ & $c P 4$ & $P m \overline{3} m$ \\
\hline 4 & $(\mathrm{ch})_{2}$ & $006 ; 3$ & $\mathrm{TiNi}_{3}$ & $h P 16$ & $P 6_{3} / m m c$ \\
\hline $6 \mathrm{~b}$ & $\left(h c_{2}\right)_{2}$ & $006 ; 3$ & $\mathrm{PuAl}_{3}, \mathrm{VCo}_{3}$ & $h P 24$ & $P 6_{3} / m m c$ \\
\hline $7 c$ & hchc $_{4}$ & $006 ; 3$ & $\mathrm{Ti}\left(\mathrm{Pt}_{0.89} \mathrm{Ni}_{0.11}\right)_{3}$ & $h P 28$ & $P \overline{3} m 1$ \\
\hline $9 a$ & $\left(\operatorname{ch}_{2}\right)_{3}$ & $006 ; 3$ & $\mathrm{BaPb}_{3}$ & $h R 12$ & $R \overline{3} m$ \\
\hline 10 & $\left(\operatorname{chch}_{2}\right)_{2}$ & $006 ; 3$ & $\gamma-\mathrm{Ta}\left(\mathrm{Pd}_{0.67} \mathrm{Rh}_{0.33}\right)_{3}$ & $h P 40$ & $P 6_{3} / m m c$ \\
\hline 12 & $\left(h_{2} c_{2}\right)_{3}$ & $006 ; 3$ & $\mathrm{PuGa}_{3}$ & $h R 16$ & $R \overline{3} m$ \\
\hline 14 & $\left(\mathrm{ch}_{2} \mathrm{ch}_{3}\right)_{2}$ & $006 ; 3$ & $\mathrm{Ba}\left(\mathrm{Pb}_{0.8} \mathrm{Tl}_{0.2}\right)_{3}$ & $h P 56$ & $P 6_{3} / m m c$ \\
\hline 15 & $\left(\mathrm{hchc}_{2}\right)_{3}$ & $006 ; 3$ & $\mathrm{HoAl}_{3}$ & $h R 20$ & $R \overline{3} m$ \\
\hline
\end{tabular}


Table 6: Self-coordination numbers of some homologous series with different $\mathrm{r}^{*}$ and $\mathrm{k}$ values (see text).

\begin{tabular}{|c|c|c|c|c|c|}
\hline r* & $\mathrm{k}$ & $T_{i}(\mathrm{M}) ; \mathrm{r}$ & type & $T_{i}(\mathrm{M}) ; \mathbf{r}$ & type \\
\hline & & \multicolumn{2}{|c|}{ cсp } & hcp & \\
\hline 2 & 2 & $2212 ; 2 a$ & $\mathrm{MoPt}_{2}, \mathrm{~b}$ & $220 ; 2 a, b$ & \\
\hline 2 & 1 & $7418 ; 1 / 2 \mathrm{a}$ & $\mathrm{Pt}_{2} \mathrm{Mo}, \mathrm{b}$ & $741 ; 1 / 2 a, b$ & \\
\hline 2 & 0 & $12624 ; 0$ & $\mathrm{Cu}$ & $1262 ; 0$ & $\mathrm{Mg}$ \\
\hline 3 & 3 & $060 ; 3$ & $\mathrm{AuCu}_{3}$ & $062 ; 3$ & $\mathrm{SnNi}_{3}$ \\
\hline 3 & 2 & $468 ; 1$ & $\mathrm{CuAu}$ & $462 ; 1$ & $\mathrm{AuCd}$ \\
\hline 3 & 1 & $8616 ; 1 / 3$ & $\mathrm{Cu}_{3} \mathrm{Au}$ & $862 ; 1 / 3$ & $\mathrm{Ni}_{3} \mathrm{Sn}$ \\
\hline 3 & 0 & $12624 ; 0$ & $\mathrm{Cu}$ & $1262 ; 0$ & $\mathrm{Mg}$ \\
\hline 4 & 4 & $028 ; 4$ & $\mathrm{MoNi}_{4}$ & & \\
\hline 4 & 3 & $3312 ; 3 / 2$ & & & \\
\hline 4 & 2 & $6416 ; 2 / 3$ & & & \\
\hline 4 & 1 & $9520 ; 1 / 4$ & $\mathrm{Ni}_{4} \mathrm{Mo}$ & & \\
\hline
\end{tabular}


Table 7: Composition, space group (SG), Pearson symbol (PS), layer and stacking sequences of [111] $M_{x} N_{y} R_{z}$ layered compounds with $h, c, s_{2}, t_{2}$ notation (Fig.19). The distance between layers $\Delta \mathrm{z} c / a$ varies in $\mathrm{N}$ (normal), I (interstitial alloys) and $\mathrm{B}$ form (body-centered cubic alloys).

\begin{tabular}{|c|c|c|c|c|}
\hline $\mathrm{M}_{\mathrm{x}} \mathrm{N}_{\mathrm{y}} \mathrm{R}_{\mathrm{z}}$ & SG & PS & layer sequ. & stacking sequ. \\
\hline \multicolumn{5}{|c|}{$\begin{array}{l}\mathbf{R M}, \mathbf{R M R},(\mathbf{R M})_{2} \mathbf{R} \text { or }(\mathbf{R M})_{3} \mathbf{R} \text { chains } \\
\text { (I form with } 0.2 \leq \Delta \mathrm{z} c / a \leq 0.8)\end{array}$} \\
\hline $\mathrm{ZnS} 3 \mathrm{C}$ & 216 & $c F 8$ & RM & $t_{2}$ \\
\hline $\mathrm{ZnS} 2 \mathrm{H}$ & 186 & $h P 4$ & $\mathrm{RM}$ & $\mathrm{s}_{2}$ \\
\hline $\mathrm{ZnS} 4 \mathrm{H}$ & 186 & $h P 8$ & $\mathrm{RM}$ & $\mathrm{s}_{2} \mathrm{t}_{2}$ \\
\hline $\mathrm{ZnS} 6 \mathrm{H}$ & 186 & $h P 12$ & $\mathrm{RM}$ & $\mathrm{s}_{2} t_{4}$ \\
\hline $\mathrm{ZnS} 12 \mathrm{R}$ & 160 & $h R 24$ & $\mathrm{RM}$ & $\mathrm{s}_{4} \mathrm{t}_{4}$ \\
\hline $\mathrm{NaCl}$ & 225 & $c F 8$ & RM & $c_{2}$ \\
\hline $\mathrm{TiC}$ & 225 & $c F 8$ & $\mathrm{RM}$ & $c_{2}$ \\
\hline NiAs & 194 & $h P 4$ & $\mathrm{RM}$ & hc \\
\hline $\mathrm{NNb} \delta^{\prime}$ & 194 & $h P 4$ & $\mathrm{RM}$ & hc \\
\hline TiS HT & 166 & $h R 18$ & RM & hc hc $c_{2}$ \\
\hline $\mathrm{C}_{2} \mathrm{Mo}_{3}$ & 194 & $h P 12$ & $\mathrm{RM}$ & $h c_{5}$ \\
\hline $\mathrm{TiP}$ & 194 & $h P 8$ & $\mathrm{RM}$ & $\mathrm{hc}_{3}$ \\
\hline $\mathrm{N}(\mathrm{Ta}, \mathrm{Mn})$ & 194 & $h P 8$ & RM & $\mathrm{hc}_{3}$ \\
\hline $\mathrm{MoS}_{2} 2 \mathrm{H}$ & 194 & $h P 6$ & RMR & $h_{3}$ \\
\hline $\mathrm{CdI}_{2} \mathrm{C} 6$ & 164 & $h P 3$ & RMR & $c_{3}$ \\
\hline $\mathrm{CTa}_{2}$ & 164 & $h P 3$ & RMR & $c_{3}$ \\
\hline $\mathrm{NbS}_{2} 2 \mathrm{H} \mathrm{HT}$ & 194 & $h P 6$ & RMR & chc \\
\hline $\mathrm{CdCl}_{2} \mathrm{C} 19$ & 166 & $h R 9$ & RMR & hch \\
\hline $\mathrm{CaF}_{2}$ & 225 & $c F 12$ & RMR & hch \\
\hline $\mathrm{SnMg}_{2}$ & 225 & $c F 12$ & RMR & hch \\
\hline $\mathrm{LaH}_{2}$ & 225 & $c F 12$ & RMR & hch \\
\hline $\mathrm{CdI}_{2} \mathrm{C} 27$ & 186 & $h P 6$ & RMR & $\mathrm{hc}_{2}$ \\
\hline $\mathrm{N}(\mathrm{ScTa})$ & 186 & $h P 6$ & RMR & $\mathrm{hc}_{2}$ \\
\hline $\mathrm{MoS}_{2} 3 \mathrm{R}$ & 160 & $h R 9$ & RMR & $\mathrm{ch}_{2}$ \\
\hline $\mathrm{TaSe}_{2} 4 \mathrm{H}(\mathrm{b})$ & 194 & $h P 12$ & RMR & $c_{3} \operatorname{chc}$ \\
\hline $\operatorname{ThI}_{2} \beta$ & 194 & $h P 12$ & RMR & $\mathrm{h}_{3} \mathrm{hch}$ \\
\hline $\mathrm{NbSe}_{2} 4 \mathrm{~s}(\mathrm{~d} 2)$ & 187 & $h P 12$ & RMR & $\mathrm{c}_{3} \mathrm{~h}_{3} \mathrm{c}_{3} \mathrm{chc}$ \\
\hline $\mathrm{NbSe}_{2} 4 \mathrm{HIT}$ & 187 & $h P 12$ & RMR & $\operatorname{chch}_{2} \operatorname{cchcch}_{2}$ \\
\hline $\mathrm{TaSe}_{2} 4 \mathrm{~s}(\mathrm{c})$ & 186 & $h P 12$ & RMR & $\mathrm{chc} \mathrm{ch}_{2}$ \\
\hline $\mathrm{NbSe}_{2} 4 \mathrm{~s}(\mathrm{~d} 1)$ & 156 & $h P 12$ & RMR & $\mathrm{ch}_{2} \mathrm{c}_{2}$ hchc $\mathrm{c}_{3}$ \\
\hline $\mathrm{TaS}_{2} 6 \mathrm{~s}$ & 160 & $h R 18$ & RMR & $\mathrm{ch}_{2} \mathrm{c}_{3}$ \\
\hline $\mathrm{SnS}_{2} 18 \mathrm{R}$ & 166 & $h R 27$ & RMR & $\mathrm{c}_{3} \mathrm{hc}_{2}$ chc \\
\hline $\mathrm{Bi}_{2} \mathrm{Te}_{3} \mathrm{I}$ & 166 & $h R 15$ & RMRMR & $c_{5}$ \\
\hline $\mathrm{Pt}_{2} \mathrm{Sn}_{3}$ & 194 & $h P 10$ & RMRMR & hchch \\
\hline $\mathrm{In}_{2} \mathrm{~S}_{3} \gamma$ & 164 & $h P 5$ & RMRMR & $h c_{3} h$ \\
\hline $\mathrm{La}_{2} \mathrm{O}_{3}$ & 164 & $h P 5$ & RMRMR & $\mathrm{hc}_{3} \mathrm{~h}$ \\
\hline
\end{tabular}


Table 7 (continued)

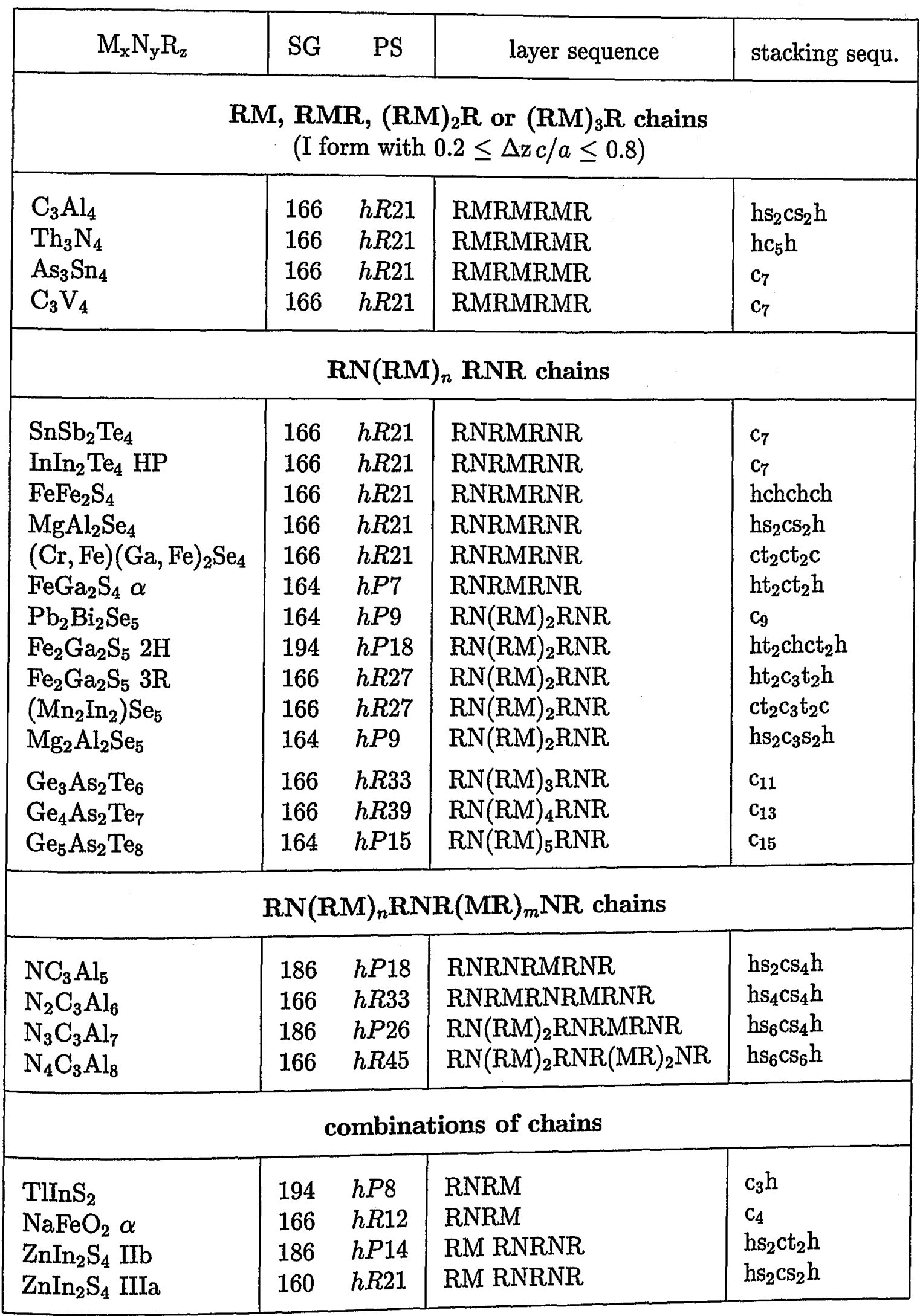


Table 7 (continued)

\begin{tabular}{|c|c|c|c|c|}
\hline $\mathrm{M}_{\mathrm{x}} \mathrm{N}_{\mathrm{y}} \mathrm{R}_{\mathrm{z}}$ & SG & PS & layer sequence & stacking sequ. \\
\hline \multicolumn{5}{|c|}{ combinations of chains } \\
\hline $\mathrm{ZnIn}_{2} \mathrm{~S}_{4} \mathrm{I}$ & 156 & $h P 7$ & RM RNRNR & $\mathrm{ht}_{2} \mathrm{ct}_{2} \mathrm{~h}$ \\
\hline $\mathrm{ZnIn}_{2} \mathrm{~S}_{4} \mathrm{VIa}$ & 160 & $h R 42$ & RM RNRNR & $\mathrm{hs}_{2} \mathrm{Ct}_{2} \mathrm{~h} \mathrm{ht}_{2} \mathrm{cs}_{2} \mathrm{~h}$ \\
\hline $\mathrm{ZnIn}_{2} \mathrm{~S}_{4} \mathrm{IIa}$ & 164 & $h P 14$ & RNRNR MR & $\mathrm{ht}_{2} \mathrm{ct}_{2} \mathrm{~h}$ \\
\hline & & & RM RNRNR & \\
\hline $\mathrm{Zn}_{2} \mathrm{In}_{2} \mathrm{~S}_{5} \mathrm{IIa}(1)$ & 186 & $h P 18$ & RM RNRMRNR & $\mathrm{ht}_{2} \mathrm{ct}_{2} \mathrm{~s}_{2} \mathrm{~h}$ \\
\hline$\left(\mathrm{Zn}_{2} \mathrm{In}_{2}\right) \mathrm{S}_{5} \mathrm{IIa}(2)$ & 186 & $h P 18$ & RM RNRMRNR & $\mathrm{hs}_{2} \mathrm{Cs}_{4} \mathrm{~h}$ \\
\hline $\mathrm{Zn}_{2} \mathrm{In}_{2} \mathrm{~S}_{5}$ IIIa & 160 & $h R 27$ & RM RNRMRNR & $\mathrm{hs}_{2} \mathrm{Ct}_{2} \mathrm{~S}_{2} \mathrm{~h}$ \\
\hline$\left(\mathrm{Zn}_{3} \mathrm{In}_{2}\right) \mathrm{S}_{6} \mathrm{Ia}$ & 164 & $h P 11$ & RM RM RNRMRNR & $\mathrm{hs}_{2} \mathrm{t}_{2} \mathrm{ct}_{2} \mathrm{~s}_{2} \mathrm{~h}$ \\
\hline $\mathrm{Zn}_{3} \operatorname{In}_{2} \mathrm{~S}_{6} \mathrm{Ib}$ & 156 & $h P 11$ & RM RM RNRMRNR & $\mathrm{hs}_{2} \mathrm{t}_{2} \mathrm{ct}_{2} \mathrm{~s}_{2} \mathrm{~h}$ \\
\hline $\mathrm{GeBi}_{4} \mathrm{Te}_{7}$ & & $h P 12$ & RNRMRNR RNRNR & $\mathrm{c}_{12}$ \\
\hline \multicolumn{5}{|c|}{ chains containing vacancies at $\mathrm{M}^{\prime}$ or $\mathrm{N}^{\prime}$ positions } \\
\hline$(\mathrm{Ga}, \operatorname{In})_{3-x} \operatorname{In} S_{5}$ & 186 & $h P 18$ & $\mathrm{RM}^{\prime} \mathrm{RNRM} \mathrm{M}^{\prime} \mathrm{M}^{\prime} \mathrm{R}$ & $\mathrm{hs}_{2} \mathrm{Cs}_{4} \mathrm{~h}$ \\
\hline$(\mathrm{Zn}, \mathrm{In})_{4-\mathrm{x}}(\mathrm{Se}, \mathrm{S})_{5} \mathrm{IIb}$ & 164 & $h P 18$ & $\mathrm{RN}^{\prime} \mathrm{RM}^{\prime} \mathrm{RNRN}$ & $\mathrm{hs}_{2} \mathrm{t}_{2} \mathrm{ct} \mathrm{t}_{2} \mathrm{~h}$ \\
\hline$(\operatorname{In}, \mathrm{Ga})_{4.67} \mathrm{~S}_{7}$ & 164 & $h P 14$ & $\mathrm{RN}^{\prime} \mathrm{RMRN} N^{\prime} \mathrm{R}\left(\mathrm{RM}^{\prime}\right)_{2} \mathrm{R}$ & $\mathrm{ht}_{2} \mathrm{ct}_{2} \mathrm{~h} \mathrm{hc_{3 } \mathrm { h }}$ \\
\hline$(\mathrm{In}, \mathrm{Ga})_{7.33} \mathrm{~S}_{11}$ & 166 & $h R 72$ & $\begin{array}{l}\mathrm{RM}^{\prime} \mathrm{RMRNR}\left(\mathrm{RM}^{\prime}\right)_{2} \mathrm{R} \\
\mathrm{RN}^{\prime} \mathrm{RMRM}^{\prime} \mathrm{R}\end{array}$ & 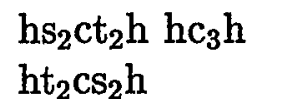 \\
\hline \multicolumn{5}{|c|}{ M-M bonding } \\
\hline $\mathrm{ZrCl}$ & 166 & $h R 12$ & $\mathrm{RM}_{2} \mathrm{R}$ & $c_{4}$ \\
\hline PtTe & 12 & $m C 8$ & $\mathrm{RM}_{2} \mathrm{R}$ & $\mathrm{c}_{4}$ \\
\hline $\mathrm{GaS} 2 \mathrm{H}(\beta)$ & 194 & $h P 8$ & $\mathrm{RM}_{2} \mathrm{R}$ & $\mathrm{hs}_{2} \mathrm{~h}$ \\
\hline GaSe 3R $(\gamma)$ & 160 & $h R 12$ & $\mathrm{RM}_{2} \mathrm{R}$ & $\mathrm{cs}_{2} \mathrm{~h}$ \\
\hline GaSe $4 \mathrm{H}(\delta)$ & 186 & $h P 16$ & $\mathrm{RM}_{2} \mathrm{R}$ & $\mathrm{cs}_{2} \mathrm{hhs}_{2} \mathrm{~h}$ \\
\hline GaSe $2 \mathrm{H}(\varepsilon)$ & 187 & $h P 8$ & $\mathrm{RM}_{2} \mathrm{R}$ & $\mathrm{Cs}_{2} \mathrm{chs}_{2} \mathrm{~h}$ \\
\hline $\mathrm{Ta}_{2} \mathrm{CS}_{2}$ & 166 & $h R 15$ & RMNMR & $c_{5}$ \\
\hline $\mathrm{Ta}_{2} \mathrm{CS}_{2} \mathrm{LT}$ & 164 & $h P 5$ & RMNMR & $\mathrm{hc}_{3} \mathrm{~h}$ \\
\hline $\mathrm{Pt}_{3} \mathrm{Te}_{4}$ & 166 & $h R 21$ & $\mathrm{RM}_{2} \mathrm{R} \mathrm{RMR}$ & $c_{7}$ \\
\hline $\mathrm{Pt}_{2} \mathrm{Te}_{3}$ & 166 & $h R 30$ & $\mathrm{RMR} \mathrm{RM} \mathrm{R}_{2} \mathrm{R} \mathrm{RMR}$ & $\mathrm{c}_{10}$ \\
\hline BiSe & 164 & $h P 12$ & RMRMR RMRMR $\mathrm{M}_{2}$ & $\mathrm{c}_{12}$ \\
\hline $\mathrm{Bi}_{4} \mathrm{Se}_{3}$ & 166 & $h R 21$ & RMRMR $\mathrm{M}_{2}$ & $c_{7}$ \\
\hline $\mathrm{Bi}_{2} \mathrm{Te}$ & 166 & $h R 27$ & RMRMR $\mathrm{M}_{2} \mathrm{M}_{2}$ & $c_{9}$ \\
\hline As & 166 & $h R 6$ & $\mathrm{M}_{2}$ & $\mathrm{c}_{2}$ \\
\hline $\mathrm{Bi}_{2} \mathrm{Te}_{3} \mathrm{II}$ & 160 & $h R 5$ & $\mathrm{M}_{2} \mathrm{R}_{3}$ & $c_{5}$ \\
\hline
\end{tabular}


Table 7 (continued)

\begin{tabular}{|c|c|c|c|c|}
\hline $\mathrm{M}_{\mathrm{x}} \mathrm{N}_{\mathrm{y}} \mathrm{R}_{\mathrm{z}}$ & SG & PS & layer sequence & stacking sequ. \\
\hline \multicolumn{5}{|c|}{$\begin{array}{c}-\mathrm{M}-\mathrm{M}-\text { or }-\mathrm{M}-\mathrm{N}-\text { bonding } \\
\quad(\mathrm{N} \text { form with } \Delta \mathrm{z} c / a \approx 0.8)\end{array}$} \\
\hline $\begin{array}{l}\mathrm{LiRh} \\
\mathrm{CuPt} \\
\mathrm{ZnAl}{ }_{2} \\
\alpha-\mathrm{Nd} \\
\mathrm{Tb} \mathrm{HP} \\
\alpha-\mathrm{Sm}\end{array}$ & $\begin{array}{l}187 \\
166 \\
164 \\
194 \\
194 \\
166\end{array}$ & $\begin{array}{l}h P 2 \\
h R 6 \\
h P 3 \\
h P 4 \\
h P 6 \\
h R 9\end{array}$ & $\begin{array}{l}\mathrm{MN} \\
\mathrm{MN} \\
\mathrm{MN}_{2} \\
\mathrm{M}_{\infty} \\
\mathrm{M}_{\infty} \\
\mathrm{M}_{\infty}\end{array}$ & $\begin{array}{l}\mathrm{h}_{2} \\
\mathrm{c}_{2} \\
\mathrm{c}_{3} \\
\mathrm{ch} \\
\mathrm{hc}_{2} \\
\mathrm{ch}_{2}\end{array}$ \\
\hline \multicolumn{5}{|c|}{$\begin{array}{c}\mathrm{MN}_{n}, \mathrm{M}_{2} \mathrm{~N}_{n} \\
\text { (B form with } \Delta \mathrm{z} c / a \approx 0.2(\text { Zalkin, Ramsey, 1956; } \\
\text { Zalkin et al., 1956)) }\end{array}$} \\
\hline $\mathrm{CsCl}$ & 221 & $c P 2$ & MN & $c_{2}$ \\
\hline MgLa & 221 & $c P 2$ & MN & $c_{2}$ \\
\hline $\mathrm{CeCd}_{2}$ & 164 & $h P 3$ & $\mathrm{MN}_{2}$ & $c_{3}$ \\
\hline $\mathrm{BiF}_{3}, \mathrm{AlFe}_{3}$ & 225 & $c F 16$ & $\mathrm{MN}_{3}$ & $c_{4}$ \\
\hline $\mathrm{LaH}_{3}$ & 225 & $c F 16$ & $\mathrm{MN}_{3}$ & $\mathrm{c}_{4}$ \\
\hline $\mathrm{NaTl}$ & 227 & $c F 16$ & $\mathrm{M}_{2} \mathrm{~N}_{2}$ & $\mathrm{c}_{4}$ \\
\hline $\mathrm{AlMnCu}_{2}$ & 225 & $c F 16$ & MRNR & $\mathrm{c}_{4}$ \\
\hline $\mathrm{AgSbLi}_{2}$ & 216 & $c F 16$ & $\mathrm{MNR}_{2}$ & $\mathrm{c}_{4}$ \\
\hline $\mathrm{Al}_{2} \mathrm{Li}_{3}$ & 166 & $h R 15$ & $\mathrm{M}_{2} \mathrm{~N}_{3}$ & $c_{5}$ \\
\hline $\mathrm{SiFe}_{2} \mathrm{HT}$ & 164 & $h P 6$ & $\mathrm{MNMN}_{3}$ & $\mathrm{c}_{6}$ \\
\hline $\mathrm{Ni}_{2} \square \mathrm{Al}_{3}$ & 164 & $h P 5$ & MRMRNR & $c_{6}$ \\
\hline $\mathrm{Ni}_{2} \mathrm{Al}_{3}$ & 164 & $h P 5$ & RMRMR & $\mathrm{hc}_{3} \mathrm{~h}$ \\
\hline $\mathrm{Si}_{2} \mathrm{Li}_{5}$ & 166 & $h R 21$ & $\mathrm{MN}_{2} \mathrm{MN}_{3}$ & $\mathrm{c}_{7}$ \\
\hline $\mathrm{Ga}_{4} \mathrm{Li}_{5}$ & 164 & $h P 9$ & $\mathrm{M}_{2} \mathrm{~N}_{2} \mathrm{M}_{2} \mathrm{~N}_{3}$ & $c_{9}$ \\
\hline $\mathrm{Ge}_{2} \mathrm{Cu}_{2} \mathrm{Li}_{5}$ & 164 & $h P 9$ & $\mathrm{MNR}_{2} \mathrm{NMR}_{3}$ & $c_{9}$ \\
\hline $\mathrm{Pb}_{3} \mathrm{Li}_{8}$ & 166 & $h R 33$ & $\mathrm{MN}_{2} \mathrm{MN}_{3} \mathrm{MN}_{3}$ & $c_{11}$ \\
\hline $\mathrm{Pb}_{2} \mathrm{Li}_{7}$ & 164 & $h P 9$ & $\mathrm{MN}_{3} \mathrm{MN}_{4}$ & $\mathbf{c}_{11}$ \\
\hline
\end{tabular}


Table 8: Composition, layer sequence and $T_{i}(\mathrm{M})$ values of fcc ( $\mathrm{V}$ form), bcc ( $\mathrm{v}$ form) and $\mathrm{CaF}_{2}$ (I form) and related compounds with stacking of square layers in [001] direction.

\begin{tabular}{|c|c|c|c|c|}
\hline \multicolumn{2}{|c|}{$\mathrm{M}_{\mathrm{x}} \mathrm{N}_{\mathrm{y}} \mathrm{R}_{\mathrm{z}}$} & SG & PS & $T_{i}(\mathrm{M}) \mathrm{bcc} / \mathrm{ccp} / \mathrm{pc}$ \\
\hline \multicolumn{5}{|c|}{$\begin{array}{c}\text { layered [001] structures (Schubert et al., 1960) (Fig.21) } \\
\text { (v form with } \Delta \mathrm{z} c / d \approx 0.6, \mathrm{bcc})\end{array}$} \\
\hline $\mathrm{CsCl}$ & $a(\mathrm{MN})$ & 221 & $c P 2$ & $0612 ; 1$ \\
\hline $\mathrm{MoSi}_{2}$ & $\mathrm{~b}\left(\mathrm{MN}_{2}\right)$ & 139 & $t I 6$ & $044 ; 2$ \\
\hline $\mathrm{ReSi}_{2}$ & $\mathrm{~b}$ & 71 & $o I 6$ & $044 ; 2$ \\
\hline $\mathrm{VAu}_{2}$ & $\mathbf{b}$ & $63 / 38$ & $o C 12$ & $044 ; 2$ \\
\hline $\mathrm{AlAu}_{2}$ & $\mathrm{~b}$ & 62 & $o P 12$ & $044 ; 2$ \\
\hline $\mathrm{AB}_{3}$ & c $\left(\mathrm{MN}_{3}\right)$ & 123 & $t P 4$ & $044 ; 3$ \\
\hline$\gamma-\mathrm{TiCu}$ & $\mathrm{d}\left(\mathrm{M}_{2} \mathrm{~N}_{2}\right)$ & 129 & $t P 4$ & $444 ; 1$ \\
\hline $\mathrm{Au}_{2} \mathrm{Nb}_{3}$ & e $\left(\mathrm{M}_{2} \mathrm{~N}_{3}\right)$ & 139 & $t I 10$ & $444 ; 1.5$ \\
\hline $\mathrm{AB}_{2}$ & $\mathrm{f}\left(\mathrm{M}_{2} \mathrm{~N}_{4}\right)$ & 129 & $t P 6$ & $444 ; 2$ \\
\hline $\mathrm{AB}$ & $\mathrm{g}\left(\mathrm{M}_{4} \mathrm{~N}_{4}\right)$ & 129 & $t P 8$ & $658 ; 1$ \\
\hline $\mathrm{Ti}_{3} \mathrm{Cu}_{4}$ & bd & 139 & $t I 14$ & $2.744 ; 1.3$ \\
\hline $\mathrm{Ti}_{2} \mathrm{Cu}_{3}$ & $\mathrm{bdb}$ & 129 & $t P 10$ & $244 ; 1.5$ \\
\hline $\mathrm{Os}_{2} \mathrm{Al}_{3}$ & $a b$ & 139 & $t I 10$ & $058 ; 1.5$ \\
\hline $\mathrm{Ti}_{3} \mathrm{Pd}_{5}$ & $\mathrm{bab}$ & 123 & $t P 8$ & $04.76 .7 ; 1.7$ \\
\hline $\operatorname{ReAl}(\operatorname{Re}, A l)_{2}$ & MRNR & 123 & $t P 4$ & $\begin{array}{c}044 ; 3(\mathrm{M}, \mathrm{N}) \\
0612 ; 1(\mathrm{R}) \\
\end{array}$ \\
\hline \multicolumn{5}{|c|}{$\begin{array}{l}\mathrm{CuAu} \text { related structures in [001] projection with composition } \\
\text { of } \mathrm{Cu} / \mathrm{Au} \text { layer ( } \mathrm{V} \text { form with } \Delta \mathrm{z} c / d \approx 0.7, \mathrm{ccp} \text { ) }\end{array}$} \\
\hline $\mathrm{Ti}_{2} \mathrm{Ga}_{3}$ & $\mathrm{M}_{4} \mathrm{~N} / \mathrm{N}_{5}$ & 83 & $t P 10$ & $356 ; 1.5$ \\
\hline $\mathrm{MN}_{3}$ & $\mathrm{MN} / \mathrm{N}_{2}$ & 65 & $\circ C 4$ & $224 ; 3 a$ \\
\hline $\mathrm{PdCu}_{4}$ & $\mathrm{M}_{2} \mathrm{~N}_{3} / \mathrm{N}_{5}$ & 84 & $t P 20$ & $114 ; 4$ \\
\hline$\square_{4} \mathrm{Co}_{5} \mathrm{Ge}_{7}$ & $\begin{array}{l}\mathrm{N}_{5} \mathrm{R}_{3} / \\
\mathrm{M}_{4} \mathrm{R}_{4}\end{array}$ & 107 & $t I 24$ & $\begin{array}{rl}2 & 24 ; 3 / \\
1.64 .83 .2 ; 2.2 /\end{array}$ \\
\hline $\mathrm{CuAu}$ & $\mathrm{a}(\mathrm{MN})$ & 123 & $t P 4$ & $\begin{array}{l}4.02 .19 .1 ; 1.0 \\
468 ; 1\end{array}$ \\
\hline $\mathrm{CuZr}_{2}$ & $\mathrm{~b}\left(\mathrm{MN}_{2}\right)$ & 139 & $t I 6$ & $440 ; 2$ \\
\hline TiCd & $\mathrm{d}\left(\mathrm{M}_{2} \mathrm{~N}_{2}\right)$ & 129 & $t P 4$ & $848 ; 1$ \\
\hline \multicolumn{5}{|c|}{ layered [001] structures ( $\mathrm{I}$ form with $\Delta \mathrm{z} c / d \approx 1, \mathbf{p c}$ ) } \\
\hline Po & $\mathrm{M}$ & 221 & $c P 1$ & $6128 ;(1)$ \\
\hline $\mathrm{Pb} \square \mathrm{O}$ & $\mathrm{a}(\mathrm{MN})$ & 129 & $t P 4$ & $440 ; 1$ \\
\hline $\mathrm{M}_{3} \square_{2} \mathrm{O}_{4}$ & $\mathrm{~b}\left(\mathrm{MN}_{2}\right)$ & 139 & $t I 14$ & $440 ; 2$ \\
\hline $\mathrm{M}_{2} \square \mathrm{O}_{3}$ & c $\left(\mathrm{MN}_{3}\right)$ & 129 & $t P 10$ & $440 ; 3$ \\
\hline $\mathrm{M} \square \mathrm{O}$ & $\mathrm{d}\left(\mathrm{M}_{2} \mathrm{~N}_{2}\right)$ & 123 & $t P 8$ & $584 ; 1$ \\
\hline $\mathrm{M}_{3} \square_{2} \mathrm{O}_{4}$ & ac & 129 & $t P 14$ & $440 ; 2$ \\
\hline $\mathrm{M} \square \mathrm{O}$ & ad & 99 & $t P 12$ & $4.76 .72 .7 ; 1$ \\
\hline
\end{tabular}


Table 9a: Self-coordination numbers $T_{i}(\mathrm{M})$ of $\mathrm{M}$ atoms with $\mathrm{M}$ atoms in $i=1-3$ shells and $y / x$ of $M_{x} N_{y}$ composition of $M$ and $N$ atoms in ordered alloys and related structures (Section 18). The structures are ordered for different structure families with sequences of structural units or a two-dimensional tiling of units shown in square brackets. Square net, structural units from Fig.8.

\begin{tabular}{|c|c|c|c|}
\hline $\begin{array}{l}T_{i}(\mathrm{M}) ; \mathrm{y} / \mathrm{x} \\
\quad \text { square }\end{array}$ & $\begin{array}{l}\text { struct. } \\
\text { units }\end{array}$ & $\begin{array}{c}\text { ccp alloy } \\
{[001]}\end{array}$ & $\begin{array}{c}\text { bcc alloy } \\
{[001]}\end{array}$ \\
\hline $\begin{array}{rl}T_{2} & =2 T_{1}-4 \\
& 444 ;(1) \\
3 & 22 ; 1 \\
2 & 04 ; 1 \\
2 & 03.3 ; 1.3 \\
3 & 22 ; 1.5 \\
2 & 02 ; 2\end{array}$ & $\begin{array}{l}\quad a, b, c \\
a, b \\
a c b c \\
c_{2} \\
c_{3} b c_{3} \\
\text { bcacb } \\
\text { cbc }\end{array}$ & $\begin{array}{l}\mathrm{Cu}, \mathrm{NaCl} \\
\mathrm{CuPt} a, b \\
\mathrm{MoPt}_{2}\end{array}$ & $\begin{array}{l}\mathrm{W}, \mathrm{CsCl} \\
\gamma-\mathrm{TiCu} \\
\mathrm{Ti}_{3} \mathrm{Cu}_{4} \\
\mathrm{Os}_{2} \mathrm{Al}_{3}\end{array}$ \\
\hline 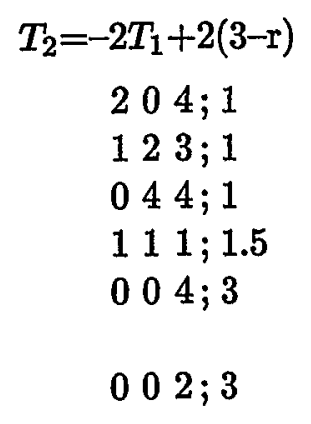 & $\begin{array}{l}\mathrm{b}^{*}, \mathrm{c}^{*}, \mathrm{~s}^{*}, \mathrm{t}^{*} \\
\mathrm{c}^{*} \\
\mathrm{c}_{2}^{*} \mathrm{t}_{2}^{*} \\
\mathrm{t}_{2}^{*} \\
{\left[\mathrm{c}^{*} \mathrm{~s}^{* *} \mathrm{t}\right]} \\
\mathrm{s}_{2}^{*} \\
\mathrm{~s}_{2}^{* *}\end{array}$ & $\begin{array}{l}\mathrm{CuPt} a, b \\
\mathrm{UPb} \\
\mathrm{PdCu}_{4} \\
\mathrm{GeCa}_{7}, \\
\mathrm{Ga}_{3} \mathrm{Pt}_{5}\end{array}$ & $\mathrm{NaTl}$ \\
\hline $\begin{array}{c}T_{1}=0 \\
044 ; 1 \\
\\
\end{array}$ & $\begin{array}{l}\quad b, c, s, t \\
t_{2} \\
\\
t_{3} s_{2} \\
s_{2} t \\
t s b s \\
{\left[b_{4} s_{12} t_{4}\right]} \\
{\left[b_{4}\right]} \\
{\left[b_{5} s_{4}\right]}\end{array}$ & $\begin{array}{l} \\
\mathrm{CuZr}_{2} \\
\mathrm{TiAl}_{3} \\
\mathrm{Mn}_{9} \mathrm{Au}_{31} \\
\mathrm{MoNi}_{4} \\
\mathrm{Ti}_{2} \mathrm{Ga}_{3} \\
\mathrm{TiPt}_{8}\end{array}$ & $\begin{array}{l}\mathrm{NaTl}, \mathrm{AlFe}_{3} \\
\mathrm{AuMn}_{3}, \mathrm{GaLi}_{2}, \\
(\mathrm{Mo}, \mathrm{U}) \mathrm{U}_{3}\end{array}$ \\
\hline 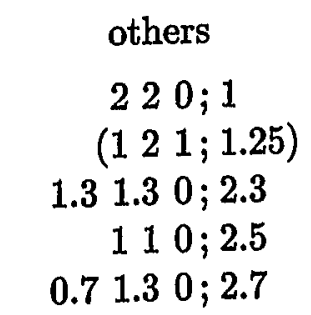 & $\begin{array}{l}\mathrm{d}_{2} \mathrm{~s}_{2} \\
{\left[\mathrm{bc}_{4} \mathrm{t}_{4}\right]} \\
{\left[\mathrm{b}_{3} \mathrm{c}_{2} \mathrm{ds}_{3} \mathrm{t}\right]} \\
{\left[\mathrm{b}_{2} \mathrm{c}_{2} \mathrm{ts}_{2}\right]} \\
{\left[\mathrm{b}_{3} \mathrm{c}_{2} \mathrm{t}_{2} \mathrm{~s}_{4}\right]}\end{array}$ & $\begin{array}{l}\mathrm{TiCd} \\
\mathrm{V}_{4} \mathrm{Zn}_{5} \\
\mathrm{Mn}_{2} \mathrm{Au}_{5} \\
\mathrm{Mo}_{3} \mathrm{Al}_{8}\end{array}$ & $\begin{array}{l}\mathrm{Au}(\mathrm{Zn}, \mathrm{Au})_{3} \\
\mathrm{Sb}_{2} \mathrm{Tl}_{7}(\mathrm{z}=0) \\
\mathrm{Sn}_{3} \mathrm{Li}_{7}\end{array}$ \\
\hline
\end{tabular}


Table 9b: Same as Table 9a, except for hexagonal net, structural units from Fig.6.

\begin{tabular}{|c|c|c|c|c|}
\hline $\begin{array}{l}T_{i}(\mathrm{M}) ; \mathrm{y} / \mathrm{x} \\
\text { hex. plan. }\end{array}$ & $\begin{array}{l}\text { struct. } \\
\text { units }\end{array}$ & $\begin{array}{c}\text { ccp alloy } \\
{[111]}\end{array}$ & $\begin{array}{c}\text { hcp alloy } \\
\text { [0001] }\end{array}$ & $\begin{array}{c}\mathrm{bcc} \\
{[111]}\end{array}$ \\
\hline $\begin{array}{l}666 ;(1) \\
4222 ; 1 \\
2226 ; 1 \\
2202 ; 2\end{array}$ & $\begin{array}{l}\quad \mathrm{a}, \mathrm{b}, \mathrm{c} \\
\mathrm{a}, \mathrm{b} \\
\mathrm{acbc} \\
\mathrm{c}_{2} \\
\mathrm{cbc}\end{array}$ & $\begin{array}{l}\mathrm{Cu} \\
\mathrm{TiCu} \\
\mathrm{CuAu} \\
\mathrm{MoSi}_{2} \\
\mathrm{MoPt}_{2}\end{array}$ & $\begin{array}{l}\mathrm{Mg} \\
\mathrm{AuCd}\end{array}$ & $\mathrm{NaTl}$ \\
\hline $\begin{array}{c}226 ; 1 \\
060 ; 2 \\
\\
041 ; 2.5 \\
03.31 .3 ; 2.7 \\
022 ; 3 \\
242 ; 1 \\
020 ; 4 \\
040 ; 4 \\
020 ; 6\end{array}$ & $\begin{array}{l}c^{*}, e-i \\
c^{*} \\
e \\
\text { ef } \\
e f_{2} \\
f \\
g, g^{\prime} \\
h \\
e i \\
i\end{array}$ & $\begin{array}{l}\mathrm{CuAu} \\
\mathrm{MoPt}_{2} \\
\mathrm{Mn}_{2} \mathrm{Au}_{5} \\
\mathrm{Mo}_{3} \mathrm{Al}_{8} \\
\mathrm{TiAl}_{3} \\
\mathrm{MoNi}_{4}\end{array}$ & $\begin{array}{l}\mathrm{AuCd} \\
\mathrm{SbAg}_{3} \\
\mathrm{TaPt}_{2} \\
\mathrm{SnAu}_{5} \\
\mathrm{TiCu}_{3} \\
\mathrm{ZrAu}_{4}\end{array}$ & \\
\hline $\begin{aligned} 226 ; 1 \\
22.45 .2 ; 1 \\
242 ; 1 \\
1.51 .54 .5 ; 1.5 \\
114 ; 2 \mathrm{a} \\
114 ; 2 \mathrm{~b} \\
006 ; 3\end{aligned}$ & $\begin{array}{l}\quad c^{\prime}, c^{\prime \prime}, k \\
c^{\prime}, c^{\prime \prime} \\
c_{9}^{\prime} c^{\prime \prime} \\
c^{\prime} c^{\prime \prime} \\
{\left[c_{3}^{\prime} c_{3}^{\prime \prime} k_{2}\right]} \\
c^{\prime} k \\
c^{\prime} k c^{\prime \prime} k \\
k\end{array}$ & $\begin{array}{l}\mathrm{CuAu} \\
\mathrm{CuAu} \text { II } \\
\\
\mathrm{Ti}_{2} \mathrm{Ga}_{3} \\
\mathrm{ZrGa}_{2} \\
\mathrm{HfGa}_{2} \\
\mathrm{AuCu}_{3}\end{array}$ & $\mathrm{AuCd}$ & \\
\hline 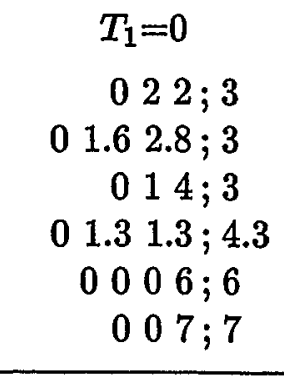 & $\begin{array}{l}\mathrm{b}, \mathrm{f}^{\prime}, \mathrm{k}, \mathrm{k}^{\prime} \\
\mathrm{f}^{\prime} \\
\mathrm{f}_{4}^{\prime} \mathrm{k} \\
\mathrm{f}^{\prime} \mathrm{k} \\
{\left[\mathrm{b}_{4} \mathrm{k}_{12}^{\prime}\right]} \\
{\left[\mathrm{b}_{8} \mathrm{k}_{8}^{\prime}\right]}\end{array}$ & $\begin{array}{l}\mathrm{TiAl}_{3} \\
\mathrm{ZrAl}_{3}\end{array}$ & $\begin{array}{l}\mathrm{TiCu}_{3} \\
\mathrm{SnCu}_{3} \\
\mathrm{CdAu}_{3}\end{array}$ & $\mathrm{Pu}_{3} \mathrm{Pd}_{4}$ \\
\hline
\end{tabular}


Table 9b (continued)

\begin{tabular}{|r|l|l|l|l|}
\hline $\begin{array}{c}T_{i}(\mathrm{M}) ; \mathrm{y} / \mathrm{x} \\
\text { hex. plan. }\end{array}$ & $\begin{array}{c}\text { struct. } \\
\text { units }\end{array}$ & \multicolumn{1}{c|}{$\begin{array}{c}\text { ccp alloy } \\
{[111]}\end{array}$} & $\begin{array}{c}\text { hcp alloy } \\
{[0001]}\end{array}$ & $\begin{array}{c}\mathrm{bcc} \\
{[111]}\end{array}$ \\
\hline $\begin{aligned} \text { others } \\
2.51 .52 .5 ; 1.25\end{aligned}$ & & & & \\
$222 ; 1.3$ & & $\mathrm{~V}_{4} \mathrm{Zn}_{5}$ & & \\
$(122 ; 2 \mathrm{a})$ & $\mathrm{c}^{\prime} \mathrm{f}^{\prime}$ & $\mathrm{ZrSi}_{2}$ & & \\
$(122 ; 2 \mathrm{~b})$ & $\mathrm{c}^{\prime} \mathrm{f}^{\prime} \mathrm{c}^{\prime \prime} \mathrm{f}^{\prime}$ & $(\mathrm{Zr}, \mathrm{Al})(\mathrm{Zr}, \mathrm{Si})_{2} \mathrm{Pd}_{7}$ & & \\
$(0.422 ; 2.6)$ & $\mathrm{c}^{\prime} \mathrm{f}_{4}^{\prime}$ & $\mathrm{Nb}_{5} \mathrm{Ga}_{13}$ & & \\
$(02.32 .8 ; 2.8)$ & {$\left[\mathrm{e}_{3} \mathrm{f}_{6} \mathrm{k}_{4}\right]$} & & & \\
\hline
\end{tabular}


Table 10a: Composition $\mathrm{M}_{\mathrm{x}} \mathrm{N}_{\mathrm{y}} \mathrm{R}_{\mathrm{z}}$ and different types of chains $\mathrm{RM},(\mathrm{RM})_{n} \mathrm{R},(n=1$ -3) and RNRM, which can be formed by atoms with one, two or three bonds, number of valence electrons $\mathrm{e}_{i}$ of $i=\mathrm{M}, \mathrm{N}$ and $\mathrm{R}$ atoms and some experimental or theoretical examples (in brackets).

\begin{tabular}{|c|c|c|c|}
\hline$M_{x} N_{y} R_{z}$ & $\mathrm{e}_{\mathrm{M}} \mathrm{e}_{\mathrm{N}} \mathrm{e}_{\mathrm{R}}$ & covalent chain & ionic chain \\
\hline $\mathrm{RM}$ & 17 & $\mathrm{I}-\mathrm{Cu}$ & $\overline{|\mathrm{I}|} \mathrm{Cu}$ \\
\hline $\mathrm{RM}$ & 26 & $\mathrm{~S}=\mathrm{Zn}$ or $-\mathrm{S}-\mathrm{Zn}-$ & $\overline{|\mathrm{S}|} \mathrm{Zn}$ \\
\hline RM & 35 & $\mathrm{~N} \equiv \mathrm{Al}$ or $-\mathrm{N}=\mathrm{Al}-$ or $=\mathrm{N}-\mathrm{Al}=$ & $\overline{|\mathrm{N}|} \mathrm{Al}$ \\
\hline $\mathrm{RM}$ & 44 & $-\mathrm{C} \equiv \mathrm{Si}-$ or $=\mathrm{C}=\mathrm{Si}=$ & $\overline{|\mathrm{C}|} \mathrm{Si}$ \\
\hline RMR & $027_{2}$ & $\mathrm{I}-\mathrm{Cd}-\mathrm{I}$ & $\overline{|\mathrm{I}|} \mathrm{Cd} \overline{\underline{I I}}$ \\
\hline RMR & $061_{2}$ & $(\mathrm{Cu}-\mathrm{S}-\mathrm{Cu})$ & $\mathrm{Cu} \overline{|\mathrm{S}|} \mathrm{Cu}$ \\
\hline RMR & $046_{2}$ & $\mathrm{~S}=\mathrm{Sn}=\mathrm{S}$ & $\overline{|\mathrm{S}|} \mathrm{Sn} \overline{\mathrm{S} \mid}$ \\
\hline RMR & $042_{2}$ & $(\mathrm{Mg}=\mathrm{C}=\mathrm{Mg})$ & $\mathrm{Mg} \overline{\lceil\mathrm{C} \mid} \mathrm{Mg}$ \\
\hline$(\mathrm{RM})_{2} \mathrm{R}$ & $03_{2} 6_{3}$ & $S=I n-S-I n=S$ & $\overline{|\mathrm{S}|} \operatorname{In} \overline{|\mathrm{S}|}$ In $\overline{|\mathrm{S}|}$ \\
\hline$(\mathrm{RM})_{2} \mathrm{R}$ & $05_{2} 2_{3}$ & $(\mathrm{Mg}=\mathrm{N}-\mathrm{Mg}-\mathrm{N}=\mathrm{Mg})$ & $\mathrm{Mg} \overline{\overline{\mathrm{N} \mid}} \mathrm{Mg} \overline{|\mathrm{N}|} \mathrm{Mg} \overline{\overline{|N|}} \mathrm{Mg}$ \\
\hline$(\mathrm{RM})_{3} \mathrm{R}$ & $04_{3} 5_{4}$ & $\mathrm{~N} \equiv \mathrm{Th}-\mathrm{N}=\mathrm{Th}=\mathrm{N}-\mathrm{Th} \equiv \mathrm{N}$ & $\overline{|\mathrm{N}|} \operatorname{Th} \overline{|\mathrm{N}|} \mathrm{Th} \overline{|\mathrm{N}|} \mathrm{Th} \overline{\mathrm{N} \mid}$ \\
\hline$(\mathrm{RM})_{3} \mathrm{R}$ & $04_{3} 3_{4}$ & $\mathrm{Al} \equiv \mathrm{C}-\mathrm{Al}=\mathrm{C}=\mathrm{Al}-\mathrm{C} \equiv \mathrm{Al}$ & $\mathrm{Al} \overline{|\mathrm{C}|} \mathrm{Al} \overline{|\mathrm{C}|} \mathrm{Al} \overline{|\mathrm{C}|} \mathrm{Al}$ \\
\hline RNRM & $245_{2}$ & $\mathrm{~N} \equiv \mathrm{Th}-\mathrm{N}=\mathrm{Mg}$ or $-\mathrm{N}=\mathrm{Th}=\mathrm{N}-\mathrm{Mg}-$ & $\overline{|\mathrm{N}|} \mathrm{Th} \overline{|\mathrm{N}|} \mathrm{Mg}$ \\
\hline RNRM & $643_{2}$ & $\mathrm{Al} \equiv \mathrm{C}-\mathrm{Al}=\mathrm{S}$ or $-\mathrm{Al}=\mathrm{C}=\mathrm{Al}-\mathrm{S}-$ & $\mathrm{Al} \overline{|\mathrm{C}|} \mathrm{Al} \overline{|\mathrm{S}|}$ \\
\hline
\end{tabular}

Table 10b: Different elements with $e_{i}$ valence electrons in the order of increasing size (Schubert, 1964). The elements shown in brackets contain also other electrons. Zn, $\mathrm{Al}, \mathrm{Ga}$ and $\mathrm{N}$ atoms (underlined) occupy tetrahedral, $\mathrm{Mn}, \mathrm{In}, \mathrm{C}$ and trivalent $\mathrm{Fe}$ (bold face) tetrahedral or octahedral sites.

\begin{tabular}{|l|l|}
\hline $\mathrm{e}_{i}$ & \\
\hline 1 & $\mathrm{Cu} \mathrm{Ag} \mathrm{Li}$ \\
2 & (Ni Co Fe Mn) $\underline{\mathrm{Zn}}(\mathrm{Ge}) \mathrm{Cd} \mathrm{Hg}(\mathrm{Sn} \mathrm{Mg} \mathrm{Pb} \mathrm{Th})$ \\
3 & (Ni Fe Cr) $\mathrm{Al} \mathrm{Ga} \mathrm{In}(\mathrm{Bi})$ \\
4 & $\mathrm{C} \mathrm{Si} \mathrm{(Pt} \mathrm{Mo} \mathrm{W} \mathrm{Ta} \mathrm{Nb)} \mathrm{Sn}(\mathrm{Zr})$ \\
5 & N P As \\
6 & $\mathrm{~S} \mathrm{Se} \mathrm{Te}$ \\
7 & $\mathrm{Cl} \mathrm{BrI}$ \\
\hline
\end{tabular}


Table 11a: Self-coordination numbers $T_{i}(\mathrm{~A})$ and $\mathrm{y} / \mathrm{x}$ of ccp alloys $\mathrm{M}_{\mathrm{x}} \mathrm{N}_{\mathrm{y}}$ and $\mathrm{NaCl}$, $\mathrm{BiF}_{3}$ or $\mathrm{ZnS}$ (sphalerite) related structures, which can be characterized by the $T_{i}(\mathrm{~A})$ of square planes or structural units a - y (Fig.12).

\begin{tabular}{|c|c|c|c|c|c|}
\hline $\begin{array}{l}T_{i}(\mathrm{~A}) ; \mathrm{y} / \mathrm{x} \\
\quad \text { ccp }\end{array}$ & $\begin{array}{l}T_{i}(\mathrm{~A}) ; \mathrm{y} / \mathrm{x} \\
\text { square }\end{array}$ & $\begin{array}{c}\mathrm{Na}_{n} \square_{\mathrm{x}} \mathrm{Cl}_{n-\mathrm{x}} \\
\left(\mathrm{BiF}_{2}\right)_{n} \square_{\mathrm{x}} \mathrm{F}_{n-\mathrm{x}}^{\prime}\end{array}$ & $\begin{array}{l}\mathrm{M}_{\mathrm{x}} \mathrm{N}_{\mathrm{y}} \\
(\mathrm{Cu})\end{array}$ & $\begin{array}{l}M_{x} N_{y} S_{x+y} \\
\quad(Z n S)\end{array}$ & $\begin{array}{l}\text { struct. } \\
\text { units }\end{array}$ \\
\hline $6012 ; 1 \mathrm{a}$ & $204 ; 1$ & $\mathrm{Gd}_{2} \square \mathrm{C}$ & $\mathrm{CuPt}, \mathrm{a}$ & $\operatorname{In}(\mathrm{Ga}, \mathrm{Al}) \mathrm{P}_{2}$ & a \\
\hline $6012 ; 1 \mathrm{~b}$ & $204 ; 1$ & $\mathrm{Ti}_{2} \square \mathrm{C}$ & $\mathrm{CuPt}, \mathrm{b}$ & & \\
\hline $4416 ; 1$ & $044 ; 1$ & $\begin{array}{l}\mathrm{Pd}_{2} \square \mathrm{H}, \mathrm{Ti}_{2} \square \mathrm{N} / \\
\left(\mathrm{NdH}_{2}\right) \mathrm{H}_{0.5} \square_{0.5}\end{array}$ & $\mathrm{UPb}$ & $\mathrm{CuFeS}_{2}$ & $\mathrm{aa}^{\prime} / \mathrm{eg}$ \\
\hline $408 ; 1.7$ & & $\mathrm{Ti}_{8} \square_{3} \mathrm{C}_{5}$ & $\mathrm{M}_{3} \mathrm{~N}_{5}$ & & \\
\hline $606 ; 2$ & $202 ; 2$ & $\mathrm{~S}_{3} \square \mathrm{In}_{2}$ & $\mathrm{ZnAl}_{2}$ & & $\mathrm{MN}_{2}$ \\
\hline $3010 ; 2 a$ & $102 ; 2$ & $\mathrm{Cl}_{3} \mathrm{Ti}_{2}$ & $\mathrm{MN}_{2}$ & & \\
\hline $3010 ; 2 b$ & $103 ; 2$ & $\mathrm{Cl}_{3} \mathrm{Al}_{2}$ & $\mathrm{MN}_{2}$ & & $\mathrm{ac}^{\prime}$ \\
\hline $3010 ; 2 c$ & $102 ; 2$ & $\mathrm{~S}_{3} \square \mathrm{Sc}_{2}$ & $\mathrm{MN}_{2}$ & & \\
\hline $\begin{array}{l}2212 ; 2 \mathrm{a} \\
2212 ; 2 \mathrm{c}\end{array}$ & $202 ; 2$ & $\square \mathrm{Ti}_{2} \square \mathrm{O}_{2}$ & $\mathrm{MoPt}_{2}$ & $\begin{array}{l}\mathrm{GeCu}_{2} \mathrm{Se}_{3} \\
\beta-\square \mathrm{Ga}_{2} \mathrm{Se}_{3}\end{array}$ & \\
\hline $1310 ; 2.5$ & & & $\mathrm{Mn}_{2} \mathrm{Au}_{5}$ & $\mathrm{Si}_{2}\left(\mathrm{NiCu}_{4}\right) \mathrm{S}_{7}$ & $\mathrm{gh}$ \\
\hline $204 ; 3$ & $204 ; 1$ & $\begin{array}{l}\mathrm{Se}_{4} \square \mathrm{Tm}_{3}, \\
\mathrm{Cl}_{4} \mathrm{Os}_{3}\end{array}$ & $\mathrm{CuPt}_{3}$ & & $c^{\prime}$ \\
\hline $208 ; 3$ & $004 ; 3$ & $\mathrm{I}_{4} \mathrm{Hf} \square_{3}$ & $\mathrm{MN}_{3}$ & & \\
\hline $048 ; 3$ & $044 ; 1$ & $\begin{array}{l}\mathrm{Nb}_{4} \square \mathrm{N}_{3}, \\
\mathrm{~F}_{4} \mathrm{Sn}_{3} \\
\left(\mathrm{CeH}_{2}\right) \mathrm{H}_{0.25} \square_{0.75}\end{array}$ & $\mathrm{TiAl}_{3}$ & $\mathrm{SbCu}_{3} \mathrm{~S}_{4}$ & $\mathrm{c} / \mathrm{h}$ \\
\hline $060 ; 3$ & $044 ; 1$ & $\begin{array}{l}\square \mathrm{Nb}_{3} \square \mathrm{O}_{3}, \\
\mathrm{Nb}_{4} \square \mathrm{C}_{3}\end{array}$ & $\mathrm{AuCu}_{3}$ & $\mathrm{Cd}\left(\square \mathrm{In}_{2}\right) \mathrm{Se}_{4}$ & $\mathrm{u}$ \\
\hline $107 ; 4$ & $000 ; 4$ & $\mathrm{Cl}_{5} \mathrm{U}_{4}$ & $\mathrm{MN}_{4}$ & & $i$ \\
\hline $028 ; 4$ & $000 ; 4$ & $\begin{array}{l}\mathrm{F}_{5} \mathrm{U}_{4} \\
\mathrm{Pd}_{5} \square \mathrm{H}_{4} \\
\mathrm{O}_{5} \square \mathrm{Ti}_{4}\end{array}$ & $\mathrm{MoNi}_{4}$ & & 1 \\
\hline $008 ; 5 a$ & $002 ; 5$ & $\mathrm{~S}_{6} \square \mathrm{Lu}_{5}$ & $\mathrm{MN}_{5}$ & & \\
\hline $008 ; 5 \mathrm{~b}$ & $000 ; 5$ & $\mathrm{~V}_{6} \square \mathrm{C}_{5}$ III & $\mathrm{MN}_{5}$ & & \\
\hline $008 ; 5 \mathrm{e}$ & $002 ; 5$ & $\begin{array}{l}\mathrm{V}_{6} \square \mathrm{C}_{5} \mathrm{I}, \\
\mathrm{S}_{6} \square \mathrm{Sc}_{5}\end{array}$ & $\mathrm{MN}_{5}$ & & \\
\hline $008 ; 5 f$ & & $\begin{array}{l}\mathrm{V}_{6} \square \mathrm{C}_{5} \mathrm{II} \\
\mathrm{O}_{6} \mathrm{ReLi}_{5}\end{array}$ & $\mathrm{MN}_{5}$ & & \\
\hline $006 ; 7$ & $000 ; 7$ & $\mathrm{~V}_{8} \square \mathrm{C}_{7}$ & $\begin{array}{l}\mathrm{MN}_{7} \\
\mathrm{MN}_{7}\end{array}$ & & \\
\hline $000 ; 7$ & $004 ; 3$ & $\mathrm{O}_{8} \mathrm{Mg}_{6} \mathrm{Mn} \square$ & $\mathrm{MN}_{7}$ & & \\
\hline
\end{tabular}


Table 11b: $T_{i}(\mathrm{~A})$ values and structural units of hcp alloys $\mathrm{M}_{\mathrm{x}} \mathrm{N}_{\mathrm{y}}$ and $\mathrm{Ni}_{2} \mathrm{In}$ or $\mathrm{ZnS}$ (wurtzite) related structures with structural units of Fig.10, 28.

\begin{tabular}{|c|c|c|c|c|c|c|}
\hline $\begin{array}{c}T_{i}(\mathrm{~A}) ; \mathrm{y} / \mathrm{x} \\
\mathrm{hcp}\end{array}$ & $\begin{array}{l}T_{i}(\mathrm{~A}) ; \mathrm{j} \\
\text { hex. pl }\end{array}$ & $y / x$ & $\begin{array}{c}\mathrm{Ni}_{n} \operatorname{In}_{n} \square_{\mathrm{x}} \mathrm{Ni}_{n-\mathrm{x}}^{\prime} \\
\left(\mathrm{Ni}_{2} \mathrm{In}\right)\end{array}$ & $\begin{array}{c}\mathrm{M}_{\mathrm{x}} \mathrm{N}_{\mathrm{y}} \\
(\mathrm{Mg})\end{array}$ & $\begin{array}{l}M_{x} N_{y} S_{x+y} \\
\quad(Z n S)\end{array}$ & $\begin{array}{l}\text { struct. } \\
\text { units }\end{array}$ \\
\hline 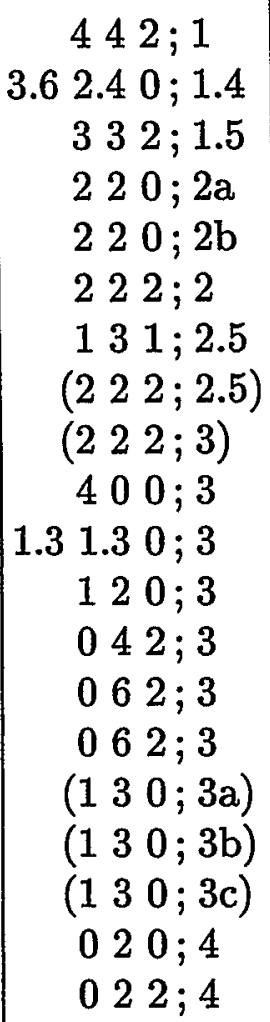 & $\begin{array}{lll}2 & 4 & 2 ; 1 \\
0 & 6 & 0 ; 2 \\
1 & 5 & 1 ; 1.5 \\
0 & 6 & 0 ; 2 \\
0 & 6 & 0 ; 2 \\
0 & 6 & 0 ; 2 \\
0 & 4 & 1 ; 2.5 \\
0 & 4 & 1 ; 2.5 \\
0 & 2 & 2 ; 3 \\
2 & 2 & 6 ; 1 \\
0 & 6 & 0 ; 2 \\
0 & 4 & 0 ; 3 \\
0 & 2 & 2 ; 3 \\
0 & 0 & 6 ; 3 \\
0 & 0 & 6 ; 3 \\
0 & 0 & 6 ; 3 \\
0 & 0 & 6 ; 3 \\
0 & 0 & 6 ; 3 \\
0 & 2 & 0 ; 4 \\
0 & 4 & 0 ; 4\end{array}$ & $\begin{array}{l}x \bar{x} \\
\bar{x} x \bar{y} y \\
5 x 5 \bar{x} \\
z \bar{x} \\
z \bar{y} x \bar{y} y \bar{x} \\
x \bar{x} \\
2 x 2 \bar{z} \\
y \bar{y} \\
y \bar{y} \\
x \bar{x} \\
2 x \bar{z} 2 \bar{y} \\
2 \bar{z} 2 x \\
2 \bar{y} 2 y \\
2 \bar{y} 2 y \\
2 \bar{y} 2 y \\
2 \bar{y} \bar{x} \\
2 \bar{y} \bar{y} 2 \bar{y} \bar{z} \\
2 \bar{y} \bar{x} \\
2 \bar{x} 2 z \\
6 x 6 \bar{x}\end{array}$ & \begin{tabular}{|l|}
$\mathrm{Ni}_{2} \mathrm{Sn}_{2} \square \mathrm{Ni}$ \\
$\mathrm{Ni}_{12} \mathrm{Ge}_{12} \square_{5} \mathrm{Ni}_{7}$ \\
$\mathrm{Mn}_{5} \mathrm{Sn}_{5} \square_{2} \mathrm{Mn}_{3}$ \\
$\mathrm{Ni}_{3} \mathrm{Ge}_{3} \square \mathrm{Ni}_{2}$ \\
$\mathrm{M}_{3} \mathrm{~N}_{3} \square \mathrm{M}_{2}$ \\
$\mathrm{M}_{3} \mathrm{~N}_{3} \square \mathrm{M}_{2}$ \\
$\mathrm{M}_{7} \mathrm{~N}_{7} \square_{2} \mathrm{M}_{5}$ \\
$\mathrm{Mn}_{7} \mathrm{Sn}_{7} \square_{2} \mathrm{Mn}_{5}$ \\
$\mathrm{M}_{4} \mathrm{~N}_{4} \square \mathrm{M}_{3}$ \\
$\mathrm{M}_{4} \mathrm{~N}_{4} \mathrm{M}_{3}$ \\
$\eta^{6}-\mathrm{Cu}_{4} \mathrm{Sn}_{4} \mathrm{Cu} \square_{3}$ \\
$\eta^{8}-\mathrm{Cu}_{4} \mathrm{Sn}_{4} \mathrm{Cu} \square_{3}$ \\
$\mathrm{M}_{4} \mathrm{~N}_{4} \mathrm{M}_{3}$ \\
$\mathrm{Fe}_{4} \mathrm{Ge}_{4} \square \mathrm{Fe}_{3}$ \\
$\mathrm{Pd}_{7} \mathrm{Tl}_{9} \square_{2} \mathrm{Pd}_{6}$ \\
$\mathrm{M}_{8} \mathrm{~N}_{8} \square_{2} \mathrm{M}_{6}$ \\
$\mathrm{Pd}_{7} \mathrm{~Pb}_{9} \square_{2} \mathrm{Pd}_{6}$ \\
$\mathrm{Pt}_{7} \mathrm{In}_{9} \square_{2} \mathrm{Pt}_{6}$ \\
$\eta^{\prime}-\mathrm{Cu}_{5} \mathrm{Sn}_{5} \mathrm{Cu}_{4}$ \\
$\mathrm{M}_{5} \mathrm{~N}_{5} \mathrm{M}_{4}$
\end{tabular} & $\begin{array}{l}\mathrm{MN}_{3}(\mathrm{~b}) \\
\mathrm{MN}_{3}(\mathrm{c}) \\
\\
\mathrm{TiCu}_{3} \\
\mathrm{SnNi}_{3} \\
\mathrm{SnNi}_{3}\end{array}$ & $\left\{\begin{array}{l}\beta-\mathrm{NaFeO}_{2} \\
\\
\mathrm{M}_{2} \mathrm{~N}_{3} \mathrm{~S}_{5} \\
\alpha^{\prime}-\square \mathrm{Ga}_{2} \mathrm{~S}_{3} \\
\alpha-\square \mathrm{Al}_{2} \mathrm{~S}_{3} \\
\mathrm{SiLi}_{2} \mathrm{O}_{3} \\
\mathrm{Si}_{2} \mathrm{Cu}_{5} \mathrm{~S}_{7}\end{array}\right.$ & $\begin{array}{l}\mathrm{xy} / \mathrm{aba} \mathrm{b}^{\mathrm{b}} \\
\mathrm{pq} \\
\mathrm{ac} \mathrm{aba}^{\prime} \mathrm{ca}^{\prime} \mathrm{b} \\
\mathrm{r} \\
\mathrm{t} / \mathrm{ac}^{\prime} / \mathrm{a}^{\prime} \mathrm{c} \\
\mathrm{tt} \\
\mathrm{tt}^{\prime} \\
\mathrm{hh}^{\prime} \\
\mathrm{kh}^{\prime} \mathrm{h}^{\prime} \\
\mathrm{khkh} \\
\mathrm{cc} / \mathrm{u} \\
\mathrm{v} \\
\mathrm{v} \\
\mathrm{v}^{\prime} \mathrm{v}^{\prime \prime} \mathrm{v}^{\prime \prime \prime} \\
\mathrm{v}^{\prime \prime} \mathrm{v}^{\prime \prime \prime} \\
\mathrm{v}^{\prime} \\
\mathrm{s} \\
\mathrm{ecec}\end{array}$ \\
\hline
\end{tabular}
(a) layer sequence $\mathrm{M}_{2} \mathrm{~N},\left(\mathrm{MN}_{2}\right)_{3}$
(b) layer sequence $(\mathrm{MN})_{2},\left(\mathrm{~N}_{2}\right)_{2}$
(c) layer sequence $\left(\mathrm{MN}_{2}\right)_{3}, \mathrm{~N}_{3}$ 
Table 12: Sequences of $\mathrm{CN}$ values of structure families with structural units (Figs. $12,23,26,28,34)$. The structure families are named by the pioneers who discovered the first compound of the family. The underlined values $\mathrm{CN} 126$ for $\mathrm{CaTiO}_{3}, 84$ for $(\mathrm{Sr}, \mathrm{Ca}) \mathrm{CuO}_{2}$ and $6^{\prime}$ for $\mathrm{SrO}$ can segregate in long sequences or combinations. The number $\mathrm{p}_{\mathrm{M}}$ of $\mathrm{M}=\mathrm{Fe}, \mathrm{Tb}, \mathrm{Dy}, \ldots$ atoms next to each $\mathrm{O}$ atom in antistructures like $\mathrm{NaFeO}_{2}$, or $\mathrm{Ni}^{\prime}$ next to each In atom in $\mathrm{Ni}_{2} \mathrm{In}$ related structures, in brackets.

\begin{tabular}{|c|c|c|c|}
\hline struct. unit & $T_{i}(\mathrm{~A}) ; \mathrm{y} / \mathrm{x}$ & $\begin{array}{c}\mathrm{M}_{n} \mathrm{O}_{\mathrm{x} n-\mathrm{x}} \square_{\mathrm{x}} \\
\left(\mathrm{M}_{n} \mathrm{O}_{\mathrm{x}} \square_{\mathrm{x} n-\mathrm{x}}\right)\end{array}$ & $\mathrm{CN}$ \\
\hline \multicolumn{3}{|c|}{ 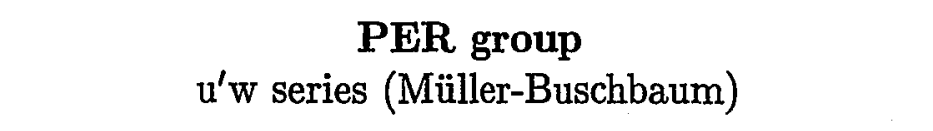 } & $\underline{84_{2}} 7_{2} \underline{6}_{n}^{\prime}$ \\
\hline $\begin{array}{l}\mathrm{w}_{2} \\
\mathrm{u}^{\prime} \mathrm{w}^{\prime} \mathrm{u}^{\prime \prime \prime} \\
\mathrm{u}_{2}^{\prime}\end{array}$ & $\begin{array}{r}008 ; 2 \\
02.72 .7 ; 2 \\
040 ; 2\end{array}$ & $\begin{array}{l}\left(\mathrm{VH}_{2}, \mathrm{SrO}_{2}\right) \\
\mathrm{CuSr}_{2} \mathrm{O}_{3} \square_{6} \\
(\mathrm{Sr}, \mathrm{Ca}) \mathrm{CuO}_{2}\end{array}$ & $\begin{array}{l}6^{\prime} \\
47_{2} \\
84\end{array}$ \\
\hline \multicolumn{3}{|c|}{ uv series (Er-Rakho, Raveau) } & $\underline{612_{2}} 5_{2} \underline{8_{2}} \underline{\underline{4}}$ \\
\hline $\mathrm{uv} \mid$ & $06.40 ; 1.4$ & $\mathrm{Ba}(\mathrm{Cu}, \mathrm{Fe})_{2} \mathrm{YO}_{5}$ & $125_{2} 8$ \\
\hline $\mathrm{v}_{2}$ & \multicolumn{2}{|l|}{$\mathrm{vu}^{\prime}$ series } & $\begin{array}{l}126 \\
\underline{126_{2}} 10_{2} \underline{4_{2} 8}\end{array}$ \\
\hline $\mathrm{vu}^{\prime} \mid$ & $\begin{array}{c}06.40 ; 1.4 \\
\text { 'v series (Raveau }\end{array}$ & $\begin{array}{l}\mathrm{M}_{4} \mathrm{O}_{5} \square_{7} \\
\mathrm{Chu})\end{array}$ & $\begin{array}{l}610_{2} 4 \\
\underline{84_{2}} 10_{2} 5_{2} \underline{8_{2} 4}\end{array}$ \\
\hline$u^{\prime} v u \mid$ & $05.70 ; 1.6$ & $\mathrm{CuBa}_{2} \mathrm{Cu}_{2} \mathrm{YO}_{7}$ & $410_{2} 5_{2} 8$ \\
\hline \multicolumn{3}{|c|}{ vw series (Ruddlesden-Popper, Müller-Bednorz) } & $\underline{126_{2}} 9_{2} \underline{6_{n}^{\prime}}$ \\
\hline $\begin{array}{l}\mathrm{vw}^{\prime} \mathrm{v}^{\prime} \mid \\
\mathrm{vw}^{\prime} \mid \\
\mathrm{vw}_{3}^{\prime} \mathrm{v}^{\prime} \mid \\
\mathrm{v}_{2} \mathrm{w}^{\prime} \mathrm{v}_{2}^{\prime} \mid\end{array}$ & $\begin{array}{rr} & 062 ; 1.25 \\
04.83 .2 ; 1.4 \\
044 ; 1.5 \\
06.91 .1 ; 1.14\end{array}$ & $\begin{array}{l}\mathrm{CuLa}_{2} \mathrm{O}_{4} \\
\mathrm{CuBa}_{2} \mathrm{TlO}_{5} \\
\mathrm{CuSr}_{2} \mathrm{Bi}_{2} \mathrm{O}_{6} \\
\mathrm{LaCu}_{2} \mathrm{La}_{2} \mathrm{O}_{7}\end{array}$ & $\begin{array}{l}69_{2} \\
69_{2} 6^{\prime} \\
69_{2} 6_{2}^{\prime} \\
126_{2} 9_{2}\end{array}$ \\
\hline \multicolumn{3}{|c|}{ uvw' series (Nguyen, Cava) } & $\underline{84_{2}} 8_{2} 5_{2} 9_{2} \underline{6^{\prime}}$ \\
\hline $\begin{array}{l}\mathrm{uvw} w^{\prime} v^{\prime} \mathrm{u}^{\prime \prime} \\
\mathrm{uvw} \mathrm{w}^{\prime} \\
\mathrm{u}^{\prime \prime} \mathrm{v}^{\prime} \mathrm{w}_{3}^{\prime} \mathrm{vu}\end{array}$ & $\begin{array}{r}5.331 .33 ; 1.5 \\
4.572 .29 ; 1.57 \\
043 ; 1.625\end{array}$ & $\begin{array}{l}\mathrm{CaCu}_{2} \mathrm{La}_{2} \mathrm{O}_{6} \\
\mathrm{CaCu}_{2} \mathrm{Ba}_{2} \mathrm{TlO}_{7} \\
\mathrm{CaCu}_{2} \mathrm{Sr}_{2} \mathrm{Bi}_{2} \mathrm{O}_{8}\end{array}$ & $\begin{array}{l}85_{2} 9 \\
85_{2} 9_{2} 6^{\prime} \\
85_{2} 9_{2} 6_{2}^{\prime}\end{array}$ \\
\hline
\end{tabular}


Table 12 (continued)

\begin{tabular}{|c|c|c|c|}
\hline struct. unit & $T_{i}(\mathrm{~A}) ; \mathrm{y} / \mathrm{x}$ & $\begin{array}{c}\mathrm{M}_{n} \mathrm{O}_{\mathrm{xn-x}} \square_{\mathrm{x}} \\
\left(\mathrm{M}_{n} \mathrm{O}_{\mathrm{x}} \square_{\mathrm{x} n-\mathrm{x}}\right)\end{array}$ & CN \\
\hline \multicolumn{3}{|c|}{$\begin{array}{l}\text { hcp } / \mathbf{N i}_{2} \text { In interstitial alloys } \\
\text { p - t (Ellner et al., 1971) }\end{array}$} & \multirow[b]{2}{*}{$\begin{array}{l}\left(23_{4}\right) \\
\left(3_{2} 4\right) \\
(1)\end{array}$} \\
\hline $\begin{array}{l}\mathrm{pq} \\
\mathrm{r} \\
\mathrm{s}\end{array}$ & $\begin{array}{l}3.62 .40 ; 1.4 \\
\quad 220 ; 2 \mathrm{a} \\
\quad 020 ; 4\end{array}$ & $\begin{array}{l}\mathrm{Ni}_{12} \mathrm{Ge}_{12} \square_{5} \mathrm{Ni}_{7} \\
\mathrm{Ni}_{3} \mathrm{Ge}_{3} \square \mathrm{Ni}_{2} \\
\eta^{\prime}-\mathrm{Cu}_{5} \mathrm{Sn}_{5} \mathrm{Cu} \square_{4}\end{array}$ & \\
\hline \multicolumn{3}{|c|}{$\begin{array}{c}\text { ccp interstitial alloys } \\
\mathrm{a}-\mathrm{d}, \mathrm{k}, 1 \text { (Ketelaar, 1935) }\end{array}$} & \\
\hline $\begin{array}{l}\mathrm{a} \\
1 l^{\prime} \\
\mathrm{aaa}^{\prime} \mathrm{a}^{\prime} \\
\mathrm{ac}^{\prime} \\
\mathrm{ac}^{\prime} \mathrm{a}^{\prime} \mathrm{c}^{\prime} \\
\mathrm{c}^{\prime} \mathrm{dc}^{\prime} \mathrm{d}\end{array}$ & 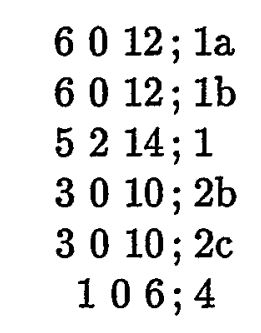 & $\begin{array}{l}\alpha-\mathrm{NaFeO}_{2} \\
\mathrm{LiTbS} \\
\mathrm{NaDyO}_{2} \\
\square_{2} \mathrm{AlCl}_{3} \\
\beta-\mathrm{Na}_{2} \mathrm{PtO}_{3} \\
\mathrm{~K}_{4} \mathrm{UO}_{5}\end{array}$ & $\begin{array}{l}(3) \\
(3) \\
(3) \\
(2) \\
(2) \\
\left(1_{2} 2\right)\end{array}$ \\
\hline \multicolumn{3}{|c|}{ e-i (Forsyth, Gran, 1962) } & \\
\hline $\begin{array}{l}\mathrm{g} \\
\mathrm{i}\end{array}$ & $\begin{array}{r}2212 ; 2 \mathrm{a} \\
028 ; 4 \mathrm{a}\end{array}$ & $\begin{array}{l}\mathrm{Li}_{2} \mathrm{ZrO}_{3} \\
\mathrm{Na}_{4} \mathrm{UO}_{5}\end{array}$ & $\begin{array}{l}(2) \\
\left(1_{2} 2\right)\end{array}$ \\
\hline \multicolumn{3}{|c|}{$\mathrm{u}-\mathrm{y}$ (Johansson, Linde, 1936; Brauer, 1939) } & \\
\hline $\begin{array}{l}x y^{\prime} x^{\prime} y \\
u_{2} u_{2}^{\prime} \\
u\end{array}$ & $\begin{array}{r}4416 ; 1 \\
048 ; 3 \\
060 ; 3\end{array}$ & $\begin{array}{l}\gamma-\mathrm{LiFeO}_{2} \\
\mathrm{Nb}_{4} \mathrm{~N}_{3} \square \\
\mathrm{Nb}_{4} \mathrm{C}_{3} \square\end{array}$ & $\begin{array}{l}(3) \\
{\left[4_{2} 5_{2}\right]} \\
{\left[4_{3} 6\right]}\end{array}$ \\
\hline
\end{tabular}


Table 12 (continued)

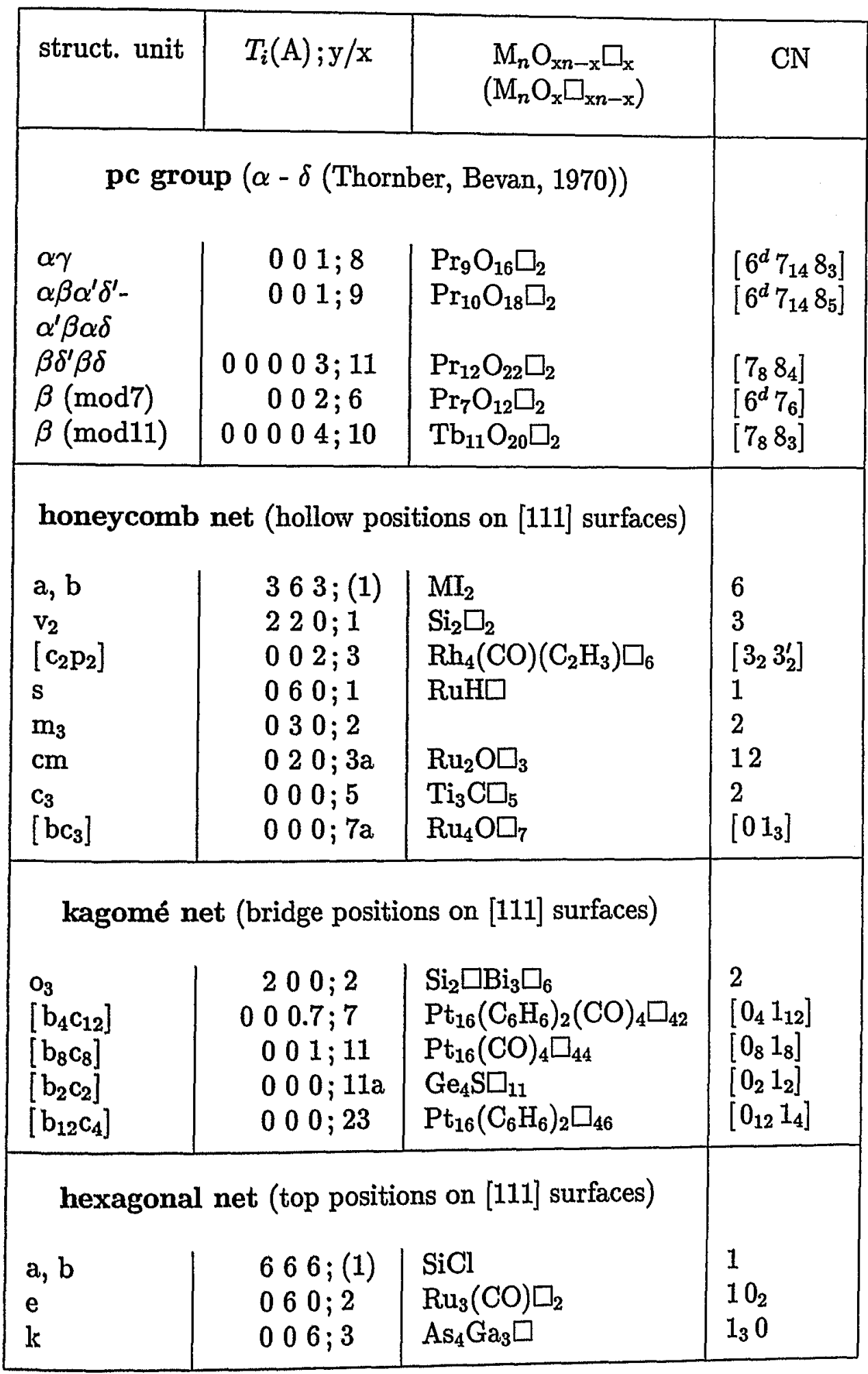


Table 12 (continued)

\begin{tabular}{|c|c|c|c|}
\hline struct. unit & $T_{i}(\mathrm{~A}) ; \mathrm{y} / \mathrm{x}$ & $\begin{array}{c}\mathrm{M}_{n} \mathrm{O}_{\mathrm{xn-x}} \square_{\mathrm{x}} \\
\left(\mathrm{M}_{n} \mathrm{O}_{\mathrm{x}} \square_{\mathrm{x} n-\mathrm{x}}\right)\end{array}$ & $\mathrm{CN}$ \\
\hline \multicolumn{3}{|c|}{$\begin{array}{c}\text { square lattice } \\
\text { hollow positions on }[001] \text { surfaces }\end{array}$} & \multirow[b]{2}{*}{$\begin{array}{l}4 \\
2 \\
2 \\
2_{3} 1_{2} \\
1\end{array}$} \\
\hline $\begin{array}{l}a, b \\
c \\
t \\
t_{3} s_{2} \\
s\end{array}$ & $\begin{array}{l}4444 ;(1) \\
204 ; 1 \\
044 ; 1 \\
0302 ; 1.5 \\
002 ; 3\end{array}$ & $\begin{array}{l}\mathrm{NiAl} \\
\mathrm{Si}_{2} \mathrm{Co} \square \\
\square \mathrm{Cu}_{3} \mathrm{O}_{2} \square_{2} \\
\mathrm{Cu}_{5} \mathrm{~Pb}_{3} \square_{2} \\
\mathrm{Cu}_{4} \mathrm{I}_{3}\end{array}$ & \\
\hline \multicolumn{3}{|c|}{ bridge positions on [001] surfaces } & \multirow{3}{*}{$\begin{array}{l}4 \\
2 \\
2101 \\
1 \\
210_{3} 1\end{array}$} \\
\hline $\begin{array}{l}a, b \\
t \\
t s b s \\
s \\
\text { tsb }_{3} s\end{array}$ & $\begin{array}{l}444 ;(1) \\
0444 ; 1 \\
020 ; 3 \\
002 ; 3 \\
020 ; 5\end{array}$ & $\begin{array}{l}\mathrm{NiSi}_{2} \\
\mathrm{Si}_{2} \mathrm{Sb}_{2} \square_{2} \\
\mathrm{Ge}_{2} \mathrm{~S}_{3} \\
\mathrm{Pd}_{2}(\mathrm{CO}) \square_{3} \\
\mathrm{Ta}_{3} \mathrm{O} \square_{5}\end{array}$ & \\
\hline \multicolumn{3}{|c|}{ top positions on [001] surfaces } & \\
\hline $\mathrm{cbc}$ & $202 ; 2$ & $\mathrm{Si}_{2} \square$ & 101 \\
\hline
\end{tabular}


Table 13a: Hexagonal close-packed interstitial alloys with different occupation at octahedral sites, $T_{i} ; \mathrm{y} / \mathrm{x}$ values of the primitive hexagonal lattice ph $(i=1-7)$ and hexagonal planes with translations $\mathrm{x}, \overline{\mathrm{x}}$ or $2 \mathrm{x}$ to next layer. $T_{1} T_{2} \mathrm{~T}_{3}$ (hex.plane) $\widehat{=}$ $T_{2} T_{5} T_{7}(\mathrm{ph}), T_{1} T_{2} T_{3}\left(\mathrm{ph}^{\prime}\right) \bumpeq T_{1}+T_{2} T_{3} T_{5}(\mathrm{ph})$.

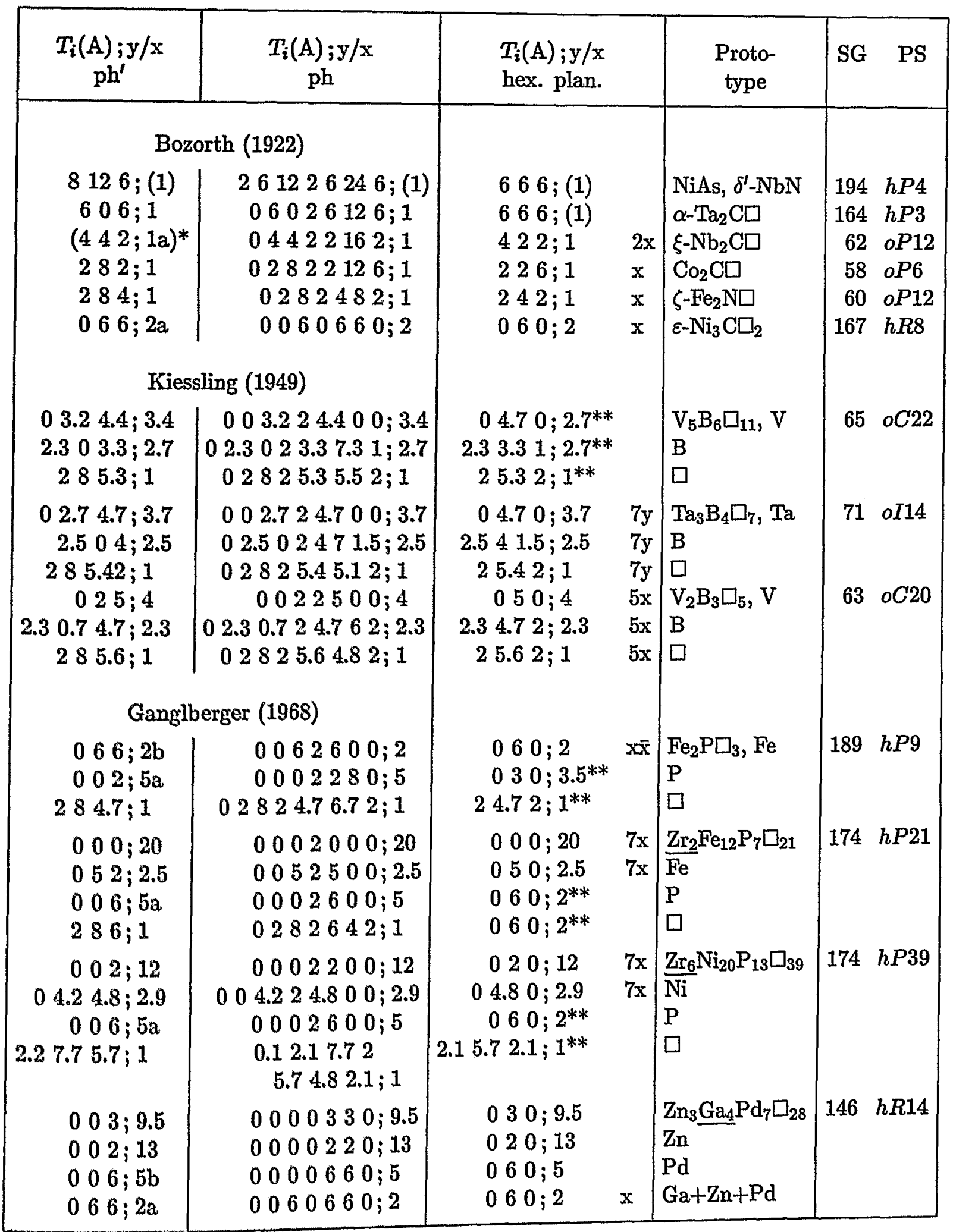


Table 13a (continued)

\begin{tabular}{|c|c|c|c|c|c|}
\hline $\begin{array}{c}T_{i}(\mathrm{~A}) ; \mathrm{y} / \mathrm{x} \\
\mathrm{ph}^{\prime}\end{array}$ & $\begin{array}{l}T_{i}(\mathrm{~A}) ; \mathrm{y} / \mathrm{x} \\
\mathrm{ph}\end{array}$ & $\begin{array}{l}T_{i}(\mathrm{~A}) ; \mathrm{y} / \mathrm{x} \\
\text { hex. plan. }\end{array}$ & $\begin{array}{l}\text { Proto- } \\
\text { type }\end{array}$ & $\mathrm{SG}$ & PS \\
\hline \multicolumn{2}{|c|}{ other structures } & & \multirow{6}{*}{$\varepsilon-\mathrm{Fe}_{2} \mathrm{~N}$} & \multirow{6}{*}{164} & \multirow{6}{*}{$h P 9$} \\
\hline $286 ; 1$ & $0282642 ; 1$ & $060 ; 2^{* *}$ & & & \\
\hline$(3.35 .32 ; 1)$ & $03.35 .32214 .73 .3 ; 1$ & 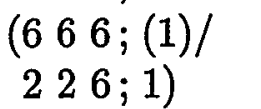 & & & \\
\hline$(2.76 .72 ; 1)$ & $0.726 .70 .7213 .36 ; 1$ & $226 ; 1 \times x x 0$ & & & \\
\hline$(443.3 ; 1)$ & $22423.310 .73 .3 ; 1$ & $\begin{array}{r}(242 ; 1 / \\
226 ; 1)\end{array}$ & & & \\
\hline$(2.76 .74 ; 1)$ & $0.726 .70 .74122 ; 1$ & $242 ; 1 \times x 0$ & & & \\
\hline $066 ; 2 b$ & $0062600 ; 2$ & $060 ; 2 \times \bar{x}$ & $\varepsilon-\mathrm{Fe}_{3} \mathrm{~N}$ & 182 & $h P 8$ \\
\hline $306 ; 2$ & $0302663 ; 2$ & $060 ; 2^{* * *}$ & $\mathrm{AlB}_{2} \square 3, \square$ & 191 & $h P 3$ \\
\hline $066 ; 2 \mathrm{~b}$ & $0062600 ; 2$ & $060 ; 2 \mathrm{x} \overline{\mathrm{x}}$ & $\mathrm{Co}_{2} \mathrm{P} \square_{2}, \mathrm{Co}$ & 62 & $o P 12$ \\
\hline $002 ; 5 b$ & $0002280 ; 5$ & $020 ; 5$ & $\mathrm{P}$ & & \\
\hline $042 ; 3 a$ & $0042202 ; 3$ & $022 ; 3 \mathrm{x}$ & & & \\
\hline $042 ; 3 b$ & $0042242 ; 3$ & $022 ; 3 \mathrm{y} \bar{z}$ & & & \\
\hline $022 ; 4$ & $0020280 ; 4$ & $020 ; 4 \mathrm{x}$ & & & \\
\hline $006 ; 5 a$ & $0002600 ; 5$ & $060 ; 2^{* *}$ & $\mathrm{AlB}_{2}, \mathrm{Al}$ & 191 & $h P 3$ \\
\hline $004 ; 6$ & $0002440 ; 6$ & $040 ; 63 \mathrm{x}$ & $\mathrm{CrB}, \mathrm{Cr}$ & 63 & $o C 8$ \\
\hline $202 ; 6$ & $0202240 ; 6$ & $220 ; 63 x$ & $\mathrm{~B}$ & & \\
\hline
\end{tabular}

* on the borders of the $T_{1} T_{2} T_{3} ; \mathrm{y} / \mathrm{x}$ polyhedron

** containing different hexagonal layers 
Table 13b: All ph structures with single $T_{i}(\mathrm{~A})$ and single $T_{i}(\mathrm{~B})$ values from the reduced cells of Section 33 (structures in curly brackets are homometric), SG and PS, electroneutral clusters (clusters without central atom in brackets).

\begin{tabular}{|c|c|c|c|c|c|}
\hline $\begin{array}{c}T_{i}(\mathrm{~A}) ; \mathrm{y} / \mathrm{x} \\
\mathrm{ph}^{\prime}\end{array}$ & $\begin{array}{c}T_{i}(\mathrm{~A}) ; \mathrm{y} / \mathrm{x} \\
\mathrm{ph}\end{array}$ & $\begin{array}{l}T_{i}(\mathrm{~A}) ; \mathrm{y} / \mathrm{x} \\
\text { hex. plan. }\end{array}$ & SG & PS & $\begin{array}{c}x+y \\
\text { neutr. clust. }\end{array}$ \\
\hline 812620242436824241848 ; (1) & 261226246 ; (1) & $666 ;(1)$ & 191 & $h P 1$ & \\
\hline$(6828128126168624 ; 1)$ & $24822122 ; 1$ & $422 ; 1$ & 51 & $o P 4$ & \\
\hline $60681224126024624 ; 1$ & $06026126 ; 1$ & $666 ;(1)$ & 191 & $h P 2$ & \\
\hline$(562810122051216824 ; 1 a)\}$ & $14602102 ; 1$ & $422 ; 1 \mathrm{z}$ & 12 & $m C 8$ & (26) \\
\hline$(562810122051216824 ; 1 \mathrm{~b})\}$ & $23622102 ; 1$ & $322 ; 1$ & 12 & $m C 16$ & (26) \\
\hline$(462812162041281024 ; 1)$ & $13602122 ; 1$ & $322 ; 1$ y & 2 & $a P 8$ & $(20,204,252)$ \\
\hline$(444128162048161016 ; 1) *$ & $22424122 ; 1$ & $242 ; 1$ & 51 & $o P 4$ & $(8)$ \\
\hline$(443121212204812824 ; 1)$ & $22423104 ; 1$ & $234 ; 1$ & 12 & $m C 16$ & $(8,46,70,94)$ \\
\hline$(44212168204881032 ; 1 \mathrm{a})^{*}$ & $04422162 ; 1$ & $422 ; 12 z$ & 63 & $\circ C 8$ & (8) \\
\hline$\left.(44212168204881032 ; 1 b)^{*}\right\}$ & $2242286 ; 1$ & $226 ; 1$ & 47 & $o P 2$ & (8) \\
\hline$\left.(36481412203128824 \text {; } 1 \mathrm{a})^{*}\right\}$ & $12604142 ; 1 \mathrm{a}\}$ & $242 ; 1 \overline{\mathrm{z}}$ & 12 & $m C 8$ & $(26,70,94)$ \\
\hline$\left.(36481412203128824 ; 1 b)^{*}\right\}$ & $12604142 ; 1 \mathrm{~b}\}$ & $242 ; 10 x$ & 63 & $o C 16$ & $(26,70,94)$ \\
\hline 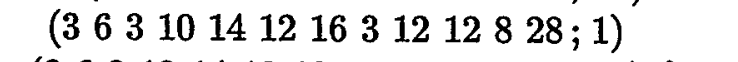 & $12603144 ; 1$ & $234 ; 12 \bar{z}$ & 2 & $a P 8$ & \\
\hline 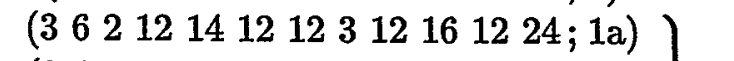 & $12602146 ; 1 \mathrm{a}\}$ & $226 ; 10 y$ & 71 & $o I 8$ & $(46)$ \\
\hline$(36212141212312161224 ; 1 \mathrm{~b})$ & $12602146 ; 1 \mathrm{~b}\}$ & $226 ; 1$ cycl. & 70 & $o F 32$ & $(46)$ \\
\hline 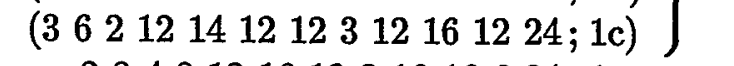 & $03622142 ; 1$ & $322 ; 12 y$ & 12 & $m C 16$ & $(46)$ \\
\hline $284812161221616624 ; 1$ & $0282482 ; 1$ & $242 ; 1 \times$ & 63 & $o C 8$ & $(20,204,252)$ \\
\hline $283128122021612824 ; 1$ & $02823104 ; 1$ & $234 ; 1 \times$ & 12 & $m C 16$ & $(20,26,130)$ \\
\hline $2821648282168148 ; 1$ & $02822126 ; 1$ & $226 ; 1 \mathrm{y}$ & 71 & $o I 4$ & \\
\hline$(4428812204816616 ; 4 / 3 a)\}$ & $2242282 ; 4 / 3 a\}$ & $222 ; 4 / 3$ & 10 & $m P 7$ & 21 \\
\hline$(4428812204816616 ; 4 / 3 b)\}$ & $2242282 ; 4 / 3 b\}$ & $222 ; 4 / 3$ & 174 & $h P 7$ & 21 \\
\hline $6066012081201824 ; 2$ & $0600606 ; 2$ & $666 ;(1)$ & 191 & $h P 3$ & 21 \\
\hline$(440484128168020 ; 2)$ & $2242042 ; 2$ & $202 ; 2$ & 65 & $o C 6$ & 27 \\
\hline$(240881012848014 ; 2)$ & $02400102 ; 2$ & $202 ; 2 \bar{y}$ & 12 & $m C 6$ & 9,21 \\
\hline $20614012081201824 ; 2$ & $20026120 ; 2$ & $060 ; 2$ & 191 & $h P 3$ & 9,27 \\
\hline $0660120188012180 ; 2$ & $0060660 ; 2$ & $060 ; 2 y$ & 166 & $h R 3$ & 21 \\
\hline$(32154916341438 ; 2.5)$ & $2122141 ; 2.5$ & $111 ; 2.5$ & 10 & $m P 7$ & \\
\hline $200812012200624 ; 3$ & $2002006 ; 3$ & $006 ; 3$ & 191 & $h P 4$ & \\
\hline $20020612201200 ; 6$ & $2002000 ; 6$ & $000 ; 6$ & 175 & $h P 7$ & 21 \\
\hline
\end{tabular}


Table 14: Interstitial alloys $M_{z} \square_{x} I_{y}$ with single configuration of tetrahedral or octahedral sites (Fig.27).

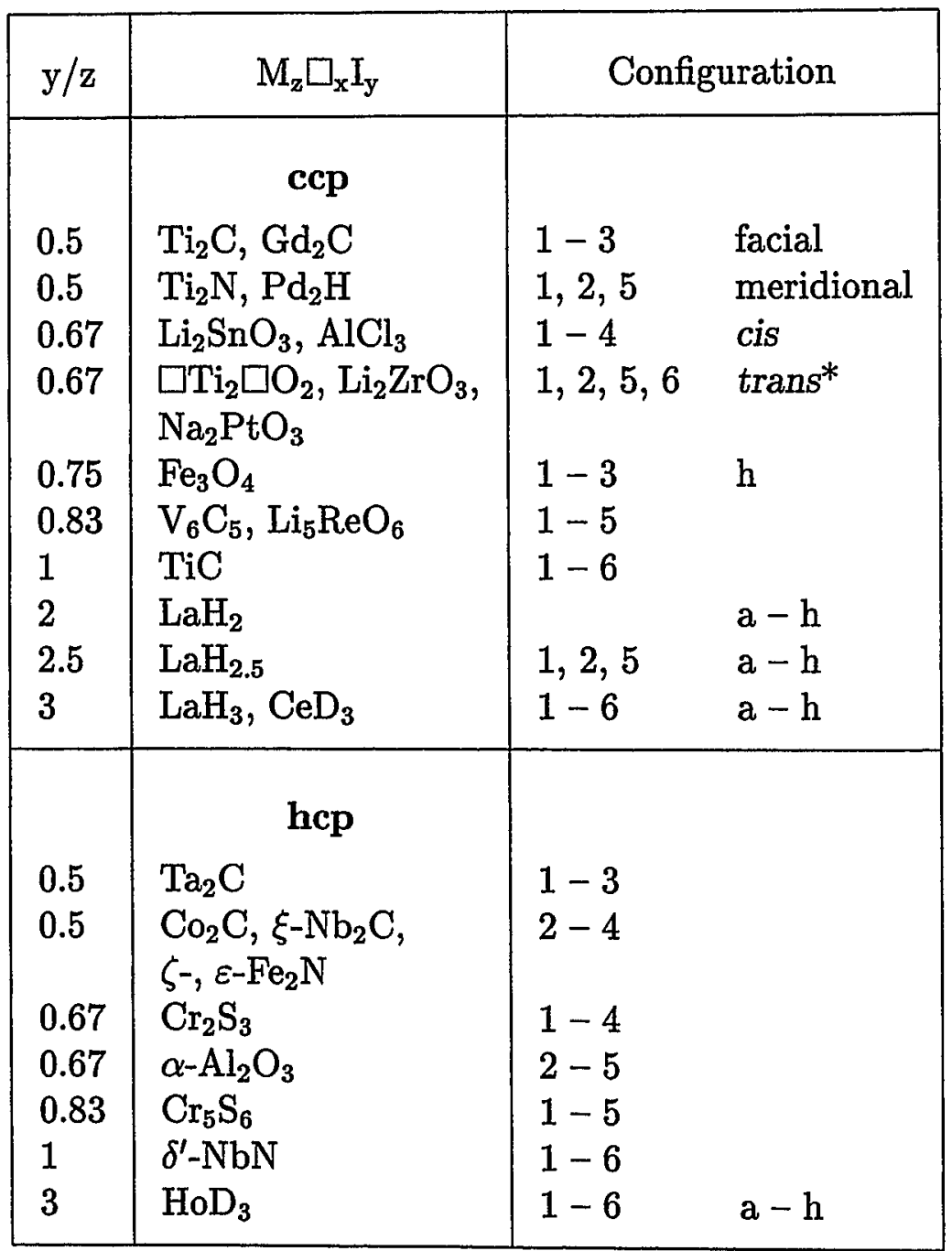

* Some atoms of $\square \mathrm{Ti}_{2} \square \mathrm{O}_{2}$, etc., have a cis configuration. 
Table 15: The $T_{i}(\mathrm{I})$ or $T_{i}(\mathrm{M})$ values of different groups of structures with composition $M_{n} I_{x} \square_{x n-x}$ or $I_{n} M_{x} \square_{x n-x}$ of the antitype indicating the number of shared polyhedra.

\begin{tabular}{|c|c|c|c|c|c|c|}
\hline group & $\square$ at & polyhedra & \multicolumn{3}{|c|}{$\begin{array}{l}\text { number of shared } \\
\text { faces edges corners }\end{array}$} & system \\
\hline $\mathrm{NaCl}$ & $\mathrm{Na} / \mathrm{Cl}$ & octahedra & - & $T_{1}$ & $T_{2}$ & сср \\
\hline $\mathrm{CaF}_{2}$ & & tetrahedra & - & $T_{1}$ & $T_{2}$ & $\mathrm{pc}$ \\
\hline $\mathrm{CaF}_{2}$ & $\mathrm{Ca}$ & cubes & - & $T_{1}$ & - & сcp \\
\hline $\mathrm{ZnS}$ & $\mathrm{Zn} / \mathrm{S}$ & tetrahedra & - & - & $T_{1}$ & $\mathrm{ccp} / \mathrm{hcp}$ \\
\hline $\mathrm{NbN}$ & $\mathrm{N}$ & octahedra & $T_{1}$ & $T_{2}$ & $T_{3}$ & $\mathrm{ph}$ \\
\hline $\mathrm{NiAs}$ & As & prisms & - & $T_{1}$ & $T_{2}$ & hcp \\
\hline $\mathrm{CaTiO}_{3}$ & $\mathrm{O}$ & distorted octah. & $T_{2}$ & $T_{3}, T_{4}$ & $T_{5}$ & PER \\
\hline $\mathrm{CaTiO}_{3}$ & $\mathrm{M}$ & CN 18 & $T_{2}$ & - & $T_{3}$ & bcc \\
\hline $\mathrm{NbH}$ & $\mathrm{H}$ & distorted tetrah. & - & $T_{1}, T_{2}$ & $T_{3}$ & PER \\
\hline $\mathrm{CsCl}$ & $\mathrm{Cs} / \mathrm{Cl}$ & cubes & $T_{2}$ & $T_{3}$ & $T_{5}$ & bcc \\
\hline $\mathrm{Ni}_{2} \mathrm{In}$ & $\mathrm{Ni}^{\prime}$ & CN 11 (Edsham.) & $T_{1}$ & - & $T_{2}$ & hcp \\
\hline $\mathrm{BiF}_{3}$ & $\mathrm{~F}^{\prime}$ & CN 14 (dodecah.) & $T_{1}$ & - & $T_{2}$ & ccp \\
\hline
\end{tabular}


Table 16: s-CN values $T_{i}(\oplus)$ and $T_{i}(\mathrm{M})$ of $\mathrm{M}$ atoms with $\oplus$ or $\ominus$ spin in compounds with composition $\mathrm{R}_{n} \mathrm{M}_{\mathrm{x}} \mathrm{M}_{\mathrm{y}}^{\prime}$ with $T_{i}(\mathrm{M})=T_{i}(\oplus+\ominus)$. The non-magnetic atoms $\mathrm{R}$ are not considered in the different systems. s-CN values, which are not at the borders of structure maps, in brackets. The $T_{i}(\mathrm{~N})$ values of the non-magnetic $\mathrm{R}$ atoms or vacancies are underlined, if the magnetic atoms are the majority components with $\mathrm{y} / \mathrm{x}$ $<1$.

\begin{tabular}{|c|c|c|}
\hline $\mathrm{R}_{n} \mathrm{M}_{\mathrm{x}} \mathrm{M}_{\mathrm{y}}^{\prime}$ & $T_{i}(\oplus) ; \mathrm{r}$ & $T_{i}(\mathrm{M}) ; \mathrm{r}$ \\
\hline \multicolumn{3}{|l|}{ pc } \\
\hline $\mathrm{ZrAlCo}_{2}$ & $6128 ;(1)$ & - \\
\hline$\beta_{1}-\mathrm{ZnMn} / \mathrm{O}_{3} \mathrm{LaCr} / \mathrm{O}_{3} \mathrm{ErFe}$ & $0120 ; 1$ & $6128 ;(1)$ \\
\hline $\mathrm{Pt}_{3} \mathrm{Fe}$ (a) $/ \mathrm{O}_{3} \mathrm{CrEr} / \mathrm{O}_{3} \mathrm{FeEr}$ & $248 ; 1$ & $6128 ;(1)$ \\
\hline $\mathrm{HoRh}$ (i)/AuMn/O ${ }_{3} \mathrm{NdMn}$ & $440 ; 1$ & $6128 ;(1)$ \\
\hline $\mathrm{O}_{3} \mathrm{AlDy}(\searrow)$ & $040 ; 3$ & - \\
\hline $\mathrm{O}_{3} \mathrm{AlDy}(\bar{K})$ & $248 ; 1$ & _ \\
\hline $\mathrm{O}_{3} \mathrm{AlDy}(\searrow \nearrow)$ & $440 ; 1$ & - \\
\hline $\mathrm{O}_{3} \mathrm{AlDy}(\searrow \swarrow)$ & $0120 ; 1$ & $6128 ;(1)$ \\
\hline $\mathrm{O}_{3} \mathrm{CrDy}(\downarrow)$ & $008 ; 3$ & - \\
\hline $\mathrm{O}_{3} \operatorname{CrDy}(\downarrow \uparrow),(\downarrow \leftarrow)$ & $248 ; 1$ & $6128 ;(1)$ \\
\hline \multicolumn{3}{|l|}{ fcc } \\
\hline $\mathrm{Cu}_{2} \mathrm{AlMn} / \mathrm{OEu}$ & 12624 ; (1) & _- \\
\hline NDy & $11.15 .120 .6 ; 1$ & $12624 ;$ (1) \\
\hline $\mathrm{As}_{0.5} \mathrm{Se}_{0.5} \mathrm{U}$ & $(10.75 .318 .7 ; 1)$ & $12624 ;(1)$ \\
\hline $\mathrm{As}_{0.67} \mathrm{Se}_{0.33} \mathrm{U}$ & $(10516 ; 1)$ & 12624 ; (1) \\
\hline $\mathrm{AsU}$ & $(848 ; 1)$ & $12624 ;(1)$ \\
\hline $\mathrm{PHo} / \mathrm{O}_{2} \mathrm{~Tb} / \mathrm{Pd}_{2} \mathrm{InMn}$ & $6012 ; 1 a$ & $12624 ;(1)$ \\
\hline $\mathrm{Cl}_{6} \mathrm{~K}_{2} \mathrm{Ir} / \mathrm{S}_{2} \mathrm{Mn}$ & $4416 ; 1$ & 12624 ; (1) \\
\hline $\mathrm{Se}_{2} \mathrm{Mn}$ & $44.713 .3 ; 1$ & 12624 ; (1) \\
\hline $\mathrm{Te}_{2} \mathrm{Mn} / \gamma-\mathrm{Mn} / \mathrm{PU} / \mathrm{O}_{2} \mathrm{U}$ & $468 ; 1$ & $12624 ;(1)$ \\
\hline $\mathrm{As}_{0.6} \mathrm{Se}_{0.4} \mathrm{U} / \mathrm{Cl}_{6} \mathrm{~K}_{2} \operatorname{Re}$ & $(848 ; 1.5)$ & 12624 ; (1) \\
\hline $\mathrm{Au}_{2} \mathrm{AlMn}$ & $(440 ; 7)$ & 12624 ; (1) \\
\hline $\mathrm{O}_{4} \mathrm{MgV}_{2}(1)$ & $208 ; 3$ & $6012 ; 1 \mathrm{~b}$ \\
\hline $\mathrm{O}_{4} \mathrm{ZnCr}_{2}(\nearrow)$ & $104.5 ; 7$ & - \\
\hline $\mathrm{O}_{4} \mathrm{ZnCr}_{2}(\nearrow \swarrow)$ & $406 ; 3$ & - \\
\hline $\mathrm{O}_{4} \mathrm{ZnCr}_{2}(\nearrow \nwarrow)$ & $208 ; 3$ & - \\
\hline $\mathrm{O}_{4} \mathrm{ZnCr}_{2}(\swarrow \nwarrow)$ & $207 ; 3$ & _ \\
\hline $\mathrm{O}_{4} \mathrm{ZnCr}_{2}(\nearrow \swarrow \nwarrow)$ & $4.309 .5 ; 1.7$ & $6012 ; 1 b$ \\
\hline $\mathrm{O}_{4} \mathrm{CoCr}_{2}(\nearrow),(\leftarrow),(\downarrow)$ & $004 ; 11$ & - \\
\hline $\mathrm{O}_{4} \mathrm{CoCr}_{2}(\nwarrow),(\searrow)$ & $00012 ; 7$ & - \\
\hline $\mathrm{O}_{4} \mathrm{CoCr}_{2}\left(\nearrow_{\leftarrow}\right)$ & $104 ; 5$ & - \\
\hline $\mathrm{O}_{4} \mathrm{CoCr}_{2}(\nearrow \nwarrow)$ & $1.604 .8 ; 3.8$ & - \\
\hline $\mathrm{O}_{4} \mathrm{CoCr}_{2}(\nwarrow \searrow)$ & $204 ; 3$ & - \\
\hline
\end{tabular}


Table 16 (continued)

\begin{tabular}{|c|c|c|}
\hline $\mathrm{R}_{n} \mathrm{M}_{\mathrm{x}} \mathrm{M}_{\mathrm{y}}^{\prime}$ & $T_{i}(\oplus) ; \mathrm{r}$ & $T_{i}(\mathrm{M}) ; \mathrm{r}$ \\
\hline 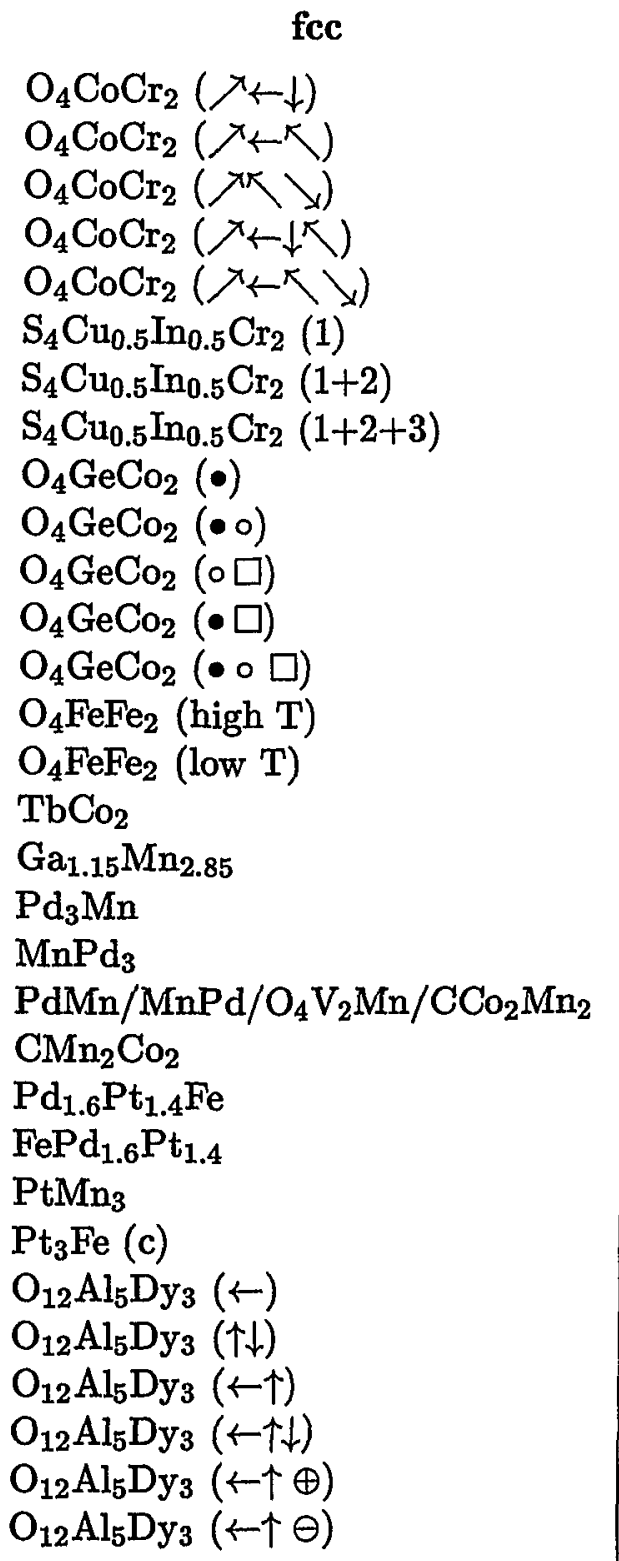 & $\begin{array}{c}204 ; 3 \\
2.906 .9 ; 2.43 \\
3.508 ; 2 \\
408 ; 1.7 \\
4.8010 .4 ; 1.4 \\
300 ; 7 \\
404 ; 3 \\
508 ; 1.7 \\
102 ; 7 \\
204 ; 3 \\
406 ; 3 \\
206 ; 3 \\
4.308 .7 ; 1.7 \\
208 ; 3 \\
004 ; 7 \\
6012 ; 1 \mathrm{~b} \\
048 ; 3 \\
050 ; 7 \\
(64.58 ; 3) \\
060 ; 3 \\
468 ; 1 \\
0204 ; 7 \\
060 ; 3 \\
2.7410 .7 ; 1.7 \\
(5410 ; 1) \\
T_{10}=4 ; 63 \\
T_{8}=2 ; 31 \\
001 ; 31 \\
001.3 ; 20.3 \\
002 ; 20.3 \mathrm{a} \\
002 ; 20.3 \mathrm{~b}\end{array}$ & $\begin{array}{c}- \\
- \\
- \\
6012 ; 1 \mathrm{~b} \\
- \\
- \\
6012 ; 1 \mathrm{~b} \\
- \\
- \\
- \\
- \\
6012 ; 1 \mathrm{~b} \\
6012 ; 1 \mathrm{~b} \\
6012 ; 1 \mathrm{~b} \\
- \\
048 ; 3 \\
054 ; 3 \\
(64.510 ; 1) \\
468 ; 1 \\
- \\
060 ; 3 \\
- \\
060 ; 3 \\
- \\
- \\
- \\
- \\
- \\
- \\
004 ; 9.7\end{array}$ \\
\hline $\begin{array}{l}\quad \text { bcc } \\
\mathrm{O}_{12} \mathrm{Ca}_{3} \mathrm{Ge}_{3} \mathrm{Mn}_{2} / \mathrm{Cl}_{2} \mathrm{Cr} \\
\mathrm{O}_{4} \mathrm{Al}_{2} \mathrm{Co} \\
\mathrm{Al}_{2} \mathrm{Dy} / \mathrm{Co}_{2} \mathrm{~Tb} \\
\mathrm{GePd}_{2} \mathrm{Mn}\left(\mathrm{Mn}_{0.04}\right) \\
\quad\left(\mathrm{Mn}_{0.96}\right) \\
\mathrm{F}_{4} \mathrm{~K}_{2} \mathrm{Ni} \quad \\
\mathrm{O}_{4} \mathrm{Ca}_{2} \mathrm{Mn}\end{array}$ & 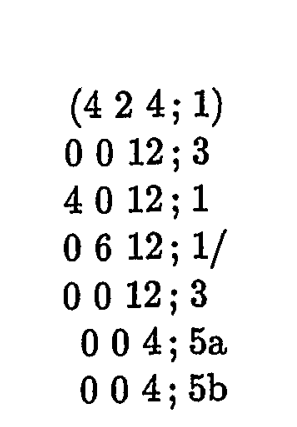 & $\begin{array}{c}8612 ;(1) \\
4012 ; 1 \\
\quad- \\
0012 ; 3\end{array}$ \\
\hline
\end{tabular}


Table 16 (continued)

\begin{tabular}{|c|c|c|}
\hline $\mathrm{R}_{n} \mathrm{M}_{\mathrm{x}} \mathrm{M}_{\mathrm{y}}^{\prime}$ & $T_{i}(\oplus) ; \mathrm{r}$ & $T_{i}(\mathrm{M}) ; \mathrm{r}$ \\
\hline \multicolumn{3}{|l|}{ ph } \\
\hline $\mathrm{TiO}_{3} \mathrm{Fe}(\mathrm{a}) / \alpha-\mathrm{Fe}_{2} \mathrm{O}_{3}$ & $0301693 ; 2$ & $\underline{00606660 ; 2}$ \\
\hline $\mathrm{TiO}_{3} \mathrm{Co}(\mathrm{b})$ & $10306120 ; 2$ & $0060660 ; 2$ \\
\hline $\mathrm{TiO}_{3} \mathrm{Mn}(\mathrm{c}) / \mathrm{O}_{3} \mathrm{Cr}_{2}$ & $0060660 ; 2$ & $\underline{00} 0600660 ; 2$ \\
\hline $\mathrm{S}_{3} \mathrm{Cr}_{2}(\oplus)$ & $0060660 ; 2$ & - \\
\hline $\mathrm{S}_{3} \mathrm{Cr}_{2}(\Theta)$ & $0301693 ; 2$ & $\underline{0301693} ; 2$ \\
\hline$(\mathrm{OH})_{2} \mathrm{Mn}(\uparrow)$ & $0002600 ; 5$ & - \\
\hline$(\mathrm{OH})_{2} \mathrm{Mn}(\uparrow \downarrow)$ & $20026120 ; 2$ & - \\
\hline$(\mathrm{OH})_{2} \mathrm{Mn}(\uparrow \nearrow),(\uparrow \nwarrow),(\nearrow \searrow)$ & $0062600 ; 2$ & - \\
\hline$(\mathrm{OH})_{2} \mathrm{Mn}\left(\uparrow_{\swarrow}\right),(\uparrow \searrow),(\nearrow \nwarrow)$ & 0302663 ; 2 & - \\
\hline$(\mathrm{OH})_{2} \mathrm{Mn}(\uparrow \downarrow \nearrow)$ & $1.32426122 ; 1$ & - \\
\hline$(\mathrm{OH})_{2} \mathrm{Mn}(\uparrow \nearrow \searrow)$ & $0282642 ; 1$ & - \\
\hline$(\mathrm{OH})_{2} \mathrm{Mn}(\uparrow \swarrow \searrow)$ & $06026126 ; 1$ & 261226246 ; (1) \\
\hline \multicolumn{3}{|l|}{ hep } \\
\hline $\mathrm{O}_{2} \mathrm{SYb}_{2}$ & $930 ; 1$ & $1262 ;(1)$ \\
\hline $\mathrm{O}_{2} \mathrm{SDy}_{2}$ & $(530 ; 1)$ & $1262 ;(1)$ \\
\hline $\mathrm{O}_{2} \mathrm{SHo}_{2}$ & $602 ; 1$ & $1262 ;(1)$ \\
\hline$\beta-\mathrm{SMn}$ & $442 ; 1$ & $1262 ;(1)$ \\
\hline $\mathrm{O}_{3} \mathrm{YMn}(\uparrow)$ & $002 ; 5$ & - \\
\hline 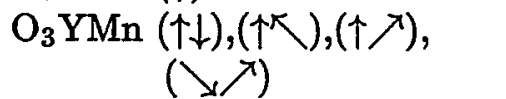 & $222 ; 2$ & - \\
\hline $\mathrm{O}_{3} \mathrm{YMn}(\uparrow \searrow),(\uparrow \swarrow),(\searrow \swarrow)$ & $302 ; 2$ & - \\
\hline $\mathrm{O}_{3} \mathrm{YMn}(\uparrow \downarrow \searrow)$ & $4.72 .72 ; 1$ & - \\
\hline $\mathrm{O}_{3} \mathrm{YMn}(\uparrow \searrow \swarrow)$ & $602 ; 1$ & $1262 ;(1)$ \\
\hline
\end{tabular}


Table 17: Constants $a_{i}, b_{i}, c_{i}$ of different relations between $T_{1}, T_{2}$ values for borders B1, .., B7 (Section 22).

$$
\begin{array}{lll}
\text { B1 : } & T_{1}=\mathrm{a}_{1}, & \\
\text { B2 }: & T_{2}=\mathrm{a}_{2} T_{1}-\mathrm{b}_{2}, & \\
\text { B3 }: & T_{2}=\mathrm{a}_{3} T_{1}-\mathrm{b}_{3}, & 1 \leq \mathrm{r} \leq \mathrm{a}_{4} / \mathrm{b}_{4}, \\
\text { B4: } & T_{2}=\mathrm{a}_{4}-\mathrm{b}_{4} \mathrm{r}, & 1 \leq \mathrm{r} \leq \mathrm{a}_{4} / \mathrm{b}_{4}, \\
\text { B5 : } & T_{2}=-\mathrm{a}_{5} T_{1}+\mathrm{b}_{5}\left(\mathrm{c}_{5}-\mathrm{r}\right), & 1 \leq \mathrm{r} \leq \mathrm{c}_{5}, \\
\text { B6 : } & T_{2}=-\mathrm{a}_{6} T_{1}+\mathrm{b}_{6}\left(\mathrm{c}_{6}-\mathrm{r}\right), & 1 \leq \mathrm{r} \leq \mathrm{c}_{6}, \\
\text { B7 }: & T_{1}=\mathrm{a}_{7}-\mathrm{b}_{7} \mathrm{r}, & 1 \leq \mathrm{r} \leq \mathrm{a}_{7} / \mathrm{b}_{7} .
\end{array}
$$

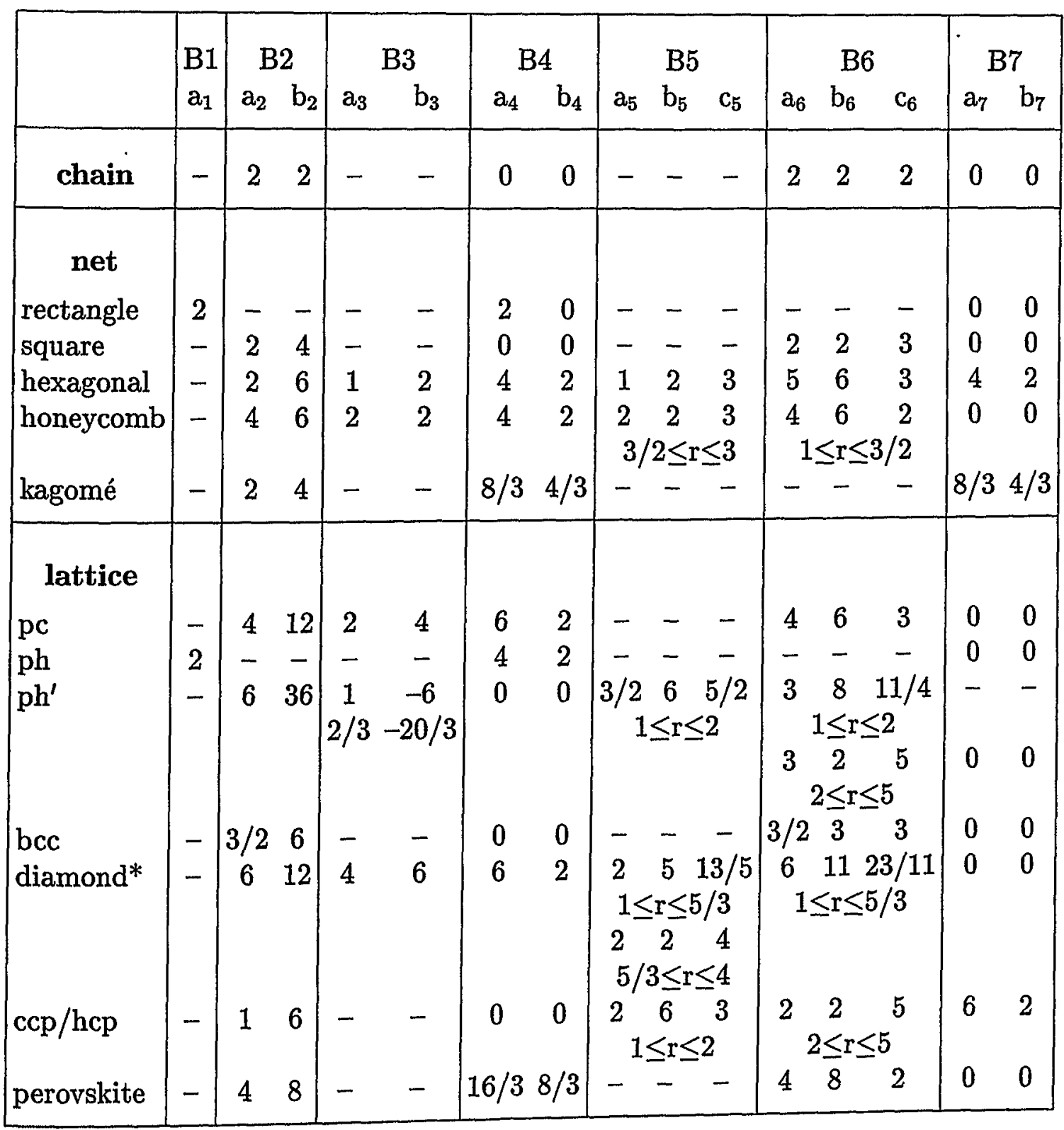

* Additional border B3': $T_{2}=2 T_{1}+2-2 \mathrm{r}, 1 \leq \mathrm{r} \leq 2, T_{2}=2 T_{1}-2, \mathrm{r} \geq 2$. 


\section{Appendix A: Relative ordering numbers, valence electrons, pseudopotential radii and coordina- tion numbers of the elements}

Table 18: Relative ordering number $\mathcal{M}$ for structure maps (Pettifor, 1994), number of valence electrons $\mathrm{VE}$, electronegativity $\mathrm{X}$ and pseudopotential radii sum $\left(r_{s}+r_{p}\right)$ of the elements for the $\Delta \mathrm{VE}, \Delta \mathrm{X}$ and $\Delta\left(r_{s}+r_{p}\right)$ coordinates of the three-dimensional structure-stability plots (Villars, 1994). No VE, X and $r_{s}+r_{p}$ values are given for $\mathcal{M}$ $=1-6(\mathrm{He}-\mathrm{Rn})$ and $\mathcal{M}=34-41(\mathrm{Lr}-\mathrm{Cm})$.

\begin{tabular}{|c|c|c|c|c|c|c|c|c|c|c|c|c|c|c|}
\hline $\mathcal{M}$ & El. & VE & $\mathrm{X}$ & $r_{s}+r_{p}$ & $\mathcal{M}$ & E1. & VE & $\mathrm{X}$ & $r_{s}+r_{p}$ & $\mathcal{M}$ & El. & VE & $\mathrm{X}$ & $r_{s}+r_{p}$ \\
\hline 7 & Fr & 1 & 0.70 & 4.37 & 45 & $\mathrm{U}$ & 3 & 1.7 & 4.72 & 75 & Cd & 12 & 1.40 & 2.215 \\
\hline 8 & Cs & 1 & 0.77 & 4.31 & 46 & $\mathrm{~Pa}$ & 3 & 1.5 & 4.96 & 76 & $\mathrm{Zn}$ & 12 & 1.44 & 1.88 \\
\hline 9 & $\mathrm{Rb}$ & 1 & 0.80 & 4.10 & 47 & $\mathrm{Th}$ & 4 & 1.3 & 4.98 & 77 & $\mathrm{Be}$ & 2 & 1.45 & 1.08 \\
\hline 10 & $\mathbf{K}$ & 1 & 0.80 & 3.69 & 48 & Ac & 3 & 1.10 & 3.12 & 78 & $\mathrm{Tl}$ & 3 & 1.69 & 2.235 \\
\hline 11 & $\mathrm{Na}$ & 1 & 0.89 & 2.65 & 49 & $\mathrm{Zr}$ & 4 & 1.70 & 2.825 & 79 & In & 3 & 1.63 & 2.05 \\
\hline 12 & $\mathrm{Li}$ & 1 & 0.90 & 1.61 & 50 & $\mathrm{Hf}$ & 4 & 1.73 & 2.91 & 80 & $\mathrm{Al}$ & 3 & 1.64 & 1.675 \\
\hline 13 & $\mathrm{Ra}$ & 2 & 0.90 & 3.53 & 51 & $\mathrm{~T} i$ & 4 & 1.86 & 2.58 & 81 & $\mathrm{Ga}$ & 3 & 1.70 & 1.695 \\
\hline 14 & $\mathrm{Ba}$ & 2 & 1.08 & 3.402 & 52 & $\mathrm{Ta}$ & 5 & 1.94 & 2.79 & 82 & $\mathrm{~Pb}$ & 4 & 1.92 & 2.09 \\
\hline 15 & $\mathrm{Sr}$ & 2 & 1.13 & 3.21 & 53 & $\mathrm{Nb}$ & 5 & 2.03 & 2.76 & 83 & $\mathrm{Sn}$ & 4 & 1.88 & 1.88 \\
\hline 16 & $\mathrm{Ca}$ & 2 & 1.17 & 3.00 & 54 & $\mathrm{~V}$ & 5 & 2.22 & 2.43 & 84 & $\mathrm{Ge}$ & 4 & 1.99 & 1.56 \\
\hline 17 & $\mathrm{Yb}$ & 3 & 1.1 & 3.59 & 55 & W & 6 & 1.79 & 2.735 & 85 & $\mathrm{Si}$ & 4 & 1.98 & 1.42 \\
\hline 18 & $\mathrm{Eu}$ & 3 & 1.15 & 3.94 & 56 & Mo & 6 & 1.94 & 2.72 & 86 & B & 3 & 1.90 & 0.795 \\
\hline 19 & $\mathrm{Sc}$ & 3 & 1.50 & 2.75 & 57 & $\mathrm{Cr}$ & 6 & 2.00 & 2.44 & 87 & $\mathrm{Bi}$ & 5 & 2.14 & 1.997 \\
\hline 20 & $\mathrm{Lu}$ & 3 & 1.2 & 3.37 & 58 & $\mathrm{Re}$ & 7 & 2.06 & 2.68 & 88 & $\mathrm{Sb}$ & 5 & 2.14 & 1.765 \\
\hline 21 & $\mathrm{Tm}$ & 3 & 1.2 & 3.60 & 59 & $\mathrm{Tc}$ & 7 & 2.18 & 2.65 & 89 & As & 5 & 2.27 & 1.415 \\
\hline 22 & $\mathrm{Er}$ & 3 & 1.2 & 3.63 & 60 & $\mathrm{Mn}$ & 7 & 2.04 & 2.22 & 90 & $\mathrm{P}$ & 5 & 2.32 & 1.24 \\
\hline 23 & Ho & 3 & 1.2 & 3.65 & 61 & $\mathrm{Fe}$ & 8 & 1.67 & 2.11 & 91 & Po & 6 & 2.40 & 1.90 \\
\hline 24 & Dy & 3 & 1.15 & 3.67 & 62 & $\mathrm{Ru}$ & 8 & 1.97 & 2.605 & 92 & $\mathrm{Te}$ & 6 & 2.38 & 1.67 \\
\hline 25 & $Y$ & 3 & 1.41 & 2.94 & 63 & Os & 8 & 1.85 & 2.65 & 93 & $\mathrm{Se}$ & 6 & 2.54 & 1.285 \\
\hline 26 & $\mathrm{~Tb}$ & 3 & 1.2 & 3.89 & 64 & Co & 9 & 1.72 & 2.02 & 94 & $\mathbf{S}$ & 6 & 2.65 & 1.10 \\
\hline 27 & $\mathrm{Gd}$ & 3 & 1.1 & 3.91 & 65 & Rh & 9 & 1.99 & 2.52 & 95 & $\mathrm{C}$ & 4 & 2.37 & 0.64 \\
\hline 28 & $\mathrm{Sm}$ & 3 & 1.2 & 4.14 & 66 & Ir & 9 & 1.87 & 2.628 & 96 & At & 7 & 2.64 & 1.83 \\
\hline 29 & $\mathrm{Pm}$ & 3 & 1.15 & 3.99 & 67 & $\mathrm{Ni}$ & 10 & 1.76 & 2.18 & 97 & $I$ & 7 & 2.76 & 1.585 \\
\hline 30 & $\mathrm{Nd}$ & 3 & 1.2 & 3.99 & 68 & $\mathrm{Pt}$ & 10 & 1.91 & 2.70 & 98 & $\mathrm{Br}$ & 7 & 2.83 & 1.20 \\
\hline 31 & $\operatorname{Pr}$ & 3 & 1.1 & 4.48 & 69 & $P d$ & 10 & 2.08 & 2.45 & 99 & $\mathrm{Cl}$ & 7 & 2.98 & 1.01 \\
\hline 32 & $\mathrm{Ce}$ & 3 & 1.1 & 4.50 & 70 & $\mathrm{Au}$ & 11 & 1.19 & 2.66 & 100 & $\mathrm{~N}$ & 5 & 2.85 & 0.54 \\
\hline 33 & $\mathrm{La}$ & 3 & 1.35 & 3.08 & 71 & $\mathrm{Ag}$ & 11 & 1.07 & 2.375 & 101 & 0 & 6 & 3.32 & 0.465 \\
\hline 42 & $\mathrm{Am}$ & 3 & 1.3 & 4.89 & 72 & $\mathrm{Cu}$ & 11 & 1.08 & 2.04 & 102 & $\mathrm{~F}$ & 7 & 3.78 & 0.405 \\
\hline 43 & $\mathrm{Pu}$ & 3 & 1.3 & 4.91 & 73 & $\mathrm{Mg}$ & 2 & 1.31 & 2.03 & 103 & $\mathrm{H}$ & 1 & 2.10 & 1.25 \\
\hline 44 & $\mathrm{~Np}$ & 3 & 1.3 & 4.93 & 74 & $\mathrm{Hg}$ & 12 & 1.49 & 2.41 & & & & & \\
\hline
\end{tabular}


Table 19: Coordination numbers $C N$ of $M$ atoms with valencies $+1, \ldots,+8$ (VE) in the order of increasing radii (Shannon, 1976).

\begin{tabular}{|c|c|}
\hline VE & Ions \\
\hline $\begin{array}{l}1 \\
2 \\
6\end{array}$ & $\begin{array}{l}\mathrm{CN}=2 \\
\mathrm{H} \mathrm{D} \mathrm{Cu} \mathrm{Pd} \mathrm{Ag} \\
\mathrm{Hg} \\
\mathrm{U}\end{array}$ \\
\hline $\begin{array}{l}1 \\
2 \\
3 \\
4 \\
5\end{array}$ & $\begin{array}{l}\mathrm{CN}=3 \\
\mathrm{Hg} \\
\mathrm{Be} \\
\mathrm{B} \\
\mathrm{C} \mathrm{Te} \\
\mathrm{N} \\
\text { pyram. } \\
\mathrm{Sb} \\
\mathrm{Cl} \mathrm{Br} \mathrm{I}\end{array}$ \\
\hline $\begin{array}{l}1 \\
2 \\
3 \\
4 \\
5 \\
6 \\
7 \\
8\end{array}$ & $\begin{array}{l}\text { CN }=4 \\
\text { Li Cu Na } \mathrm{Ag} \mathrm{K} \\
\text { Be Ni Mg Cu Co Zn Fe Mn Cd Hg } \\
\text { B Al Ga Fe In Tl } \\
\text { C Si Mn Ge Co Cr Ti Sn Hf Zr Pb Te } \\
\text { P Mn As Cr V Mo Nb } \\
\text { S Fe Mn Cr Se Mo W Te U } \\
\text { Cl Mn Br Tc Ru Re I } \\
\text { Ru Os Xe } \\
\text { square } \\
\text { Ag } \\
\text { Ni Cu Pt Pd Fe } \mathrm{Ag} \mathrm{Pb} \\
\text { Br Ag } \mathrm{Au} \mathrm{Sb}\end{array}$ \\
\hline
\end{tabular}


Table 19 (continued)

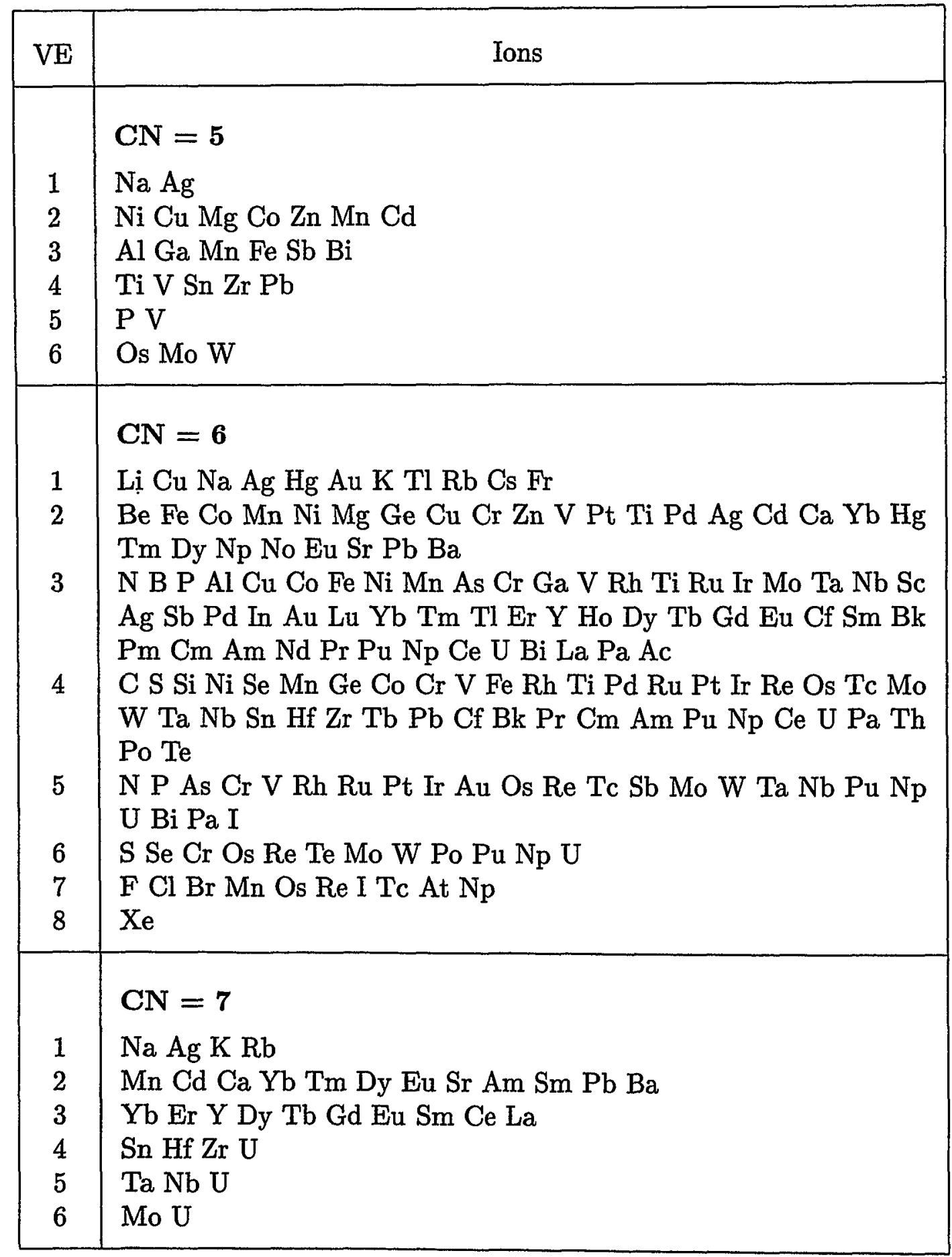


Table 19 (continued)

\begin{tabular}{|c|c|}
\hline VE & Ions \\
\hline $\begin{array}{l}1 \\
2 \\
3 \\
4 \\
5 \\
6\end{array}$ & $\begin{array}{l}\text { CN }=8 \\
\mathrm{Li} \text { Na Ag K Tl Rb Cs } \\
\mathrm{Mg} \mathrm{Zn} \text { Co Fe Mn Cd Ca Yb Hg Dy Eu Sr Am Sm Pb Nd Ba Ra } \\
\text { Fe Sc In Lu Tl Yb Tm Er Ho Y Dy Tb Gd Eu Sm Am Pm Nd Pr Ce La Bi } \\
\text { V Ti Nb Sn Hf Zr Tb Cf Bk Pb Cm Am Pu Pr Ce Np U Pa Th Po } \\
\text { Cr Ta Nb Pa } \\
\text { U }\end{array}$ \\
\hline $\begin{array}{l}1 \\
2 \\
3 \\
4 \\
5\end{array}$ & $\begin{array}{l}\text { CN }=9 \\
\mathrm{Na} \text { K Rb Cs } \\
\mathrm{Ca} \text { Eu Sr Am Sm Pb Nd Ba } \\
\text { Lu Yb Tm Er Ho Y Dy Tb Gd Eu Sm Pm Nd Pr Ce La } \\
\text { Zr U Th } \\
\mathrm{Pa}\end{array}$ \\
\hline $\begin{array}{l}1 \\
2 \\
3 \\
4\end{array}$ & $\begin{array}{l}\mathrm{CN}=\mathbf{1 0} \\
\mathrm{K} \mathrm{Rb} \mathrm{Cs} \\
\mathrm{Ca} \mathrm{Eu} \mathrm{Sr} \mathrm{Pb} \mathrm{Ba} \\
\mathrm{Ho} \mathrm{Ce} \mathrm{La} \\
\mathrm{Ce} \mathrm{Th}\end{array}$ \\
\hline $\begin{array}{l}1 \\
2 \\
4\end{array}$ & $\begin{array}{l}\mathrm{CN}=\mathbf{1 1} \\
\mathrm{Rb} \mathrm{Cs} \\
\mathrm{Pb} \mathrm{Ba} \\
\mathrm{Th}\end{array}$ \\
\hline $\begin{array}{l}1 \\
2 \\
3 \\
4\end{array}$ & $\begin{array}{l}\text { CN = } 12 \\
\mathrm{Na} \mathrm{K} \mathrm{Tl} \mathrm{Rb} \mathrm{Cs} \\
\mathrm{Cd} \mathrm{Ca} \mathrm{Sr} \mathrm{Pb} \mathrm{Ba} \mathrm{Ra} \\
\text { Sm Nd Ce La } \\
\text { Ce U Th }\end{array}$ \\
\hline 1 & $\begin{array}{l}\mathrm{CN}=14 \\
\mathrm{Rb}\end{array}$ \\
\hline
\end{tabular}




\section{Appendix B: Reduced unit cells of the hcp, ccp, bcc and pc lattice, structures with a single set of $T_{i}$ values, group-subgroup relations and isotopes}

Table 20a: Reduced unit cells and space groups (SG) of all possible hep alloys $\mathrm{MN}_{\mathrm{y}}$ $(\mathrm{y}=1,3,5, \ldots, 15)$ with self-coordination numbers $T_{i}(\mathrm{M})(i=1-6)$ of $\mathrm{M}$ atoms. The cell parameters $a^{2}, b^{2}, c^{2}, \mathbf{b c}, \mathbf{a c}$, ab should be divided by 6 to have the unit cell of the LiRh or $\mathrm{Mg}$ structure in units of $a_{0}=1, c_{0}=\sqrt{8 / 3}$ (first line). The Cartesian coordinates of $\mathbf{a}, \mathbf{b}$ and $\mathbf{c}$ for a possible representation of the Bravais lattice should be multiplied by $0.5(\mathrm{x}), \sqrt{3} / 2(\mathrm{y})$ and $\sqrt{8 / 3}$ (z-component).

\begin{tabular}{|c|c|c|c|c|c|c|c|c|c|c|c|}
\hline$\#$ & $T_{i}(\mathrm{M}) ; \mathrm{y}$ & $a^{2}$ & $b^{2}$ & $c^{2}$ & bc & ac & $\mathbf{a b}$ & SG & $\mathbf{a}$ & $\mathbf{b}$ & c \\
\hline 1 & $6026126 ; 1$ & 6 & 6 & 16 & 0 & 0 & -3 & 187 & 200 & $\overline{1} \overline{1} 0$ & 001 \\
\hline $\begin{array}{l}2 \\
3 \\
4 \\
\end{array}$ & $\begin{array}{l}600606 ; 3 \\
202246 ; 3 \\
200286 ; 3 \\
\end{array}$ & $\begin{array}{l}6 \\
6 \\
6 \\
\end{array}$ & $\begin{array}{r}6 \\
16 \\
18 \\
\end{array}$ & $\begin{array}{l}64 \\
18 \\
22 \\
\end{array}$ & $\begin{array}{r}0 \\
0 \\
-9 \\
\end{array}$ & $\begin{array}{r}0 \\
0 \\
-3 \\
\end{array}$ & $\begin{array}{r}-3 \\
0 \\
0 \\
\end{array}$ & $\begin{array}{r}187 \\
25 \\
44 \\
\end{array}$ & $\begin{array}{lll}2 & 0 & 0 \\
2 & 0 & 0 \\
2 & 0 & 0 \\
\end{array}$ & $\begin{array}{lll} & \overline{1} & 0 \\
0 & 0 & 1 \\
0 & \overline{2} & 0 \\
\end{array}$ & $\begin{array}{lll}0 & 0 & 2 \\
0 & 2 & 0 \\
1 & 1 & 1 \\
\end{array}$ \\
\hline $\begin{array}{l}5 \\
6 \\
7 \\
8 \\
9 \\
\end{array}$ & 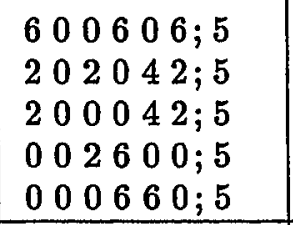 & $\begin{array}{r}6 \\
6 \\
6 \\
16 \\
18 \\
\end{array}$ & $\begin{array}{r}6 \\
16 \\
22 \\
18 \\
18 \\
\end{array}$ & $\begin{array}{r}144 \\
42 \\
34 \\
18 \\
22 \\
\end{array}$ & $\begin{array}{r}0 \\
0 \\
-7 \\
-9 \\
9 \\
\end{array}$ & $\begin{array}{r}0 \\
-3 \\
0 \\
0 \\
9 \\
\end{array}$ & $\begin{array}{r}-3 \\
0 \\
-3 \\
0 \\
9\end{array}$ & $\begin{array}{r}187 \\
35 \\
8 \\
187 \\
155 \\
\end{array}$ & $\begin{array}{lll}2 & 0 & 0 \\
2 & 0 & 0 \\
2 & 0 & 0 \\
0 & 0 & 1 \\
3 & 1 & 0 \\
\end{array}$ & $\begin{array}{lll}\overline{1} & \overline{1} & 0 \\
0 & 0 & 1 \\
\overline{1} & \overline{1} & 1 \\
\overline{3} & 1 & 1 \\
0 & 0 & 0 \\
0 & 2 & 0 \\
\end{array}$ & $\begin{array}{lll}0 & 0 & 3 \\
1 & 3 & 0 \\
0 & \overline{2} & \overline{1} \\
3 & 1 & 0 \\
1 & 1 & 1\end{array}$ \\
\hline $\begin{array}{l}10 \\
11 \\
12 \\
13 \\
14 \\
15 \\
16 \\
17 \\
18 \\
19 \\
\end{array}$ & $\begin{array}{llllll}2 & 0 & 2 & 0 & 4 & 2 ; 7 \\
2 & 0 & 0 & 2 & 0 & 6 ; 7 \\
2 & 0 & 0 & 2 & 0 & 6 ; 7 \mathrm{~b} \\
2 & 0 & 0 & 0 & 4 & 2 ; 7 \\
2 & 0 & 0 & 0 & 0 & 2 ; 7 \\
0 & 0 & 2 & 2 & 0 & 2 ; 7 \\
0 & 0 & 2 & 0 & 0 & 6 ; 7 \\
0 & 0 & 0 & 2 & 4 & 2 ; 7 \\
0 & 0 & 0 & 2 & 4 & 2 ; 7 \mathrm{~b} \\
0 & 0 & 0 & 0 & 4 & 6 ; 7 \\
\end{array}$ & $\begin{array}{r}6 \\
6 \\
6 \\
6 \\
6 \\
16 \\
16 \\
18 \\
18 \\
22 \\
\end{array}$ & $\begin{array}{l}16 \\
18 \\
18 \\
22 \\
34 \\
18 \\
24 \\
22 \\
22 \\
22 \\
\end{array}$ & $\begin{array}{l}72 \\
64 \\
70 \\
58 \\
34 \\
24 \\
24 \\
24 \\
22 \\
24 \\
\end{array}$ & $\begin{array}{r}0 \\
0 \\
-9 \\
4 \\
-2 \\
0 \\
-12 \\
-6 \\
-10 \\
-6 \\
\end{array}$ & $\begin{array}{r}0 \\
0 \\
-3 \\
3 \\
0 \\
0 \\
0 \\
0 \\
0 \\
-6 \\
\end{array}$ & $\begin{array}{r}0 \\
0 \\
0 \\
3 \\
0 \\
0 \\
0 \\
-9 \\
0 \\
-10 \\
\end{array}$ & $\begin{array}{r}25 \\
25 \\
44 \\
8 \\
35 \\
25 \\
187 \\
5 \\
35 \\
42 \\
\end{array}$ & $\begin{array}{lll}2 & 0 & 0 \\
2 & 0 & 0 \\
2 & 0 & 0 \\
2 & 0 & 0 \\
2 & 0 & 0 \\
0 & 0 & 1 \\
0 & 0 & 1 \\
3 & 1 & 0 \\
3 & 1 & 0 \\
1 & 1 & 1 \\
\end{array}$ & $\begin{array}{lll}0 & 0 & 1 \\
0 & \overline{2} & 0 \\
0 & \overline{2} & 0 \\
1 & \overline{1} & 1 \\
0 & \overline{2} & 1 \\
\overline{3} & \overline{1} & 0 \\
\overline{4} & 0 & 0 \\
\overline{1} & \overline{1} & 1 \\
\overline{1} & 1 & \overline{1} \\
1 & 1 & \overline{1} \\
\end{array}$ & $\begin{array}{lll}0 & 4 & 0 \\
0 & 0 & 2 \\
\overline{1} & 1 & 2 \\
1 & 3 & 1 \\
0 & 2 & 1 \\
2 & 2 & 0 \\
2 & 2 & 0 \\
\overline{2} & 2 & 0 \\
\overline{1} & 1 & 1 \\
\overline{4} & 0 & 0\end{array}$ \\
\hline $\begin{array}{l}20 \\
21 \\
22 \\
23 \\
24 \\
25 \\
\end{array}$ & $\begin{array}{llllll}2 & 0 & 2 & 0 & 4 & 2 ; 9 \\
2 & 0 & 0 & 0 & 4 & 2 ; 9 \\
2 & 0 & 0 & 0 & 0 & 2 ; 9 \\
0 & 0 & 2 & 2 & 0 & 0 ; 9 \\
0 & 0 & 0 & 2 & 4 & 0 ; 9 \\
0 & 0 & 0 & 2 & 2 & 0 ; 9 \\
\end{array}$ & $\begin{array}{r}6 \\
6 \\
6 \\
16 \\
18 \\
18 \\
\end{array}$ & $\begin{array}{l}16 \\
22 \\
34 \\
18 \\
22 \\
22 \\
\end{array}$ & $\begin{array}{r}114 \\
88 \\
58 \\
42 \\
40 \\
34 \\
\end{array}$ & $\begin{array}{r}0 \\
-2 \\
-11 \\
-9 \\
-10 \\
-7 \\
\end{array}$ & $\begin{array}{r}-3 \\
0 \\
-3 \\
0 \\
0 \\
-9 \\
\end{array}$ & $\begin{array}{r}0 \\
-3 \\
0 \\
0 \\
-9 \\
0 \\
\end{array}$ & $\begin{array}{r}35 \\
8 \\
8 \\
35 \\
5 \\
5\end{array}$ & $\begin{array}{lll}2 & 0 & 0 \\
2 & 0 & 0 \\
2 & 0 & 0 \\
0 & 0 & 1 \\
3 & 1 & 0 \\
3 & 1 & 0 \\
\end{array}$ & $\begin{array}{lll}0 & 0 & 1 \\
\overline{1} & \overline{1} & 1 \\
0 & \overline{2} & 1 \\
\overline{3} & \overline{1} & 0 \\
\overline{1} & \overline{1} & 1 \\
\overline{1} & 1 & \overline{1} \\
\end{array}$ & $\begin{array}{lll}\overline{1} & 5 & 0 \\
0 & 4 & 1 \\
\overline{1} & 3 & 1 \\
4 & \overline{2} & 0 \\
2 & \overline{2} & \overline{1} \\
\overline{3} & 1 & 1 \\
\end{array}$ \\
\hline $\begin{array}{l}26 \\
27 \\
28 \\
29 \\
30 \\
31\end{array}$ & $\begin{array}{llllll}2 & 0 & 2 & 0 & 4 & 2 ; 11 \\
2 & 0 & 0 & 2 & 0 & 6 ; 11 a \\
2 & 0 & 0 & 2 & 0 & 6 ; 11 \mathrm{~b} \\
2 & 0 & 0 & 0 & 4 & 2 ; 11 \\
2 & 0 & 0 & 0 & 0 & 2 ; 11 \mathrm{a} \\
2 & 0 & 0 & 0 & 0 & 2 ; 11 \mathrm{~b}\end{array}$ & $\begin{array}{l}6 \\
6 \\
6 \\
6 \\
6 \\
6\end{array}$ & $\begin{array}{l}16 \\
18 \\
18 \\
22 \\
34 \\
42\end{array}$ & $\begin{array}{r}162 \\
144 \\
150 \\
130 \\
82 \\
64\end{array}$ & $\begin{array}{r}0 \\
0 \\
-9 \\
8 \\
-14 \\
0\end{array}$ & $\begin{array}{r}0 \\
0 \\
-3 \\
3 \\
0 \\
0\end{array}$ & $\begin{array}{r}0 \\
0 \\
0 \\
3 \\
0 \\
-3\end{array}$ & $\begin{array}{r}25 \\
25 \\
44 \\
8 \\
6 \\
35\end{array}$ & $\begin{array}{lll}2 & 0 & 0 \\
2 & 0 & 0 \\
2 & 0 & 0 \\
2 & 0 & 0 \\
2 & 0 & 0 \\
2 & 0 & 0\end{array}$ & $\begin{array}{lll}0 & 0 & 1 \\
0 & \overline{2} & 0 \\
0 & \overline{2} & 0 \\
1 & \overline{1} & 1 \\
0 & \overline{2} & 1 \\
\overline{1} & \overline{3} & 0\end{array}$ & $\begin{array}{lll}0 & 6 & 0 \\
0 & 0 & 3 \\
\overline{1} & 1 & 3 \\
1 & \overline{5} & \overline{1} \\
0 & \overline{2} & \overline{2} \\
0 & 0 & 2\end{array}$ \\
\hline
\end{tabular}


Table 20a (continued)

\begin{tabular}{|c|c|c|c|c|c|c|c|c|c|c|c|}
\hline$\#$ & $T_{i}(\mathrm{M}) ; \mathrm{y}$ & $a^{2}$ & $b^{2}$ & $c^{2}$ & bc & ac & $\mathbf{a b}$ & SG & $\mathbf{a}$ & $\mathbf{b}$ & c \\
\hline 32 & $200002 ; 11 c$ & 6 & 42 & 70 & 15 & 3 & 3 & 8 & 200 & $1 \overline{3} 0$ & $1 \overline{1} 2$ \\
\hline 33 & $002200 ; 11$ & 16 & 18 & 54 & 0 & 0 & 0 & 25 & 001 & $\overline{3} \overline{1} 0$ & $3 \overline{3} 0$ \\
\hline 34 & $002002 ; 11$ & 16 & 24 & 42 & -6 & 0 & 0 & 6 & 001 & $\overline{4} 00$ & $1 \overline{3} 0$ \\
\hline 35 & $000600 ; 11 a$ & 18 & 18 & 64 & 0 & 0 & -9 & 187 & 310 & $\overline{3} 10$ & 002 \\
\hline 36 & $000600 ; 11 b$ & 18 & 18 & 70 & 9 & 9 & 9 & 155 & 310 & 020 & 112 \\
\hline 37 & $000240 ; 11$ & 18 & 22 & 54 & -9 & 0 & -9 & 5 & 310 & $\bar{I} \overline{1} 1$ & $\overline{3} 30$ \\
\hline 38 & $000220 ; 11$ & 18 & 22 & 40 & -4 & 0 & 0 & 3 & 310 & $\overline{1} 1 \overline{1}$ & $\overline{2} 21$ \\
\hline 39 & $000200 ; 11$ & 18 & 34 & 34 & 7 & 9 & 9 & 44 & 310 & 021 & $3 \overline{1} 1$ \\
\hline 40 & $000042 ; 11$ & 22 & 22 & 42 & -3 & -3 & -10 & 8 & 111 & $11 \overline{1}$ & $\overline{5} 10$ \\
\hline 41 & $000022 ; 11 a$ & 22 & 24 & 40 & 12 & 10 & 6 & 1 & 111 & 400 & $2 \overline{2} 1$ \\
\hline 42 & $000022 ; 11 b$ & 22 & 24 & 34 & 0 & -7 & -6 & 1 & 111 & $\overline{4} 00$ & $02 \overline{1}$ \\
\hline 43 & $200042 ; 13$ & 6 & 22 & 178 & 11 & 3 & 3 & 8 & 200 & $1 \overline{1} 1$ & 152 \\
\hline 44 & $2000002 ; 13$ & 6 & 34 & 106 & -5 & -3 & 0 & 8 & 200 & $0 \overline{2} 1$ & $\overline{1} \overline{3} \overline{2}$ \\
\hline 45 & $002200 ; 13$ & 16 & 18 & 78 & -9 & 0 & 0 & 35 & 001 & $\overline{3} \overline{1} 0$ & $5 \overline{3} 0$ \\
\hline 46 & $002000 ; 13$ & 16 & 42 & 42 & -21 & 0 & 0 & 174 & 001 & $\overline{5} 0$ & $4 \overline{2} 0$ \\
\hline 47 & $000240 ; 13$ & 18 & 22 & 70 & -7 & 0 & -9 & 5 & 310 & $\overline{1} \overline{1} 1$ & $3 \overline{3} \overline{1}$ \\
\hline 48 & $000220 ; 13$ & 18 & 22 & 58 & -1 & -9 & 0 & 5 & 310 & $\overline{1} 1 \overline{1}$ & $\overline{4} 21$ \\
\hline 49 & $000200 ; 13$ & 18 & 34 & 40 & -2 & 0 & -9 & 5 & 310 & $\overline{3} 11$ & $2 \overline{2} 1$ \\
\hline 50 & $000020 ; 13$ & 22 & 34 & 40 & 16 & 10 & 7 & 1 & 111 & $\overline{3} \overline{1} 1$ & $2 \overrightarrow{2} 1$ \\
\hline 51 & $200042 ; 15$ & 6 & 22 & 226 & -5 & 0 & -3 & 8 & 200 & $\overline{1} \overline{1} 1$ & $0 \overline{6} \overline{2}$ \\
\hline 52 & $200002 ; 15$ & 6 & 34 & 136 & -4 & 0 & 0 & 6 & 200 & $0 \overline{2} 1$ & 042 \\
\hline 53 & $002200 ; 15$ & 16 & 18 & 96 & 0 & 0 & 0 & 25 & 001 & $\overline{3} \overline{1} 0$ & $4 \overline{40}$ \\
\hline 54 & $002002 ; 15 a$ & 16 & 24 & 72 & 0 & 0 & 0 & 25 & 001 & 400 & 040 \\
\hline 55 & $002002 ; 15 b$ & 16 & 24 & 78 & -12 & 0 & 0 & 35 & 001 & 400 & 240 \\
\hline 56 & $002000 ; 15$ & 16 & 42 & 42 & -6 & 0 & 0 & 35 & 001 & $\overline{5} 10$ & $\overline{1} \overline{3} 0$ \\
\hline 57 & $000240 ; 15$ & 18 & 22 & 94 & 10 & 9 & 9 & 5 & 310 & 111 & $5 \overline{3} 1$ \\
\hline 58 & $000220 ; 15$ & 18 & 22 & 70 & -2 & 0 & 0 & 3 & 310 & 111 & $3 \overline{3} 1$ \\
\hline 59 & $000202 ; 15 a$ & 18 & 24 & 64 & 0 & 0 & 0 & 25 & 310 & $\overline{2} 20$ & 002 \\
\hline 60 & $000202 ; 15 b$ & 18 & 24 & 70 & -6 & -9 & 0 & 5 & 310 & $\overline{2} 20$ & $\overline{1} 12$ \\
\hline 61 & $000202 ; 15 c$ & 18 & 24 & 70 & -12 & 0 & 0 & 35 & 310 & $\overline{2} 20$ & $1 \tilde{1} 2$ \\
\hline 62 & $000200 ; 15 a$ & 18 & 34 & 58 & 11 & 9 & 9 & 5 & 310 & $02 \overline{1}$ & $\overline{1} 31$ \\
\hline 63 & $000200 ; 15 b$ & 18 & 40 & 40 & -8 & 0 & 0 & 35 & 310 & $\overline{2} 21$ & $2 \overline{2} 1$ \\
\hline 64 & $000042 ; 15 a$ & 22 & 22 & 78 & -6 & -6 & -10 & 42 & 111 & $11 \overline{1}$ & $\overline{7} 10$ \\
\hline 65 & $000042 ; 15 b$ & 22 & 22 & 72 & 0 & 0 & -10 & 35 & 111 & $11 \overline{1}$ & $\overline{6} 20$ \\
\hline 66 & $000022 ; 15 a$ & 22 & 24 & 58 & -6 & -1 & -6 & 1 & 111 & 400 & $13 \overline{1}$ \\
\hline 67 & $000022 ; 15 b$ & 22 & 24 & 58 & 6 & 4 & 6 & 1 & 111 & 400 & $1 \overline{3} 1$ \\
\hline 68 & $000020 ; 15 a$ & 22 & 40 & 40 & 4 & 10 & 10 & 5 & 111 & $\overline{4} 01$ & $2 \overline{2} 1$ \\
\hline 69 & $000020 ; 15 b$ & 22 & 34 & 42 & 9 & 3 & 7 & 1 & 111 & $0 \overline{2} 1$ & $5 \overline{10}$ \\
\hline 70 & $000006 ; 15 a$ & 24 & 24 & 64 & 0 & 0 & -12 & 187 & 400 & $\overline{2} \overline{2} 0$ & 002 \\
\hline 71 & $000006 ; 15 b$ & 24 & 24 & 70 & 6 & 12 & 12 & 42 & 400 & $2 \overline{2} 0$ & 202 \\
\hline 72 & $000002 ; 15 a$ & 24 & 34 & 34 & -2 & 0 & 0 & 35 & 400 & $0 \overline{2} 1$ & 021 \\
\hline 73 & $0000002 ; 15 b$ & 24 & 34 & 40 & -2 & -12 & 0 & 8 & 400 & $0 \overline{2} 1$ & $\overline{2} 21$ \\
\hline 74 & $000002 ; 15 \mathrm{c}$ & 24 & 40 & 40 & 8 & 12 & 12 & 44 & 400 & 221 & 221 \\
\hline 75 & $000000 ; 15$ & 34 & 34 & 40 & -16 & -16 & -2 & 42 & 311 & $\overline{3} \overline{1} 1$ & $2 \overline{2} \overline{1}$ \\
\hline 76 & $000600 ; 17$ & 18 & 18 & 144 & 0 & 0 & -9 & 187 & 310 & $\overline{3} 10$ & 003 \\
\hline
\end{tabular}


Table 20b: Reduced unit cells and space groups (SG) of all ccp alloys $\mathrm{MN}_{\mathrm{y}}(\mathrm{y}=1$ 8) with self-coordination numbers $T_{i}(\mathrm{M})(i=1-3)$ of $\mathrm{M}$ atoms. The cell parameters $a^{2}, b^{2}, c^{2}, \mathbf{b c}, \mathbf{a c}, \mathbf{a b}$ are in units of $a_{0} / 2$ of fcc $\mathrm{Cu}$ structure. The Cartesian coordinates of $\mathbf{a}, \mathbf{b}$ and $\mathbf{c}$ for a possible representation of the Bravais lattice in the same units.

\begin{tabular}{|c|c|c|c|c|c|c|c|c|c|c|c|}
\hline$\#$ & $T_{i}(\mathrm{M}) ; \mathrm{y}$ & $a^{2}$ & $b^{2}$ & $c^{2}$ & bc & ac & $a b$ & SG & $\mathbf{a}$ & $\mathbf{b}$ & c \\
\hline 1 & $12624 ;(1)$ & 2 & 2 & 2 & 1 & 1 & 1 & 225 & 110 & 011 & 101 \\
\hline 2 & $6012 ; 1 \mathrm{a}$ & 2 & 2 & 6 & 1 & 1 & 1 & 166 & 110 & $01 \overline{1}$ & $\overline{1} 21$ \\
\hline 3 & $468 ; 1$ & 2 & 2 & 4 & 0 & 0 & 0 & 123 & 110 & $\overline{1} 10$ & 002 \\
\hline 4 & $606 ; 2$ & 2 & 2 & 12 & 0 & 0 & -1 & 164 & 110 & $\overline{1} 0 \overline{1}$ & $\overline{222}$ \\
\hline 5 & $440 ; 2$ & 2 & 2 & 10 & -1 & -1 & 0 & 139 & 110 & $\overline{1} 10$ & $0 \overline{1} 3$ \\
\hline 6 & $2212 ; 2 a$ & 2 & 4 & 6 & -2 & -1 & 0 & 71 & 110 & $00 \overline{2}$ & $\overline{2} 11$ \\
\hline 7 & $606 ; 3$ & 2 & 2 & 22 & 1 & 1 & 1 & 166 & 110 & 011 & $3 \overline{2} 3$ \\
\hline 8 & $40 ; 3$ & 2 & 2 & 16 & 0 & 0 & 0 & 123 & 110 & $\overline{1} 10$ & 004 \\
\hline $\mathbf{9}$ & $224 ; 3$ & 2 & 4 & 8 & 0 & 0 & 0 & 47 & 110 & 002 & $\overline{2} 20$ \\
\hline 10 & $206 ; 3$ & 2 & 6 & 6 & -1 & 0 & -1 & 12 & 110 & $\overline{2} 11$ & $1 \overline{1} 2$ \\
\hline 11 & $204 ; 3$ & 2 & 6 & 6 & -2 & 0 & 0 & 65 & 110 & $\overline{1} 1 \overline{2}$ & $\overline{1} 12$ \\
\hline 12 & $060 ; 3$ & 4 & 4 & 4 & 0 & 0 & 0 & 221 & 200 & 020 & 002 \\
\hline 13 & $048 ; 3$ & 4 & 4 & 6 & -2 & -2 & 0 & 139 & 200 & $0 \overline{2} 0$ & $\overline{1} 12$ \\
\hline 14 & $606 ; 4$ & 2 & 2 & 34 & 1 & 1 & 1 & 166 & 110 & $01 \overline{1}$ & $\overline{3} 43$ \\
\hline 15 & $440 ; 4$ & 2 & 2 & 26 & -1 & -1 & 0 & 139 & 110 & 110 & $0 \overline{1} 5$ \\
\hline 16 & $224 ; 4$ & 2 & 4 & 14 & -2 & -1 & 0 & 71 & 110 & $00 \overline{2}$ & $\overline{3} 21$ \\
\hline 17 & $204 ; 4$ & 2 & 6 & 10 & 2 & 1 & 1 & 12 & 110 & 121 & 103 \\
\hline 18 & $028 ; 4$ & 4 & 6 & 6 & 1 & 2 & 2 & 87 & 200 & $1 \overline{2} 1$ & 112 \\
\hline 19 & $606 ; 5$ & 2 & 2 & 48 & 0 & 0 & -1 & 164 & 110 & 10 & $\overline{4} 44$ \\
\hline 20 & $440 ; 5$ & 2 & 2 & 36 & 0 & 0 & 0 & 123 & 110 & $\overline{1} 10$ & 006 \\
\hline 21 & $224 ; 5$ & 2 & 4 & 18 & 0 & 0 & 0 & 47 & 110 & 002 & $\overline{3} 30$ \\
\hline 22 & $204 ; 5$ & 2 & 6 & 14 & 2 & 1 & 1 & 12 & 110 & $\overline{1} 2 \overline{1}$ & $\overline{1} 23$ \\
\hline 23 & $202 ; 5 a$ & 2 & 6 & 12 & 0 & 0 & 0 & 10 & 110 & $\overline{1} 1 \overline{2}$ & $\overline{2} 22$ \\
\hline 24 & $202 ; 5 b$ & 2 & 6 & 14 & -3 & -1 & 0 & 12 & 110 & $\overline{1} 1 \overline{2}$ & $\overline{2} 13$ \\
\hline 25 & $200 ; 5$ & 2 & 8 & 10 & -2 & -1 & 0 & 12 & 110 & $\overline{2} 20$ & $0 \overline{1} 3$ \\
\hline 26 & $040 ; 5$ & 4 & 4 & 10 & 0 & -2 & 0 & 65 & 200 & $0 \overline{2} 0$ & $\overline{1} 03$ \\
\hline 27 & $024 ; 5$ & 4 & 6 & 8 & -2 & 0 & -2 & 12 & 200 & $\overline{1} \overline{2} 1$ & 022 \\
\hline 28 & $008 ; 5$ & 6 & 6 & 6 & 1 & 2 & 3 & 12 & 211 & $1 \overline{1} 2$ & $2 \overline{1} \overline{1}$ \\
\hline 29 & $606 ; 6$ & 2 & 2 & 66 & 1 & 1 & 1 & 166 & 110 & 011 & $5 \overline{4} 5$ \\
\hline 30 & $224 ; 6$ & 2 & 4 & 26 & -2 & -1 & 0 & 71 & 110 & $00 \overline{2}$ & $\overline{4} 31$ \\
\hline 31 & $204 ; 6$ & 2 & 6 & 18 & -1 & 0 & -1 & 12 & 110 & $\overline{2} 1 \overline{1}$ & $\overline{1} 14$ \\
\hline 32 & $200 ; 6$ & 2 & 10 & 12 & -4 & 0 & -1 & 12 & 110 & $\overline{1} 0 \overline{3}$ & $\overline{2} 22$ \\
\hline 33 & $024 ; 6$ & 4 & 6 & 10 & -1 & 0 & -2 & 12 & 200 & $\overline{1} \overline{2} 1$ & $0 \overline{1} \overline{3}$ \\
\hline 34 & $006 ; 6$ & 6 & 6 & 6 & -1 & -1 & -1 & 148 & 211 & $\overline{1} \overline{1} 2$ & $1 \overline{2} \overline{1}$ \\
\hline
\end{tabular}


Table 20b (continued)

\begin{tabular}{|c|c|c|c|c|c|c|c|c|c|c|c|}
\hline$\#$ & $T_{i}(\mathrm{M}) ; \mathrm{y}$ & $a^{2}$ & $b^{2}$ & $c^{2}$ & bc & ac & $\mathbf{a b}$ & SG & $\mathbf{a}$ & $\mathbf{b}$ & c \\
\hline 35 & $606 ; 7$ & 2 & 2 & 86 & 1 & 1 & 1 & 166 & 110 & $01 \overline{1}$ & 565 \\
\hline 36 & $224 ; 7$ & 2 & 4 & 32 & 0 & 0 & 0 & 47 & 110 & 002 & $\overline{4} 40$ \\
\hline 37 & $204 ; 7$ & 2 & 6 & 24 & -2 & 0 & -1 & 12 & 110 & $\overline{2} 11$ & $2 \overline{2} 4$ \\
\hline 38 & $202 ; 7 \mathrm{a}$ & 2 & 6 & 22 & -2 & 0 & 0 & 10 & 110 & $\overline{1} 12$ & $3 \overline{3} 2$ \\
\hline 39 & $202 ; 7 \mathrm{~b}$ & 2 & 6 & 22 & -1 & -1 & 0 & 12 & 110 & $\overline{1} 1 \overline{2}$ & $\overline{3} 23$ \\
\hline 40 & $2004 ; 7 a$ & 2 & 8 & 16 & 0 & 0 & 0 & 47 & 110 & $\overline{2} 20$ & $\begin{array}{llll}0 & 0 & 4\end{array}$ \\
\hline 41 & $2004 ; 7 \mathrm{~b}$ & 2 & 8 & 18 & -4 & 0 & 0 & 65 & 110 & $\overline{2} 20$ & $1 \overline{1} 4$ \\
\hline 42 & $2002 ; 7 a$ & 2 & 10 & 14 & 1 & 1 & 1 & 12 & 110 & 013 & $3 \overline{2} 1$ \\
\hline 43 & $2002 ; 7 \mathrm{~b}$ & 2 & 12 & 12 & -4 & 0 & 0 & 65 & 110 & $\overline{2} 22$ & $2 \overline{2} 2$ \\
\hline 44 & $040 ; 7 \mathrm{a}$ & 4 & 4 & 16 & 0 & 0 & 0 & 123 & 200 & 020 & 004 \\
\hline 45 & $040 ; 7 \mathrm{~b}$ & 4 & 4 & 18 & -2 & -2 & 0 & 139 & 200 & $0 \overline{2} 0$ & 114 \\
\hline 46 & $024 ; 7$ & 4 & 6 & 14 & 2 & 2 & 2 & 12 & 200 & $1 \overline{2} 1$ & $1 \overline{2} \overline{3}$ \\
\hline 47 & $0204 ; 7$ & 4 & 8 & 8 & 0 & 0 & 0 & 123 & 200 & $0 \overline{2} 2$ & 022 \\
\hline 48 & $0202 ; 7$ & 4 & 8 & 10 & -4 & 0 & 0 & 65 & 200 & $0 \overline{2} 2$ & 031 \\
\hline 49 & $0044 ; 7$ & 6 & 6 & 8 & 0 & 0 & -2 & 65 & 211 & $\overline{2} 11$ & $0 \overline{2} 2$ \\
\hline 50 & $0042 ; 7 a$ & 6 & 6 & 8 & 0 & -2 & -1 & 2 & 211 & $\overline{1} 2 \overline{1}$ & $\overline{2} 02$ \\
\hline 51 & $0042 ; 7 \mathrm{~b}$ & 6 & 6 & 10 & -2 & -2 & -2 & 69 & 211 & $\overline{2} 11$ & $0 \overline{3} 1$ \\
\hline 52 & $002 ; 7$ & 6 & 8 & 8 & 4 & 2 & 2 & 12 & 211 & $2 \overline{2} 0$ & $20 \overline{2}$ \\
\hline 53 & $000012 ; 7$ & 8 & 8 & 8 & 4 & 4 & 4 & 225 & 220 & 022 & 202 \\
\hline 54 & $606 ; 8$ & 2 & 2 & 108 & 0 & 0 & -1 & 12 & 110 & $\overline{1} 0 \bar{I}$ & $\overline{6} 66$ \\
\hline 55 & $224 ; 8$ & 2 & 4 & 42 & -2 & -1 & 0 & 71 & 110 & $00 \overline{2}$ & $\overline{5} 41$ \\
\hline 56 & $204 ; 8$ & 2 & 6 & 30 & 1 & 1 & 1 & 164 & 110 & 121 & $2 \overline{1} 5$ \\
\hline 57 & $2002 ; 8 \mathrm{a}$ & 2 & 10 & 18 & -3 & 0 & -1 & 65 & 110 & 103 & $3 \overline{3} 0$ \\
\hline 58 & $2002 ; 8 \mathrm{~b}$ & 2 & 12 & 14 & 0 & -1 & 0 & 12 & 110 & $\overline{2} 2 \overline{2}$ & $\overline{2} 13$ \\
\hline 59 & $024 ; 8$ & 4 & 6 & 18 & 3 & 2 & 2 & 12 & 200 & $1 \overline{2} \overline{1}$ & $11 \overline{4}$ \\
\hline 60 & $0200 ; 8 \mathrm{a}$ & 4 & 10 & 10 & 1 & 2 & 2 & 139 & 200 & $1 \overline{3} 0$ & $10 \overline{3}$ \\
\hline 61 & $0200 ; 8 \mathrm{~b}$ & 4 & 10 & 10 & -3 & 0 & -2 & 12 & 200 & $\overline{1} \overline{3} 0$ & $0 \underline{1} \overline{3}$ \\
\hline 62 & $0060 ; 8 \mathrm{a}$ & 6 & 6 & 12 & 0 & 0 & -3 & 2 & 211 & $\overline{1} \overline{2} 1$ & $2 \overline{2} \overline{2}$ \\
\hline 63 & $0060 ; 8 \mathrm{~b}$ & 6 & 6 & 14 & 3 & 3 & 3 & 164 & 211 & $1 \overline{1} 2$ & $3 \underline{2} \overline{1}$ \\
\hline 64 & $004 ; 8$ & 6 & 6 & 10 & 1 & 2 & 1 & 166 & 211 & $\overline{1} 21$ & $0 \overline{1} 3$ \\
\hline
\end{tabular}


Table 20c: Reduced unit cells and space groups (SG) of all bcc alloys $\mathrm{MN}_{\mathrm{y}}(\mathrm{y}=1-$ 8) with self-coordination numbers $T_{i}(\mathrm{M})(i=1-3)$ of $\mathrm{M}$ atoms. The cell parameters $a^{2}, b^{2}, c^{2}, \mathbf{b c}, \mathbf{a c}$, ab are in units of $a_{0} / 2$ of the bcc W structure. The Cartesian coordinates of $\mathbf{a}, \mathbf{b}$ and $\mathbf{c}$ for a possible representation of the Bravais lattice in the same units.

\begin{tabular}{|c|c|c|c|c|c|c|c|c|c|c|c|}
\hline$\#$ & $T_{i}(\mathrm{M}) ; \mathrm{y}$ & $a^{2}$ & $b^{2}$ & $c^{2}$ & bc & ac & $\mathbf{a b}$ & SG & $\mathbf{a}$ & $\mathbf{b}$ & c \\
\hline 1 & $8612 ;(1)$ & 3 & 3 & 3 & -1 & -1 & -1 & 229 & 111 & $1 \overline{1} \overline{1}$ & $\overline{1} 1 \overline{1}$ \\
\hline 2 & $424 ; 1 a$ & 3 & 3 & 8 & 0 & 0 & -1 & 65 & 111 & $\overline{1} \overline{1} 1$ & $2 \overline{2} 0$ \\
\hline 3 & $0612 ; 1$ & 4 & 4 & 4 & 0 & 0 & 0 & 221 & 200 & 020 & 002 \\
\hline 4 & $422 ; 2 a$ & 3 & 3 & 19 & -1 & -1 & -1 & 69 & 111 & $\overline{1} \overline{1} 1$ & $3 \overline{3} \overline{1}$ \\
\hline 5 & $206 ; 2$ & 3 & 8 & 8 & -4 & 0 & 0 & 164 & 111 & $\overline{2} 02$ & $2 \overline{2} 0$ \\
\hline 6 & $044 ; 2$ & 4 & 4 & 11 & -2 & -2 & 0 & 139 & 200 & 020 & $1 \overline{13}$ \\
\hline 7 & $422 ; 3$ & 3 & 3 & 32 & 0 & 0 & -1 & 65 & 111 & $\overline{1} \overline{1} 1$ & $4 \overline{4} 0$ \\
\hline 8 & $202 ; 3$ & 3 & 8 & 11 & 0 & -1 & 0 & 10 & 111 & $\overline{2} 02$ & $1 \overline{3} 1$ \\
\hline 9 & $200 ; 3$ & 3 & 11 & 11 & -5 & -1 & -1 & 166 & 111 & $\overline{3} 11$ & $1 \overline{3} 1$ \\
\hline 10 & $044 ; 3$ & 4 & 4 & 16 & 0 & 0 & 0 & 123 & 200 & 020 & 004 \\
\hline 11 & $024 ; 3$ & 4 & 8 & 8 & 0 & 0 & 0 & 123 & 200 & 022 & $02 \overline{2}$ \\
\hline 12 & $022 ; 3$ & 4 & 8 & 11 & -4 & -2 & 0 & 71 & 200 & $0 \overline{2} \overline{2}$ & $\overline{1} 3 \overline{1}$ \\
\hline 13 & $0012 ; 3$ & 8 & 8 & 8 & 4 & 4 & 4 & 225 & 220 & 022 & 202 \\
\hline 14 & $422 ; 4$ & 3 & 3 & 51 & -1 & -1 & -1 & 69 & 111 & $\overline{1} \overline{1} 1$ & $5 \overline{5} \overline{1}$ \\
\hline 15 & $202 ; 4$ & 3 & 8 & 19 & -4 & -1 & 0 & 12 & 111 & $\overline{2} 20$ & $\overline{1} \overline{3} 3$ \\
\hline 16 & $044 ; 4$ & 4 & 4 & 27 & -2 & -2 & 0 & 139 & 200 & 020 & $\overline{1} \overrightarrow{1} 5$ \\
\hline 17 & $020 ; 4$ & 4 & 11 & 11 & 1 & 2 & 2 & 87 & 200 & $1 \overline{3} \overline{1}$ & $11 \overline{3}$ \\
\hline 18 & $006 ; 4$ & 8 & 8 & 11 & 4 & 4 & 4 & 166 & 220 & $02 \overline{2}$ & $\overline{1} 31$ \\
\hline 19 & $422 ; 5$ & 3 & 3 & 72 & 0 & 0 & -1 & 65 & 111 & $\overline{1} \overline{1} 1$ & $6 \overline{6} 0$ \\
\hline 20 & $202 ; 5$ & 3 & 8 & 24 & 0 & 0 & 0 & 10 & 111 & $2 \overline{2} 0$ & $22 \overline{4}$ \\
\hline 21 & $200 ; 5$ & 3 & 11 & 19 & 3 & 1 & 1 & 2 & 111 & $\overline{1} \overline{1} 3$ & $3 \overline{3} 1$ \\
\hline 22 & $044 ; 5$ & 4 & 4 & 36 & 0 & 0 & 0 & 123 & 200 & 020 & 006 \\
\hline 23 & $022 ; 5 a$ & 4 & 8 & 19 & 0 & -2 & 0 & 65 & 200 & $0 \overline{2} \overline{2}$ & $\overline{1} 3 \overline{3}$ \\
\hline 24 & $022 ; 5 b$ & 4 & 8 & 20 & -4 & 0 & 0 & 65 & 200 & $0 \underline{2} \overline{2}$ & $04 \overline{2}$ \\
\hline 25 & $020 ; 5$ & 4 & 11 & 16 & -4 & 0 & -2 & 12 & 200 & $\overline{1} \overline{3} \overline{1}$ & 004 \\
\hline 26 & $006 ; 5$ & 8 & 8 & 12 & 0 & 0 & -4 & 164 & 220 & $\overline{2} 0 \overline{2}$ & $\overline{2} 22$ \\
\hline 27 & $004 ; 5$ & 8 & 8 & 11 & 0 & -4 & 0 & 65 & 220 & $\overline{2} 20$ & $\overline{1} \overline{1} 3$ \\
\hline 28 & $002 ; 5$ & 8 & 11 & 11 & 5 & 4 & 4 & 12 & 220 & 131 & 113 \\
\hline 29 & $422 ; 6$ & 3 & 3 & 99 & -1 & -1 & -1 & 69 & 111 & $\overline{1} \overline{1} 1$ & $7 \overline{7} \overline{1}$ \\
\hline 30 & $202 ; 6$ & 3 & 8 & 35 & -4 & -1 & 0 & 12 & 111 & $\overline{2} 02$ & $3 \overline{5} 1$ \\
\hline 31 & $200 ; 6$ & 3 & 19 & 19 & -9 & -1 & -1 & 148 & 111 & $\overline{3} \overline{1} 3$ & $3 \overline{3} \overline{1}$ \\
\hline 32 & $044 ; 6$ & 4 & 4 & 51 & -2 & -2 & 0 & 139 & 200 & 020 & $\overline{1} \overline{1} 7$ \\
\hline 33 & $020 ; 6$ & 4 & 11 & 20 & -2 & 0 & -2 & 12 & 200 & $\overline{1} \overline{3} \overline{1}$ & $02 \overline{4}$ \\
\hline 34 & $006 ; 6$ & 8 & 8 & 19 & 4 & 4 & 4 & 166 & 220 & 022 & $3 \overline{1} 3$ \\
\hline 35 & $002 ; 6$ & 8 & 11 & 11 & -1 & 0 & -4 & 12 & 220 & $\overline{3} 11$ & $1 \overline{1} 3$ \\
\hline
\end{tabular}


Table 20c (continued)

\begin{tabular}{|c|c|c|c|c|c|c|c|c|c|c|c|}
\hline \# & $T_{i}(\mathrm{M}) ; \mathrm{y}$ & $a^{2}$ & $b^{2}$ & $c^{2}$ & bc & ac & $a b$ & SG & a & b & c \\
\hline 36 & $422 ; 7$ & 3 & 3 & 128 & 0 & 0 & -1 & 65 & 111 & $\overline{1} \overline{1} 1$ & $8 \overline{8} 0$ \\
\hline 37 & $202 ; 7$ & 3 & 8 & 43 & 0 & -1 & 0 & 10 & 111 & $\overline{2} 20$ & $\overline{3} \overline{3} 5$ \\
\hline 38 & $2002 ; 7 a$ & 3 & 11 & 32 & 0 & 0 & -1 & 10 & 111 & $\overline{3} 11$ & $0 \overline{4} 4$ \\
\hline 39 & $2002 ; 7 \mathrm{~b}$ & 3 & 11 & 35 & -5 & -1 & -1 & 12 & 111 & $\overline{3} 11$ & 153 \\
\hline 40 & $2000 ; 7$ & 3 & 19 & 19 & 3 & 1 & 1 & 12 & 111 & $\overline{3} 13$ & $1 \overline{3} 3$ \\
\hline 41 & $044 ; 7$ & 4 & 4 & 64 & 0 & 0 & 0 & 123 & 200 & 020 & 008 \\
\hline 42 & $022 ; 7 \mathrm{a}$ & 4 & 8 & 32 & 0 & 0 & 0 & 47 & 200 & 022 & $04 \overline{4}$ \\
\hline 43 & $022 ; 7 \mathrm{~b}$ & 4 & 8 & 35 & -4 & -2 & 0 & 71 & 200 & $0 \overline{2} \overline{2}$ & $\overline{1} 5 \overline{3}$ \\
\hline 44 & $0204 ; 7$ & 4 & 11 & 27 & 3 & 2 & 2 & 12 & 200 & 131 & $1 \overline{1} 5$ \\
\hline 45 & $0200 ; 7 \mathrm{a}$ & 4 & 16 & 16 & 0 & 0 & 0 & 123 & 200 & 040 & 004 \\
\hline 46 & $0200 ; 7 \mathrm{~b}$ & 4 & 16 & 20 & -8 & 0 & 0 & 65 & 200 & 040 & $\underline{0} \overline{2} 4$ \\
\hline 47 & $006 ; 7$ & 8 & 8 & 24 & 4 & 4 & 4 & 166 & 220 & $02 \overline{2}$ & $\overline{2} 42$ \\
\hline 48 & $004 ; 7 \mathrm{a}$ & 8 & 8 & 16 & 0 & 0 & 0 & 123 & 220 & $\overline{2} 20$ & 004 \\
\hline 49 & $004 ; 7 \mathrm{~b}$ & 8 & 8 & 20 & -4 & -4 & 0 & 139 & 220 & $\overline{2} 20$ & $0 \overline{2} 4$ \\
\hline 50 & $0024 ; 7$ & 8 & 11 & 16 & -4 & 0 & -4 & 12 & 220 & $\overline{3} 1 \overline{1}$ & 004 \\
\hline 51 & $0022 ; 7$ & 8 & 11 & 12 & -2 & 0 & 0 & 10 & 220 & $\overline{1} 1 \overline{3}$ & $\overline{2} 22$ \\
\hline 52 & $0020 ; 7$ & 8 & 12 & 12 & -4 & 0 & 0 & 65 & 220 & $\overline{2} 22$ & $2 \overline{2} 2$ \\
\hline 53 & $0006 ; 7$ & 11 & 11 & 11 & -1 & -1 & -5 & 12 & 311 & $\overline{1} \overline{3} 1$ & $1 \overline{1} \overline{3}$ \\
\hline 54 & $0004 ; 7$ & 11 & 11 & 12 & -2 & -2 & -5 & 12 & 311 & $\overline{1} 1 \overline{3}$ & $\overline{2} 22$ \\
\hline 55 & $\begin{array}{lllll}0 & 0 & 0 & 0 & 8 ; 7\end{array}$ & 12 & 12 & 12 & -4 & -4 & -4 & 229 & 222 & $\overline{2} \overline{2} 2$ & $2 \overline{2} \overline{2}$ \\
\hline 56 & $2024 ; 8$ & 3 & 8 & 56 & -4 & 0 & 0 & 65 & 111 & 202 & $4 \overline{6} 2$ \\
\hline 57 & $00 ; 8 a$ & 3 & 19 & 24 & -4 & 0 & -1 & 2 & 111 & & $\overline{2} \overline{2} 4$ \\
\hline 58 & $000 ; 8 \mathrm{~b}$ & 3 & 24 & 24 & -12 & 0 & 0 & 164 & 111 & $\overline{4} 22$ & $2 \overline{4} 2$ \\
\hline 59 & $0204 ; 8$ & 4 & 11 & 35 & 5 & 2 & 2 & 12 & 200 & $1 \overline{3} 1$ & $1 \overline{3} \overline{5}$ \\
\hline 60 & $0200 ; 8 \mathrm{a}$ & 4 & 19 & 19 & 1 & 2 & 2 & 139 & 200 & $1 \overline{3} \overline{3}$ & $13 \overline{3}$ \\
\hline 61 & $0200 ; 8 \mathrm{~b}$ & 4 & 19 & 20 & -6 & 0 & -2 & 12 & 200 & $\overline{1} \overline{3} \overline{3}$ & $04 \overline{2}$ \\
\hline 62 & $24 \cdot 8$ & 8 & 11 & 19 & -3 & 0 & -4 & 12 & 220 & $\overline{1} \overline{1} \overline{3}$ & $\overline{3} 31$ \\
\hline 63 & $024 ; 8 b$ & 8 & 11 & 20 & 2 & 4 & 4 & 69 & 220 & $\overline{1} 3 \overline{1}$ & 024 \\
\hline 64 & $0060 ; 8$ & 8 & 8 & 27 & 0 & 0 & -4 & 164 & 220 & $\overline{2} 0 \overline{2}$ & $\overline{3} 33$ \\
\hline 65 & & 8 & 11 & 19 & -5 & -4 & 0 & 12 & 220 & & $\overline{3} 13$ \\
\hline 66 & $0006 ; 8$ & 11 & 11 & 11 & -1 & -1 & -1 & 166 & 311 & 亩高 3 & $1 \overline{3} \overline{1}$ \\
\hline
\end{tabular}


Table 20d: Reduced unit cells and space groups (SG) of all pc alloys $\mathrm{MN}_{\mathrm{y}}(\mathrm{y}=1-$ 8) with self-coordination numbers $T_{i}(\mathrm{M})(i=1-3)$ of $\mathrm{M}$ atoms. The cell parameters $a^{2}, b^{2}, c^{2}, \mathrm{bc}, \mathrm{ac}, \mathrm{ab}$ are in units of $a_{0}$ of the pc structure or $a_{0} / 2$ of the $\mathrm{F}$ atoms of the $\mathrm{CaF}_{2}$ structure. The Cartesian coordinates of $\mathbf{a}, \mathbf{b}$ and $\mathbf{c}$ for a possible representation of the Bravais lattice in the same units.

\begin{tabular}{|c|c|c|c|c|c|c|c|c|c|c|c|}
\hline \# & $T_{i}(\mathrm{M}) ; \mathrm{y}$ & $a^{2}$ & $b^{2}$ & $c^{2}$ & bc & ac & $\mathbf{a b}$ & SG & a & b & c \\
\hline 1 & $6128 ;(1)$ & 1 & 1 & 1 & 0 & 0 & 0 & 221 & 100 & 010 & 0001 \\
\hline 2 & $440 ; 1$ & 1 & 1 & 4 & 0 & 0 & 0 & 123 & 100 & 010 & 002 \\
\hline 3 & $248 ; 1$ & 1 & 2 & 2 & 0 & 0 & 0 & 123 & 100 & 011 & $0 \overline{1} 1$ \\
\hline 4 & $0120 ; 1$ & 2 & 2 & 2 & 1 & 1 & 1 & 225 & 110 & $10 \overline{1}$ & $01 \overline{1}$ \\
\hline 5 & $440 ; 2$ & 1 & 1 & 9 & 0 & 0 & 0 & 123 & 100 & 010 & 003 \\
\hline 6 & $224 ; 2$ & 1 & 2 & 5 & -1 & 0 & 0 & 65 & 100 & 011 & $0 \overline{2} 1$ \\
\hline 7 & $062 ; 2$ & 2 & 2 & 3 & 0 & 0 & -1 & 164 & 110 & $0 \overline{1} 1$ & $1 \overline{1} \overline{1}$ \\
\hline 8 & $440 ; 3$ & 1 & 1 & 16 & 0 & 0 & 0 & 123 & 100 & 010 & $\begin{array}{lll}0 & 0 & 4\end{array}$ \\
\hline 9 & $224 ; 3$ & 1 & 2 & 8 & 0 & 0 & 0 & 47 & 100 & 011 & $0 \overline{2} 2$ \\
\hline 10 & $2006 ; 3$ & 1 & 4 & 4 & 0 & 0 & 0 & 123 & 100 & 020 & 002 \\
\hline 11 & $2004 ; 3$ & 1 & 4 & 5 & -2 & 0 & 0 & 65 & 100 & 020 & $0 \overline{1} 2$ \\
\hline 12 & $060 ; 3$ & 2 & 2 & 6 & 1 & 1 & 1 & 166 & 110 & 101 & $2 \overline{1} \overline{1}$ \\
\hline 13 & $0406 ; 3$ & 2 & 2 & 4 & 0 & 0 & 0 & 123 & 110 & $1 \overline{1} 0$ & $00 \overline{2}$ \\
\hline 14 & $0404 ; 3$ & 2 & 2 & 5 & -1 & -1 & 0 & 139 & 110 & $1 \overline{1} 0$ & $\overline{1} 0 \overline{2}$ \\
\hline 15 & $024 ; 3$ & 2 & 3 & 3 & -1 & 0 & 0 & 65 & 110 & $1 \overline{1} \overline{1}$ & $\overline{1} 1 \overline{1}$ \\
\hline 16 & $008 ; 3$ & 3 & 3 & 3 & -1 & -1 & -1 & 229 & 111 & $1 \overline{1} \overline{1}$ & $\overline{1} 1 \overline{1}$ \\
\hline 17 & $440 ; 4$ & 1 & 1 & 25 & 0 & 0 & 0 & 123 & 100 & 010 & $\begin{array}{lll}0 & 0 & 5\end{array}$ \\
\hline 18 & $224 ; 4$ & 1 & 2 & 13 & -1 & 0 & 0 & 65 & 100 & 011 & $0 \overline{3} 2$ \\
\hline 19 & $200 ; 4$ & 1 & 5 & 5 & 0 & 0 & 0 & 83 & 100 & 021 & $0 \overline{1} 2$ \\
\hline 20 & $060 ; 4$ & 2 & 2 & 9 & 1 & 1 & 1 & 166 & 110 & $10 \overline{1}$ & $\overline{1} 2 \overline{2}$ \\
\hline 21 & $022 ; 4$ & 2 & 3 & 5 & -1 & -1 & 0 & 12 & 110 & $1 \overline{1} 1$ & $0 \overline{1} \overline{2}$ \\
\hline 22 & $440 ; 5$ & 1 & 1 & 36 & 0 & 0 & 0 & 123 & 100 & 010 & 006 \\
\hline 23 & $224 ; 5$ & 1 & 2 & 18 & 0 & 0 & 0 & 47 & 100 & 011 & $0 \overline{3} 3$ \\
\hline 24 & $2004 ; 5 a$ & 1 & 4 & 9 & 0 & 0 & 0 & 47 & 100 & 020 & $\begin{array}{llll}0 & 0 & 3\end{array}$ \\
\hline 25 & $2004 ; 5 b$ & 1 & 4 & 10 & -2 & 0 & 0 & 65 & 100 & 020 & $0 \overline{1} 3$ \\
\hline 26 & $2002 ; 5$ & 1 & 5 & 8 & -2 & 0 & 0 & 10 & 100 & 021 & $0 \overline{2} 2$ \\
\hline 27 & $060 ; 5$ & 2 & 2 & 12 & 0 & 0 & -1 & 164 & 110 & 0 Iิ 1 & $2 \overline{2} \overline{2}$ \\
\hline 28 & $040 ; 5 a$ & 2 & 2 & 9 & 0 & 0 & 0 & 123 & 110 & $1 \overline{1} 0$ & $\begin{array}{llll}0 & 0 & \overline{3}\end{array}$ \\
\hline 29 & $040 ; 5 \mathrm{~b}$ & 2 & 2 & 10 & -1 & -1 & 0 & 139 & 110 & 1 I 0 & $\overline{1} 0 \overline{3}$ \\
\hline 30 & $022 ; 5$ & 2 & 3 & 6 & 0 & 0 & 0 & 10 & 110 & $1 \overline{1} 1$ & $1 \overline{1} \overline{2}$ \\
\hline 31 & $0202 ; 5 a$ & 2 & 4 & 5 & 0 & -1 & 0 & 65 & 110 & 002 & $1 \overline{2} 0$ \\
\hline 32 & $0202 ; 5 b$ & 2 & 4 & 6 & -2 & -1 & 0 & 71 & 110 & 002 & $1 \overline{2} \overline{1}$ \\
\hline 33 & $0200 ; 5$ & 2 & 5 & 5 & 2 & 1 & 1 & 12 & 110 & 2 I 0 & $10 \overline{2}$ \\
\hline 34 & $004 ; 5$ & 3 & 3 & 5 & 1 & 1 & 1 & 12 & 111 & 111 & 120 \\
\hline 35 & $224 ; 6$ & 1 & 2 & 25 & -1 & 0 & 0 & 65 & 100 & 011 & $0 \widetilde{4} 3$ \\
\hline 36 & $200 ; 6$ & 1 & 5 & 10 & -1 & 0 & 0 & 10 & 100 & $02 \overline{1}$ & 013 \\
\hline
\end{tabular}


Table 20d (continued)

\begin{tabular}{|c|c|c|c|c|c|c|c|c|c|c|c|}
\hline$\#$ & $T_{i}(\mathrm{M}) ; \mathrm{y}$ & $a^{2}$ & $b^{2}$ & $c^{2}$ & bc & ac & $\mathbf{a b}$ & SG & $\mathbf{a}$ & b & c \\
\hline 37 & $060 ; 6$ & 2 & 2 & 17 & 1 & 1 & 1 & 166 & 110 & 101 & $3 \overline{2} \overline{2}$ \\
\hline 38 & $022 ; 6$ & 2 & 3 & 9 & -1 & -1 & 0 & 12 & 110 & $1 \overline{1} \overline{1}$ & $\overline{2} 1 \overline{2}$ \\
\hline 39 & $020 ; 6$ & 2 & 5 & 6 & 1 & 1 & 1 & 12 & 110 & $10 \overline{2}$ & $\overline{1} 2 \overline{1}$ \\
\hline 40 & $002 ; 6$ & 3 & 5 & 5 & -2 & -1 & -1 & 148 & 111 & $10 \overline{2}$ & $\overline{2} 10$ \\
\hline 41 & $224 ; 7$ & 1 & 2 & 32 & 0 & 0 & 0 & 47 & 100 & 011 & $0 \overline{4} 4$ \\
\hline 42 & $2004 ; 7 \mathrm{a}$ & 1 & 4 & 16 & 0 & 0 & 0 & 47 & 100 & 020 & 004 \\
\hline 43 & $2004 ; 7 \mathrm{~b}$ & 1 & 4 & 17 & -2 & 0 & 0 & 65 & 100 & 020 & $0 \overline{1} 4$ \\
\hline 44 & $2002 ; 7 a$ & 1 & 5 & 13 & -1 & 0 & 0 & 10 & 100 & 021 & $0 \overline{2} 3$ \\
\hline 45 & $2002 ; 7 b$ & 1 & 8 & 8 & 0 & 0 & 0 & 123 & 100 & 022 & $0 \overline{2} 2$ \\
\hline 46 & $2002 ; 7 c$ & 1 & 8 & 10 & -4 & 0 & 0 & 65 & 100 & 022 & $0 \overline{3} 1$ \\
\hline 47 & $060 ; 7$ & 2 & 2 & 22 & 1 & 1 & 1 & 166 & 110 & $10 \overline{1}$ & $\overline{2} 3 \overline{3}$ \\
\hline 48 & $040 ; 7 a$ & 2 & 2 & 16 & 0 & 0 & 0 & 123 & 110 & $1 \overline{1} 0$ & $00 \overline{4}$ \\
\hline 49 & $040 ; 7 \mathrm{~b}$ & 2 & 2 & 17 & -1 & -1 & 0 & 139 & 110 & $1 \overline{1} 0$ & $\overline{1} 0 \overline{4}$ \\
\hline 50 & $022 ; 7$ & 2 & 3 & 11 & -1 & 0 & 0 & 10 & 110 & $1 \overline{1} 1$ & $1 \overline{1} \overline{3}$ \\
\hline 51 & $0202 ; 7 a$ & 2 & 4 & 8 & 0 & 0 & 0 & 47 & 110 & 002 & $2 \overline{2} 0$ \\
\hline 52 & $0202 ; 7 \mathrm{~b}$ & 2 & 4 & 9 & -2 & 0 & 0 & 65 & 110 & 002 & $2 \overline{2} \overline{1}$ \\
\hline 53 & $0200 ; 7 a$ & 2 & 5 & 8 & -2 & 0 & -1 & 12 & 110 & $0 \overline{1} \overline{2}$ & $\overline{2} \underset{2}{2} 0$ \\
\hline 54 & $0200 ; 7 \mathrm{~b}$ & 2 & 6 & 6 & -2 & 0 & 0 & 65 & 110 & $1 \overline{1} 2$ & $1 \overline{1} \overline{2}$ \\
\hline 55 & $0200 ; 7 \mathrm{c}$ & 2 & 6 & 6 & -1 & 0 & -1 & 12 & 110 & $1 \overline{2} \overline{1}$ & $\overline{1} 1 \overline{2}$ \\
\hline 56 & $004 ; 7 a$ & 3 & 3 & 8 & 0 & 0 & -1 & 65 & 111 & $1 \overline{1} \overline{1}$ & $02 \overline{2}$ \\
\hline 57 & $004 ; 7 \mathrm{~b}$ & 3 & 3 & 9 & -1 & -1 & -1 & 69 & 111 & $1 \overline{1} \overline{1}$ & $\overline{1} 2 \overline{2}$ \\
\hline 58 & $002 ; 7$ & 3 & 5 & 5 & 1 & 1 & 1 & 12 & 111 & $20 \overline{1}$ & $02 \overline{1}$ \\
\hline 59 & $0006 ; 7$ & 4 & 4 & 4 & 0 & 0 & 0 & 221 & 200 & 020 & 002 \\
\hline 60 & $0004 ; 7 a$ & 4 & 4 & 5 & 0 & -2 & 0 & 65 & 200 & 020 & 102 \\
\hline 61 & $0004 ; 7 \mathrm{~b}$ & 4 & 4 & 6 & -2 & -2 & 0 & 139 & 200 & 020 & $\overline{1} \overrightarrow{1} 2$ \\
\hline 62 & $0002 ; 7 a$ & 4 & 5 & 5 & 1 & 2 & 2 & 139 & 200 & 120 & 102 \\
\hline 63 & $0002 ; 7 \mathrm{~b}$ & 4 & 5 & 5 & -2 & 0 & -2 & 12 & 200 & $\overline{1} 20$ & $0 \overline{1} 2$ \\
\hline 64 & $22420 ; 8$ & 1 & 2 & 41 & -1 & 0 & 0 & 65 & 100 & 011 & $0 \overline{5} 4$ \\
\hline 65 & $20022 ; 8$ & 1 & 5 & 17 & -2 & 0 & 0 & 10 & 100 & $02 \overline{1}$ & 0114 \\
\hline 66 & $20020 ; 8 a$ & 1 & 9 & 10 & -3 & 0 & 0 & 10 & 100 & 030 & $0 \overline{1} 3$ \\
\hline 67 & $20020 ; 8 b$ & 1 & 9 & 9 & 0 & 0 & 0 & 123 & 100 & 030 & 003 \\
\hline 68 & $06000 ; 8$ & 2 & 2 & 27 & 0 & 0 & -1 & 164 & 110 & $0 \overline{1} 1$ & $3 \overline{3} \overline{3}$ \\
\hline 69 & $02204 ; 8$ & 2 & 3 & 14 & 0 & -1 & 0 & 65 & 110 & $1 \overline{1} 1$ & $1 \overline{2} \overline{3}$ \\
\hline 70 & $02004 ; 8 a$ & 2 & 5 & 10 & 2 & 1 & 1 & 12 & 110 & $2 \overline{1} 0$ & $10 \overline{3}$ \\
\hline 71 & $02004 ; 8 \mathrm{~b}$ & 2 & 5 & 9 & 0 & 0 & -1 & 65 & 110 & $1 \overline{2} 0$ & $00 \overline{3}$ \\
\hline 72 & $02000 ; 8$ & 2 & 6 & 9 & 3 & 1 & 1 & 12 & 110 & $2 \overrightarrow{1} 1$ & $2 \overline{1} \overline{2}$ \\
\hline 73 & $00202 ; 8$ & 3 & 5 & 6 & -1 & 0 & -1 & 2 & 111 & $1 \overline{2} 0$ & $11 \overline{2}$ \\
\hline 74 & $00200 ; 8$ & 3 & 6 & 6 & -3 & 0 & 0 & 164 & 111 & $2 \overline{1} \overline{1}$ & $\overline{1} 2 \bar{I}$ \\
\hline 75 & $00006 ; 8$ & 5 & 5 & 5 & 2 & 2 & 2 & 166 & 210 & $10 \overline{2}$ & $02 \overline{1}$ \\
\hline 76 & $00000 ; 15 a$ & 6 & 6 & 10 & -2 & -2 & -2 & 69 & 211 & $\overline{2} 11$ & $0 \overline{3} 1$ \\
\hline 77 & $00000 ; 15 b$ & 8 & 8 & 8 & 4 & 4 & 4 & 225 & 220 & 022 & 202 \\
\hline
\end{tabular}


Table 21a: Hexagonal close-packed structures with a single set of $T_{i}(\mathrm{M})$ and $T_{i}(\mathrm{~N})$ values (homogeneous sphere packing with $T_{1}(\mathrm{M})$ or $T_{1}(\mathrm{~N})$ contacts). Homometric structures $a, b, \ldots$ with brackets, $T_{i}$ values of hexagonal or square plane, space group (SG), Pearson symbol (PS), number of reduced unit cell from Table 20a $-\mathrm{d}$ and positions of $M$ atoms in addition to $M_{1}$ at origin. $\left(T_{i} ; r\right)$ values are not at the borders of the structure map.

\begin{tabular}{|c|c|c|c|c|c|c|c|c|c|c|c|c|}
\hline $\begin{array}{c}T_{i}(\mathrm{M}) ; \mathrm{y} / \mathrm{x} \\
\mathrm{hcp}\end{array}$ & $\begin{array}{l}T_{i}(\mathrm{M}) ; \mathrm{r} \\
\text { hex.plan. }\end{array}$ & SG & PS & $\#$ & & $\begin{array}{l}\mathrm{M}_{2} \\
\mathrm{M}_{5}\end{array}$ & & & $\begin{array}{l}\mathrm{M}_{3} \\
\mathrm{M}_{6}\end{array}$ & \multicolumn{3}{|c|}{$\mathrm{M}_{4}$} \\
\hline $1262 ;(1)$ & $666 ;(1)$ & 194 & $h P 2$ & 1 & $1 / 3$ & $2 / 3$ & $1 / 2$ & & & & & \\
\hline $930 ; 1$ & $666 ;(1)$ & 164 & $h P 4$ & 2 & $1 / 3$ & $2 / 3$ & $1 / 4$ & & & & & \\
\hline$(730 ; 1 a)$ ? & $422 ; 1$ & 10 & $m P 8$ & 14 & 0 & $5 / 12$ & $1 / 12$ & $1 / 2$ & $1 / 6 \quad 1 / 3$ & $1 / 2$ & $1 / 4$ & $3 / 4$ \\
\hline$(730 ; 1 b)\}$ & $422 ; 1$ & 11 & $m P 8$ & 14 & 0 & $5 / 12$ & $1 / 12$ & $1 / 2$ & $3 / 4 \quad 1 / 4$ & $1 / 2$ & $1 / 6$ & $1 / 3$ \\
\hline$(622 ; 1)^{*}$ & $242 ; 1$ & 57 & $o P 8$ & 15 & $1 / 2$ & $2 / 3$ & 0 & 0 & $1 / 21 / 4$ & $1 / 2$ & $1 / 6$ & $1 / 4$ \\
\hline $602 ; 1 a$ & $666 ;(1)$ & 187 & $h P 2$ & 1 & & & & & & & & \\
\hline $602 ; 1 \mathrm{~b}$ & $226 ; 1$ & 59 & $o P 4$ & 3 & $1 / 2$ & $1 / 2$ & $1 / 6$ & & & & & \\
\hline$(530 ; 1 a)^{*}$ ] & $226 ; 1$ & 12 & $m C 8$ & 4 & $3 / 4$ & $1 / 12$ & $1 / 2$ & & & & & \\
\hline$\left.(530 ; 1 b)^{*}\right\}$ & $226 ; 1$ & 43 & $o F 32$ & 19 & $11 / 12$ & $5 / 12$ & $1 / 3$ & 0 & $\begin{array}{ll}0 & 1 / 2\end{array}$ & $5 / 12$ & $11 / 12$ & $5 / 6$ \\
\hline$\left.(530 ; 1 d)^{*}\right\}$ & $242 ; 1$ & 13 & $m P 8$ & 18 & $5 / 6$ & $1 / 2$ & 0 & $1 / 2$ & $1 / 4 \quad 1 / 4$ & $1 / 3$ & $3 / 4$ & $1 / 4$ \\
\hline$(530 ; 1 \mathrm{e}$ & $242 ; 1$ & 15 & $m C 16$ & 18 & $5 / 6$ & $1 / 2$ & 0 & $1 / 2$ & $1 / 4 \quad 1 / 4$ & $1 / 3$ & $1 / 4$ & $3 / 4$ \\
\hline $462 ; 1$ & $226 ; 1$ & 51 & $o P 4$ & 3 & 0 & $1 / 2$ & $2 / 3$ & & & & & \\
\hline $442 ; 1$ & $242 ; 1$ & 62 & $o P 8$ & 15 & $1 / 2$ & $1 / 6$ & $1 / 4$ & $1 / 2$ & $2 / 31 / 2$ & 0 & $1 / 2$ & $3 / 4$ \\
\hline $600 ; 2$ & $666 ;(1)$ & 194 & $h P 6$ & 5 & $1 / 3$ & $2 / 3$ & $1 / 2$ & & & & & \\
\hline $222 ; 2$ & $060 ; 2$ & 63 & $o C 12$ & 8 & $1 / 2$ & $2 / 3$ & 0 & & & & & \\
\hline $220 ; 2 a\}$ & $0660 ; 2$ & 15 & $m C 12$ & 9 & $1 / 2$ & $1 / 6$ & $1 / 2$ & & & & & \\
\hline $220 ; 2 b\}$ & $060 ; 2$ & 178 & $h P 18$ & 76 & $1 / 3$ & 0 & $1 / 6$ & $2 / 3$ & $1 / 3 \quad 1 / 3$ & $2 / 3$ & $2 / 3$ & $1 / 2$ \\
\hline & & & & & $1 / 3$ & $2 / 3$ & $2 / 3$ & 0 & $1 / 3 \quad 5 / 6$ & & & \\
\hline $062 ; 3$ & $0 \begin{array}{lll}0 & 6 ; 3\end{array}$ & 194 & $h P 8$ & 16 & $1 / 2$ & $2 / 3$ & $1 / 3$ & & & & & \\
\hline
\end{tabular}

* on the borders of the $T_{1} T_{2} T_{3} ; \mathrm{y} / \mathrm{x}$ polyhedron 
Table 21b: Same as Table 21a, except for ccp.

\begin{tabular}{|c|c|c|c|c|c|c|c|}
\hline $\begin{array}{l}T_{i}(\mathrm{M}) ; \mathrm{y} / \mathrm{x} \\
\quad \text { ccp }\end{array}$ & $\begin{array}{c}T_{i}(\mathrm{M}) ; \mathrm{y} / \mathrm{x} \\
\text { square }\end{array}$ & SG & PS & $\#$ & $\mathrm{M}_{2}$ & $\mathrm{M}_{3}$ & $\mathrm{M}_{4}$ \\
\hline $12624 ;(1)$ & $444 ;(1)$ & 225 & $c F 4$ & 1 & & & \\
\hline $9312 ; 1$ & $666 ;(1)^{* *}$ & 166 & $h R 4$ & 7 & 101 & & \\
\hline$(848 ; 1)^{*}$ & $444 ;(1)$ & 129 & $t P 4$ & 8 & 011 & & \\
\hline$(7210 ; 1)^{*}$ & $322 ; 1$ & 11 & $m P 8$ & 43 & 011 & 101 & $1 \overline{1} 2$ \\
\hline$(6212 ; 1 a)\}$ & $204 ; 1$ & 59 & $o P 4$ & 9 & $0 \underline{11}$ & & \\
\hline$(6212 ; 1 b)\}$ & $204 ; 1$ & 131 & $t P 8$ & 47 & $0 \overline{1} 1$ & 101 & 112 \\
\hline$(6112 ; 1)$ & $204 ; 1$ & 11 & $m P 8$ & 41 & 011 & 103 & 002 \\
\hline $6012 ; 1 \mathrm{a}\}$ & $204 ; 1$ & 166 & $h R 2$ & 2 & & & \\
\hline $6012 ; 1 \mathrm{~b}\}$ & $204 ; 1$ & 227 & $c F 32$ & 53 & 110 & 011 & 101 \\
\hline$(5410 ; 1)$ & $122 ; 1$ & 11 & $m P 8$ & 48 & 121 & 101 & 011 \\
\hline$(5312 ; 1 a)\}$ & $322 ; 1$ & 12 & $m C 8$ & 10 & 002 & & \\
\hline$(5312 ; 1 b)\}$ & $222 ; 1$ & 15 & $m C 16$ & 52 & 200 & $3 \overline{1} 0$ & $10 \bar{I}$ \\
\hline $5214 ; 1$ & $122 ; 1$ & 15 & $m C 16$ & 51 & $\overline{1} \overline{1} 2$ & $\overline{1} 01$ & $0 \overline{1} 1$ \\
\hline $468 ; 1$ & $444 ;(1)$ & 123 & $t P 2$ & 3 & & & \\
\hline $4512 ; 1 \mathrm{a}$ & $044 ; 1$ & 74 & $o I 16$ & 45 & $0 \overline{1} 3$ & $0 \overline{1} 1$ & 002 \\
\hline $4416 ; 1$ & $044 ; 1$ & 141 & $t I 8$ & 13 & $0 \overline{1} 1$ & & \\
\hline $4212 ; 1.3 a\}$ & $222 ; 1.3^{* *}$ & 2 & $a P 7$ & 34 & $1 \overline{1} 0$ & $0 \overline{1} 1$ & \\
\hline $4212 ; 1.3 \mathrm{~b}\}$ & $222 ; 1.3^{* *}$ & 146 & $h R 7$ & 34 & $1 \overline{2} 1$ & $1 \overline{1} 0$ & \\
\hline $3312 ; 1.5$ & $111 ; 1.5$ & 12 & $m C 10$ & 18 & 101 & & \\
\hline $606 ; 2$ & 666 ; (1)** & 164 & $h P 3$ & 4 & & & \\
\hline$(440 ; 2)^{*}$ & $444 ;(1)$ & 139 & $t I 6$ & 5 & & & \\
\hline $2212 ; 2 a\}$ & $202 ; 2$ & 71 & $o I 6$ & 6 & & & \\
\hline $2212 ; 2 b\}$ & $060 ; 2^{* *}$ & 151 & $h P 9$ & 62 & $1 \overline{1} 0$ & $1 \overline{2} \overline{1}$ & \\
\hline $2212 ; 2 \mathrm{c}$ & $060 ; 2^{* *}$ & 2 & $a P 6$ & 63 & $3 \overline{1} 0$ & $2 \overline{1} 1$ & \\
\hline $219 ; 2.5$ & $111 ; 2.5^{* *}$ & 2 & $a P 7$ & 34 & $0 \overline{1} 1$ & & \\
\hline $060 ; 3$ & $044 ; 1$ & 221 & $c P 4$ & 12 & & & \\
\hline $028 ; 4 a$ & $0004 ; 4$ & 87 & $t I 10$ & 18 & & & \\
\hline $006 ; 6$ & $0006 ; 6^{* *}$ & 166 & $h R 7$ & 34 & & & \\
\hline
\end{tabular}

* on the borders of the $T_{1} T_{2} T_{3} ; \mathrm{y} / \mathrm{x}$ polyhedron ** hexagonal planar 
Table 21c: Same as Table 21a, except for bcc.

\begin{tabular}{|c|c|c|c|c|c|c|c|}
\hline $\begin{array}{c}T_{i}(\mathrm{M}) ; \mathrm{y} / \mathrm{x} \\
\mathrm{bcc}\end{array}$ & $\begin{array}{c}T_{i}(\mathrm{M}) ; \mathrm{y} / \mathrm{x} \\
\text { square }\end{array}$ & SG & PS & $\#$ & $\mathrm{M}_{2}$ & $\mathrm{M}_{3}$ & $\mathrm{M}_{4}$ \\
\hline $8612 ;(1)$ & 444 ; (1) & 229 & $c I 2$ & 1 & & & \\
\hline$(646 ; 1)^{*}$ & $220 ; 1$ & 67 & $o C 8$ & 7 & $1 \overline{1} 1$ & & \\
\hline$(535 ; 1)^{*}$ & $124 ; 1$ & 2 & $a P 8$ & 40 & $\overline{1} 13$ & $\overline{2} 04$ & 002 \\
\hline$(444 ; 1 \mathrm{a})^{*}$, & $444 ;(1)$ & 129 & $t P 4$ & 10 & 111 & & \\
\hline$\left.(444 ; 1 \mathrm{~b})^{*}\right\}$ & $204 ; 1$ & 131 & $t P 8$ & 45 & 002 & 131 & 111 \\
\hline$(434 ; 1)^{*}$ & $122 ; 1$ & 11 & $m P 8$ & 46 & $1 \overline{1} 3$ & 111 & 002 \\
\hline$(426 ; 1 a)\}$ & $220 ; 1$ & 11 & $m P 4$ & 8 & $0 \overline{2} 2$ & & \\
\hline$(426 ; 1 b)\}$ & $220 ; 1$ & 66 & $o C 16$ & 52 & 202 & $\overline{1} 11$ & 111 \\
\hline$(425 ; 1)$ & $124 ; 1$ & 2 & $a P 8$ & 39 & $\overline{2} 02$ & $\overline{1} \overline{3} 3$ & $0 \overline{2} 2$ \\
\hline$\left.(424 ; 1 a)^{*}\right\}$ & $204 ; 1$ & 65 & $o C 4$ & 2 & & & \\
\hline$\left.(424 ; 1 \mathrm{~b})^{*}\right\}$ & $204 ; 1$ & 141 & $t I 16$ & 55 & $\overline{1} \overline{1} 1$ & $1 \overline{1} \overline{1}$ & 200 \\
\hline$(418 ; 1)^{*}$ & $044 ; 1$ & 74 & $o I 16$ & 49 & $\overline{1} 13$ & $\overline{1} 11$ & 002 \\
\hline $4012 ; 1$ & $044 ; 1$ & 227 & $c F 16$ & 13 & 111 & & \\
\hline$(335 ; 1)^{*}$ & $124 ; 1$ & 2 & $a P 8$ & 53 & $0 \overline{2} 0$ & $1 \overline{3} \overline{1}$ & $1 \overline{1} \overline{1}$ \\
\hline$(246 ; 1)^{*}$ & $220 ; 1$ & 74 & $o I 8$ & 12 & $00 \overline{2}$ & & \\
\hline $0612 ; 1$ & $444 ;(1)$ & 221 & $c P 2$ & 3 & & & \\
\hline$(424 ; 1.3 a)\}$ & $222 ; 1.3^{* *}$ & 2 & $a P 7$ & 31 & $1 \overline{1} 1$ & $2 \overline{2} 0$ & \\
\hline$(424 ; 1.3 b)\}$ & $222 ; 1.3^{* *}$ & 146 & $h R 7$ & 31 & 002 & $2 \overline{2} 0$ & \\
\hline$(233 ; 1.5)$ & $115 ; 1.5$ & 12 & $m C 10$ & 17 & $1 \overline{1} \overline{1}$ & & \\
\hline$\left.(422 ; 2 a)^{*}\right)$ & 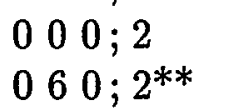 & 69 & $o F 12$ & 4 & & & \\
\hline$\left.(422 ; 2 b)^{*}\right)$ & $060 ; 2^{* *}$ & 151 & $h P 9$ & 58 & 002 & $1 \overline{1} 1$ & \\
\hline$(222 ; 2)^{*}$ & $060 ; 2^{* *}$ & 2 & $a P 9$ & 66 & $0 \overline{2} 2$ & $1 \overline{3} 1$ & \\
\hline $206 ; 2$ & $666 ;(1)^{* *}$ & 164 & $h P 3$ & 5 & & & \\
\hline $044 ; 2$ & $444 ;(1)$ & 139 & $t I 6$ & 6 & & & \\
\hline$(312 ; 2.5)$ & $111 ; 2.5^{* *}$ & 2 & $a P 7$ & 31 & $2 \overline{2} 0$ & & \\
\hline $200 ; 3$ & $006 ; 3^{* *}$ & 166 & $h R 4$ & 9 & & & \\
\hline $020 ; 4$ & $000 ; 4$ & 87 & $t I 10$ & 17 & & & \\
\hline $200 ; 6$ & $000 ; 6^{* *}$ & 148 & $h R 7$ & 31 & & & \\
\hline
\end{tabular}

* on the borders of the $T_{1} T_{2} T_{3} ; \mathrm{y} / \mathrm{x}$ polyhedron

** hexagonal planar 
Table 21d: Same as Table 21a, except for pc.

\begin{tabular}{|c|c|c|c|c|c|c|c|c|}
\hline $\begin{array}{c}T_{i}(\mathrm{M}) ; \mathrm{y} / \mathrm{x} \\
\mathrm{pc}\end{array}$ & $\begin{array}{c}T_{i}(\mathrm{M}) ; \mathrm{y} / \mathrm{x} \\
\text { square }\end{array}$ & SG & PS & $\#$ & $\begin{array}{l}\mathrm{M}_{2} \\
\mathrm{M}_{6}\end{array}$ & $\begin{array}{l}M_{3} \\
M_{7}\end{array}$ & $\begin{array}{l}\mathrm{M}_{4} \\
\mathrm{M}_{8}\end{array}$ & $\mathrm{M}_{5}$ \\
\hline $6128 ;(1)$ & 444 ; (1) & 221 & $c P 1$ & 1 & & & & \\
\hline $584 ; 1$ & $444 ;(1)$ & 123 & $t P 4$ & 8 & 001 & & & \\
\hline$\left.(464 ; 1 a)^{*}\right\}$ & $220 ; 1 a$ & 51 & $o P 4$ & 9 & 001 & & & \\
\hline$\left.(464 ; 1 b)^{*}\right\}$ & $220 ; 1 b$ & 123 & $t P 8$ & 45 & $0 \overline{1} 1$ & 001 & 012 & \\
\hline$(452 ; 1)^{*}$ & $212 ; 1$ & 10 & $m P 8$ & 46 & $0 \overline{1} 2$ & 001 & 002 & \\
\hline $440 ; 1$ & $444 ;(1)$ & 123 & $t P 2$ & 2 & & & & \\
\hline$(364 ; 1 a)$ & $666 ;(1)^{* *}$ & 166 & $h R 12$ & 12 & 100 & & & \\
\hline$(364 ; 1 b)$ & $220 ; 1 a$ & 63 & $o C 16$ & 54 & $1 \overline{1} \overline{1}$ & 100 & 101 & \\
\hline \multirow{2}{*}{$(364 ; 1 c)$} & $220 ; 1 b$ & 225 & $c F 64$ & 77 & 011 & 101 & 110 & 111 \\
\hline & & & & & 122 & 212 & 221 & \\
\hline \multirow[t]{2}{*}{$(364 ; 1 d)$} & $220 ; 1 b$ & 160 & $h R 48$ & 77 & 011 & 101 & 110 & 111 \\
\hline & & & & & 122 & 212 & 223 & \\
\hline \multirow[t]{2}{*}{$(364 ; 1 e))$} & $220 ; 1 b$ & 141 & $t I 32$ & 77 & 011 & 101 & 110 & 111 \\
\hline & & & & & 122 & 223 & 232 & \\
\hline$(354 ; 1 a)$ & $212 ; 1$ & 2 & $a P 8$ & 58 & 110 & $12 \overline{1}$ & 120 & \\
\hline \multirow[t]{2}{*}{$(354 ; 1 b)$} & $212 ; 1$ & 5 & $m C 32$ & 76 & $0 \overline{1} 1$ & $0 \overline{1} 2$ & 001 & $1 \overrightarrow{1} 1$ \\
\hline & & & & & $1 \overline{1} 2$ & 101 & 111 & \\
\hline \multirow[t]{2}{*}{$(354 ; 1 c)$} & $212 ; 1$ & 2 & $a P 16$ & 76 & $0 \underline{1} 2$ & 002 & 012 & $1 \overline{1} 1$ \\
\hline & & & & & $1 \overline{1} 2$ & 101 & 111 & \\
\hline \multirow[t]{2}{*}{$(354 ; 1 d)$} & $212 ; 1$ & 43 & $o F 64$ & 76 & $0 \overline{1} 1$ & $0 \overline{1} 2$ & 012 & $1 \overline{1} 1$ \\
\hline & & & & & $1 \overline{1} 2$ & 101 & 111 & \\
\hline \multirow[t]{2}{*}{$(354 ; 1 \mathrm{e})$} & $212 ; 1$ & 15 & $m C 32$ & 76 & $0 \overline{1} 2$ & 001 & 002 & $1 \overline{1} 1$ \\
\hline & & & & & $1 \overline{1} 2$ & 101 & 111 & \\
\hline \multirow[t]{2}{*}{$(354 ; 1 f)$} & $212 ; 1$ & 12 & $m C 32$ & 76 & $0 \overline{2} 1$ & 002 & 012 & $1 \overline{1} 1$ \\
\hline & & & & & $1 \overline{1} 2$ & 101 & 111 & \\
\hline $344 ; 1 a\}$ & $322 ; 1$ & 65 & $o C 8$ & 11 & 001 & & & \\
\hline $344 ; 1 \mathrm{~b}\}$ & $204 ; 1$ & 141 & $t I 16$ & 61 & 001 & 010 & 101 & \\
\hline$\left.(264 ; 1 a)^{*}\right\}$ & $220 ; 1 a$ & 63 & $o C 8$ & 15 & $01 \overline{1}$ & & & \\
\hline$\left.(264 ; 1 \mathrm{~b})^{*}\right\}$ & $220 ; 1 \mathrm{~b}$ & 139 & $t I 16$ & 62 & 101 & 110 & 211 & \\
\hline$(256 ; 1)$ & $212 ; 1$ & 12 & $m C 16$ & 57 & $01 \overline{2}$ & $01 \overline{1}$ & $10 \overline{1}$ & \\
\hline $248 ; 1$ & $044 ; 1$ & 123 & $t P 2$ & 3 & & & & \\
\hline $184 ; 1$ & $044 ; 1$ & 123 & $t P 4$ & 14 & $00 \overline{1}$ & & & \\
\hline $0120 ; 1$ & $044 ; 1$ & 225 & $c F 8$ & 4 & & & & \\
\hline$(244 ; 1.3 a)^{*}$ & $222 ; 1.3^{* *}$ & 146 & $h R 21$ & 40 & 010 & 110 & & \\
\hline$\left.(244 ; 1.3 \mathrm{~b})^{*}\right\}$ & $222 ; 1.3^{* *}$ & 2 & $a P 7$ & 40 & $11 \overline{1}$ & 110 & & \\
\hline $332 ; 1.5$ & $111 ; 1.5$ & 10 & $m P 5$ & 19 & 001 & & & \\
\hline
\end{tabular}


Table 21d (continued)

\begin{tabular}{|c|c|c|c|c|c|c|c|c|}
\hline $\begin{array}{c}T_{i}(\mathrm{M}) ; \mathrm{y} / \mathrm{x} \\
\mathrm{pc}\end{array}$ & $\begin{array}{c}T_{i}(\mathrm{M}) ; \mathrm{y} / \mathrm{x} \\
\text { square }\end{array}$ & SG & PS & $\#$ & $\begin{array}{l}\mathrm{M}_{2} \\
\mathrm{M}_{6}\end{array}$ & $\begin{array}{l}\mathrm{M}_{3} \\
\mathrm{M}_{7}\end{array}$ & $\begin{array}{l}\mathrm{M}_{4} \\
\mathrm{M}_{8}\end{array}$ & $\mathrm{M}_{5}$ \\
\hline $440 ; 2$ & $202 ; 2$ & 123 & $t P 3$ & 5 & & & & \\
\hline $224 ; 2 \mathrm{a}\}$ & $020 ; 2$ & 51 & $o P 3$ & 6 & & & & \\
\hline $224 ; 2 b\}$ & $060 ; 2^{* *}$ & 151 & $h P 9$ & 74 & 010 & 110 & & \\
\hline $222 ; 2$ & $060 ; 2^{* *}$ & 2 & $a P 9$ & 75 & $11 \overline{2}$ & $12 \overline{2}$ & & \\
\hline $062 ; 2$ & $666 ;(1)^{* *}$ & 164 & $h P 3$ & 7 & & & & \\
\hline$(123 ; 2.5)^{*}$ & $111 ; 2.5^{* *}$ & 2 & $a P 7$ & 40 & 010 & & & \\
\hline $008 ; 3$ & $004 ; 3$ & 229 & $c I 8$ & 16 & & & & \\
\hline $200 ; 4$ & $0004 ; 4$ & 83 & $t P 5$ & 19 & & & & \\
\hline $002 ; 6$ & $0006 ; 6^{* *}$ & 148 & $h R 21$ & 40 & & & & \\
\hline
\end{tabular}

* on the borders of the $T_{1} T_{2} T_{3} ; \mathrm{y} / \mathrm{x}$ polyhedron

** hexagonal planar 
Table 22: Group-subgroup relations of homogeneous sphere packings with s-CN values, space groups (SG) and Pearson symbols (PS) of the ccp, bcc and pc structures from Table $21 \mathrm{~b}-\mathrm{d}$. Structures with underlined $T_{i} ; \mathrm{r}$ values are stable sphere packings with contact numbers $k=T_{1}$ (or $T_{2}$, if $T_{1}=0$ ) (Koch and Fischer, 1992). All sphere packings with $k=T_{1} \geq 4\left(T_{2} \geq 4\right.$, if $\left.T_{1}=0\right)$ except $6012 ; 1 \mathrm{a}, 9312 ; 1$ (ccp) are stable. Structures in double brackets have the same projection pattern, underlined $T_{i}$ structures in square brackets are structures with a stable homogeneous packing of $A$ or $B$ atoms (only $B$ atoms at $y / x>1$ ).

\begin{tabular}{|c|c|c|c|c|}
\hline SG & PS & $T_{i}(\mathrm{M})_{\mathrm{bcc}}$ & $T_{i}(\mathrm{M})_{\mathrm{fcc}}$ & $T_{i}(\mathrm{M})_{\mathrm{pc}}$ \\
\hline 229 & $c I 2$ & $\underline{8612} ;(1)$ & & \\
\hline 229 & $c I 8$ & & & $008 ; 3$ \\
\hline 227 & $c F 32$ & & $6012 ; 1 b$ & \\
\hline 227 & $c F 16$ & $4012 ; 1$ & $((\underline{416} ; 1))$ & \\
\hline 225 & $c F 64$ & & & $364 ; 1 c$ \\
\hline 225 & $c F 8$ & & & $\underline{0120} ; 1$ \\
\hline 225 & $c F 4$ & & 12624 ; (1) & \\
\hline 221 & $c P 4$ & & $060 ; 3$ & \\
\hline 221 & $c P 2$ & $0612 ; 1$ & $((468 ; 1))$ & \\
\hline 221 & $c P 1$ & & & $\underline{6128} ;$ (1) \\
\hline 166 & $h R 7$ & & $\underline{006} ; 6$ & \\
\hline 166 & $h R 4$ & {$\left[\begin{array}{lll}2 & 0 & 0\end{array} ; 3\right]$} & $9312 ; 1$ & $364 ; 1 a$ \\
\hline 166 & $h R 2$ & & $6012 ; 1 a$ & \\
\hline 164 & $h P 3$ & 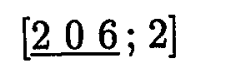 & $606 ; 2$ & $062 ; 2$ \\
\hline 160 & $h R 16$ & & & $364 ; 1 d$ \\
\hline 151 & $h P 9$ & {$[\underline{422} ; 2 \mathrm{~b}]$} & {$[\underline{2} \quad 2 \quad 12 ; 2 b]$} & {$[\underline{2} 244 ; 2 \mathrm{~b}]$} \\
\hline 148 & $h R 7$ & {$[200 ; 6]$} & & {$\left[\begin{array}{lll}0 & 0 & 2\end{array} ; 6\right]$} \\
\hline 146 & $h R 7$ & $424 ; 1.33 \mathrm{a}$ & $\underline{4212} ; 1.33 \mathrm{~b}$ & {$[244 ; 1.33 \mathrm{a}]$} \\
\hline 141 & $t T 32$ & & & $364 ; 1 \mathrm{e}$ \\
\hline 141 & $t I 16$ & $\underline{424} ; 1 \mathrm{~b}$ & & $344 ; 1 b$ \\
\hline 141 & $t I 8$ & & $4416 ; 1$ & \\
\hline 139 & $t I 16$ & & & $264 ; 1 b$ \\
\hline 139 & $t I 6$ & {$\left[\begin{array}{llll}0 & 4 & 4\end{array} ; 2\right]$} & $440 ; 2$ & \\
\hline 131 & $t P 8$ & $444 ; 1 b$ & $6212 ; 1 \mathrm{~b}$ & \\
\hline 129 & $t P 4$ & $444 ; 1 \mathrm{a}$ & $848 ; 1$ & \\
\hline 123 & $t P 8$ & & & $\begin{array}{l}184 ; 1 \\
464 ; 1 \mathrm{~b}\end{array}$ \\
\hline 123 & $t P 4$ & & $468 ; 1$ & $584 ; 1$ \\
\hline 123 & $t P 3$ & & & $440 ; 2$ \\
\hline 123 & $t P 2$ & & & $440 ; 1$ \\
\hline
\end{tabular}


Table 22 (continued)

\begin{tabular}{|c|c|c|c|c|}
\hline $\mathrm{SG}$ & PS & $T_{i}(\mathrm{M})_{\mathrm{bcc}}$ & $T_{i}(\mathrm{M})_{\mathrm{fcc}}$ & $T_{i}(\mathrm{M})_{\mathrm{pc}}$ \\
\hline 87 & $t P 2$ & \multirow{3}{*}[\begin{array}{lll}{0}&{2}&{0}\end{array};4]{} & \multirow{3}{*}{ 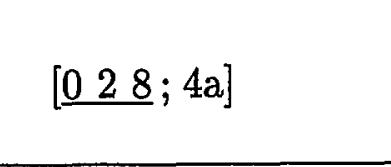 } & $248 ; 1$ \\
\hline 87 & $t I 10$ & & & \\
\hline 83 & $t P 5$ & & & {$\left[\begin{array}{lll}2 & 0 & 0\end{array} ; 4\right]$} \\
\hline 74 & $o I 16$ & \multirow{3}{*}{$\begin{array}{l}418 ; 1 \\
246 ; 1\end{array}$} & \multirow{7}{*}{ 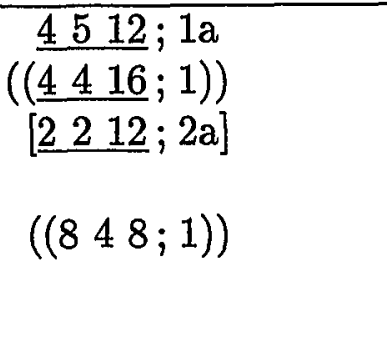 } & \multirow{14}{*}{$\begin{array}{l}{[\underline{2} 24 ; 2 \mathrm{a}]} \\
464 ; 1 \mathrm{a} \\
354 ; 1 \mathrm{~d}\end{array}$} \\
\hline 74 & $o I 8$ & & & \\
\hline 71 & $o I 6$ & & & \\
\hline 69 & $o F 12$ & \multirow{4}{*}{$\begin{array}{l}422 ; 2 \mathrm{a} \\
646 ; 1 \\
\underline{426} ; 1 \mathrm{~b}\end{array}$} & & \\
\hline 67 & $o C 8$ & & & \\
\hline 66 & $o C 16$ & & & \\
\hline 65 & $o C 8$ & & & \\
\hline 65 & $o C 4$ & \multirow[t]{7}{*}{$424 ; 1 \mathrm{a}$} & \multirow[t]{3}{*}{$\begin{array}{c}((468 ; 1))(\text { in } z) \\
((6012 ; 1 a))(\text { in } x)\end{array}$} & \\
\hline 63 & $o C 16$ & & & \\
\hline 63 & $o C 8$ & & & \\
\hline 59 & $o P 4$ & & \multirow[t]{4}{*}{$6212 ; 1 \mathrm{a}$} & \\
\hline 51 & $o P 6$ & & & \\
\hline 51 & $o P 4$ & & & \\
\hline 43 & $o F 64$ & & & \\
\hline 15 & $m C 32$ & \multirow{12}{*}[\underline{2}333;1.5]{} & \multirow{12}{*}{ 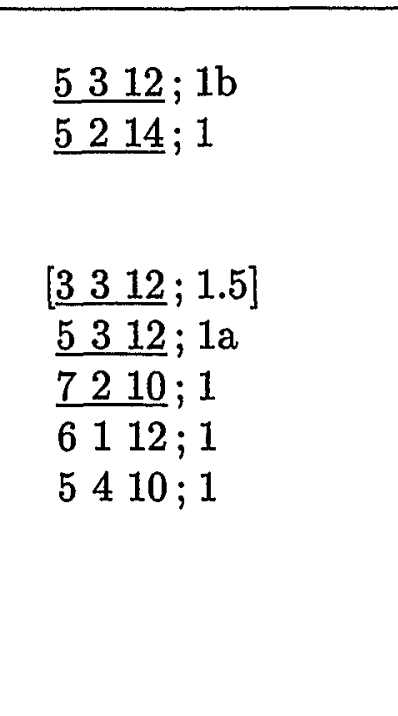 } & $354 ; 1 e$ \\
\hline 15 & $m C 16$ & & & \\
\hline 12 & $m C 32$ & & & 354 ; 1f \\
\hline 12 & $m C 16$ & & & $256 ; 1$ \\
\hline 12 & $m C 10$ & & & \\
\hline 12 & $m C 8$ & & & \\
\hline \multirow[t]{2}{*}{11} & $m P 8$ & & & \\
\hline & & & & \\
\hline 11 & $m P 4$ & & & \\
\hline 10 & $m P 8$ & & & $452 ; 1$ \\
\hline 10 & $m P 5$ & & & $332 ; 1.5$ \\
\hline 3 & $m P 16$ & & & $354 ; 1 \mathrm{~b}$ \\
\hline 2 & $a P 16$ & & & $354 ; 1 c$ \\
\hline 2 & $a P 9$ & {$[\underline{2} 22 ; ; 2]$} & & $222 ; 2$ \\
\hline 2 & $a P 8$ & $335 ; 1$ & & $354 ; 1 \mathrm{a}$ \\
\hline & & $\begin{array}{l}425 ; 1 \\
535 ; 1\end{array}$ & & \\
\hline 2 & $a P 7$ & $\underline{53 \mathrm{~b}} ; 1$ & $\underline{4212} ; 1.33 \mathrm{a}$ & {$[244 ; 1.33 b]$} \\
\hline & & {$[312 ; 2.5]$} & {$[219 ; 2.5]$} & {$[123 ; 2.5]$} \\
\hline 2 & $a P 6$ & & {$\left[\begin{array}{lll}2 & 2 & 12\end{array} ; 2 c\right]$} & \\
\hline
\end{tabular}


Table 23a: Self-coordination numbers $T_{i}^{\mathrm{M}}(i=1-I)$ of $\mathrm{M}$ particles in the hcp lattice with identical values of all $\mathrm{M}$ (in the bulk), neutral clusters $\mathrm{M}_{\mathrm{x}} \mathrm{N}_{\mathrm{y}}$ with closed shells and theoretical structures of isotopes with closed shells $T_{i}$. Structures with $T_{i}$ in brackets are not at the border of the structure maps (Fig.9). Structures with $T_{i}$ and an asterisk (*) are on the border of the $T_{1} T_{2} T_{3} ; \mathrm{y} / \mathrm{x}$ polyhedron. In the last column clusters without central atom are in brackets.

\begin{tabular}{|c|c|c|c|c|c|c|c|c|c|c|c|c|c|c|c|c|c|c|c|}
\hline$T_{1}$ & $T_{2}$ & $T_{3}$ & $T_{4} 1$ & $T_{5} 7$ & $T_{6} T$ & $T_{7} T$ & $T_{8} ?$ & $T_{9} T$ & 10 & $T_{11}$ & $T_{12} I$ & 13 & $T_{14}$ & $T_{15}$ & $T_{16}$ & $T_{17}$ & $T_{18} ; \mathrm{r}$ & Isotopes & $\begin{array}{c}\mathrm{x}+\mathrm{y} \\
\text { neutr. clust. }\end{array}$ \\
\hline 12 & 6 & 2 & 181 & 12 & $\begin{array}{lll}61 & 1\end{array}$ & 121 & 12 & 6 & 6 & 12 & 24 & 6 & 12 & 12 & 24 & 12 & $12 ; 0$ & & \\
\hline 9 & 3 & 0 & 12 & 0 & 6 & 6 & 0 & 3 & 3 & 0 & 18 & 3 & 6 & 9 & 0 & 6 & $6 ; 1$ & $\begin{array}{l}{ }^{51} \mathrm{Mn},{ }^{81} \mathrm{Rb},{ }^{87} \mathrm{Zr},{ }^{93} \mathrm{Tc},{ }^{105} \mathrm{Tc} \\
{ }^{183} \mathrm{Au}\end{array}$ & \\
\hline$(7$ & 3 & 0 & 8 & 4 & 2 & 6 & 8 & 3 & 3 & 8 & 10 & 3 & 6 & 7 & 16 & 6 & $6 ; 1 \mathrm{a}, \mathrm{b})$ & ${ }^{13} \mathrm{O},{ }^{21} \mathrm{Na},{ }^{39} \mathrm{~K},{ }^{51} \mathrm{~V},{ }^{57} \mathrm{Mn}$ & $(20,104)$ \\
\hline$(6$ & 2 & 2 & 8 & 4 & 2 & 8 & 8 & 6 & 4 & 4 & 12 & 2 & 4 & 6 & 16 & 4 & $8 ; 1)^{*}$ & $\begin{array}{l}{ }^{13} \mathrm{~N},{ }^{19} \mathrm{~F},{ }^{21} \mathrm{Na},{ }^{39} \mathrm{~K},{ }^{51} \mathrm{~V} \\
{ }^{57} \mathrm{Mn},{ }^{69} \mathrm{As}\end{array}$ & $(12,20,80)$ \\
\hline 6 & 0 & 2 & 6 & 12 & 6 & 0 & 12 & 0 & 0 & 12 & 12 & 0 & 0 & 6 & 24 & 0 & $0 ; 1$ & ${ }^{13} \mathrm{~N},{ }^{19} \mathrm{~N},{ }^{21} \mathrm{~F},{ }^{69} \mathrm{As}$ & $(12)$ \\
\hline 6 & 0 & 2 & 10 & 4 & 6 & 8 & 4 & 0 & 4 & 12 & 12 & 0 & 8 & 6 & 8 & 0 & $8 ; 1$ & ${ }^{13} \mathrm{~N},{ }^{19} \mathrm{~N},{ }^{21} \mathrm{~F},{ }^{39} \mathrm{~K},{ }^{51} \mathrm{~V},{ }^{87} \mathrm{Nb}$ & $(12,56,80)$ \\
\hline$(5$ & 3 & 0 & 8 & 8 & 6 & 6 & 8 & 3 & 3 & 0 & 10 & 3 & 6 & 5 & 16 & 6 & $6 ; 1 \mathrm{a}, \mathrm{b})^{*}$ & ${ }^{13} \mathrm{C},{ }^{19} \mathrm{~F},{ }^{21} \mathrm{~F},{ }^{39} \mathrm{Cl},{ }^{51} \mathrm{Mn}$ & \\
\hline$(5$ & 3 & 0 & 10 & 8 & 2 & 6 & 4 & 3 & 3 & 8 & 14 & 3 & 6 & 5 & 8 & 6 & $6 ; 1 \mathrm{~d}, \mathrm{e})^{*}$ & ${ }^{13} \mathrm{C},{ }^{19} \mathrm{~F},{ }^{21} \mathrm{~F},{ }^{39} \mathrm{~K}$ & $(56,68,104)$ \\
\hline 4 & 6 & 2 & 6 & 4 & 6 & 4 & 4 & 6 & 2 & 12 & 8 & 6 & 4 & 4 & 8 & 12 & $4 ; 1$ & ${ }^{13} \mathrm{~B},{ }^{39} \mathrm{~K},{ }^{51} \mathrm{~V},{ }^{69} \mathrm{As},{ }^{81} \mathrm{Rb}$ & $(56,128)$ \\
\hline 4 & 4 & 2 & 12 & 4 & 2 & 4 & 8 & 0 & 2 & 4 & 16 & 4 & 8 & 4 & 16 & 8 & $4 ; 1$ & $\begin{array}{l}{ }^{13} \mathrm{~B},{ }^{19} \mathrm{~F},{ }^{21} \mathrm{Na},{ }^{69} \mathrm{As},{ }^{87} \mathrm{Nb} \\
{ }^{93} \mathrm{Tc},{ }^{105} \mathrm{Ag}\end{array}$ & $(20,56,80)$ \\
\hline 6 & 0 & 0 & 6 & 0 & 6 & 0 & 0 & 0 & 6 & 0 & 12 & 6 & 12 & 6 & 0 & 0 & $12 ; 2$ & ${ }^{13} \mathrm{~N},{ }^{19} \mathrm{~N},{ }^{21} \mathrm{~N}$ & $21,39,57,183$ \\
\hline 2 & 2 & 2 & 10 & 0 & 0 & 4 & 12 & 2 & 2 & 0 & 4 & 2 & 4 & 8 & 0 & 4 & $4 ; 2$ & ${ }^{21} \mathrm{~N},{ }^{39} \mathrm{Cl},{ }^{81} \mathrm{As},{ }^{87} \mathrm{Br},{ }^{93} \mathrm{Rb}$ & 21,51 \\
\hline 2 & 2 & 0 & 10 & 6 & 0 & 4 & 0 & 2 & 2 & 6 & 4 & 2 & 4 & 8 & 12 & 4 & $4 ; 2 a, b$ & ${ }^{51} \mathrm{Sc}$ & 105,159 \\
\hline 0 & 6 & 2 & 0 & 0 & 6 & 0 & 0 & 6 & 0 & 12 & 0 & 6 & 0 & 0 & 0 & 12 & $0 ; 3$ & ${ }^{19} \mathrm{~N},{ }^{21} \mathrm{~F}$ & \\
\hline
\end{tabular}


Table 23b: Same as Table 23a, except for ccp.

\begin{tabular}{|c|c|c|c|c|c|c|c|c|c|}
\hline \multicolumn{8}{|c|}{$\begin{array}{lllllllllllll}T_{1} & T_{2} & T_{3} & T_{4} & T_{5} & T_{6} & T_{7} & T_{8} & T_{9} & T_{10} & T_{11} ; \mathrm{r}\end{array}$} & \multirow[t]{2}{*}{ Isotopes } & $\begin{array}{c}x+y \\
\text { neutr. clust. }\end{array}$ \\
\hline 12 & 624 & 412 & 24 & 848 & 636 & 24 & $24 ; 0$ & & \\
\hline 9 & 312 & 26 & 6 & 424 & 015 & 12 & $12 ; 1$ & ${ }^{79} \mathrm{Rb},{ }^{87} \mathrm{Nb}$ & \\
\hline$(8$ & $4 \varepsilon$ & 4 & 16 & 016 & 624 & 16 & $8 ; 1)^{*}$ & $\begin{array}{l}{ }^{43} \mathrm{Sc}{ }^{55} \mathrm{Mn},{ }^{87} \mathrm{Nb},{ }^{135} \mathrm{La}, \\
{ }^{141} \mathrm{Eu}\end{array}$ & \\
\hline$(7$ & 210 & 06 & 10 & 428 & 221 & 16 & $10 ; 1)^{*}$ & 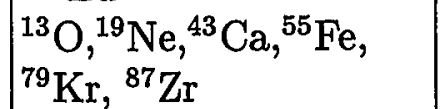 & $(18,134)$ \\
\hline$(6$ & $21:$ & 24 & 12 & 824 & 618 & 8 & $12 ; 1 a, b)$ & $\begin{array}{l}{ }^{13} \mathrm{~N},{ }^{19} \mathrm{~F},{ }^{43} \mathrm{Sc},{ }^{55} \mathrm{Mn}, \\
{ }^{79} \mathrm{Rb}\end{array}$ & $(12,200,224)$ \\
\hline$(6$ & 11 & 28 & 12 & 424 & 418 & 12 & $12 ; 1)$ & ${ }^{13} \mathrm{~N},{ }^{19} \mathrm{O},{ }^{43} \mathrm{Ca}$ & $(12,54,78,86,134)$ \\
\hline 6 & 01 & 212 & 12 & 024 & 618 & 0 & $12 ; 1 \mathrm{a}, \mathrm{b}$ & ${ }^{13} \mathrm{~N},{ }^{19} \mathrm{~N},{ }^{43} \mathrm{~K}$ & (12) \\
\hline$(5$ & 411 & 06 & 14 & 428 & 215 & 8 & $10 ; 1)$ & ${ }^{13} \mathrm{C},{ }^{19} \mathrm{Ne},{ }^{43} \mathrm{Ca},{ }^{55} \mathrm{Fe}$ & $(18,78,86,176)$ \\
\hline (5 & 31 & 6 & 14 & 424 & 019 & 12 & $12 ; 1 \mathrm{a}, \mathrm{b})$ & ${ }^{13} \mathrm{C},{ }^{19} \mathrm{~F},{ }^{43} \mathrm{Sc},{ }^{55} \mathrm{Co}$ & \\
\hline 5 & 21 & 14 & 14 & 420 & 215 & 16 & $14 ; 1$ & ${ }^{13} \mathrm{C},{ }^{19} \mathrm{O},{ }^{43} \mathrm{Ti}$ & $(42,54,224)$ \\
\hline 4 & $6 \varepsilon$ & 812 & & 816 & 612 & 24 & $8 ; 1$ & ${ }^{13} \mathrm{~B},{ }^{43} \mathrm{~K}$ & $(224)$ \\
\hline 4 & 512 & 128 & 8 & 424 & 420 & 12 & $12 ; 1$ & ${ }^{13} \mathrm{~B},{ }^{19} \mathrm{Ne},{ }^{43} \mathrm{Ti},{ }^{79} \mathrm{Sr}$ & $(18,42)$ \\
\hline 4 & 416 & 164 & 8 & 032 & 612 & 16 & $16 ; 1$ & ${ }^{13} \mathrm{~B},{ }^{19} \mathrm{~F},{ }^{79} \mathrm{Rb},{ }^{87} \mathrm{Rb}$ & \\
\hline 4 & $21:$ & 4 & 12 & 420 & 212 & 12 & $8 ; 1.33 a, b$ & $\begin{array}{l}{ }^{13} \mathrm{~B},{ }^{19} \mathrm{~N},{ }^{43} \mathrm{~K},{ }^{79} \mathrm{Br} \\
{ }^{87} \mathrm{Y},{ }^{135} \mathrm{Pr},{ }^{141} \mathrm{Pm},{ }^{177} \mathrm{Ta} \\
{ }^{201} \mathrm{At}\end{array}$ & \\
\hline 3 & 31 & 123 & 9 & 224 & 9 & 9 & $6 ; 1.5$ & $\mid \begin{array}{l}{ }^{19} \mathrm{~N},{ }^{43} \mathrm{~K},{ }^{79} \mathrm{Ga},{ }^{87} \mathrm{As} \\
{ }^{135} \mathrm{La},{ }^{141} \mathrm{Nd}{ }^{201} \mathrm{Pt}\end{array}$ & 55 \\
\hline 6 & 0 & 6 & 0 & 224 & 018 & 12 & $0 ; 2$ & ${ }^{13} \mathrm{~N},{ }^{19} \mathrm{~N}$ & 135,225 \\
\hline$(4$ & 4 & 04 & 16 & 016 & 412 & & $16 ; 2)^{*}$ & ${ }^{13} \mathrm{~B},{ }^{19} \mathrm{~F}$ & 87,135 \\
\hline 2 & 21 & 122 & 8 & 48 & 224 & 4 & $8 ; 2 a, b$ & & \\
\hline 2 & 21 & 12 & 8 & 214 & 212 & 10 & $8 ; 2 c$ & & 201,225 \\
\hline 2 & 1 & $\begin{array}{ll}9 & 2\end{array}$ & 9 & $\begin{array}{ll}313 \\
\text { S }\end{array}$ & 16 & 9 & $4 ; 2.5$ & & \\
\hline 0 & 6 & $\begin{array}{ll}0 & 12\end{array}$ & 0 & 80 & 0 & 24 & $0 ; 3$ & ${ }^{19} \mathrm{~N}$ & \\
\hline 0 & 2 & 80 & 4 & $\begin{array}{ll}016 \\
\text {. }\end{array}$ & 20 & 4 & $0 ; 4$ & & 55 \\
\hline 0 & 0 & 0 & 6 & 26 & $\begin{array}{ll}0 & 0\end{array}$ & 6 & $0 ; 6$ & & \\
\hline
\end{tabular}


Table 23c: Same as Table 23a, except for bcc.

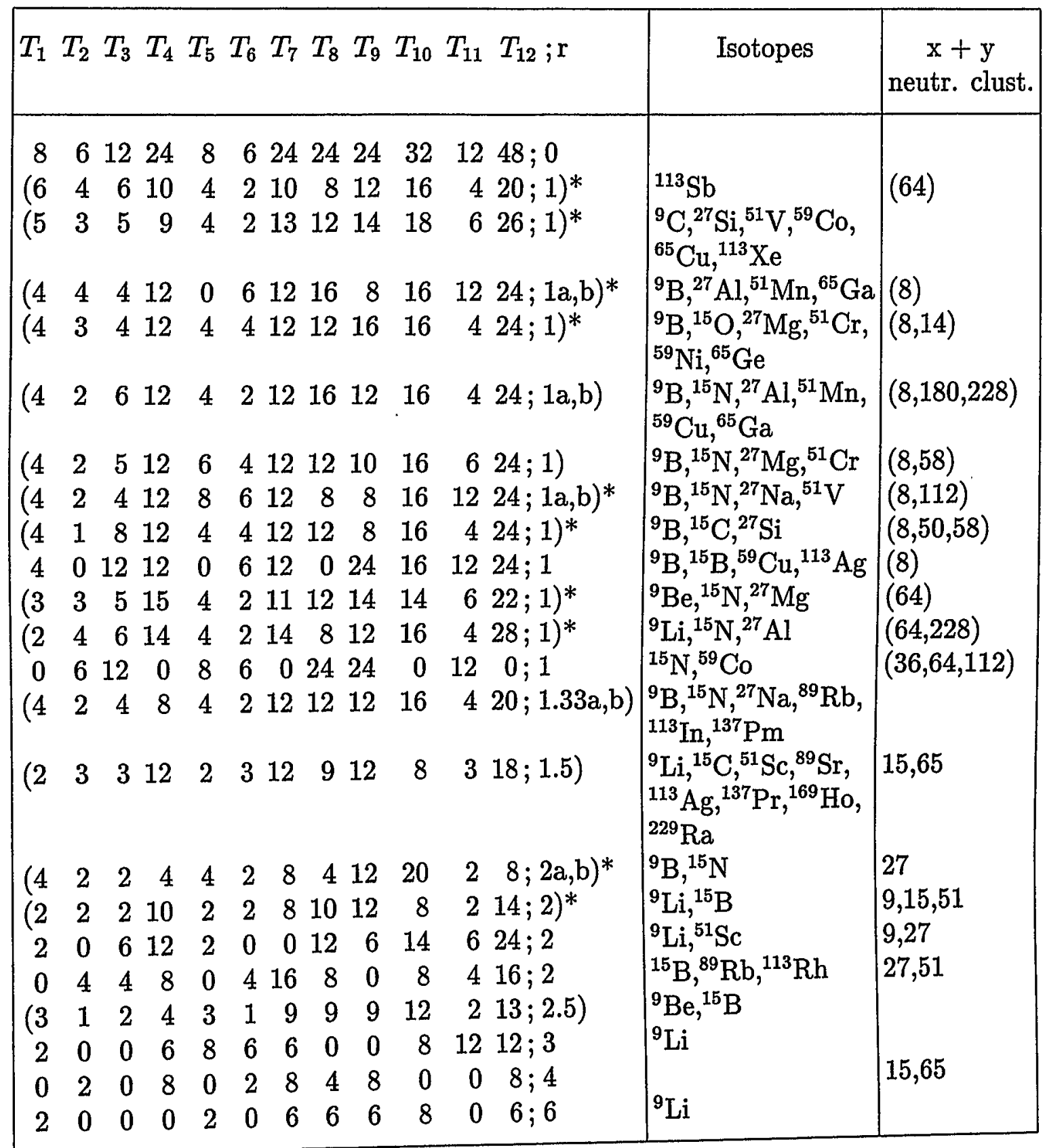


Table 23d: Same as Table 23a, except for pc.

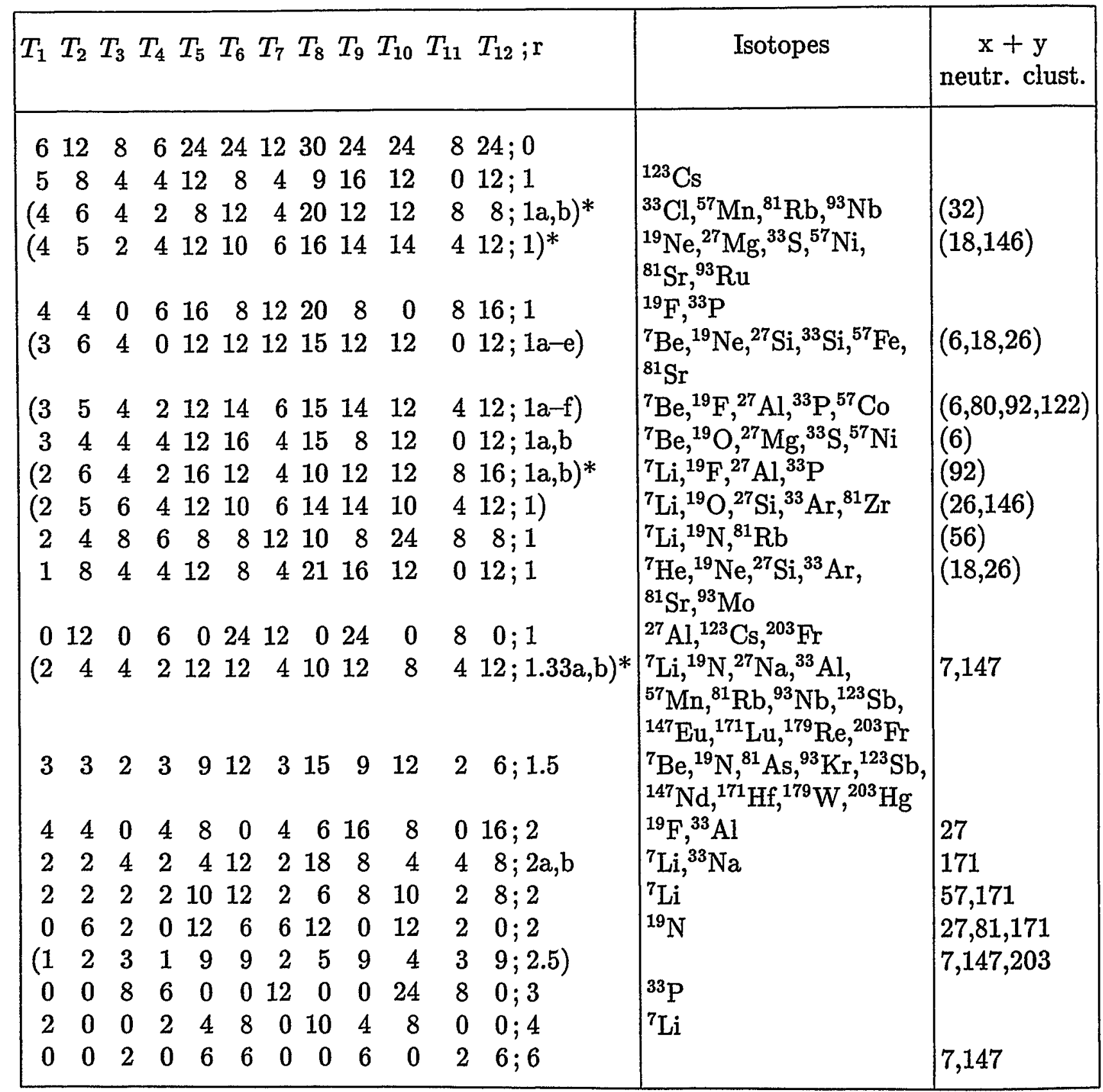


Table 24: Characterization of nucleic acids by the number of adenine (A), thymine or uracyl (T), cytosine (C) and guanine (G) units (first number), the clusters per mill (second number) and the location on the structure map (Fig.16).

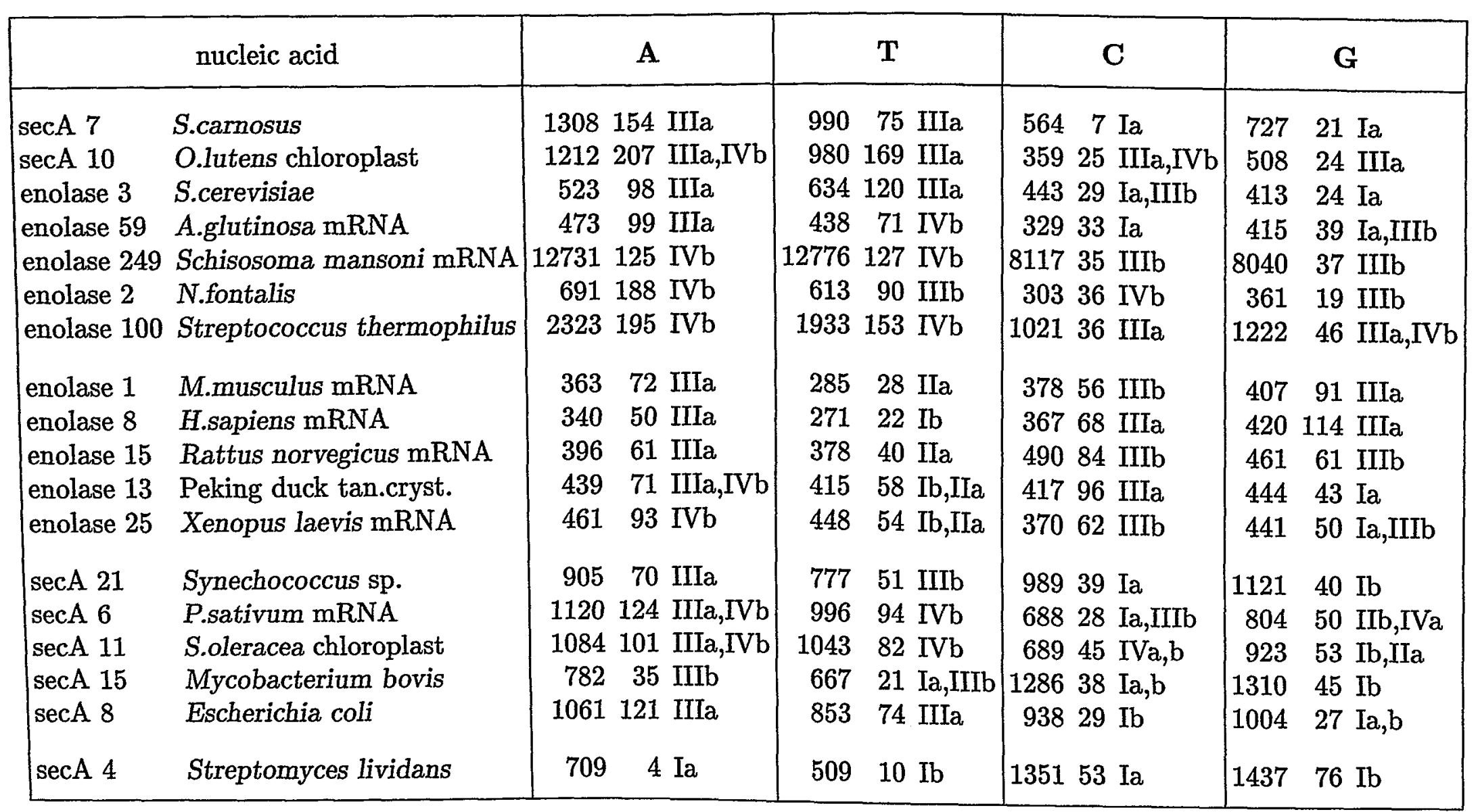


Table 25a: Lattices with highest density (lowest $r$ values) from different positions $a$ - $f$ of the cubic space groups (SG) $195-230$ without variable $x, y, z$ parameters and a selection of structure types $\mathrm{R}_{n} \mathrm{M}_{\mathrm{x}}$ or $\mathrm{R}_{n} \mathrm{M}_{\mathrm{x}} \mathrm{N}_{\mathrm{y}}$ with $T_{i}(\mathrm{M})$ of $\mathrm{M}$ atoms or $T_{i}(\mathrm{~N})$ of $\mathrm{N}$ atoms. The majority positions are occupied for $\underline{T_{i}} ; \mathrm{r}$ values.

\begin{tabular}{|c|c|c|c|c|c|c|c|}
\hline SG & $a$ & $b$ & $c$ & $d$ & $e$ & $f$ & further SG \\
\hline 195 & $\mathrm{pc}$ & $\mathrm{pc}$ & fcc 1 & fcc 1 & & & $200,207,215,219,221,226$ \\
\hline 196 & fcc & fcc & fcc & fcc & & & 216 \\
\hline 197 & bcc & pc 1 & & & & & \\
\hline 201 & bcc & fcc & fcc & pc 1 & & & \\
\hline 202 & fcc & fcc & $\mathrm{pc}$ & fcc 1 & & & 209,225 \\
\hline 203 & bcc 1 & bcc 1 & fcc 2 & fcc 2 & & & 210,227 \\
\hline 204 & bcc & pc 1 & $\mathrm{pc}$ & & & & \\
\hline 205 & fcc & fcc & & & & & \\
\hline 206 & $\mathrm{pc}$ & $\mathrm{pc}$ & & & & & \\
\hline 208 & bcc & fcc & fcc & pc 1 & fcc 5 & fcc 5 & \\
\hline 211 & bcc & pc 1 & $\mathrm{pc}$ & fcc 3 & & & 222,229 \\
\hline 212 & fcc 6 & fcc 6 & & & & & 213 \\
\hline 214 & fcc 4 & fcc 4 & fcc 8 & fcc 8 & & & \\
\hline 217 & bcc & pc 1 & - & fcc 3 & & & \\
\hline 218 & bcc & $\mathrm{pc} 1$ & fcc 5 & fcc 5 & & & \\
\hline 220 & fcc 9 & fcc 9 & & & & & \\
\hline 223 & bcc & pc 1 & fcc 5 & fcc 5 & $\mathrm{pc}$ & & \\
\hline 224 & bcc & fcc & fcc & pc 1 & - & fcc 3 & \\
\hline 228 & bcc & fcc & fcc & bcc & & & \\
\hline 230 & bcc & pc 2 & fcc $7 a$ & fcc $7 b$ & & & \\
\hline
\end{tabular}

pc (6 $128 ;(1)):$ Po $\alpha, \underline{\mathrm{ClCs}}, \mathrm{Cu}_{3} \mathrm{Au}, \mathrm{O}_{3} \mathrm{Re}, \mathrm{Fe}_{4} \mathrm{C}, \mathrm{O}_{3} \mathrm{CaTi}, \mathrm{B}_{6} \mathrm{Ca}, \mathrm{Cu}_{3} \mathrm{~S} 4 \mathrm{~V}, \mathrm{NaPt}_{3} \mathrm{O}_{4}$, $\mathrm{Pr}_{3} \mathrm{Sn}_{13} \mathrm{Rh}_{4}, \mathrm{PtHg}_{4}, \mathrm{Pt}_{3} \mathrm{O}_{4}, \mathrm{As}_{3} \mathrm{Co}, \mathrm{P}_{12} \mathrm{LaFe}_{4}, \mathrm{Re}_{7} \mathrm{Si}_{6} \mathrm{U}_{4}, \mathrm{CaF}_{2}, \mathrm{D}_{6} \mathrm{RuSr}_{2}, \mathrm{~S}_{4} \mathrm{Cu}_{3} \mathrm{Sb}$, $\mathrm{Zn}_{13} \mathrm{Na}, \mathrm{B}_{198} \mathrm{Th}_{3} \mathrm{O}, \mathrm{Cu}_{6.5} \mathrm{Al}_{6.5} \mathrm{Ca}, \mathrm{B}_{66} \mathrm{ThO}_{0.33}, \mathrm{PdGa}_{12} \mathrm{Y}_{4}$, (Si, Re) ${ }_{13} \mathrm{U}_{4}, \mathrm{Ce}_{3} \mathrm{Ni}_{6} \mathrm{Si}_{2}, \mathrm{~Tb}_{3}$ $\mathrm{Ni}_{6} \mathrm{H}_{0.5} \mathrm{Al}_{2}, \mathrm{NiD}_{4} \mathrm{Mg}_{2},\left(\mathrm{FeCu}_{5}\right) \mathrm{S}_{4}, \mathrm{Ni}_{2.67} \mathrm{Sn}_{5.44} \mathrm{Gd}, \mathrm{Pt}_{12} \mathrm{Sn}_{25} \mathrm{Ce}_{4}, \mathrm{LaSn}_{3} \mathrm{Ru}, \mathrm{Yb}_{3} \mathrm{Sn}_{13} \mathrm{Rh}_{4}$, $\mathrm{Y}_{3} \mathrm{Ge}_{13} \mathrm{Co}_{4}, \mathrm{AlMnCu}_{2}, \mathrm{MnGa}_{6} \mathrm{Gd}_{2}, \mathrm{Ni}_{3} \mathrm{Ga}_{4}, \mathrm{Ga}_{4} \mathrm{Ni}_{3}$

pc $1(\underline{0} 08$; 3$): \mathrm{Ag}_{2} \mathrm{O}_{3}, \mathrm{Bi}_{2} \mathrm{O}_{3}, \mathrm{La}_{2} \mathrm{O}_{3}, \mathrm{O}_{4} \mathrm{Pt}_{3}, \mathrm{~S}_{4} \mathrm{VTl}_{3}, \mathrm{Ge}_{4} \mathrm{Pt}_{4} \mathrm{Na}_{3}, \mathrm{P}_{2} \mathrm{Mg}_{3}, \mathrm{~B}_{14} \mathrm{Fe}_{62} \mathrm{Y}_{3}$ pc $2(032 ; 3)$

pc $3(2.72 .70 ; 2.55)(229 b+d): \mathrm{SAg}_{2}$

pc $4 \mathrm{a}(03.430 ; 3.57)(229 a+d): \mathrm{U}_{4} \mathrm{Si}_{6} \mathrm{Re}_{7}$

pc $4 \mathrm{~b}\left(\begin{array}{llll}0 & 0 & 0 & 1.5 ; 7\end{array}\right)(218 a+d):(\mathrm{Al}, \mathrm{Ge})_{23} \mathrm{Na}_{4}, \mathrm{As}_{4} \mathrm{Ba}_{4} \mathrm{Si}_{,} \mathrm{Li}_{7} \mathrm{~N}_{4} \mathrm{Mn}, \mathrm{H}_{3} \mathrm{U}, \mathrm{RuSn} \mathrm{La}_{3}$ $\mathrm{Al}_{8} \mathrm{Ga}_{15} \mathrm{Ba}_{4}, \mathrm{Ge}_{11} \mathrm{~K}, \mathrm{Si}_{23} \mathrm{~K}_{4}$ 
Table 25a (continued)

fcc (12 $624 ;(1)): \mathrm{OAg}_{2}, \mathrm{O}_{3} \mathrm{Ag}_{2}, \mathrm{O}_{3} \mathrm{Bi}_{2} \delta, \mathrm{S}_{2} \mathrm{Fe}, \mathrm{S}_{9} \mathrm{~Pb}_{4} \mathrm{Sb}_{2} \mathrm{Sn}, \mathrm{Cu}, \mathrm{SZn}, \underline{\mathrm{ClNa}}$, LiSiAl, $\mathrm{AsMgAg}, \mathrm{F}_{2} \mathrm{Ca}, \mathrm{Hg}_{2} \mathrm{TiCu}, \mathrm{F}_{3} \mathrm{Bi}, \mathrm{Be}_{5} \mathrm{Au}, \mathrm{Cu}_{3} \mathrm{Te}_{2}, \mathrm{Ca} 7 \mathrm{Ge}, \mathrm{C}_{2} \mathrm{La}, \mathrm{D}_{6} \mathrm{Sr}_{2} \mathrm{Ru}, \mathrm{D}_{3} \mathrm{Ce}, \mathrm{Cu}_{9} \mathrm{Se}_{5}$, $\mathrm{S}_{8} \mathrm{Mo}_{4} \mathrm{Ga}, \mathrm{B}_{12} \mathrm{U}, \mathrm{S}_{4} \mathrm{Zr}_{3}, \mathrm{~S}_{6} \mathrm{Cu}_{9} \mathrm{Bi}, \mathrm{Sb}_{4} \mathrm{Co}_{5} \mathrm{Mn}_{4}, \mathrm{~S}_{4} \mathrm{Cu}_{7}, \mathrm{Se}_{6} \mathrm{Ag}_{9} \mathrm{Ga}, \mathrm{Te}_{6} \mathrm{Ag}_{8} \mathrm{Ge}_{2} \mathrm{Mg}_{2} \mathrm{D}_{4} \mathrm{Ni}$, $\mathrm{CuFe}_{5} \mathrm{~S}_{4} \mathrm{HT}, \mathrm{Cl}_{14} \mathrm{Mo}_{6} \mathrm{Hg}, \mathrm{Rb}_{7} \mathrm{Ge}_{8} \mathrm{Na}, \mathrm{S}_{9}\left(\mathrm{~Pb}_{0.67} \mathrm{Sb}_{0.33}\right)_{6} \mathrm{Sn}, \mathrm{Li}_{2} \mathrm{AgSb}, \mathrm{LiMgPdSn}, \mathrm{Cu}_{4} \mathrm{Sn}$

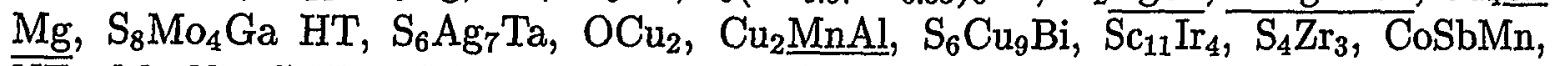
$\overline{N F}_{4}, \mathrm{Mo}_{3} \mathrm{~N}_{2}, \mathrm{C}_{3} \mathrm{Nb}_{4}, \mathrm{~N}_{2} \mathrm{Mo}_{3}, \mathrm{~S}_{3} \mathrm{U}_{4}, \mathrm{Pt}_{7} \mathrm{Cu} \cong \mathrm{Ca}_{7} \mathrm{Ge}, \mathrm{Cl}_{6} \mathrm{~K}_{2} \mathrm{Pt}, \mathrm{O}_{8} \mathrm{Mg}_{6} \mathrm{Mn}, \mathrm{Fe}_{7} \mathrm{C}_{0.45} \mathrm{Cr}$, $\mathrm{S}_{8}(\mathrm{Fe}, \mathrm{Ni})_{8} \mathrm{Ag}$

fcc $1(\underline{060} ; 3): \mathrm{AuCu}_{3}, \mathrm{ReO}_{3}, \mathrm{CaTiO}_{3} \widehat{=} \mathrm{AlCMn}_{3}, \mathrm{ONb}, \mathrm{U}_{4} \mathrm{~S}_{3}, \mathrm{Nb}_{4} \mathrm{C}_{3}, \mathrm{~S}_{4} \mathrm{VCu}_{3}$, $\mathrm{Hg}_{11} \mathrm{Ba}, \mathrm{S}_{4} \mathrm{SbCu}_{3}, \mathrm{Bi}_{4} \mathrm{Cu}_{4} \mathrm{Mn}_{3}, \mathrm{Rb}_{6} \mathrm{O}, \mathrm{Ag}_{4} \mathrm{Al}_{7} \mathrm{Ca}, \mathrm{S}_{26} \mathrm{ClK}_{6} \mathrm{Fe}_{23} \mathrm{Li}_{1}, \mathrm{Ca}_{4} \mathrm{Mg}_{4} \mathrm{H}_{22} \mathrm{Fe}_{3}, \mathrm{NCu}_{3}$, $\mathrm{Ga}_{3} \mathrm{TmRu}$

fcc 2 (6 $012 ; 1 \mathrm{~b}): \mathrm{MgCu}_{2}, \mathrm{Ti}_{2} \mathrm{C}, \mathrm{O}_{4} \mathrm{MgAl}_{2}, \mathrm{C}_{4} \mathrm{~N}_{4} \mathrm{ZnK}_{2}, \mathrm{Fe}_{3} \mathrm{~W}_{3} \mathrm{C}, \mathrm{D}_{7} \mathrm{Zr}_{2} \mathrm{Cr}_{4}, \mathrm{Ge}_{2} \mathrm{~S}_{5} \mathrm{Ba}$, $\mathrm{D}_{9} \mathrm{Zr}_{2} \mathrm{~V}_{4}, \mathrm{HCa}_{2} \mathrm{~N}, \mathrm{Al}_{10} \mathrm{~V}, \mathrm{Al}_{18} \mathrm{Mg}_{3} \mathrm{Cr}_{2}, \mathrm{~W}_{4} \mathrm{Co}_{2} \mathrm{C}, \mathrm{Ni}_{7} \mathrm{Zn}_{6} \mathrm{Ge}_{2}, \mathrm{H}_{3.7} \mathrm{ZrV}_{2} \mathrm{HT}, \mathrm{CeAl}_{20} \mathrm{Cr}_{2}$, $\mathrm{Al}_{11} \mathrm{~V}, \mathrm{Ca}_{2}(\mathrm{Ca}, \mathrm{Ge}) \cong \mathrm{Ti}_{2} \mathrm{C}, \mathrm{O}_{2} \underline{\mathrm{LiTi}}, \mathrm{S}_{4} \mathrm{CuLi}_{2} \mathrm{Ti}_{2}, \mathrm{H}_{7} \mathrm{ThZr}_{2}, \mathrm{Mn}_{3} \mathrm{Ni}_{2} \mathrm{Si}$

fcc $2 b(\underline{0} 0012 ; 7)(225 b+d): \mathrm{GeCa}_{7} \widehat{=} \mathrm{CuPt}_{7}, \mathrm{Zr}_{6} \mathrm{Cu}_{16} \mathrm{H}_{9} \mathrm{Al}_{7}, \mathrm{Mg}_{6} \mathrm{Cu}_{16} \mathrm{Si}_{7}$

fcc 3 (4 2 8; 1.67): $\mathrm{V}_{2} \mathrm{D} \alpha, \mathrm{As}_{7} \mathrm{~S}_{12} \mathrm{Hg}_{4}, \mathrm{P}_{17} \mathrm{La}_{6} \mathrm{Ni}_{6}, \mathrm{~S}_{12}(\mathrm{Cs}, \mathrm{Tl})_{2} \mathrm{As}_{4} \mathrm{Hg}_{5}, \mathrm{P}_{17} \mathrm{La}_{6} \mathrm{Ni}_{6}$

fcc $4(306 ; 3): \mathrm{Te}_{2} \mathrm{AuAg}_{3} \mathrm{LT}$

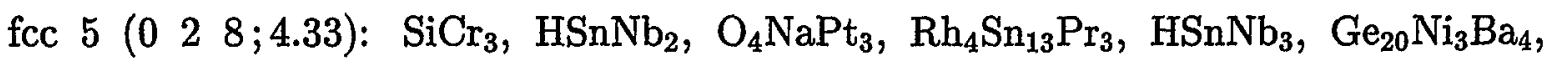
$\mathrm{Co}_{4} \mathrm{Ge}_{13} \mathrm{Y}_{3}$

fcc $6(006 ; 7): \mathrm{Si}_{2} \mathrm{Sr}, \mathrm{Al}_{2} \mathrm{Mo}_{3} \mathrm{C}, \mathrm{Te}_{10} \mathrm{Al}_{6} \mathrm{Ca}, \mathrm{O}_{23} \mathrm{P}_{8} \mathrm{Na}_{3} \mathrm{Fe}$

fcc $7 \mathrm{a}(004 ; 9.67 \mathrm{a}): \mathrm{Bi}_{4} \mathrm{Rh} \alpha, \mathrm{O}_{12} \mathrm{Al}_{2} \mathrm{Si}_{3} \mathrm{Ca}_{3}$

fcc $7 \mathrm{~b}(004 ; 9.67 \mathrm{~b}): \mathrm{O}_{12} \mathrm{Al}_{2} \mathrm{Ca}_{3} \mathrm{Si}_{3}$

fcc $8(004 ; 20.33)$

fcc $9\left(T_{7}=8 ; 20.33\right): \mathrm{P}_{4} \mathrm{Th}_{3}$, Ga II HP, $\mathrm{Sb}_{4} \underline{\mathrm{Au}}_{3} \underline{\mathrm{Y}}_{3}, \mathrm{Li}_{12} \mathrm{Si}_{4} \mathrm{Mg}_{3}, \mathrm{~S}_{8} \mathrm{Ba}_{3} \mathrm{Sn}_{2} \mathrm{Cd}$ bcc (8 $612 ;(1)): \mathrm{Ag}_{2} \mathrm{O}, \mathrm{Cr}_{3} \mathrm{Si}, \mathrm{HNb}_{2} \mathrm{Sn}, \mathrm{O}_{4} \mathrm{Pt}_{3} \mathrm{Na}, \mathrm{W}, \mathrm{O}_{3} \mathrm{La}_{2}, \mathrm{Hg}_{4} \mathrm{Pt}, \mathrm{DV}_{2} \alpha, \mathrm{S}_{4} \mathrm{Tl}_{3} \mathrm{~V}$, $\mathrm{Ag}_{2} \mathrm{~S}, \mathrm{Al}_{12} \mathrm{~W}, \mathrm{P}_{12} \mathrm{Fe}_{4} \mathrm{La}, \mathrm{Y}_{4} \mathrm{Ga}_{12} \mathrm{Pd}, \mathrm{Ni}_{3} \mathrm{Ga}_{3} \mathrm{Zn}, \mathrm{O}_{12} \mathrm{Ca}_{3} \mathrm{Si}_{3} \mathrm{Al}_{2}, \mathrm{Gd}_{4} \mathrm{Ni}_{12} \mathrm{Sn}_{24}(\mathrm{Gd}, \mathrm{Sn})$, $\mathrm{F}_{4} \mathrm{Si}, \mathrm{Hg}_{5} \mathrm{As}_{4} \mathrm{~S}_{12}, \mathrm{Nb}_{3} \mathrm{HSn}, \mathrm{O}_{4} \mathrm{Pt}_{3} \mathrm{Na}$

bcc $1(4012 ; 1): C, \mathrm{CuTiHg}_{2}, \mathrm{NaTl}, \mathrm{Cu}_{2} \mathrm{Mg}, \mathrm{O}_{4} \mathrm{Al}_{2} \mathrm{Mg}, \mathrm{S}_{4} \mathrm{Yb}_{2} \mathrm{Fe}, \mathrm{C}_{4} \mathrm{~K}_{2} \mathrm{~N}_{4} \mathrm{Zn}_{2} \mathrm{Mo}_{6} \mathrm{Ni}_{6} \mathrm{C}$, $\mathrm{Cr}_{4} \mathrm{D}_{7} \mathrm{Zr}_{2}, \mathrm{D}_{9} \mathrm{~V}_{4} \mathrm{Zr}_{2}, \mathrm{Al}_{20} \mathrm{Cr}_{2} \mathrm{Ce}, \mathrm{Zn}_{22} \mathrm{Zr}, \mathrm{O}_{2} \mathrm{Si}$ (cristoballite), $\mathrm{Na}_{8} \mathrm{Sb}_{4} \mathrm{Sn}, \mathrm{W}_{6} \mathrm{Fe}_{6} \mathrm{C}, \mathrm{N}_{2} \mathrm{Cs}$ $\underline{\mathrm{Ta}}, \mathrm{W}_{3} \mathrm{Fe}_{3} \mathrm{C}, \mathrm{V}_{2} \mathrm{H}_{3.7} \mathrm{Zr} \mathrm{HT}, \mathrm{S}_{4} \mathrm{Li}_{2} \mathrm{Ti}_{2} \mathrm{Cu}, \mathrm{H}_{7} \mathrm{Zr}_{2} \mathrm{Th}, \mathrm{Mo}_{2} \mathrm{~S}_{4} \mathrm{Al}_{0.55}, \mathrm{AgSbLi}_{2}, \mathrm{~S}_{8} \mathrm{Mo}_{4} \mathrm{Al}$ bcc $2(\underline{0} 012 ; 3)(225 b+c): \mathrm{BiF}_{3}, \mathrm{AlFe}_{3}, \mathrm{P}_{11} \mathrm{Cs}_{3} \mathrm{HT}, \mathrm{Co}_{20} \mathrm{~B}_{6} \mathrm{Al}_{3}, \mathrm{GePd}_{2} \mathrm{Mn}, \mathrm{C}_{60} \mathrm{~K}_{3}$ bcc $3 a(203 ; 3)(227 a+b+d): \mathrm{Ti}_{2} \mathrm{NiH}$ bcc 3b $(004 ; 4.33)(227 a+d): \mathrm{S}_{4} \mathrm{Co}_{3}, \mathrm{Li}_{13} \mathrm{In}_{3}, \mathrm{Si}_{17} \mathrm{Na}_{1.25}, \mathrm{Si}_{94} \mathrm{Na}, \mathrm{Pd}_{7} \mathrm{Te}_{2}$ bcc 3c $(2.704 ; 4.33)(227 a+c): \mathrm{S}_{4}(\mathrm{Cr}, \mathrm{Sn})_{2} \mathrm{Cu}_{3}, \mathrm{Nb}_{8} \mathrm{Zn}_{4} \mathrm{C}_{3}, \mathrm{Cr}_{2} \mathrm{Al}_{18} \mathrm{Mg}_{3}$ 
Table 25b: Same as Table 25a, except for trigonal and hexag. space groups $143-194$

\begin{tabular}{|l|lllllll|l|}
\hline SG & $a$ & $b$ & $c$ & $d$ & $e$ & $f$ & $g$ & further SG \\
\hline 147 & ph & ph & - & - & ph 2a & ph 2a & & 164 \\
148 & ph 1f & ph 1f & - & ph 3c & ph 3c & & & 166 \\
149 & ph & ph & ph & ph & ph & ph & & $174,187,188$ \\
150 & ph & ph & & & & & & \\
155 & ph 1f & ph 1f & & & & & & \\
162 & ph & ph & ph 1b & ph 1b & - & ph 2a & ph 2a & $175,177,191,192$ \\
163 & ph & ph & ph 1e & ph 1e & - & - & ph 2a & 176,194 \\
165 & ph & ph & - & - & ph 2a & & & \\
167 & ph 1f & ph 1f & - & ph 3c & & & & \\
180 & ph & ph & ph 2d & ph 2d & & & & 181 \\
182 & ph & ph & ph 1e & ph 1e & & & & 190 \\
189 & ph & ph & ph 1b & ph 1b & & & & \\
193 & ph & ph & ph 1b & ph 1b & - & ph 2a & & \\
\hline
\end{tabular}

ph (26 1226246 ; (1)) (147a): $\underline{\mathrm{LiRh}}, \underline{\mathrm{CW}}, \mathrm{Cd}_{2} \mathrm{Ce}, \mathrm{I}_{2} \mathrm{Cd}, \mathrm{Zn}_{2} \mathrm{Cu}, \underline{\mathrm{BaLiSi}}, \mathrm{B}_{2} \mathrm{Al}, \mathrm{Fe}_{2} \mathrm{~N}$, $\mathrm{S}_{2} \mathrm{CuSc}, \mathrm{O}_{3} \mathrm{U}, \mathrm{Li}_{3} \mathrm{~N}, \mathrm{AsNi}, \mathrm{Se}_{2} \mathrm{CuEr}, \mathrm{Be}_{2} \mathrm{H}_{2} \mathrm{Zr}_{2}, \mathrm{Zn}_{1.7} \mathrm{Pt}, \mathrm{S}_{2} \mathrm{Cu}_{3} \mathrm{Cs}, \mathrm{Cu}_{5} \mathrm{Ca}, \mathrm{SFe}, \mathrm{In}_{2} \mathrm{Ca}$, $\mathrm{S}_{2} \mathrm{Nb}, \mathrm{S}_{4} \mathrm{Ga}_{2} \mathrm{Fe}, \mathrm{C}_{6} \mathrm{Li}, \mathrm{Cu}_{7} \mathrm{~Tb}, \mathrm{P}_{2} \mathrm{NiMo}, \mathrm{B}_{3} \mathrm{Re}, \mathrm{S}_{2} \mathrm{TaSn}, \mathrm{AlCr}_{2} \mathrm{C}, \mathrm{O}_{6} \mathrm{As}_{2} \mathrm{Ca}, \mathrm{Zr}_{6} \mathrm{Al}_{2} \mathrm{Co}$, $\mathrm{D}_{3} \mathrm{Zr}_{4} \mathrm{~N}, \mathrm{Mn}_{12} \mathrm{Pt}_{4} \mathrm{~N}, \mathrm{BaS}_{3} \mathrm{~V}, \mathrm{Se}_{6} \mathrm{AlNb}_{3}, \mathrm{~S}_{6} \mathrm{Zn}_{3} \mathrm{In}_{2}, \mathrm{Ru}_{3} \mathrm{Si}_{2} \mathrm{La}, \mathrm{Al}_{9} \mathrm{Co}_{2} \mathrm{Sr}, \mathrm{BCo}_{4} \mathrm{Ce}, \mathrm{B}_{2} \mathrm{Pt}_{3} \mathrm{Ba}$, $\mathrm{Fe}_{6} \mathrm{Ge}_{6}, \mathrm{Mg}, \mathrm{Co}_{5} \mathrm{Y}, \mathrm{C}_{6} \mathrm{Eu}, \mathrm{Nb}_{6} \mathrm{Se}_{8} \mathrm{Tl}, \mathrm{S}_{12} \mathrm{Ti}_{9} \mathrm{~K}, \mathrm{Cu}_{13} \mathrm{Yb}_{2}, \mathrm{Se}_{8} \mathrm{Nb}_{4} \mathrm{Cr}, \mathrm{S}_{8} \mathrm{Nb}_{4} \mathrm{Mn}, \mathrm{Zn}_{11} \mathrm{Sm}$, $\mathrm{Fe}_{8} \mathrm{Ho}, \mathrm{Ni}_{19} \mathrm{Th}_{2}, \mathrm{Co}_{17} \mathrm{Ho}_{2}, \mathrm{O}_{27} \mathrm{Fe}_{18} \mathrm{Ba}, \mathrm{Se}_{2} \mathrm{Ta}, \mathrm{C}_{8} \mathrm{Cs}, \mathrm{Al}_{3} \mathrm{Fe}_{14} \mathrm{~Tb}_{2}, \mathrm{~B}_{2} \mathrm{Ru}_{3} \mathrm{U}, \mathrm{Si}_{3} \mathrm{Ce}_{5} \mathrm{Ni}_{2}$, $\mathrm{D}_{7} \mathrm{Ni}_{5} \mathrm{La}$

ph 1a $(\underline{0} 0626000 ; 2)(194 b+d): \mathrm{C}, \mathrm{BaCu}, \mathrm{Ni}_{17} \mathrm{Th}_{2}, \mathrm{Ti}_{3} \mathrm{O}, \mathrm{Se}_{24} \mathrm{Zr}_{12} \mathrm{Fe}_{5}, \mathrm{~B}_{4} \mathrm{~W}, \mathrm{H}_{9} \mathrm{~K}_{2} \mathrm{Re}$, $\mathrm{Zn}_{17} \mathrm{U}_{2}$

ph 1b $(\underline{20026120} ; 2)(162 c): \mathrm{AlB}_{2}, \mathrm{ZrH}_{2} \mathrm{Be}_{2}, \mathrm{NdRh}_{3} \mathrm{~B}_{2}, \mathrm{NaPd}_{3} \mathrm{Sn}_{2}, \mathrm{O}_{6} \mathrm{CaAs}_{2}$, $\mathrm{S}_{6} \mathrm{Na}_{35} \mathrm{Sn}_{2}, \mathrm{Zr}_{6} \mathrm{CoAl}_{2}, \mathrm{Al}_{9} \mathrm{SrCo}_{2}, \mathrm{CeCo}_{4} \mathrm{~B}, \mathrm{~S}_{3} \mathrm{PLi}$

ph 1c $\left(\begin{array}{lllllll}0 & 0 & 0 & 2 & 6 & 0 & 0\end{array} ; 5\right)(191 a+c+d): \mathrm{Zn}_{10} \mathrm{U}, \mathrm{W}_{12} \mathrm{C}_{5}$

ph 1d $(0282642 ; 1)(191 b+c): \mathrm{NLi}_{3}, \mathrm{NTa}, \mathrm{CoSn}, \mathrm{V}_{2} \mathrm{~N}, \mathrm{Fe}_{2} \mathrm{P}, \mathrm{Ga}_{5} \mathrm{Ni}_{5} \mathrm{Ho}_{2}, \mathrm{Fe}_{6} \mathrm{Ge}_{6} \mathrm{Li}$, La

ph 1e (0 $0626600 ; 2)$ (194c) (hcp): $\mathrm{Mg}, \mathrm{CuZn}_{2}, \mathrm{NFe}_{2}, \mathrm{ZnS}, \mathrm{NiAs}, \mathrm{Ni}_{2} \mathrm{Si}, \mathrm{Ni}_{7} \mathrm{Ge}_{4}$, $\mathrm{B}_{3} \mathrm{Re}, \mathrm{S}_{2} \mathrm{Mo}, \mathrm{Ni}_{2} \mathrm{In}, \mathrm{S}_{2} \mathrm{InTl}, \mathrm{Se}_{6} \mathrm{Nb}_{3} \mathrm{~K}_{2}, \mathrm{Ni}_{3} \mathrm{Sn}, \mathrm{S}_{2} \mathrm{SnTa}, \mathrm{CCr}_{2} \mathrm{Al}, \mathrm{Na}_{3} \mathrm{As}, \mathrm{Mn}_{12} \mathrm{NPt}_{4}$, $\mathrm{S}_{3} \mathrm{VBa}, \mathrm{Se}_{4} \mathrm{CuNb}_{2}, \mathrm{C}_{6} \mathrm{Ba}, \mathrm{Fe}_{3} \mathrm{Te}_{3} \mathrm{Tl}, \mathrm{Cu}_{2} \mathrm{~S}, \mathrm{~B}_{4} \mathrm{Ir}_{3} \mathrm{Zr}_{2} \mathrm{Fe}_{4} \mathrm{Si}_{9} \mathrm{Y}_{2}, \mathrm{~S}_{6} \mathrm{Nb}_{3} \mathrm{Co}, \mathrm{S}_{12} \mathrm{Ti}_{9} \mathrm{~K}$

ph 1f $(00606660$;2) (166a/195a, 196a, 197a) (pc, fcc, bcc): Hg, Po, OBi, HgIn, $\underline{\mathrm{PtCu}}, \mathrm{S}_{2} \mathrm{Mo}, \mathrm{N}_{2} \mathrm{~W}, \mathrm{~S}_{4} \mathrm{NaTi}_{2}, \mathrm{~N}_{3} \mathrm{Na}, \mathrm{S}_{2} \mathrm{NaV}, \mathrm{S}_{2} \mathrm{NaCr} \widehat{=} \mathrm{O}_{2} \mathrm{NaFe} \widehat{=} \mathrm{Cu}_{2} \mathrm{SrGa}, \mathrm{S}_{2} \overline{\mathrm{CuCr}}$, $\mathrm{Se}_{2} \mathrm{AgCr}, \mathrm{Ni}_{3} \mathrm{C}, \mathrm{S}_{8} \mathrm{Mo}_{8} \mathrm{~Pb}, \mathrm{~S}_{16} \mathrm{Mo}_{12} \mathrm{FeSn}_{2}, \mathrm{Rh}_{3} \mathrm{Si}_{7} \mathrm{Sc}, \mathrm{S}_{2} \mathrm{Ni}_{3} \mathrm{~Pb}_{2}, \mathrm{~B}_{6} \mathrm{Ni}_{12} \mathrm{Sr}, \mathrm{Mg}_{2} \mathrm{Ni}_{3} \mathrm{Si}$, $\mathrm{K}_{5} \mathrm{Sb}_{2} \mathrm{Cu}, \mathrm{Se}_{3} \mathrm{Ga}_{2} \mathrm{Na}_{2}, \mathrm{Te}_{4} \mathrm{Sb}_{2} \mathrm{Ge}, \mathrm{Au}_{5} \mathrm{Sn}, \mathrm{S}_{2} \mathrm{Ta}_{2} \mathrm{C}, \mathrm{Bi}_{2} \mathrm{Te}_{2} \mathrm{~S}, \mathrm{Cl}_{2} \mathrm{Cd}, \mathrm{F}_{2} \mathrm{HNa}, \mathrm{CuSe}_{1-x}$, $\mathrm{SSc}_{1-x}, \mathrm{Bi}_{2} \mathrm{Te}_{2} \mathrm{~S}, \mathrm{~S}_{2} \mathrm{Ta}_{2} \underline{\mathrm{CFe}}, \mathrm{P}_{2} \mathrm{~K}_{4} \mathrm{Cd}, \mathrm{P}_{2} \mathrm{Cu}_{4} \mathrm{Ca}, \mathrm{Se}_{4} \mathrm{Al}_{2} \mathrm{Mg}, \mathrm{Fe}_{6} \mathrm{Ge}_{4} \mathrm{Zr}, \mathrm{S}_{3} \mathrm{KPt}_{2}, \mathrm{Mn}_{3} \mathrm{Bi}$, $\mathrm{B}_{6} \mathrm{Ni}_{12} \mathrm{Sr}$ 
Table 25b (continued)

ph $1 \mathrm{~g}(0061630 ; 2)(194 a+c)$ : TiAs, $\mathrm{Ni}_{3} \mathrm{Ti}, \mathrm{SiNi}_{2}, \mathrm{InNi}_{2}, \mathrm{Be}_{3} \mathrm{~N}_{2}, \mathrm{Se}_{4} \mathrm{Nb}_{2} \mathrm{Cr}$ ph 1h $(0040640 ; 3)(225 b+c)$ (bcc 2):

ph 1i $(00300600 ; 5)(203 a)$ (bcc 1):

ph $2 \mathrm{a}(\underline{2002006} ; 3)(164 e): \mathrm{S}_{2} \mathrm{CsCu}_{3}, \mathrm{SnCo}, \mathrm{NaSn}_{2} \mathrm{Pd}_{3}, \mathrm{~S}_{8} \mathrm{P}_{2} \mathrm{Pd}_{3}, \mathrm{Si}_{16} \mathrm{U}_{20} \mathrm{C}_{3}$

ph $2 \mathrm{~b}(03623126 ; 1)(164 a+f): \mathrm{Se}_{6} \mathrm{Ti}_{3} \mathrm{Fe}, \mathrm{Ge}_{6} \mathrm{Ni}_{6} \mathrm{Sc}$

ph 2c $(12612126 ; 1)(180 b+d): \mathrm{Mg}_{2} \mathrm{Ni}$

ph 2d $(0040086 ; 3)(180 c)$ : $\mathrm{O}_{2} \mathrm{Si}$ (high quartz), $\mathrm{Si}_{2} \mathrm{Cr}, \mathrm{Si}_{2} \mathrm{Nb}$

ph 3a $(004.822 .401 .2 ; 1.4)(191 c+g): \mathrm{CaCu}_{5}, \mathrm{DLaNi}_{5}$

ph 3b $(005.105 .15 .10 ; 2.43)(225 b+d)($ fcc $2 b)$ :

ph 3c (0 $040440 ; 3)(166 e / 197 b, 195 c)$ (pc 1, fcc 1): $\mathrm{S}_{2} \mathrm{~Pb}_{2} \mathrm{Ni}_{3}, \mathrm{P}_{2} \mathrm{~K}_{3} \mathrm{Cu}_{3}, \mathrm{Se}_{8} \mathrm{Rh}_{3}$, $\mathrm{SiMg}_{2} \mathrm{Ni}_{3}, \mathrm{~B}_{2} \mathrm{ThC}$

ph 3d $(002.404 .84 .80 ; 3.8)(166 a+b+d): \mathrm{Ti}_{8} \mathrm{C}_{5}$

ph 3e $(0030330 ; 5)(203 c, 166 b+e)$ (fcc 2): $\mathrm{TbFe}_{2}$

ph $3 f(0031.5300 ; 5)(194 c+g): \mathrm{SiO}_{2}, \mathrm{~W}_{20} \mathrm{Co}_{6} \mathrm{C}_{7}$

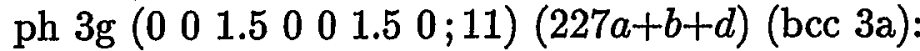

ph $3 \mathrm{~h}(00000200 ; 15)(227 a+d)(\mathrm{bcc} 3 \mathrm{~b}): \mathrm{S}_{2} \mathrm{Ni}_{3} \mathrm{~Pb}_{2}$

ph 3i $(002020200 ; 15)(227 a+c)(b c c 3 c)$ :

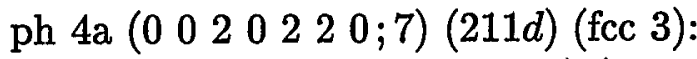

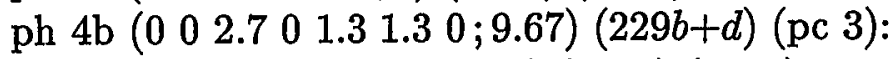

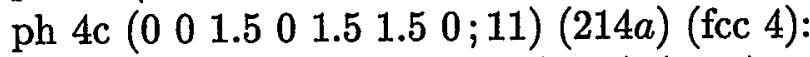

ph 4d $\left(\begin{array}{lllllll}0 & 0 & 0 & 0 & 1.5 & 1.5 & 0\end{array} ; 11\right)(230 b)(\mathrm{pc} 2)$ :

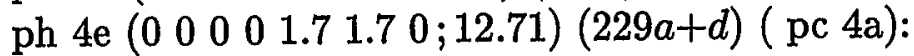

ph $4 \mathrm{f}\left(\begin{array}{lllllllll}0 & 0 & 0 & 0 & 0 & 2 & 0 & ; & 15\end{array}\right)(218 c)(\mathrm{fcc} 5)$ :

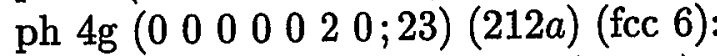

ph $4 \mathrm{~h}\left(T_{11}=1.5 ; 23\right)(218 a+d)($ pc $4 b)$ :

ph $5 \mathrm{a}(000000010 ; 31 \mathrm{a})(230 c)(\mathrm{fcc} 7 \mathrm{a})$ :

ph $5 \mathrm{~b}\left(\begin{array}{lllllll}0 & 0 & 0 & 0 & 0 & 1 & 0 ; 31 \mathrm{~b}) \\ (230 d)(\mathrm{fcc} 7 \mathrm{~b}) \text { : }\end{array}\right.$

ph $5 \mathrm{c}\left(\begin{array}{lllllll}0 & 0 & 0 & 0 & 0 & 1 & 0 ; 63)(214 c)(\mathrm{fcc} 8) \text { : }\end{array}\right.$

ph $5 \mathrm{~d}\left(T_{14}=2 ; 63\right)(220 a)(\mathrm{fcc} 9)$ : 
Table 25c: Same as Table 25a, except for tetragonal space groups 75 - 142

\begin{tabular}{|c|c|c|c|c|c|c|c|}
\hline SG & $a$ & $b$ & $c$ & $d$ & $e$ & $f$ & further SG \\
\hline 81 & $\mathrm{pc}^{\prime}$ & $\mathrm{pc}^{\prime}$ & $\mathrm{pc}^{\prime}$ & $\mathrm{pc}$ & & & $115,116,120,127,135$ \\
\hline 82 & $\mathrm{bcc}^{\prime}$ & $\mathrm{bcc}^{\prime}$ & $\mathrm{bcc}^{\prime}$ & $\mathrm{bcc}^{\prime}$ & & & $86,118,119$ \\
\hline 83 & $\mathrm{pc}^{\prime}$ & $\mathrm{pc}^{\prime}$ & $\mathrm{pc}^{\prime}$ & $\mathrm{pc}^{\prime}$ & $\mathrm{pc} c^{\prime}$ & $\mathrm{pc}^{\prime}$ & $89,111,123,124,125,132$ \\
\hline 84 & $\mathrm{pc}^{\prime}$ & $\mathrm{pc}^{\prime}$ & $\mathrm{bcc}^{\prime}$ & $\mathrm{bcc}^{\prime}$ & $\mathrm{pc}^{\prime}$ & $\mathrm{pc}^{\prime}$ & 93,131 \\
\hline 85 & $\mathrm{pc}^{\prime}$ & $\mathrm{pc}^{\prime}$ & - & $\mathrm{pc}^{\prime}$ & $\mathrm{pc}^{\prime}$ & & 129 \\
\hline 87 & $\mathrm{bcc}^{\prime}$ & $\mathrm{bcc}^{\prime}$ & $\mathrm{pc}^{\prime}$ & $\mathrm{pc}^{\prime}$ & - & $\mathrm{pc}^{\prime}$ & 126,139 \\
\hline 88 & bcc 1 & bcc 1 & fcc 2 & fcc 2 & & & 141 \\
\hline 90 & $\mathrm{pc}^{\prime}$ & $\mathrm{pc}^{\prime}$ & & & & & 113 \\
\hline 94 & $\mathrm{bcc}^{\prime}$ & bcc $^{\prime}$ & & & & & 114 \\
\hline 97 & $\mathrm{bcc}^{\prime}$ & $\mathrm{bcc}^{\prime}$ & $\mathrm{pc}^{\prime}$ & $\mathrm{pc} c^{\prime}$ & & & $117,121,128,136$ \\
\hline 98 & bc̣c 1 & bcc 1 & & & & & 122 \\
\hline 112 & $\mathrm{pc}^{\prime}$ & $\mathrm{bcc}^{\prime}$ & $\mathrm{pc}^{\prime}$ & $\mathrm{bcc}^{\prime}$ & $\mathrm{pc} c^{\prime}$ & $\mathrm{bcc}^{\prime}$ & \\
\hline 130 & $\mathrm{pc}^{\prime}$ & $\mathrm{pc}^{\prime}$ & - & $\mathrm{pc}^{\prime}$ & & & \\
\hline 133 & $\mathrm{pc}^{\prime}$ & $\mathrm{pc} c^{\prime}$ & $\mathrm{pc}^{\prime}$ & $\mathrm{pc}^{\prime}$ & $\mathrm{pc}^{\prime}$ & & 140 \\
\hline 134 & $\mathrm{bcc}^{\prime}$ & $\mathrm{bcc}^{\prime}$ & $\mathrm{pc}^{\prime}$ & $\mathrm{pc}^{\prime}$ & $\mathrm{bcc}^{\prime}$ & $\mathrm{bcc}^{\prime}$ & \\
\hline 137 & $\mathrm{bcc}^{\prime}$ & $\mathrm{bcc}^{\prime}$ & - & - & $\mathrm{pc}^{\prime}$ & & \\
\hline 138 & $\mathrm{pc}^{\prime}$ & $\mathrm{pc}^{\prime}$ & bcc $^{\prime}$ & $\mathrm{bcc}^{\prime}$ & & & \\
\hline 142 & $\mathrm{bcc}^{\prime}$ & $\mathrm{bcc}^{\prime}$ & $\mathrm{bcc}^{\prime}$ & & & & \\
\hline
\end{tabular}

$\mathrm{pc}^{\prime}(4248420 ;(1)): \mathrm{S}_{6} \mathrm{P}_{2} \mathrm{Zr}, \mathrm{Te}_{3} \mathrm{Ga}, \mathrm{S}_{4} \mathrm{BaFe}_{2} \beta, \mathrm{Se}_{4} \mathrm{In}_{2} \mathrm{Cd} \alpha, \mathrm{Se}_{8} \mathrm{In}_{5} \mathrm{Ag} \mathrm{LT}, \mathrm{Cl}_{4} \mathrm{AlCu}$, $\mathrm{Se}_{2} \mathrm{FeCu}, \mathrm{ClO}_{2}\left(\mathrm{NH}_{4}\right), \mathrm{S}_{3} \mathrm{Ba}, \mathrm{Cr}_{3} \mathrm{GeN}, \mathrm{O}_{7} \mathrm{Si}_{2} \mathrm{Ca}_{2} \mathrm{Mg}, \mathrm{S}_{8} \mathrm{Cu}_{6} \mathrm{SnFe}_{2}, \mathrm{~B}_{25} \mathrm{AlCu}_{0.79}, \mathrm{Sn}_{2} \mathrm{Au}_{4} \mathrm{~K}$, $\mathrm{I}_{4} \mathrm{HgCu}_{2} \beta, \mathrm{S}_{4} \mathrm{SnFeCu}_{2}, \underline{\mathrm{TiCu}} \delta, \underline{\mathrm{CuAu}}, \mathrm{Hg}_{2} \mathrm{Pt}, \mathrm{Si}_{2} \mathrm{Fe} \alpha, \underline{\mathrm{NFeNi}}, \mathrm{Pb}_{3} \mathrm{Sr}, \mathrm{Ti}_{3} \mathrm{Cu}, \mathrm{Pt}_{2} \underline{\mathrm{ZnCd}}$, $\mathrm{Si}_{2} \mathrm{Mg}_{2} \mathrm{Ce}, \mathrm{Mn}_{2} \mathrm{Co}_{2} \mathrm{C}, \mathrm{Cl}_{4} \mathrm{~K}_{2} \mathrm{Pt}, \mathrm{Ga}_{5} \underline{\mathrm{CoHo}}, \mathrm{Pd}_{5} \underline{\mathrm{AsTl}}, \mathrm{Ga}_{6} \underline{\mathrm{CuCe}}, \mathrm{S}_{3} \mathrm{Cu}_{4} \mathrm{~K}, \mathrm{H}_{5} \mathrm{Sr}_{2} \underline{\mathrm{LiPd}}$, $\mathrm{Ga}_{8} \mathrm{Ho}_{2} \mathrm{Co}, \overline{\mathrm{Ga}_{6} \mathrm{LaNi}}, \mathrm{Te}_{4} \mathrm{Nb} \mathrm{\textrm {HT }}, \mathrm{Nb}_{4} \underline{\mathrm{SiCo}}, \mathrm{Pb}_{4} \mathrm{Pt}, \mathrm{Se}_{4} \mathrm{KCe}, \mathrm{Ga}_{6} \mathrm{Pu}, \mathrm{Te}_{4} \mathrm{Al}_{2} \mathrm{Ba}, \mathrm{S}_{4} \mathrm{SrFe}_{2}$, $\mathrm{B}_{2} \mathrm{Mo}_{2} \mathrm{Fe}, \mathrm{F}_{5} \mathrm{Rb}_{3} \mathrm{Pd}, \mathrm{Mg}_{2} \mathrm{Ir}_{5} \mathrm{~B}_{2} \mathrm{Si}, \mathrm{Ni}_{9} \mathrm{In}_{2} \mathrm{Y}, \mathrm{Mn}_{6} \mathrm{Ni}_{5} \mathrm{Ce}, \mathrm{I}_{6} \mathrm{TeRb}_{2} \mathrm{LT}, \mathrm{PbO}, \mathrm{PbCl} \overline{\mathrm{F}}, \mathrm{Al}-$ GeMn, PLiMn, $\mathrm{C}_{2} \mathrm{ScCo}, \mathrm{Si}_{2} \mathrm{HfCu}$, AsZrSiCu, PtS, CYYCo, $\mathrm{C}_{2} \mathrm{~B}_{2} \mathrm{Y}, \mathrm{Te}_{2} \mathrm{Cu}_{3} \mathrm{Tl}, \mathrm{VSnRh}_{2}$, $\mathrm{Se}_{2} \mathrm{TlCu}_{2}$, TeUGe, $\mathrm{H}_{4} \mathrm{PtNa}_{2} \mathrm{LT}, \mathrm{Al}_{4} \mathrm{YbMo}_{2}, \mathrm{Bi}_{2} \mathrm{SrZn}, \mathrm{H}_{5} \mathrm{IrSr}_{2} \mathrm{LT}, \mathrm{S} 2 \mathrm{Sr}, \mathrm{Al}_{2} \mathrm{Cu} \theta$, $\mathrm{Ir}_{3} \mathrm{Si}, \mathrm{U}_{3} \mathrm{Si}$, SeTl, TeInNa, $\mathrm{Cu}_{2} \mathrm{CeIn}_{2}, \mathrm{D}_{5} \mathrm{CoMg}_{2}, \mathrm{~N}_{2} \mathrm{CoLiSr}_{2}, \mathrm{D}_{4} \mathrm{PtK}{ }_{2}, \mathrm{D}_{3} \mathrm{~K}_{3} \mathrm{Pd}, \mathrm{ThH}_{2}$, $\mathrm{Ga}_{2} \mathrm{CeAl}_{2}, \mathrm{Te}_{4} \mathrm{Al}_{2} \mathrm{Sr}, \mathrm{N}_{3} \mathrm{~K}, \mathrm{Se}_{2} \mathrm{InGa}, \mathrm{NCr}_{3} \mathrm{As}, \mathrm{Ga}_{5} \mathrm{Pd}, \mathrm{U}_{6} \mathrm{Mn}, \mathrm{H}_{4} \mathrm{Th}_{2} \mathrm{Al}, \mathrm{Nb}_{5} \mathrm{Sn}_{2} \mathrm{Si}$, $\mathrm{La}_{2} \mathrm{NiGe}, \mathrm{Mo}_{5} \mathrm{~B}_{2} \mathrm{Si}, \mathrm{Te}_{3} \mathrm{Tl}_{4} \mathrm{~Pb}, \mathrm{Te}_{5} \mathrm{Tl}_{2} \mathrm{Sn}, \mathrm{C}_{15} \mathrm{Cs}_{3} \mathrm{Co}, \mathrm{Ge}_{4} \mathrm{Co}_{9} \mathrm{Pr}, \mathrm{Si}{ }_{4}(\mathrm{Ni}, \mathrm{Si}) \mathrm{Ni}_{8} \mathrm{Ce}, \mathrm{Nb}_{9}$ $\mathrm{Co}_{4} \mathrm{Ge}, \mathrm{H}_{3} \mathrm{Zr}_{2} \mathrm{Ni}, \mathrm{Pr}_{6} \mathrm{Fe}_{13} \mathrm{Ge}, \mathrm{La}_{6} \mathrm{Co}_{9}(\mathrm{Co}, \mathrm{Ga})_{4} \mathrm{Ga}, \mathrm{Tb}_{6} \mathrm{Al}_{3} \mathrm{Si}, \mathrm{H}_{4.8} \mathrm{Zr}_{2} \mathrm{Ni} \mathrm{HT}, \mathrm{S}_{4} \mathrm{PPr}$ pc $1(\underline{0} 08$; 3$)(126 a+c): \mathrm{Se}_{4} \mathrm{Al}_{2} \mathrm{Ba}$ fcc $1(\underline{060} ; 3 \widehat{=}$ bcc $\underline{02} 4 ; 3)(123 c+e): \mathrm{SrPb}_{3}, \mathrm{CuTi}_{3}, \mathrm{Mn}_{3} \mathrm{Au}$

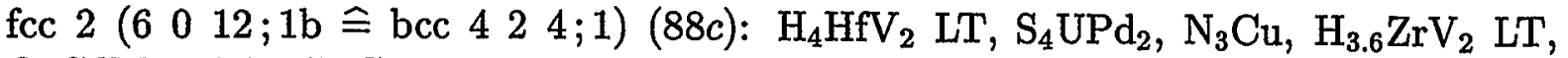
$\mathrm{O}_{4} \mathrm{CdMn}_{2}, \mathrm{Mn}_{11} \mathrm{LaC}_{2}$ 
Table 25c (continued)

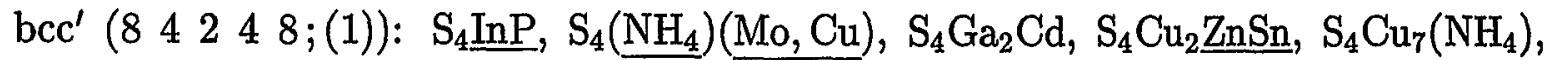
$\mathrm{C}_{4} \mathrm{~W}_{4} \mathrm{U}, \mathrm{S}_{4}(\mathrm{Pt}, \mathrm{Ni})_{3} \mathrm{Pd}, \mathrm{B}_{4} \mathrm{Co}_{4} \mathrm{Nd}, \mathrm{Ni}_{4} \mathrm{Mo}, \mathrm{S}_{4} \mathrm{Fe}_{2} \mathrm{Ba} \beta, \mathrm{H}_{4} \mathrm{Li}_{4} \mathrm{Rh}_{2} \mathrm{C}_{4} \mathrm{Cr}_{4} \mathrm{U}, \mathrm{Br}_{6} \mathrm{Rb}_{2} \mathrm{Te}$ LT, $\mathrm{Br}_{6} \mathrm{TeRb}_{2} \mathrm{LT}, \mathrm{P}_{4} \mathrm{Cu}_{8} \mathrm{Ba}, \mathrm{Cu}_{2} \mathrm{~S}$, GeP HP, $\mathrm{B}_{25} \mathrm{~N}, \mathrm{Cl}_{4} \mathrm{CuAl}, \mathrm{Pd}_{4} \mathrm{Se}, \mathrm{S}_{4} \mathrm{Na}_{4} \mathrm{Sn}, \mathrm{H}_{1.33} \mathrm{Pd}$, (Ga, $\mathrm{Ag}) \mathrm{Te}, \mathrm{Te}_{2} \mathrm{AgTl}, \mathrm{I}_{4} \mathrm{Cu}_{2} \mathrm{Hg} \beta, \mathrm{S}_{4} \mathrm{Cu}_{2} \mathrm{SnFe}, \mathrm{S}_{4} \mathrm{Cu}_{3} \mathrm{Sb}, \mathrm{Sn}_{6} \mathrm{Ru}_{4} \mathrm{Y}, \mathrm{I}_{6} \mathrm{Rb}_{2} \mathrm{Te} \mathrm{LT}, \mathrm{B}_{13} \mathrm{Er}_{4}$ $\mathrm{Ni}, \mathrm{S}_{11}\left(\mathrm{H}_{2} \mathrm{O}\right)_{2}\left(\mathrm{NH}_{4}\right)_{2} \mathrm{Pd}, \mathrm{Al}_{4} \mathrm{Ba}, \mathrm{Ga}_{2} \mathrm{Al}_{2} \mathrm{Ce}, \mathrm{Se}_{2} \mathrm{Cu}_{2} \mathrm{Tl}, \mathrm{F}_{4} \mathrm{~K}_{2} \mathrm{Ni}, \mathrm{H}_{4} \mathrm{Na}_{2} \mathrm{Pt} \mathrm{LT}, \mathrm{Al}_{4} \mathrm{Mo}_{2} \mathrm{Yb}$, $(\mathrm{Mn}, \mathrm{Cu})_{6} \mathrm{Ce}, \mathrm{Ni}_{2} \mathrm{Al}_{5} \mathrm{Zr}, \mathrm{Pt}_{8} \mathrm{Ti}, \mathrm{H}_{5} \mathrm{Sr}_{2} \mathrm{Ir} \mathrm{LT}, \mathrm{Fe}_{8} \mathrm{~N}, \mathrm{SPt}, \mathrm{C}_{2} \mathrm{Th} \mathrm{HT}, \mathrm{YCoC}, \mathrm{B}_{24} \mathrm{TiC}$, $\mathrm{B}_{25} \mathrm{Ti}, \mathrm{O}_{2} \mathrm{Ti}$ (rutile), $\mathrm{Li}_{3} \mathrm{~N}_{2} \mathrm{~B} \alpha, \mathrm{N}_{2} \mathrm{Sr}_{2} \mathrm{LiCo}, \mathrm{Si}_{2} \mathrm{Fe}_{4} \mathrm{Zr}, \mathrm{D}_{4} \mathrm{~K}_{2} \mathrm{Pt}$ LT, $\mathrm{V}_{8} \mathrm{~N}, \mathrm{I}_{2} \mathrm{Hg} \beta$, $\mathrm{B}_{4} \mathrm{Co}_{4} \mathrm{Ce}, \mathrm{N}_{4} \mathrm{Li}_{6} \mathrm{Mo}$, In, Pa LT, Mn $\gamma, \mathrm{C}_{0.05} \mathrm{Fe}, \underline{\mathrm{OCo}}, \mathrm{H}_{2} \mathrm{Th}, \mathrm{C}_{2} \mathrm{Ca}, \mathrm{Si}_{2} \mathrm{Mo}, \mathrm{Zr}_{2} \mathrm{Cu}$, $\mathrm{Rh}_{2} \underline{\mathrm{SnV}}, \mathrm{Al}_{3} \mathrm{Ti}, \mathrm{Al}_{2} \underline{\mathrm{UCu}}, \mathrm{O}_{2} \mathrm{Na}_{2} \mathrm{Hg}, \mathrm{S}_{4} \mathrm{Cu}_{6} \mathrm{Tl}, \mathrm{Ga}_{10} \mathrm{Ce}_{2} \mathrm{Ni}, \mathrm{Al}_{8} \mathrm{Mn}_{4} \mathrm{Ca}, \mathrm{Fe}_{10} \mathrm{Mo}_{2} \mathrm{Nd}$, $\mathrm{Mn}_{12} \mathrm{Th}, \mathrm{P}_{4} \mathrm{Eu}_{2} \mathrm{Pt}_{7} \mathrm{Al}, \mathrm{S}_{4}(\mathrm{Cu}, \mathrm{Fe}, \mathrm{Ag})_{6.35}(\mathrm{Tl}, \mathrm{K})_{2} \mathrm{Sb}, \mathrm{Fe}_{11} \mathrm{TiN}_{0.5} \mathrm{Y}, \mathrm{Ga}_{17} \mathrm{Ce}_{4} \mathrm{Ni}_{2}, \mathrm{~S}_{16} \mathrm{Ni}_{18}$ $\mathrm{Bi}_{3} \mathrm{As}, \mathrm{B}_{4} \mathrm{Ru}_{4} \mathrm{Lu}, \mathrm{Sb}_{11} \mathrm{Ca}_{14} \mathrm{Al}$

bcc $1(4012 ; 1)$ : $\mathrm{Cl}_{4} \mathrm{Th} \beta, \mathrm{O}_{4} \mathrm{SiZr}, \mathrm{Ca}_{4} \mathrm{In}_{2} \mathrm{~N}, \mathrm{Li}_{4} \mathrm{~N}_{2} \mathrm{Sr}, \mathrm{O}_{4} \mathrm{Mn}_{2} \mathrm{Cd}, \mathrm{Cd}_{11} \mathrm{Ba}, \mathrm{Si}_{2.4} \mathrm{Ni}_{8.6} \mathrm{Ce}$, $\mathrm{Cl}_{2} \mathrm{Zn} \alpha, \mathrm{N}_{2} \mathrm{CaGe}, \mathrm{S}_{2} \underline{\mathrm{CuFe}}, \mathrm{Se}_{4}(\underline{\mathrm{Sn}}, \mathrm{Ga}) \underline{\mathrm{Ag}}, \mathrm{Sn} \beta, \underline{\mathrm{UPb}}, \mathrm{Si}_{2} \mathrm{Sr}, \mathrm{Si}_{2} \mathrm{Th} \alpha, \mathrm{Mo}_{2} \mathrm{~N} \beta, \mathrm{O}_{2} \mathrm{Ti}$, anatase $\mathrm{Ti}_{2} \mathrm{~N}, \mathrm{C}_{0.5} \mathrm{SiFe}_{10} \mathrm{Nd}, \mathrm{Mn}_{11} \mathrm{C}_{2} \mathrm{La}$

bcc $2(\underline{0012} ; 3 \widehat{=}$ fcc $\underline{048} ; 3)(139 b+d): \mathrm{N}_{2} \mathrm{BLi}_{3} \alpha, \mathrm{TiAl}_{3}, \mathrm{NbN} \gamma$

bcc $3 \mathrm{~b}(004 ; 4.33 \cong$ fcc $01.338 ; 4.33)(88 b+c): \mathrm{SrPb}_{3}, \mathrm{CuTi}_{3}, \mathrm{Mn}_{3} \mathrm{Au}$ 
Forschungszentrum Jülich

Jül-3732

January 2000

ISSN 0944-2952 